SAND94-2316 - UC -610

Unlimited Release

Printed December 1994

\title{
MELCOR 1.8.3 Assessment: CSE Containment Spray Experiments
}

\author{
L. N. Kmetyk
}

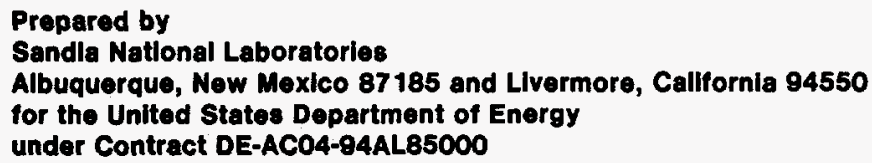

Approved for public release; distribution is unlimited. ,

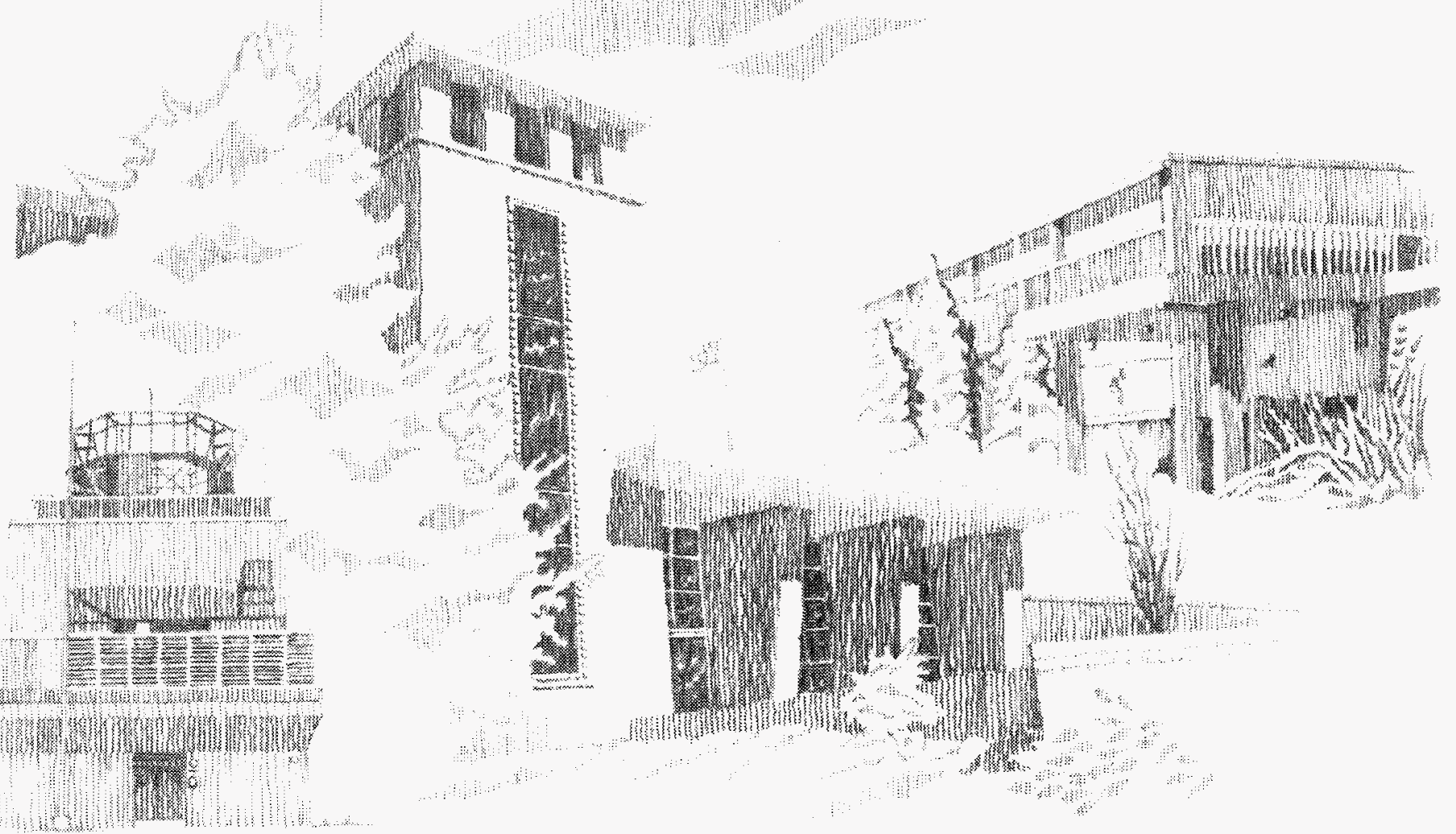


Issued by Sandia National Laboratories, operated for the United States Department of Energy by Sandia Corporation.

NOTICE: This report was prepared as an account of work sponsored by an agency of the United States Government. Neither the United States Government nor any agency thereof, nor any of their employees, nor any of their contractors, subcontractors, or their employees, makes any warranty, express or implied, or assumes any legal liability or responsibility for the accuracy, completeness, or usefulness of any information, apparatus, product, or process disclosed, or represents that its use would not infringe privately owned rights. Reference herein to any specific commercial product, process, or service by trade name, trademark, manufacturer, or otherwise, does not necessarily constitute or imply its endorsement, recommendation, or favoring by the United States Government, any agency thereof or any of their contractors or subcontractors. The views and opinions expressed herein do not necessarily state or reflect those of the United States Government, any agency thereof or any of their contractors.

Printed in the United States of America. This report has been reproduced directly from the best available copy.

Available to DOE and DOE contractors from

Office of Scientific and Technical Information

PO Box 62

Oak Ridge, TN 37831

Prices available from (615) 576-8401, FTS 626-8401

Available to the public from

National Technical Information Service

US Department of Commerce

5285 Port Royal RD

Springfield, VA 22161

NTIS price codes

Printed copy: A13

Microfiche copy: A06 


\section{DISCLAIMER}

Portions of this document may be illegible in electronic image products. Images are produced from the best available original document. 
Distribution

Category UC-610

SAND94-2316

Unlimited Release

Printed December 1994

\title{
MELCOR 1.8.3 Assessment: CSE Containment Spray Experiments
}

\author{
L. N. Kmetyk \\ Thermal/Hydraulic Analysis Department \\ Sandia National Laboratories \\ Albuquerque, NM 87185
}

\begin{abstract}
MELCOR is a fully integrated, engineering-level computer code, being developed at Sandia National Laboratories for the USNRC, that models the entire spectrum of severe accident phenomena in a unified framework for both BWRs and PWRs. As part of an ongoing assessment program, the MELCOR computer code has been used to analyze a series of containment spray tests performed in the Containment Systems Experiment (CSE) vessel to evaluate the performance of aqueous sprays as a means of decontaminating containment atmospheres. Basecase MELCOR results are compared with test data, and a number of sensitivity studies on input modelling parameters and options in both the spray package and the associated aerosol washout and atmosphere decontamination by sprays modelled in the radionuclide package have been done. Time-step and machinedependency calculations were done to identify whether any numeric effects exist in these CSE assessment analyses. A significant time-step dependency due to an error in the spray package coding was identified and eliminated. A number of other code deficiencies and inconveniences also are noted.
\end{abstract}

\section{MASTER}




\section{Contents}

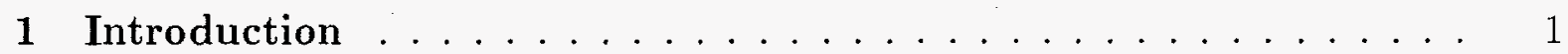

2 Facility and Test Description . . . . . . . . . . . . 2

3 MELCOR Input Model . . . . . . . . . . . . . . . . . . . . . . . 13

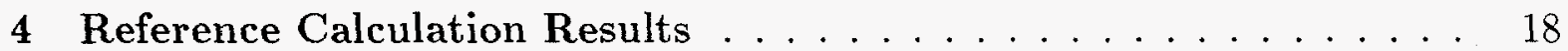

4.1 Thermal/Hydraulic Response . . . . . . . . . . . . 18

4.2 Aerosol Response . . . . . . . . . . . . . . . . . . 26

4.3 Iodine Vapor Response . . . . . . . . . . . . . . . . . . 40

5 Experimental Parameter Studies . . . . . . . . . . . . . 45

5.1 Effect of Spray Flow Rate (A-6 vs A-9) . . . . . . . . . 45

5.2 Effect of Spray Droplet Size (A-6 vs A-8) $\ldots \ldots \ldots$

5.3 Effect of Atmosphere Conditions (A-6 vs A-4) . . . . . . . 64

5.4 Effect of Spray Chemistry $(\mathbf{A}-6$ vs A-7) $\ldots \ldots \ldots 73$

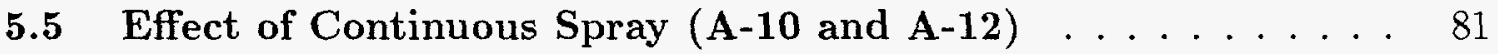

6 Spray Modelling Sensitivity Studies _. . . . . . . . . . . . . 99

6.1 Spray Fraction Interacting with Atmosphere . . . . . . . . 99

6.2 Spray Droplet Size . . . . . . . . . . . . . . . . . . . 100

6.3 Spray Droplet Drag Coefficient and Terminal Velocity . . . . 108

6.4 Spray Droplet Mass Transfer . . . . . . . . . . . . . . . 114

7 Aerosol Modelling Sensitivity Studies . . . . . . . . . . . . . . . . 124

7.1 Number of MAEROS Components . . . . . . . . . . . . . 124

7.2 Number of MAEROS Sections . . . . . . . . . . . . . . 132

7.3 Aerosol Density . . . . . . . . . . . . . . . . . . . 137

7.4 Aerosol Particle Initial Size . . . . . . . . . . . . . . . . 141

7.5 Condensation/Evaporation on Aerosols . . . . . . . . . . 147

8 Vapor Modelling Sensitivity Studies . . . . . . . . . . . . . . . 164

8.1 Partition Coefficient . . . . . . . . . . . . . . . . 164

8.2 Re-evolution from Pools . . . . . . . . . . . . . . . 167

9 Time Step Effects and Machine Dependency . . . . . . . . . . 168

9.1 Machine Dependencies . . . . . . . . . . . . . . . 168

9.2 Time Step Effects . . . . . . . . . . . . . . . 168 
10 Code Problems Identified . . . . . . . . . . . . . . . . 187

10.1 Recirculating Sprays . . . . . . . . . . . . . 187

10.2 Sensitivity Coefficients Not Connected . . . . . . . . . . 187

10.3 Iodine Re-evolution . . . . . . . . . . . . . . . . . 187

10.4 Time Step Dependency . . . . . . . . . . . . . . 188

10.5 I/O Improvements Needed . . . . . . . . . . . . . . 188

10.6 Evaporation/Condensation . . . . . . . . . . . . 189

11 Summary and Conclusions ................... 191

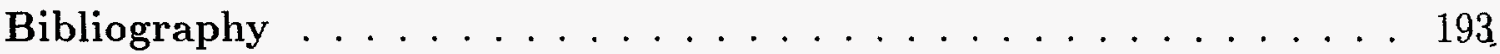

A CSE A-9 Reference Calculation Input Deck . . . . . . . . . 195 


\section{List of Figures}

2.1 Schematic of Test Facility for CSE Spray Tests (from [19]) . . . . . . . 3

2.2 Schematic of Maypack Used in CSE Spray Tests (from [19]) . . . . . . 4

3.1 Reference MELCOR Model for CSE Containment Spray Experiments Test Vessel . . . . . . . . . . . . . . . . . . . . . . . 14

4.1 Spray Flow Rate for CSE Test A-9 - Reference Calculation _ . . . . . 19

4.2 Spray Temperature for CSE Test A-9 - Reference Calculation . . . . . 20

4.1.1 Vessel Pressure for CSE Test A-9 - Reference Calculation . . . . . . . . 21

4.1.2 Atmosphere Total and Partial Pressures in Vessel Dome (upper left), in Lower Drywell (upper right), in Middle Room (lower left) and in Vessel Sump (lower right) for CSE Test A-9 - Reference Calculation . . . . . . 23

4.1.3 Vessel Temperatures for CSE Test A-9 - Reference Calculation . . . . . 24

4.1.4 Atmosphere, Pool, Saturation and Wall Temperatures in Vessel Dome for CSE Test A-9 - Reference Calculation . . . . . . . . . . . . . 27

4.1.5 Atmosphere, Pool, Saturation and Wall Temperatures in Lower Drywell for CSE Test A-9 - Reference Calculation . . . . . . . . . . . . . .

4.1.6 Atmosphere, Pool, Saturation and Wall Temperatures in Middle Room for CSE Test A-9 - Reference Calculation . . . . . . . . . . . . . . .

4.1.7 Atmosphere, Pool, Saturation and Wall Temperatures in Lower Room for CSE Test A-9 - Reference Calculation . . . . . . . . . . . . . .

4.1.8 Lower Drywell and Sump Pool Masses for CSE Test A-9 - Reference Calculation . . . . . . . . . . . . . . . . . . . . .

4.2.1 Cesium Aerosol Airborne Concentrations for CSE Test A-9 - Reference

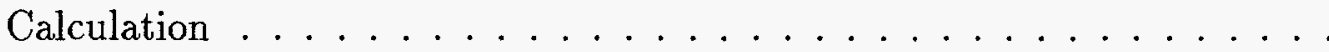

4.2.2 Uranium Aerosol Airborne Concentrations for CSE Test A-9 - Reference

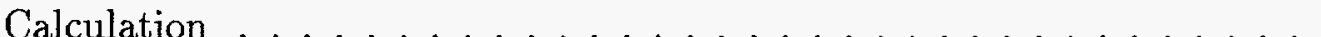

4.2.3 Airborne Aerosol AMMDs in Vessel Dome (upper left), in Lower Drywell (upper right), in Middle Room (lower left) and in Vessel Sump (lower right) for CSE Test A-9 - Reference Calculation ... . . . . . . . . . . . .

4.2.4 Airborne Aerosol GSDs in Vessel Dome (upper left), in Lower Drywell (upper right), in Middle Room (lower left) and in Vessel Sump (lower right) for CSE Test A-9 - Reference Calculation . . . . . . . . . . . . . .

4.3.1 Iodine Vapor Airborne Concentrations for CSE Test A-9 - Reference Calculation . . . . . . . . . . . . . . . . . . . 41

5.1.1 Spray Flow Rate for CSE Test A-6 - Reference Calculation . . . . . . . 46 
5.1.2 Spray Temperature for CSE Test A-6 - Reference Calculation . . . . . . 47

5.1.3 Vessel Pressure for CSE Test A-6 - Reference Calculation . . . . . . . . 49

5.1.4 Vessel Temperatures for CSE Test A-6 - Reference Calculation . . . . . 50

5.1.5 Cesium Aerosol Airborne Concentrations for CSE Test A-6 - Reference Calculation ......................... 51

5.1.6 Uranium Aerosol Airborne Concentrations for CSE Test A-6 - Reference Calculation

5.1.7 Todine Vapor Airborne Concentrations for CSE Test A-6 - Reference Cal-

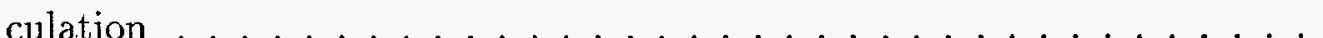

5.2.1 Spray Flow Rate for CSE Test A-8 - Reference Calculation . . . . . . 56

5.2.2 Spray Temperature for CSE Test A-8 - Reference Calculation . . . . . . . 57

5.2 .3 Vessel Pressure for CSE Test A-8 - Reference Calculation . . . . . . . . 58

5.2.4 Vessel Temperatures for CSE Test A-8 - Reference Calculation . . . . . 59

5.2.5 Cesium Aerosol Airborne Concentrations for CSE Test A-8 - Reference Calculation ....................... 60

5.2.6 Uranium Aerosol Airborne Concentrations for CSE Test A-8 - Reference

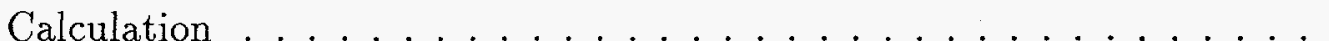

5.2.7 Iodine Vapor Airborne Concentrations for CSE Test A-8 - Reference Calculation

5.3.1 Spray Flow Rate for CSE Test A-4 - Reference Calculation . . . . . . . . 65

5.3.2 Spray Temperature for CSE Test A-4 - Reference Calculation . . . . . 66

5.3.3 Vessel Pressure for CSE Test A-4 - Reference Calculation . . . . . . . . 67

5.3.4 Vessel Temperatures for CSE Test A-4 - Reference Calculation . . . . . . 68

5.3.5 Cesium Aerosol Airborne Concentrations for CSE Test A-4 - Reference Calculation ..................... 69

5.3.6 Uranium Aerosol Airborne Concentrations for CSE Test A-4 - Reference Calculation

5.3.7 Iodine Vapor Airborne Concentrations for CSE Test A-4 - Reference Cal-

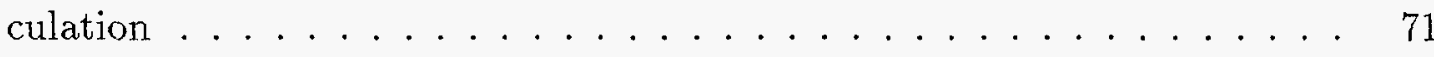

5.4.1 Spray Flow Rate for CSE Test A-7 - Reference Calculation . . . . . . 74

5.4.2 Spray Temperature for CSE Test A-7 - Reference Calculation . . . . . . 75

5.4.3 Vessel Pressure for CSE Test A-7 - Reference Calculation . . . . . . . 76

5.4.4 Vessel Temperatures for CSE Test A-7 - Reference Calculation . . . . . 77

5.4.5 Cesium Aerosol Airborne Concentrations for CSE Test A-7 - Reference Calculation 
5.4.6 Uranium Aerosol Airborne Concentrations for CSE Test A-7 - Reference Calculation .......................

5.4.7 Iodine Vapor Airborne Concentrations for CSE Test A-7 - Reference Calculation

5.5.1 Spray Flow Rate for CSE Test A-10 - Reference Calculation . . . . . . 83

5.5.2 Spray Temperature for CSE Test A-10 - Reference Calculation . . . . . 84

5.5.3 Vessel Pressure for CSE Test A-10 - Reference Calculation . . . . . . . 85

5.5.4 Vessel Temperatures for CSE Test A-10 - Reference Calculation . . . . . 86

5.5.5 Cesium Aerosol Airborne Concentrations for CSE Test A-10 - Reference

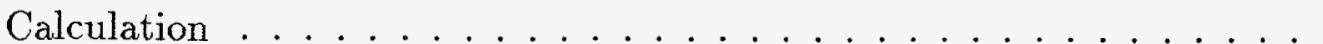

5.5.6 Uranium Aerosol Airborne Concentrations for CSE Test A-10 - Reference Calculation . . . . . . . . . . . . . . . 89

5.5.7 Iodine Vapor Airborne Concentrations for CSE Test A-10 - Reference Calculation ................................ 90

5.5.8 Spray Flow Rate for CSE Test A-12 - Reference Calculation . . . . . . . 91

5.5.9 Spray Temperature for CSE Test A-12 - Reference Calculation . . . . . 92

5.5.10 Vessel Pressure for CSE Test A-12 - Reference Calculation . . . . . . . . 93

5.5.11 Vessel Temperatures for CSE Test A-12 - Reference Calculation . . . . . 94

5.5.12 Cesium Aerosol Airborne Concentrations for CSE Test A-12 - Reference

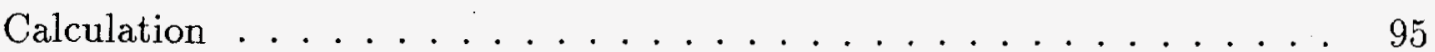

5.5.13 Uranium Aerosol Airborne Concentrations for CSE Test A-12 - Reference Calculation

5.5.14 Iodine Vapor Airborne Concentrations for CSE Test A-12 - Reference Calculation

6.1.1 Vessel Pressure for CSE Test A-9 - Spray Fraction Interacting with Atmosphere Sensitivity Study . . . . . . . . . . . . . . . . . . 101

6.1.2 Vessel Dome Temperatures for CSE Test A-9 - Spray Fraction Interacting with Atmosphere Sensitivity Study . . . . . . . . . . . . . . . 102

6.1.3 Cesium Aerosol Airborne Concentrations for CSE Test A-9 - Spray Fraction Interacting with Atmosphere Sensitivity Study

6.1.4 Iodine Vapor Airborne Concentrations for CSE Test A-9 - Spray Fraction Interacting with Atmosphere Sensitivity Study

6.2.1 Vessel Pressure for CSE Test A-9 - Spray Droplet Size Distribution Sensitivity Study ......................... 106

6.2.2 Vessel Dome Temperatures for CSE Test A-9 - Spray Droplet Size Distribution Sensitivity Study 
6.2.3 Cesium Aerosol Airborne Concentrations for CSE Test A-9 - Spray Droplet Size Distribution Sensitivity Study . . . . . . . . . . . . .

6.2.4 Iodine Vapor Airborne Concentrations for CSE Test A-9 - Spray Droplet Size Distribution Sensitivity Study . . . . . . . . . . . . .

6.3.1 Vessel Pressure for CSE Test A-9 - Spray Droplet Drag Coefficient and Terminal Velocity Sensitivity Study . . . . . . . . . . . . .

6.3.2 Vessel Dome Temperatures for CSE Test A-9 - Spray Droplet Drag Coefficient and Terminal Velocity Sensitivity Study . . . . . . . . . .

6.3.3 Cesium Aerosol Airborne Concentrations for CSE Test A-9 - Spray Droplet Drag Coefficient and Terminal Velocity Sensitivity Study . . . . . . . .

6.3.4 Iodine Vapor Airborne Concentrations for CSE Test A-9 - Spray Droplet Drag Coefficient and Terminal Velocity Sensitivity Study . . . . . . .

6.4.1 Vessel Pressure for CSE Test A-9 - Spray Droplet Mass Transfer Coefficient Sensitivity Study ....................

6.4.2 Vessel Dome Temperatures for CSE Test A-9 - Spray Droplet Mass Transfer Coefficient Sensitivity Study . . . . . . . . . . . . .

6.4.3 Cesium Aerosol Airborne Concentrations for CSE Test A-9 - Spray Droplet Mass Transfer Coefficient Sensitivity Study . . . . . . . . . . . . .

6.4.4 Iodine Vapor Airborne Concentrations for CSE Test A-9 - Spray Droplet Mass Transfer Coefficient Sensitivity Study . . . . . . . . . . .

7.1.1 Iodine Vapor Airborne Concentrations for CSE Test A-9 - MAEROS Component Sensitivity Study . . . . . . . . . . . . . . 125

7.1.2 Cesium Aerosol Airborne Concentrations for CSE Test A-9 - MAEROS Component Sensitivity Study . . . . . . . . . . . . . . . . 12

7.1.3 Uranium Aerosol Airborne Concentrations for CSE Test A-9 - MAEROS Component Sensitivity Study

7.1.4 Cesium (left) and Uranium (right) Aerosol Airborne Concentration AMMDs (top) and GSDs (bottom) in Dome for CSE Test A-9 - MAEROS Component Sensitivity Study

7.1.5 Fog Water Aerosol Airborne Concentration AMMDs (top) and GSDs (bottom) in Dome for CSE Test A-9 - MAEROS Component Sensitivity Study 131

7.2.1 Iodine Vapor Airborne Concentrations for CSE Test A-9 - MAEROS Sections Sensitivity Study . . . . . . . . . . . . .

7.2.2 Cesium Aerosol Airborne Concentrations for CSE Test A-9 - MAEROS Sections Sensitivity Study . . . . . . . . . . . . . . 134

7.2.3 Uranium Aerosol Airborne Concentrations for CSE Test A-9 - MAEROS Sections Sensitivity Study 
7.2.4 Cesium (left) and Uranium (right) Aerosol Airborne Concentration AMMDs (top) and GSDs (bottom) in Dome for CSE Test A-9 - MAEROS Sections Sensitivity Study . . . . . . . . . . . . . . . . .

7.2.5 Fog Water Aerosol Airborne Concentration AMMDs (top) and GSDs (bottom) in Dome for CSE Test A-9 - MAEROS Sections Sensitivity Study . .

7.3.1 Iodine Vapor Airborne Concentrations for CSE Test A-9 - Aerosol Density Sensitivity Study . . . . . . . . . . . . . . . . . . .

7.3.2 Cesium Aerosol Airborne Concentrations for CSE Test A-9 - Aerosol Density Sensitivity Study . . . . . . . . . . . . . . . . . . .

7.3.3 Uranium Aerosol Airborne Concentrations for CSE Test A-9 - Aerosol Density Sensitivity Study . . . . . . . . . . . . . . .

7.3.4 Cesium (left) and Uranium (right) Aerosol Airborne Concentration AMMDs (top) and GSDs (bottom) in Dome for CSE Test A-9 - Aerosol Density Sensitivity Study . . . . . . . . . . . . . . . . .

7.3.5 Fog Water Aerosol Airborne Concentration AMMDs (top) and GSDs (bottom) in Dome for CSE Test A-9 - Aerosol Density Sensitivity Study . . .

7.4.1 Iodine Vapor Airborne Concentrations for CSE Test A-9 - Aerosol Particle Initial Size Sensitivity Study . . . . . . . . . . . . . . . . . 148

7.4.2 Cesium Aerosol Airborne Concentrations for CSE Test A-9 - Aerosol Particle Initial Size Sensitivity Study . . . . . . . . . . . . . . . . . 149

7.4.3 Uranium Aerosol Airborne Concentrations for CSE Test A-9 - Aerosol Particle Initial Size Sensitivity Study . . . . . . . . . . . . . .

7.4.4 Cesium (left) and Uranium (right) Aerosol Airborne Concentration AMMDs (top) and GSDs (bottom) in Dome for CSE Test A-9 - Aerosol Particle Initial Size Sensitivity Study . . . . . . . . . . . . . .

7.4.5 Fog Water Aerosol Airborne Concentration AMMDs (top) and GSDs (bottom) in Dome for CSE Test A-9 - Aerosol Particle Initial Size Sensitivity Study . . . . . . . . . . . . . . . . .

7.5.1 Vessel Pressures for CSE Test A-9 - Aerosol Condensation/Evaporation Sensitivity Study . . . . . . . . . . . . . . . . .

7.5.2 Vessel Dome Temperatures for CSE Test A-9 - Aerosol Condensation/Evaporation Sensitivity Study . . . . . . . . . . . . . . . . 156

7.5.3 Iodine Vapor Airborne Concentrations for CSE Test A-9 - Aerosol Condensation/Evaporation Sensitivity Study . . . . . . . . . . . . 157

7.5.4 Cesium Aerosol Airborne Concentrations for CSE Test A-9 - Aerosol Condensation/Evaporation Sensitivity Study . . . . . . . . . . . . 159

7.5.5 Uranium Aerosol Airborne Concentrations for CSE Test A-9 - Aerosol Condensation/Evaporation Sensitivity Study . . . . . . . . . . . . . . 160 
7.5.6 Cesium (left) and Uranium (right) Aerosol Airborne Concentration AMMDs (top) and GSDs (bottom) in Dome for CSE Test A-9 - Aerosol Condensation/Evaporation Sensitivity Study . . . . . . . . . . . . . .

7.5.7 Fog Water Aerosol Airborne Concentration AMMDs (top) and GSDs (bottom) in Dome for CSE Test A-9 - Aerosol Condensation/Evaporation Sensitivity Study . . . . . . . . . . . . . . . . .

8.1.1 Iodine Vapor Airborne Concentrations for CSE Test A-9 - Iodine Partition Coefficient Sensitivity Study . . . . . . . . . . . . . . . 165

9.1.1 Vessel Pressure for CSE Test A-9 - Machine Dependency Sensitivity Study 169

9.1.2 Vessel Dome Temperature for CSE Test A-9 - Machine Dependency Sensitivity Study . . . . . . . . . . . . . . . . . . 170

9.1.3 Cesium Aerosol Airborne Concentrations for CSE Test A-9 - Machine Dependency Sensitivity Study . . . . . . . . . . . . .

9.1.4 Uranium Aerosol Airborne Concentrations for CSE Test A-9 - Machine Dependency Sensitivity Study . . . . . . . . . . . . . . .

9.1.5 Iodine Vapor Airborne Concentrations for CSE Test A-9 - Machine Dependency Sensitivity Study . . . . . . . . . . . . . . . 173

9.1.6 Total Run Times for CSE Test A-9 - Machine Dependency Sensitivity Study174

9.1.7 Total and Package Run Times for CSE Test A-9 - Reference Calculation . 175

9.2.1 Time Steps for CSE Test A-9 - Time Step Sensitivity Study . . . . . . . 177

9.2.2 Vessel Pressure for CSE Test A-9 - Time Step Sensitivity Study (Version

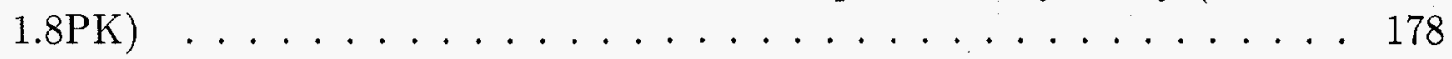

9.2.3 Vessel Dome Temperature for CSE Test A-9 - Time Step Sensitivity Study

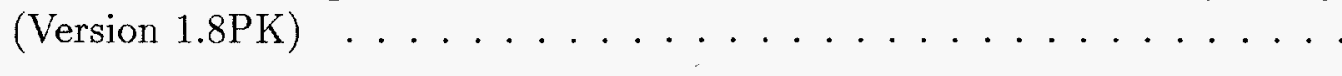

9.2.4 Cesium Aerosol Airborne Concentrations for CSE Test A-9 - Time Step Sensitivity Study (Version 1.8PK) . . . . . . . . . . . . 180

9.2.5 Iodine Vapor Airborne Concentrations for CSE Test A-9 - Time Step Sensitivity Study (Version 1.8PK) . . . . . . . . . . . . . . 181

9.2.6 Vessel Pressure for CSE Test A-9 - Time Step Sensitivity Study (Version

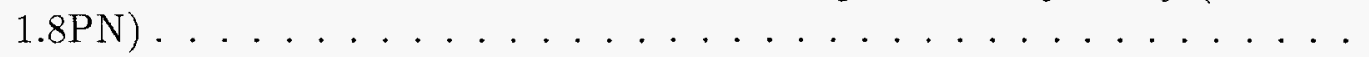

9.2.7 Vessel Dome Temperature for CSE Test A-9 - Time Step Sensitivity Study

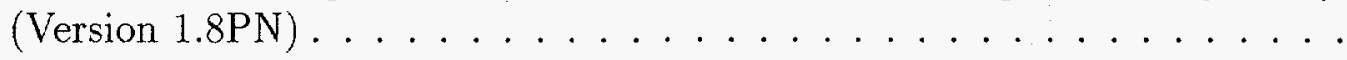

9.2.8 Cesium Aerosol Airborne Concentrations for CSE Test A-9 - Time Step Sensitivity Study (Version 1.8PN) . . . . . . . . . . . . .

9.2.9 Iodine Vapor Airborne Concentrations for CSE Test A-9 - Time Step Sensitivity Study (Version 1.8PN) . . . . . . . . . . . . . 186 


\section{List of Tables}

2.1 Physical Conditions Common to All CSE Spray Experiments (from [19,21]) 6

2.2 Experimental Conditions in CSE Spray Experiments (from [19,21]) . . . 7

2.3 Spray Flow Rates and Solutions Used in CSE Spray Experiments (from $[19,21]) \ldots \ldots \ldots \ldots \ldots \ldots \ldots$

2.4 Timing of Spray Periods in CSE Spray Experiments (from [19,21]) . . 9

2.5 Atmospheric Conditions in CSE Spray Experiments (from [19,21]) . . . . 10

2.6 Typical Initial Fission Product Simulant Concentrations in the Vapor Space in CSE Spray Experiments (from $[19,21]$ ) . . . . . . . . . . . . . . . 11

2.7 Test Conditions for Continuous Spray CSE Experiments (from [21]) . . . 12

3.1 Droplet Size Distribution Used in Reference MELCOR Model for CSE Containment Spray Experiment A-9 . . . . . . . . . . . 17

4.2.1 Cesium Aerosol Washout Rates for CSE Test A-9 - Reference Calculation 36

4.2.2 Uranium Aerosol Washout Rates for CSE Test A-9 - Reference Calculation 36

4.3.1 Iodine Vapor Washout Rates for CSE Test A-9 - Reference Calculation . . 44

5.1.1 Washout Rates for CSE Tests - Effect of Spray Flow Rate.(A-6 vs A-9) . . 54

5.2.1 Washout Rates for CSE Tests - Effect of Spray Droplet Size (A-6 vs A-8) 63

5.3.1 Washout Rates for CSE Tests - Effect of Atmosphere Conditions (A-6 vs

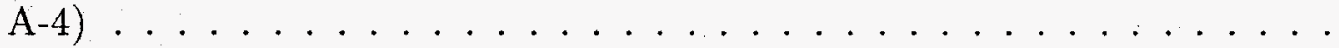

5.4.1 Cesium Aerosol Washout Rates for CSE Tests - Effect of Spray Chemistry

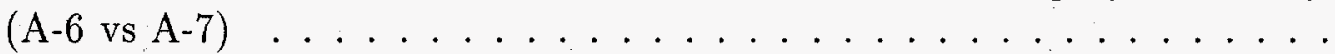

6.1.1 Washout Rates for CSE Test A-9 - Spray Fraction Interacting with Atmosphere Sensitivity Study . . . . . . . . . . . . . 105

6.2.1 Washout Rates for CSE Test A-9 - Spray Droplet Size Distribution Sensitivity Study . . . . . . . . . . . . . . . . 111

6.3.1 Washout Rates for CSE Test A-9 - Spray Droplet Drag Coefficient and Terminal Velocity Sensitivity Study . . . . . . . . . . . . 117

6.4.1 Washout Rates for CSE Test A-9 - Spray Droplet Mass Transfer Coefficient Sensitivity Study . . . . . . . . . . . . . . 123

7.1.1 Washout Rates for CSE Test A-9 - MAEROS Component Sensitivity Study 129

7.2.1 Washout Rates for CSE Test A-9 - MAEROS Sections Sensitivity Study . 136

7.3.1 Washout Rates for CSE Test A-9 - Aerosol Density Sensitivity Study . . . 144

7.4.1 Washout Rates for CSE Test A-9 - Aerosol Particle Initial Size Sensitivity

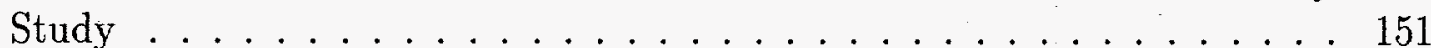


7.5.1 Washout Rates for CSE Test A-9 - Aerosol Condensation/Evaporation Sensitivity Study . . . . . . . . . . . . . . . . . . 161

8.1.1 Washout Rates for CSE Test A-9 - Iodine Partition Coefficient Sensitivity Study . . . . . . . . . . . . . . . 166 


\section{Introduction}

MELCOR [1] is a fully integrated, engineering-level computer code being developed at Sandia National Laboratories for the U. S. Nuclear Regulatory Commission (USNRC), that models the progression of severe accidents in light water reactor (LWR) nuclear power plants. A spectrum of severe accident phenomena, including reactor coolant system and containment thermal/hydraulic response, core heatup, degradation and relocation, and fission product release and transport, is treated in MELCOR in a unified framework for both boiling water reactors and pressurized water reactors. The MELCOR computer code has been developed to the point that it is now being applied in severe accident analyses.

Some limited technical assessment activities were performed early in the MELCOR development process [2]; more recently, a systematic program of verification and validation has been under way. To this end, a number of assessment calculations have been and are being done [3-13]. One of these assessment activities is analysis of a series of eight large-scale containment spray experiments performed in the Containment Systems Experiment (CSE) vessel [14], to evaluate the performance of aqueous sprays as a means of decontaminating containment atmospheres. Measurements were obtained which provide a suitable basis for judging the ability of various mathematical models to predict spray performance in large nuclear power plant buildings.

MELCOR version $1.8 \mathrm{PN}$ was used for all the calculations described in this report. Note that these MELCOR calculations were done as an open posttest study, with the experimental data available to guide the selection of code input.

The test facility, experimental configuration and experimental procedure are outlined briefly in Section 2. Section 3 describes the input used for these MELCOR assessment analyses. The results of our reference calculation for test A-9 (the experiment analysis used as the base case for our sensitivity studies) are given in Section 4 . Section 5 gives results for other experiments in the CSE series with experimental parameters varied. Sensitivity studies on modelling variations affecting the spray (SPR) package are presented in Section 6, while Sections 7 and 8 present results obtained varying parameters and options affecting the aerosol and vapor modelling, respectively, in the radionuclide $(\mathrm{RN})$ package. Section 9 contains the results of our time step and machine dependency sensitivity studies. Section 10 summarizes the code and modelling problems identified during these assessment analyses. A summary and conclusions of this MELCOR assessment study are presented in Section 11. The input used for the CSE test A-9 reference calculation is listed in Appendix A. 


\section{$2 \quad$ Facility and Test Description}

Eight experiments have been performed in the CSE containment vessel to evaluate the performance of aqueous sprays as a means of decontaminating containment atmospheres. Measurements were obtained which provide a suitable basis for judging the ability of various mathematical models to predict spray performance in large nuclear power plant buildings. The test facility is briefly described in this section, along with the tests

performed and important test parameters. These descriptions are taken primarily from $[19,21]$.

All eight experiments were performed in the main CSE containment vessel, which was $7.62 \mathrm{~m}(25 \mathrm{ft})$ in diameter by $20.33 \mathrm{~m}(66.7 \mathrm{ft})$ high. Figure 2.1 is a schematic drawing showing most of the important experimental features. A fresh, room-temperature spray solution was made up in an exterior, stainless steel storage tank. About $7570 l$ (2000 gal) were used in most of the experiments, which was about $1.3 \%$ of the gas volume in the main room. The spray manifold near the top dome was arranged for either 3 or 12 nozzles at a uniform spacing. About $80 \%$ of the gas space in the main room was washed by spray in all tests except A-3, in which only $50 \%$ of the space was covered.

Other features shown schematically in Figure 2.1 are the system for generating fission product iodine and aerosol simulants [15], the boiler house steam line used for establishing the desired post-accident atmosphere, a $360^{\circ}$ wall trough near the deck for measuring wall liquid runoff rate and fission product concentrations in the wall film, funnels to catch falling drops, the liquid pool sampling systems, a viewing window, and the gas sampling locations. The gas was sampled at 14 different locations by Maypack clusters [16]. Figure 2.2 is a schematic diagram of a single Maypack showing the trapping components in the sequence used. Twelve Maypacks were installed in each cluster, each with its own solenoid valve. A detailed description of the experimental equipment is given in [17].

Two kinds of materials were aerosolized in the CSE experiments to represent solids that could be released during postulated accidents. $\mathrm{UO}_{2}$ fuel elements clad with stainless steel or zircaloy were heated inductively to temperatures high enough to form appreciable quantities of aerosol, which was probably converted to $\mathrm{U}_{3} \mathrm{O}_{8}$; this uranium oxide aerosol simulated core materials that have very low vapor pressures and low solubilities in water. Cesium carbonate was heated by means of an electrical resistance heater. This material volatilizes at a relatively low temperature and forms aerosols of cesium hydroxide, and possibly $\mathrm{Cs}_{2} \mathrm{CO}_{3}$, in humid atmospheres. This cesium aerosol simulated fission products classed as volatile solids and is highly soluble in water.

Iodine was injected in two forms: elemental iodine and methyl iodide. When release was desired, a flask containing elemental iodine (equilibrated with an I-131 tracer) was heated electrically and air swept through the flask carried the elemental iodine through the hot zone of the $\mathrm{UO}_{2}$ melt furnace. Some particulate-associated iodine and organic iodides were always produced. Additional iodine in the form of reagent grade methyl iodide was equilibrated with I-131 in methyl alcohol in a stainless steel U-tube; when release was desired, air was passed through the U-tube to sweep the methyl iodide directly into the test vessel (bypassing the $\mathrm{UO}_{2}$ furnace). 


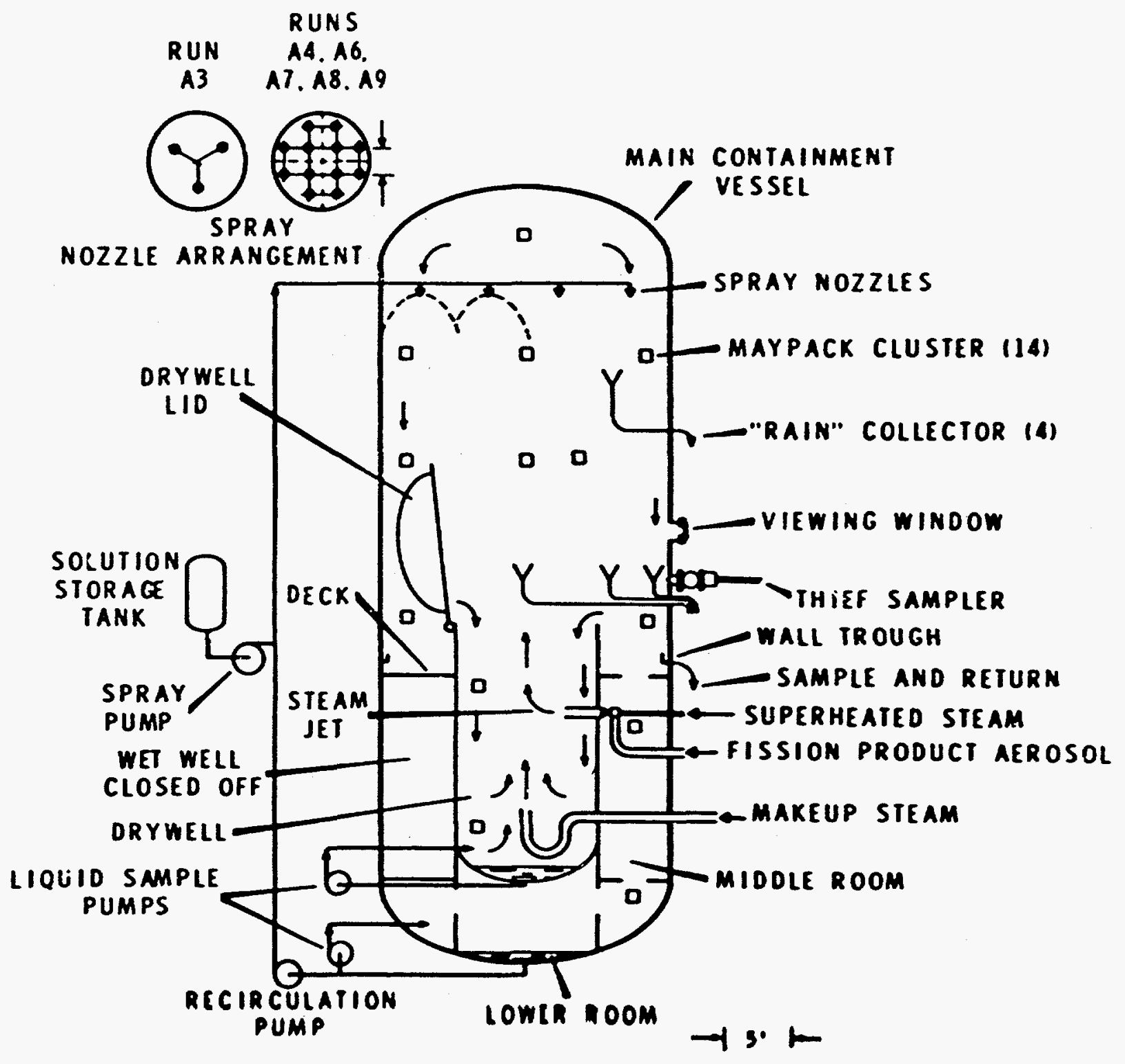

Figure 2.1. Schematic of Test Facility for CSE Spray Tests (from [19]) 


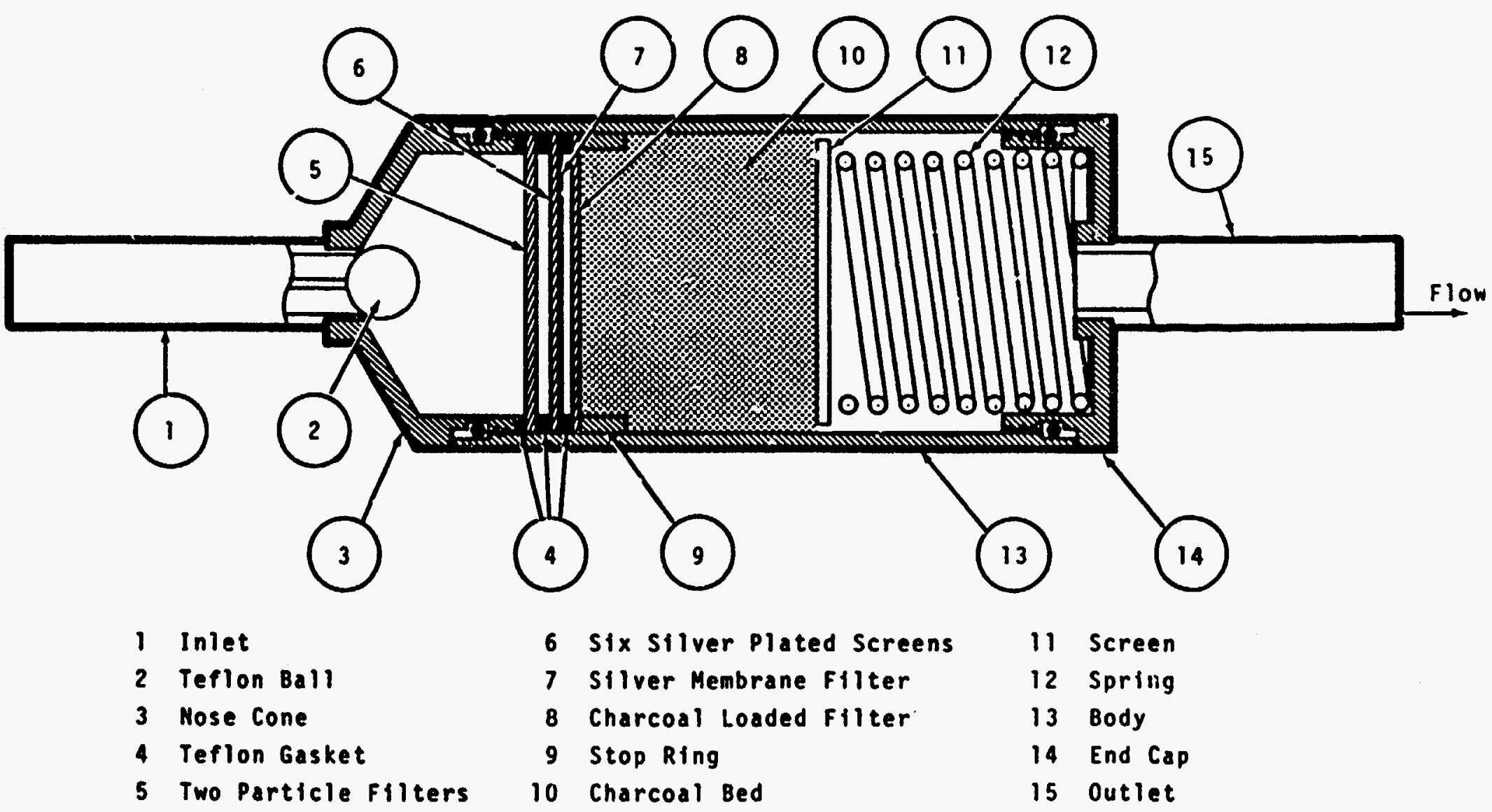

Figure 2.2. Schematic of Maypack Used in CSE Spray Tests (from [19]) 
The experimental procedure was to establish the desired atmospheric temperature and pressure in the containment vessel by using boiler house steam. (In two tests the atmosphere was room-temperature air at ambient pressure.) When the atmosphere reached the desired temperature, the steam feed rate was reduced to a point where thermal equilibrium was maintained and left at this rate for the duration of the experiment. The condensate produced by warming the steel vessel was discarded. Then the fission product simulant was injected in a $10 \mathrm{~min}$ period. Time was referenced to the start of aerosol injection. Samples were taken during a short waiting period to determine the removal rate by natural effects. Then the fresh spray was started. In six of the experiments the sprays were operated intermittently; in two tests they operated continuously, with recirculation from the sump after all the fresh solution had entered the vessel.

Gas samples were taken at all 14 locations simultaneously by operating electrical solenoid valves from an adjoining laboratory. The gas sample rate was maintained at $0.5 \pm 0.05 \mathrm{ft}^{3} / \mathrm{min}$ (STP) for $3.0 \pm 0.07 \mathrm{~min}$ for all samples. Liquid samples were taken more frequently early in the test when concentrations changed rapidly.

Exact timing of the spray periods was accomplished by priming the spray manifold before the test and by the use of an electric ball valve for on-off control. Visual observations confirmed that sprays started and stopped within $3 \mathrm{~s}$ of the specified times. The spraying rate was controlled to that which gave the specified pressure drop across the spray nozzles.

The experimental conditions and test parameters are listed in Tables 2.1 through 2.7. Table 2.1 gives the physical conditions common to all the experiments, while Table 2.2 gives the values of parameters which were varied from test to test. Table 2.3 lists the total spray flow rates and chemical composition for each spray period. Table 2.4 lists the start and stop times for the six tests in which sprays operated intermittently.

In the tests with a steam-air atmosphere, the cold fresh spray rapidly reduced the temperature and pressure within the vessel. Table 2.5 summarizes the atmospheric conditions immediately before and after each spray period. The measured temperature for these CSE tests is the arithmetic average reading of 5 Chromel-Alumel (Type K) thermocouples located in the main room vapor space. The pressure was recorded by visual readings of a precision absolute pressure gauge.

The masses of iodine, cesium and uranium released into the containment vessel varied slightly between experiments, but the nominal initial concentrations are given in Table 2.6. The initial concentration of iodine was about the maximum expected in a large PWR with a $50 \%$ release of the iodine inventory in the core.

Two experiments (A-10 and A-12) were done in which the sprays were started shortly before fission product simulants were released, and continued without interruption until all the fresh solution had been sprayed into the containment vessel [19]. After a $10 \mathrm{~min}$ period to arrange valves, recirculation from the sump was started and continued for about $20 \mathrm{hr}$. Table 2.7 lists the conditions for these two experiments. 
Table 2.1. Physical Conditions Common to All CSE Spray Experiments (from $[19,21])$

Volume above deck including drywell

Surface area above deck including drywell

Surface area/Volume

Cross-sectional area in main vessel

Cross-sectional area in drywell

Volume in middle room

Surface area in middle room

Volume in lower room

Surface area in lower room

Total volume in all rooms

Total surface area in all rooms

Drop fall height to deck

Drop fall height to drywell bottom

Surface coating

Thermal insulation
$595 \mathrm{~m}^{3}$
$569 \mathrm{~m}^{2}$
$21,005 \mathrm{ft}^{3}$
$6,140 \mathrm{ft}^{2}$
$0.958 / \mathrm{m}$
$0.293 / \mathrm{ft}$
$45.5 \mathrm{~m}^{2}$
$490 \mathrm{ft}^{2}$
$8.8 \mathrm{~m}^{2}$
$95 \mathrm{ft}^{2}$
$59 \mathrm{~m}^{3}$
$127 \mathrm{~m}^{2}$
$2,089 \mathrm{ft}^{3}$
$1,363 \mathrm{ft}^{2}$
$96 \mathrm{~m}^{3}$
$191 \mathrm{~m}^{2}$
$3,384 \mathrm{ft}^{3}$
$2,057 \mathrm{ft}^{2}$
$751 \mathrm{~m}^{3}$
$888 \mathrm{~m}^{2}$
$26,477 \mathrm{ft}^{3}$
$9,560 \mathrm{ft}^{2}$
$10.3 \mathrm{~m}$
$33.8 \mathrm{ft}$
$15.4 \mathrm{~m}$
$50.5 \mathrm{ft}$

All interior surfaces coated with phenolic paint;

Two coats phenolic $302^{a}$

over one coat phenolic $300^{a}$

All exterior surfaces covered with 1 in Fiberglas ${ }^{b}$ insulation; $\mathrm{k}=0.027 \mathrm{Btu} / \mathrm{hr}-\mathrm{ft}-{ }^{\circ} \mathrm{F}$ at $200^{\circ} \mathrm{F}$, Type PF-615

${ }^{a}$ The Carboline Co., St. Louis, Missouri

${ }^{b}$ Owens-Corning Fiberglas Corp. 
Table 2.2. Experimental Conditions in CSE Spray Experiments (from $[19,21]$ )

\begin{tabular}{|c|c|c|c|c|c|c|}
\hline Parameter & $A-3$ & A-4 & A-6 & $A-7$ & A-8 & A-9 \\
\hline Atmosphere & Air & Air & Steam-air & Steam-air & Steam-air & Steam-air \\
\hline Temperature (K) & 298 & 298 & 394 & 394 & 394 & 394 \\
\hline Pressure $(\mathrm{kPa})$ & 100.66 & 100.66 & 303.36 & 344.73 & 330.94 & 303.36 \\
\hline Nozzle type & $\begin{array}{l}3 / 47 \mathrm{G} 3, \\
\text { full cone }\end{array}$ & $\begin{array}{l}3 / 47 \mathrm{G} 3 \text {, } \\
\text { full cone } \mathrm{e}^{a}\end{array}$ & $\begin{array}{l}\text { 3/4 } 7 \mathrm{G} 3 \text {, } \\
\text { full cone }\end{array}$ & $\begin{array}{l}3 / 47 \mathrm{G} 3 \\
\text { full cone }\end{array}$ & $\begin{array}{l}3 / 8 \text { A20, } \\
\text { hollow cone }\end{array}$ & $\begin{array}{c}\text { 3/4 A50, } \\
\text { hollow cone }\end{array}$ \\
\hline Droplet $\mathrm{MMD}^{b}(\mu \mathrm{m})$ & 1210 & 1210 & 1210 & 1210 & 770 & 1220 \\
\hline Droplet $\mathrm{GSD}^{b}$ & 1.53 & 1.53 & 1.53 & 1.53 & 1.50 & 1.50 \\
\hline Number of nozzles & 3 & 12 & 12 & 12 & 12 & 12 \\
\hline Spray rate $(l / \mathrm{s})$ & 0.8064 & 3.0744 & 3.087 & 3.087 & 3.1815 & 9.135 \\
\hline Total spray volume $(l)$ & 1928 & 7371 & 7409 & 7409 & 7636 & 8694 \\
\hline \multicolumn{7}{|l|}{ Spray solution } \\
\hline Boron concentration (ppm) & 525 & 525 & 3000 & 3000 & 3000 & 3000 \\
\hline Boron form & $\mathrm{H}_{3} \mathrm{BO}_{3}$ & $\mathrm{H}_{3} \mathrm{BO}_{3}$ & $\mathrm{H}_{3} \mathrm{BO}_{3}$ & $\mathrm{H}_{3} \mathrm{BO}_{3}$ & $\mathrm{H}_{3} \mathrm{BO}_{3}$ & $\mathrm{H}_{3} \mathrm{BO}_{3}$ \\
\hline Boron carrier & $\mathrm{NaOH}$ & $\mathrm{NaOH}$ & $\mathrm{NaOH}$ & demineralized water & $\mathrm{NaOH}$ & $\mathrm{NaOH}$ \\
\hline $\mathrm{pH}$ & 9.5 & 9.5 & 9.5 & 5 & 9.5 & 9.5 \\
\hline
\end{tabular}


Table 2.3. Spray Flow Rates and Solutions Used in CSE Spray Experiments (from $[19,21])$

Spray Period

First

Total flow rate $(l / \mathrm{s})$

Volume sprayed $(l)$

Spraying pressure $(\mathrm{kPad})$

Solution
A-3 $\quad$ A-4 $\quad$ A- $6 \quad$ A-7 $\quad$ A- 8

A -9

Second

Total flow rate $(l / \mathrm{s})$

Volume sprayed $(l)$

Spraying pressure $(\mathrm{kPad})$

Solution

$\begin{array}{cccccc}0.8064 & 3.087 & 3.087 & 3.087 & 3.150 & 9.324 \\ 484 & 1852 & 1852 & 1852 & 567 & 1678 \\ 276 & 276 & 276 & 276 & 276 & 352 \\ a & a & b & c & b & b\end{array}$

Third

Total flow rate $(l / \mathrm{s})$

0.7875

2.646

1.008

2.8665

2.961

9.387

Volume sprayed $(l)$

2778

Spraying pressure $(\mathrm{kPad})$

Solution

276
$d$

$7144 \quad 3251$

10319

10660

5632

$\begin{array}{cc}200 & 28 \\ e & e\end{array}$

252
$e$

252

352

Fourth

Total flow rate $(l / \mathrm{s})$

Volume sprayed $(l)$

$\begin{array}{cccccc}- & - & - & 3.0618 & 3.1752 & 8.694 \\ - & - & - & 9178 & 9526 & 31298 \\ - & - & - & 276 & 276 & 338 \\ - & - & - & f & f & e\end{array}$

Solution

${ }^{a}$ Fresh, room temperature, $525 \mathrm{ppm}$ boron as $\mathrm{H}_{3} \mathrm{BO}_{3}$ in demineralized water, $\mathrm{NaOH}$ added to $\mathrm{pH}$ of 9.5

${ }^{b}$ Fresh, room temperature, $3000 \mathrm{ppm}$ boron as $\mathrm{H}_{3} \mathrm{BO}_{3}$ in demineralized water, $\mathrm{NaOH}$ added to $\mathrm{pH}$ of 9.5

${ }^{c}$ Fresh, room temperature, $3000 \mathrm{ppm}$ boron as $\mathrm{H}_{3} \mathrm{BO}_{3}$ in demineralized water, no $\mathrm{NaOH}$ added, $\mathrm{pH}$ of 5

${ }^{d}$ Fresh, room temperature, demineralized water

${ }^{e}$ Solution in main vessel sump recirculated, no heat exchanger used

${ }^{f}$ Fresh, room temperature, $1 \%$ wt $\mathrm{Na}_{2} \mathrm{~S}_{2} \mathrm{O}_{3}, 3000$ ppm boron as $\mathrm{H}_{3} \mathrm{BO}_{3}$ in demineralized water, $\mathrm{NaOH}$ added to $\mathrm{pH}$ of 9.4 
Table 2.4. Timing of Spray Periods in CSE Spray Experiments (from $[19,21]$ )

Time after Start of lodine Release ( $\mathrm{s}$ )

Spray Period A-3 A-4 A-6 $\quad$ A-7 $\quad$ A-8 $\quad$ A-9

First

$\begin{array}{lcccccc}\text { Start } & 2400 & 2430 & 1800 & 1800 & 1800 & 1800 \\ \text { Stop } & 3000 & 3030 & 2400 & 2400 & 1980 & 1980 \\ \text { Duration } & 600 & 600 & 600 & 600 & 180 & 180\end{array}$

Second

$\begin{array}{lllllll}\text { Start } & 8400 & 8400 & 4800 & 4800 & 4800 & 3300\end{array}$

Stop $\quad \begin{array}{llllll}10200 & 10200 & 6600 & 6600 & 7020 & 3480\end{array}$

$\begin{array}{lllllll}\text { Duration } & 1800 & 1800 & 1800 & 1800 & 2220 & 180\end{array}$

Third

Start

$\begin{array}{llllll}88380 & 72300 & 93900 & 79380 & 12000 & 5400\end{array}$

Stop 91980

75000

97500

Duration 3600

2700 3600

$82980 \quad 15600 \quad 6000$

Fourth

$\begin{array}{lcccccc}\text { Start } & - & - & - & 86580 & 81000 & 12600 \\ \text { Stop } & - & - & - & 89580 & 84000 & 16200 \\ \text { Duration } & - & - & - & 3000 & 3000 & 3600\end{array}$


Table 2.5. Atmospheric Conditions in CSE Spray Experiments (from $[19,21]$ )

\begin{tabular}{|c|c|c|c|c|c|c|}
\hline & $A-3$ & A-4 & $A-6$ & $A-7$ & A-8 & A-9 \\
\hline Containment vessel insulated & no & no & yes & yes & yes & yes \\
\hline Forced air circulation ${ }^{a}$ & yes & yes & no & no & no & no \\
\hline \multicolumn{7}{|l|}{ Start of First Spray } \\
\hline Vapor temperature (K) & 298 & 298 & 397.0 & 393.5 & 394.3 & 393.7 \\
\hline Pressure $(\mathrm{kPa})$ & 100.66 & 100.66 & 304.74 & 344.73 & 349.55 & 303.36 \\
\hline Relative humidity (\%) & 70 & 88 & 100 & 100 & 100 & 100 \\
\hline
\end{tabular}

Stop of First Spray

$\begin{array}{lcccccc}\text { Vapor temperature }(\mathrm{K}) & 298 & 298 & 382.6 & 385.6 & 390.4 & 383.1 \\ \text { Pressure }(\mathrm{kPa}) & 100.66 & 100.66 & 266.13 & 306.12 & 332.32 & 265.44\end{array}$

Start of Second Spray

Vapor temperature $(\mathrm{K})$

$\begin{array}{cccccc}298 & 298 & 387.0 & 388.7 & 390.4 & 385.9 \\ 100.66 & 100.66 & 281.30 & 317.15 & 339.90 & 272.34\end{array}$

Stop of Second Spray

$\begin{array}{lllllll}\text { Vapor temperature }(\mathrm{K}) & 298 & 298 & 367.6 & 368.1 & 359.8 & 376.5\end{array}$

Pressure $(\mathrm{kPa})$

$\begin{array}{llllll}100.66 & 100.66 & 203.39 & 248.20 & 235.10 & 237.86\end{array}$

Start of Third Spray

$\begin{array}{lllllll}\text { Vapor temperature (K) } & 298 & 298 & 392.0 & 393.1 & 375.9 & 379.3\end{array}$

$\begin{array}{lllllll}\text { Pressure }(\mathrm{kPa}) & 100.66 & 100.66 & 302.67 & 322.67 & 286.12 & 248.20\end{array}$

Stop of Third Spray

$\begin{array}{lllllll}\text { Vapor temperature }(\mathrm{K}) & 298 & 298 & 384.8 & 383.1 & 376.5 & 355.9\end{array}$

$\begin{array}{lllllll}\text { Pressure }(\mathrm{kPa}) & 100.66 & 100.66 & 280.61 & 288.19 & 222.00 & 186.84\end{array}$

Start of Fourth Spray

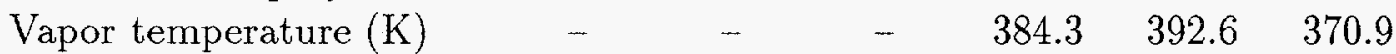

Pressure $(\mathrm{kPa})$

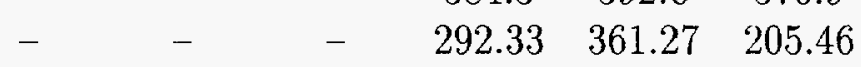

Stop of Fourth Spray

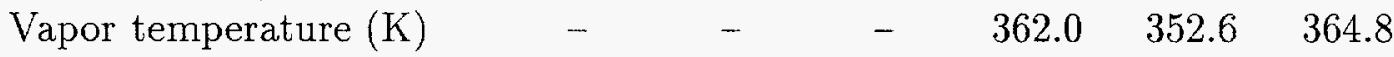

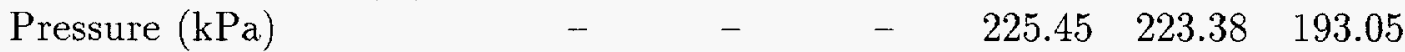

${ }^{a}$ Fan without duct located in bottom of drywell; $2400 \mathrm{ft}^{3} / \mathrm{min}$ discharge. 
Table 2.6. Typical Initial Fission Product Simulant Concentrations in the Vapor Space in CSE Spray Experiments (from [19,21])

Species

Elemental iodine

Particulate-associated iodine

Methyl iodide

Cesium

Uranium
$C_{0}\left(\mathrm{mg} / \mathrm{m}^{3}\right)$

100

5

2

5

5 
Table 2.7. Test Conditions for Continuous Spray CSE Experiments (from [21])

Parameter

Solution composition

$\mathrm{pH}$

Nozzle type

Number of nozzles

Spraying pressure (kPad)

Droplet MMD $(\mu \mathrm{m})$

Droplet GSD

Total spray rate $(l / \mathrm{s})$

Wall flow rate $(l / \mathrm{s})$

Total spray volume $(l)$

Fresh spray

Start time (s)

End time (s)

Recirculating spray

Start time (s)

End time (s)

Initial conditions

Vapor temperature $(\mathrm{K})$

Pressure (kPa)

At end of fresh spray period

Vapor temperature (K)

Pressure $(\mathrm{kPa})$

During recirculation

Average temperature $(\mathrm{K})$

Average pressure $(\mathrm{kPa})$

Fission product release

Start time (s)

End time (s)

Mass of iodine released ( $\mathrm{g}$ )

Mass of cesium released $(\mathrm{g})$

Mass of uranium released $(\mathrm{g})$
A-10

A-12

2750 ppm

$\mathrm{B}, \mathrm{NaOH}$
2750 ppm B, $\mathrm{NaOH}$, $1 \%$ wt $\mathrm{Na}_{2} \mathrm{~S}_{2} \mathrm{O}_{3}$

9.4

9.2

Spraying Systems No. $7 \mathrm{G} 3$

12

276

1210

1.53

3.087

0.145

7787

3.150

0.145

8316

$-120$

$-480$

2400

2400

3000

3000

68400

78600

393.7

395.9

337.83

324.04

360.9

375.9

216.49

237.17

394.3

391.5

386.10

324.04

$\begin{array}{cc}0 & 0 \\ 600 & 600 \\ 99 & 100 \\ 7.1 & 4.7 \\ \sim 2 & \sim 2\end{array}$




\section{MELCOR Input Model}

The MELCOR input model used for these CSE containment spray experiment calculations is shown in Figure 3.1. The MELCOR model had 7 control volumes ( 6 for the containment test vessel and 1 for the environment), 5 flow paths (all internal to the test vessel), and 18 heat structures (12 internal to the test vessel and 6 representing the vessel exterior walls). Two sprays were defined, one for the (multiple) fresh sprays and one for the spray recirculating from the test vessel sump. Twelve tabular functions were used: 9 for material properties (for steel, paint and insulation), 2 to define the fresh and recirculating spray flow rates, and 1 to define the fission product injection. Over a hundred (106) control functions were used: 2 to model the external steam feed mass and energy sources, 13 to model the spray injection (flow rates, temperature, etc.), 1 to define the fission product injection and 90 to track the cesium and uranium oxide particle and iodine inventories in the various vessel subcompartments. A listing of the input for the test A-9 base case calculation is included as an appendix, for reference.

Five control volumes are used in the test vessel, representing the dome above and below the spray injection ring, the lower drywell region, the middle room, the lower room (also referred to as the sump volume), and the wetwell (which in this problem is isolated from the rest of the vessel). The other control volume is a time-independent volume used to model a constant, ambient environment serving as the outer boundary volume for the vessel walls. All control volumes were specified to use nonequilibrium thermodynamics and were specified to be vertical volumes.

Five flow paths were provided for flow within the test vessel: from the doine to the lower drywell, from the dome to the middle room, from the middle room to the lower room, between the two dome regions (divided at the spray injection elevation) and for recirculation from the lower drywell up to the upper vessel dome. Most of the junctions were defined to be either normal vertical flow paths or normal horizontal flow paths as determined by the system geometry. SPARC bubble rise physics was turned off at all junctions (the default). The recirculation flow path was provided to allow for the fact that in the actual facility there can be downflow in some fraction of the dome with upflow in the remaining dome region. The area of that recirculation flow path was set to $1 \mathrm{~m}^{2}$, about $10 \%$ of the total cross-sectional flow area in the dome. (Studies showed little or no sensitivity to the exact value of the flow area used, as long as a non-zero recirculation flow path area was used.)

All heat structures used the steady-state temperature-gradient self-initialization option. The heat structures were specified to use the "external" set of heat transfer coefficient correlations with the heat structure length or height input as the characteristic length. The critical pool fractions for pool and atmosphere heat transfer were set to 0.0 and 1.0 respectively. Radiation heat transfer between structure and atmosphere was modelled, with the emissivities set to 0.80 .

The default radionuclide class structure and properties in the MELCOR RN package were used. The fission product simulants were specified to be class $2(\mathrm{CsOH})$, class $4\left(\mathrm{I}_{2}\right)$ 


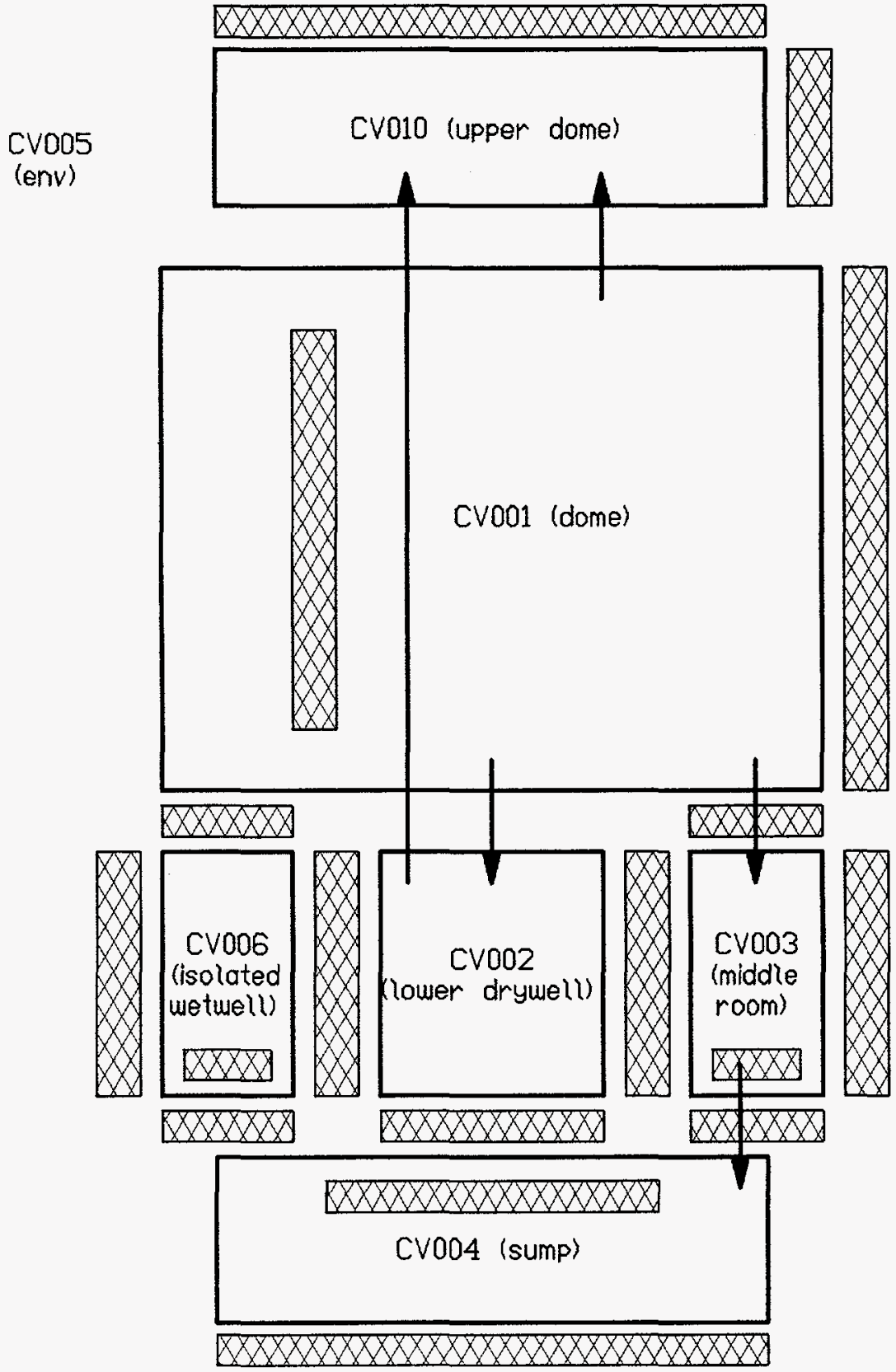

Figure 3.1. Reference MELCOR Model for CSE Containment Spray Experiments Test Vessel 
and class $10(\mathrm{U})$, and the water droplets were in class $14\left(\mathrm{H}_{2} \mathrm{O}\right)$. Most of our CSE analyses were done specifying one MAEROS component (the default), with 10 aerosol distribution size bins from $0.1 \mu \mathrm{m}$ to $50 \mu \mathrm{m}$. (Sensitivity studies were done with separate MAEROS components for each class, as discussed in Section 7.1, and with both 5 and 20 aerosol distribution size bins, as discussed in Section 7.2.) The fission product simulants were assumed to be nonradioactive and no decay heat (DCH) package input was included.

The aerosol density in the base case calculations was set to $2500 \mathrm{~kg} / \mathrm{m}^{3}\left(2.5 \mathrm{~g} / \mathrm{cm}^{3}\right)$. Because MELCOR allows only a single aerosol density (regardless of how many MAEROS components are specified), that aerosol density must represent two quite different particle sets. The value used is intended to represent a compromise between the cesium particles, with $\mathrm{CsOH}$ having a nominal density of just under $1.8 \mathrm{~g} / \mathrm{cm}^{3}$ (and CsI having a density of $3.14 \mathrm{~g} / \mathrm{cm}^{3}$ ), and the uranium oxide particles, with $\mathrm{U}_{3} \mathrm{O}_{8}$ having a nominal density of just under $8 \mathrm{~g} / \mathrm{cm}^{3}$. The default aerosol density in MELCOR is $1000 \mathrm{~kg} / \mathrm{m}^{3}=1 \mathrm{~g} / \mathrm{cm}^{3}$, i.e., water density. (To evaluate the effect of this parameter, a sensitivity study was done in which the aerosol density assumed was varied, with the results summarized in Section 7.3.)

These fission products were specified to be injected over a $10 \mathrm{~min}$ period at the start of the calculation. The cesium and uranium were injected as aerosols, while the iodine was injected in vapor form. There is not much information available in the test reports on the particle size distribution; based upon data in [19], the cesium aerosol was sourced in as a log-normal particle distribution with an average mass median diameter (AMMD) of $0.5 \mu \mathrm{m}$ and a geometric standard deviation (GSD) of 1.5, while the uranium aerosol was sourced in as somewhat larger particles using a log-normal distribution with an AMMD of $1.0 \mu \mathrm{m}$ and GSD of 1.5. (Because the data on particle size distribution is considered to have a large uncertainty, a sensitivity study was done in which the initial particle size distributions assumed were varied, with the results summarized in Section 7.4.)

For most of the tests, two sprays were defined: one representing fresh spray water from an exterior source at a constant specified temperature, and the other representing spray water recirculated from the lower room sump at local temperature. Both the spray on/off timings and the flow rates were specified using sets of control functions and tabular functions. Both the fresh and the recirculating sprays were specified to have a five-size droplet distribution (the finest resolution allowed in MELCOR), with equal numbers of droplets in each bin and with the droplet AMMD and GSD taken from the test data as given in Tables 2.2 and 2.7. The droplet size distributions used for the test A-9 base case analysis is given in Table 3.1, for reference. (A sensitivity study was done for test A-9 in which the droplet size distribution assumed was varied, with results summarized in Section 6.2.)

MELCOR assumes that the spray droplets are well mixed and interact completely with the adjacent atmosphere. In reality, some of the spray hits the vessel walls (measured to be from $1 \%$ to $11 \%$ in the various tests [19]). Also, only a fraction of the gas volume in the dome was washed by the sprays; based upon the known spray height and envelope diameter for the nozzle arrangements used, that fraction was estimated to be $50 \%$ in test A-3 and $80 \%$ in the other tests [19]. In most of our calculations, $70 \%$ of the spray 
flow rate was assumed to interact fully with the adjacent volume atmosphere, and the remainder was specified to go directly to the liquid pool. The fraction of spray interacting with the atmosphere was reduced partly to represent the fact that not all the volume cross-sectional area was washed by spray (measured to be about $80 \%$ in most tests), and partly to represent the lack of complete mixing immediately below the spray injection, where the sprays first fan out from the injection nozzles. (A sensitivity study was done in which the spray fraction assumed to interact with the dome atmosphere was varied, with the results summarized in Section 6.1.)

MELCOR currently does not include iodine chemistry modelling. However, a userinput parameter is available to define different iodine partition coefficients for different spray types, to help account for chemical interaction effects as reagents such as water and borax solution (boric acid neutralized with sodium hydroxide to a $\mathrm{pH}>9$ ) react reversibly with iodine so that equilibria are established. The partition coefficient is defined as the ratio of the concentration of iodine in the liquid droplets (elemental iodine and its reaction products) to the concentration of iodine in the gas under equilibrium conditions. It is normally much greater than 1.0 (the default value in MELCOR), and recommended best-estimate values are 5000 for sodium hydroxide and hydrazine sprays, 100,000 for sodium thiosulfate (which reacts instantaneously and essentially irreversibly with iodine) and 2500 for boric acid sprays. This parameter was set to 5000 in our base case MELCOR model. (A sensitivity study was done in which the iodine partition coefficient used was varied, with the results summarized in Section 8.1.)

The calculations were begun at $t=-18000 \mathrm{~s}(-5 \mathrm{hr})$, with $t=0$ taken as the start of the $10 \mathrm{~min}$ aerosol injection period. This was done to allow time for fog droplets to grow larger than the minimum aerosol particle size. (The effect of this is discussed in more detail in Section 7.5, which describes sensitivity studies on evaporation/condensation.)

The user-specified maximum time step in these calculations was $2 \mathrm{~s}$ during the spray injection periods and $20 \mathrm{~s}$ between the spray injection periods. The results of a time step study, both using code-determined time steps and reducing the user-specified maximum time steps further, are given in Section 9.2. The majority of these calculations were run on an HP9000 Model 755 workstation. Results of a machine-dependency study are given in Section 9.1. 
Table 3.1. Droplet Size Distribution Used in Reference MELCOR Model for CSE Containment Spray Experiment A-9

$\begin{array}{ccc}\begin{array}{c}\text { Size } \\ \text { Section }\end{array} & \begin{array}{c}\text { Diameter } \\ (\mu \mathrm{m})\end{array} & \begin{array}{c}\text { Fraction of } \\ \text { Spray Drops }(\%)\end{array} \\ & & \\ 1 & 420 & 20 \\ 2 & 580 & 20 \\ 3 & 725 & 20 \\ 4 & 980 & 20 \\ 5 & 1250 & 20\end{array}$




\section{Reference Calculation Results}

This section gives base case MELCOR assessment analysis results for CSE containment spray experiment A-9. This experiment was selected as the base case for analysis because it had the highest spray flow rates into a prototypic containment atmosphere and because it had the most test data documented [21] and thus available for comparison. Results of our MELCOR analyses simulating the other intermittent spray tests and the continuous-spray tests are given later in Section 5 .

The primary purpose of test A-9 was to demonstrate the removal of iodine and aerosol particles from containment atmospheres at a high spray flow rate $(145 \mathrm{gal} / \mathrm{min})$. The atmosphere initially was a saturated steam-air mixture at 3 bars and $394 \mathrm{~K}$, about $2 / 3$ steam and $1 / 3$ air. The iodine, methyl iodide, cesium and uranium oxide were released into the vessel and allowed to mix for $30 \mathrm{~min}$. Fresh room-temperature water with caustic ( $\mathrm{pH} 9.4$ ) boric acid was used; the spray system was operated for $3 \mathrm{~min}$, and a second $3 \mathrm{~min}$ spray and then a 10 min spray were performed. Finally, the spray liquid in the test vessel sump was recirculated for $1 \mathrm{hr}$. Figures 4.1 and 4.2 show the spray flow rates and spray temperatures for both the three fresh sprays and the recirculating spray used in test A-9. (Section 2 gives more detail on the initial and boundary conditions of the experiment.)

\subsection{Thermal/Hydraulic Response}

The effect of the sprays on containment atmosphere response is shown in Figure 4.1.1, which compares calculated MELCOR results with test data for the test vessel pressure.

The individual fresh spray periods (at 1800-1980 s, 3300-3480 s and 5400-6000 s) are predicted to cause rapid declines in the test vessel pressure, which is in qualitative agreement with test data, with the test vessel pressure recovering somewhat between sprays, owing both to the residual steam feed and to heat transfer from the walls. The pressure drops caused by the fresh spray in the calculation are visibly greater than those measured. This is probably due to the code's overprediction of steam condensation by the fresh sprays because MELCOR assumes that the spray immediately becomes wellmixed in the volume and that the only difference in velocities between the atmosphere and the spray droplets is the droplet fall velocity, which results in rapid equilibration of the droplets with the atmosphere (within $<1 \mathrm{~m}$ fall height in this problem, out of a total fall height of $\leq 10 \mathrm{~m}$ ). In reality, the spray does not mix completely in the volume immediately after leaving the nozzles, because the spray droplets disperse in a cone geometry, and because the droplets within the spray cone are partially shielded from the volume atmosphere by droplets nearer the surface of the spray cone. There may be a significant additional velocity differential owing to internal recirculation flow in the atmosphere caused by local cooling but, even though such recirculation flow can be modelled in MELCOR, the spray model does not take into account the resulting atmosphere velocity. The discrepancy is most noticable for the third spray period both because that spray period is longer than the first two and because small changes in 


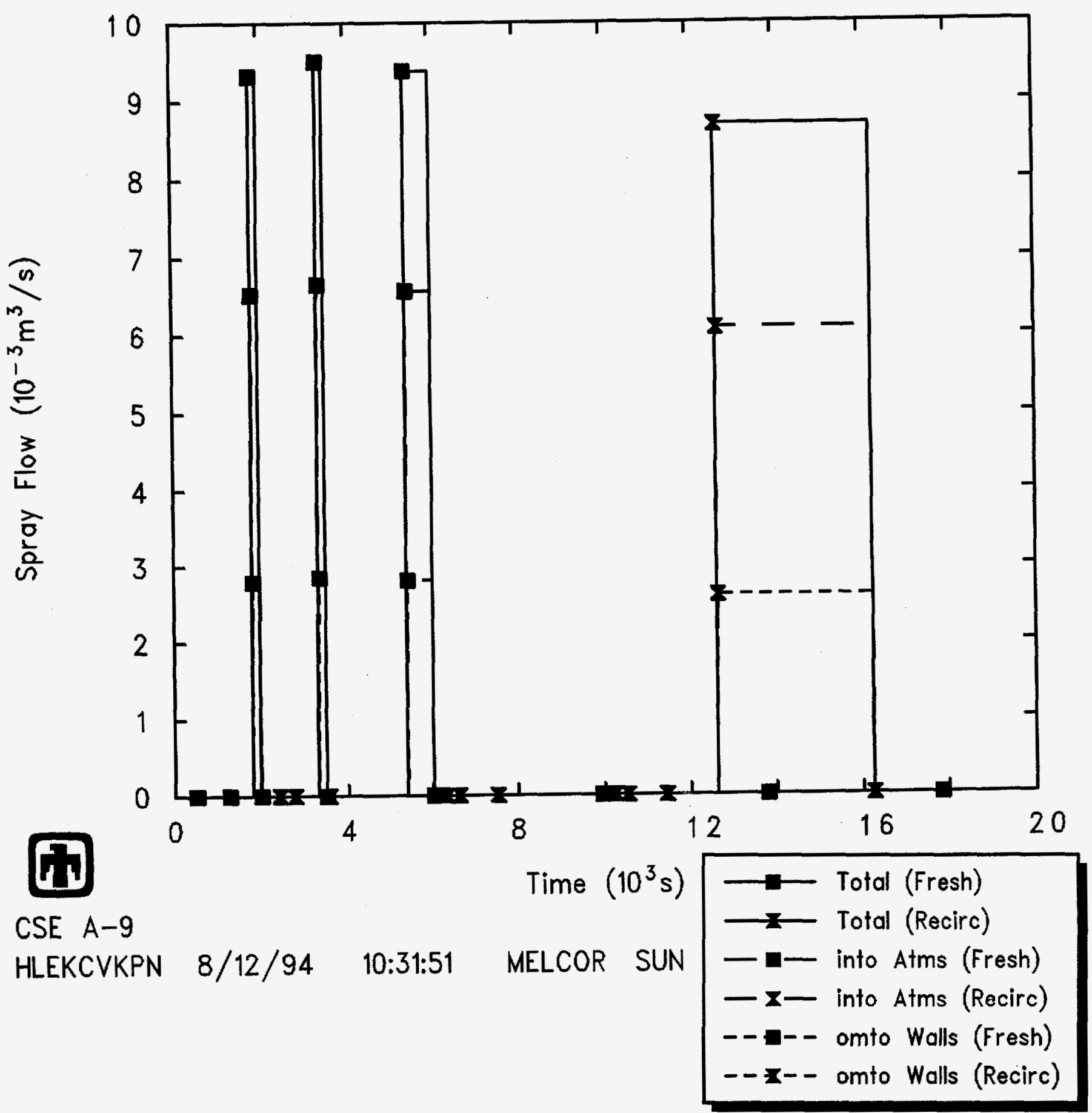

Figure 4.1. Spray Flow Rate for CSE Test A-9 - Reference Calculation 


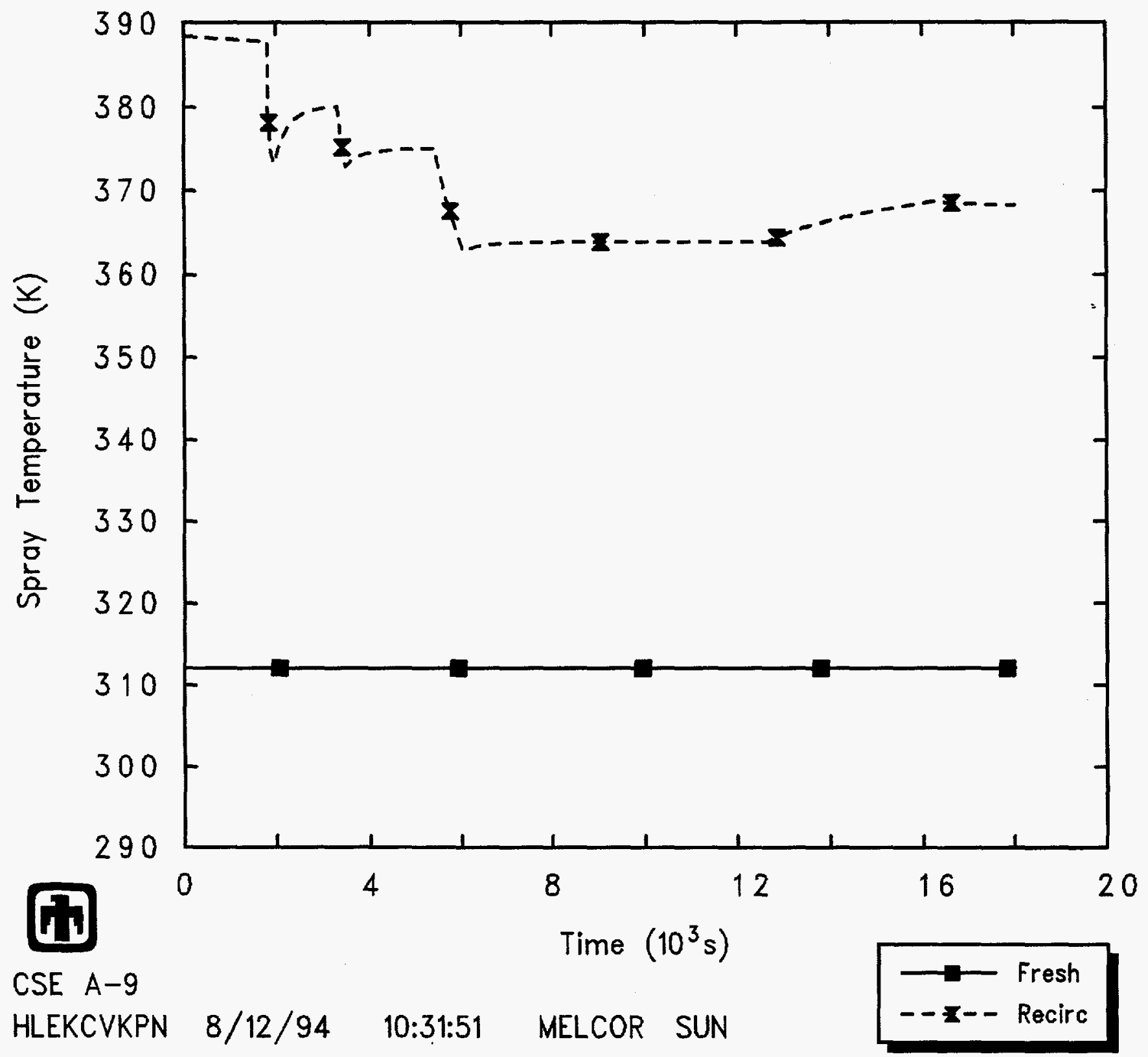

Figure 4.2. Spray Temperature for CSE Test A-9 - Reference Calculation 


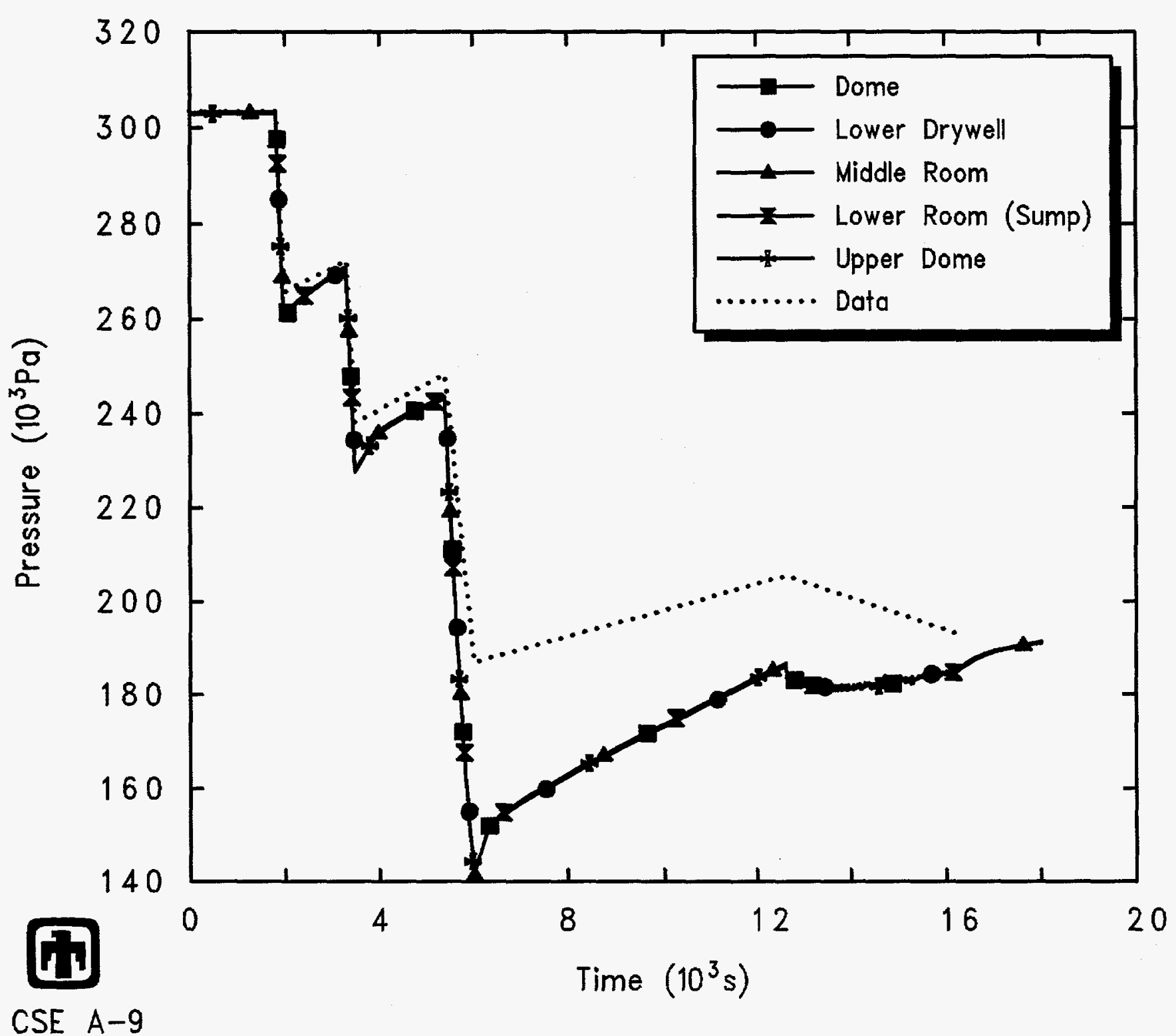

HLEKCVKPN $\quad 8 / 12 / 94 \quad 10: 31: 51 \quad$ MELCOR SUN

Figure 4.1.1. Vessel Pressure for CSE Test A-9 - Reference Calculation 
steam condensation have relatively larger effects at the lower pressures found later in this transient.

The pressure increases calculated between the fresh spray periods are also somewhat greater than measured, most noticably late in the transient after the third and longest fresh spray period at about $6000 \mathrm{~s}$; this could be caused by small differences in the continuous steam feed flow rate and/or temperature used in the experiment and the calculation, to differences in heat transfer from the interior of the vessel wall, and to differences in the residual heat loss through the insulated vessel exterior. (No exact values for the continuous steam feed were given in the test report. The enthalpy used for the steam source in the MELCOR calculation was the enthalpy of saturated steam at the initial conditions in the test vessel; the mass flow rate used for the steam source was the rate needed to offset the vessel heat loss and maintain the test vessel temperature nearly constant before the first spray began.)

That most of the pressure drop predicted in the vessel is due to condensation of steam in the atmosphere is demonstrated in Figure 4.1.2, which gives the partial pressures of steam, nitrogen and oxygen in the atmospheres of the control volumes representing the test vessel, together with the total pressures (with the experimental data included for reference). In most of the vessel, especially in the dome and lower drywell volumes, there is very little change in the partial pressure of the air (i.e., the nitrogen and oxygen); almost all of the pressure change is due to decreases in the partial pressure of steam. There is a somewhat bigger change in the partial pressures of the air in the lower volumes (the middle room and the sump), probably caused by displacement of the air from the lower volumes (middle room and sump) into the upper volumes (dome and upper dome) to maintain uniform pressure.

The effect of the sprays on containment atmosphere temperature is shown in Figure 4.1.3, which shows calculated MELCOR results compared with test data. Calculated temperatures are given for the vapor atmospheres in the five active control volumes modelling the test vessel, not including the isolated wetwell volume. Temperature histories are also included for the liquid pools accumulating in the lower drywell and lower room sump volumes. In MELCOR, the pool temperature in a control volume can be, unequal to the atmosphere temperature only when a liquid pool is present in the volume. In this problem, liquid pools accumulate only in the lower drywell and in the sump; the dome and middle room volumes do not have any significant and persistent pools, only small amounts of liquid water during and immediately after the spray injection periods which correspond to spray droplets and condensate draining to the lower drywell and sump pools.

As found for the vessel pressure, the three fresh spray periods are predicted to cause rapid declines in the test vessel temperature, in qualitative agreement with test data, with the test vessel temperature recovering somewhat between sprays, owing both to the residual steam feed and to heat transfer from the walls. The temperature increases calculated between the fresh spray periods are slightly greater than measured. This occurred with the pressures also, and is consistent with the speculation that the discrepancies between sprays are due to small differences in the continuous steam feed flow rate and/or 

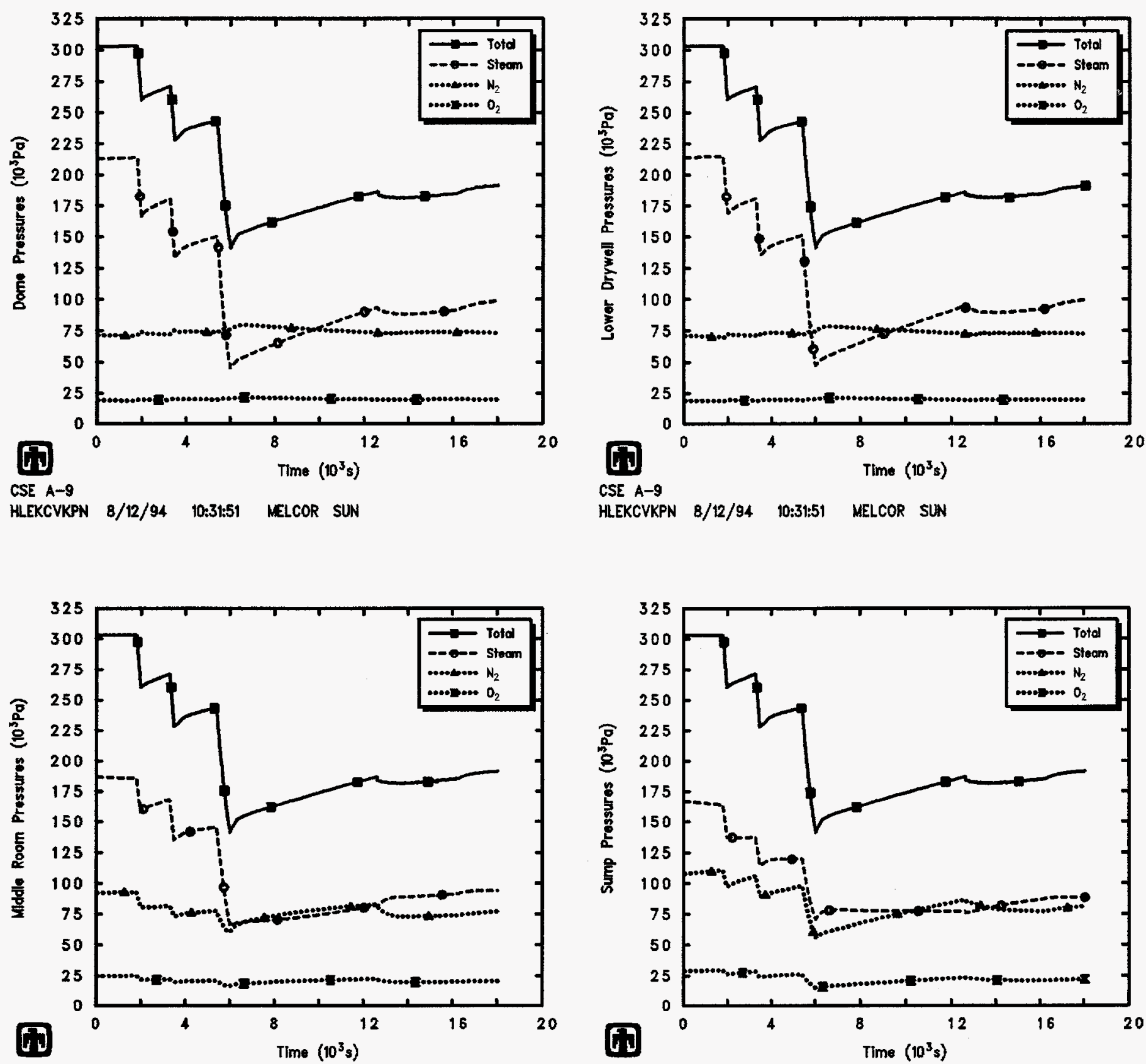

CSE A-9

HLEKCVKPN $8 / 12 / 94$ 10:31:51 MELCOR SUN

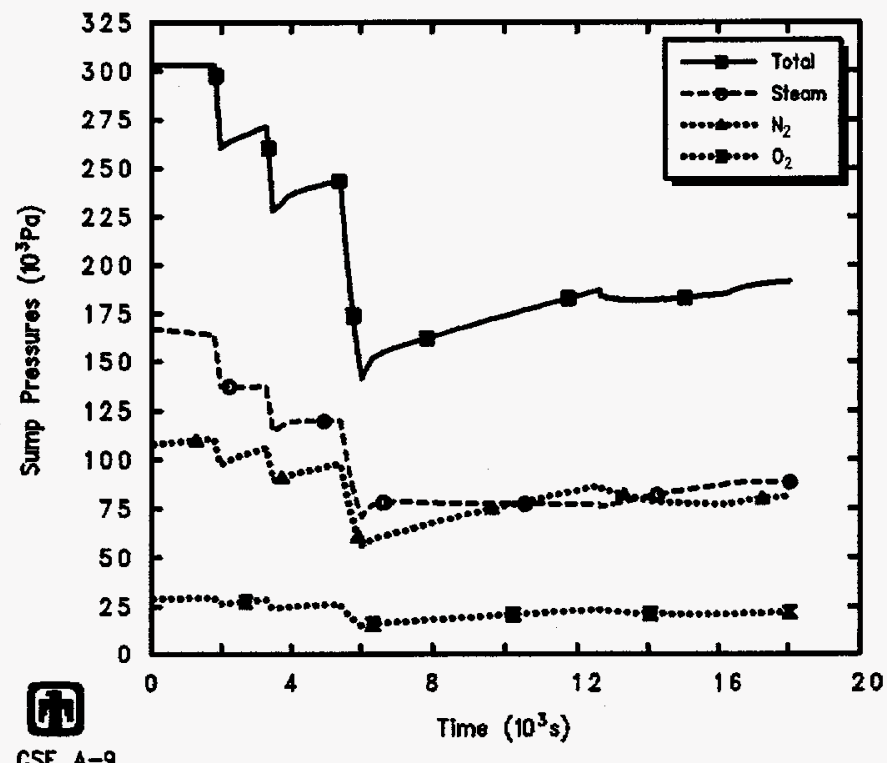

CSE A-9

HLEKCVKPN $8 / 12 / 94 \quad 10: 31: 51 \quad$ MELCOR SLN

Figure 4.1.2. Atmosphere Total and Partial Pressures in Vessel Dome (upper left), in Lower Drywell (upper right), in Middle Room (lower left) and in Vessel Sump (lower right) for CSE Test A-9 - Reference Calculation 


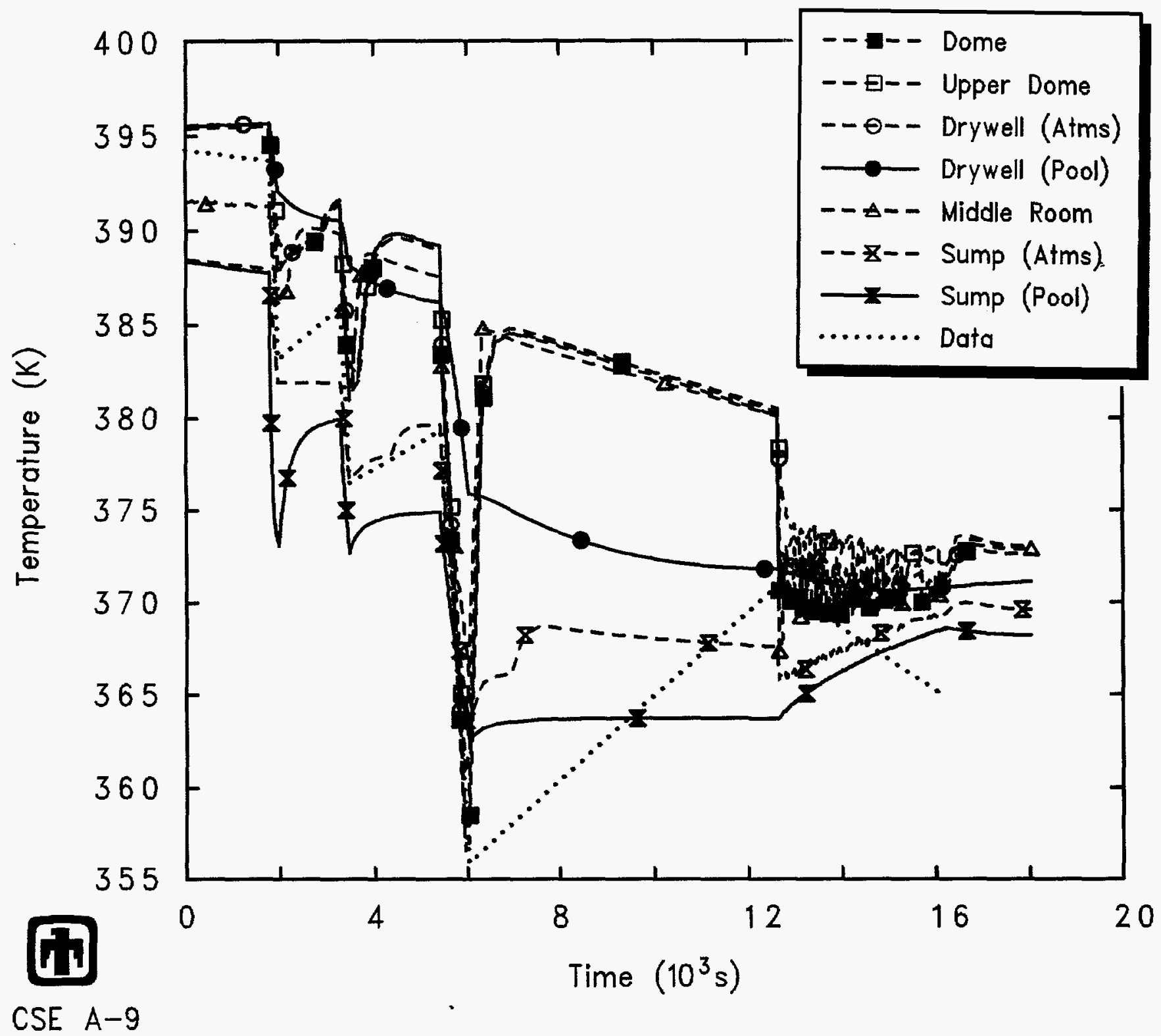

HLEKCVKPN $\quad 8 / 12 / 94 \quad 10: 31: 51 \quad$ MELCOR SUN

Figure 4.1.3. Vessel Temperatures for CSE Test A-9 - Reference Calculation 
temperature used in the experiment and the calculation, to differences in interior heat transfer and/or exterior residual heat loss, or to small differences in all of these parameters. The calculation shows a rapid temperature recovery after the temperature drops caused by fresh spray injection followed by a quasi-adiabatic period.

The recirculating spray was observed in the experiment to produce a small pressure increase and a temperature decline. The pressure increase is also found in the MELCOR results, but the calculated vessel temperatures remain nearly constant or increase slightly. This qualitative difference most likely occurs because the recirculating spray period starts with different conditions in the vessel in the experiment and in the simulation. In particular, the sump water could be cooler in the test than the vessel atmosphere because there is less interaction with the steam in the atmosphere, while in the calculation the spray droplets come to full equilibration with the vessel atmosphere; recirculating water that is at the same temperature as the atmosphere should not change the atmosphere temperature noticably, as seen in the calculation, while continually decreasing the vessel vapor temperature, as seen in the data, should only be possible by injecting cooler spray water. (Note that the recirculating spray was modelled using a new input feature added in MELCOR 1.8.3 by which a user can explicitly specify spray to be drawn from an existing control volume pool at local conditions. Thus, there is no question of potential user errors in control functions used to model the energy sink caused by depleting the sump liquid, as would have been the case with earlier versions of MELCOR.)

The test data included in this figure represent the arithmetic average reading of 5 thermocouples located in the main room (dome) vapor space. The test data show a linear increase between sprays because test data for the vessel temperature were available only for time points corresponding to the beginning and end of spray injection; the data curve should not be interpreted as demonstrating linear response between sprays. The pressure measurement reflects global vessel conditions because the pressure differentials between different parts of the vessel are very small; the temperature measurement, in contrast, represents only localized conditions near the thermocouples and may not reflect the average response in either the dome or the remainder of the vessel. In fact, the given temperature data cannot represent isothermal conditions in all regions in the test vessel because saturation at those temperatures added to a partial pressure of air proportional to the temperatures corresponds to pressures below those measured. The pressure and temperature data can be reconciled by assuming that part (less than half) of the test vessel system remains at temperatures closer to the initial temperature (394 K) than to the cooler temperatures in the dome in the thermocouple data (which represent a region efficiently cooled by spray injection).

The calculation indicates that most of the control volumes representing the test vessel are at about the same temperature, except for the atmosphere in the lower room, which is cooled by the accumulating sump pool.

Figures 4.1.4 through 4.1.7 present the atmosphere and pool temperatures in the control volumes representing the test vessel, together with the saturation temperature corresponding to the partial pressure of steam and local wall surface temperatures. During the spray periods, the atmosphere temperatures drop to the local steam saturation 
temperature. After the spray ends, the atmosphere temperature rapidly rises, owing to the steam makeup flow and to heat transfer from the vessel structures. In volumes with an accumulating liquid pool (the lower drywell and the lower room), the floor structure surface temperature closely follows the pool temperature. In the calculation, any heat addition is generally heat transferred from interior structures; the inner surface of the exterior test vessel cylinder is usually cooler than the adjacent volume atmosphere (in the dome and middle room).

Figure 4.1 .8 compares the water masses calculated in the liquid pools in the lower drywell and in the sump to test data. The qualitative agreement is quite good, despite the quantitative discrepancies, particularly the step increases in water mass due to the fresh spray periods, the higher fraction of water in the lower room sump relative to the lower drywell accumulation, and the transfer of some water from the lower room sump to the lower drywell by the recirculating spray late in the transient. However, the calculation indicates less water accumulating than measured, and does not reproduce the drop in lower drywell pool mass after the end of both the fresh and the recirculating sprays; it is not clear how the water pool could be redistributed in the test from the lower drywell sump to the lower room sump unless the lower drywell leaks.

\subsection{Aerosol Response}

Figure 4.2.1 presents the concentrations of cesium aerosol in various regions in the test vessel atmosphere, compared with test data; the concentrations shown are the mass of airborne aerosol in the control volume atmosphere divided by the volume. The default class description for cesium in MELCOR (i.e., class 2) includes a vapor pressure characteristic of $\mathrm{CsOH}$, so that cesium could be present in either aerosol or vapor form depending on other conditions such as volume pressure and temperature; in this calculation, the conditions are such that cesium is predicted to be present only in aerosol form despite the non-zero vapor pressure curve. (Class $2, \mathrm{CsOH}$, was used rather than class 16 , CsI, because the cesium aerosol was generated by heating cesium carbonate by means of an electrical resistance heater, and this material forms aerosols of cesium hydroxide in humid atmospheres.)

The concentrations plotted are for the test vessel dome or main room, the middle room and the lower room or sump. The calculation shows virtually equal concentrations in the dome, the upper dome above the spray injection elevation, and the lower drywell, because the recirculation flow modelled keeps these volumes well mixed.

The calculated concentrations of airborne cesium aerosols agree qualitatively with the measured concentrations. The code predicts stepwise decreases in concentration in the dome atmosphere during each of the three fresh spray periods and a more gradual, linear decline during the longer, late-time recirculation period, as observed in the test. Also, the concentrations of airborne cesium aerosols in the middle room and lower room rise gradually during the first portion of the test until they approach the concentration in the dome, after which the concentrations throughout the vessel remain nearly equal as they drop uniformly. 


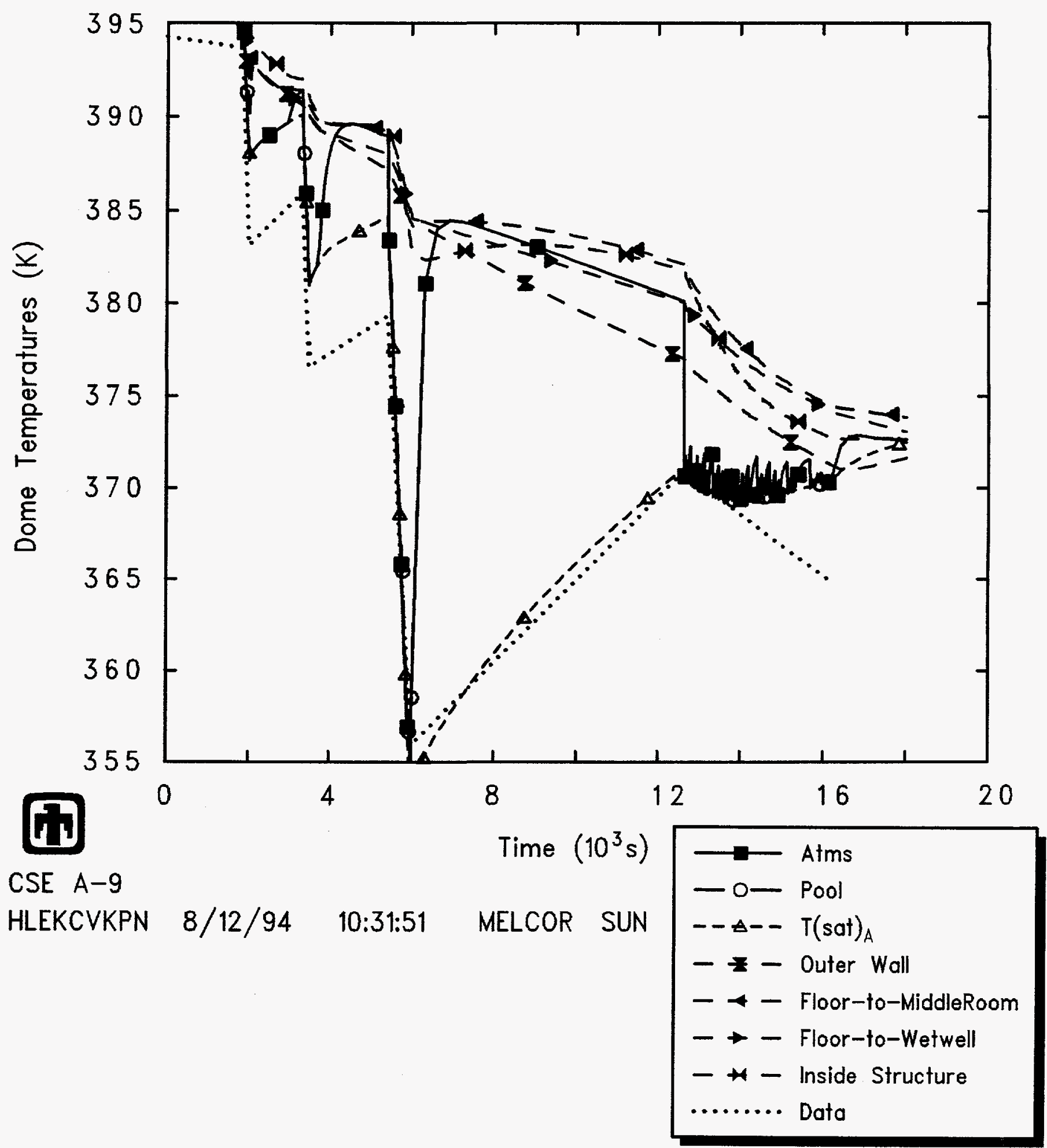

Figure 4.1.4. Atmosphere, Pool, Saturation and Wall Temperatures in Vessel Dorne for CSE Test A-9 - Reference Calculation 


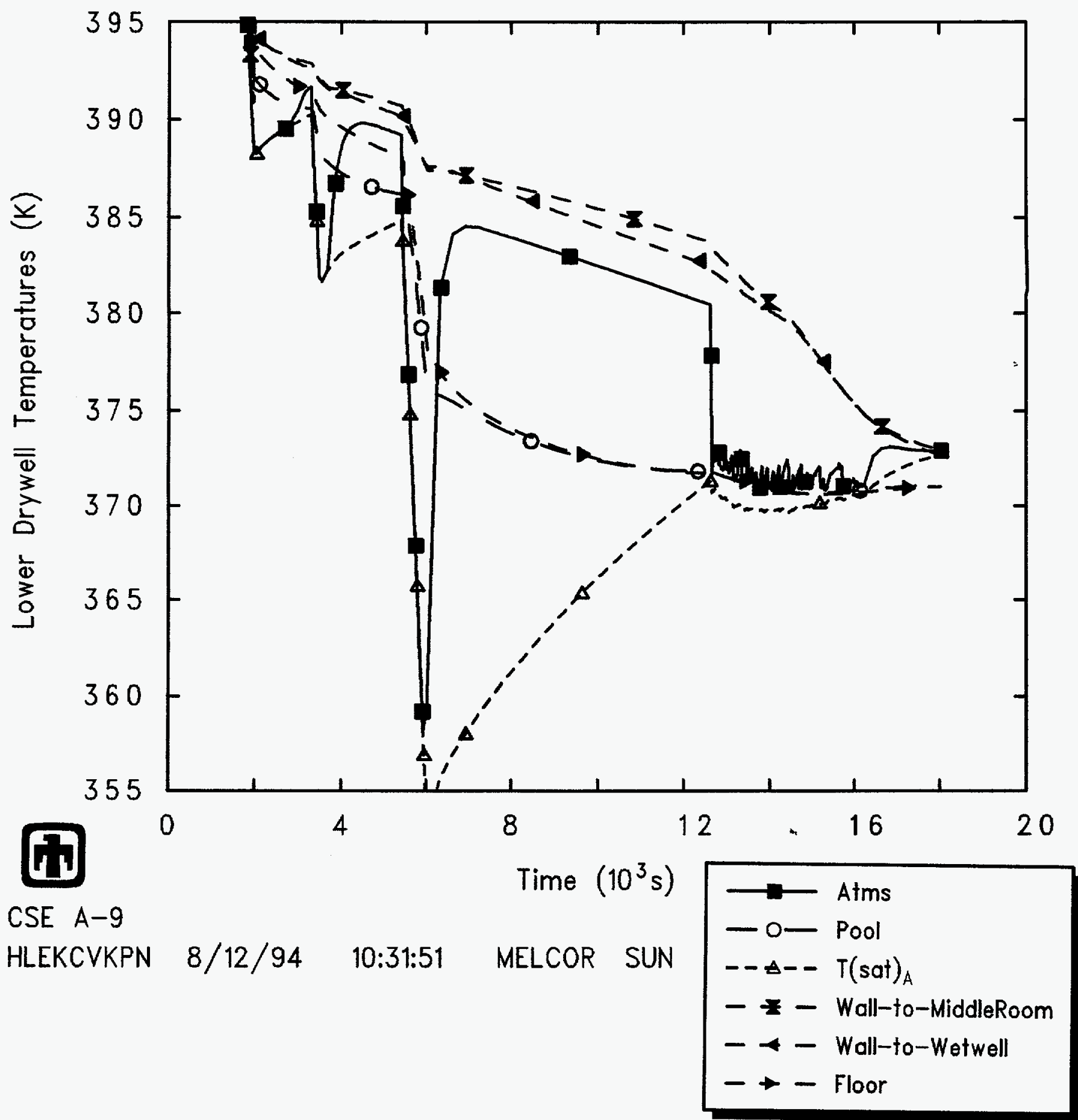

Figure 4.1.5. Atmosphere, Pool, Saturation and Wall Temperatures in Lower Drywell for CSE Test A-9 - Reference Calculation 


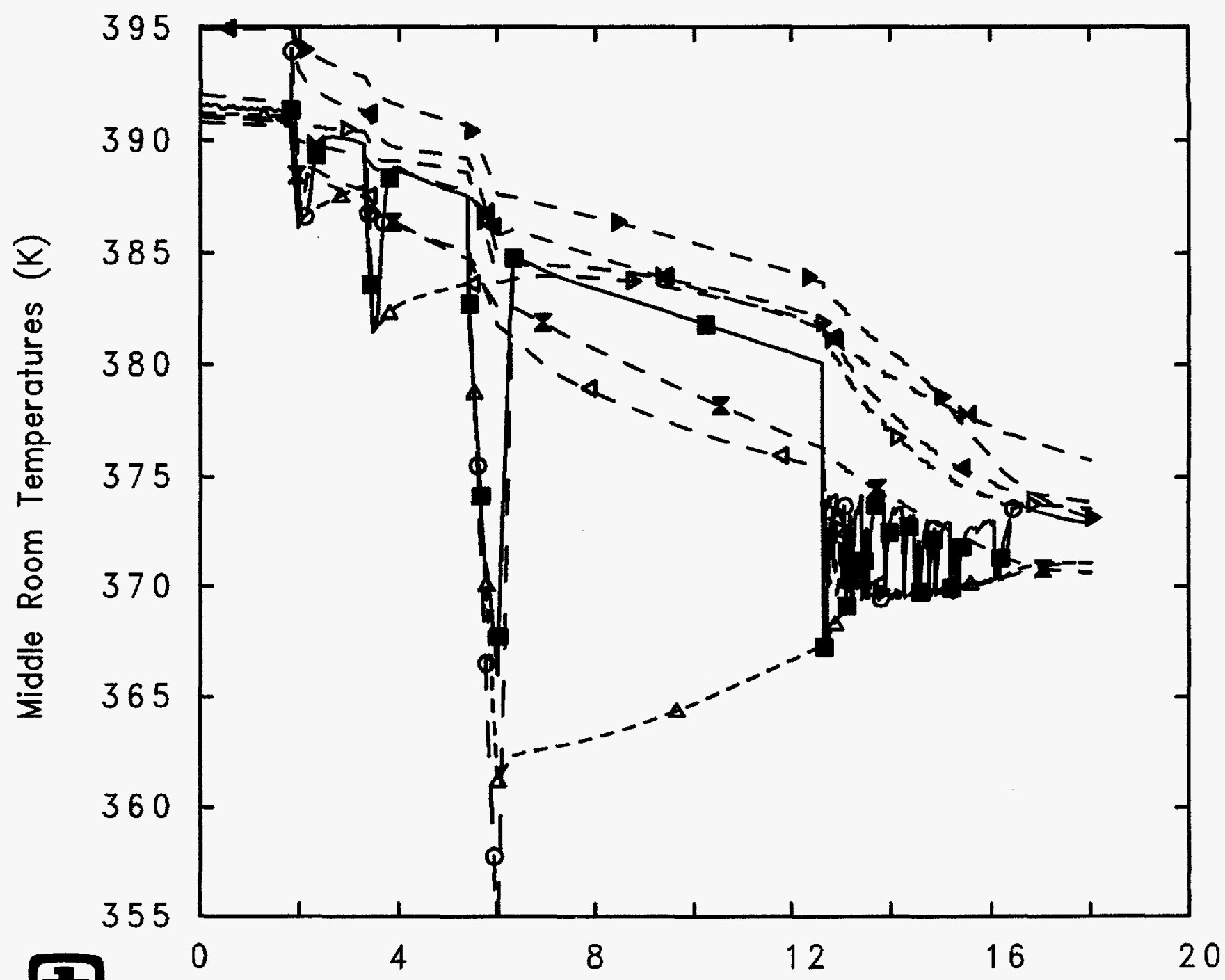

CSE A-9

Time $\left(10^{3} \mathrm{~s}\right)$

$$
\begin{aligned}
& \longrightarrow \text { Atms } \\
& -0-\text { Pool } \\
& --\rightarrow--T(\text { sat })_{A} \\
& -\neq- \text { Outer Wall } \\
& -\rightarrow-\text { Roof } \\
& -\rightarrow-\text { Inner Wall } \\
& -\rightarrow-\text { Wall-to-Wetwell } \\
& -\rightarrow-\text { Floor } \\
& -\rightarrow-\text { Inside Structure }
\end{aligned}
$$

Figure 4.1.6. Atmosphere, Pool, Saturation and Wall Temperatures in Middle Room for CSE Test A-9 - Reference Calculation 


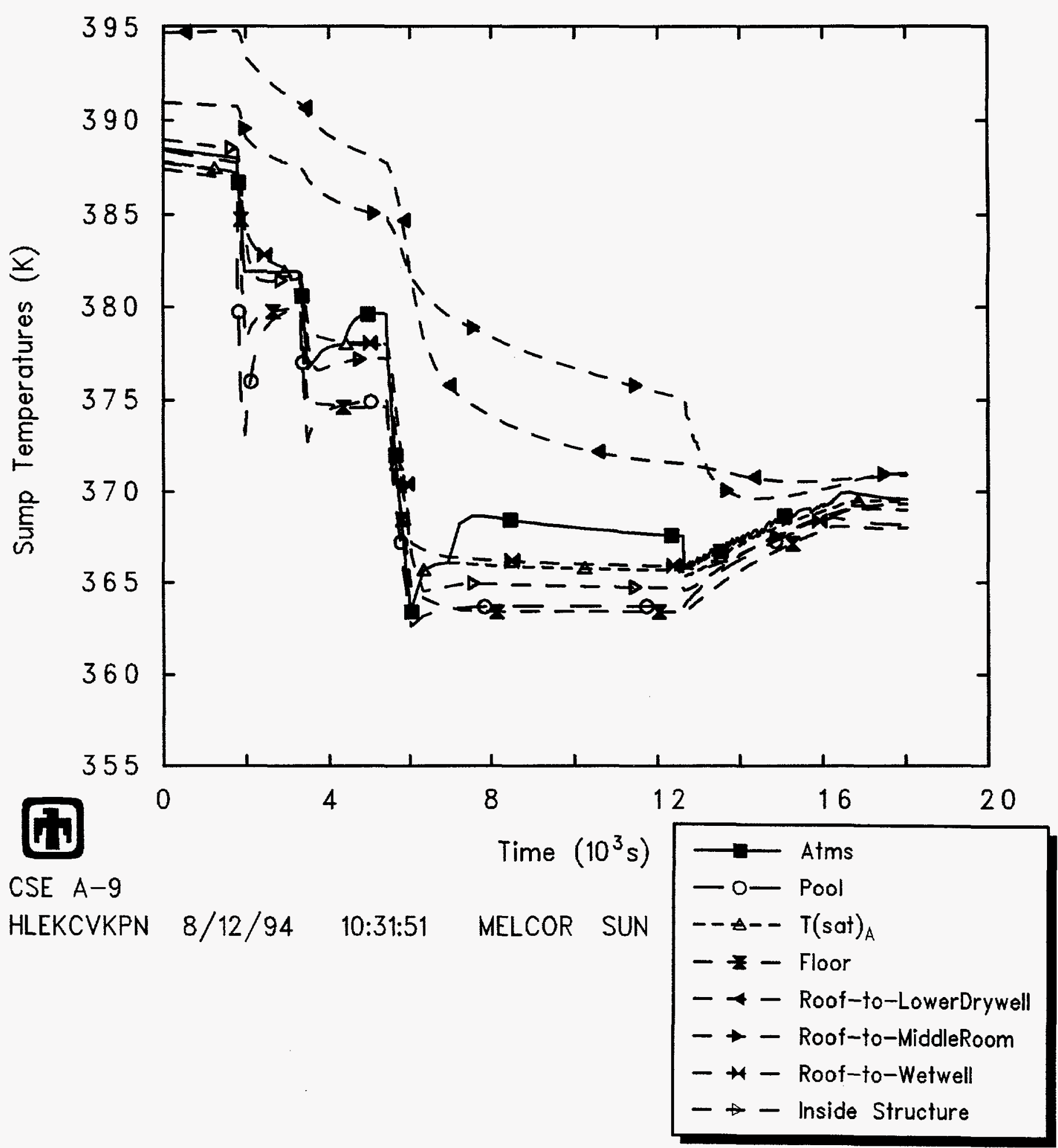

Figure 4.1.7. Atmosphere, Pool, Saturation and Wall Temperatures in Lower Room for CSE Test A-9 - Reference Calculation 


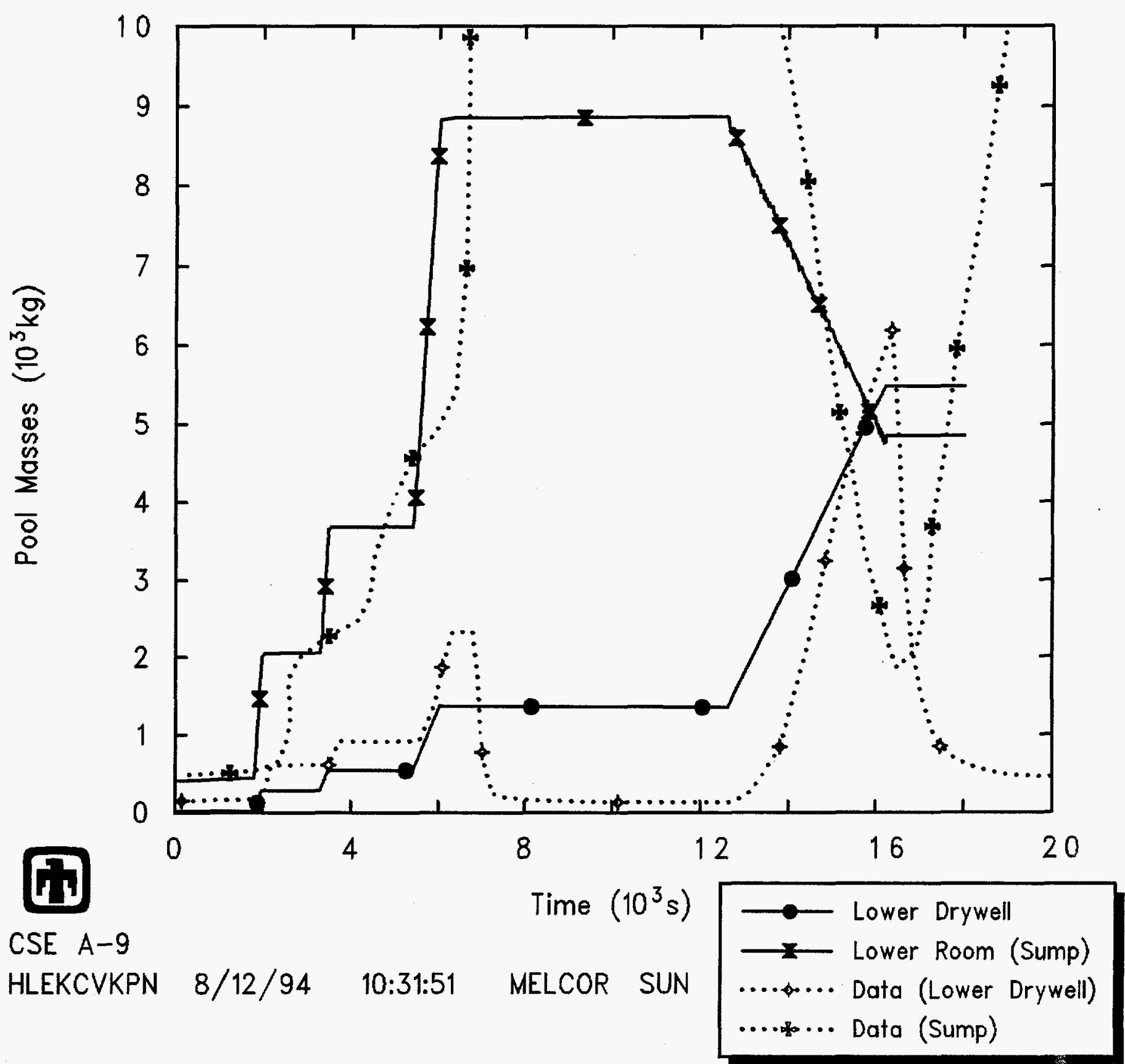

Figure 4.1.8. Lower Drywell and Sump Pool Masses for CSE Test A-9 - Reference Calculation 


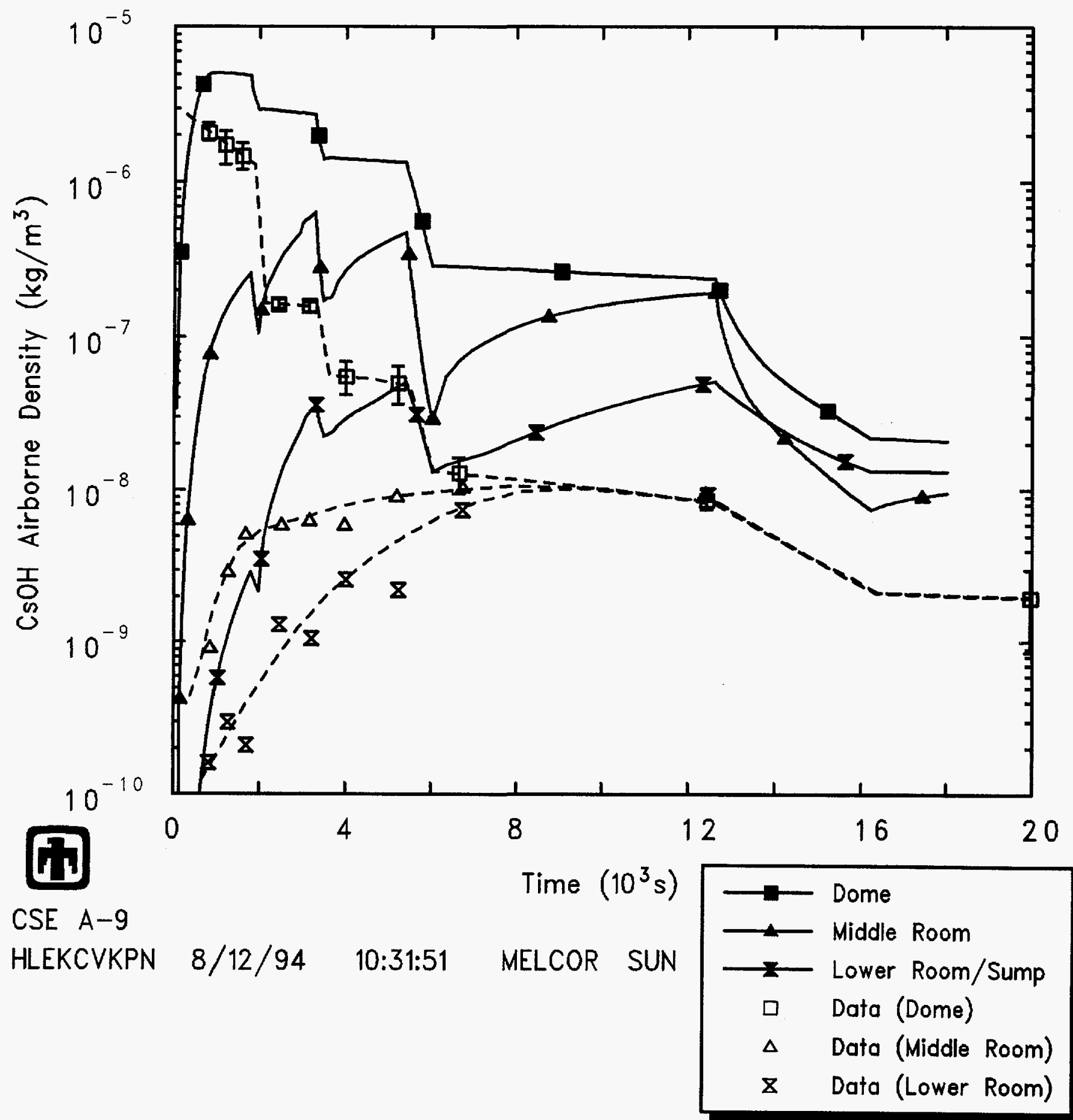

Figure 4.2.1. Cesium Aerosol Airborne Concentrations for CSE Test A-9 - Reference Calculation 
There are, however, a number of significant quantitative discrepancies in the calculated aerosol response compared with measured test data.

The calculated concentration of airborne cesium aerosols in the dome remains very nearly constant between spray periods, while the test data show a substantial decline prior to the first spray period. Further, the stepwise decreases in concentration predicted in the dome atmosphere during each of the fresh spray periods are about equal, with slightly more aerosol removal with each successive fresh spray, while the test data show much more aerosol removal during the first spray period than during subsequent sprays. Finally, the airborne concentrations in the middle and lower rooms equilibrate with the dome concentration at a much higher value in the calculation than was measured; this is simply because the calculation predicted less aerosol removal by sprays and by natural processes such as settling than was observed in the experiments.

The experimental data on spray effectiveness also has been analyzed in terms of the concentration half-lives, and the resulting washout constants for cesium aerosols are listed in Table 4.2.1. The observed half-lives during the spray periods were corrected for natural removal processes [19]; no such modifications were made to the calculated half-lives predicted in the calculation because the natural removal rates were extremely slow. (Two values are given for the washout during the recirculating spray because the MELCOR calculation indicates a substantial decline in washout during the long recirculating spray period.)

A comparison of the tabulated half-lives and washout coefficients confirms the conclusion drawn from the plotted cesium aerosol airborne concentrations in Figure 4.2.1 that the code significantly underpredicts the removal of cesium aerosols from the test vessel atmosphere compared with test data, both during sprays and between spray periods. The test data show consistently less removal during later spray periods and later in the transient, while the MELCOR aerosol removal rates appear more random during and between the various spray periods, with no obvious pattern emerging.

Figure 4.2.2 presents the calculated concentrations of uranium aerosol in the test vessel atmosphere, compared with experimental data. (The concentrations shown are the mass of airborne aerosol in the control volume atmosphere divided by the volume.) Results are shown for the main room or dome, the middle room, and the lower room sump. The MELCOR results for the upper dome control volume (above the spray injection elevation) and for the lower drywell are very similar to the results given for the dome, because the recirculation flow modelled keeps these volumes well mixed. The default class description for uranium in MELCOR (i.e., class 10) has a zero vapor pressure for $\mathrm{T}<3000 \mathrm{~K}$, so that uranium is predicted to be present only in aerosol form regardless of other conditions.

There are some obvious problems with the uranium aerosol airborne concentration measurements. The initial concentration of uranium is given as $5 \mathrm{mg} / \mathrm{m}^{3}$, which is equal to the initial cesium aerosol airborne concentration $[14,21]$; however, the uranium aerosol airborne concentration measured prior to the first spray period as given in [21] is about an order of magnitude lower than the corresponding cesium aerosol airborne concentration given for the same test. The uranium aerosol airborne concentrations measured prior to 


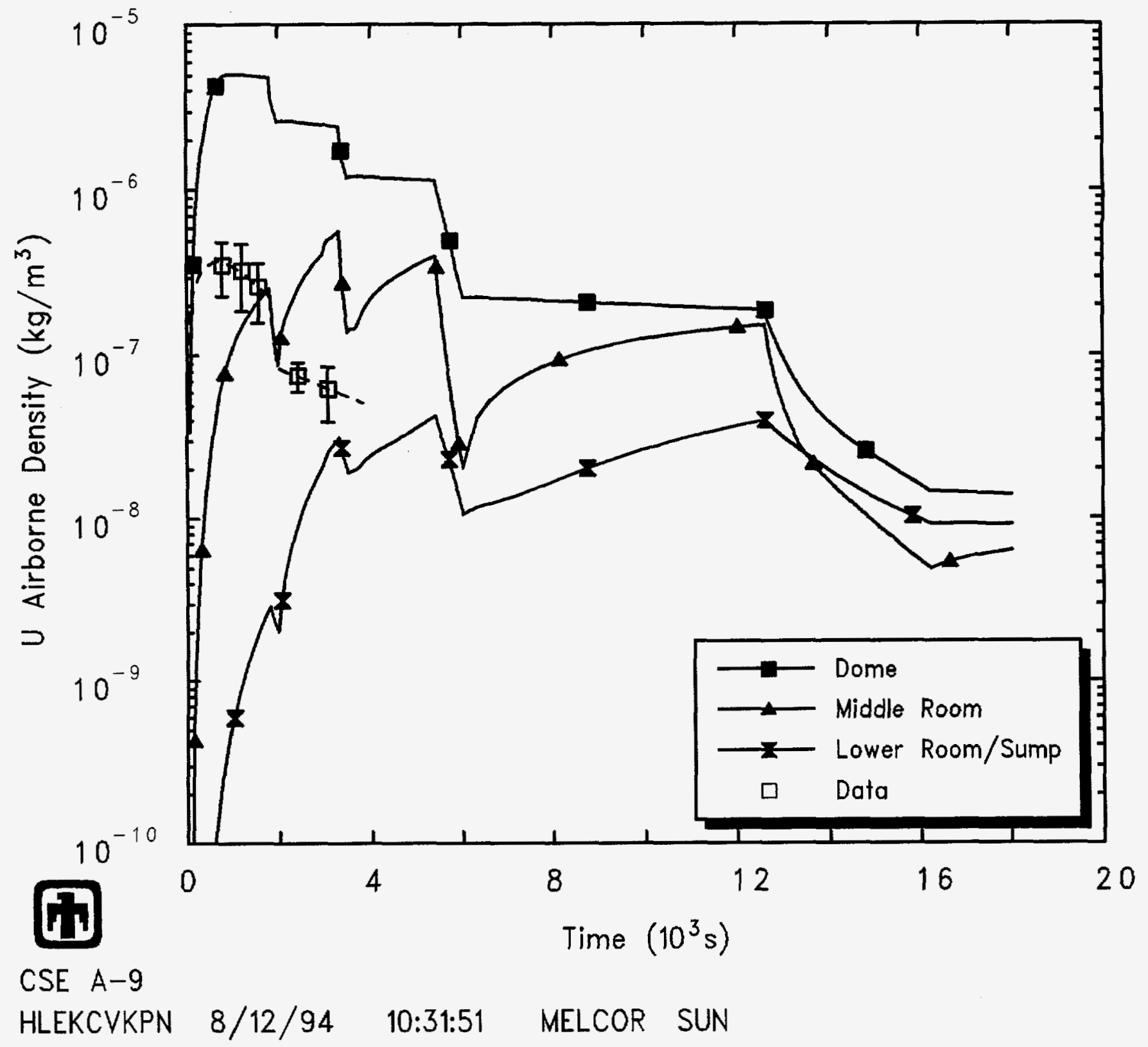

Figure 4.2.2. Uranium Aerosol Airborne Concentrations for CSE Test A-9 Reference Calculation 
the first spray period for other CSE tests [19] are generally at least an order of magnitude higher than the results given for test A-9 and more sinilar to the corresponding cesium aerosol airborne concentrations given. Since the aerosol source was nominally the same in all the intermittent-spray tests and since the only major difference between test A-9 and tests A-6, A-7 and A-8 is in the spray conditions, there is obviously an inconsistency between the given initial concentration and the early-time measurement. Also, note that samples were not analyzed for uranium after the first spray period.

The predicted response of the uranium aerosol closely resembles that already presented for the cesium aerosols. The calculated concentrations of airborne uranium aerosols agree qualitatively with the measured concentration, but there are a number of quantitative discrepancies. The calculated concentration of airborne uranium aerosols in the dome remains very nearly constant between spray periods, while the test data show a substantial decline prior to the first spray period. Also, the test data show much more aerosol removal during the first spray period than predicted in the calculation.

The concentration half-lives, and the resulting washout constants for uranium aerosols are listed in Table 4.2.2. The larger initial AMMD for the uranium aerosol compared with the cesium aerosol is reflected in the shorter half-lives and higher washout rates before and during the first spray period. The nearly equal size distributions for the cesium and uranium aerosols later in the transient are reflected in the very similar half-lives and washout rates for the uranium and cesium aerosol airborne concentrations later in the test period.

The observed significant decline in cesium aerosol airborne concentration owing to natural removal in the absence of sprays is seen only before the first spray period, and is probably due to the settling of aerosol particles growing by rapid condensation of steam onto aerosols in the humid vessel atmosphere. The lack of a further noticable decline in cesium aerosol airborne concentration due to natural removal between spray periods is probably due to less condensation potential after the first spray has cooled the vessel atmosphere somewhat. The rapid drop observed during the first spray period is due to the spray efficiently washing out selected particle sizes; the substantially slower removal of aerosols by later sprays is most likely due to the changed airborne aerosol particle distribution, with only particles left which the spray is less efficient in removing.

There are very few experimental data on particle size distribution in the CSE containment spray tests. Based on data from earlier tests in the CSE series on natural removal of suspended cesium and uranium aerosol particles [22], significantly larger particles (up to $16 \mu \mathrm{m}$ diameter) would be required to match the observed natural depletion rates such as those found before the first spray period. Particle size was measured by cascade impactors at selected intervals during the spray tests; for test A-9, the measured mass median particle diameter was $0.5 \mu \mathrm{m}$ for cesium and $0.6 \mu \mathrm{m}$ for uranium before the first spray period, and $0.4 \mu \mathrm{m}$ for both cesium and uranium after the second spray period [14]. At longer times, more of the material penetrated the impactor, indicating a shift to smaller sizes.

The limited test data indicate that initially the particles exist as large fog drops in the humid test vessel atmosphere. The introduction of a cold spray reduces the relative 
Table 4.2.1. Cesium Aerosol Washout Rates for CSE Test A-9 - Reference Calculation

$$
t_{1 / 2}(\min ) \quad \lambda_{s}\left(\min ^{-1}\right)
$$

Measured Calculated Measured Calculated

During first spray period

During second spray period

During third spray period

During fourth spray period

Prior to first spray

Between sprays 1-2

Between sprays 2-3

Between sprays $3-4$
1.08

2.0

5.4

33

24

145

170

180
5.0

4.6

4.3

$6.9-34.7^{a}$

130

300

525

400
0.643

0.34

0.13

0.021

0.0289

0.0048

0.0041

0.0039
0.14

0.15

0.16

$0.10-0.02^{a}$

0.005

0.002

0.001

0.002

${ }^{a}$ at start of spray and at end of spray

Table 4.2.2. Uranium Aerosol Washout Rates for CSE Test A-9-Reference Calculation

$$
t_{1 / 2}(\min ) \quad \lambda_{s}\left(\min ^{-1}\right)
$$

Measured Calculated Measured Calculated

During first spray period

During second spray period

During third spray period

During fourth spray period

Prior to first spray

Between sprays 1-2

Between sprays 2-3

Between sprays $3-4$
2.3

4.6

4.3

0.31

0.17

4.3

$6.9-34.7^{a}$

0.16

0.16

$0.10-0.02^{a}$

21

45

100

0.0330

0.007

100

0.0154

0.007

250

0.003

400

0.002

${ }^{a}$ at start of spray and at end of spray 
humidity within the containment atmosphere, causing evaporation of the fog drops and a reduction in the particle size. The particle size is then further reduced by evaporation of water caused by a decrease in relative humidity as the gas passes through the cascade impactor. This explanation accounts for the small size indicated by the impactor and the large size implied from the natural transport experiments.

MELCOR qualitatively predicts the correct behavior, with the aerosol particles initially growing in the humid atmosphere, and later shrinking as the sprays remove the larger particles. However, there are a number of quantitative discrepancies.

Figures 4.2.3 and 4.2.4 give the mass median diameter and geometric standard deviation, respectively, of the airborne cesium aerosol particle distributions in the atmospheres of the various control volumes representing the test vessel; the aerosols in the upper dome show very similar behavior to those in the main dome, and are not shown here.

Fog (i.e., water aerosol) is initially present in the dome, upper dome and lower drywell. Recall that the steam makeup source is introduced in the lower drywell, and that a recirculation flow path is modelled from the lower drywell to the upper dome, so that the dome, upper dome and lower drywell volumes are well mixed. The fog is generated from condensation of the steam makeup flow. No fog is calculated to be present in either the middle or lower rooms, which have no recirculation flow paths, except for brief times during and just after the spray periods.

The fog droplets are created at the minimum aerosol particle size, which in our input model was set to $0.1 \mu \mathrm{m}$. (There is no capability in MELCOR to vary the initial size of the fog drops except by changing the minimum aerosol particle size, which would affect all aerosol species at all times.) As noted in Section 3, these calculations were begun $5 \mathrm{hr}$ before the start of the $10 \mathrm{~min}$ aerosol injection period, to allow time for the fog droplets to grow larger than the minimum aerosol particle size. The fog droplets have grown to almost $2.5 \mu \mathrm{m}$ during that preconditioning period, and do not seem to be growing further when the transient is begun. The fog aerosol particles' AMMD drops by a factor of 2 to $1.25 \mu \mathrm{m}$ during the first spray period. The fog particles then grow again after the end of the first spray period until the system pressure and temperature drop sufficiently to cause the remaining fog droplets to be precipitated from the control volume atmosphere into the pool. At later times, no fog is calculated to be present in the dome or lower drywell except for brief times during and just after the spray periods.

The cesium particles are injected with an AMMD of $0.5 \mu \mathrm{m}$ and GSD set to 1.5 . The cesium aerosol mass median diameter increases to $\sim 1.4 \mu \mathrm{m}$ at the start of the first spray period in the volumes with fog initially present, but increases only to $\sim 0.8-0.9 \mu$ m before the first spray period in the volumes with no fog initially present. The mass median diameter of the cesium aerosol particles drops rapidly by about 10-20\% during the fresh spray periods, more in the dome and lower drywell volumes washed by the spray than in the downstream middle and lower rooms. The cesium particles grow slightly after the first spray period as long as fog is present, then remain nearly constant between spray periods. The diameter of the cesium aerosol particles drops more gradually, by $30-40 \%$, during the late-time recirculating spray period. 


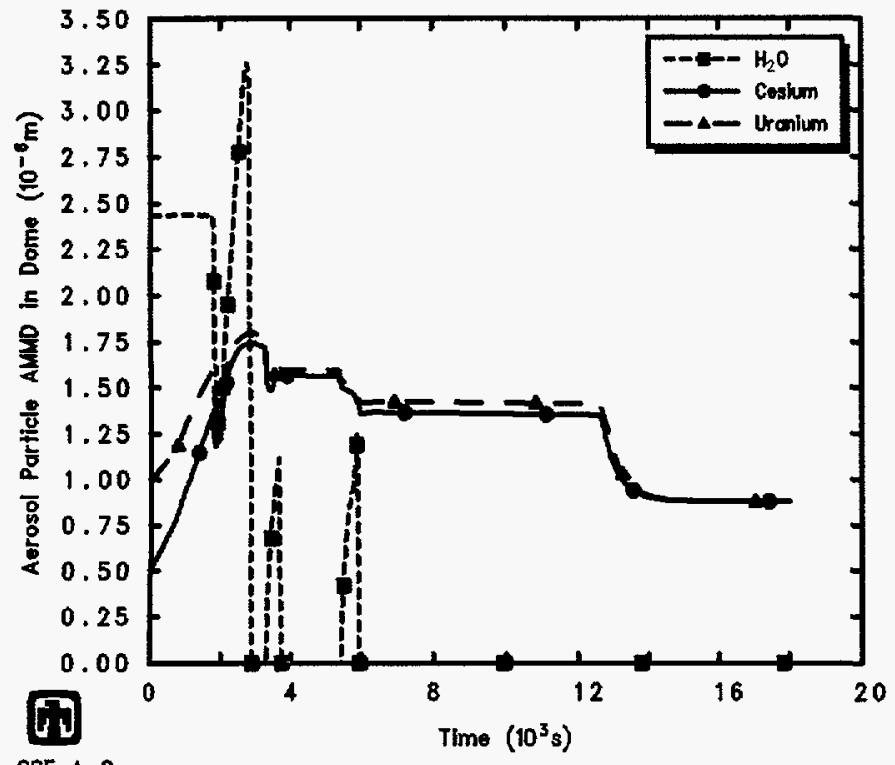

CSE A-9

HLEKCVKPN $8 / 12 / 94 \quad 10: 31: 51$ MELCOR SLN

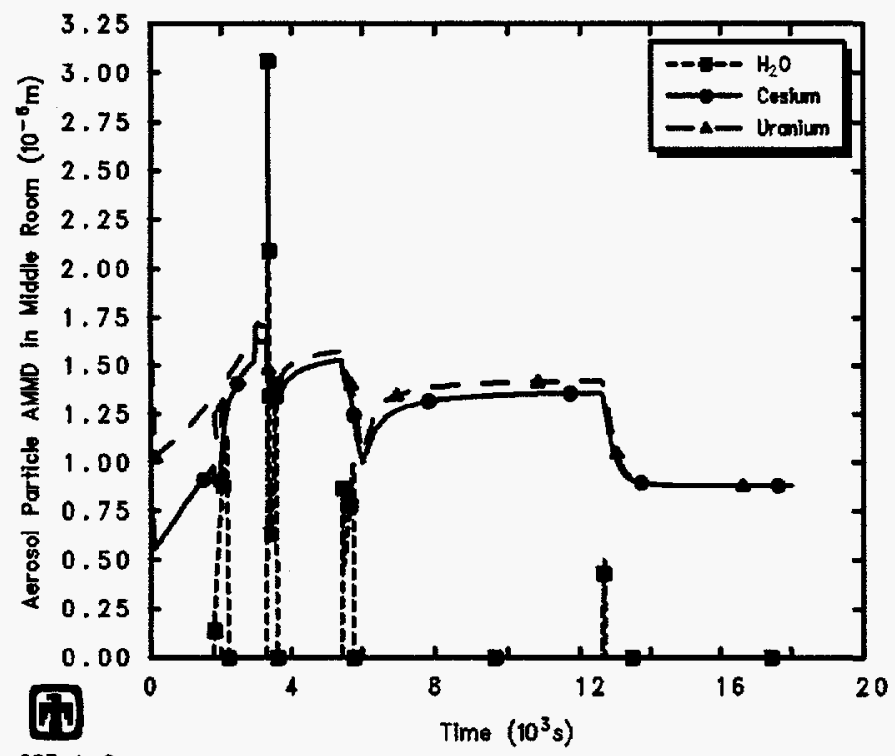

CSE A-9

HLEKCVKPN $8 / 12 / 94 \quad 10: 31: 51$ MELCOR SUN

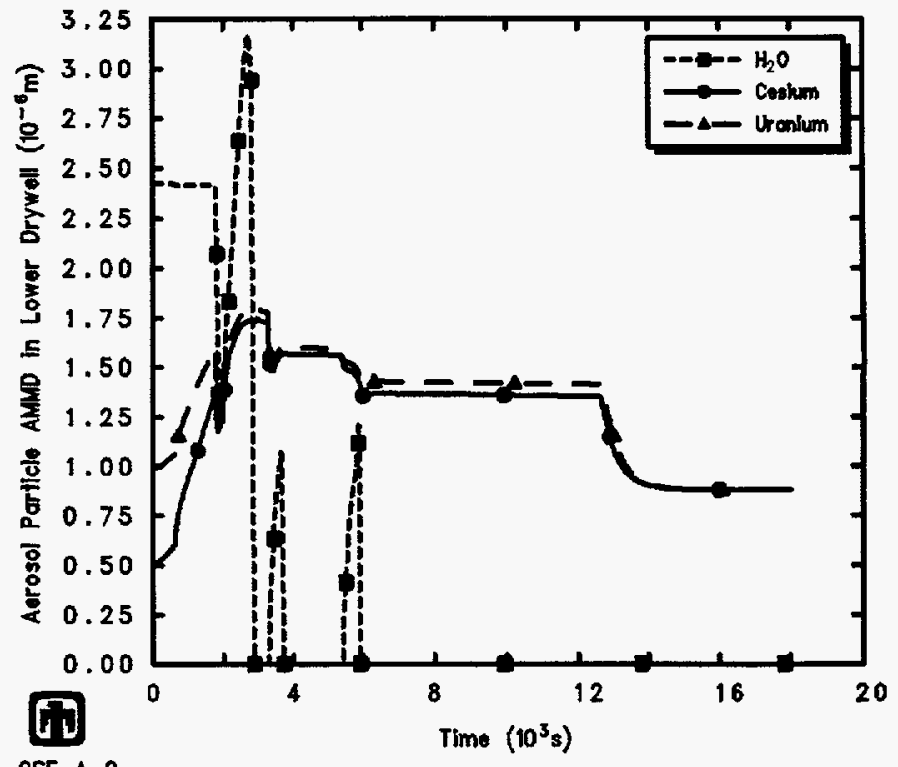

CSE A-9

HLEKCVKPN $8 / 12 / 94 \quad 10: 31: 51$ MELCOR SUN

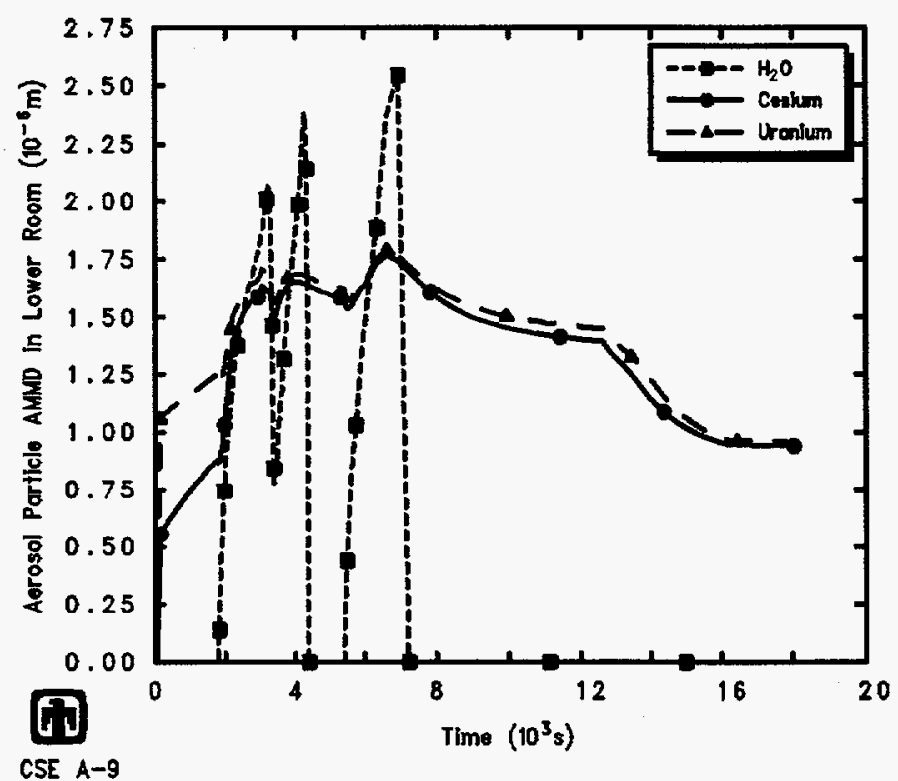

HLEKCVKPN $B / 12 / 94 \quad 10: 31: 51$ MELCOR SUN

Figure 4.2.3. Airborne Aerosol AMMDs in Vessel Dome (upper left), in Lower Drywell (upper right), in Middle Room (lower left) and in Vessel Sump (lower right) for CSE Test A-9-Reference Calculation 


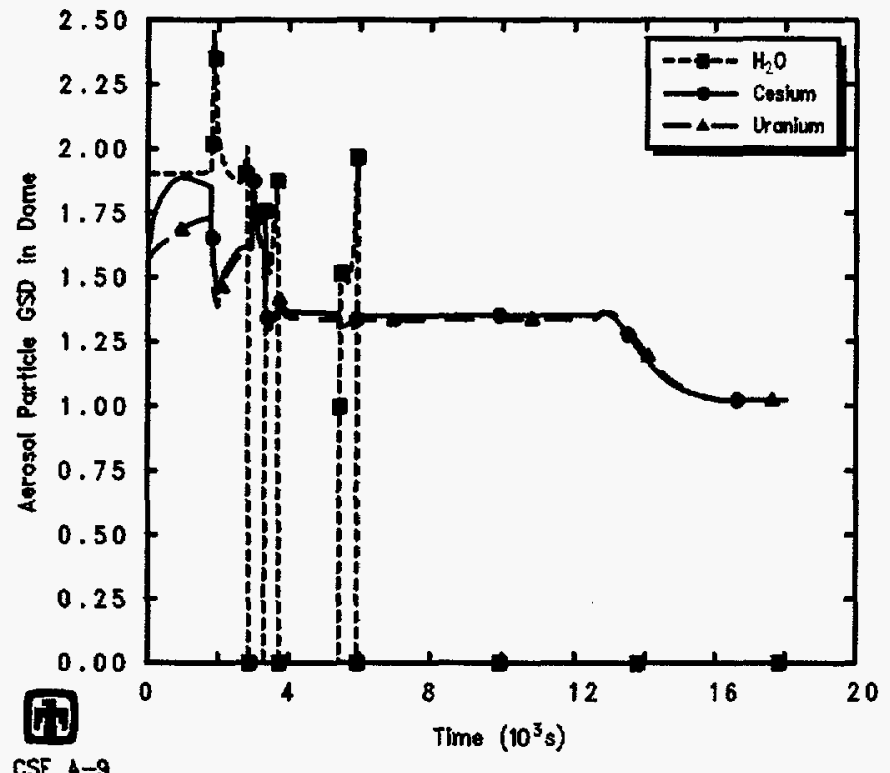

CSE A-9

HLEKCVKPN $8 / 12 / 94$ 10:31:51 MELCOR SUN

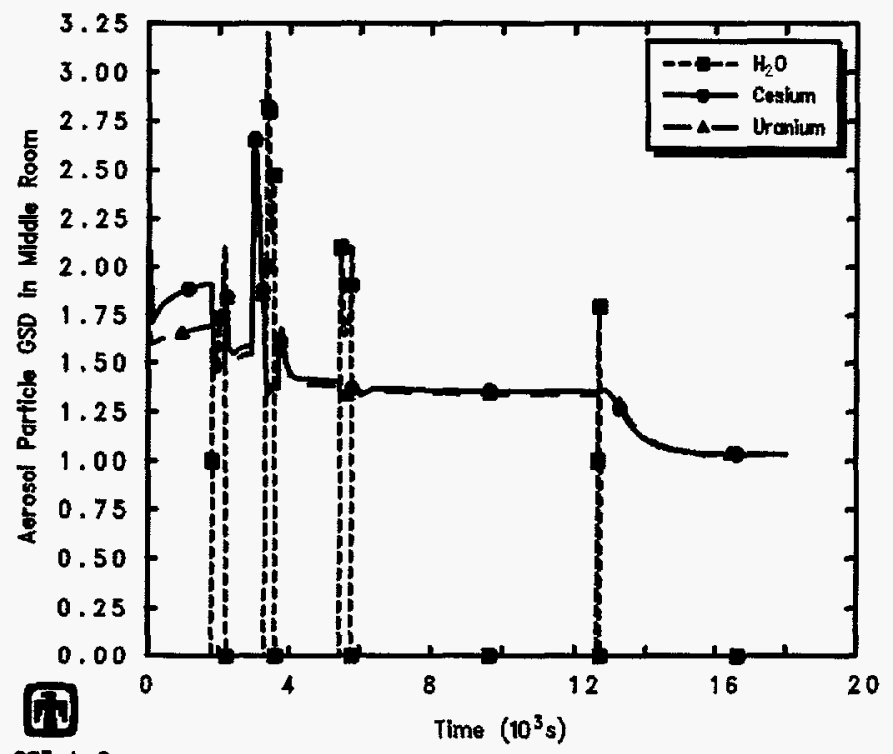

CSE A-9

HLEKCVKPN $8 / 12 / 94 \quad 10: 31: 51$ MELCOR SUN

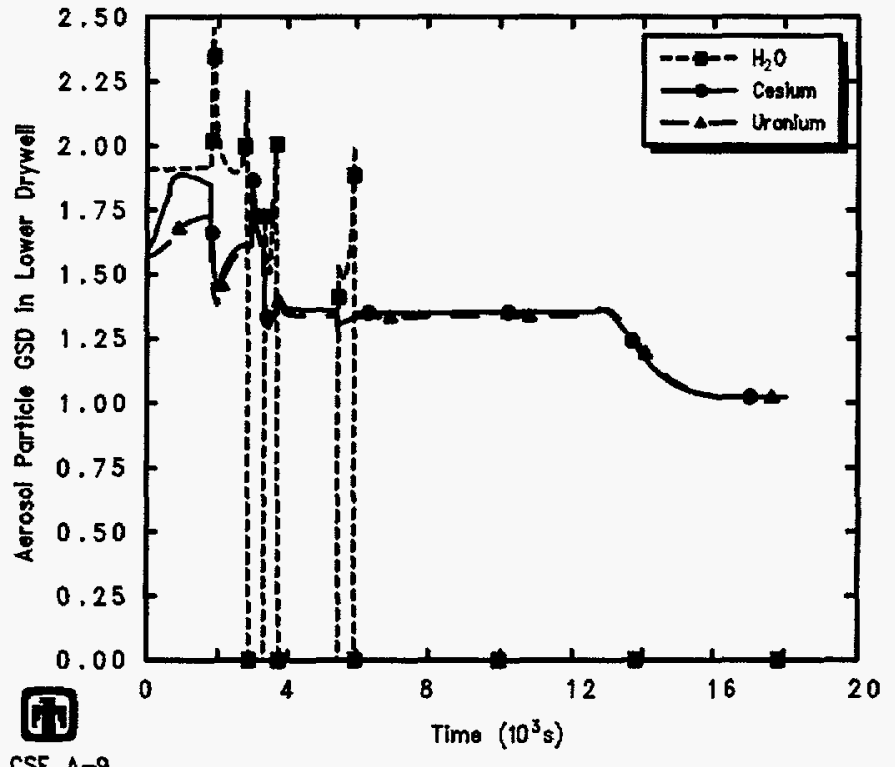

CSE A-9

HLEKCVKPN 8/12/94 10:31:51 MELCOR SUN

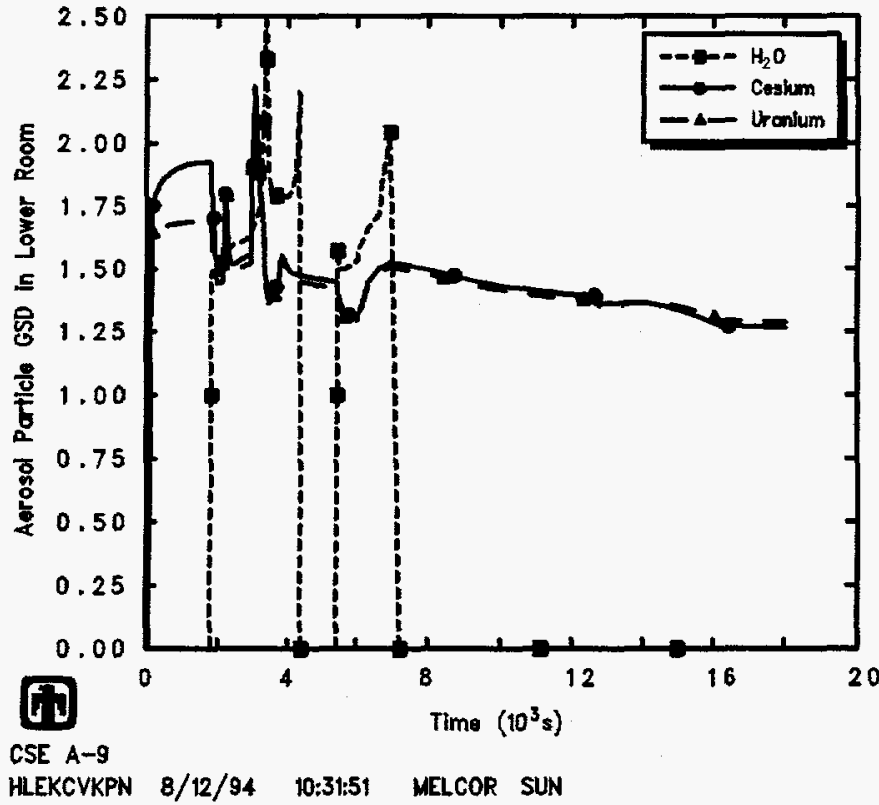

Figure 4.2.4. Airborne Aerosol GSDs in Vessel Dome (upper left), in Lower Drywell (upper right), in Middle Room (lower left) and in Vessel Sump (lower right) for CSE Test A-9 - Reference Calculation 
(The calculated GSD for the airborne cesium aerosol particles varies slightly but generally echoes some of the AMMD behavior, increasing when fog is present and when aerosol particles are growing, remaining nearly constant after the fog has disappeared and then dropping gradually during the late-time recirculating spray period. The GSD remains in the $1.0-2.0$ range throughout.)

The uranium particles were injected with an AMMD of $1.0 \mu \mathrm{m}$ and the GSD set to 1.5, i.e., somewhat larger than the cesium particles injected. Throughout the transient period simulated, the predicted mass median diameters for the uranium aerosol particles closely resemble but remain slightly greater than those predicted for the cesium aerosol particles, with the difference decreasing with time. The GSD of the uranium particle size distribution also closely resembles the GSD for the cesium aerosol particles, but generally remains slightly smaller.

Other experimental programs also have noted higher initial aerosol removal rates when sprays are started than long-term removal rates in runs where steam is present [23]; this is attributed to local subcooling and nucleation of water drops on airborne particles together with processes associated with condensation on the spray drops. The results in [23] for cesium removal in spray experiments show that the decontamination factor increases where there is more condensation, while it decreases where there is less. (The results in [23] for uranium concentrations in spray experiments are close to sensitivity limits and are of questionable accuracy, which is not dissimilar to the CSE results.)

\subsection{Iodine Vapor Response}

Figure 4.3.1 presents the concentrations of iodine vapor in the test vessel atmosphere, compared with test data. (The concentrations shown are the mass of iodine vapor in the control volume atmosphere divided by the volume.) Results are shown for the main room or dome, the middle room, and the lower room sump. The MELCOR results for the upper dome control volume (above the spray injection elevation) and for the lower drywell are very similar to the results given for the dome because the recirculation flow modelled keeps these volumes well mixed. The default class description for iodine in MELCOR (i.e., class 4) includes a vapor pressure characteristic of $\mathrm{I}_{2}$, so that iodine could potentially be present in either aerosol or vapor form depending on other conditions such as volume pressure and temperature; in this problem, the conditions are such that iodine is predicted to be present only in vapor form.

The concentration half-lives and the resulting washout constants for iodine vapor are listed in Table 4.3.1.

The calculated concentrations of airborne elemental iodine vapor show large stepwise decreases in concentration in the dome atmosphere during each of the three fresh spray periods and during the fourth recirculating spray. The calculated washout rates during the three fresh spray periods are all nearly equal, and the calculated washout rate during the recirculating spray is only slightly slower than during the fresh sprays. The test data, in contrast, show significant iodine removal only during the first fresh spray period, with 


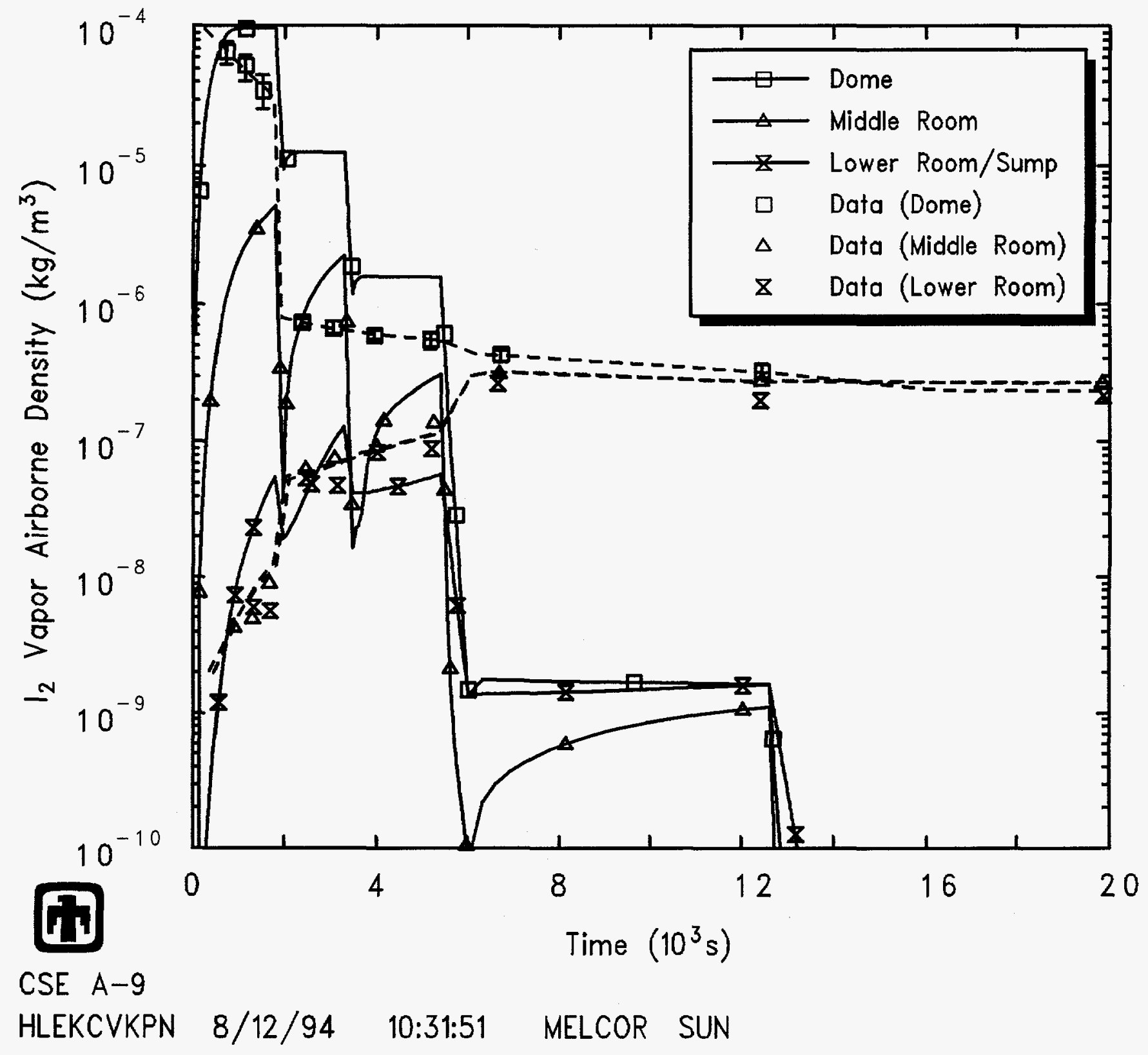

Figure 4.3.1. Iodine Vapor Airborne Concentrations for CSE Test A-9 - Reference Calculation 
little or no further removal by the later sprays. The iodine removal measured during that first spray period is much greater than calculated, as was also the case for the cesium and uranium aerosol removal rates. However, owing to the continued removal of iodine in the calculation, the predicted concentrations of airborne iodine vapor in the vessel are much lower than the measured airborne iodine concentrations late in the test; this is opposite to the case for aerosol behavior, where the code predicts higher late-time airborne aerosol concentrations than measured.

One of the chief conclusions from all eight CSE spray experiments is that the initial rapid washout of iodine did not continue after the inorganic (elemental) iodine concentration was reduced to about $1 \%$ of its initial value, so that later sprays were not very effective in lowering the concentration further. The experimentalists [14] attributed this effect to two causes: (1) the Maypack is not a perfect discriminator of iodine forms and some of the iodine called inorganic might be in forms less readily removed, and (2) absorption of inorganic iodine by aqueous sprays is a reversible process and back diffusion into the gas space can occur from liquid films which contain high concentrations of iodine.

Other experimental programs (e.g., the NSPP spray program [23]) also have noted that overall decontamination factors for iodine are higher in short-term runs than in longer term runs, i.e., that initial iodine removal rates are much higher than long-term removal rates. In that reference, analytical model studies indicate that the bulk of the iodine is collected by the first spray solution, while the last of the solution has only a little iodine to absorb and can take the concentration in the gas considerably below the equilibrium value, assuming a well-mixed solution. In long-term runs with solution recycled repeatedly through the containment atmosphere, enough recycling occurs to establish equilibrium between the gas and liquid phases and to homogenize the liquid. Also, a little of the spray solution is airborne as an aerosol of very fine, $\sim 1 \mu \mathrm{m}$ diameter droplets, which the sampling system may not be able to distinguish from vapor. The results and discussion of those NSPP spray tests are consistent with the results and discussion of the CSE spray tests.

The first calculations for these CSE assessment analyses showed removal of elemental iodine by sprays from the test vessel atmosphere as discussed above, but then predicted that the iodine vapor would re-evolve from the liquid pools in the lower drywell and lower room sumps very quickly, returning to near the initial airborne iodine vapor concentration. This occurred because the water in the pool had no capability of continuing to bind the iodine vapor chemically in the MELCOR coding. This problem was noted by the code developers and the implementation of TRAP-MELT modelling for fission product condensation and evaporation was modified to totally disallow any evaporation of fission products residing in a control volume pool. This is considered a temporary modification and is expected to by replaced by the iodine chemistry model under development for MELCOR. Until this new model is implemented, note that MELCOR versions 1.8.2 and 1.8.3 could have very different fission product vapor responses calculated in control volumes with pools and sprays neither can be expected to be "correct" because the behavior is probably intermediate between the two limiting extremes.

Also, note that MELCOR does not account for any fission product aerosol or vapor 
"loading" in recirculating sump water. A new feature was added in MELCOK 1.8.3 in the spray package, allowing the user to specify a control volume from which to extract water for recirculating sprays. In previous code versions, this had to be done by the user defining mass and energy sinks using control functions to subtract water from the control volume pool to balance the external spray injection. Since the spray is input as a volumetric flow at a specified temperature (at an undocumented reference pressure), while the mass and energy sinks are defined as a mass flow at a given enthalpy, there was significant potential for mass and energy conservation problems modelling closed, recirculating spray systems. However, any fission product aerosols and/or vapors already deposited in the pool are left behind in the pool. For example, any iodine collected by the sprays remains behind, locked in the liquid pool, as the water from that pool is recirculated by the spray package. Thus, MELCOR does not account for any pre-existing binding of iodine with reactants such as borax or sodium thiosulfate in recirculating sump water, which might further degrade its capability to remove iodine.

Finally, note that MELCOR currently has no built-in capability to model methyl iodide (a moderately soluble and less reactive vapor) or other iodine forms, or to model details of iodine chemistry such as interaction with sprays containing different additives. This capability will be added in the next code version after MELCOR 1.8.3. 
Table 4.3.1. Iodine Vapor Washout Rates for CSE Test A-9 - Reference Calculation

$$
t_{1 / 2}(\min ) \quad \lambda_{s}\left(\min ^{-1}\right)
$$

Measured Calculated Measured Calculated

During first spray period

0.58

1.1

1.193

0.65

During second spray period

42

1.4

0.017

0.50

During third spray period

34

1.0

0.020

0.70

During fourth spray period

180

1.4

0.0038

0.50

Prior to first spray

Between sprays 1-2

14.1

72

210

300

2000

0.0492

0.0004

Between sprays 2-3

Between sprays 3-4

250

0.0096

0.0028

1000

0.0033

0.0007

1000

0.0023

0.0007 


\section{Experimental Parameter Studies}

Eight experiments have been performed in the CSE containment vessel to evaluate the performance of aqueous sprays as a means of decontaminating containment atmospheres, as summarized in Section 2. Experiment parameters varied for the six intermittent spray tests included atmosphere composition, spray flow rate, spray droplet size, and spray chemistry; two tests used continuous rather than intermittent sprays.

Section 4 gave a detailed presentation of our base case MELCOR assessment results for CSE containment spray experiment A-9. The results of our MELCOR analyses simulating the other intermittent spray tests and the continuous-spray tests are given in this section, demonstrating that, while the effects of varying these experiment parameters are generally qualitatively reproduced in the MELCOR analyses, the same quantitative differences are found in the other CSE spray tests as discussed in the last section for test A-9.

\subsection{Effect of Spray Flow Rate (A-6 vs A-9)}

Test A-6 generally resembled test A-9. In both tests, the test vessel was initialized with a saturated steam-air mixture at a pressure of about 3 bars and a temperature of $390-400 \mathrm{~K}$. The major difference was that the spray flow rates used were about a factor of three higher in CSE A-9 than in CSE A-6. The timing of the sprays was also somewhat different. Test A-6 had only two fresh spray injections, while test A-9 had three fresh spray injections. The total amounts of water injected during the first spray period were quite similar in tests A-6 and A-9, but more water was injected during the second spray period in A-6 than in A-9 (but about the same amounts of water were injected during the second fresh spray in A-6 as during the third fresh spray in A-9). Also, the total amount of sump water recirculated in test A-6 was about an order of magnitude less than during test A-9. (Section 2 gives more detail on the initial and boundary conditions of the experiments.)

Figures 5.1.1 and 5.1.2 show the spray flow rates and spray temperatures for both the two fresh sprays and the recirculating spray used in test A-6. Note that the spray flow rates used in the calculation represent $70 \%$ of the spray flow rates given in Table 2.3 and are the flows assumed to interact fully with the atmosphere; also note that the recirculating spray temperature shown is simply the temperature of the water in the lower room sump.

The effects of the sprays on the response of the containment atmosphere are presented in Figures 5.1.3 and 5.1.4, which compare calculated MELCOR results with experimental data for the test vessel pressures and temperatures, respectively, for test A-6. The qualitative and quantitative agreement between calculation and experiment for test A-6 for the vessel thermal/hydraulic response is generally quite similar to the qualitative and quantitative agreement between calculation and experiment for test A-9 as discussed in detail in Section 4.1. The overall pressure is underpredicted owing to overprediction of the pressure drops during spray injection, because too much steam is being condensed by 


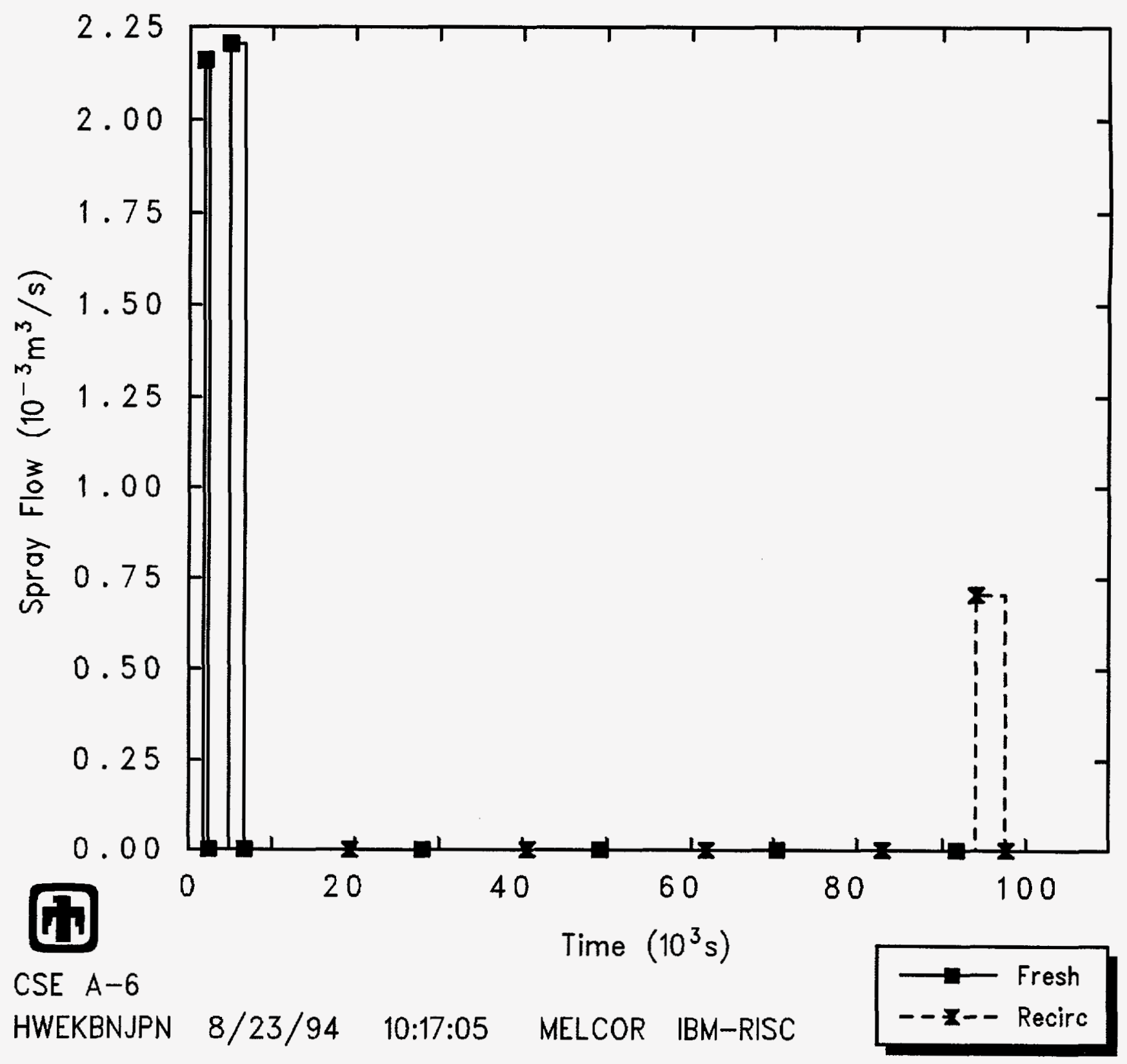

Figure 5.1.1. Spray Flow Rate for CSE Test A-6 - Reference Calculation 


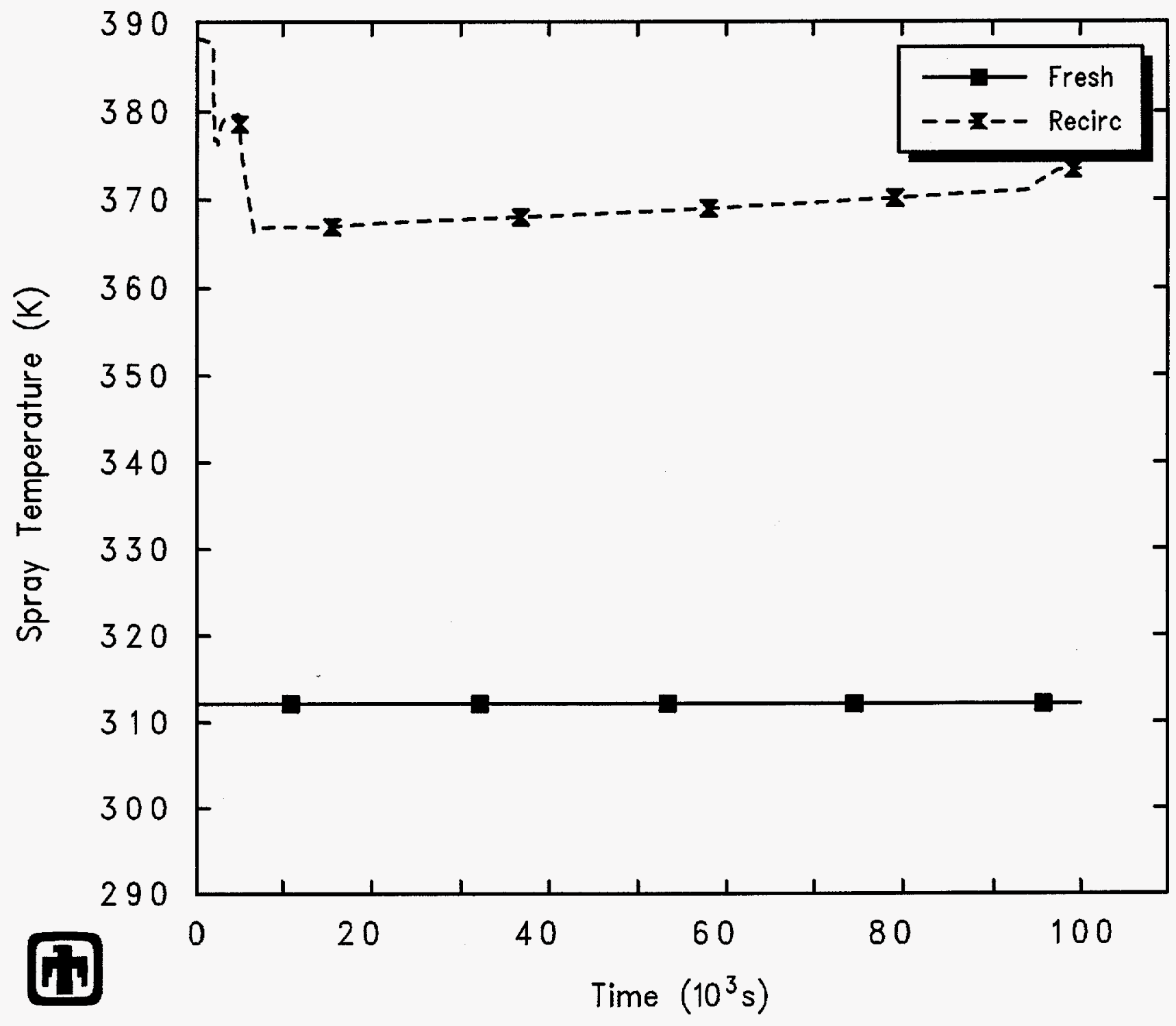

CSE A-6

HWEKBNJPN 8/23/94 10:17:05 MELCOR IBM-RISC

Figure 5.1.2. Spray Temperature for CSE Test A-6 - Reference Calculation 
the sprays; in contrast, the calculated temperatures are generally higher than measured in the test vessel dome.

Figures 5.1.5 and 5.1.6 present the concentrations of cesium and uranium aerosol, respectively, in various regions in the test vessel atmosphere, compared with test data; Figure 5.1.7 presents the concentrations of iodine vapor in the test vessel atmosphere, together with test data. The concentrations shown are the mass of airborne aerosol or vapor in the control volume atmosphere divided by the volume. The concentrations plotted are for the test vessel dome or main room, the middle room and the lower room or sump. The calculation shows virtually equal concentrations in the dome, the upper dome above the spray injection elevation and the lower drywell, because the recirculation flow modelled keeps these volumes well mixed.

The calculated concentrations of airborne cesium and uranium aerosols agree qual-: itatively with the measured concentrations. The code predicts stepwise decreases in concentration in the dome atmosphere during both fresh spray periods and a more gradual, linear decline during the late-time recirculation period, as observed in the test. The agreement with data is quite good during and between the fresh sprays if adjusted for the difference in initial concentration. The calculation also shows a decline in the airborne aerosol concentration in the vessel dome caused by natural settling during the long period between the fresh sprays early in the transient and the recirculating spray late in the test; the predicted removal rate in the dome is in good agreement with test data in the first portion of this period (before $\sim 25000 \mathrm{~s}$ ) but then more rapid than observed during the later times.

The predicted removal of iodine vapor during the first fresh spray period is less than that measured, while the predicted removal of iodine vapor during the later spray periods is significantly greater than that measured. MELCOR predicts similar iodine removal rates during the two fresh spray periods, while the test data indicate much more iodine removal by the first spray than by later sprays. This is generally the same behavior as predicted for test A-9, as discussed in Section 4.3.

Table 5.1.1 summarizes the washout rates predicted for cesium and uranium aerosol and iodine vapor in the test vessel dome for the different spray flow rates used in tests A-6 and A-9. Although the absolute aerosol and vapor removal rates do not agree quantitatively with the test data, MELCOR correctly predicts the trend of slower aerosol and vapor removal for the lower spray flow rates used in test A-6 than for the higher flow rates used in test $A-9$.

\subsection{Effect of Spray Droplet Size (A-6 vs A-8)}

Test A-8 generally resembled test A-6. In both tests, the test vessel was initialized with a saturated steam-air mixture at a pressure of about 3 bars and a temperature of 390-400 K, and the spray flow rates used were quite similar in these two tests. The major difference was that the spray flow droplets were smaller in CSE A-8 than in CSE A-6, owing to a change in spray nozzle. The timing of the sprays was also somewhat different. 


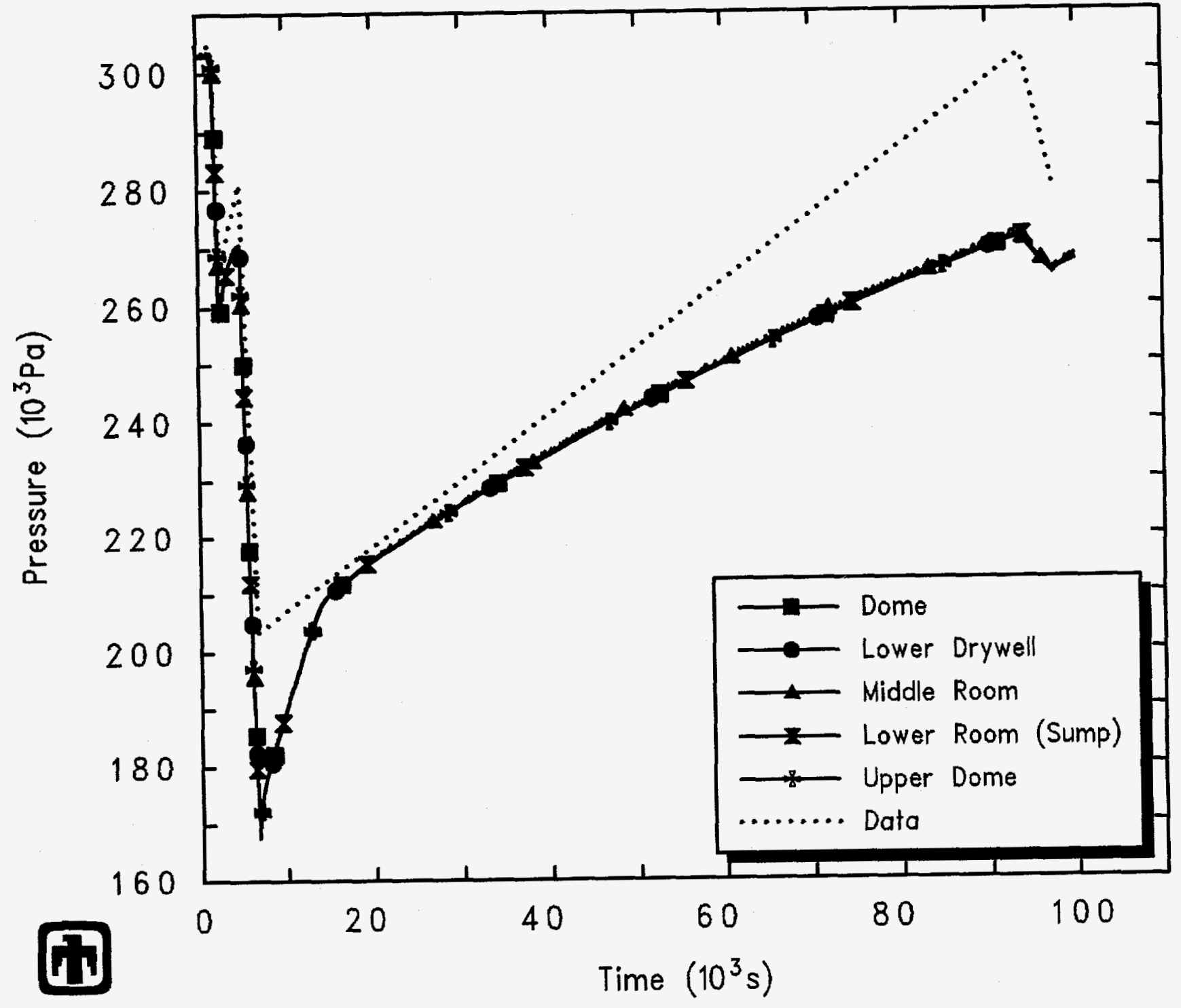

CSE A-6

HWEKBNJPN 8/23/94 10:17:05 - MELCOR IBM-RISC

Figure 5.1.3. Vessel Pressure for CSE Test A-6 - Reference Calculation 


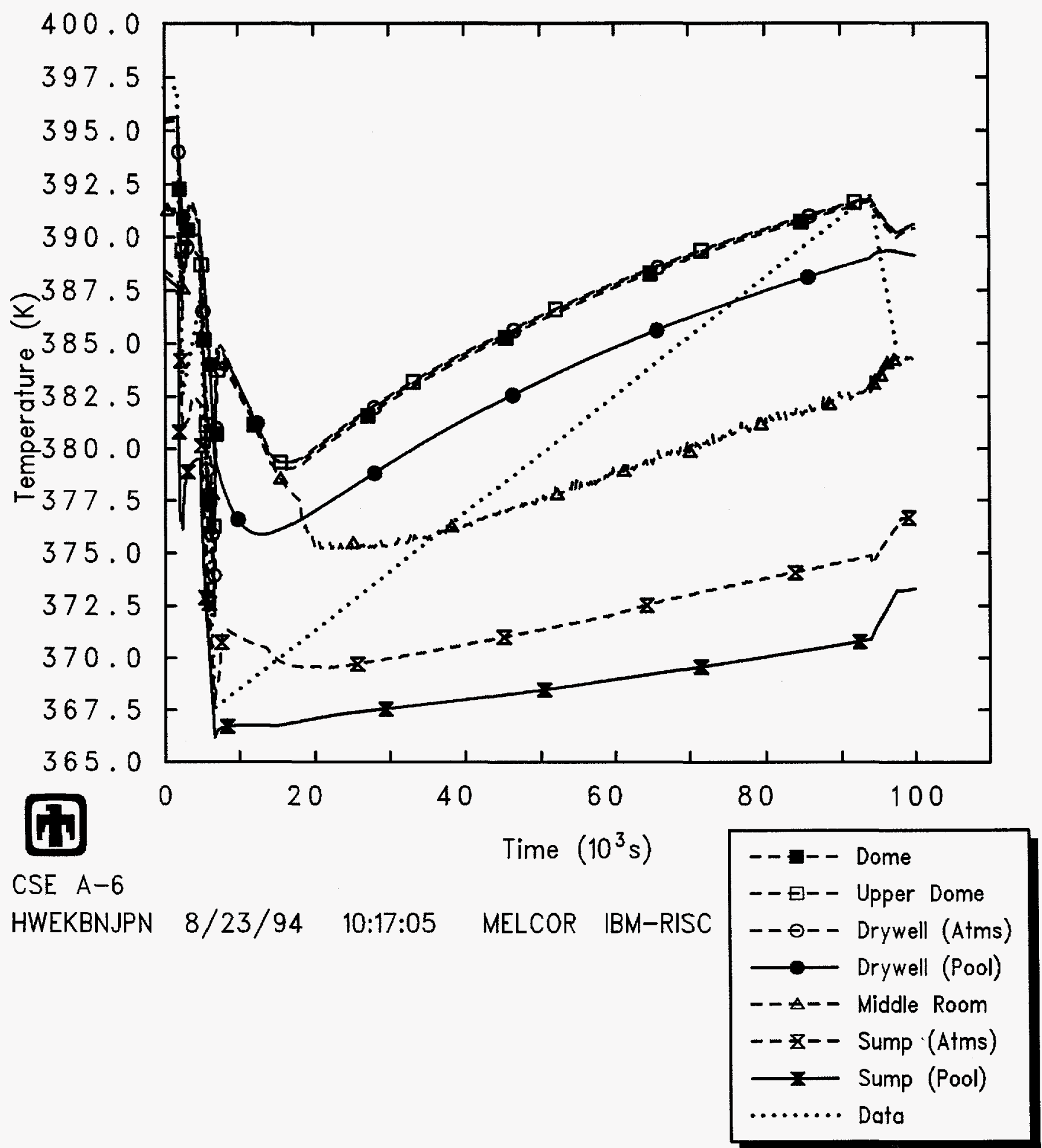

Figure 5.1.4. Vessel Temperatures for CSE Test A-6 - Reference Calculation 


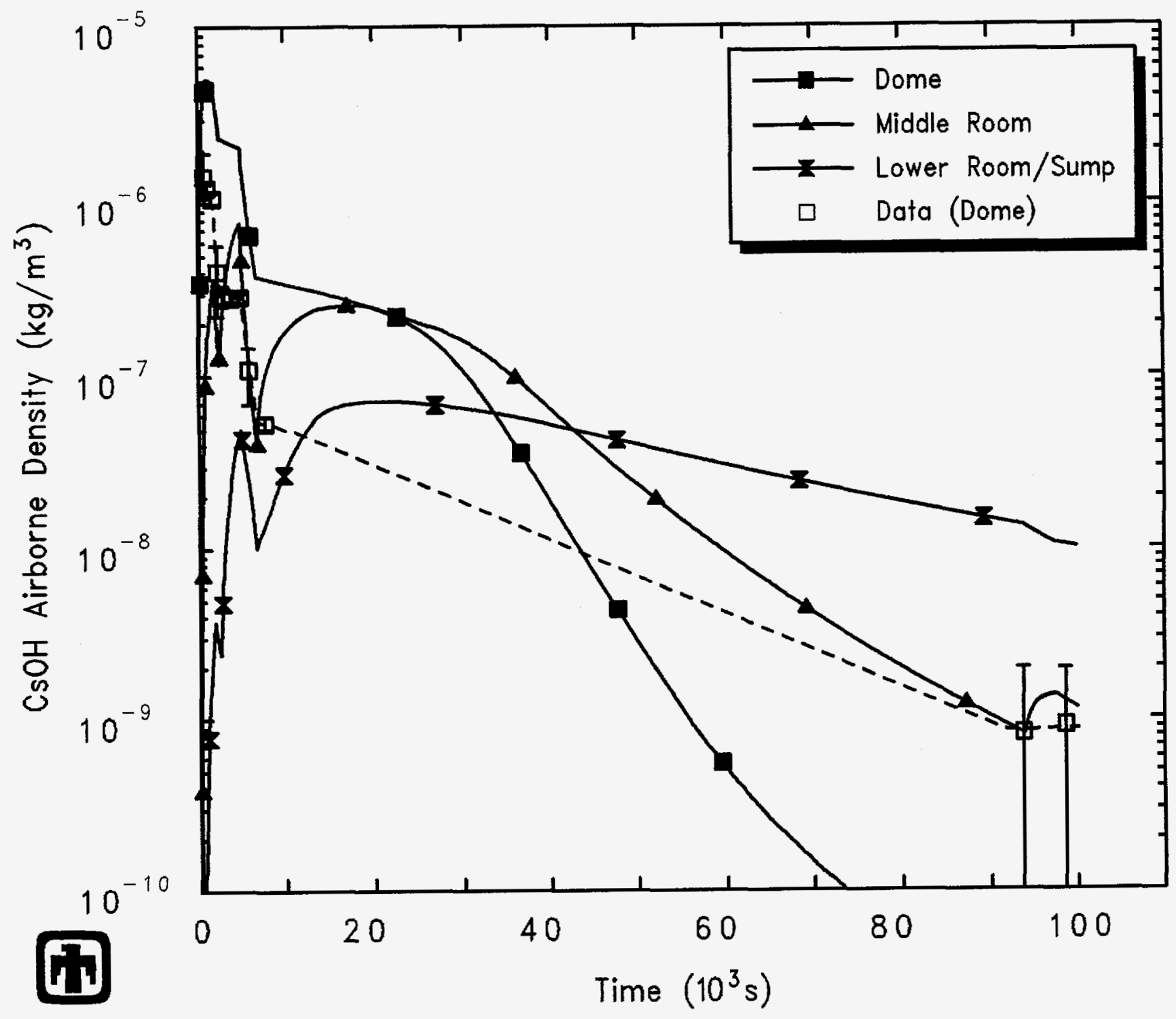

CSE $A-6$

HWEKBNJPN 8/23/94 10:17:05 MELCOR IBM-RISC

Figure 5.1.5. Cesium Aerosol Airborne Concentrations for CSE Test A-6-Reference Calculation 


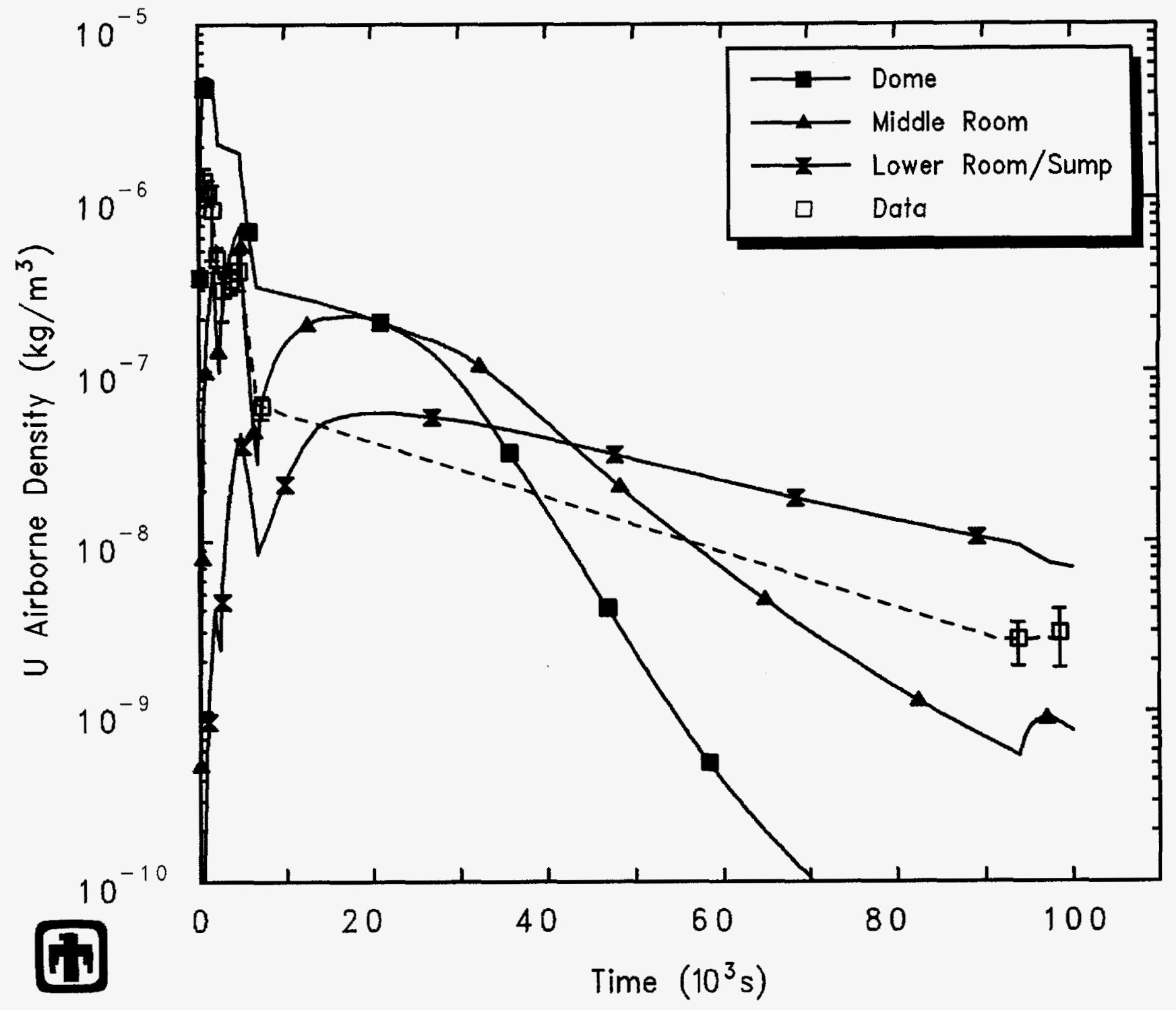

CSE A-6

HWEKBNJPN 8/23/94 10:17:05 MELCOR IBM-RISC

Figure 5.1.6. Uranium Aerosol Airborne Concentrations for CSE Test A-6 Reference Calculation 


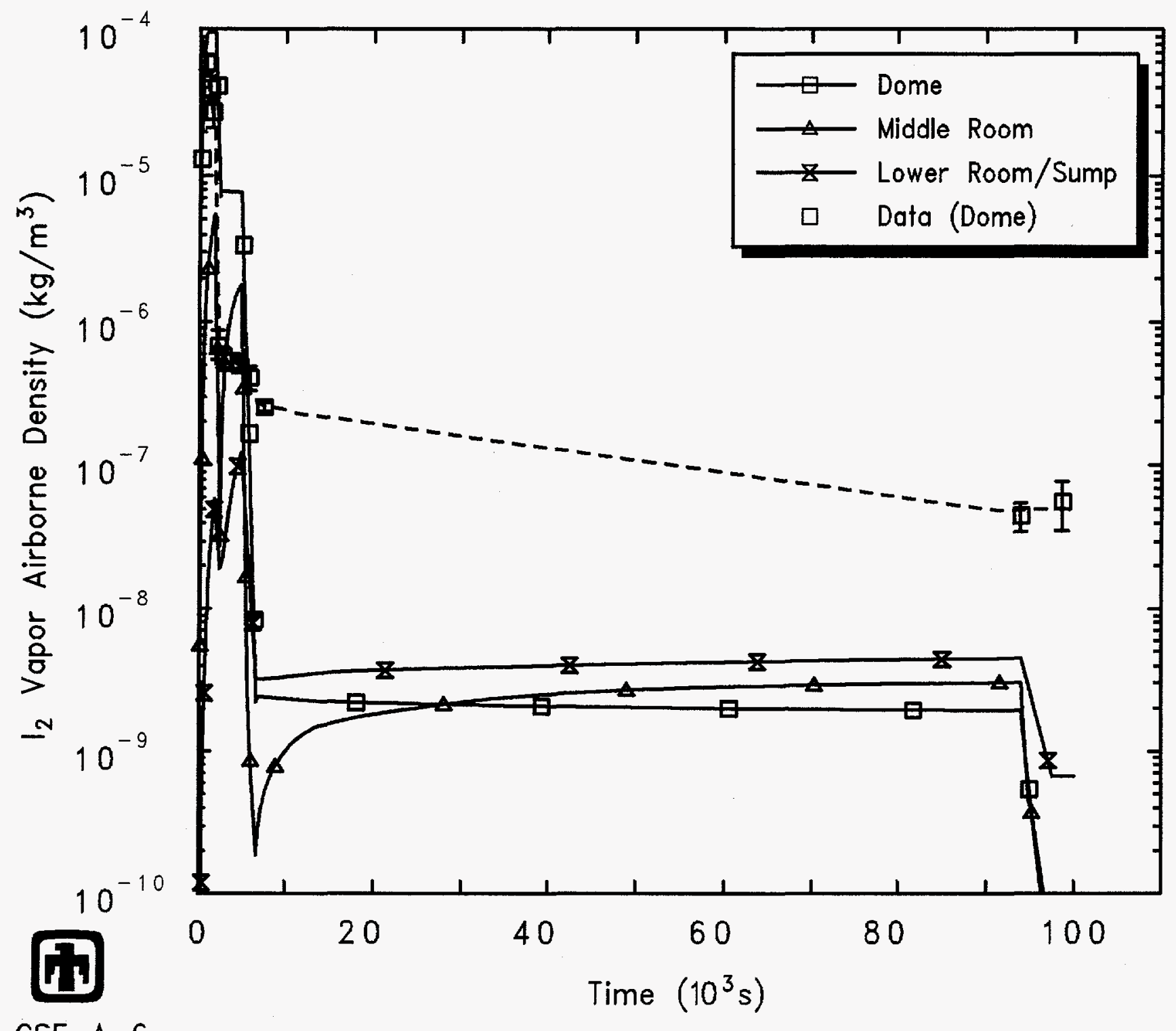

CSE A-6

HWEKBNJPN 8/23/94 10:17:05 MELCOR IBM-RISC

Figure 5.1.7. Iodine Vapor Airborne Concentrations for CSE Test A-6 - Reference Calculation 
Table 5.1.1. Washout Rates for CSE Tests - Effect of Spray Flow Rate (A-6 vs A-9)

\begin{tabular}{|c|c|c|c|c|}
\hline & \multicolumn{4}{|c|}{$t_{1 / 2}(\min )$} \\
\hline & \multicolumn{2}{|c|}{ A- 6} & \multicolumn{2}{|c|}{ A-9 } \\
\hline & Measured & Calculated & Measured & Calculated \\
\hline \multicolumn{5}{|l|}{ Cesium } \\
\hline First spray & 5.6 & 8.7 & 1.08 & 5.0 \\
\hline Second spray & 13. & 11.6 & 2.0 & 4.6 \\
\hline Third spray & $a$ & 8.7 & 5.4 & 4.3 \\
\hline Fourth spray & & & 33 & $34.7^{b}$ \\
\hline \multicolumn{5}{|l|}{ Uranium } \\
\hline First spray & 7.8 & 6.9 & 2.3 & 4.6 \\
\hline Second spray & 12.5 & 11.7 & & 4.3 \\
\hline Third spray & ${ }^{a}$ & 7.7 & & 4.3 \\
\hline Fourth spray & & & & $34.7^{b}$ \\
\hline \multicolumn{5}{|l|}{ Iodine } \\
\hline First spray & 2.1 & 2.8 & 0.58 & 1.1 \\
\hline Second spray & 35. & 2.5 & 42 & 1.4 \\
\hline Third spray & $\infty$ & 8.7 & 34 & 1.0 \\
\hline Fourth spray & & & 180 & 1.4 \\
\hline & & $\begin{array}{l}{ }^{a} \text { indet } \\
{ }^{b} \text { at en }\end{array}$ & $\begin{array}{l}\text { minant } \\
\text { of spray }\end{array}$ & \\
\hline
\end{tabular}


Both test A-6 and test A-8 had two fresh spray injections, followed by a recirculating spray period, but test A-8 also had a late-time third fresh spray injection. The total amounts of water injected during the first two fresh spray periods were quite similar in tests A-6 and A-8, but more water was injected during the first spray period in A-6 than in A-8, while less water was injected during the second spray period in A-6 than in A-8. Also, the total amount of sump water recirculated in test A-6 was about a third less than during test A-8. (Section 2 gives more detail on the experiment initial and boundary conditions.)

Figures 5.2.1 and 5.2.2 show the spray flow rates and spray temperatures for both the three fresh sprays and the recirculating spray used in test A-8. Note that the spray flow rates used in the calculation represent $70 \%$ of the flow rates given in Table 2.3 and are the flows assumed to interact fully with the atmosphere; also note that the recirculating spray temperature shown is simply the temperature of the water in the lower room sump.

The effects of the sprays on containment atmosphere response are presented in Figures 5.2.3 and 5.2.4, which compare calculated MELCOR results with test data for the test vessel pressures and temperatures, respectively, for test A-8. In general, the overall pressure is underpredicted owing to overpredicting the pressure drops during fresh spray injection because too much steam is being condensed by the sprays; in contrast, the calculated temperatures are generally higher than those measured in the test vessel dome. There is no noticable pressure drop predicted by MELCOR during the recirculating spray injection between $12000 \mathrm{~s}$ and $15600 \mathrm{~s}$, while the experimental data indicates a large depressurization comparable to the response to fresh, cold spray injection. This MELCOR result for test A-8 is consistent with the behavior predicted for the recirculating spray period in test A-9, during which little or no pressure change is calculated.

Figures 5.2.5 and 5.2.6 present the concentrations of cesium and uranium aerosol, respectively, in various regions in the test vessel atmosphere, compared with test data; Figure 5.2.7 presents the concentrations of iodine vapor in the test vessel atmosphere, together with test data. The concentrations shown are the mass of airborne aerosol or vapor in the control volume atmosphere divided by the volume. The concentrations plotted are for the test vessel dome or main room, the middle room and the lower room or sump; the calculation shows virtually equal concentrations in the dome, the upper dome above the spray injection elevation and the lower drywell because the recirculation flow modelled keeps these volumes well mixed.

The calculated concentrations of airborne cesium and uranium aerosols predicted for test A-8, and for the airborne iodine vapor as well, are very similar qualitatively to the results obtained for test A-6, presented in the previous subsection. Table 5.2.1 summarizes the washout rates predicted for cesium and uranium aerosol and iodine vapor in the test vessel dome for the different spray droplet sizes used in tests A-6 and A-8. Although the absolute aerosol and vapor removal rates do not agree quantitatively with the test data, MELCOR correctly predicts the trend of more rapid aerosol and vapor removal for the smaller spray droplets used in test A-8 than for the larger spray droplets used in test A-6. 


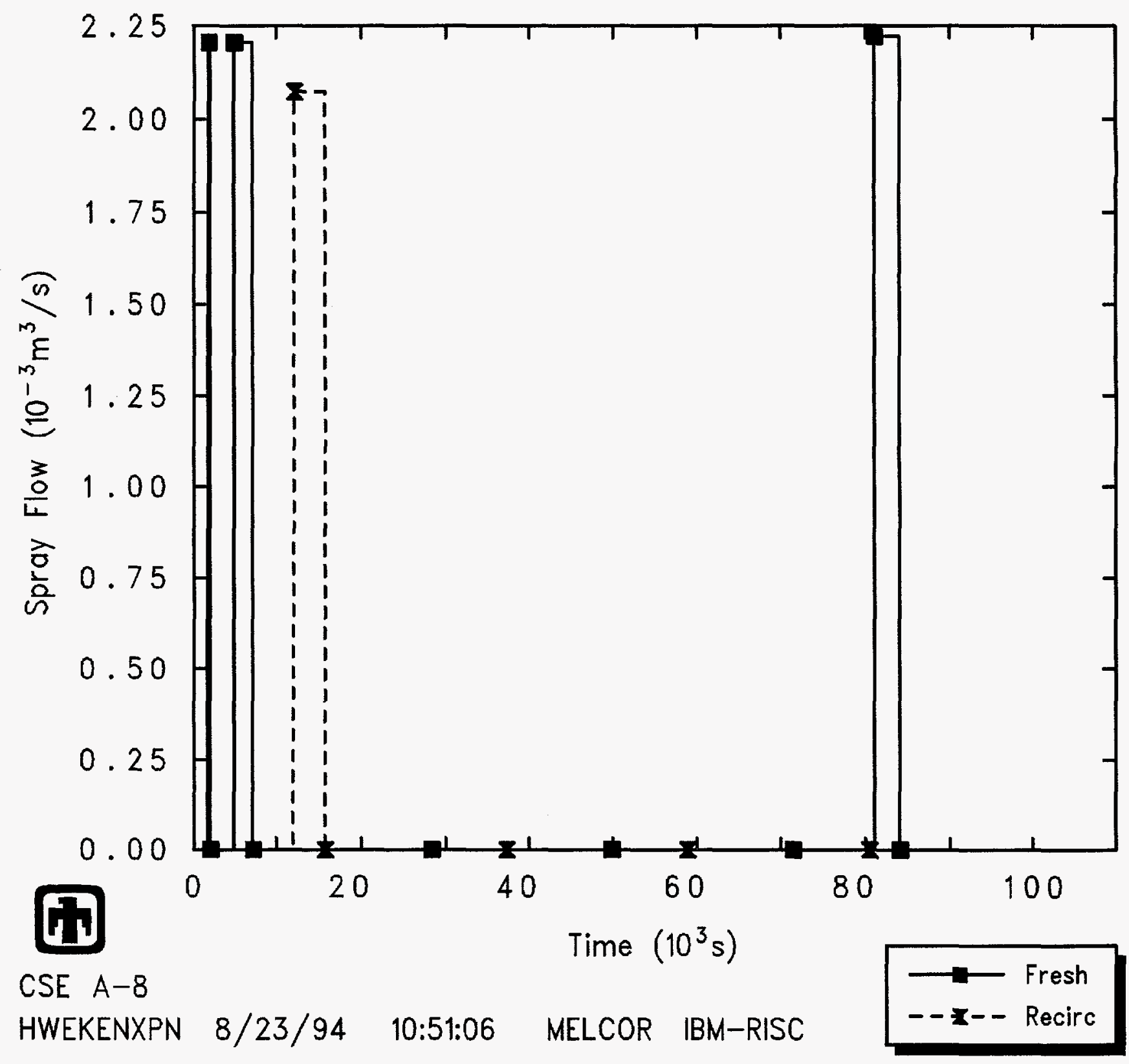

Figure 5.2.1. Spray Flow Rate for CSE Test A-8 - Reference Calculation 


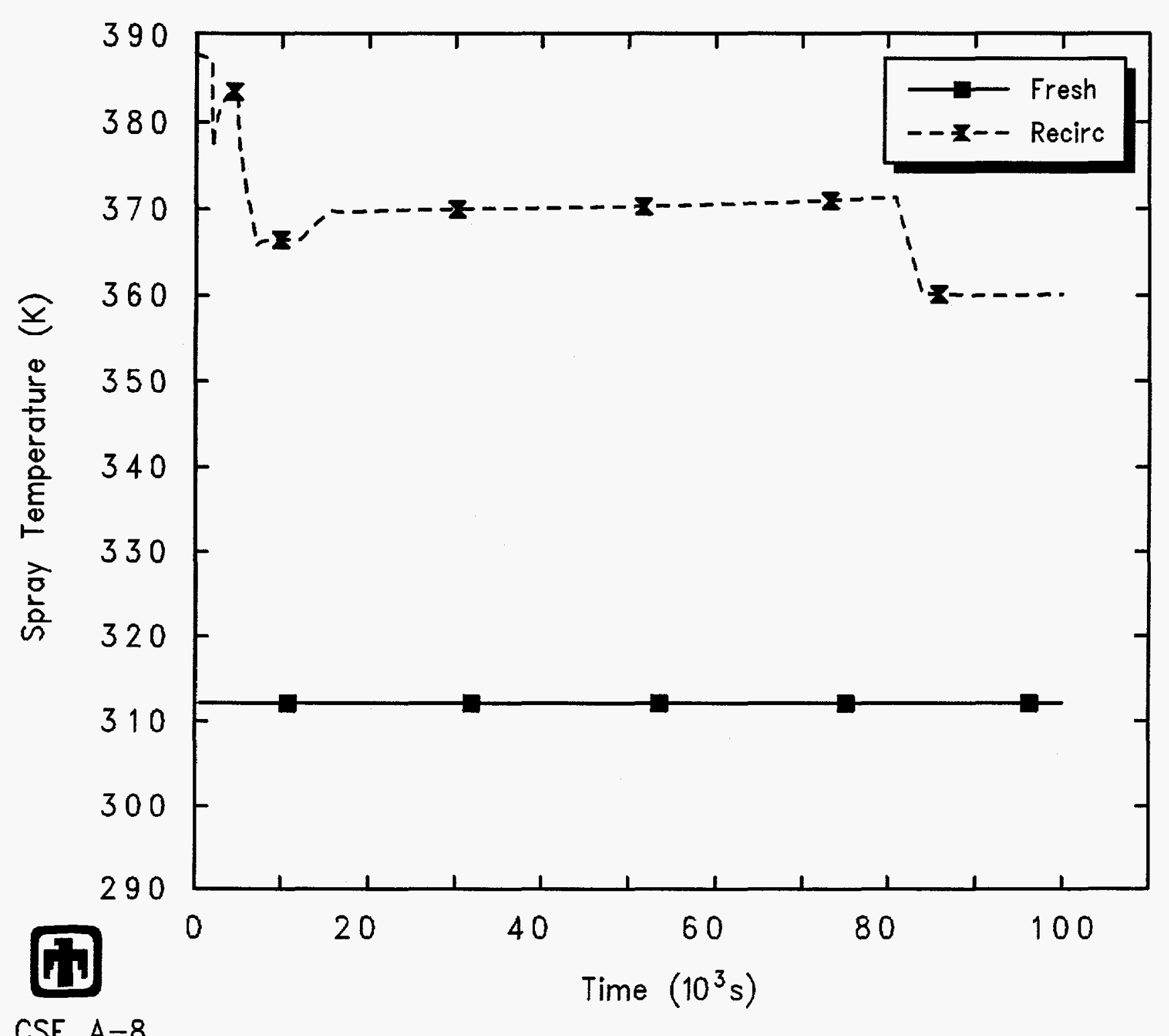

HWEKENXPN $8 / 23 / 94 \quad 10: 51: 06 \quad$ MELCOR IBM-RISC

Figure 5.2.2. Spray Temperature for CSE Test A-8 - Reference Calculation 


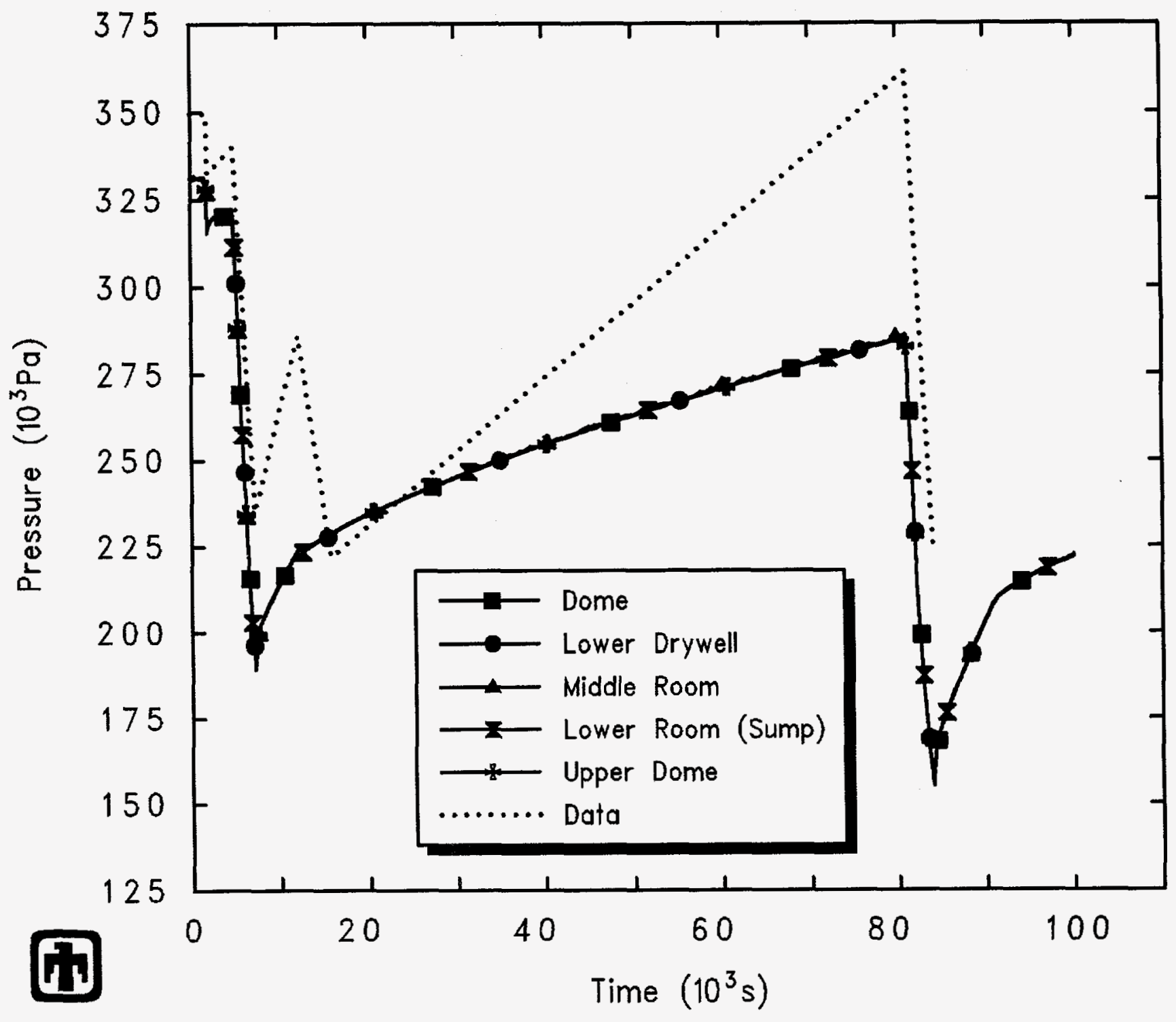

CSE $A-8$

HWEKENXPN $8 / 23 / 94 \quad 10: 51: 06 \quad$ MELCOR IBM-RISC

Figure 5.2.3. Vessel Pressure for CSE Test A-8 - Reference Calculation 


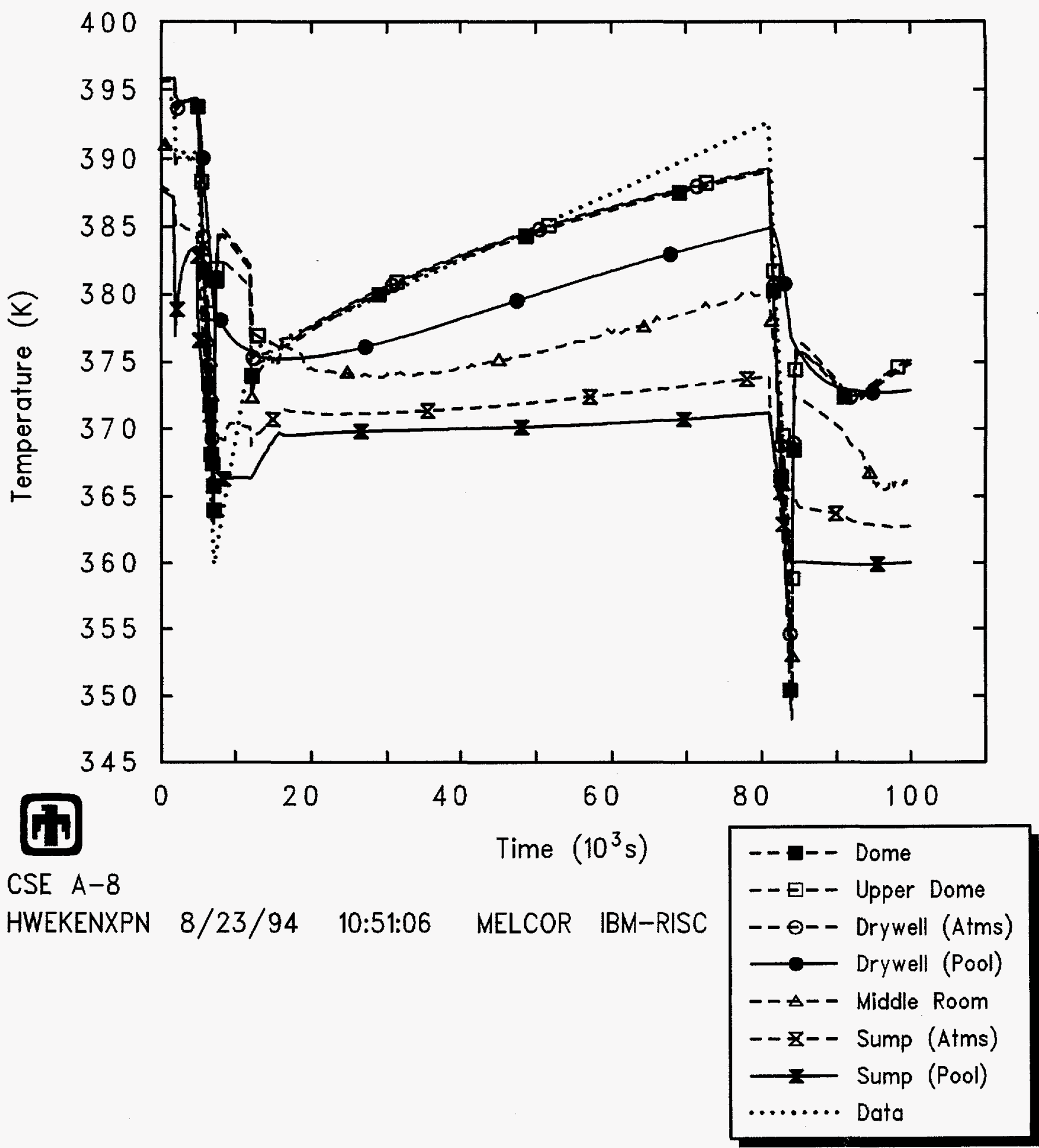

Figure 5.2.4. Vessel Temperatures for CSE Test A-8 - Reference Calculation 


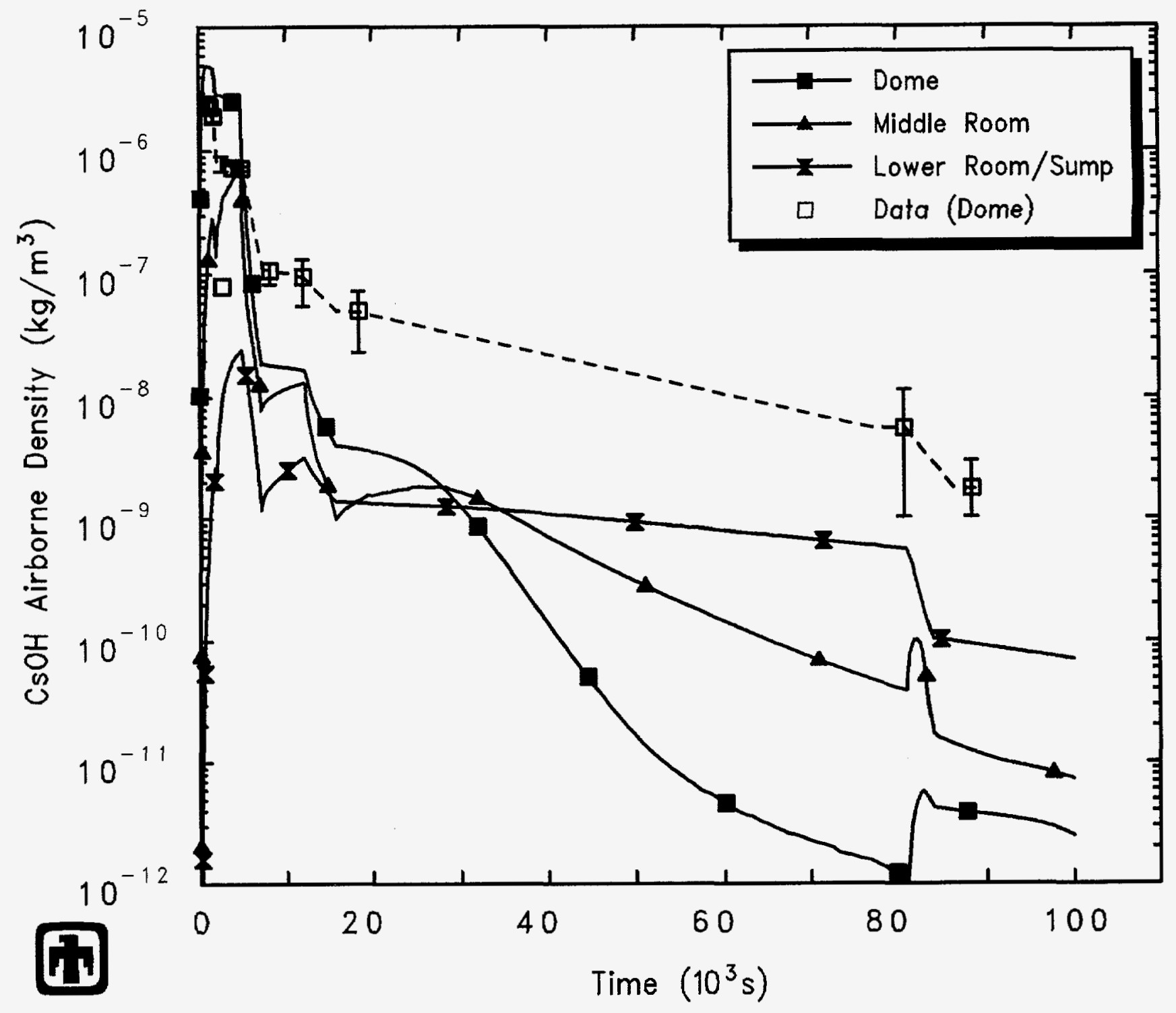

CSE A-8

HWEKENXPN $8 / 23 / 94 \quad 10: 51: 06 \quad$ MELCOR IBM-RISC

Figure 5.2.5. Cesium Aerosol Airborne Concentrations for CSE Test A-8 - Reference Calculation 


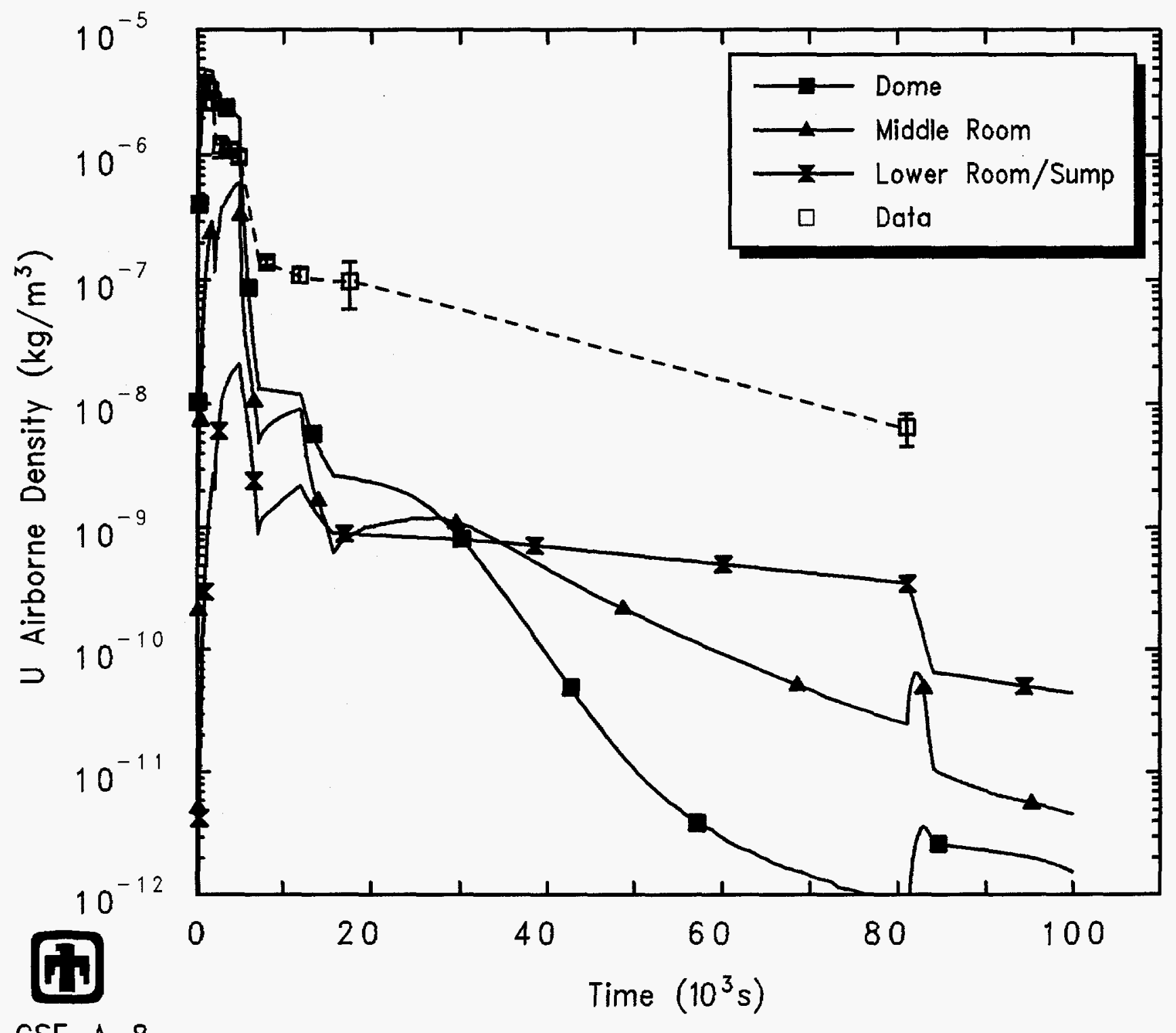

CSE A-8

HWEKENXPN 8/23/94 10:51:06 MELCOR IBM-RISC

Figure 5.2.6. Uranium Aerosol Airborne Concentrations for CSE Test A-8 Reference Calculation 


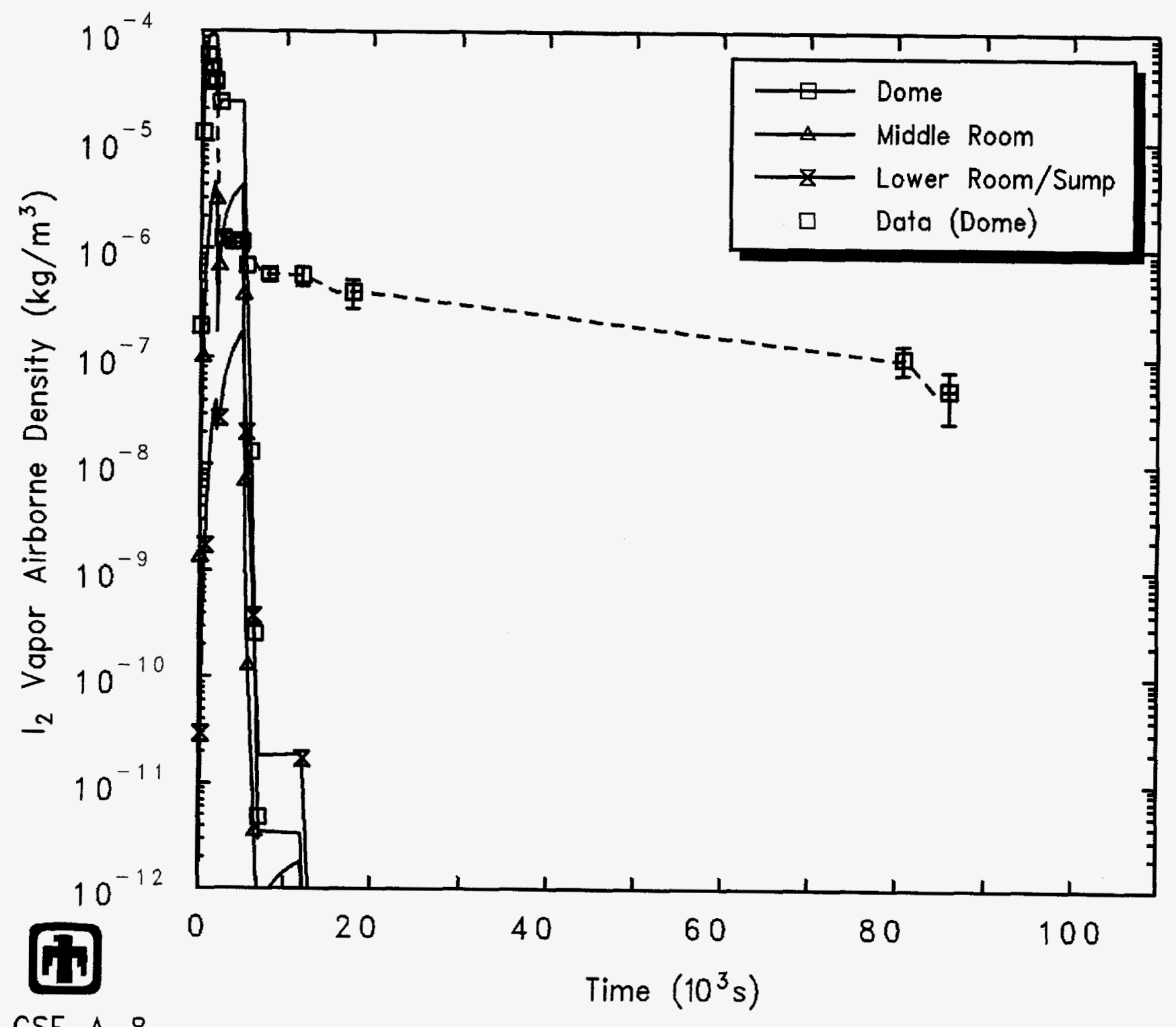

CSE A-8

HWEKENXPN 8/23/94 10:51:06 MELCOR IBM-RISC

Figure 5.2.7. Todine Vapor Airborne Concentrations for CSE Test A-8 - Reference Calculation 
Table 5.2.1. Washout Rates for CSE Tests - Effect of Spray Droplet Size (A-6 vs A-8)

\begin{tabular}{|c|c|c|c|c|}
\hline & \multicolumn{4}{|c|}{$t_{1 / 2}(\min )$} \\
\hline & \multicolumn{2}{|c|}{ A-6 } & \multicolumn{2}{|c|}{ A-8 } \\
\hline & Measured & Calculated & Measured & Calculated \\
\hline First spray & 5.6 & 8.7 & 2.6 & 5.8 \\
\hline Second spray & 13 & 11.6 & 14 & 5.0 \\
\hline Third spray & $a$ & 8.7 & 67 & 23.1 \\
\hline Fourth spray & & & 30 & 2.0 \\
\hline \multicolumn{5}{|l|}{ Uranium } \\
\hline First spray & 7.8 & 6.9 & 6.6 & 4.8 \\
\hline Second spray & 12.5 & 11.7 & 14 & 4.8 \\
\hline Third spray & $b$ & 7.7 & 350 & 17.3 \\
\hline Fourth spray & & & $b$ & 2.0 \\
\hline \multicolumn{5}{|l|}{ Iodine } \\
\hline First spray & 2.1 & 2.8 & 0.64 & 1.5 \\
\hline Second spray & 35. & 2.5 & 40 & 1.5 \\
\hline Third spray & $\infty$ & 8.7 & 125 & 1.7 \\
\hline Fourth spray & & & 50 & 2.3 \\
\hline & & $\begin{array}{r}{ }^{a} \text { concentra } \\
{ }^{b} \text { indet }\end{array}$ & $\begin{array}{l}\text { on increas } \\
\text { minant }\end{array}$ & \\
\hline
\end{tabular}




\subsection{Effect of Atmosphere Conditions (A-6 vs A-4)}

The major difference between test A-6 and test A-4 was in the initial condition of the test vessel atmosphere. In test A-6, the test vessel was initialized with a saturated steam-air mixture at a pressure of about 3 bars and a temperature of $390-400 \mathrm{~K}$, while in test A-4 the test vessel was initialized with a steam-air mixture at atmospheric pressure and about $300 \mathrm{~K}$ temperature, and about $90 \%$ humidity. The spray flow rates used were quite similar in these two tests, although the timing of the sprays was somewhat different. Both test A-6 and test A-4 had two fresh spray injections, followed by a recirculating spray period. The flow rates and total amounts of water injected during the first two fresh spray periods were quite similar in tests A-6 and A-4, but more water was injected at a higher rate during the recirculating spray period in test A-4 than in test A-6. (Section 2 gives more detail on the experiment initial and boundary conditions.)

Figures 5.3.1 and 5.3.2 show the spray flow rates and spray temperatures for both the three fresh sprays and the recirculating spray used in test A-4. Note that the spray flow rates used in the calculation represent $70 \%$ of the rates given in Table 2.3 and are the flows assumed to interact fully with the atmosphere; also note that the recirculating spray temperature shown is simply the temperature of the water in the lower room sump.

The effects of the sprays on containment atmosphere response are presented in Figures 5.3.3 and 5.3.4, which compare calculated MELCOR results with test data for the test vessel pressures and temperatures, respectively, for test A-4. There is no noticable pressure or temperature change caused by spray injection in the experimental data, while the MELCOR calculation indicates a slight pressurization and heatup. The calculated pressurization and heatup in the calculation obviously is due to the fresh spray being injected at a higher temperature than the ambient test vessel atmosphere; the fresh spray temperature was kept at $312 \mathrm{~K}$ in the MELCOR input deck in the absence of any information in the experiment documentation about spray temperature differences.

Figures 5.3.5 and 5.3.6 present the concentrations of cesium and uranium aerosols, respectively, in various regions in the test vessel atmosphere, compared with test data; Figure 5.3.7 presents the concentrations of iodine vapor in the test vessel atmosphere, together with test data. Table 5.3.1 summarizes the washout rates predicted for cesium and uranium aerosols and iodine vapor in the test vessel dome for the different containment atmosphere conditions used in tests A-6 and A-4. The experimental data in general appear to indicate generally more rapid removal of aerosol and vapor at atmospheric conditions than at elevated pressures and temperatures; the results of the MELCOR calculation indicate a similar response for the iodine vapor removal, but the opposite behavior for the aerosols. This is probably because the aerosol response is strongly affected by problems in correctly calculating evaporation/condensation onto aerosols (as discussed in more detail in Section 7.5). 


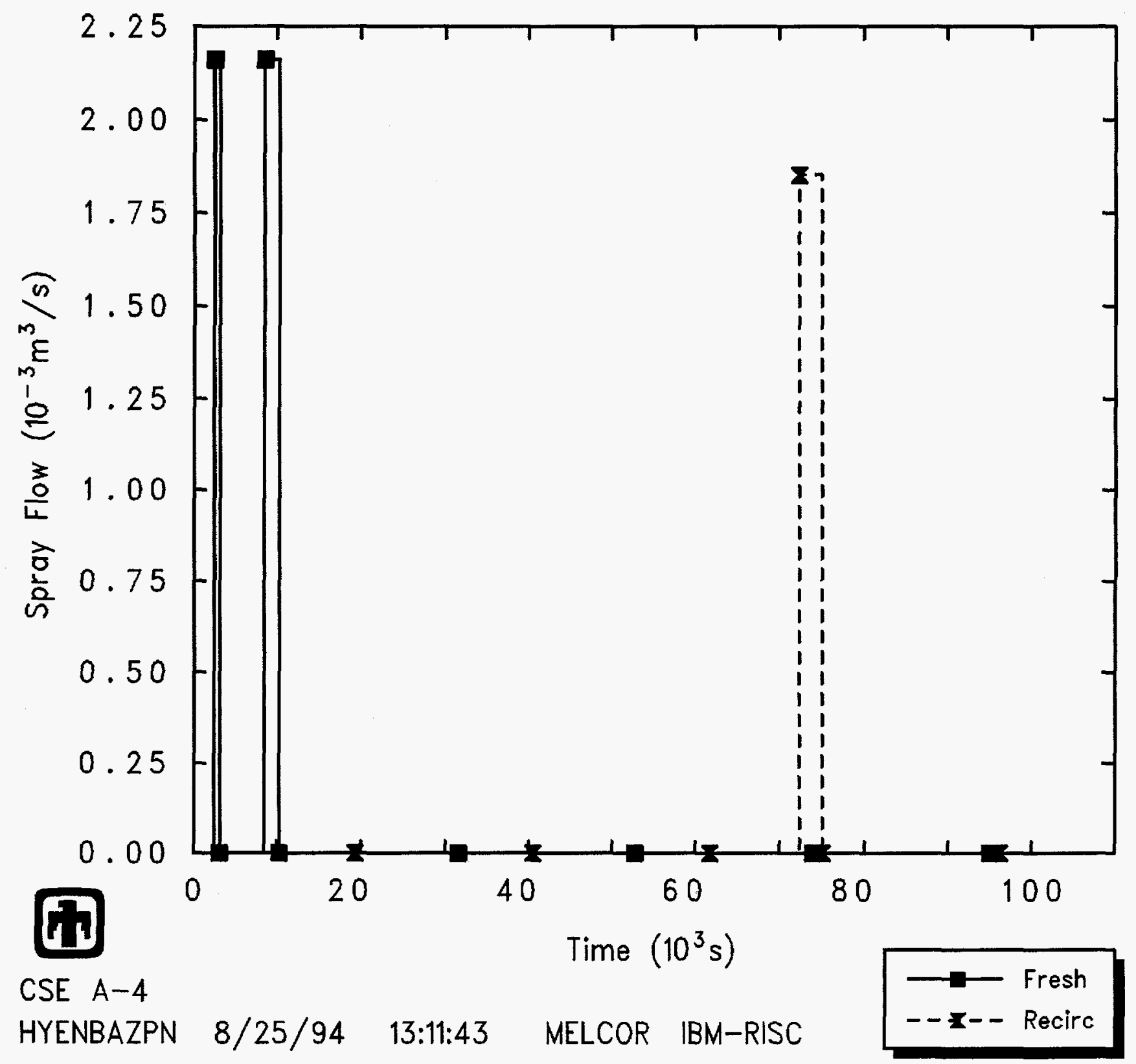

Figure 5.3.1. Spray Flow Rate for CSE Test A-4 - Reference Calculation 


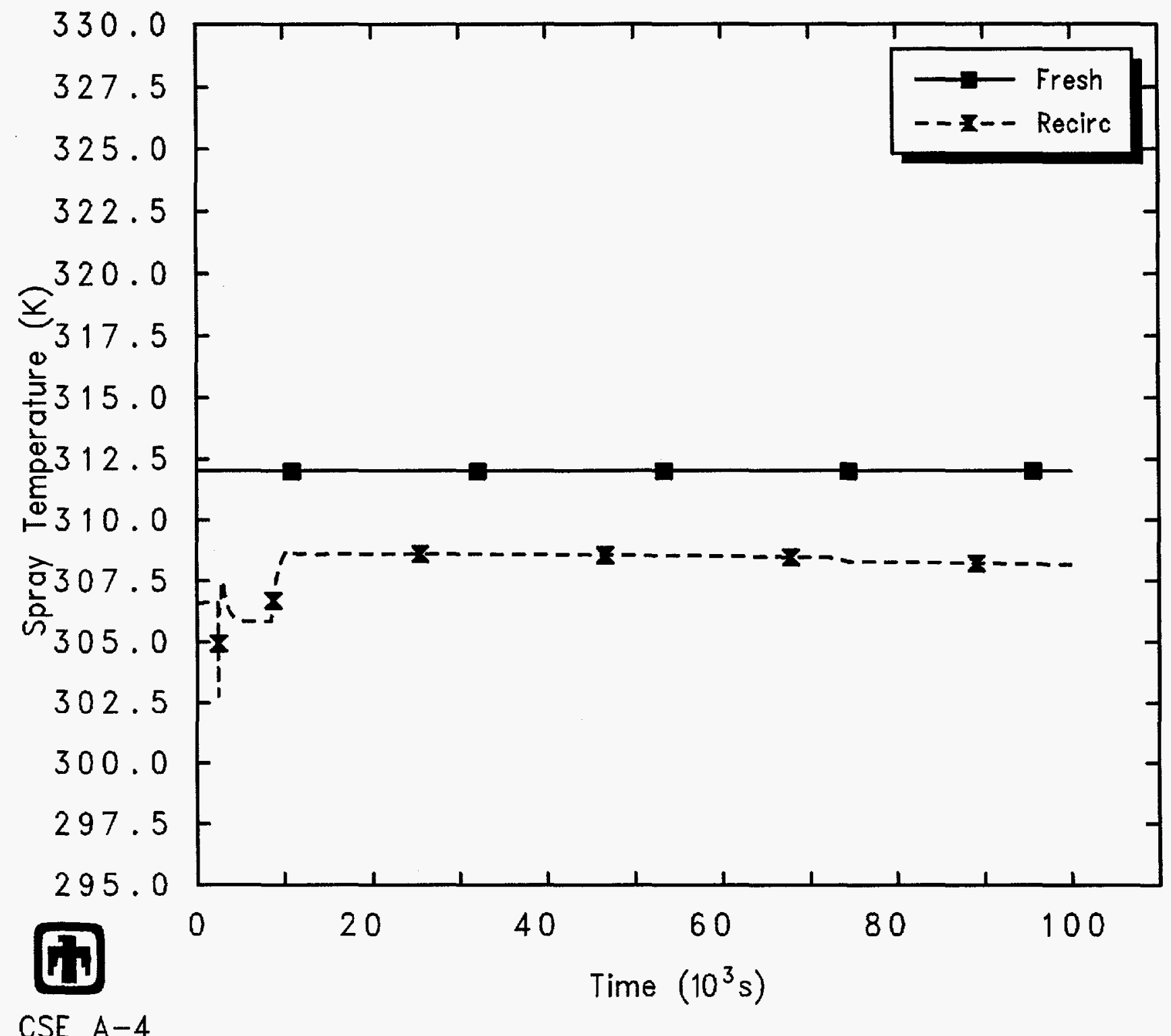

HYENBAZPN $8 / 25 / 94 \quad 13: 11: 43 \quad$ MELCOR IBM-RISC

Figure 5.3.2. Spray Temperature for CSE Test A-4 - Reference Calculation 


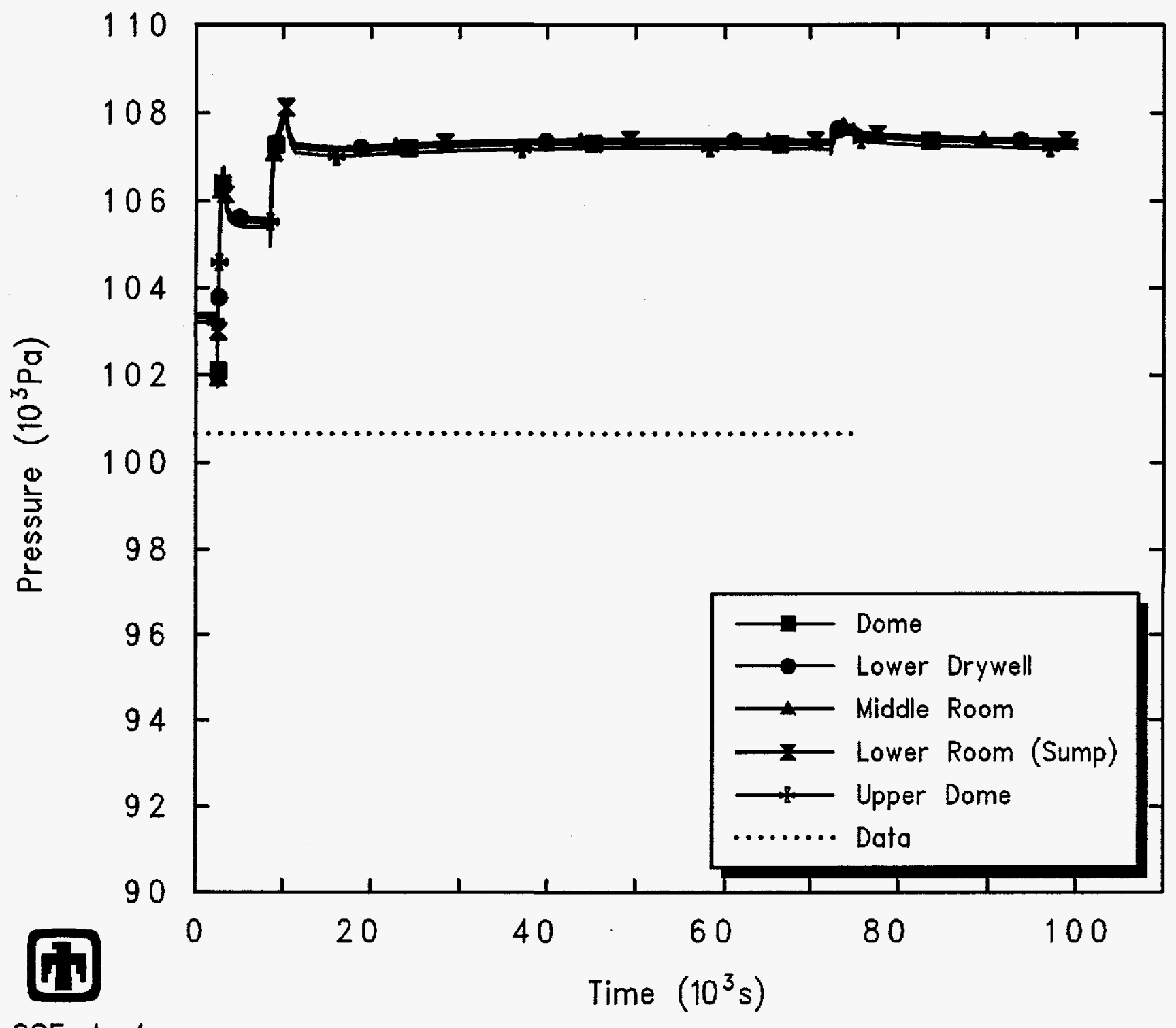

CSE A-4

HYENBAZPN $8 / 25 / 94 \quad 13: 11: 43$ MELCOR IBM-RISC

Figure 5.3.3. Vessel Pressure for CSE Test A-4 - Reference Calculation 


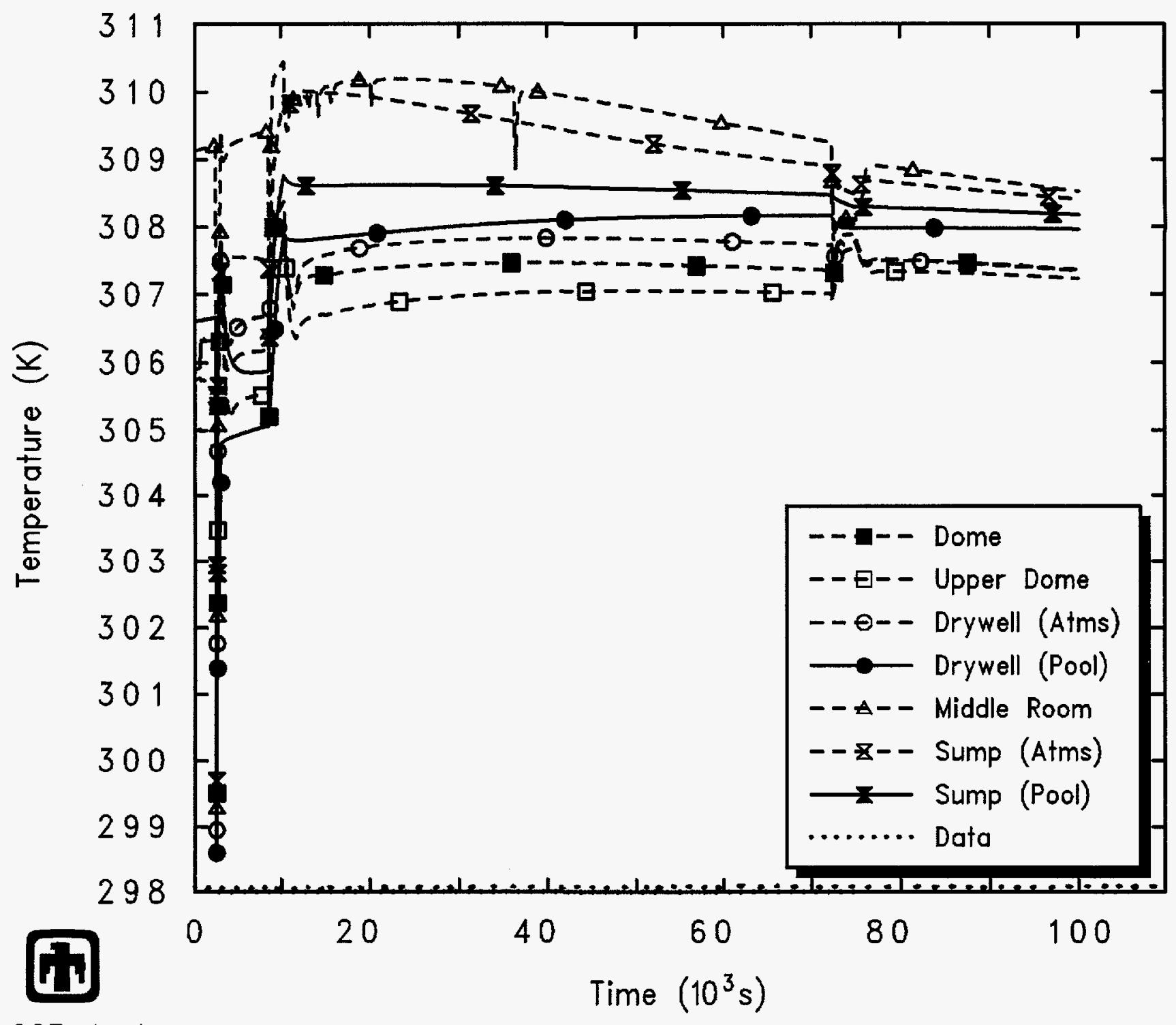

CSE $A-4$

HYENBAZPN $8 / 25 / 94 \quad 13: 11: 43 \quad$ MELCOR IBM-RISC

Figure 5.3.4. Vessel Temperatures for CSE Test A-4 - Reference Calculation 


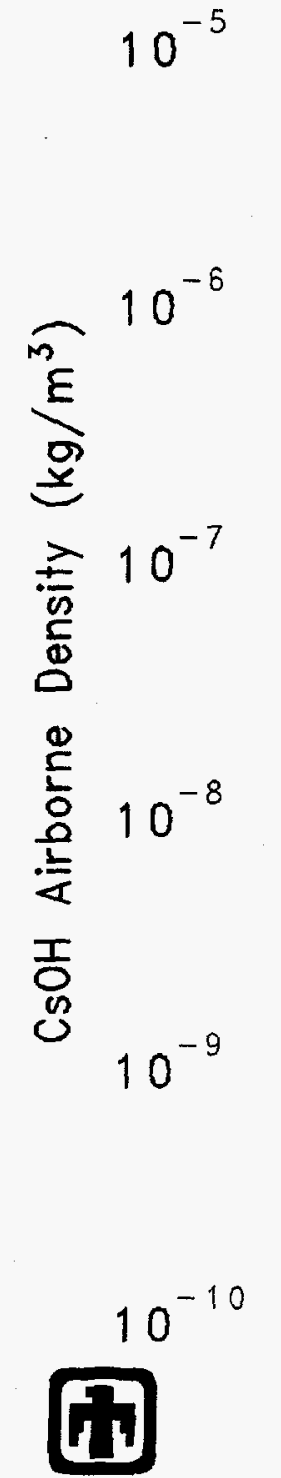

CSE A-4 HYENBAZPN

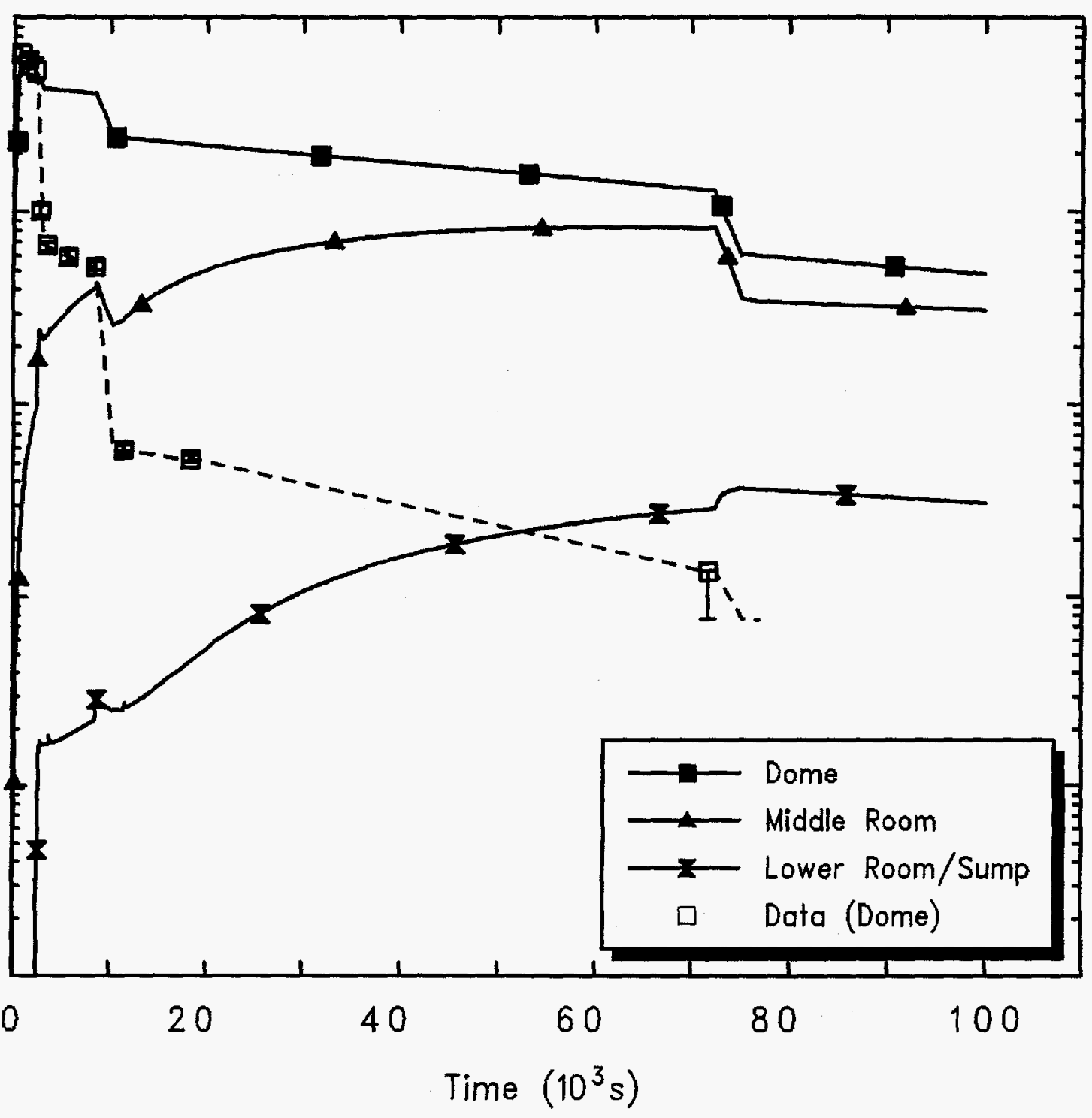

Figure 5.3.5. Cesium Aerosol Airborne Concentrations for CSE Test A-4 - Reference Calculation 


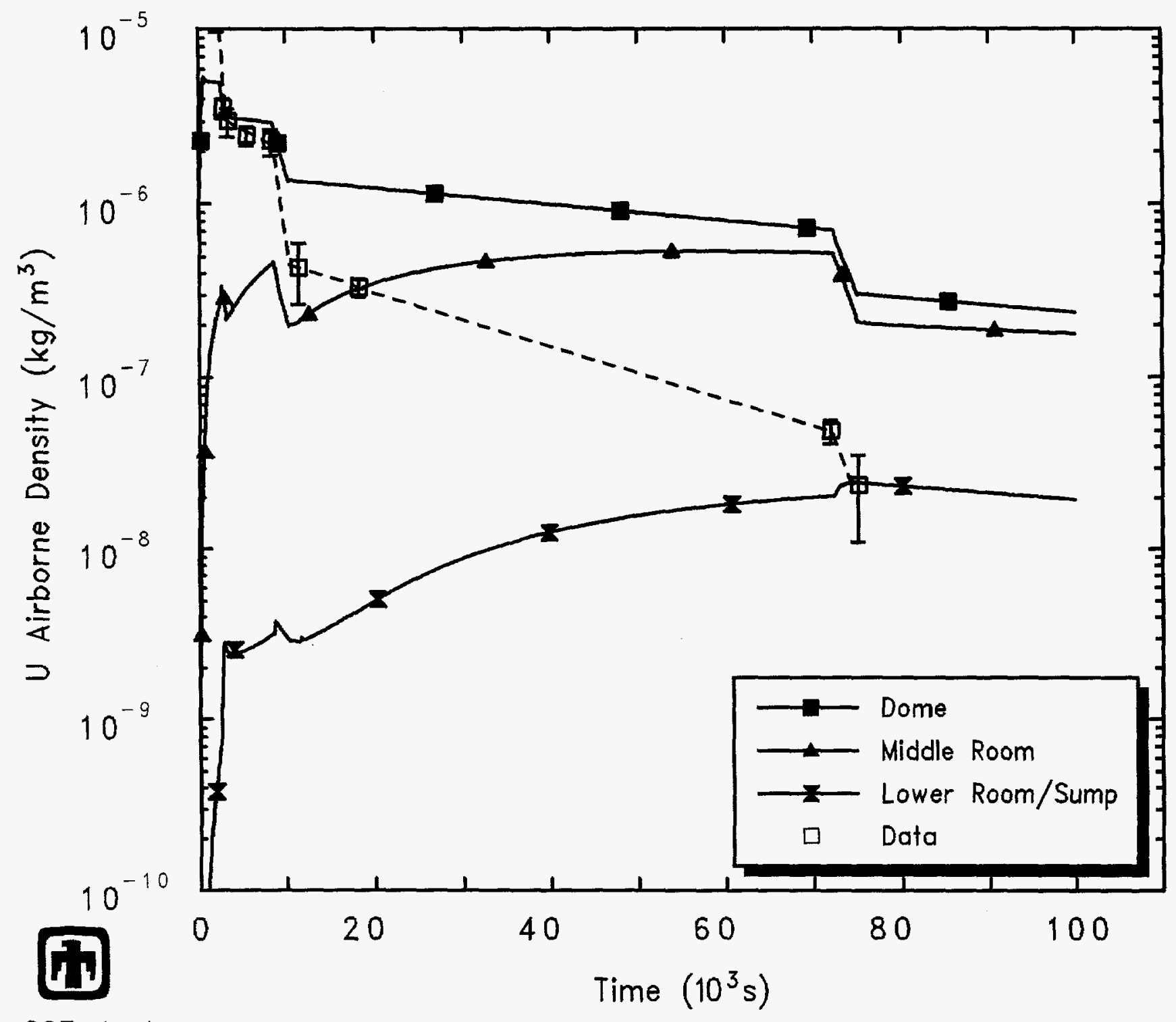

CSE A-4

HYENBAZPN $8 / 25 / 94 \quad 13: 11: 43 \quad$ MELCOR IBM-RISC

Figure 5.3.6. Uranium Aerosol Airborne Concentrations for CSE Test A-4 Reference Calculation 


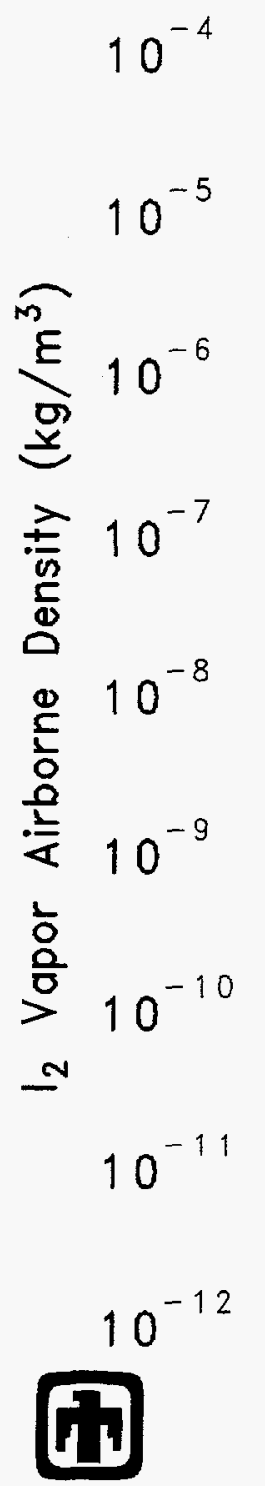

CSE A-4

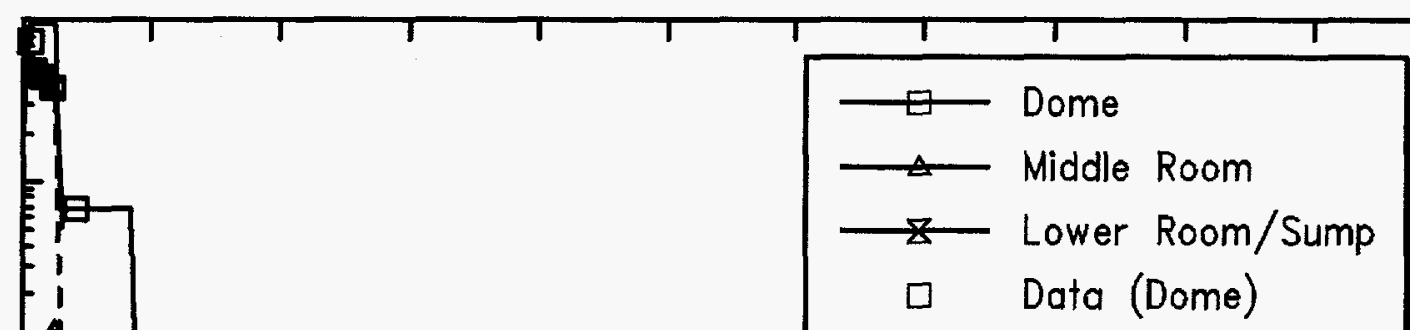

HYENBAZPN $\quad 8 / 25 / 94 \quad 13: 11: 43 \quad$ MELCOR IBM-RISC

Figure 5.3.7. Iodine Vapor Airborne Concentrations for CSE Test A-4 - Reference Calculation 
Table 5.3.1. Washout Rates for CSE Tests - Effect of Atmosphere Conditions (A-6 vs $\mathrm{A}-4$ )

\begin{tabular}{|c|c|c|c|c|}
\hline & \multicolumn{4}{|c|}{$t_{1 / 2}(\min )$} \\
\hline & \multicolumn{2}{|c|}{ A-6 } & \multicolumn{2}{|c|}{ A-4 } \\
\hline & Measured & Calculated & Measured & Calculated \\
\hline \multicolumn{5}{|l|}{ Cesium } \\
\hline First spray & 5.6 & 8.7 & 3.5 & 40 \\
\hline Second spray & 13. & 11.6 & 9.3 & 40 \\
\hline Third spray & $a$ & 8.7 & 50 & 40 \\
\hline \multicolumn{5}{|l|}{ Fourth spray } \\
\hline \multicolumn{5}{|l|}{ Uranium } \\
\hline First spray & 7.8 & 6.9 & 5.5 & 11.6 \\
\hline Second spray & 12.5 & 11.7 & 13.5 & 23 \\
\hline Third spray & $b$ & 7.7 & 45 & 35 \\
\hline \multicolumn{5}{|l|}{ Fourth spray } \\
\hline \multicolumn{5}{|l|}{ Iodine } \\
\hline First spray & 2.1 & 2.8 & 1.4 & 2.3 \\
\hline Second spray & 35. & 2.5 & 9 & 2.3 \\
\hline Third spray & $\infty$ & 8.7 & $a$ & 3.5 \\
\hline Fourth spray & & & & \\
\hline \multicolumn{5}{|c|}{$\begin{array}{c}{ }^{a} \text { concentration increased } \\
{ }^{b} \text { indeterminant }\end{array}$} \\
\hline
\end{tabular}




\subsection{Effect of Spray Chemistry (A-6 vs A-7)}

Test A-7 generally resembled test A-6. In both tests, the test vessel was initialized with a saturated steam-air mixture at a pressure of about 3 bars and a temperature of $390-400 \mathrm{~K}$, and the spray flow rates used were quite similar in these two tests. The major difference was that the boron carrier in the sprays in CSE A-7 was simply demineralized water rather than the $\mathrm{NaOH}$ solution used in CSE A-6, changing the spray $\mathrm{pH}$ from 9.5 to 5. The timing of the sprays was also somewhat different. Both test A-6 and test A-7 had two fresh spray injections, followed by a recirculating spray period, but test A-7 also had a late-time third fresh spray injection. The total amounts of water injected during the first two fresh spray periods were quite similar in tests A-6 and A-7, but the total amount of sump water recirculated in test A-6 was about a third less than during test A-7. (Section 2 gives more detail on the experiment initial and boundary conditions.)

Figures 5.4.1 and 5.4.2 show the spray flow rates and spray temperatures for both the three fresh sprays and the recirculating spray used in test A-7. Note that the spray flow rates used in the calculation represent $70 \%$ of the spray rates given in Table 2.3 and are the flows assumed to interact fully with the atmosphere; also note that the recirculating spray temperature shown is simply the temperature of the water in the lower room sump.

The effects of the sprays on containment atmosphere response are presented in Figures 5.4.3 and 5.4.4, which compare calculated MELCOR results with test data for the test vessel pressures and temperatures, respectively, for test A-7. The MELCOR result for test A-7 is consistent with and similar to the behavior predicted for test A-6 (cf. Section 5.1). In general, the overall pressure is underpredicted owing to overprediction of the pressure drops during fresh spray injection, because too much steam is being condensed by the sprays; in contrast, the calculated temperatures are generally higher than measured in the test vessel dome. A smaller pressure drop is predicted in the MELCOR calculation during the recirculating spray injection between $79380 \mathrm{~s}$ and $82890 \mathrm{~s}$ than was observed from the experimental data.

Figures 5.4.5 and 5.4.6 present the concentrations of cesium and uranium aerosols, respectively, in various regions in the test vessel atmosphere, compared with test data; Figure 5.4.7 presents the concentrations of iodine vapor in the test vessel atmosphere, together with test data. The concentrations shown are the mass of airborne aerosol or vapor in the control volume atmosphere divided by the volume. The concentrations plotted are for the test vessel dome or main room, the middle room and the lower room or sump; the calculation shows virtually equal concentrations in the dome, the upper dome above the spray injection elevation and the lower drywell, because the recirculation flow modelled keeps these volumes well mixed.

The calculated concentrations of airborne cesium and uranium aerosols predicted for test A-7, and for the airborne iodine vapor as well, are very similar qualitatively to the results obtained for test A-6, presented in Section 5.1. Table 5.4.1 summarizes the washout rates predicted for cesium and uranium aerosol and iodine vapor in the test vessel dome for the different spray chemistry used in A-6 and A-7. Although the absolute aerosol and vapor removal rates do not agree quantitatively with the test data, MELCOR 


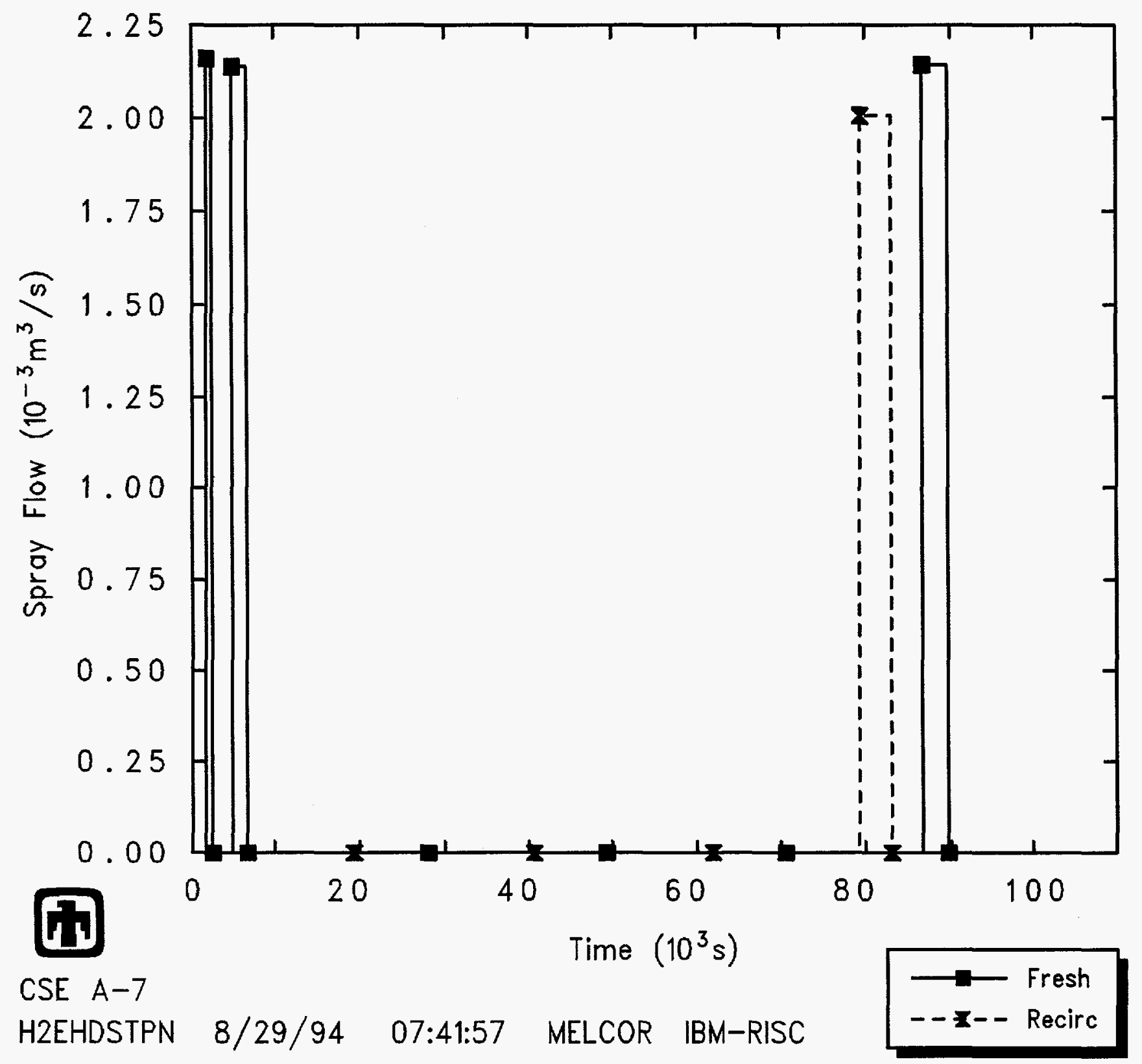

Figure 5.4.1. Spray Flow Rate for CSE Test A-7 - Reference Calculation 


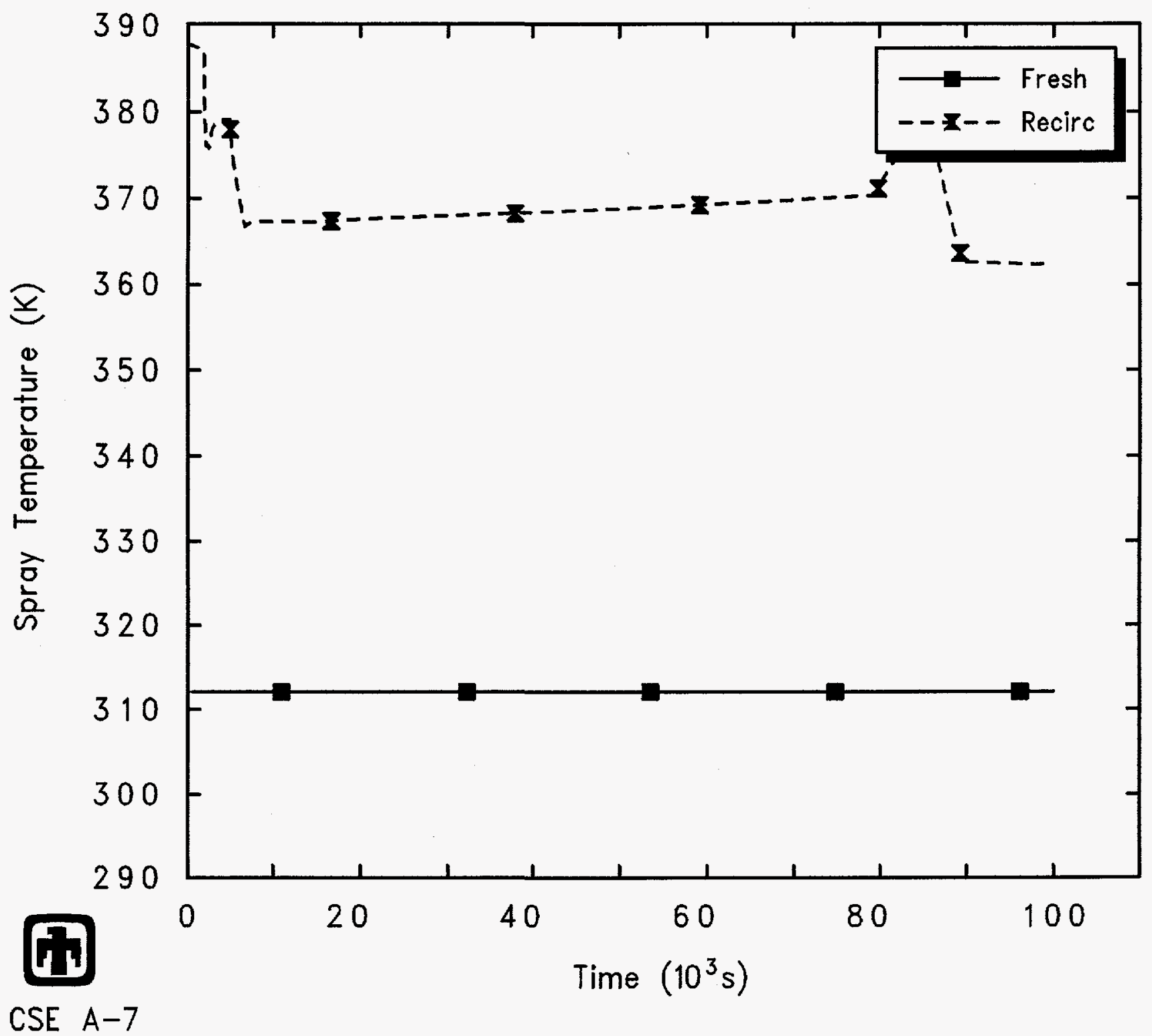

H2EHDSTPN $\quad 8 / 29 / 94 \quad 07: 41: 57 \quad$ MELCOR $\quad$ IBM-RISC

Figure 5.4.2. Spray Temperature for CSE Test A-7 - Reference Calculation 


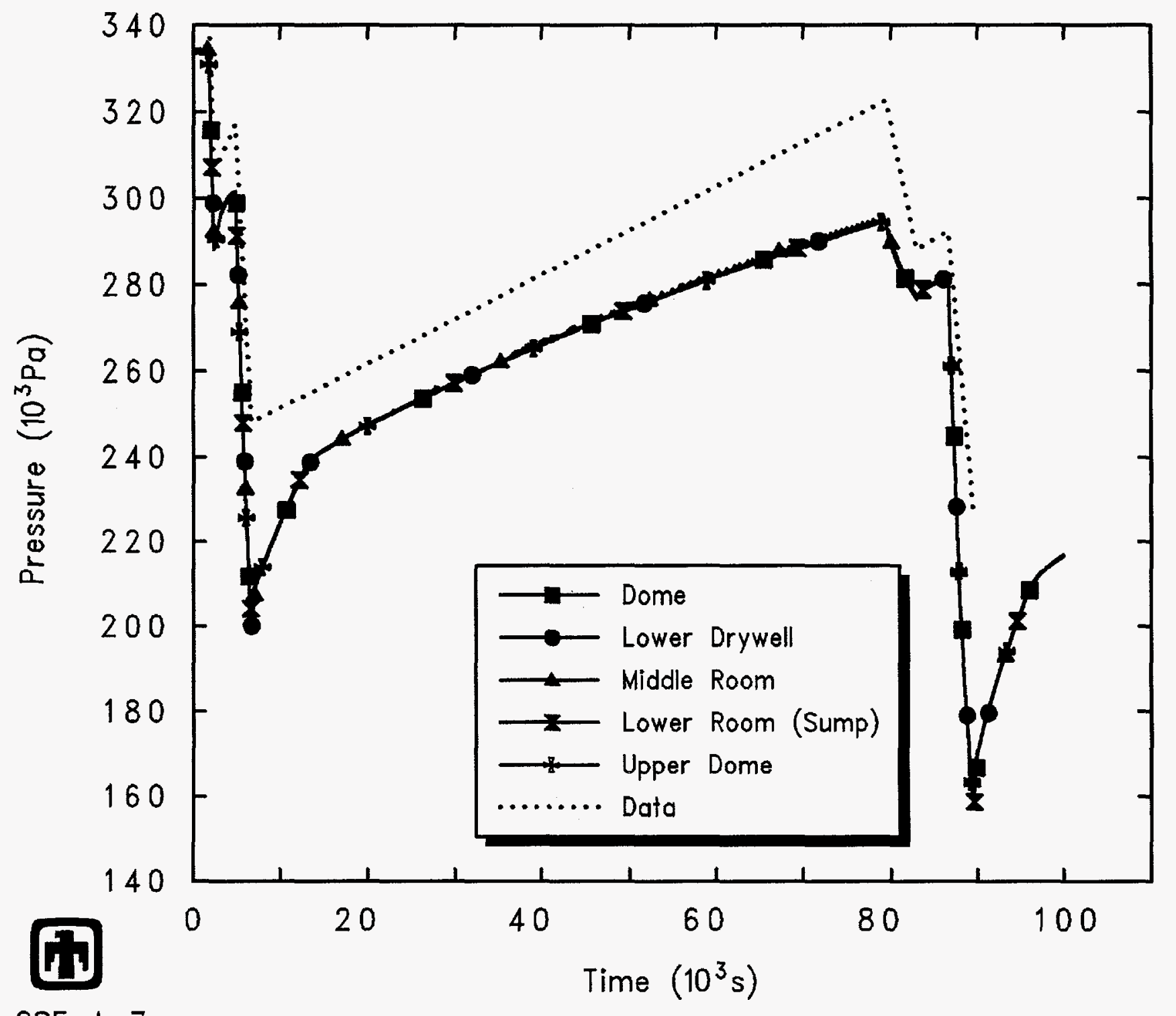

CSE A-7

H2EHDSTPN $8 / 29 / 94 \quad 07: 41: 57$ MELCOR IBM-RISC

Figure 5.4.3. Vessel Pressure for CSE Test A-7 - Reference Calculation 


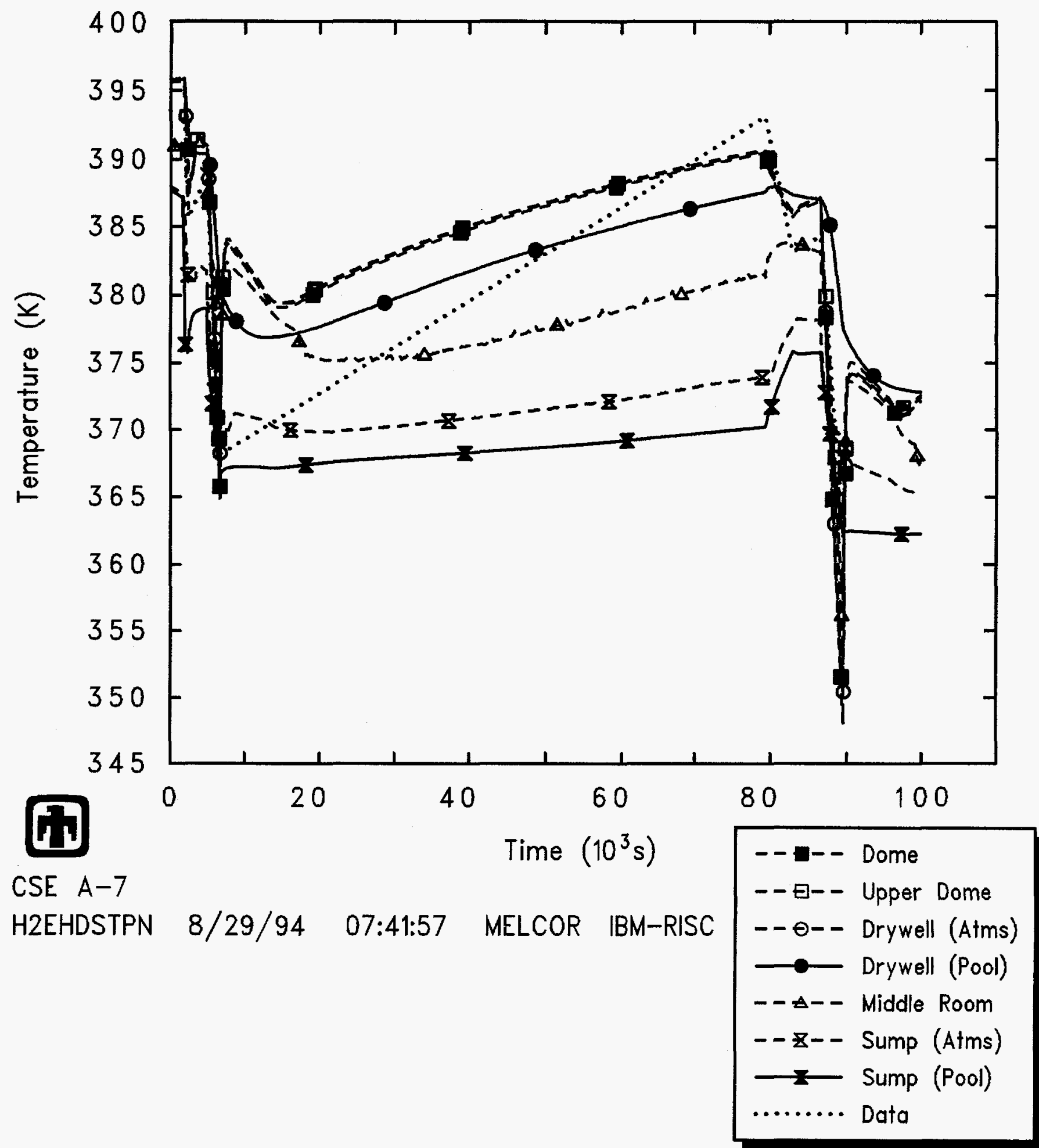

Figure 5.4.4. Vessel Temperatures for CSE Test A-7 - Reference Calculation 


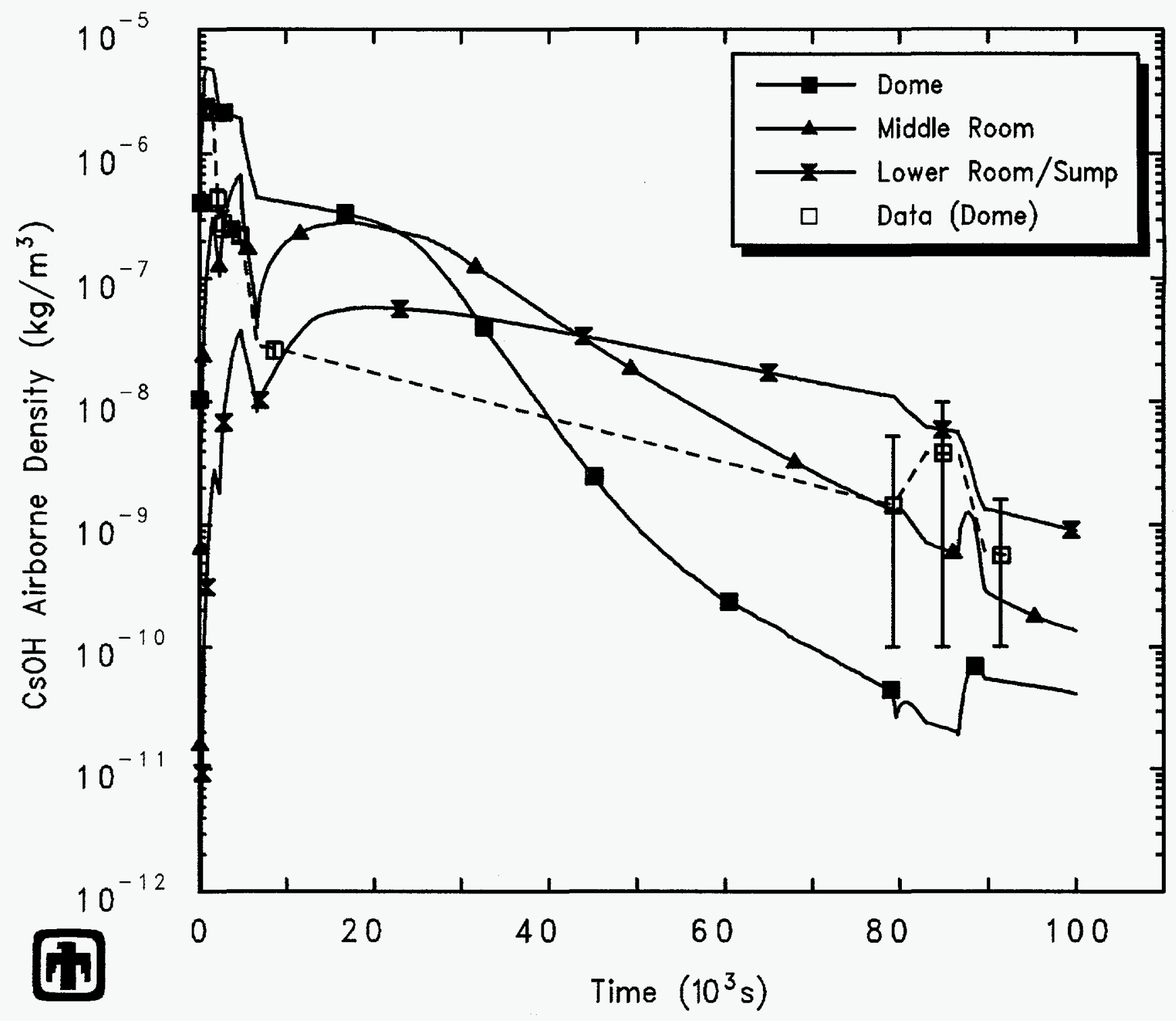

CSE $A-7$

H2EHDSTPN $8 / 29 / 94 \quad 07: 41: 57$ MELCOR IBM-RISC

Figure 5.4.5. Cesium Aerosol Airborne Concentrations for CSE Test A-7 - Reference Calculation 


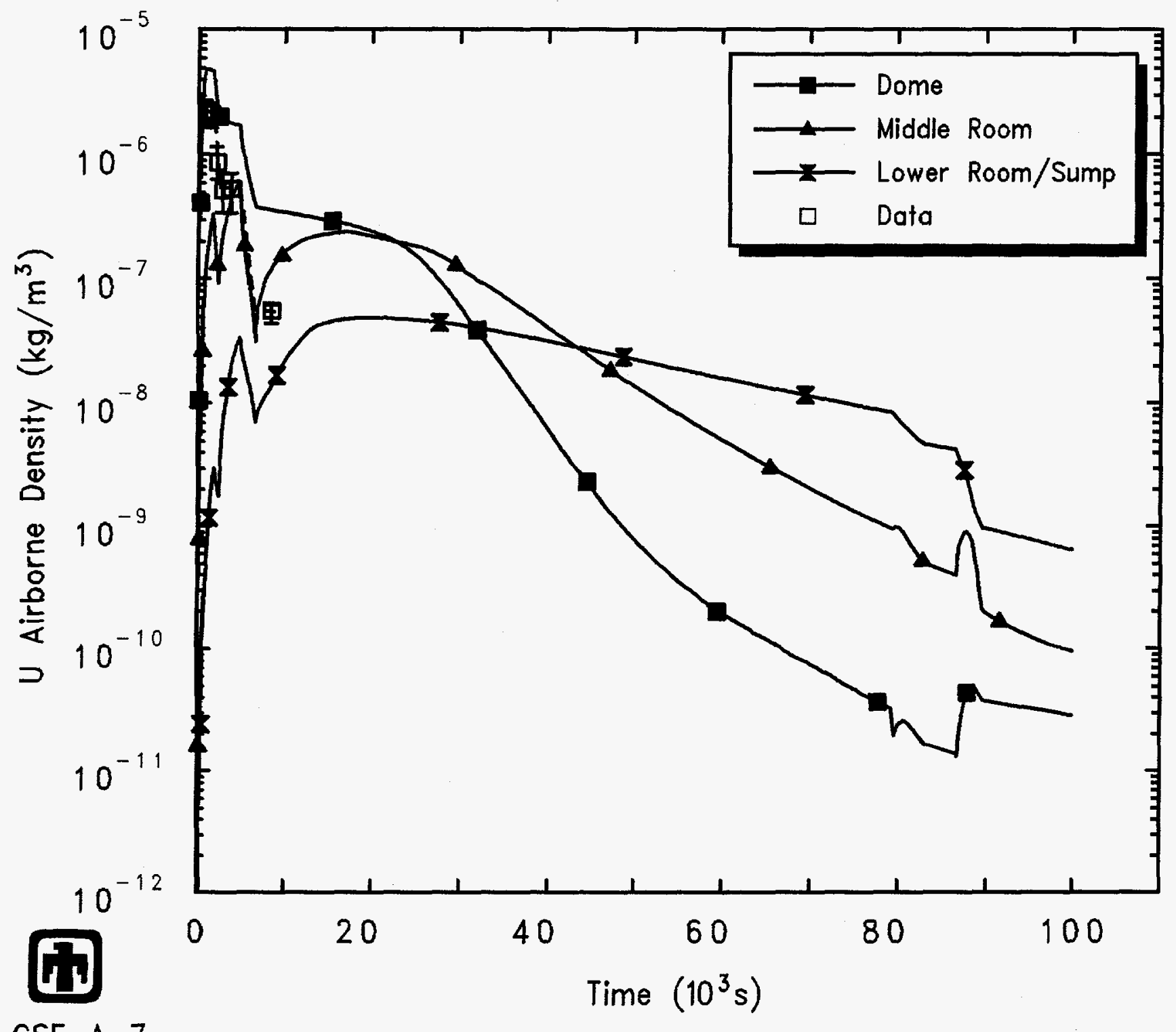

CSE A-7

H2EHDSTPN 8/29/94 07:41:57 MELCOR IBM-RISC

Figure 5.4.6. Uranium Aerosol Airborne Concentrations for CSE Test A-7 -Reference Calculation 


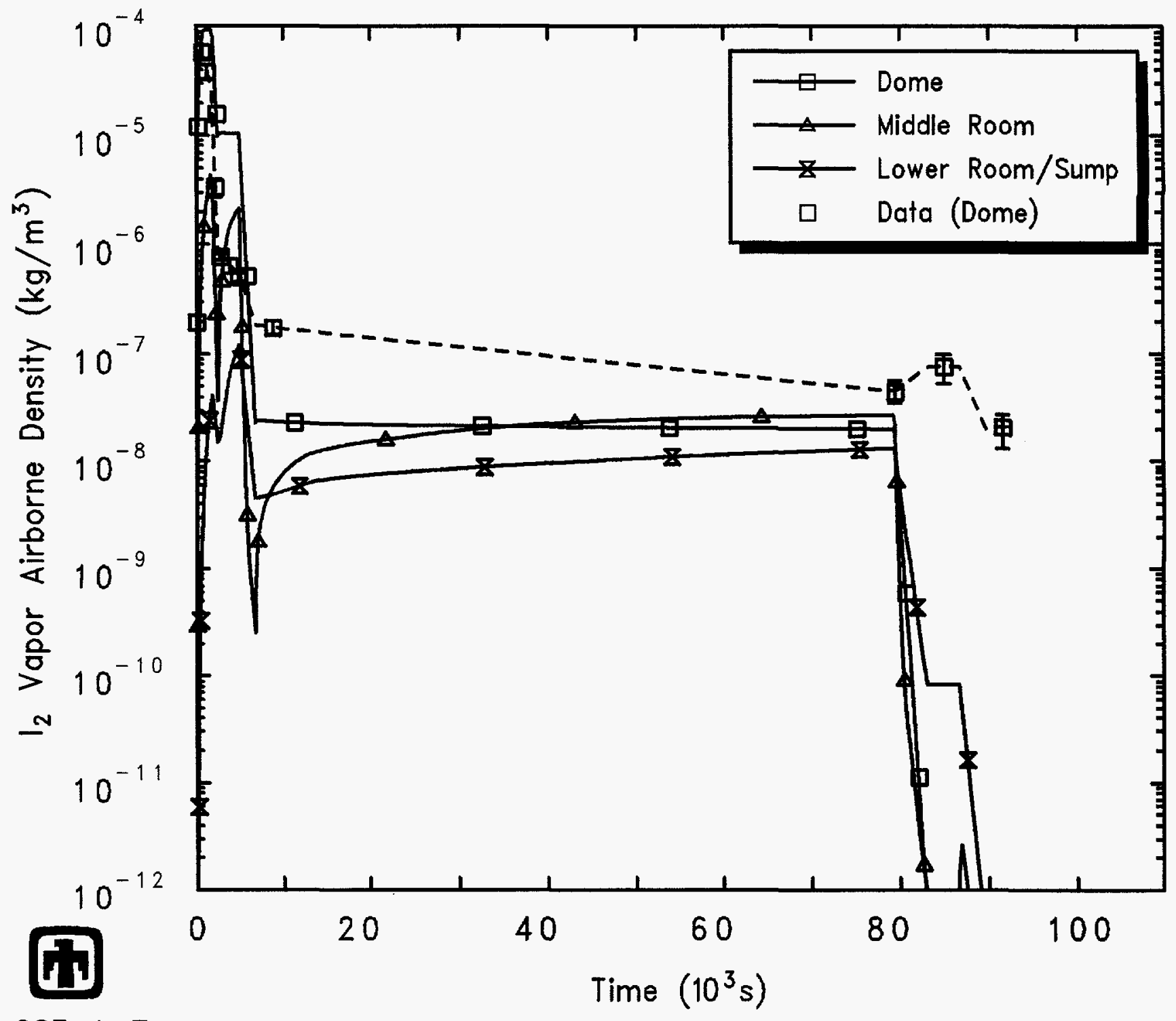

CSE A-7

H2EHDSTPN $8 / 29 / 94 \quad 07: 41: 57$ MELCOR IBM-RISC

Figure 5.4.7. Iodine Vapor Airborne Concentrations for CSE Test A-7 - Reference Calculation 
correctly predicts little or no change in the aerosol removal rate but a more rapid vapor removal with the caustic spray used in test A-6 than for the simple demineralized water spray used in test A-7. (Note that for this test A-7 MELCOR simulation the iodine partition coefficient was reduced from the value of 5000 used in the test A-6 calculation to 2500 for both the fresh and recirculation sprays, causing the slower iodine removal; these are the best-estimate values recommended in the MELCOR users guide [29] for sodium hydroxide sprays and for boric acid sprays, respectively.)

\subsection{Effect of Continuous Spray (A-10 and A-12)}

Two experiments were done in the CSE test series in which the sprays were started shortly before the start of fission product simulant release and continued without interruption until all the fresh solution had been sprayed into the containment vessel [19]. After a $10 \mathrm{~min}$ period to arrange valves, recirculation from the sump was started and continued for about $20 \mathrm{hr}$.

Table 2.7 lists the conditions for these two experiments. The conditions in the two continuous spray experiments were generally similar. The test vessel was initialized with a saturated steam-air mixture at a pressure of about 3 bars and a temperature of 390 $400 \mathrm{~K}$, and the spray flow rates were quite similar to the intermittent spray flow rates in test A-6, with the same spray nozzles and spray droplet size distribution. The major difference in these two continuous spray tests was that the sprays in CSE A-12 included sodium thiosulfate in addition to the borax solution. The timing of the sprays was also somewhat different. The fresh spray in test A-12 was begun earlier than in test A-10, and the recirculating spray in test A-12 was continued somewhat longer than in test A-10. Therefore the total amount of water injected during test A-12 was somewhat greater than in test A-10.

Figures 5.5.1 and 5.5.2 show the spray flow rates and spray temperatures for both the fresh spray and the recirculating spray used in test A-10. Note that the spray flow rates used in the calculation represent $70 \%$ of the flow rates given in Table 2.3 and are the flows assumed to interact fully with the atmosphere; also note that the recirculating spray temperature shown is simply the temperature of the water in the lower room sump.

The effects of the sprays on containment atmosphere response are presented in Figures 5.5.3 and 5.5.4, which compare calculated MELCOR results with test data for the test vessel pressures and temperatures, respectively, for test A-10. The MELCOR result for the continuous spray test, test A-10, is generally consistent with the behavior predicted for the intermittent spray tests, such as test A-6 (cf. Section 5.1). In general, the overall pressure is underpredicted owing to the pressure drop during fresh spray injection being overpredicted because too much steam is being condensed by the sprays. There is a smaller pressure recovery predicted in the MELCOR calculation during the recirculating spray injection period than observed in the experimental data. The temperature comparison reflects the pressure comparison, because the temperatures correspond to the saturation temperature of water at the partial pressure of water vapor in the atmosphere. 
Table 5.4.1. Cesium Aerosol Washout Rates for CSE Tests - Effect of Spray Chemistry (A-6 vs A-7)

\begin{tabular}{|c|c|c|c|}
\hline \multicolumn{4}{|c|}{$t_{1 / 2}(\min )$} \\
\hline & & & A-7 \\
\hline Measured & Calculated & Measured & Calculated \\
\hline
\end{tabular}

\section{Cesium}

First spray

Second spray

Third spray

$$
\begin{aligned}
& 5.6 \\
& 13 .
\end{aligned}
$$

Fourth spray

\section{Uranium}

First spray

Second spray

Third spray

7.8
12.5
$a$

6.9

11.7

8.7

11.7

8.7

3.8

8.7

10.3

$b$

7.7

9.9

19

11.6

Fourth spray

Iodine

First spray

Second spray

2.1

35 .

Third spray

$\infty$

7.7

5.0

7.7

9

7.7

a

9.9

a $\quad 11.6$

Fourth spray

2.8

2.2

22

3.2

2.5

8.7

b

3.5

3.9

27

3.9

${ }^{a}$ indeterminant

${ }^{b}$ concentration increased 


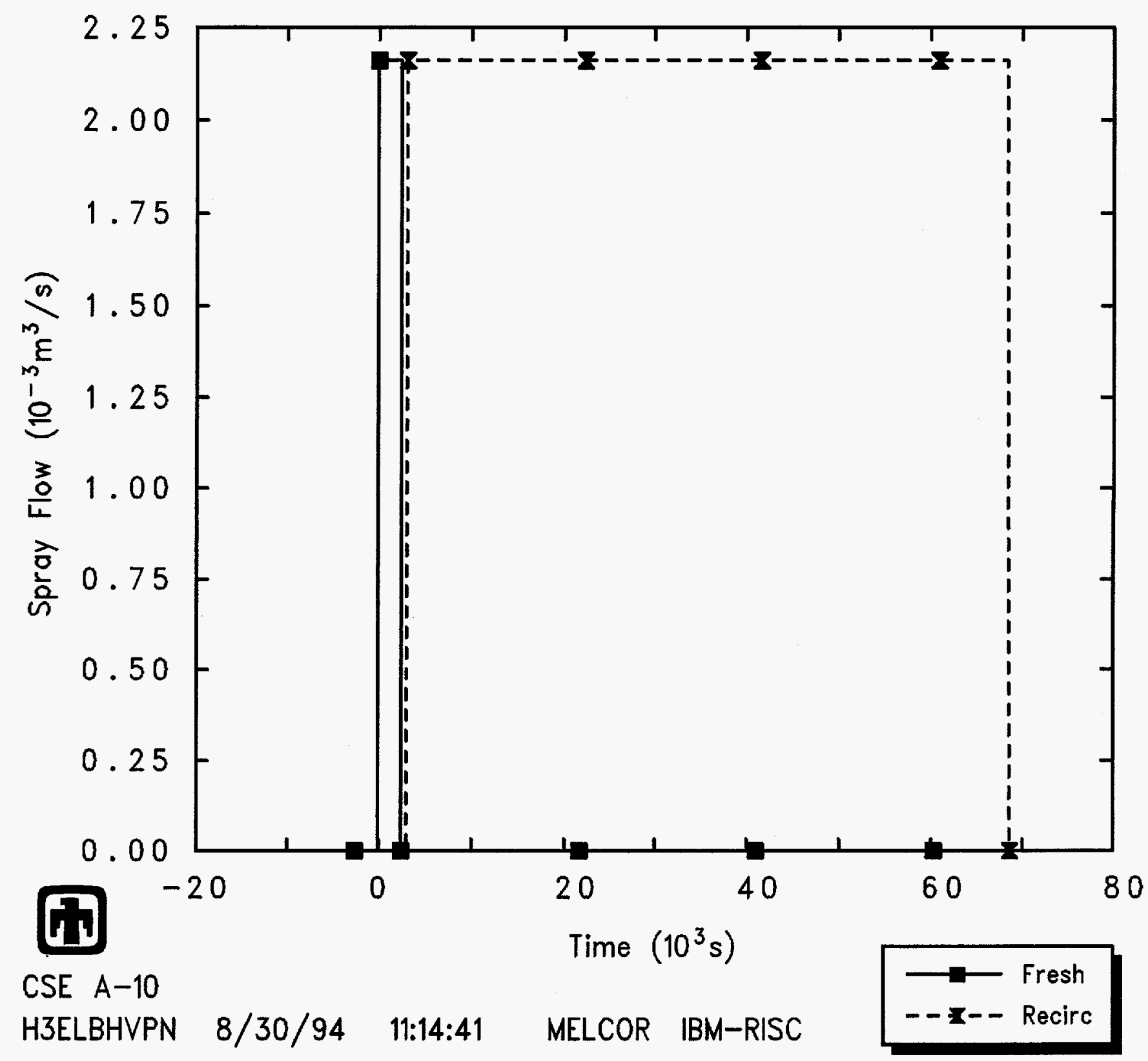

Figure 5.5.1. Spray Flow Rate for CSE Test A-10 - Reference Calculation 


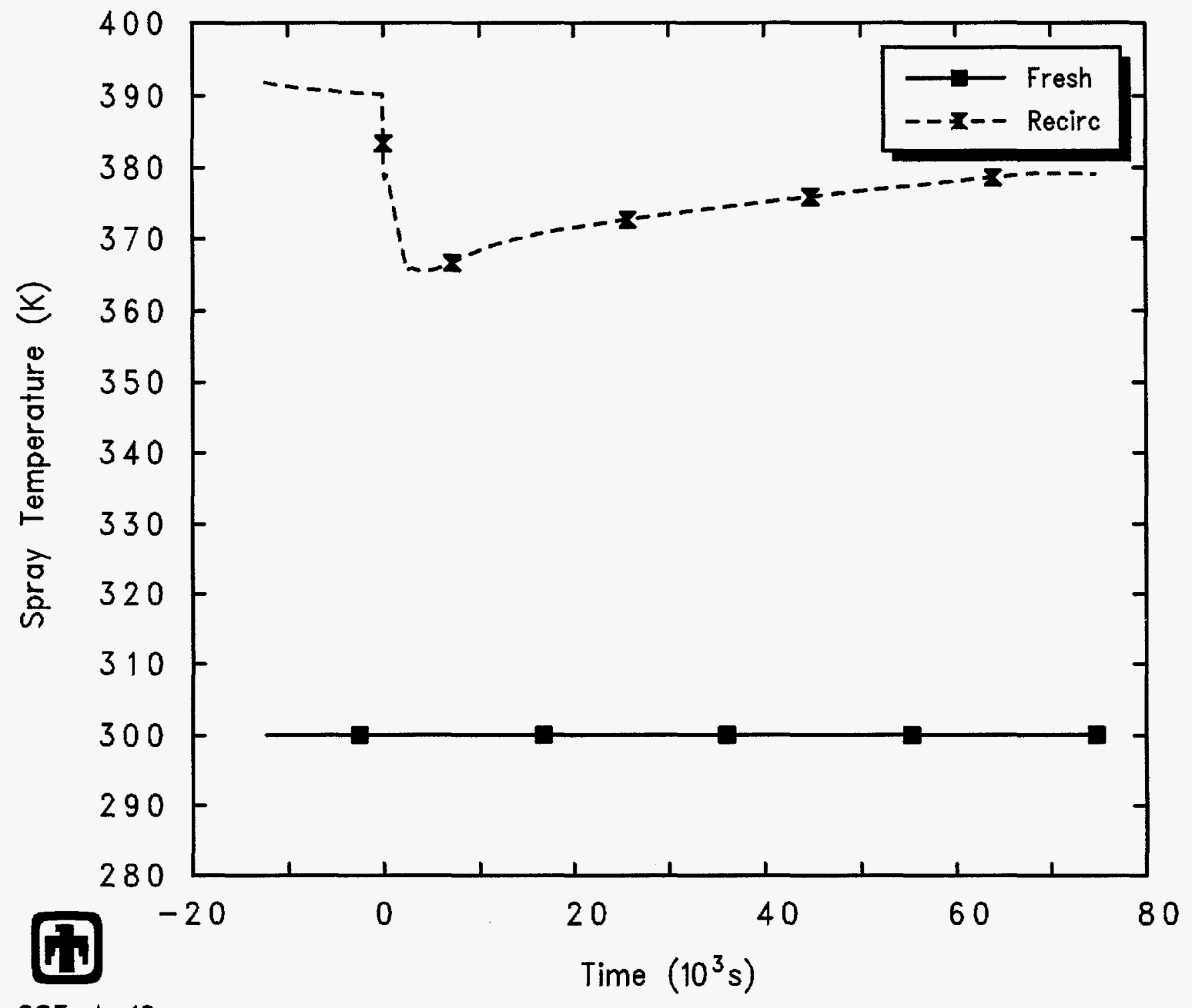

CSE $A-10$

H3ELBHVPN $\quad 8 / 30 / 94 \quad 11: 14: 41 \quad$ MELCOR IBM-RISC

Figure 5.5.2. Spray Temperature for CSE Test A-10 - Reference Calculation 


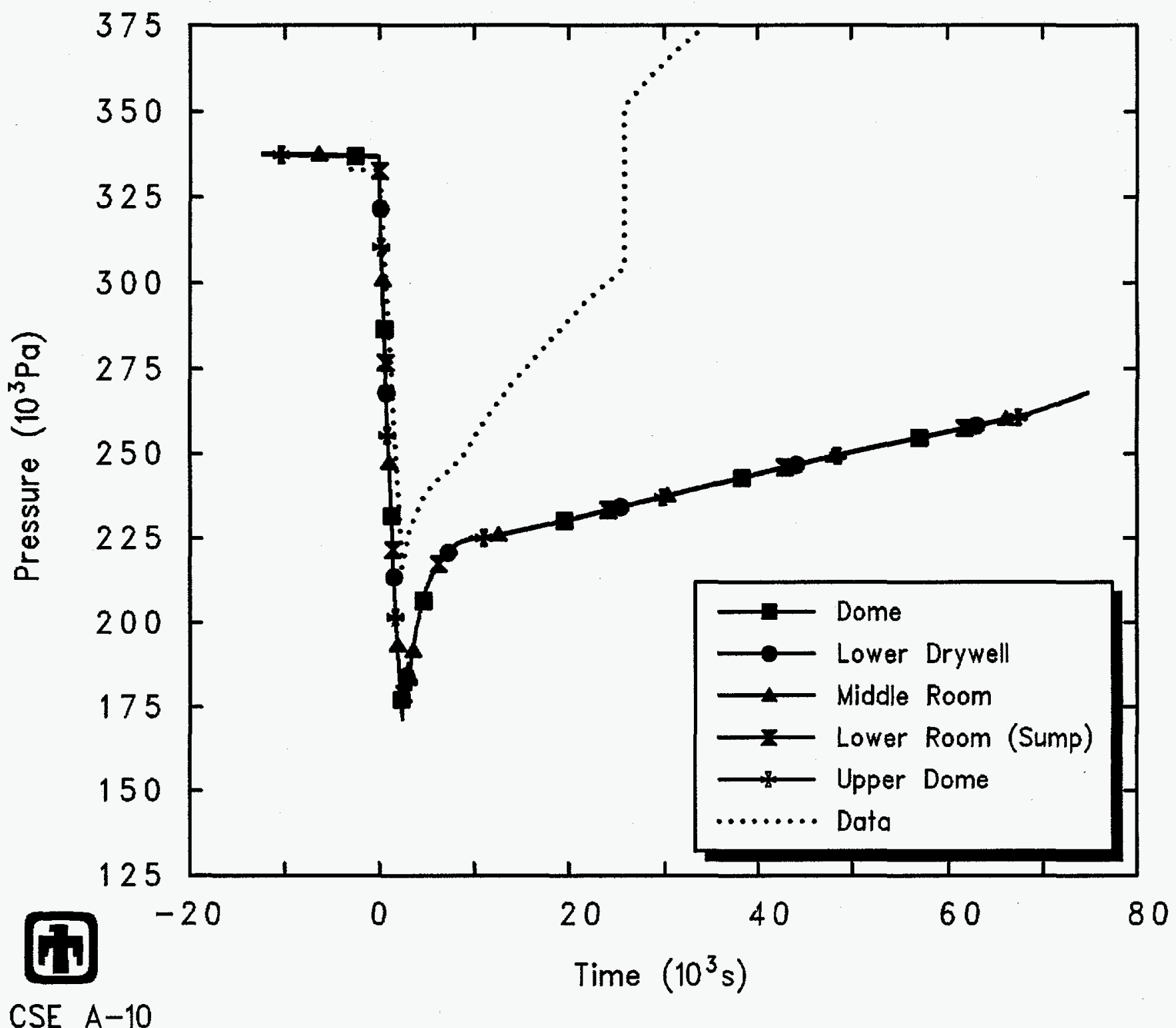

H3ELBHVPN $\quad 8 / 30 / 94 \quad 11: 14: 41 \quad$ MELCOR IBM-RISC

Figure 5.5.3. Vessel Pressure for CSE Test A-10 - Reference Calculation 


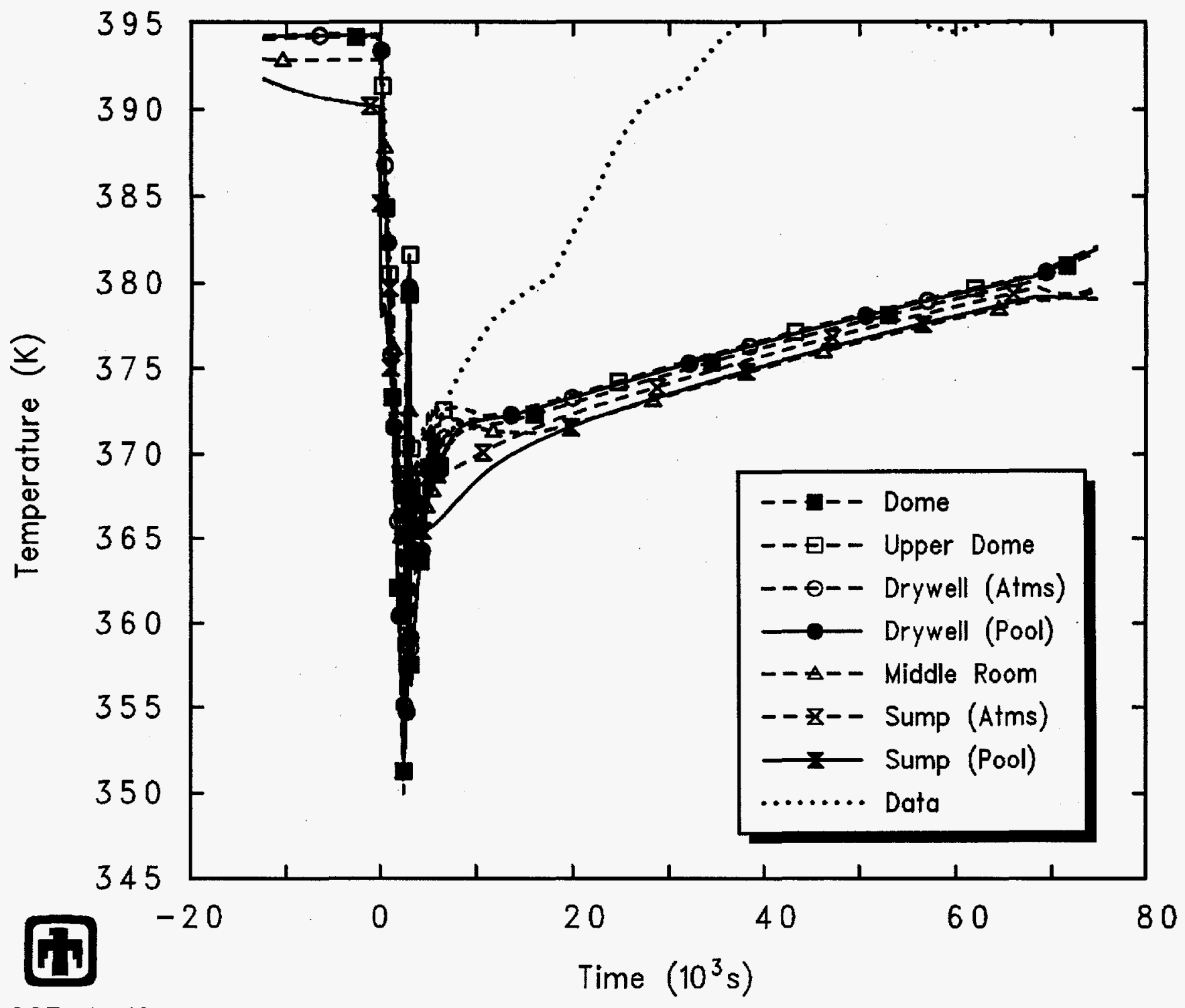

CSE $A-10$

H3ELBHVPN $\quad 8 / 30 / 94 \quad 11: 14: 41 \quad$ MELCOR IBM-RISC

Figure 5.5.4. Vessel Temperatures for CSE Test A-10 - Reference Calculation 
Figures 5.5.5 and 5.5.6 present the concentrations of cesium and uranium aerosols, respectively, in various regions in the test vessel atmosphere, compared with test data; Figure 5.5.7 presents the concentrations of iodine vapor in the test vessel atmosphere, together with test data. The concentrations shown are the mass of airborne aerosol or vapor in the control volume atmosphere divided by the volume. The concentrations plotted are for the test vessel dome or main room, the middle room and the lower room or sump; the calculation shows virtually equal concentrations in the dome, the upper dome above the spray injection elevation and the lower drywell, because the recirculation flow modelled keeps these volumes well mixed.

The calculated concentrations of airborne cesium and uranium aerosols predicted for test A-10, and for the airborne iodine vapor as well, are very similar qualitatively to the results obtained for test A-6, presented in Section 5.1. The initial removal rates calculated appear to agree reasonably well with experiment, but the removal rates calculated at later times are much greater than those observed in the experiment.

Figures 5.5.8 and 5.5.9 show the spray flow rates and spray temperatures for both the fresh spray and the recirculating spray used in test A-12. Note that the flow rates used in the calculation represent $70 \%$ of the spray flow rates given in Table 2.3 and are the flows assumed to interact fully with the atmosphere; also note that the recirculating spray temperature shown is simply the temperature of the water in the lower room sump.

The effects of the sprays on containment atmosphere response are presented in Figures 5.5.10 and 5.5.11, which compare calculated MELCOR results with test data for the test vessel pressures and temperatures, respectively, for test A-12. The MELCOR result for the continuous spray test test A-12 is quite similar to the behavior calculated for test A-10 and is generally consistent with the behavior predicted for the intermittent spray tests, such as test A-6 (cf. Section 5.1). The pressure drop during fresh spray injection is overpredicted, leading to underprediction of the late-time system pressure, because too much steam is being condensed by the sprays; also, there is a smaller pressure recovery predicted in the MELCOR calculation during the recirculating spray injection than observed in the experimental data. The temperature comparison reflects the pressure comparison because the temperatures correspond to the saturation temperature of water at the partial pressure of water vapor in the atmosphere.

Figures 5.5.12 and 5.5.13 present the concentrations of cesium and uranium aerosols, respectively, in various regions in the test vessel atmosphere, compared with test data; Figure 5.5.14 presents the concentrations of iodine vapor in the test vessel atmosphere, together with test data. The concentrations shown are the mass of airborne aerosol or vapor in the control volume atmosphere divided by the volume. The concentrations plotted are for the test vessel dome or main room, the middle room and the lower room or sump; the calculation shows virtually equal concentrations in the dome, the upper dome above the spray injection elevation and the lower drywell, because the recirculation flow modelled keeps these volumes well mixed.

The calculated concentrations of airborne cesium and uranium aerosols predicted for test A-12, and for the airborne iodine vapor as well, are very similar to the results 


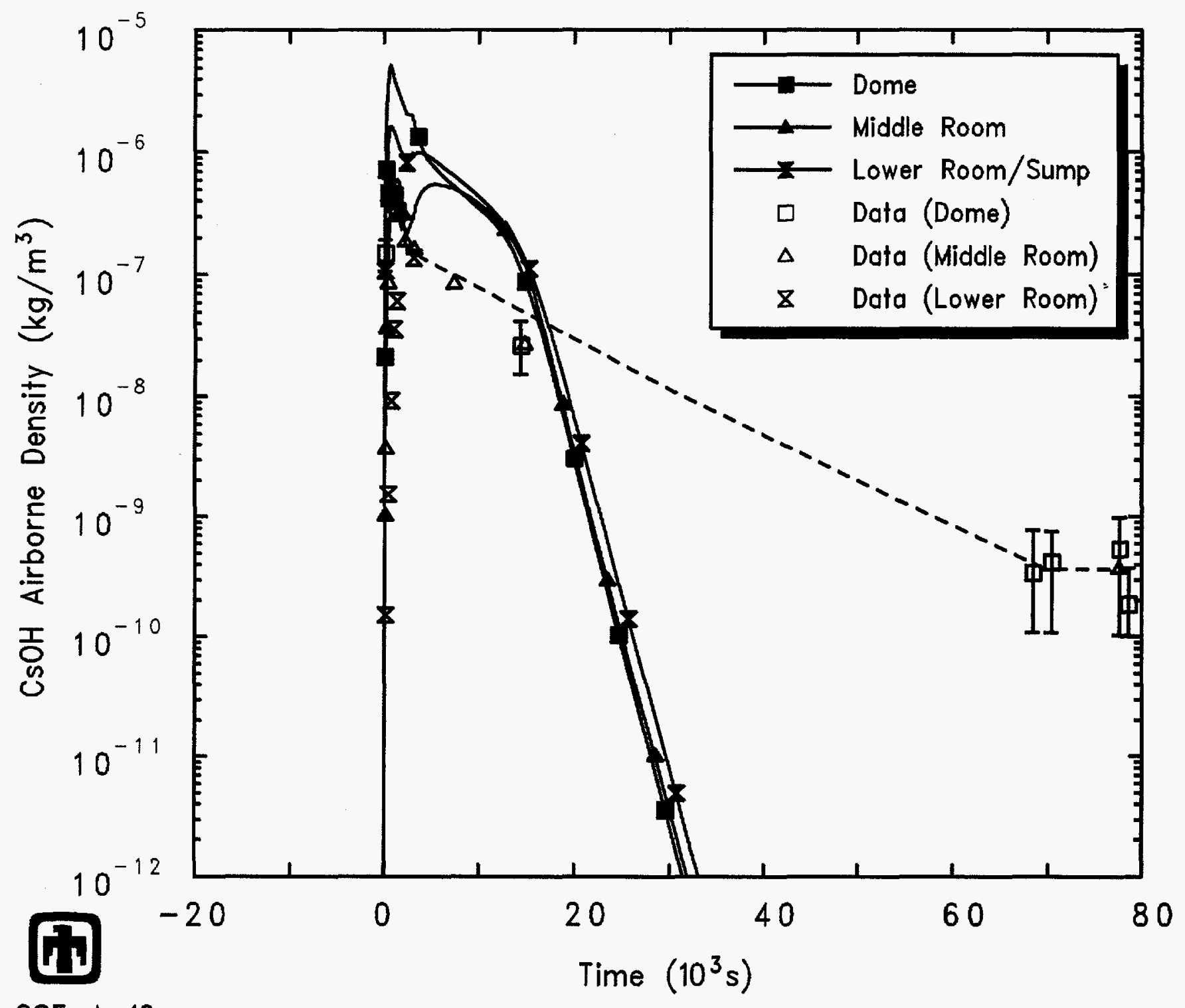

CSE $A-10$

H3ELBHVPN $8 / 30 / 94 \quad 11: 14: 41 \quad$ MELCOR IBM-RISC

Figure 5.5.5. Cesium Aerosol Airborne Concentrations for CSE Test A-10 Reference Calculation 


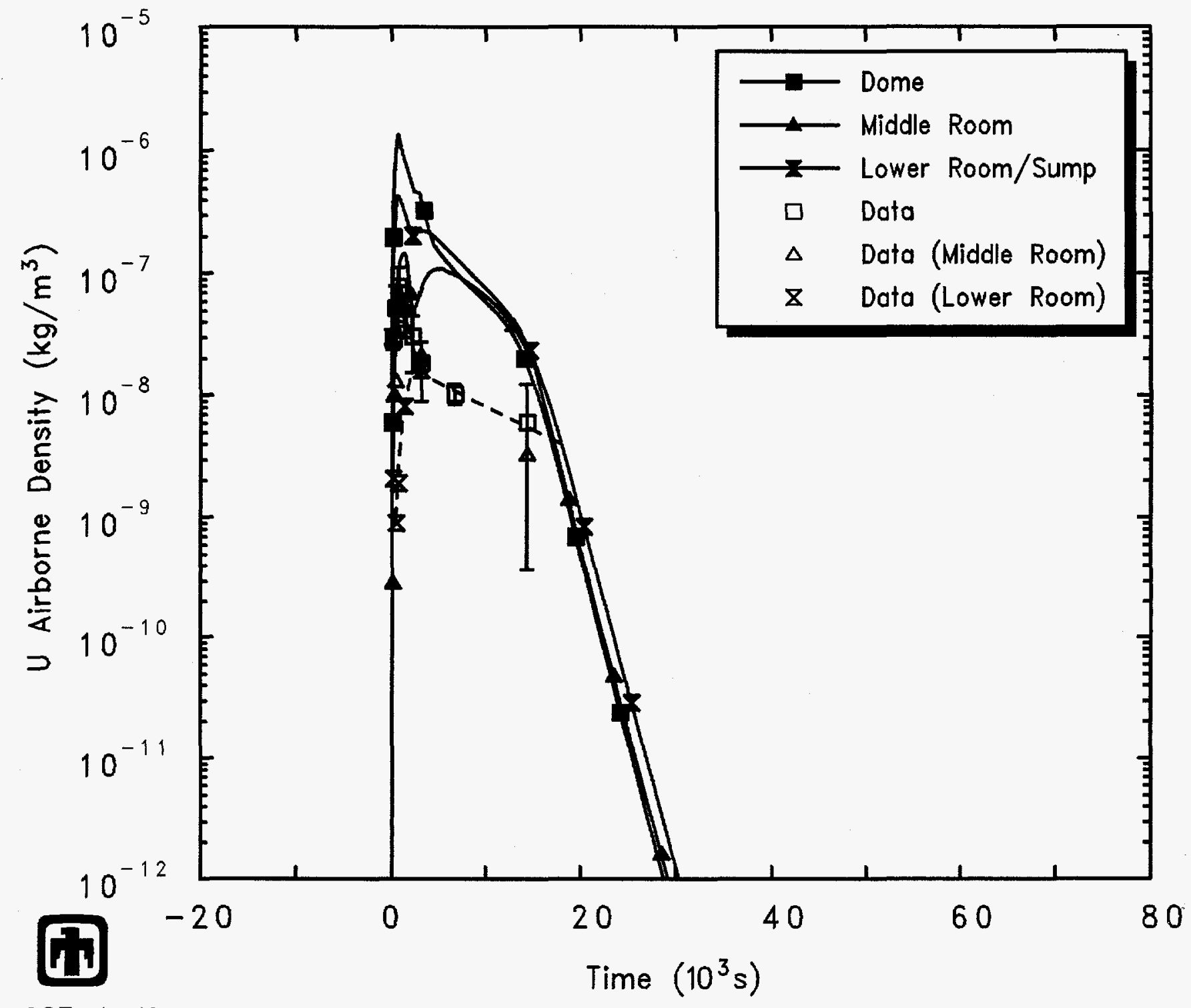

CSE $A-10$

H3ELBHVPN $8 / 30 / 94 \quad 11: 14: 41 \quad$ MELCOR IBM-RISC

Figure 5.5.6. Uranium Aerosol Airborne Concentrations for CSE Test A-10Reference Calculation 


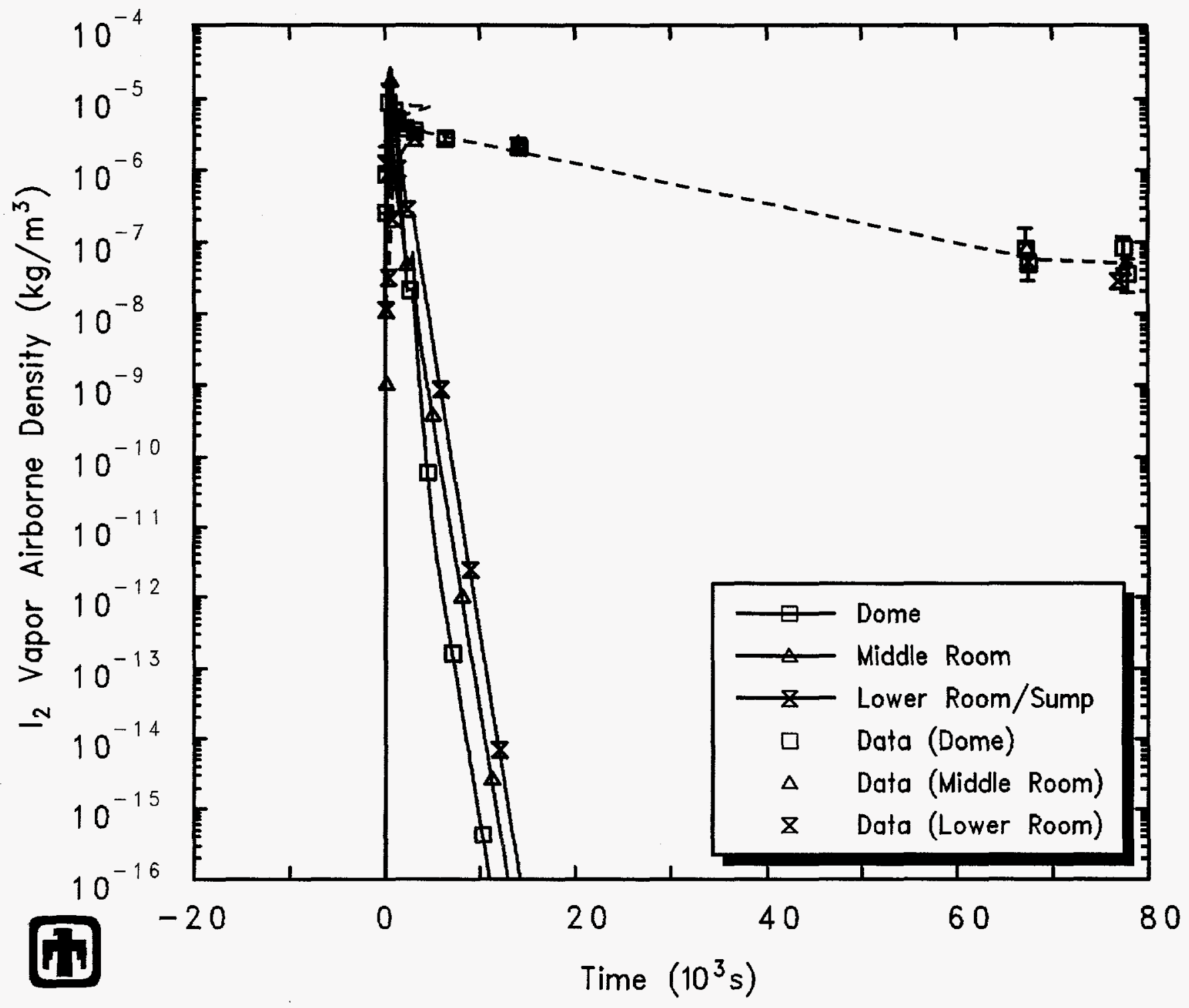

CSE A-10

H3ELBHVPN $8 / 30 / 94 \quad 11: 14: 41 \quad$ MELCOR IBM-RISC

Figure 5.5.7. Iodine Vapor Airborne Concentrations for CSE Test A-10 - Reference Calculation 


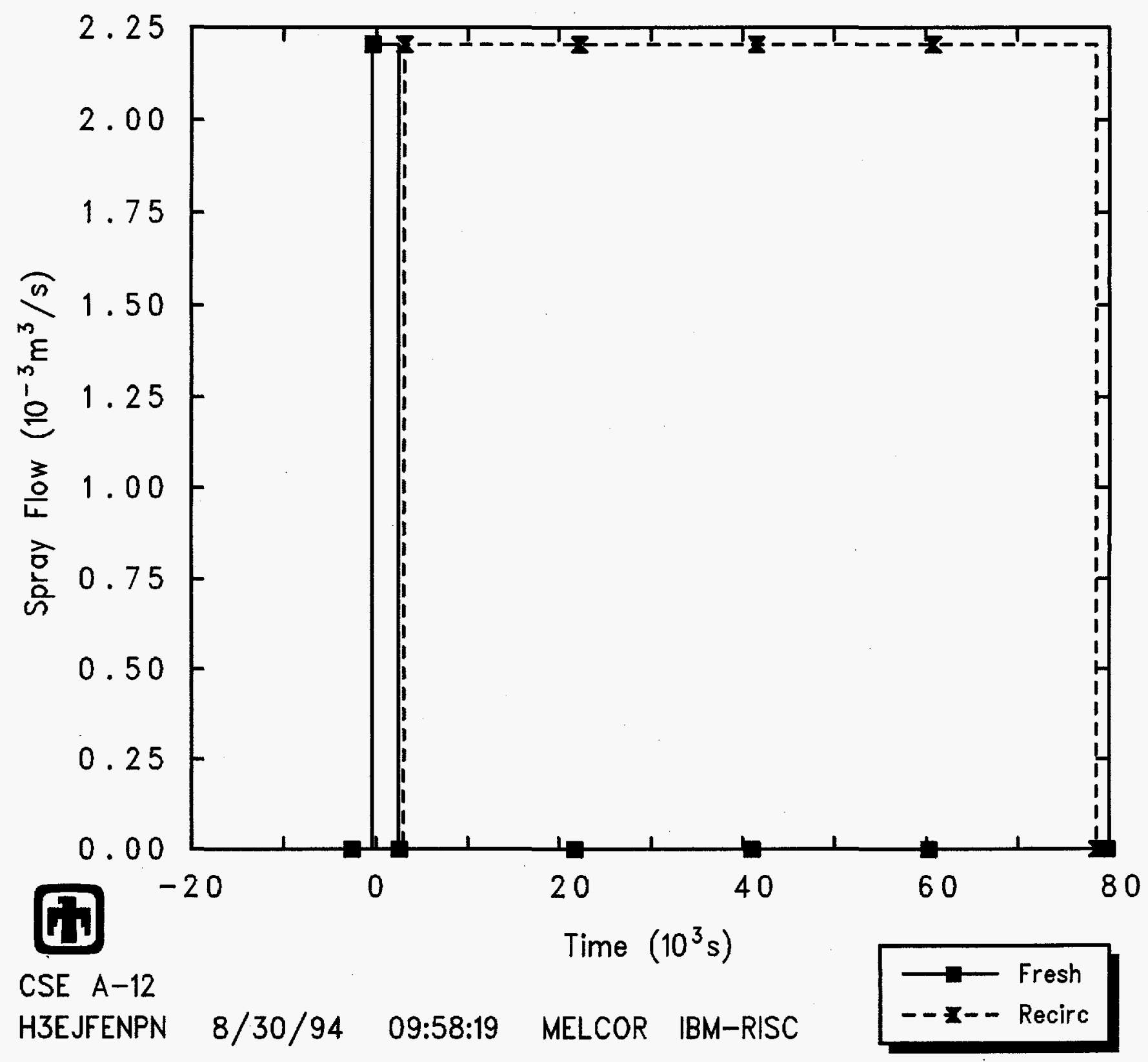

Figure 5.5.8. Spray Flow Rate for CSE Test A-12 - Reference Calculation 


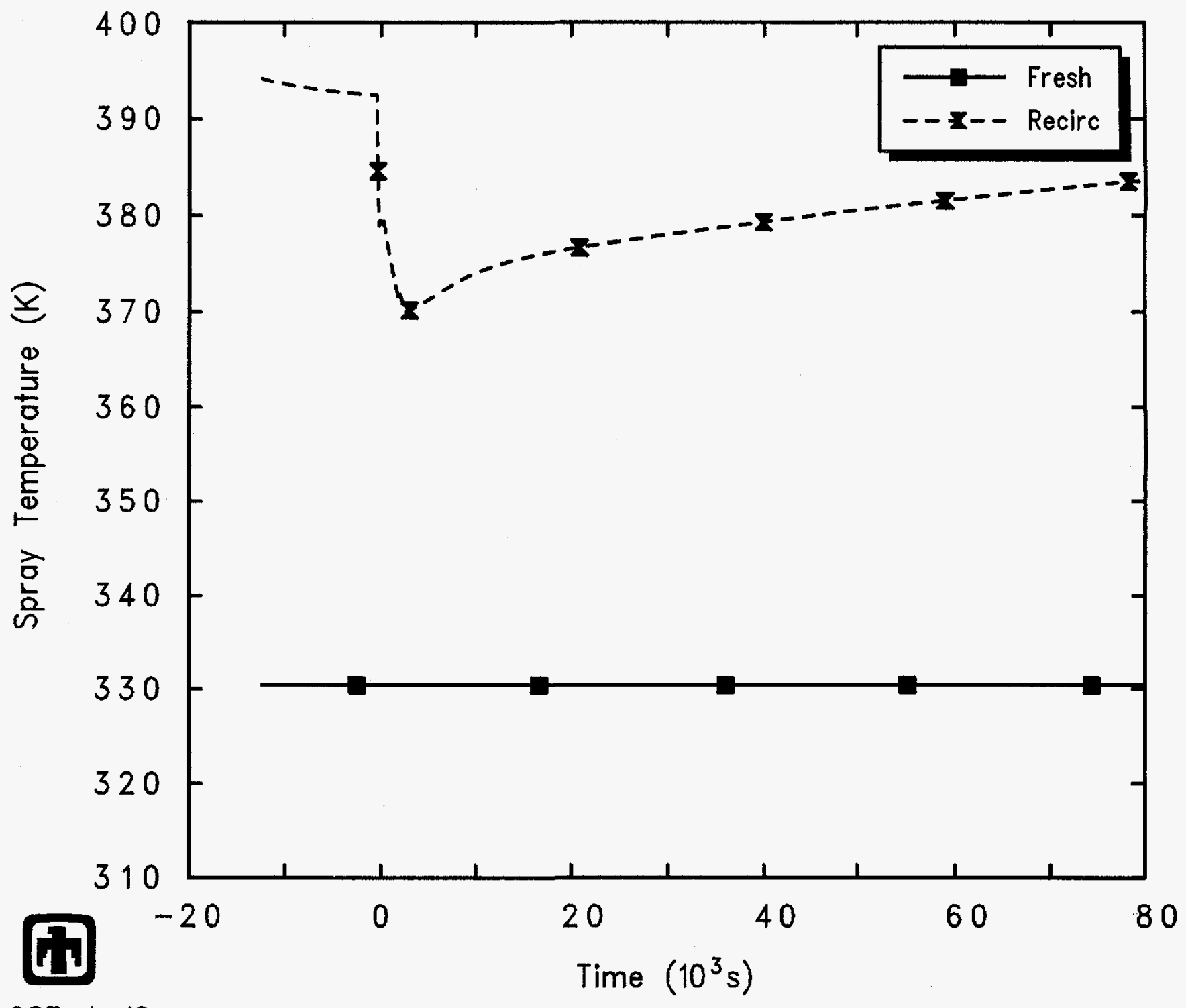

CSE $A-12$ H3EJFENPN $\quad 8 / 30 / 94 \quad 09: 58: 19 \quad$ MELCOR IBM-RISC

Figure 5.5.9. Spray Temperature for CSE Test A-12 - Reference Calculation 


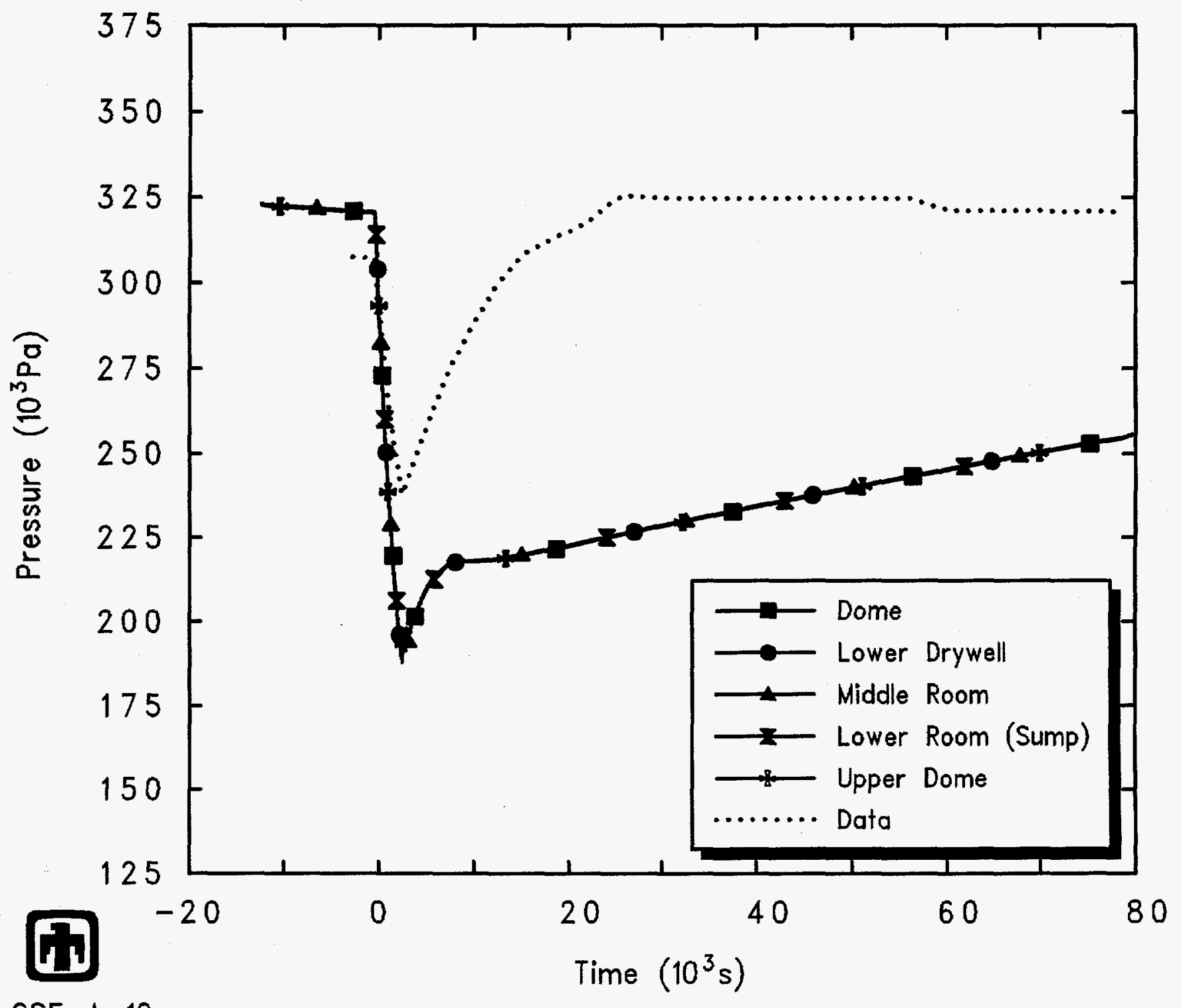

CSE $A-12$

H3EJFENPN $8 / 30 / 94 \quad 09: 58: 19$ MELCOR IBM-RISC

Figure 5.5.10. Vessel Pressure for CSE Test A-12 - Reference Calculation 


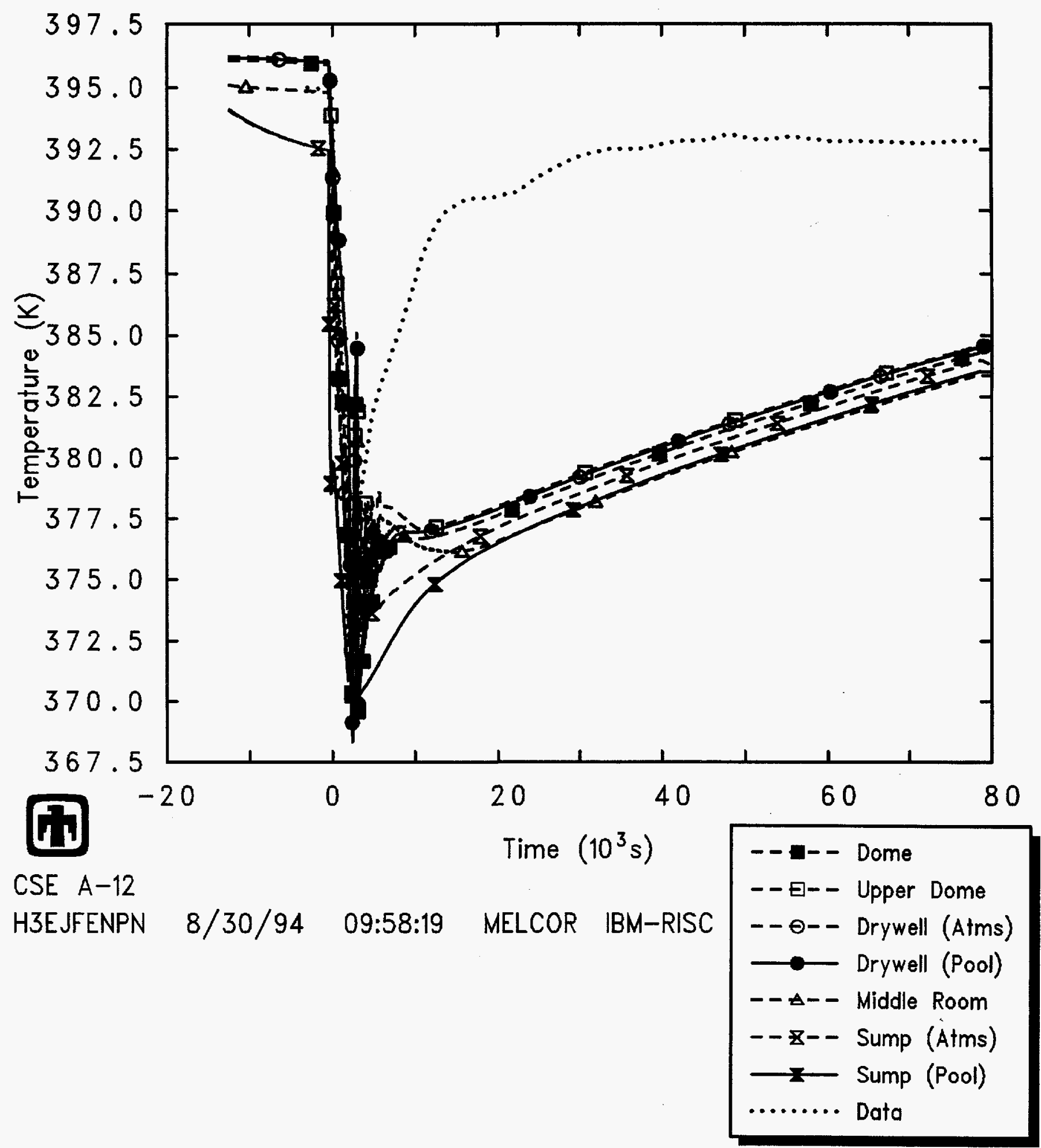

Figure 5.5.11. Vessel Temperatures for CSE Test A-12 - Reference Calculation 


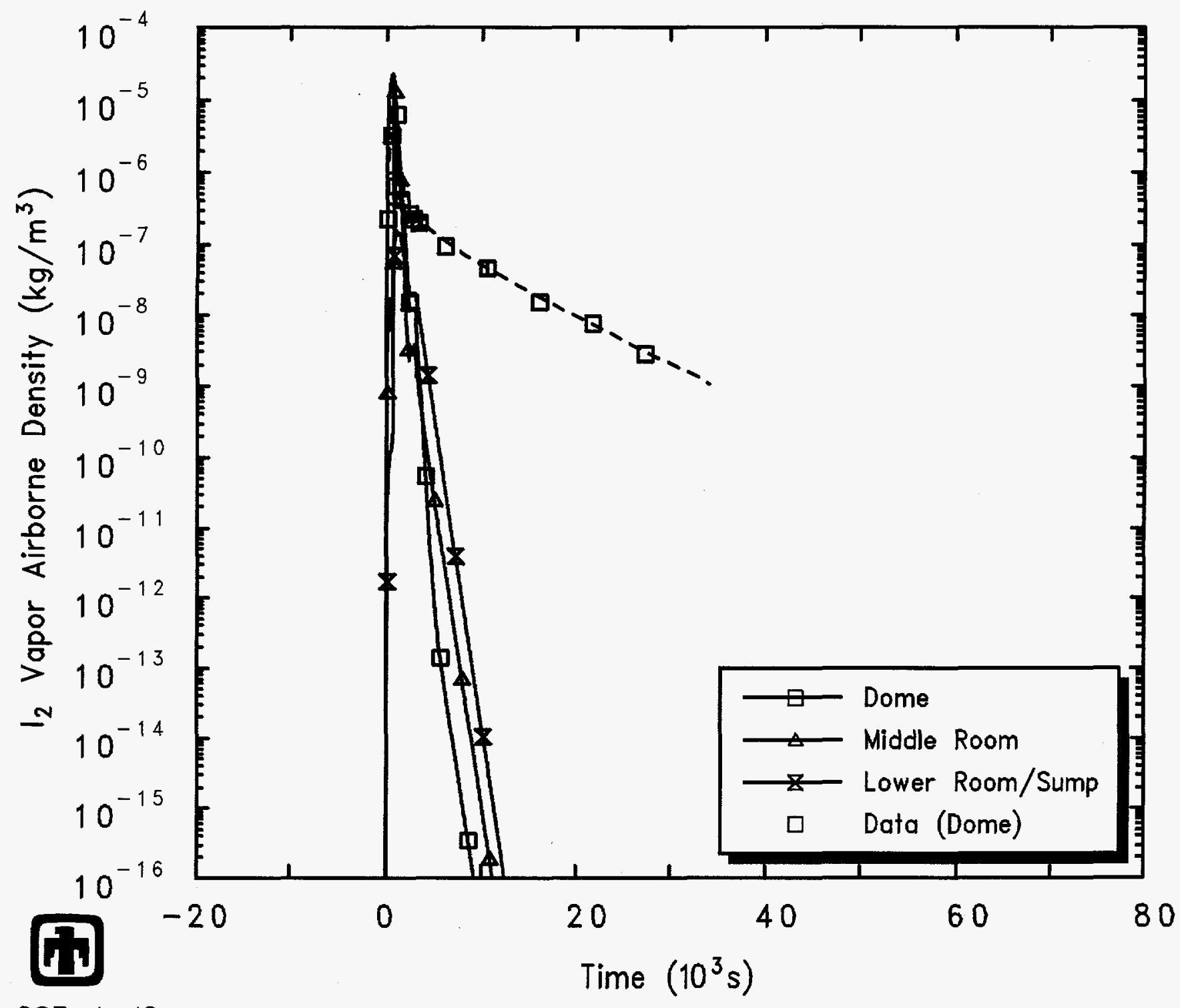

CSE $A-12$

H3EJFENPN $8 / 30 / 94 \quad 09: 58: 19$ MELCOR IBM-RISC

Figure 5.5.12. Cesium Aerosol Airborne Concentrations for CSE Test A-12 Reference Calculation 


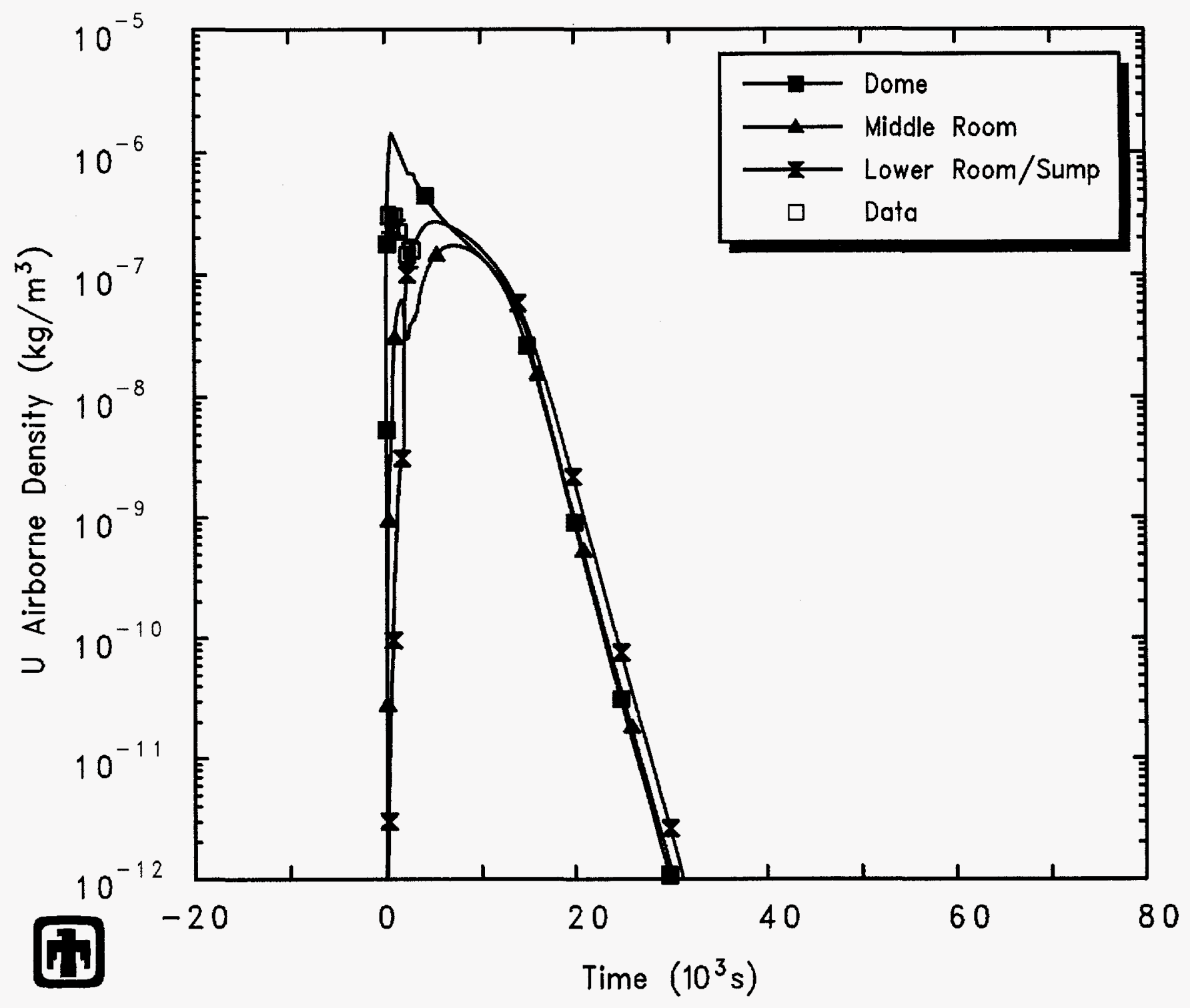

CSE $A-12$

H3EJFENPN 8/30/94 09:58:19 MELCOR IBM-RISC

Figure 5.5.13. Uranium Aerosol Airborne Concentrations for CSE Test A-12 Reference Calculation 


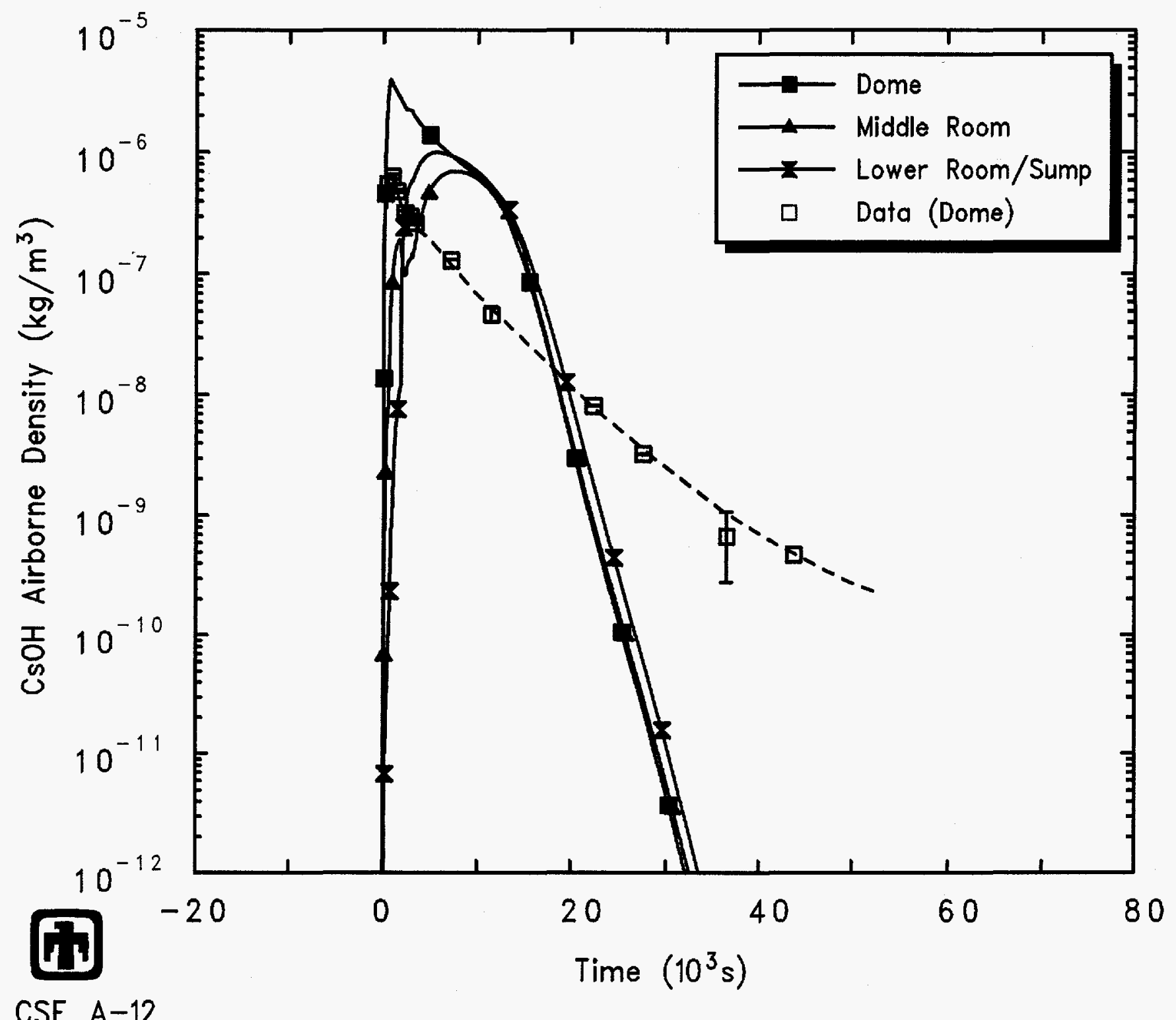

H3EJFENPN $\quad 8 / 30 / 94 \quad 09: 58: 19$ MELCOR IBM-RISC

Figure 5.5.14. Iodine Vapor Airborne Concentrations for CSE Test A-12 - Reference Calculation 
obtained for test A-10. The initial removal rates calculated appear to agree reasonably well with the experiment data, but the removal rates calculated at later times are much greater than those observed in the experiment. 


\section{$6 \quad$ Spray Modelling Sensitivity Studies}

There are options and uncertainties both in some MELCOR input values and in the modelling approach taken to represent test conditions. As described in this and the next two sections, a set of sensitivity studies has been done varying some parameters to determine how the results could be affected by such modelling variations and uncertainties.

The MELCOR Containment Spray (SPR) package models the heat and mass transfer between spray water droplets and the containment building atmosphere; the SPR package is coupled to the RN package for the calculation of aerosol washout and atmosphere decontamination by the sprays.

The modelling in the SPR package is taken virtually intact from the HECTR 1.5 code [24]. For each spray source, the user specifies an initial droplet temperature and flow rate; a droplet size distribution also is input. The model assumes that the spray droplets are spherical and isothermal, and that they fall through containment at their terminal velocity with no horizontal velocity component. Spray droplet heatup and cooldown in a steam environment are modelled using a correlation for forced convection heat transfer coefficients; similarly, evaporation and condensation are modelled using a mass transfer coefficient correlation.

This section describes modelling variations affecting the spray package, while the following sections present results varying parameters and options affecting the aerosol and vapor modelling, respectively, in the RN package. Sensitivity studies were done on the fraction of the spray flow rate interacting with the containment atmosphere, on the spray droplet size distribution, on the droplet terminal velocity, and on the mass transfer correlation used to calculate evaporation and condensation. The first two studies involve parameters which can be varied through normal input; the latter two studies involve parameters which can be varied only through sensitivity coefficients.

(The first attempt at those latter two studies identified a coding error in that the spray package sensitivity coefficients listed in the documentation could be input, but then had no effect on the calculation because there was no coding to save and implement the sensitivity coefficients in the SPR package. This was corrected in version $1.8 \mathrm{OM}$ as part of DIR 1216.)

\subsection{Spray Fraction Interacting with Atmosphere}

MELCOR assumes that the spray droplets are well mixed and interact completely with the surrounding atmosphere. In reality, some of the spray hits the vessel walls (measured to be from $1 \%$ to $11 \%$ in the various CSE tests [19]). Also, only a fraction of the gas volume in the dome was washed by the sprays; based upon the known spray height and envelope diameter for the nozzle arrangements used, that fraction was estimated to be $50 \%$ in test A-3 and $80 \%$ in the other tests [19]. In our reference calculations, we assumed $70 \%$ of the spray flow would interact fully with the adjacent volume atmosphere, with the remainder specified to go directly to the liquid pool. The selection of this fraction 
was based partly upon the experimental data and partly upon physical considerations, and was also based on the results of a sensitivity study done for test A-9 that varied the spray fraction assumed to interact with the dome atmosphere from $100 \%$ down to $50 \%$.

Figures 6.1.1 and 6.1.2 show the pressures and temperatures predicted in the test vessel dome for test A-9 when the spray fraction assumed to interact with the dome atmosphere was varied from $100 \%$ down to $50 \%$ (with the reference calculation described in Section 4 corresponding to a fraction of 0.70 in the plots in this section). As would be expected, Figure 6.1.1 demonstrates that, as more spray interacts directly with the surrounding atmosphere, more steam is condensed, with correspondingly lower pressures. Figure 6.1.2 indicates that as more of the relatively cold spray interacts directly with the surrounding atmosphere, the more the atmosphere is cooled (although the differences are quite small). Interestingly, these effects are seen only for the fresh sprays with cold water injected from an external source; once the system switches to recirculating sump water through the spray, the same late-time pressures and temperatures are predicted in all these cases.

Figures 6.1.3 and 6.1.4 present the airborne cesium aerosol and iodine vapor concentrations predicted in the test vessel dome for test A-9 with the spray fraction assumed to interact with the dome atmosphere varied from $100 \%$ down to $50 \%$. (The uranium aerosol response is very similar to the cesium aerosol response, and is not shown separately.) Again, as would be expected, as more of the spray interacts directly with the surrounding atmosphere, more aerosols are washed out and more iodine vapor is removed.

Table 6.1.1 summarizes the washout rates predicted for cesium and uranium aerosol and iodine vapor in the test vessel dome for test A-9 when the spray fraction assumed to interact with the dome atmosphere was varied from $100 \%$ down to $50 \%$. These tabular values echo the trend seen in Figures 6.1.3 and 6.1.4.

\subsection{Spray Droplet Size}

In the reference calculations, both the fresh and the recirculating sprays were specified to have a five-size droplet distribution (the finest resolution allowed in MELCOR), with equal numbers of droplets in each bin and with the droplet AMMD and GSD taken from the test data as given in Tables 2.2 and 2.7. To determine the importance of knowing the droplet size distribution, a sensitivity study was done that varied the droplet size distributions assumed. Calculations were done for test A-9 multiplying the given AMMD of $1220 \mu \mathrm{m}$ by factors of 0.5 and 2 (keeping the same GSD of about 1.5 ); calculations were also done using a single drop size set to the various droplet distribution AMMDs used in the drop size distributions (i.e., $610 \mu \mathrm{m}, 1220 \mu \mathrm{m}$ and $2440 \mu \mathrm{m}$ ).

Figures 6.2.1 and 6.2.2 show the pressures and temperatures predicted in the test vessel dome for test A-9 when the spray droplet size and size distribution was varied (with the reference calculation described in Section 4 corresponding to a droplet AMMD of $1220 \mu \mathrm{m}$ in the plots in this section). These figures demonstrate that there is no 


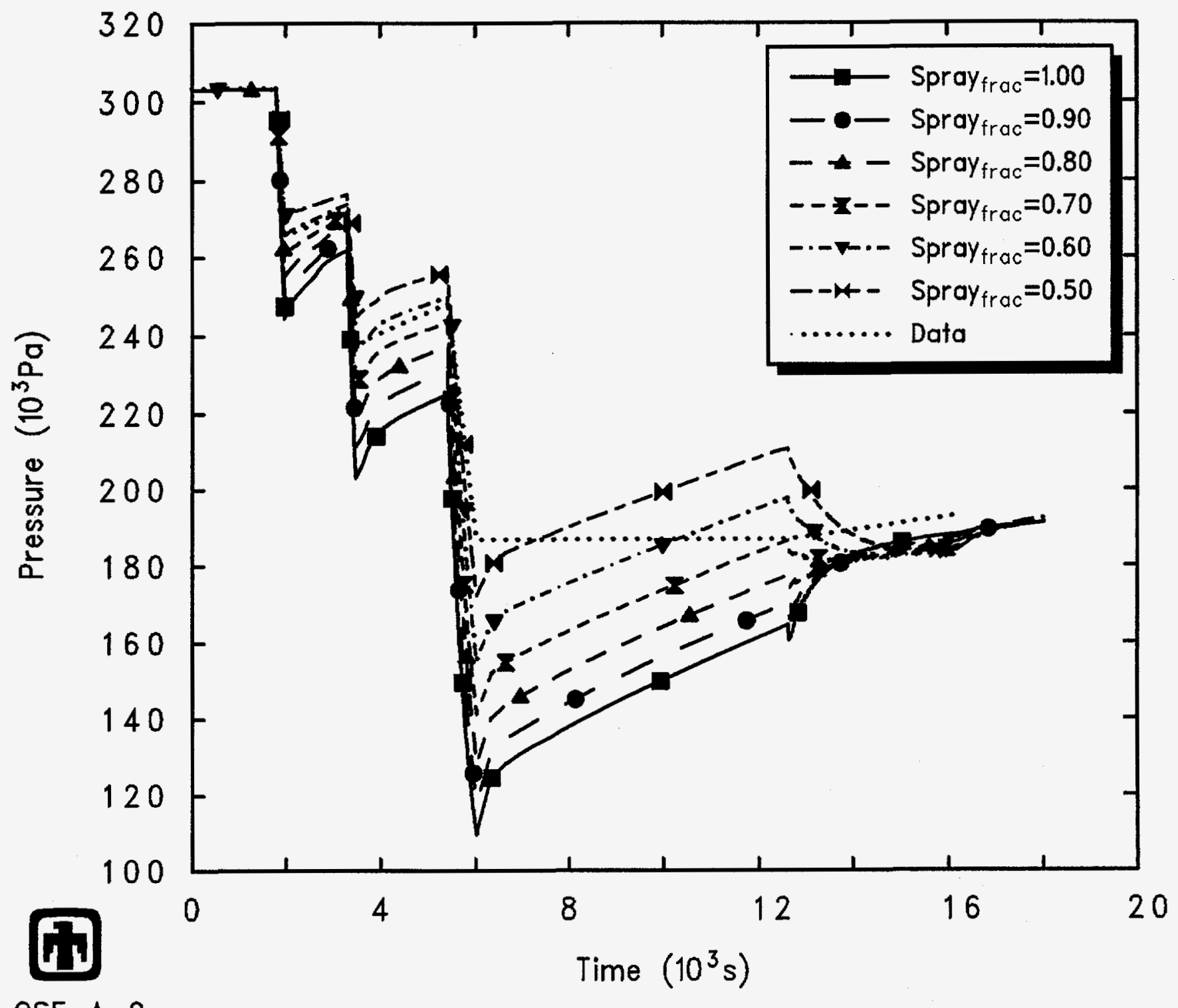

CSE A-9

HLELCGQPN $8 / 12 / 94 \quad 11: 25: 26$ MELCOR SUN

Figure 6.1.1. Vessel Pressure for CSE Test A-9 - Spray Fraction Interacting with Atmosphere Sensitivity Study 


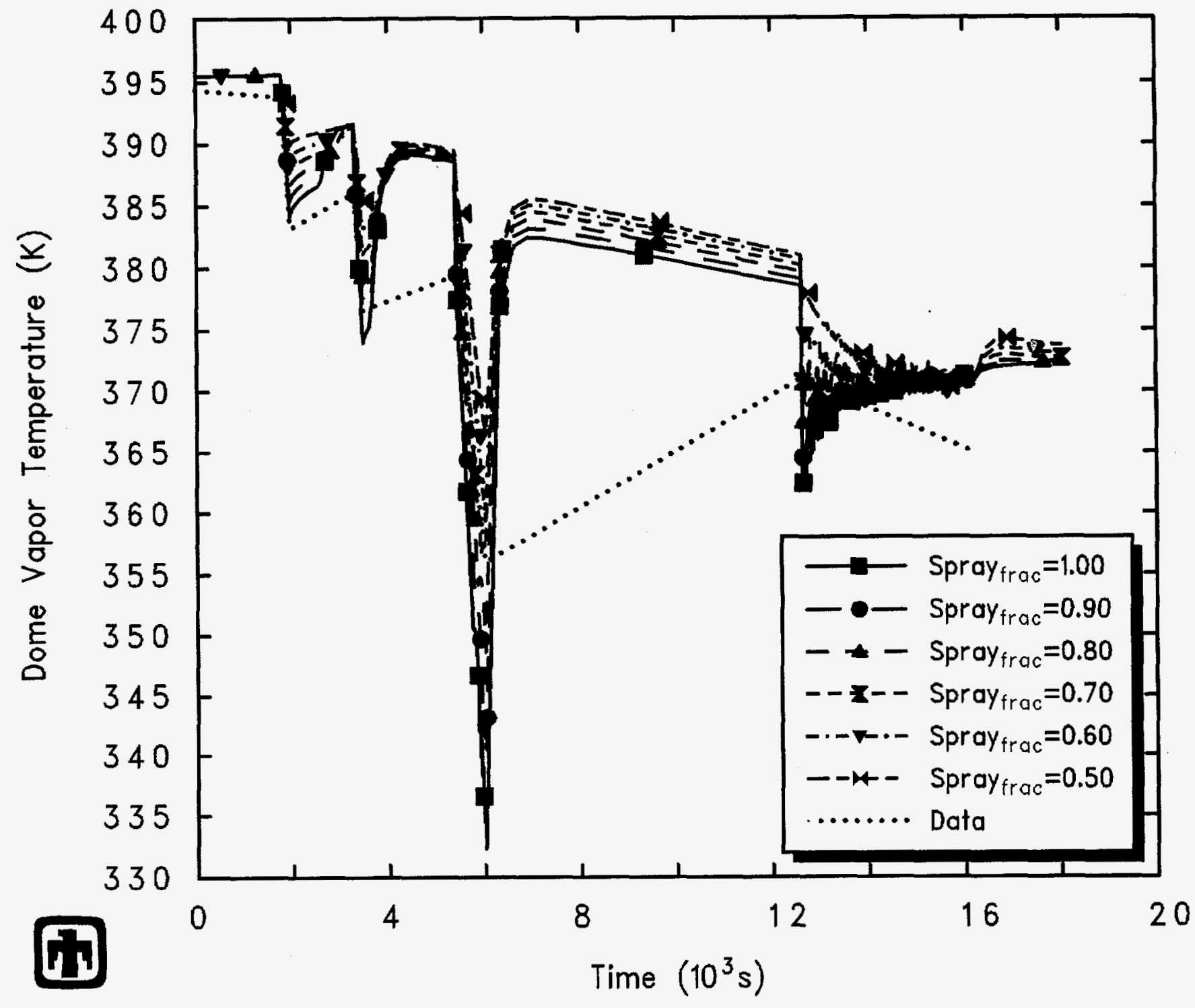

CSE A-9

HLELCGQPN $8 / 12 / 94 \quad 11: 25: 26$. MELCOR SUN

Figure 6.1.2. Vessel Dome Temperatures for CSE Test A-9 - Spray Fraction Interacting with Atmosphere Sensitivity Study 


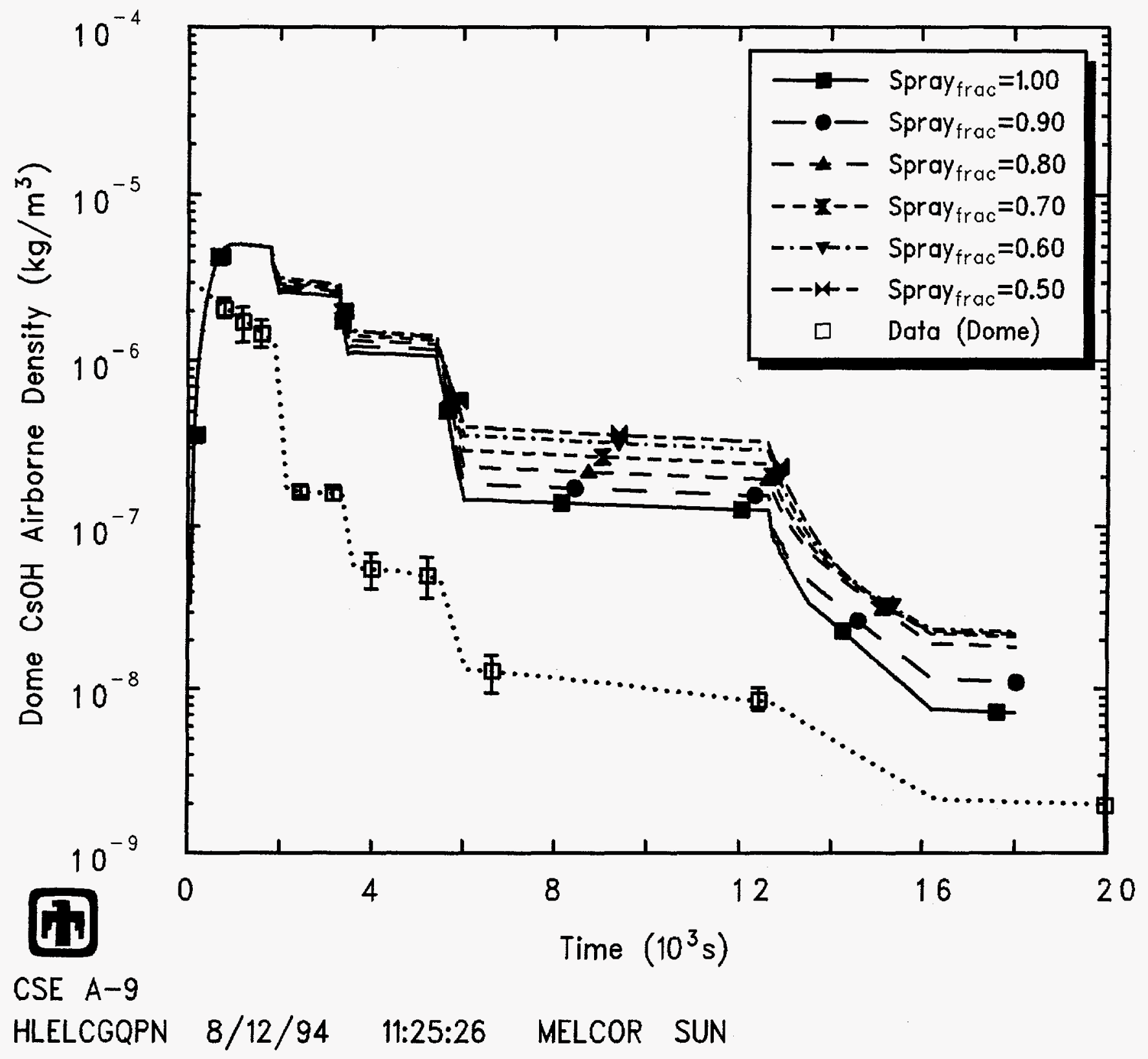

Figure 6.1.3. Cesium Aerosol Airborne Concentrations for CSE Test A-9 - Spray Fraction Interacting with Atmosphere Sensitivity Study 


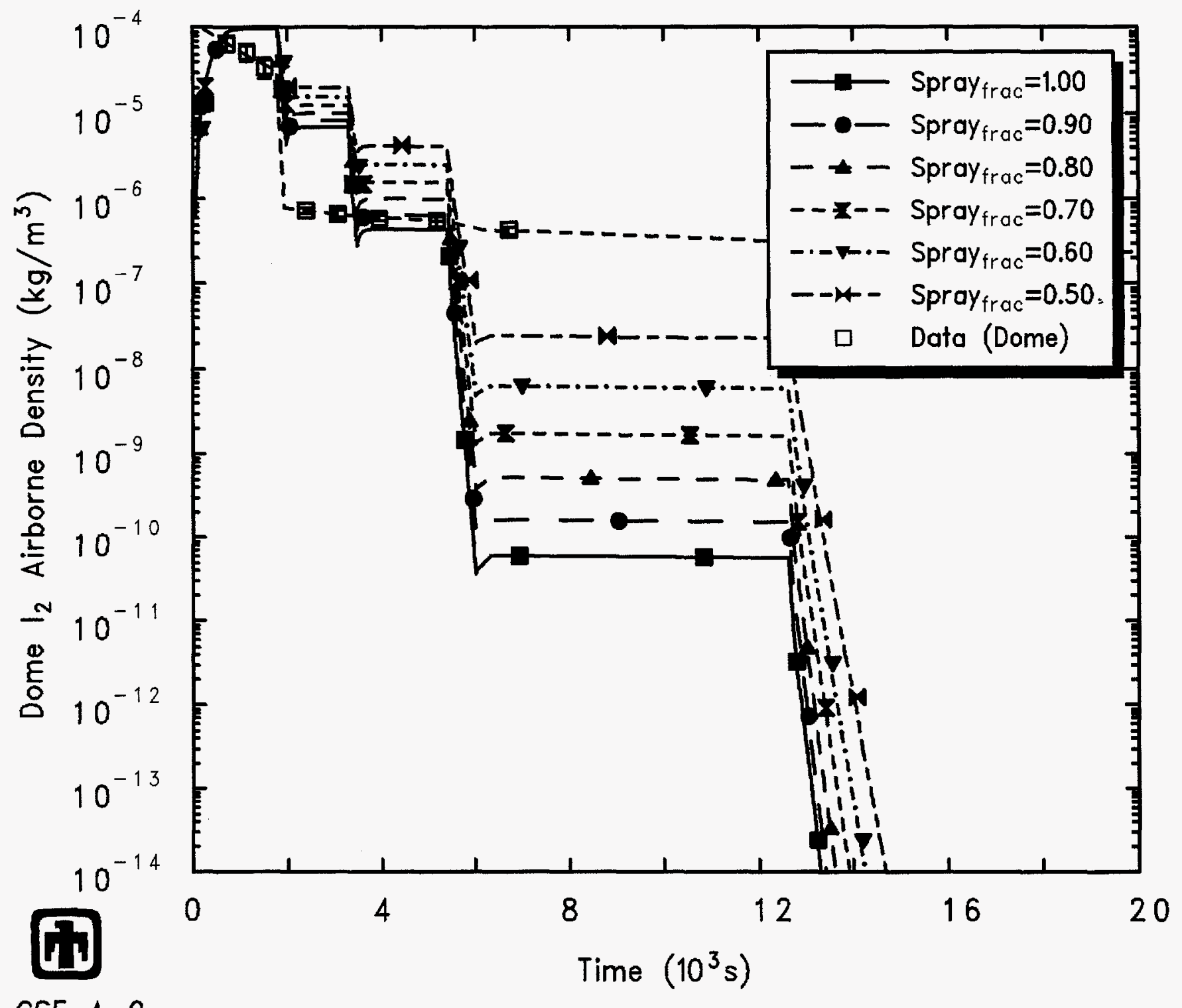

CSE A-9 HLELCGQPN 8/12/94 11:25:26 MELCOR SUN

Figure 6.1.4. Iodine Vapor Airborne Concentrations for CSE Test A-9 - Spray Fraction Interacting with Atmosphere Sensitivity Study 
Table 6.1.1. Washout Rates for CSE Test A-9 - Spray Fraction Interacting with Atmosphere Sensitivity Study

\begin{tabular}{|c|c|c|c|c|c|c|c|}
\hline & \multirow[t]{2}{*}{ Measured } & \multicolumn{6}{|c|}{$\begin{array}{c}\text { MELCOR } \\
\text { Fraction Interacting }\end{array}$} \\
\hline & & 1.0 & 0.9 & 0.8 & $0.7^{a}$ & 0.6 & 0.5 \\
\hline \multicolumn{8}{|l|}{ Cesium } \\
\hline First spray & 1.08 & 4.3 & 4.5 & 4.6 & 5.0 & 5.3 & 5.8 \\
\hline Second spray & 2.0 & 3.9 & 4.2 & 4.4 & 4.6 & 5.0 & 5.7 \\
\hline Third spray & 5.4 & 3.5 & 3.7 & 4.1 & 4.3 & 4.9 & 5.7 \\
\hline Fourth spray & 33 & $23^{b}$ & $27^{b}$ & $29^{b}$ & $35^{b}$ & $37^{b}$ & $40^{b}$ \\
\hline \multicolumn{8}{|l|}{ Uranium } \\
\hline First spray & 2.3 & 3.5 & 3.7 & 3.9 & 4.6 & 4.3 & 4.7 \\
\hline Second spray & & 3.7 & 3.9 & 4.2 & 4.3 & 4.6 & 4.4 \\
\hline Third spray & & 3.2 & 3.5 & 3.8 & 4.3 & 4.8 & 5.5 \\
\hline Fourth spray & & $23^{b}$ & $26^{b}$ & $29^{b}$ & $35^{b}$ & $37^{b}$ & $39^{b}$ \\
\hline \multicolumn{8}{|l|}{ Iodine } \\
\hline First spray & 0.58 & 0.80 & 0.87 & 0.95 & 1.1 & 1.2 & 1.4 \\
\hline Second spray & 42 & 0.84 & 0.90 & 1.2 & 1.4 & 1.5 & 1.6 \\
\hline Third spray & 34 & 0.74 & 0.80 & 0.88 & 1.0 & 1.1 & 1.3 \\
\hline Fourth spray & 180 & 0.99 & 1.0 & 1.3 & 1.4 & 1.5 & 1.7 \\
\hline
\end{tabular}




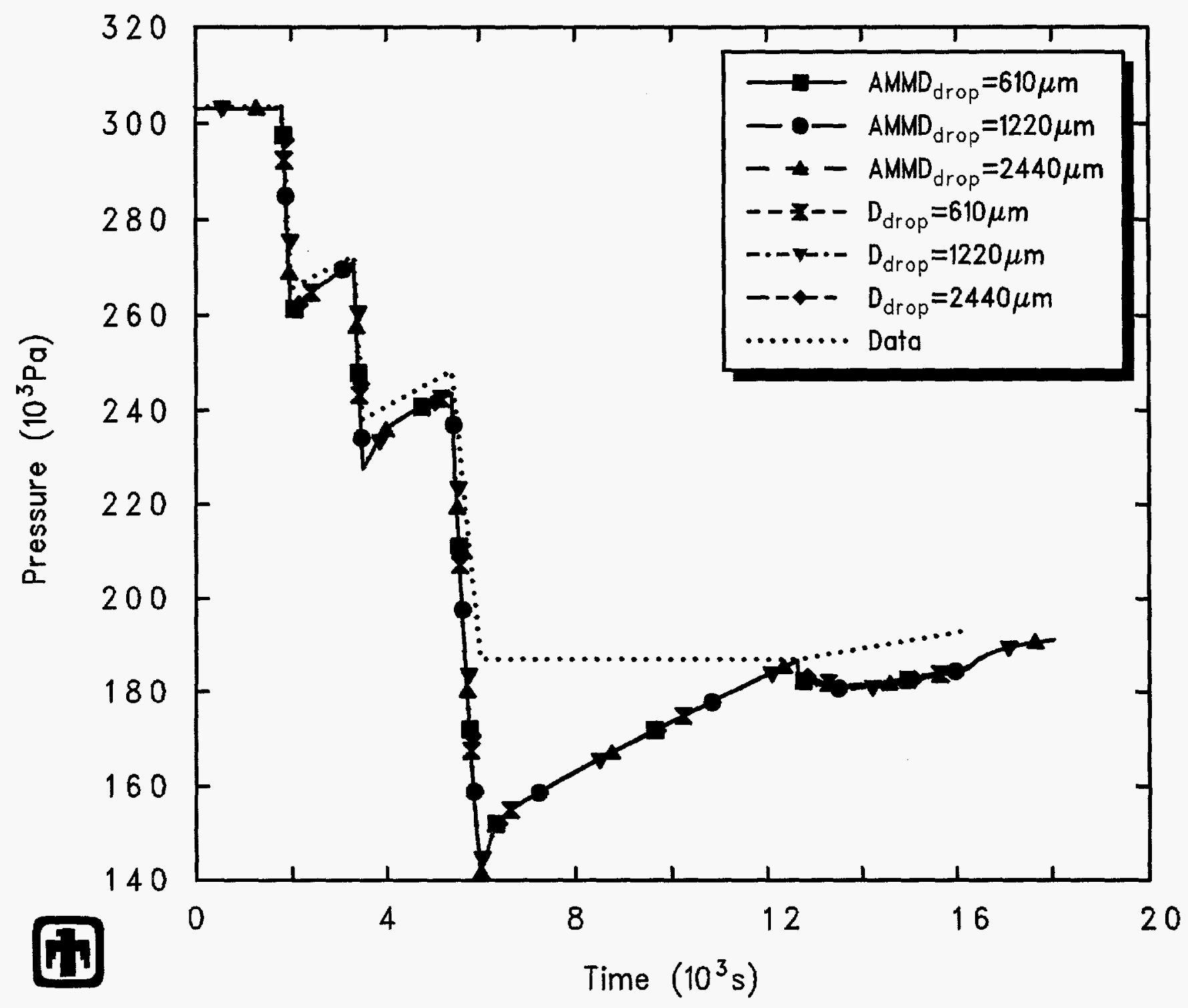

CSE A-9

HLETAMGPN $8 / 12 / 94 \quad 19: 05: 20$ MELCOR SUN

Figure 6.2.1. Vessel Pressure for CSE Test A-9 - Spray Droplet Size Distribution Sensitivity Study 


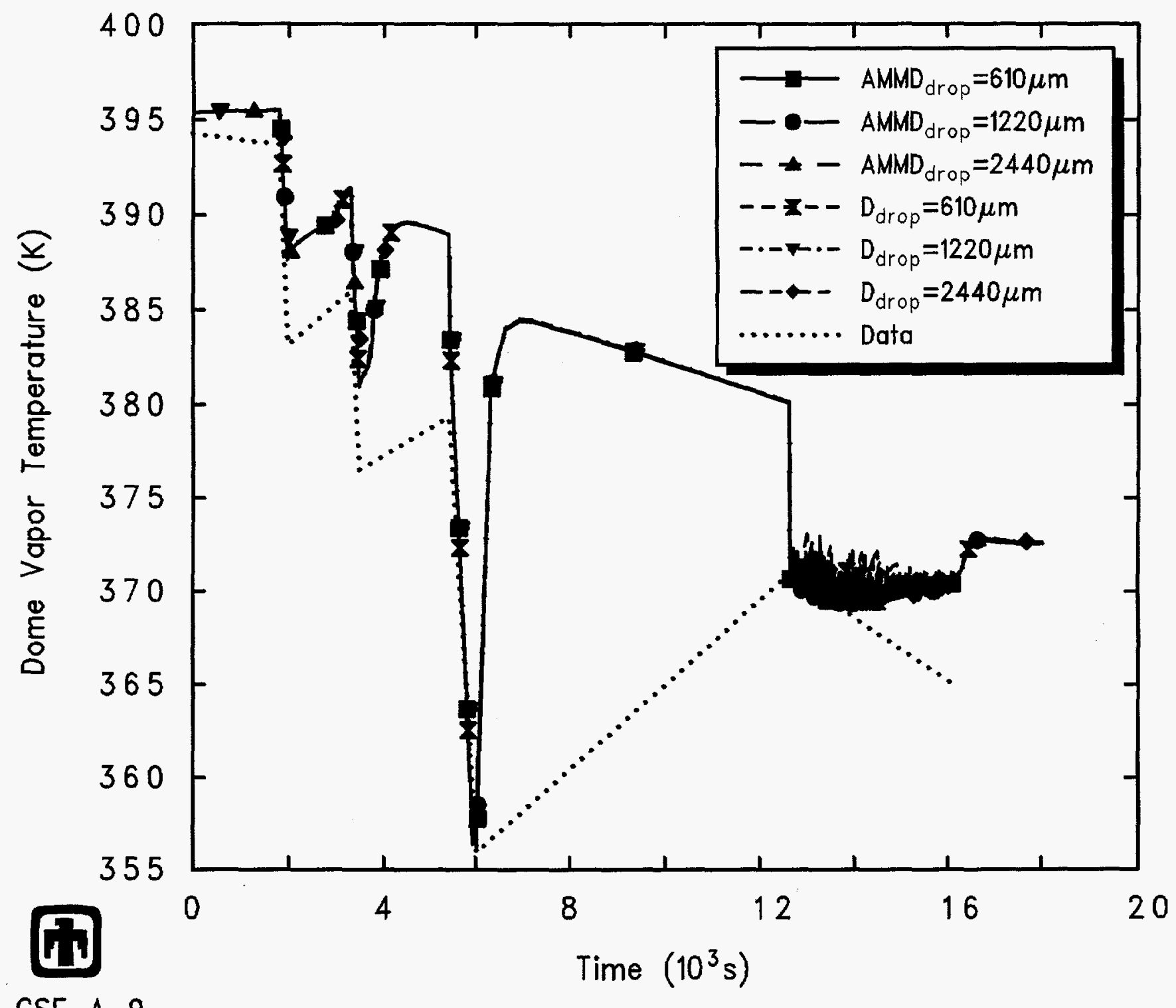

CSE A-9

HLETAMGPN 8/12/94 19:05:20 MELCOR SUN

Figure 6.2.2. Vessel Dome Temperatures for CSE Test A-9 - Spray Droplet Size Distribution Sensitivity Study 
visible effect on steam condensation varying the droplet size by small factors; a much larger change would be needed to have any effect.

The airborne cesium aerosol and iodine vapor concentrations predicted in the test vessel dome for test A-9 varying the spray droplet size and size distribution are given in. Figures 6.2 .3 and 6.2.4, respectively. (The uranium aerosol response is very similar to the cesium aerosol response, and is not shown separately.) Larger droplets are less efficient at removing both aerosols and iodine vapor, while smaller droplets remove both aerosols and vapors more efficiently than in the reference case. Droplets all a single size are less effective than a corresponding droplet size distribution that includes both smaller and larger drops, because the increased removal of the smaller drops in the distribution outweighs the decreased removal of the larger drops in the distribution.

Table 6.2.1 summarizes the washout rates predicted for cesium and uranium aerosol and iodine vapor in the test vessel dome for test A-9 varying the spray droplet size distribution assumed. These tabular values echo the trend seen in Figures 6.2.3 and 6.2.4.

\subsection{Spray Droplet Drag Coefficient and Terminal Velocity}

Sensitivity coefficients are available in MELCOR to modify the correlation used for the drag coefficient $C_{d}$ as a function of Reynolds number. Three different correlations are used for $C_{d}$ for low, medium and high Reynolds number, and the terminal droplet velocity is then proportional to $C_{d}^{1 / 2}$. Each of these three drag coefficient correlations has a leading constant multiplier, followed in some cases by a power dependence on the Reynolds number. As a sensitivity study, these leading constants in the $C_{d}$ correlations were multiplied by $10^{4}, 10^{2}, 10^{-2}$ and $10^{-4}$, which effectively multiplied the terminal droplet velocity by $0.01,0.1,10$ and 100 . The three leading constants for the three correlations used for $C_{d}$ for low, medium and high Reynolds number were multiplied by the same factor to ensure the same change in terminal fall velocity regardless of Reynolds number regime.

Figures 6.3.1 and 6.3.2 show the pressures and temperatures predicted in the test vessel dome for test A-9 varying the spray droplet size and size distribution (with the reference calculation described in Section 4 using the code default values). There are virtually no differences in results calculated when the drag coefficient was varied up or down by a factor of 100 (varying the terminal velocity by a factor of 10 either way), but some differences become visible when the drag coefficient was varied more, by a factor of $10^{4}$ (varying the terminal velocity by a factor of 100 either way). Decreasing the drag coefficient and thereby increasing the droplet terminal fall velocity would be expected to decrease condensation by decreasing the time available for the droplet to interact with the atmosphere. It is not immediately obvious why increasing the drag coefficient and thereby decreasing the droplet terminal fall velocity should have the same effect of decreasing condensation; possibly there is an increase in boundary layer thickness and an associated increased resistance to heat and mass transfer at lower velocities.

The airborne cesium aerosol and iodine vapor concentrations, respectively, predicted in the test vessel dome for test A-9 when the spray droplet drag coefficient and associated 


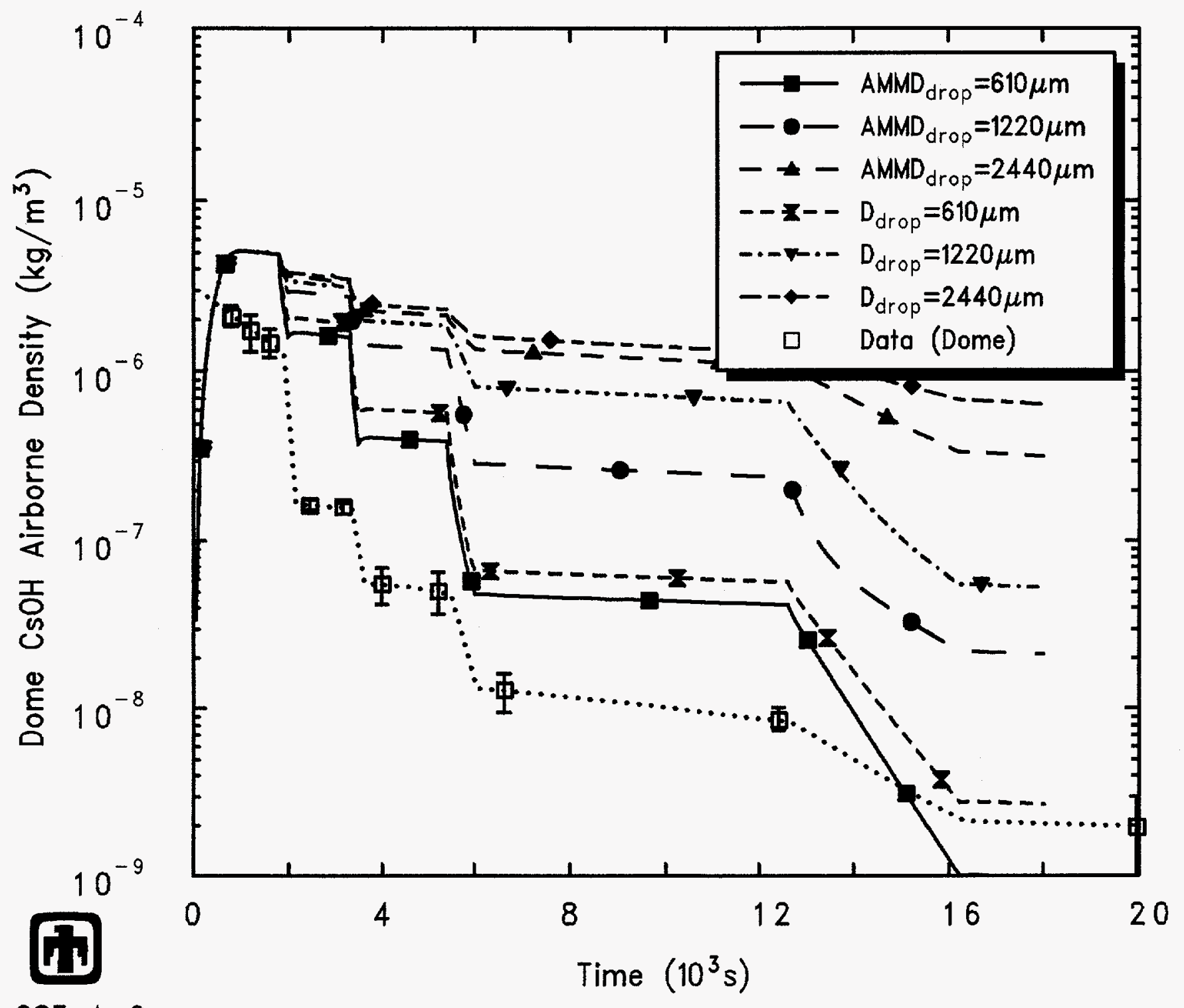

CSE A-9

HLETAMGPN $\quad 8 / 12 / 94 \quad 19: 05: 20$ MELCOR SUN

Figure 6.2.3. Cesium Aerosol Airborne Concentrations for CSE Test A-9 - Spray Droplet Size Distribution Sensitivity Study 


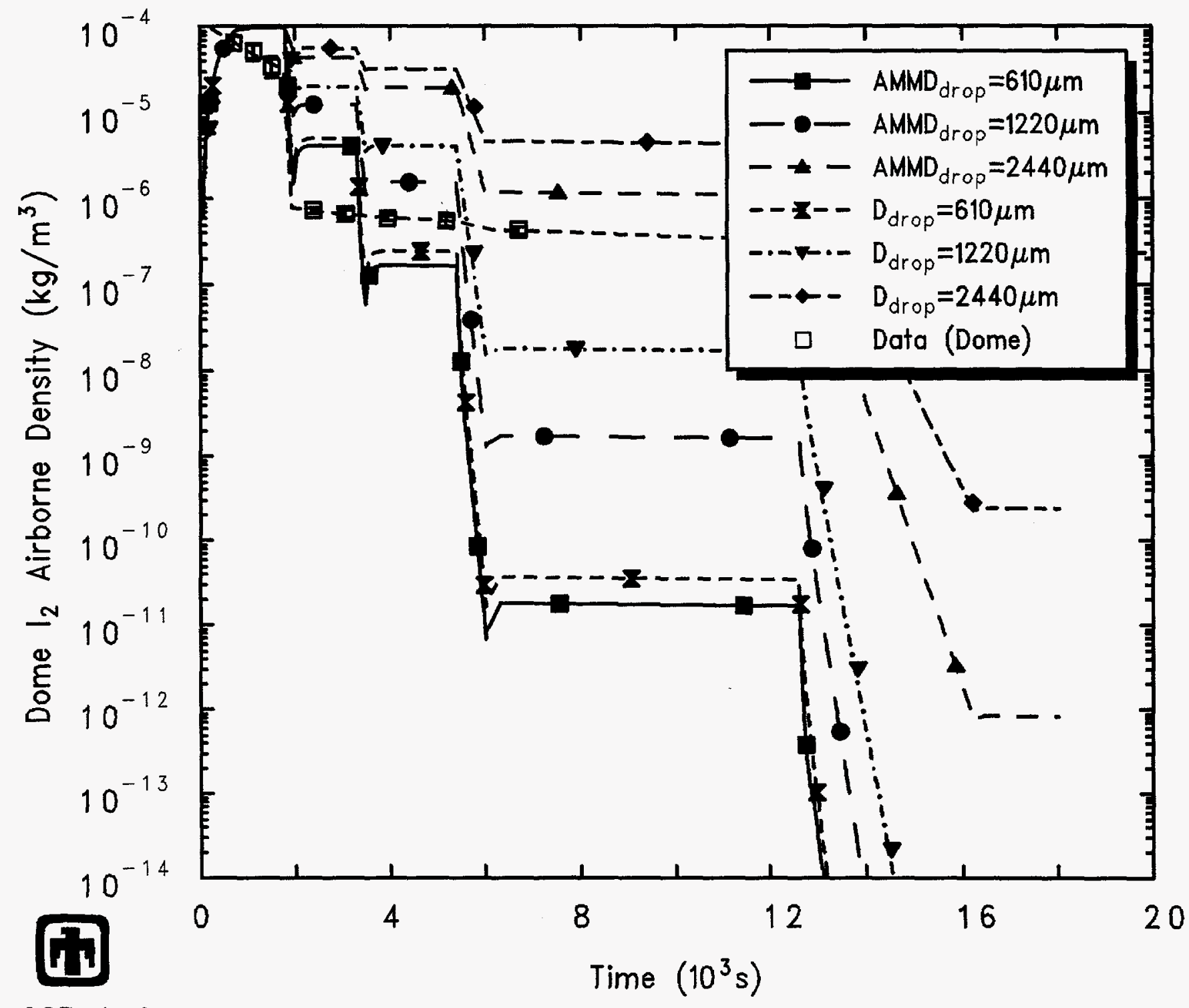

CSE $A-9$

HLETAMGPN $8 / 12 / 94 \quad 19: 05: 20$ MELCOR SUN

Figure 6.2.4. Iodine Vapor Airborne Concentrations for CSE Test A-9 - Spray Droplet Size Distribution Sensitivity Study 
Table 6.2.1. Washout Rates for CSE Test A-9 - Spray Droplet Size Distribution Sensitivity Study

$$
\text { Measured } \quad t_{1 / 2}(\min )
$$

Spray Droplet $\mathrm{AMMD}=$ Spray Droplet $\mathrm{D}=$ $610 \mu \mathrm{m} \quad 1220 \mu \mathrm{m}^{a} \quad 2440 \mu \mathrm{m} \quad 610 \mu \mathrm{m} \quad 1220 \mu \mathrm{m} \quad 2440 \mu \mathrm{m}$

Cesium

$\begin{array}{lccccccc}\text { First spray } & 1.08 & 2.3 & 5.0 & 8.3 & 2.9 & 6.7 & 9.8 \\ \text { Second spray } & 2.0 & 2.1 & 4.6 & 7.6 & 2.4 & 7.1 & 8.4 \\ \text { Third spray } & 5.4 & 2.7 & 4.3 & 15.4 & 2.8 & 8.3 & 17.8 \\ \text { Fourth spray } & 33 & 14^{b} & 35^{b} & 46^{b} & 17^{b} & 29^{b} & 69^{b}\end{array}$

Uranium

First spray

Second spray

Third spray

Fourth spray

Iodine

First spray

Second spray

Third spray

Fourth spray
2.3

1.9

1.9

2.6

$13^{b}$

4.6

4.3

4.3

$35^{b}$

1.1

1.4

1.0

1.4
1.2

0.56

0.97

0.59

180
42
6.9

7.0

14.7

$43^{b}$

3.0

3.6

2.5

3.0

${ }^{a}$ Reference calculation value

${ }^{b}$ Value at end of recirculating spray 


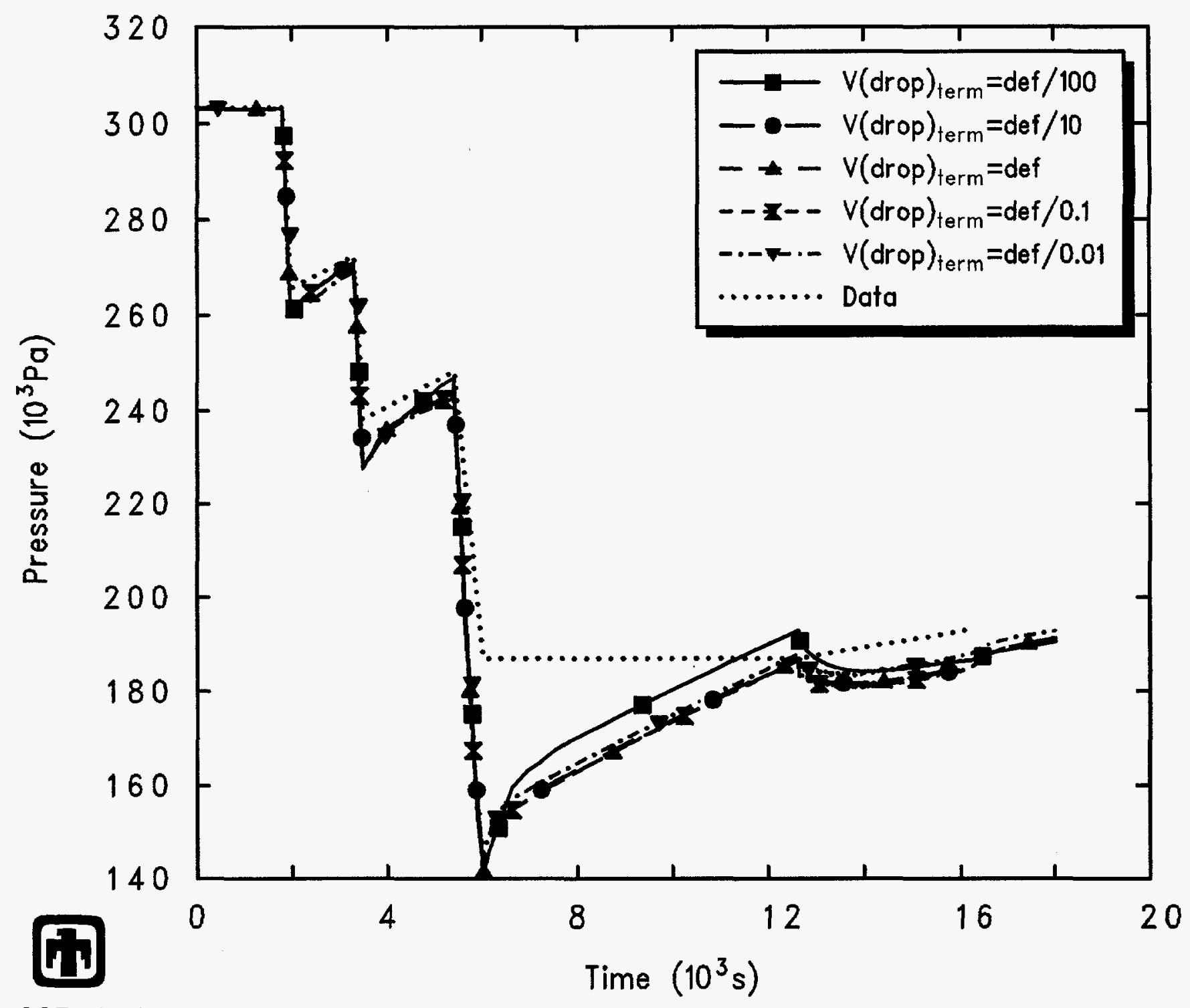

CSE A-9

HLEUCVFPN $8 / 12 / 94 \quad 20: 31: 45$ MELCOR SUN

Figure 6.3.1. Vessel Pressure for CSE Test A-9 - Spray Droplet Drag Coefficient and Terminal Velocity Sensitivity Study 


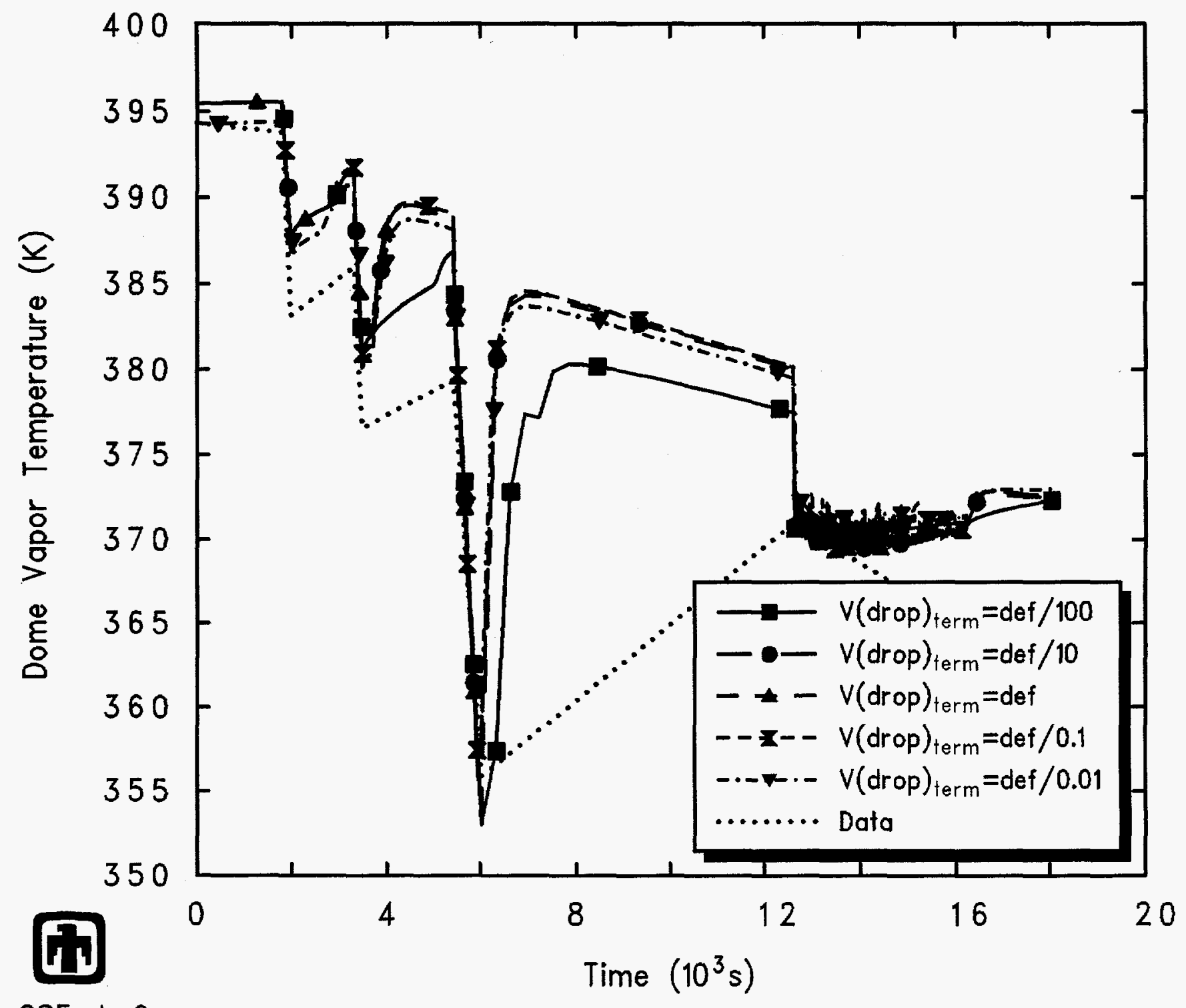

CSE A-9

HLEUCVFPN $8 / 12 / 94 \quad 20: 31: 45$ MELCOR SUN

Figure 6.3.2. Vessel Dome Temperatures for CSE Test A-9 - Spray Droplet Drag Coefficient and Terminal Velocity Sensitivity Study 
terminal velocity were varied are given in Figures 6.3.3 and 6.3.4. (The uranium aerosol response is very similar to the cesium aerosol response, and is not shown separately.) The diffusive removal rate of vapors such as iodine is simply proportional to the droplet residence time, and hence proportional to the drag coefficient and inversely proportional to the fall velocity. Aerosol removal is assumed to be due primarily to inertial impaction and interception, although diffusiophoresis and diffusion effects are also included in MELCOR. As the drag coefficient is decreased and the droplet terminal velocity increased, aerosols have less opportunity to evade the falling drops and the collection efficiency and removal rate increases; as the drag coefficient is increased and the droplet terminal velocity decreased, the aerosols can flow around the droplets without capture by interception or impaction, leaving only diffusive removal.

Table 6.3.1 summarizes the washout rates predicted for cesium and uranium aerosol and iodine vapor in the test vessel dome for test A-9 when the spray droplet drag coefficient, and hence droplet terminal velocity, were varied. These tabular values echo the trends seen in Figures 6.3.3 and 6.3.4.

\subsection{Spray Droplet Mass Transfer}

Sensitivity coefficients are available in MELCOR to modify the correlation used for the mass transfer coefficient in the spray package. One of these sensitivity coefficients is the leading multiplier in the correlation, set to -2.0 by default. As a sensitivity study, this leading constant in the mass transfer correlation was multiplied by $100,10,0.1$ and 0.01 .

Figures 6.4.1 and 6.4.2 show the pressures and temperatures predicted in the test vessel dome for test A-9 when the spray droplet mass transfer coefficient was varied (with the reference calculation described in Section 4 using the code default values). There are virtually no differences in results calculated varying the droplet mass transfer coefficient up or down by a factor of 10 , but some differences become visible when the droplet mass transfer coefficient is varied more, by a factor of 100 . Decreasing the droplet mass transfer coefficient obviously would be expected to decrease condensation. It is not obvious why increasing the droplet mass transfer coefficient should have the same effect of decreasing condensation (albeit to a slight degree).

The airborne cesium aerosol and iodine vapor concentrations predicted in the test vessel dome for test A-9 varying the spray droplet mass transfer coefficient are given in Figures 6.4 .3 and 6.4.4, respectively. (The uranium aerosol response is very similar to the cesium aerosol response, and is not shown separately.) Vapor removal is very similar in all cases with mass transfer coefficients sufficiently high to bring the spray droplets into equilibrium with the surrounding atmosphere during droplet fall, but is somewhat reduced when the mass transfer coefficient is too low to bring the spray droplets into equilibrium with the surrounding atmosphere during droplet fall.

Table 6.4.1 summarizes the washout rates predicted for cesium and uranium aerosol and iodine vapor in the test vessel dome for test A-9 varying the spray droplet evapora- 


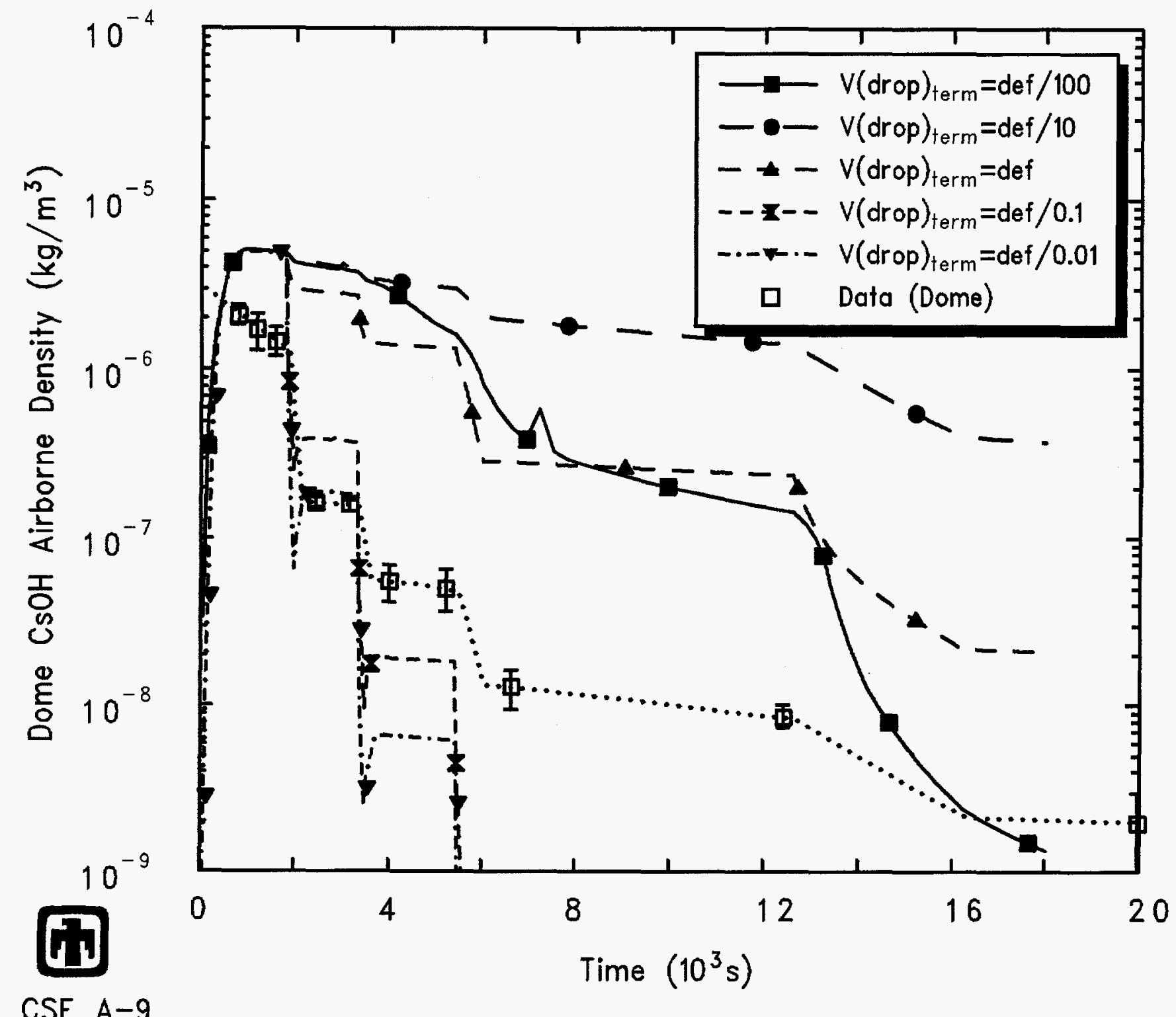

HLEUCVFPN $8 / 12 / 94 \quad 20: 31: 45$ MELCOR SUN

Figure 6.3.3. Cesium Aerosol Airborne Concentrations for CSE Test A-9 - Spray Droplet Drag Coefficient and Terminal Velocity Sensitivity Study 


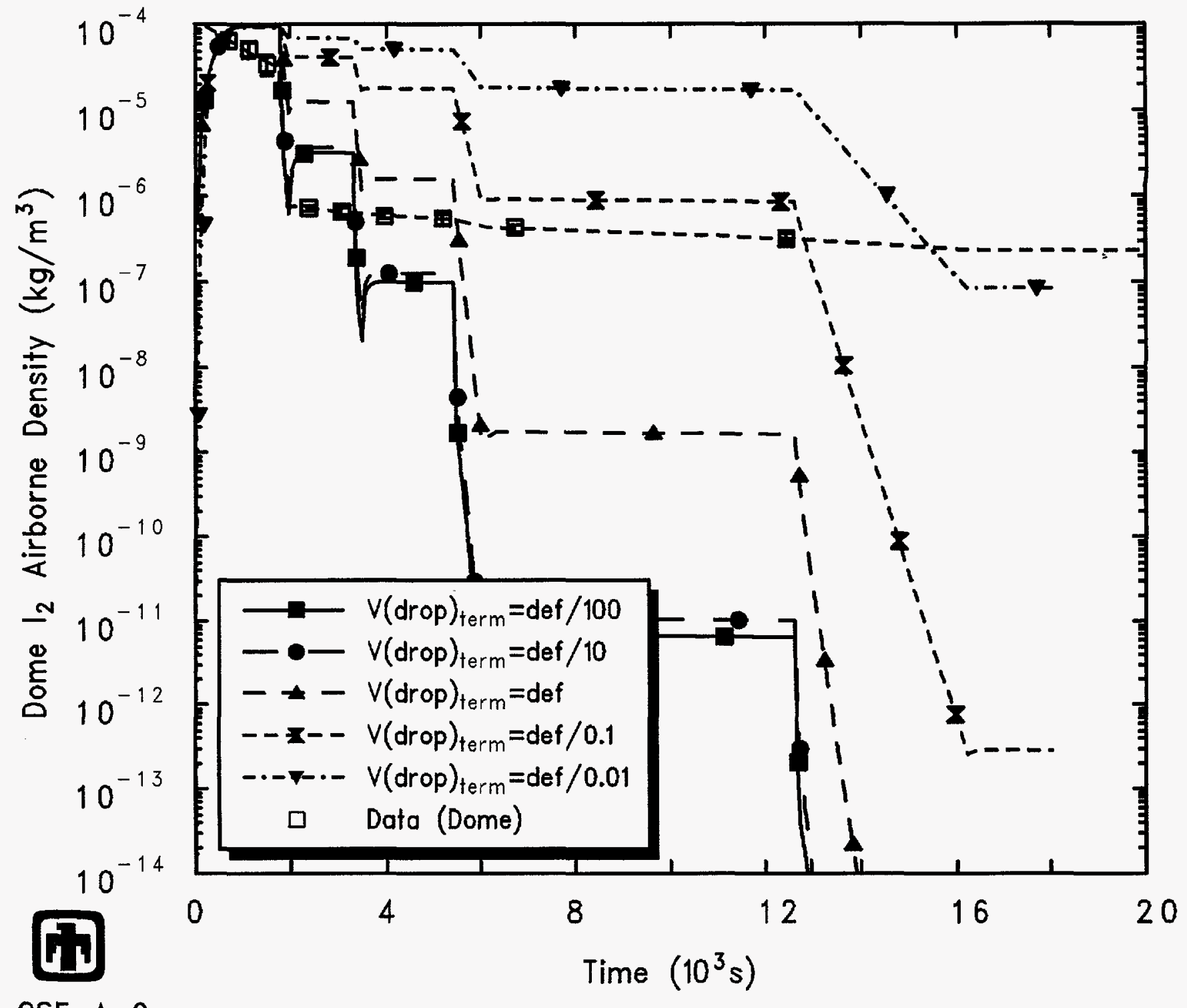

CSE A-9

HLEUCVFPN $8 / 12 / 94 \quad 20: 31: 45$ MELCOR SUN

Figure 6.3.4. Iodine Vapor Airborne Concentrations for CSE Test A-9 - Spray Droplet Drag Coefficient and Terminal Velocity Sensitivity Study 
Table 6.3.1. Washout Rates for CSE Test A-9 - Spray Droplet Drag Coefficient and Terminal Velocity Sensitivity Study

Measured

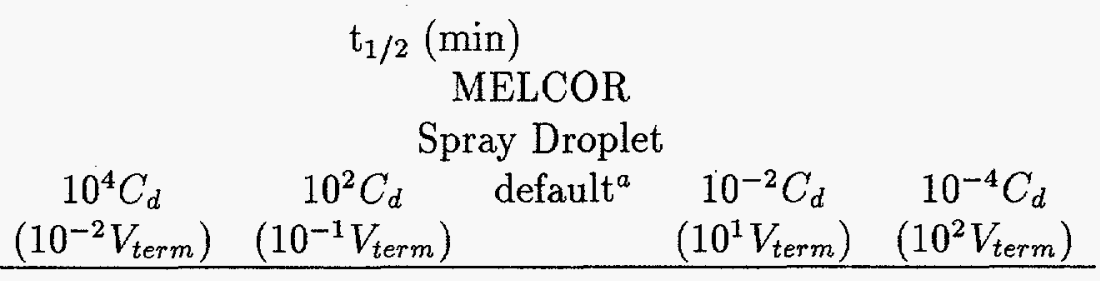

Cesium

First spray

1.08

18.5

2.0

20.6

Third spray

5.4

7.7

33

$19^{b}$

2.3

19.8

20.7

7.5

$19^{b}$

27.7

5.0

0.88

0.43

20.6

4.6

0.88

0.43

4.3

0.87

0.53

$41^{b}$

$35^{b}$

$27^{b}$

$1.4^{b}$

Uranium

First spray

Second spray

Third spray

Fourth spray

Iodine

First spray

0.58

0.45

0.82

Second spray

42

Third spray

34

0.50

24.8

4.6

0.68

0.41

Fourth spray

180

1.2

19.5

4.3

0.83

0.43

4.3

0.77

0.53

$41^{b}$

$35^{b}$

$28^{b}$

$1.4^{b}$

${ }^{a}$ Reference calculation value

${ }^{b}$ Value at end of recirculating spray 


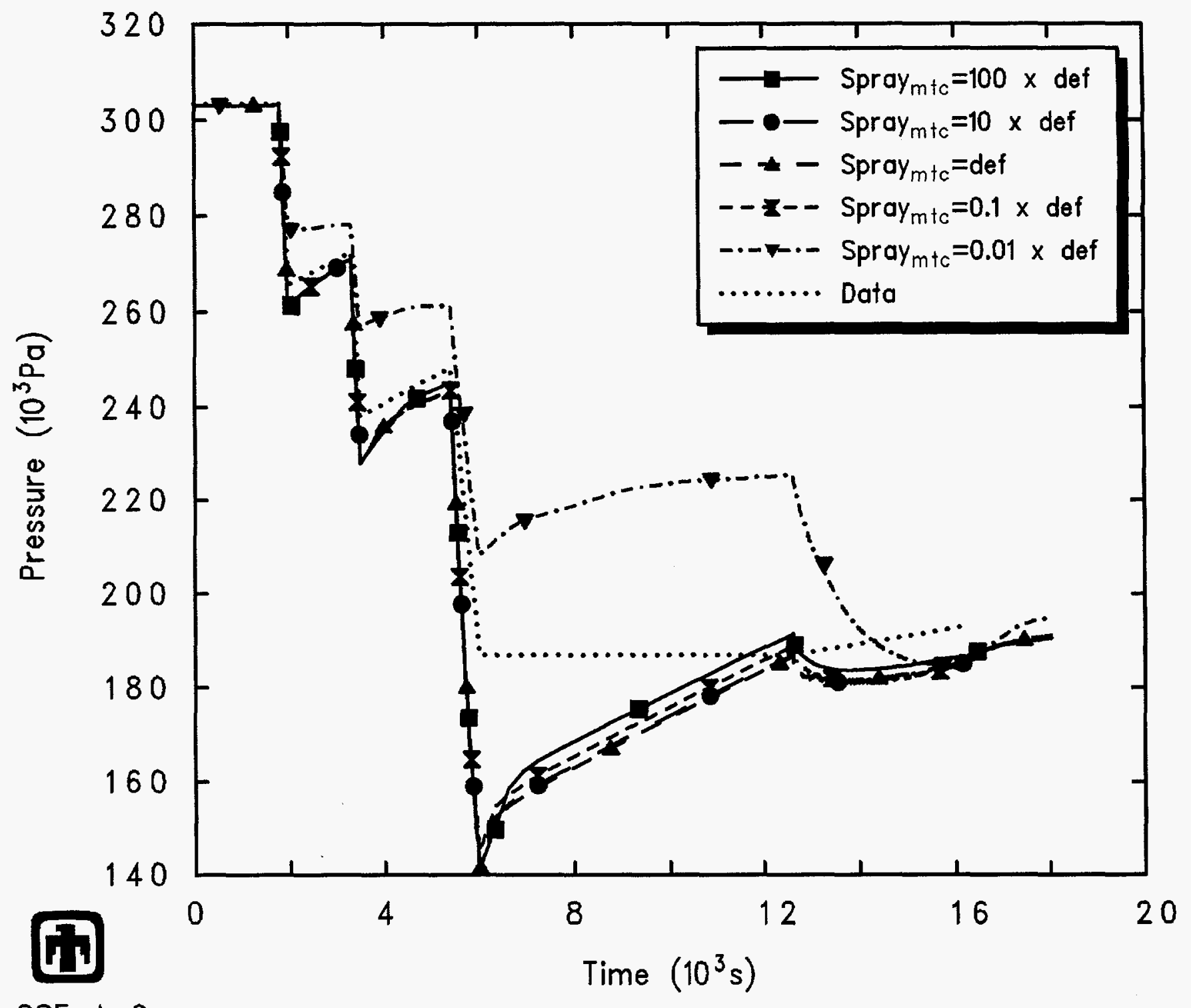

CSE A-9

HLEOFFHPN $8 / 12 / 94 \quad 14: 58: 38 \quad$ MELCOR SUN

Figure 6.4.1. Vessel Pressure for CSE Test A-9 - Spray Droplet Mass Transfer Coefficient Sensitivity Study 


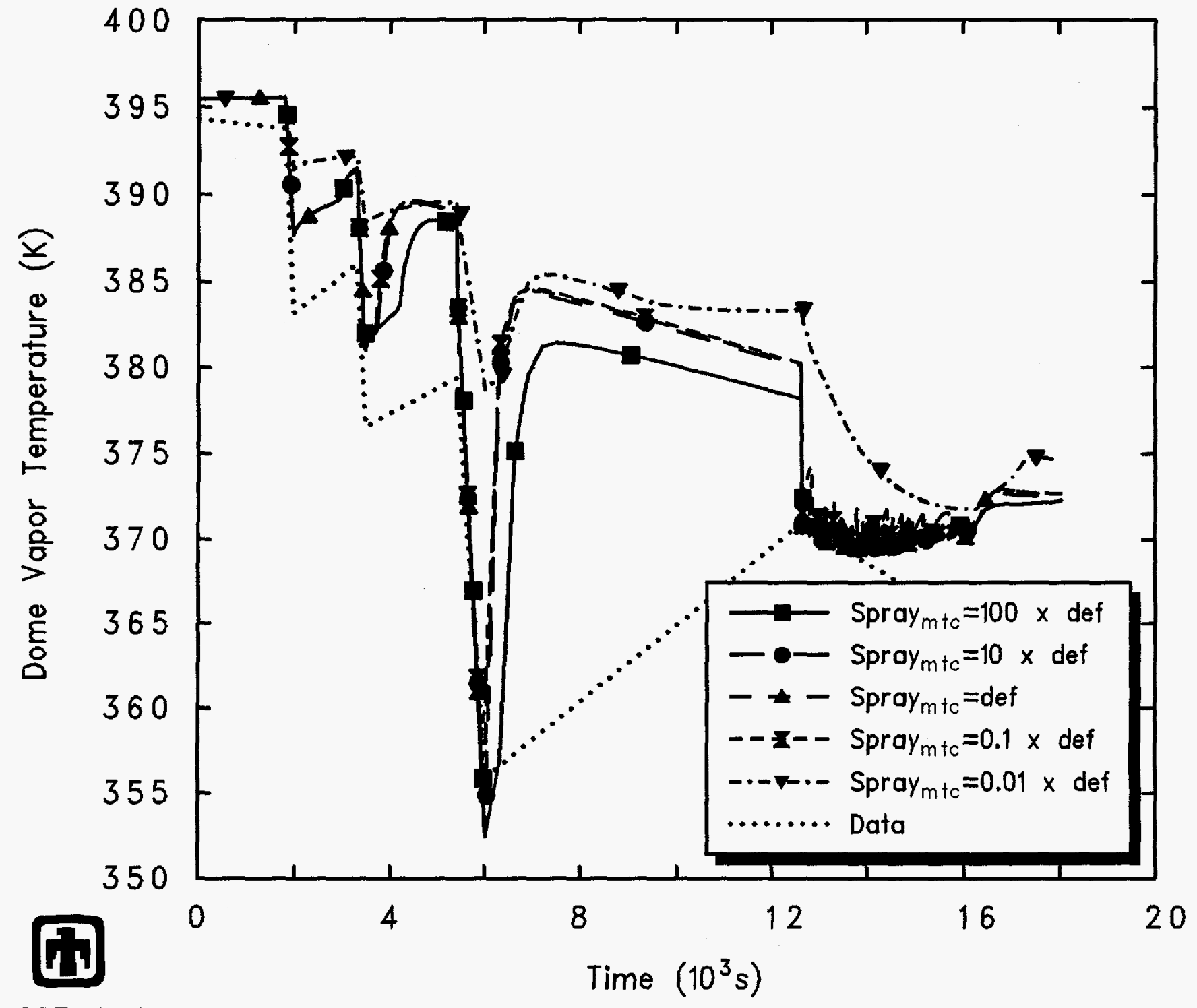

CSE A-9

HLEOFFHPN $8 / 12 / 94 \quad 14: 58: 38$ MELCOR SUN Figure 6.4.2. Vessel Dome Temperatures for CSE Test A-9-Spray Droplet Mass
Transfer Coefficient Sensitivity Study 


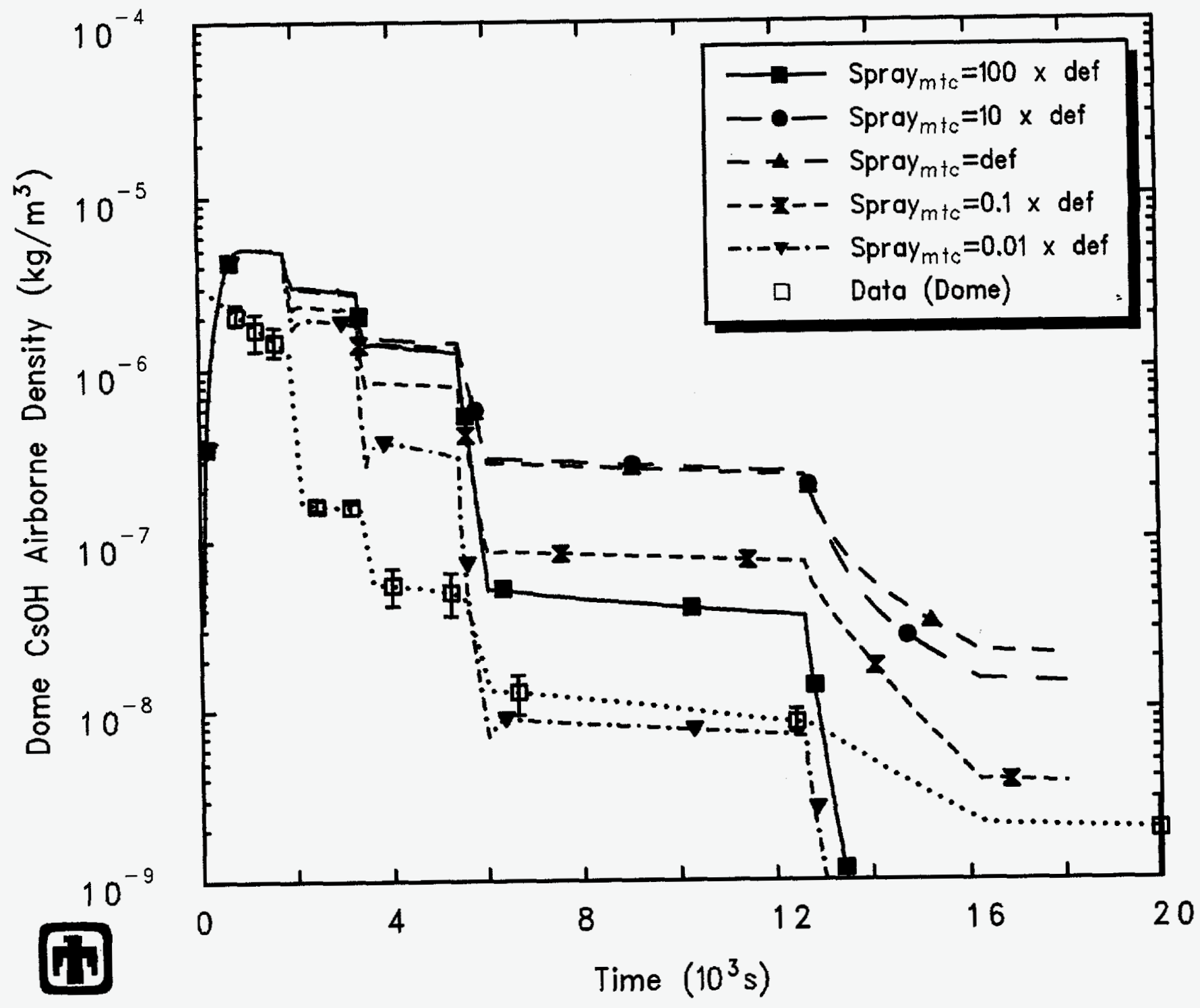

CSE A-9

HLEOFFHPN $\quad 8 / 12 / 94 \quad 14: 58: 38 \quad$ MELCOR SUN

Figure 6.4.3. Cesium Aerosol Airborne Concentrations for CSE Test A-9 - Spray Droplet Mass Transfer Coefficient Sensitivity Study 


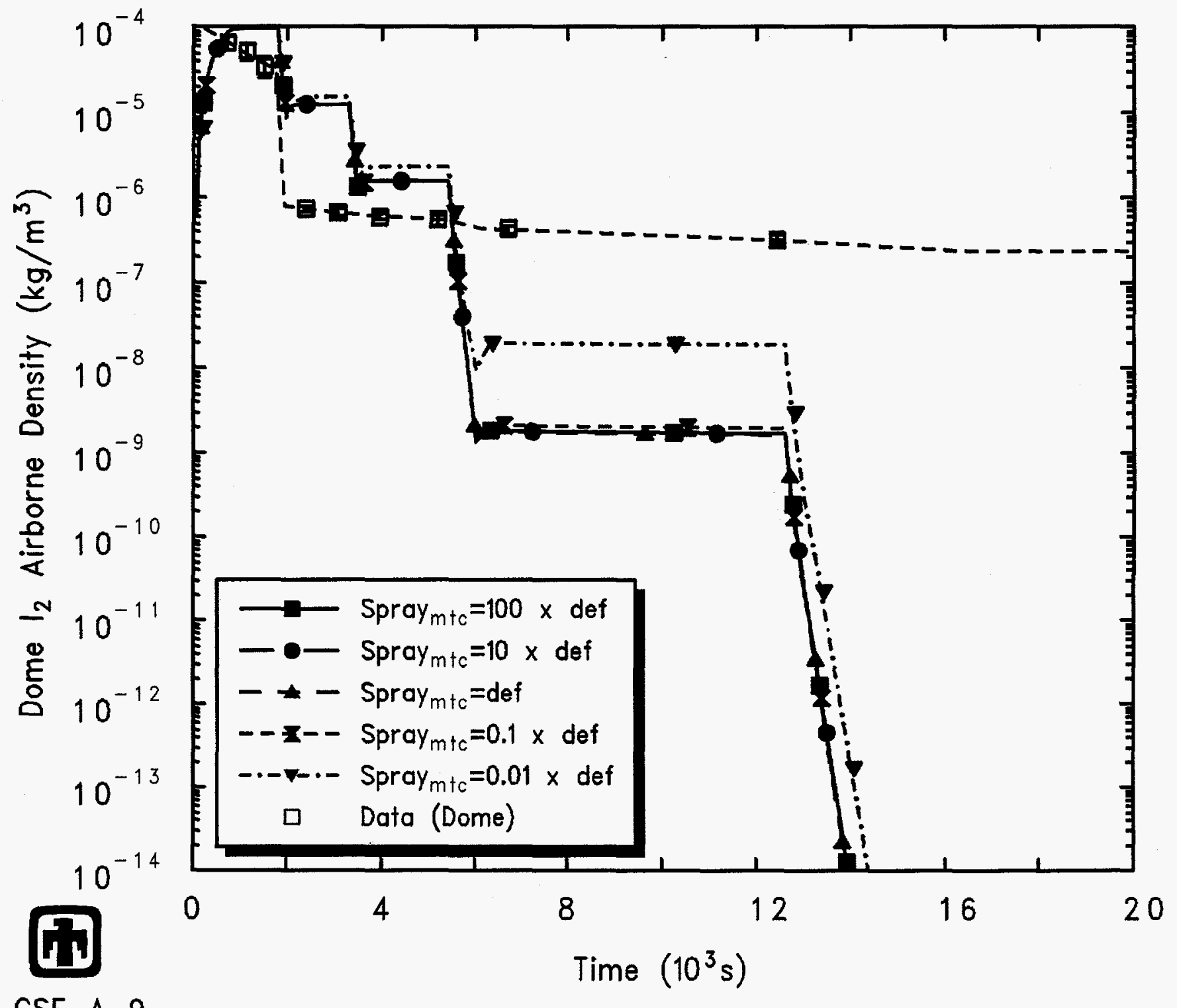

CSE A-9

HLEOFFHPN $8 / 12 / 94 \quad 14: 58: 38$ MELCOR SUN

Figure 6.4.4. Iodine Vapor Airborne Concentrations for CSE Test A-9 - Spray Droplet Mass Transfet Coefficient Sensitivity Study 
tion/condensation mass transfer coefficient. These tabular values echo the trend seen in Figures 6.4.3 and 6.4.4. 
Table 6.4.1. Washout Rates for CSE Test A-9 - Spray Droplet Mass Transfer Coefficient Sensitivity Study

\begin{tabular}{|c|c|c|c|c|c|c|}
\hline & \multicolumn{6}{|c|}{$t_{1 / 2}(\mathrm{~min})$} \\
\hline & \multirow[t]{3}{*}{ Measured } & \multicolumn{5}{|c|}{ MELCOR } \\
\hline & & \multicolumn{5}{|c|}{ Spray Droplet MTC × } \\
\hline & & 100 & 10 & $1^{a}$ & 0.1 & 0.01 \\
\hline \multicolumn{7}{|l|}{ Cesium } \\
\hline First spray & 1.08 & 5.3 & 5.2 & 5.0 & 3.6 & 2.7 \\
\hline Second spray & 2.0 & 4.3 & 4.9 & 4.6 & 3.1 & 1.7 \\
\hline Third spray & 5.4 & 2.3 & 4.5 & 4.3 & 2.9 & 1.5 \\
\hline Fourth spray & 33 & $10^{b}$ & $35^{b}$ & $35^{b}$ & $20^{b}$ & $17^{b}$ \\
\hline \multicolumn{7}{|l|}{ Uranium } \\
\hline First spray & 2.3 & 4.3 & 4.3 & 4.6 & 3.1 & 2.2 \\
\hline Second spray & & 4.0 & 4.6 & 4.3 & 2.9 & 1.7 \\
\hline Third spray & & 2.3 & 4.3 & 4.3 & 2.8 & 1.5 \\
\hline Fourth spray & & $9^{b}$ & $35^{b}$ & $35^{b}$ & $20^{b}$ & $17^{b}$ \\
\hline \multicolumn{7}{|l|}{ Iodine } \\
\hline First spray & 0.58 & 1.1 & 1.1 & 1.1 & 1.1 & 1.0 \\
\hline Second spray & 42 & 1.2 & 1.3 & 1.4 & 1.3 & 1.1 \\
\hline Third spray & 34 & 1.0 & 1.0 & 1.0 & 1.0 & 1.0 \\
\hline Fourth spray & 180 & 1.4 & 1.4 & 1.4 & 1.4 & 1.5 \\
\hline
\end{tabular}




\section{$7 \quad$ Aerosol Modelling Sensitivity Studies}

There are options and uncertainties both in some MELCOR input values and in the modelling approach taken to represent test conditions. The preceding section investigated how modelling variations would affect the response predicted by the spray (SPR) package. This section presents results varying parameter and options affecting the aerosol modelling in the RN package, i.e., MAEROS parameter studies, while the next section will discuss modelling variations affecting the vapor modelling in the $\mathrm{RN}$ package used to calculate the iodine response.

\subsection{Number of MAEROS Components}

The aerosol transport and deposition portion of MELCOR is based on the MAEROS program [25], a multicomponent aerosol dynamics code. Because a large amount of computer time usually would be needed to set each MELCOR radionuclide class to its own component, which would be expected to give the most accurate results, the MELCOR default is specification of a single component for all radionuclide classes (with multicomponent calculations remaining an available input option).

The reference analyses were run using three MAEROS aerosol components, one for any water droplets in control volumes atmospheres (i.e., any fog, also known as Class 14) and the other two for the cesium and uranium aerosols. (The iodine was predicted to exist as a vapor, not an aerosol.) This approach was taken because sensitivity studies in our earlier MELCOR assessment using the LACE LA4 aerosol test [3] indicated that at least two components (one for fog) were needed to correctly account for condensation and evaporation effects. As a sensitivity study, calculations were run for test A-9 using a single aerosol component for all classes, which is the normal MELCOR default assumption, and using two components, one for the water fog and the other for both the cesium and uranium aerosols, for comparison with the reference calculation results presented in Section 4.

The thermal/hydraulic responses calculated using either one or two aerosol components were virtually identical to each other and to the results calculated using three aerosol components, as would be expected.

Figure 7.1.1 presents the concentrations of iodine vapor in the test vessel dome atmosphere predicted using different numbers of MAEROS components, with the test data also included for reference. The comparisons are very similar for the upper dome, the lower drywell, the middle room and the lower room or sump. The iodine vapor responses calculated using either one or two aerosol components are virtually identical to each other and to the results calculated using three aerosol components, which is also as would be expected.

Figure 7.1.2 presents the concentrations of cesium aerosol in the test vessel dome atmosphere that were predicted using different numbers of MAEROS components, with the test data included for reference; the comparisons are very similar for the upper dome, 


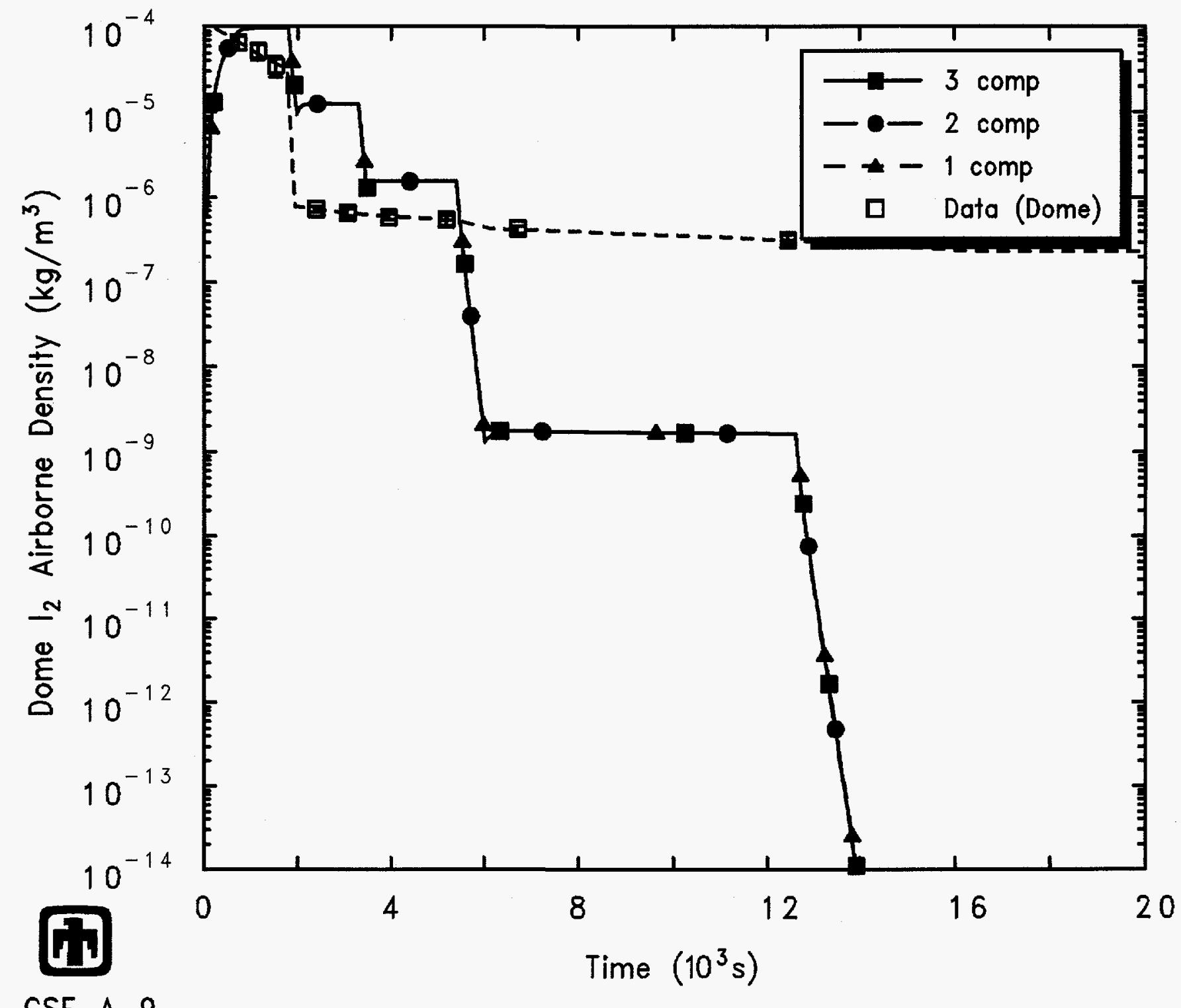

CSE A-9

HLEKCVKPN $8 / 12 / 94 \quad 10: 31: 51 \quad$ MELCOR SUN

Figure 7.1.1. Iodine Vapor Airborne Concentrations for CSE Test A-9 - MAEROS Component Sensitivity Study 
the lower drywell, the middle room and the lower room or sump. The results with two and with three components are very similar. The cesium aerosol airborne density drops slightly faster in the calculation with two components because in that case the cesium particles are combined in a single component with the slightly larger uranium aerosol particles and consequently settle out faster. The difference is greater when only a single aerosol component is used, i.e., when fog is not modelled as a separate component, especially after the fog is all gone after the second spray period.

Figure 7.1.3 presents the concentrations of uranium aerosol in the test vessel dome atmosphere predicted using different numbers of MAEROS components, with the test data included for reference. The comparisons are very similar for the upper dome, the lower drywell, the middle room and the lower room or sump. The results with two and with three components are very similar, and the reverse of the relative behavior predicted for the cesium aerosol. The uranium aerosol airborne density drops slightly slower in the calculation with two components because in that case the uranium particles are combined in a single component with the slightly smaller cesium aerosol particles and consequently settle out slower. The response when only a single aerosol component is used is very similar to the results found for the cesium.

The corresponding aerosol and vapor washout constants are listed in Table 7.1.1; again, the test data are included for reference.

Figure 7.1.4 gives the mass median diameters and geometric standard deviations of the airborne cesium and uranium aerosol particle distributions in the dome atmosphere calculated using different numbers of MAEROS components. Figure 7.1.5 presents the same results for the fog (i.e., water aerosol) particles in the dome atmosphere.

With the cesium and uranium aerosols represented by separate components, as in the reference calculation, the cesium particle AMMD is just slightly smaller and the uranium particle AMMD just a little larger than the average AMMD when both aerosols are represented by a single component; the GSDs are also very similar. Treating these two aerosols in a single component makes little difference in this case because both species are at the same density and are introduced into the system at the same time, at similar rates and at similar sizes, and are removed at similar rates by the same mechanisms. Thus, combining aerosol particles with very similar behavior in a single component results in an average response that adequately represents the individual species behavior.

A much bigger difference is found in results if the water aerosol (fog) is treated as a separate component or averaged in with the cesium and uranium aerosols because the fog particles act very differently. They are pre-existing at the start of the transient at a noticably larger size than the injected aerosols, they are affected much more by the start and stop of sprays, and they disappear relatively early in the transient, except for some intermittent fog generation associated with the intermittent spray operation. Combining aerosol particles with such different behavior in a single component results in an average response which does not represent any individual species well.

Relative to the reference calculation using 3 MAEROS components, using 2 components ( 1 for the fog) required $4.5 \%$ less cpu time, while using only 1 component (the 


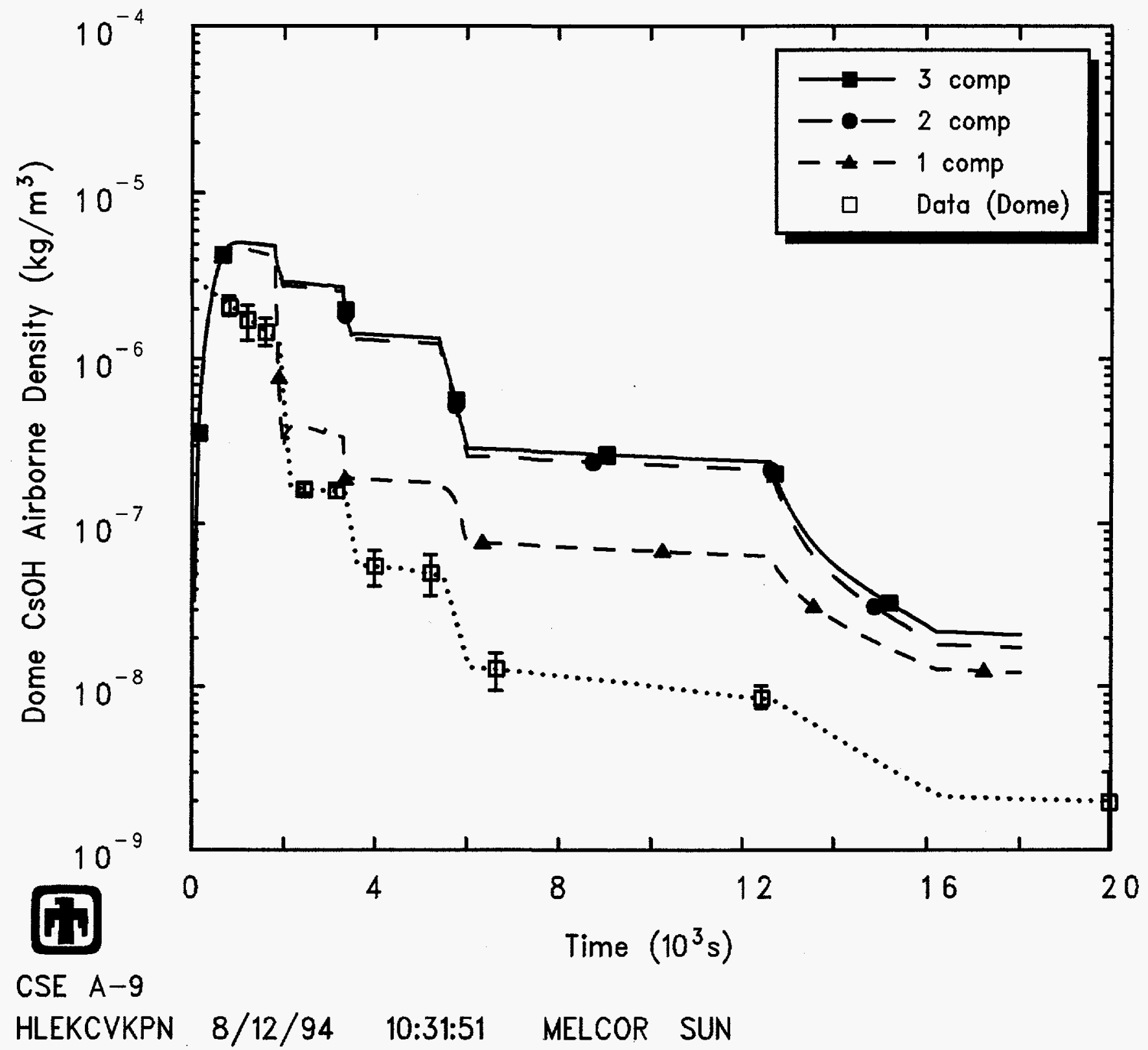

Figure 7.1.2. Cesium Aerosol Airborne Concentrations for CSE Test A-9 MAEROS Component Sensitivity Study 


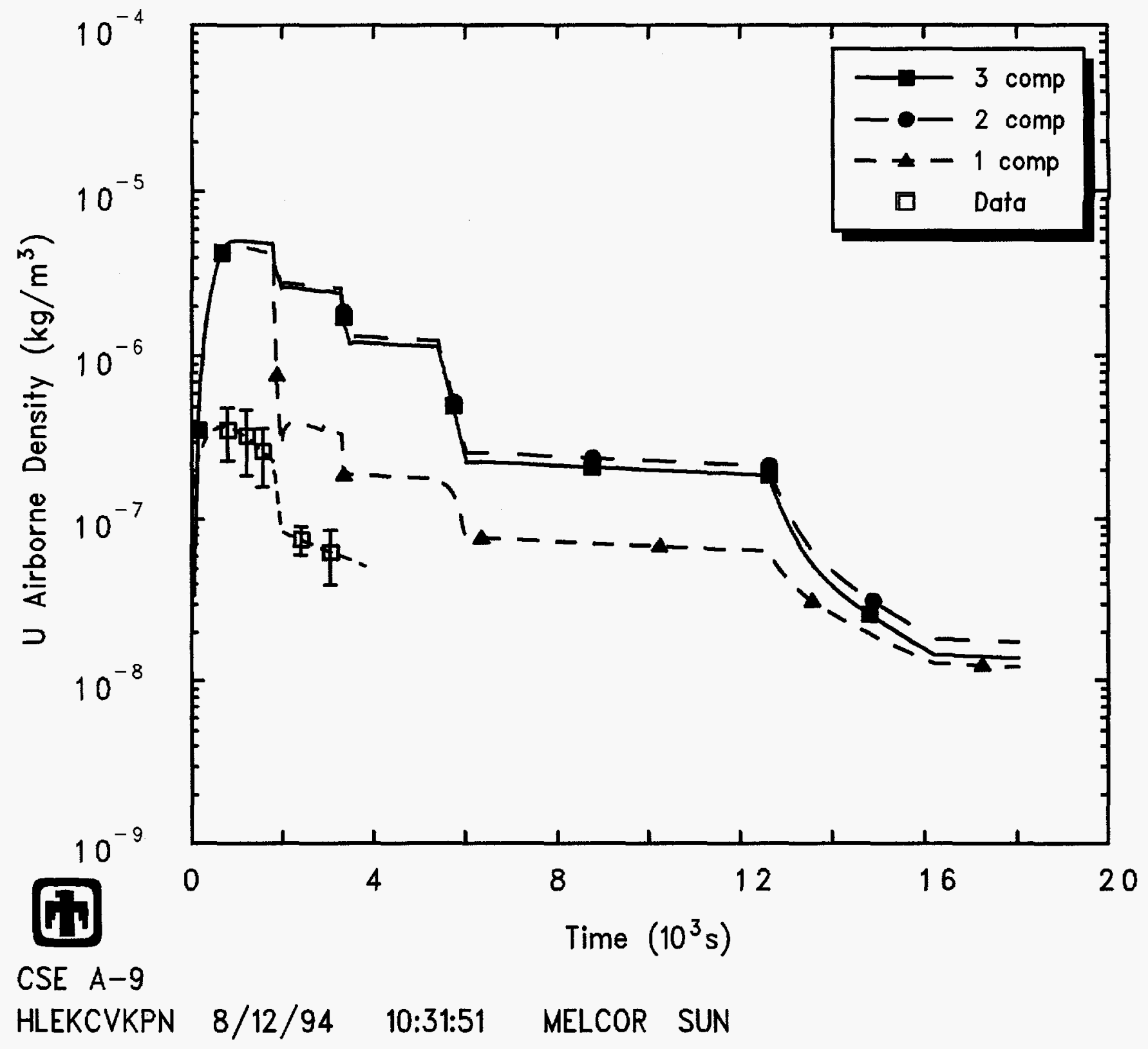

Figure 7.1.3. Uranium Aerosol Airborne Concentrations for CSE Test A-9 MAEROS Component Sensitivity Study 
Table 7.1.1. Washout Rates for CSE Test A-9 - MAEROS Component Sensitivity Study

\begin{tabular}{lcccc} 
& \multicolumn{5}{c}{$t_{1 / 2}$ (min) } \\
& Measured & \multicolumn{3}{c}{ components } \\
& & $3^{a}$ & 2 & 1 \\
\hline & & & & \\
Cesium & & & & \\
$\quad$ First spray & 1.08 & 5.0 & 4.5 & 0.83 \\
Second spray & 2.0 & 4.6 & 4.6 & 19.8 \\
Third spray & 5.4 & 4.3 & 4.4 & 5.3 \\
Fourth spray & 33 & $35^{b}$ & $35^{b}$ & $45^{b}$ \\
& & & & \\
Uranium & & & & \\
First spray & 2.3 & 4.6 & 4.5 & 0.83 \\
Second spray & & 4.3 & 4.6 & 19.8 \\
Third spray & & 4.3 & 4.4 & 5.3 \\
Fourth spray & & $35^{b}$ & $35^{b}$ & $45^{b}$ \\
& & & & \\
Iodine & & & & \\
First spray & 0.58 & 1.1 & 1.1 & 1.1 \\
Second spray & 42 & 1.4 & 1.4 & 1.4 \\
Third spray & 34 & 1.0 & 1.0 & 1.0 \\
Fourth spray & 180 & 1.4 & 1.4 & 1.4 \\
& & & & \\
& ${ }^{a}$ Reference calculation value \\
& ${ }^{b}$ Value at end of recirculating spray
\end{tabular}




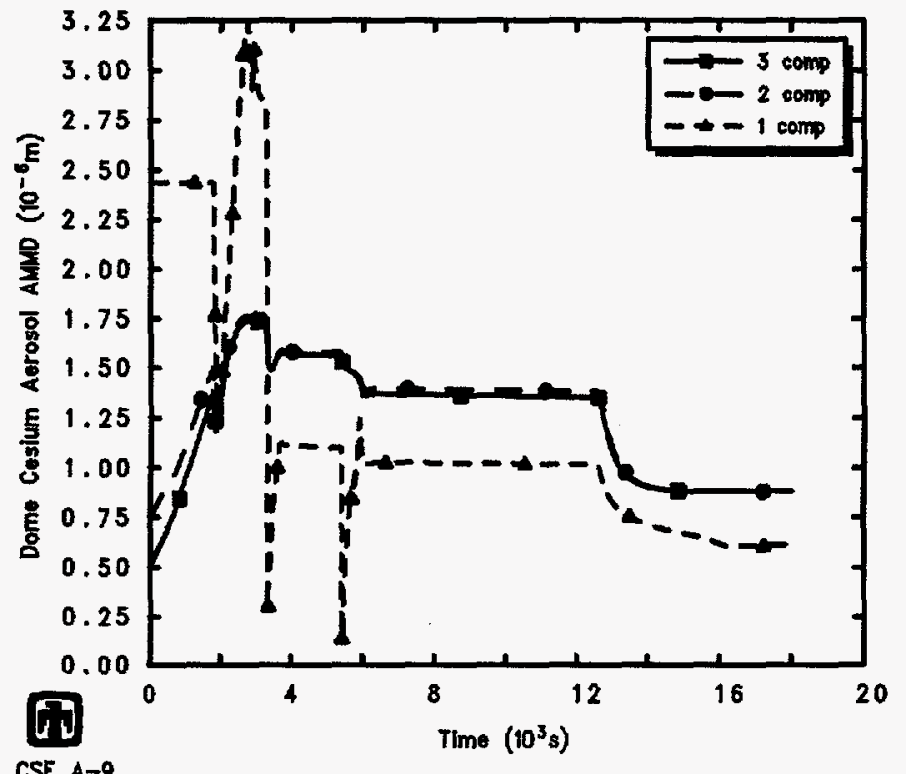

HLEKCVKPN $8 / 12 / 94 \quad 10: 31: 51$ MELCOR SUN

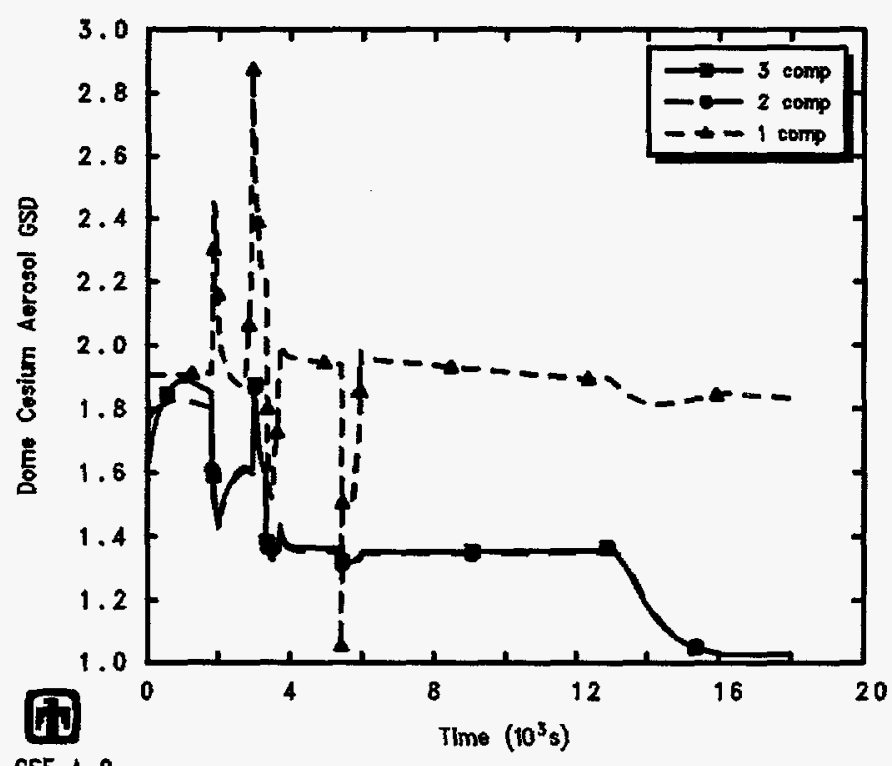

CSE $A-9$

HLEKCVKPN $8 / 12 / 94 \quad 10: 31: 51$ MELCOR SUN

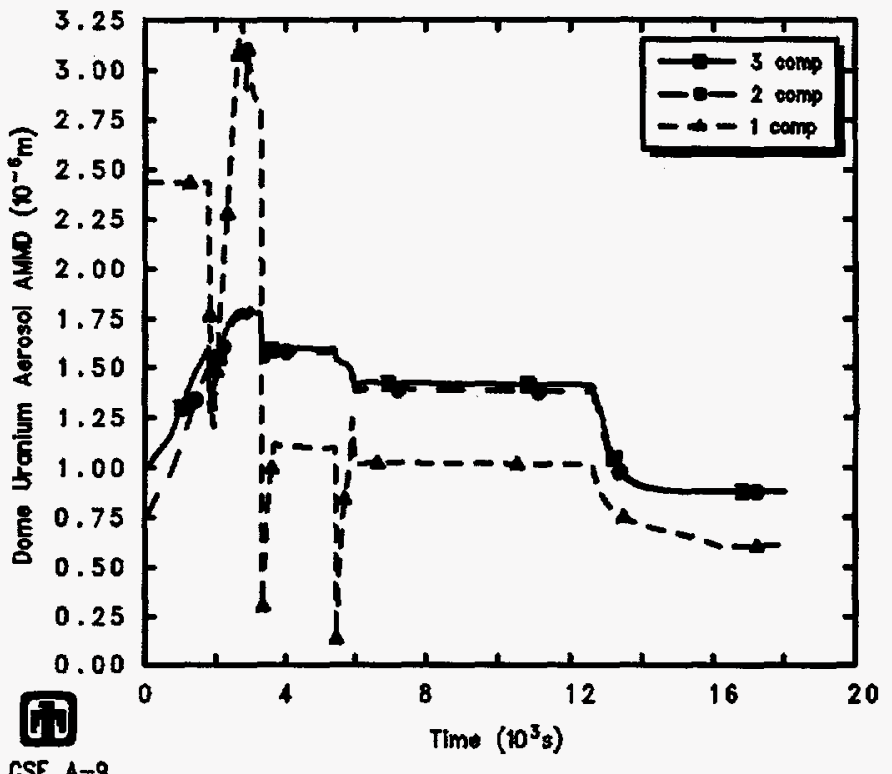

CSE A-9

HLEKCVKPN $8 / 12 / 94 \quad 10: 3 \mathrm{t}: 51$ MELCOR SUN

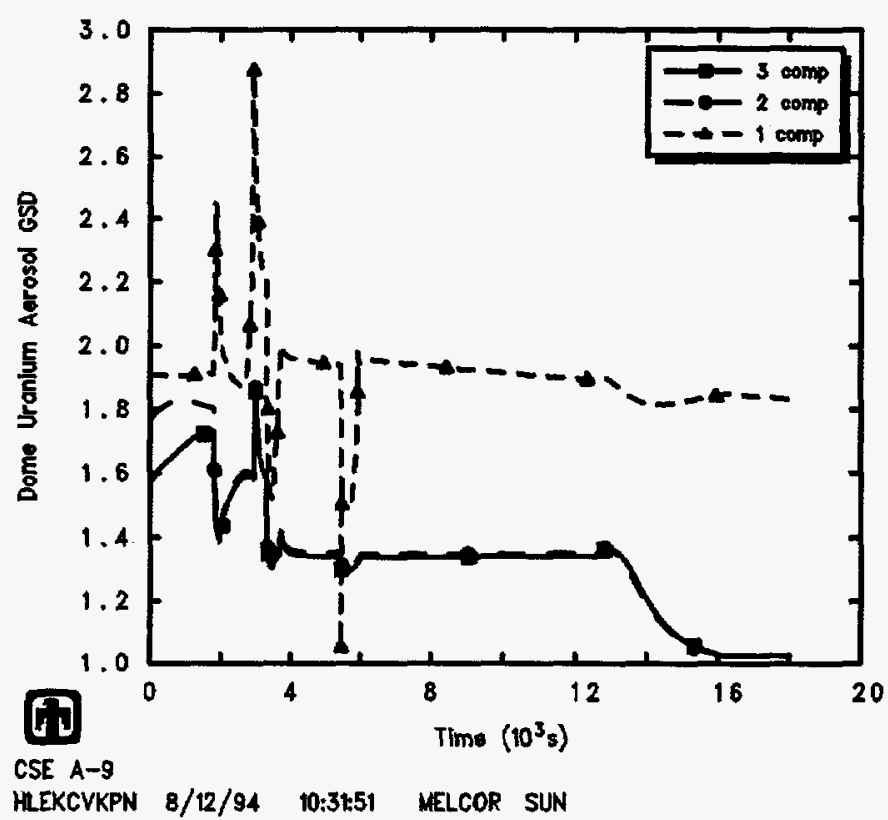

Figure 7.1.4. Cesium (left) and Uranium (right) Aerosol Airborne Concentration AMMDs (top) and GSDs (bottom) in Dome for CSE Test A-9MAEROS Component Sensitivity Study 

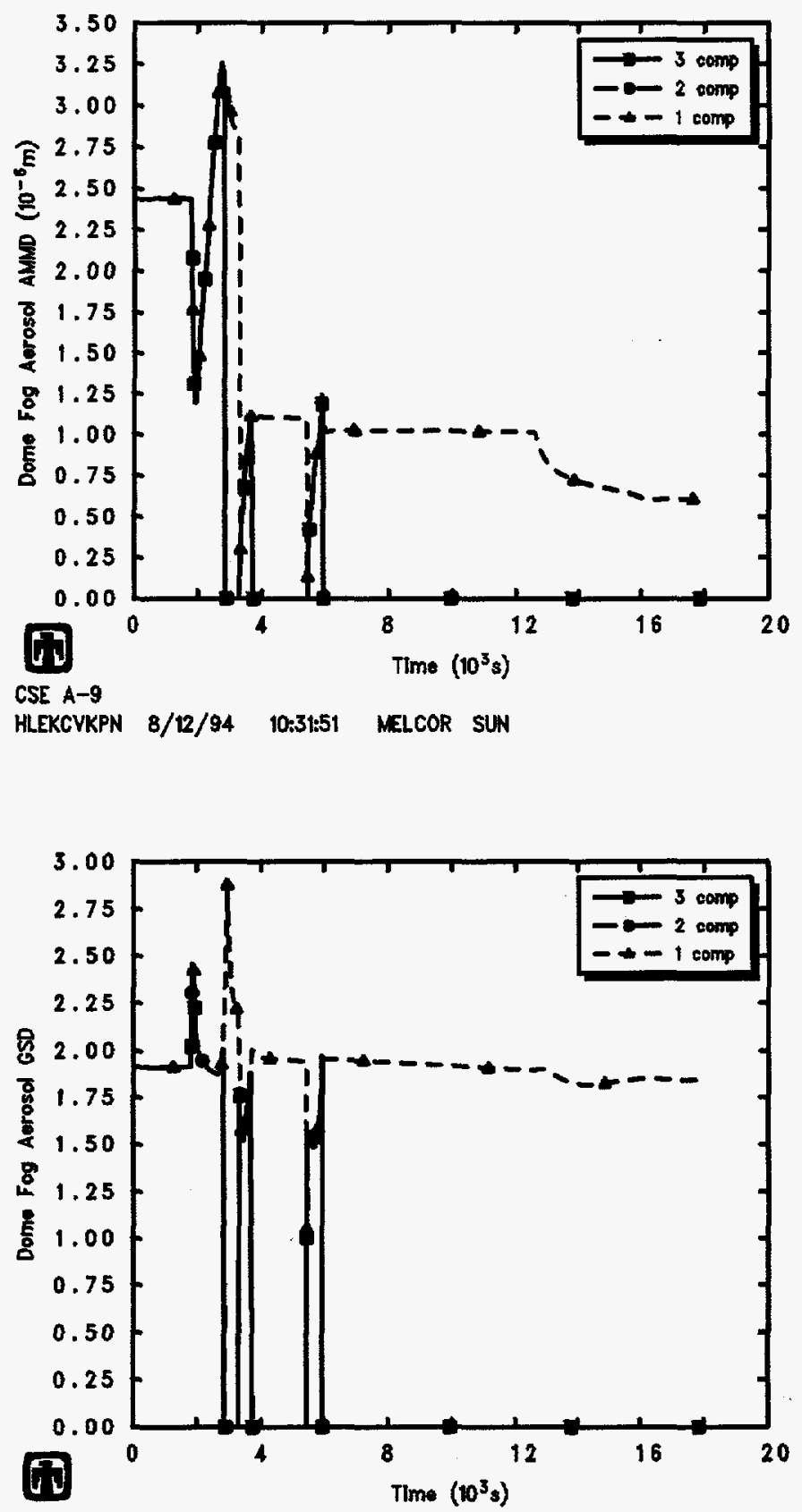

CSE $A-9$

HLEKCVKPN $8 / 12 / 94$ 10:3t:51 MELCOR SUN

Figure 7.1.5. Fog Water Aerosol Airborne Concentration AMMDs (top) and GSDs (bottom) in Dome for CSE Test A-9 - MAEROS Component Sensitivity Study 
default) took $7 \%$ less cpu time. Given the relatively small savings in run time, it is easy to justify using more components to more accurately represent differences in behavior.

(The same conclusion, that water fog droplets should usually be modelled as a separate MAEROS component in any problem in which humidity and humidity changes are expected to be a factor, was reached during an earlier MELCOR 1.8 .2 assessment using the LACE LA4 experiment [3].)

\subsection{Number of MAEROS Sections}

The MAEROS program [25], and its implementation in MELCOR, evaluates the dynamic size distribution of each component; this size distribution is described by the mass in each size bin, or section. The default in MELCOR is to use 5 sections. The: minimum and maximum diameters are default to $1 \mu \mathrm{m}$ and $50 \mu \mathrm{m}$, respectively, and the section boundaries are equally logarithmically spaced, with the restriction that the masses in adjacent sections differ by at least a factor of two.

The reference calculations were run using 10 sections, with the minimum particle diameter reduced to $0.1 \mu \mathrm{m}$ and the maximum diameter kept at the default of $50 \mu \mathrm{m}$. Sensitivity study calculations were done for test A-9 by increasing the number of sections to 20 and reducing the number of sections to the default of 5 , in both cases keeping the minimum and maximum diameters the same as in the reference calculation.

(Note that there is an inherent limit in the number of sections that can be used in MELCOR in any given problem, because of the constraint in MAEROS that the mass of particles in adjacent sections must differ by at least a factor of 2 . For these CSE analyses, given the minimum aerosol particle diameter set to $0.1 \mu \mathrm{m}$ and the maximum set to $50 \mu \mathrm{m}$, the upper limit allowed would be 26 MAEROS sections.)

The thermal/hydraulic responses calculated using more or fewer sections were virtually identical to each other and to the reference calculation results, as would be expected.

Figure 7.2.1 presents the concentrations of iodine vapor in the test vessel dome atmosphere predicted using different numbers of MAEROS sections, with the test data included for reference. The comparisons are very similar for the upper dome, the lower drywell, the middle room and the lower room or sump. The iodine vapor responses calculated using more or less aerosol sections are virtually identical, which is also as would be expected.

Figures 7.2.2 and 7.2.3 present the concentrations of cesium and uranium aerosol, respectively, in the test vessel dome atmosphere predicted using different numbers of MAEROS sections, with the test data included for reference. The comparisons are very similar for the upper dome, the lower drywell, the middle room and the lower room or sump. The corresponding washout constants are listed in Table 7.2.1, with the test data included for reference.

While the results are not completely consistent or convergent, in general there appears to be less removal of aerosols by sprays as more MAEROS sections are used to resolve the aerosol particle size distribution. 


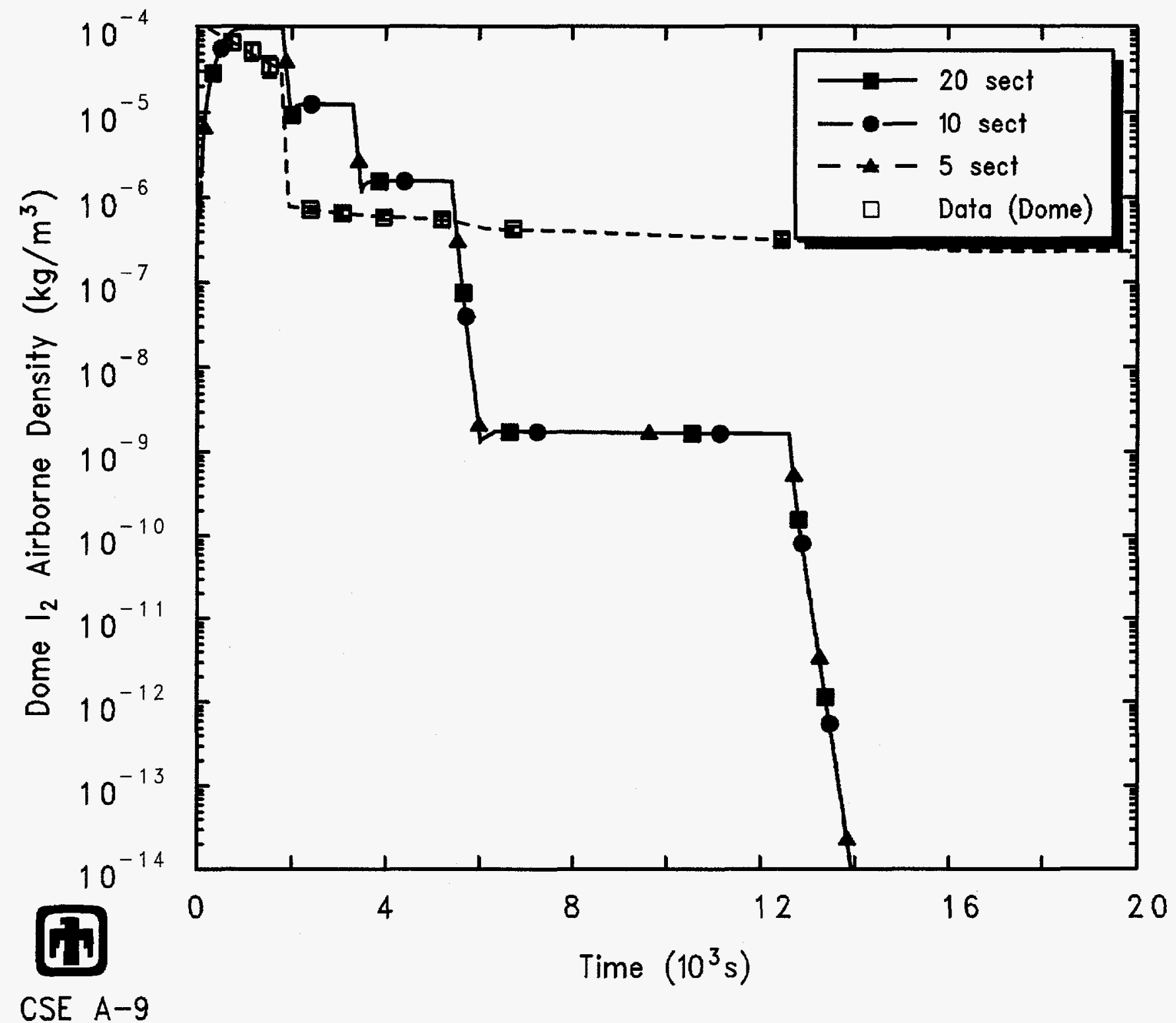

HMEAEQAPN $8 / 13 / 94 \quad 00: 52: 02$ MELCOR SUN

Figure 7.2.1. Iodine Vapor Airborne Concentrations for CSE Test A-9 - MAEROS Sections Sensitivity Study 


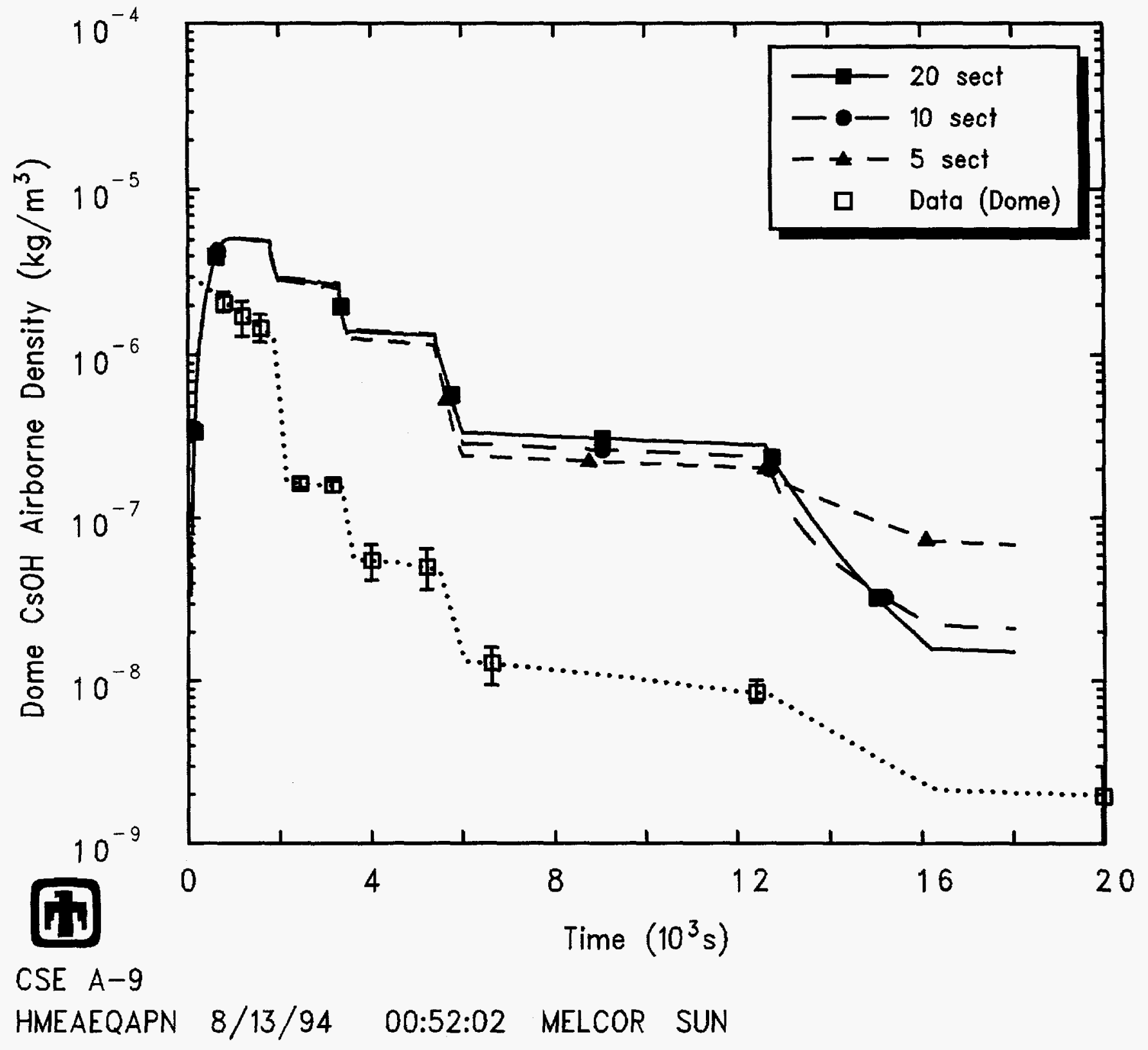

Figure 7.2.2. Cesium Aerosol Airborne Concentrations for CSE Test A-9 MAEROS Sections Sensitivity Study 


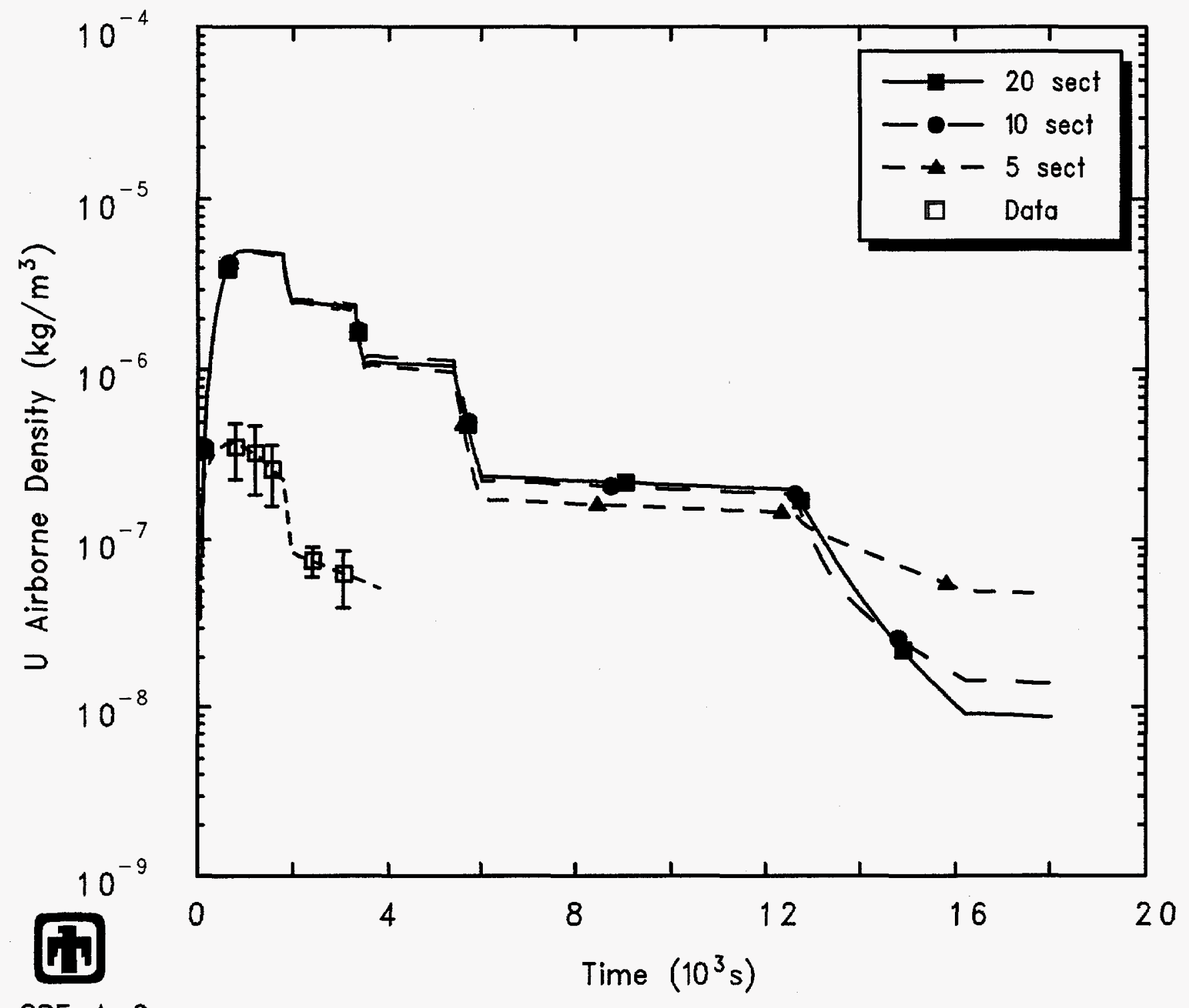

CSE A-9

HMEAEQAPN $8 / 13 / 94 \quad 00: 52: 02$ MELCOR SUN

Figure 7.2.3. Uranium Aerosol Airborne Concentrations for CSE Test A-9 MAEROS Sections Sensitivity Study 
Table 7.2.1. Washout Rates for CSE Test A-9 - MAEROS Sections Sensitivity Study

\begin{tabular}{|c|c|c|c|c|}
\hline & \multicolumn{4}{|c|}{$t_{1 / 2}(\min )$} \\
\hline & \multirow[t]{2}{*}{ Measured } & \multicolumn{3}{|c|}{ sections } \\
\hline & & 20 & $10^{a}$ & 5 \\
\hline \multicolumn{5}{|l|}{ Cesium } \\
\hline First spray & 1.08 & 4.7 & 5.0 & 4.8 \\
\hline Second spray & 2.0 & 4.4 & 4.6 & 3.7 \\
\hline Third spray & 5.4 & 5.1 & 4.3 & 3.9 \\
\hline Fourth spray & 33 & $23^{b}$ & $35^{b}$ & $51^{t}$ \\
\hline \multicolumn{5}{|l|}{ Uranium } \\
\hline First spray & 2.3 & 3.8 & 4.5 & 4.1 \\
\hline Second spray & & 3.8 & 4.6 & 3.5 \\
\hline Third spray & & 4.6 & 4.4 & 3.6 \\
\hline Fourth spray & & $23^{b}$ & $35^{b}$ & $50^{\circ}$ \\
\hline \multicolumn{5}{|l|}{ Iodine } \\
\hline First spray & 0.58 & 1.1 & 1.1 & 1.1 \\
\hline Second spray & 42 & 1.4 & 1.4 & 1.4 \\
\hline Third spray & 34 & 1.0 & 1.0 & 1.0 \\
\hline Fourth spray & 180 & 1.4 & 1.4 & 1.4 \\
\hline
\end{tabular}


Figure 7.2.4 gives the mass median diameters and geometric standard deviations of the airborne cesium and uranium aerosol particle distributions in the dome atmosphere calculated using different numbers of size bins to resolve the MAEROS aerosol size distribution. Figure 7.2.5 presents the same results for the fog (i.e., water aerosol) particles in the dome atmosphere.

The cesium and uranium aerosol particles are predicted to have generally smaller AMMDs as more MAEROS sections are used to resolve the aerosol particle size distribution, both early in the transient (before about $6000 \mathrm{~s}$, the end of the last fresh spray) and later in the transient (after about $12600 \mathrm{~s}$, the start of the recirculating spray); however, there is a period midway through the transient when the reference calculation using 10 MAEROS sections predicts larger aerosol particle sizes than calculated using either more (20) or fewer (5) sections, which does not seem physically or numerically reasonable. The comparisons of GSDs for the cesium and uranium aerosols seem even more contradictory.

In contrast to the behavior calculated for the other aerosols, the water aerosol particle sizes (when present) increase as more MAEROS sections are used to resolve the aerosol particle size distribution. Note that even for the water aerosol, the GSD calculated before the first spray period by the reference calculation using 10 MAEROS sections is larger than the corresponding values calculated using either more (20) or fewer (5) sections, which again does not seem physically or numerically reasonable.

Relative to the reference calculation using 10 MAEROS sections, using 20 sections required almost $50 \%$ more cpu time, while using only 5 sections (the default) took $24 \%$ less cpu time. Given the savings in run time, using 10 sections rather than the default 5 is probably justifiable, but more sections make the calculation noticably more expensive. However, the inconsistencies seen in some of the results of this sensitivity study suggest that there may remain some code problems affecting the accuracy and convergence of the results, which need identification and resolution in the future.

\subsection{Aerosol Density}

The default nominal aerosol density assumed in MELCOR is $1000 \mathrm{~kg} / \mathrm{m}^{3}$ (i.e., water density). The reference calculations were run with the aerosol density set to $2500 \mathrm{~kg} / \mathrm{m}^{3}$ (as discussed in Section 3). As a sensitivity study, calculations were done for test A-9 specifying nominal aerosol densities of 1000 (the default) and $5000 \mathrm{~kg} / \mathrm{m}^{3}$. (Note that in MAEROS a single density value is used for all aerosol components.)

The thermal/hydraulic responses calculated using different aerosol densities were virtually identical, as would be expected.

Figure 7.3.1 presents the concentrations of iodine vapor in the test vessel dome atmosphere predicted using different aerosol densities, with the test data included for reference. The comparisons are very similar for the upper dome, the lower drywell, the middle room and the lower room or sump. The iodine vapor responses calculated using different aerosol densities are virtually identical, also as would be expected. 


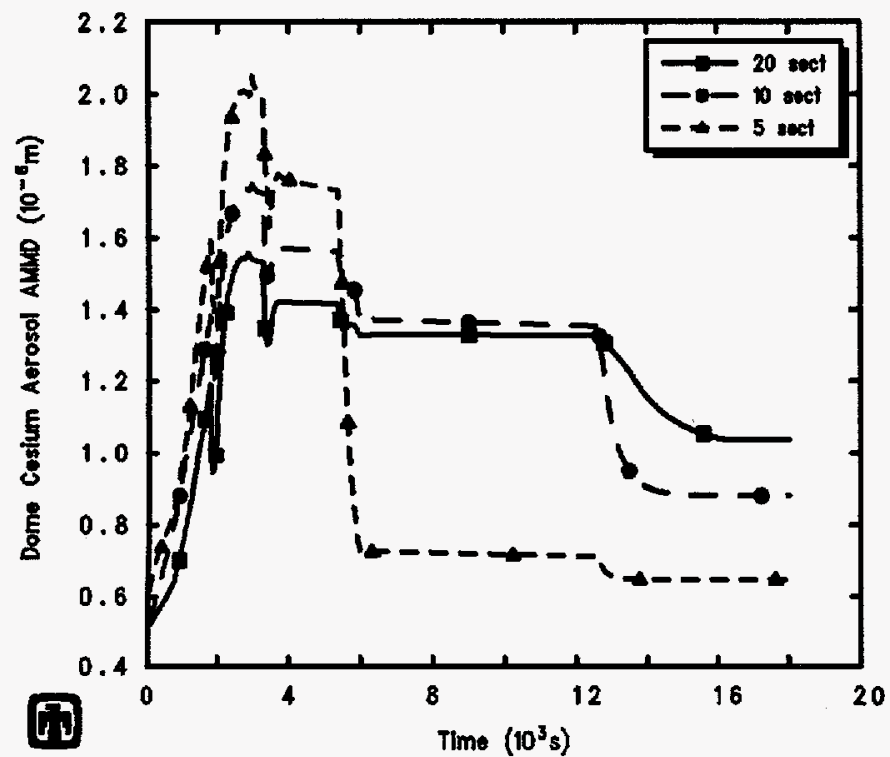

CSE A-9

HMEAEQAPN 8/13/94 00:52:02 NELCOR SUN

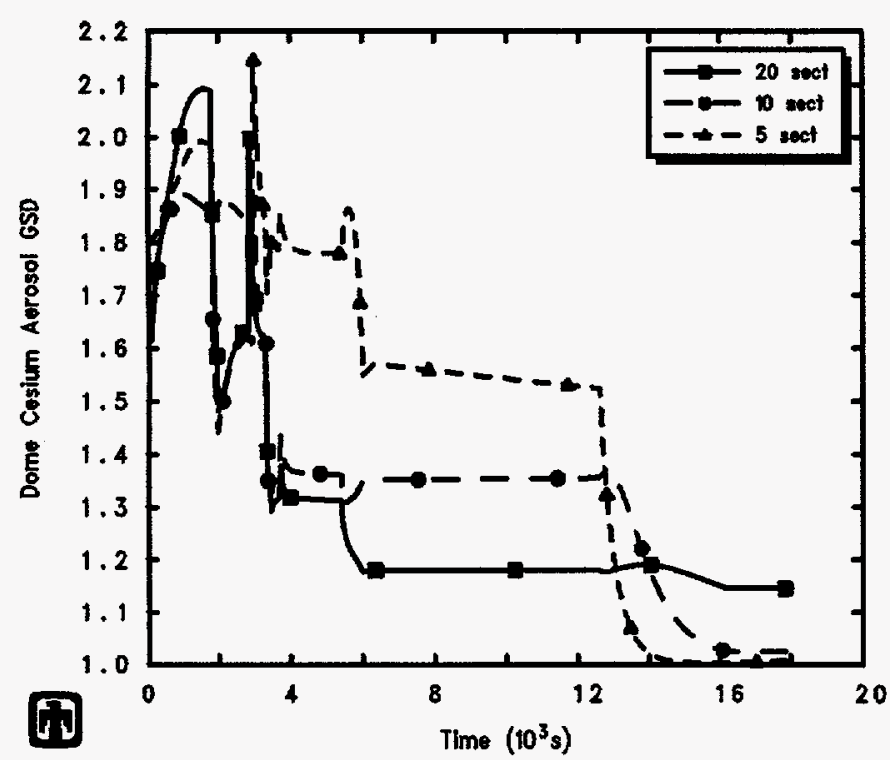

CSE A-9

HMEAEQAPN $8 / 13 / 94 \quad 00: 52: 02$ MELCOR SUN

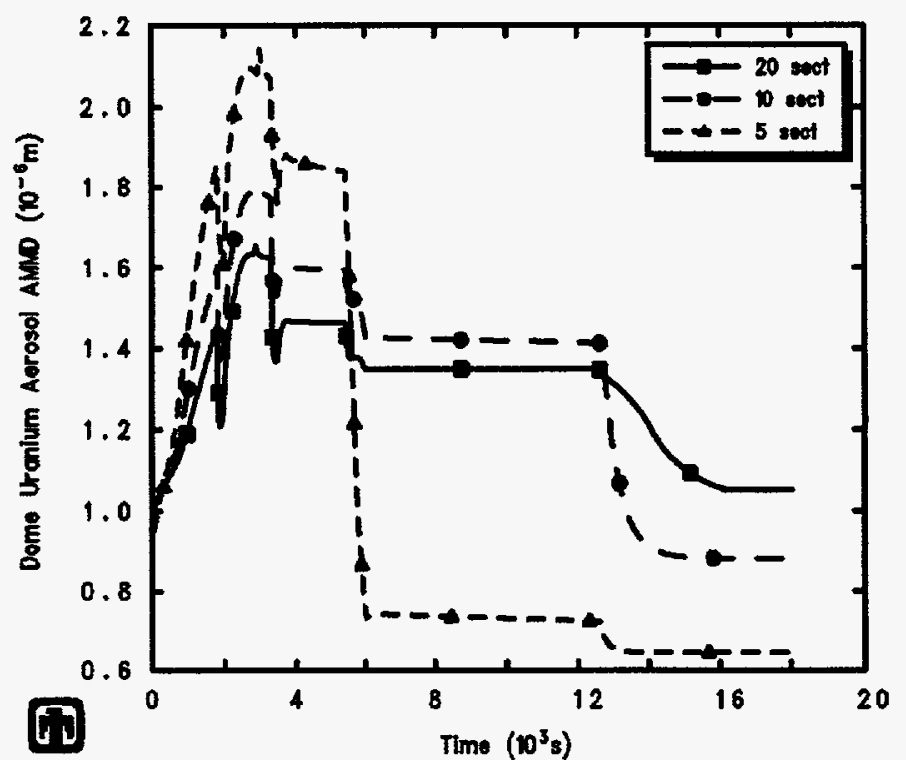

CSE A-9

HMEAEQAPN $8 / 13 / 94$ 00:52:02 MELCOR SUN

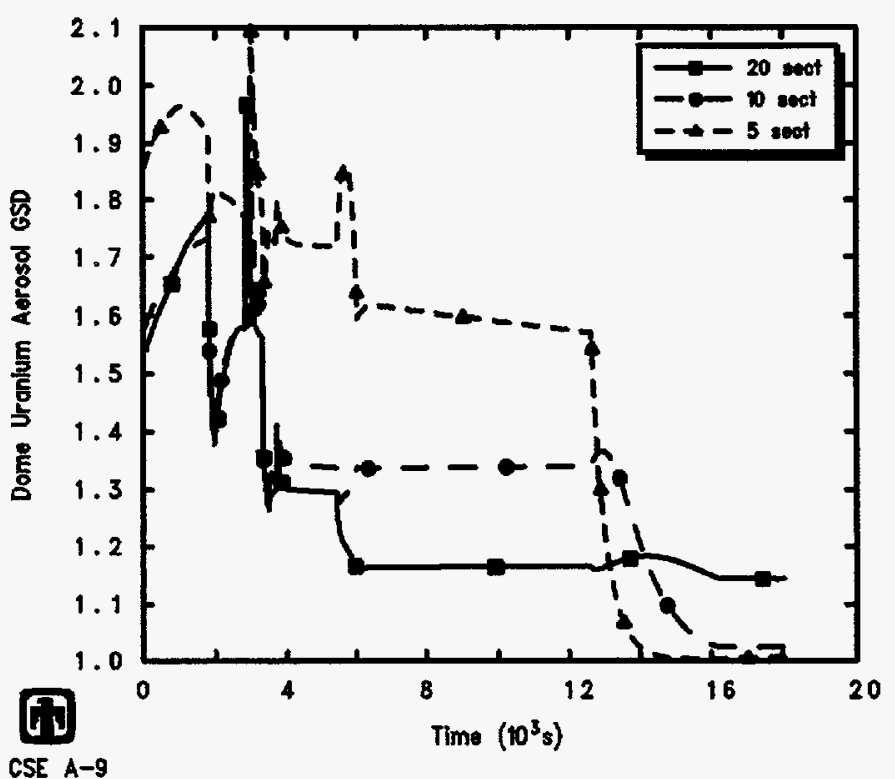

CSE $A-9$

HIEAEQAPN B/13/94 00:52:02 MELCOR SUN

Figure 7.2.4. Cesium (left) and Uranium (right) Aerosol Airborne Concentration AMMDs (top) and GSDs (bottom) in Dome for CSE Test A-9 MAEROS Sections Sensitivity Study 

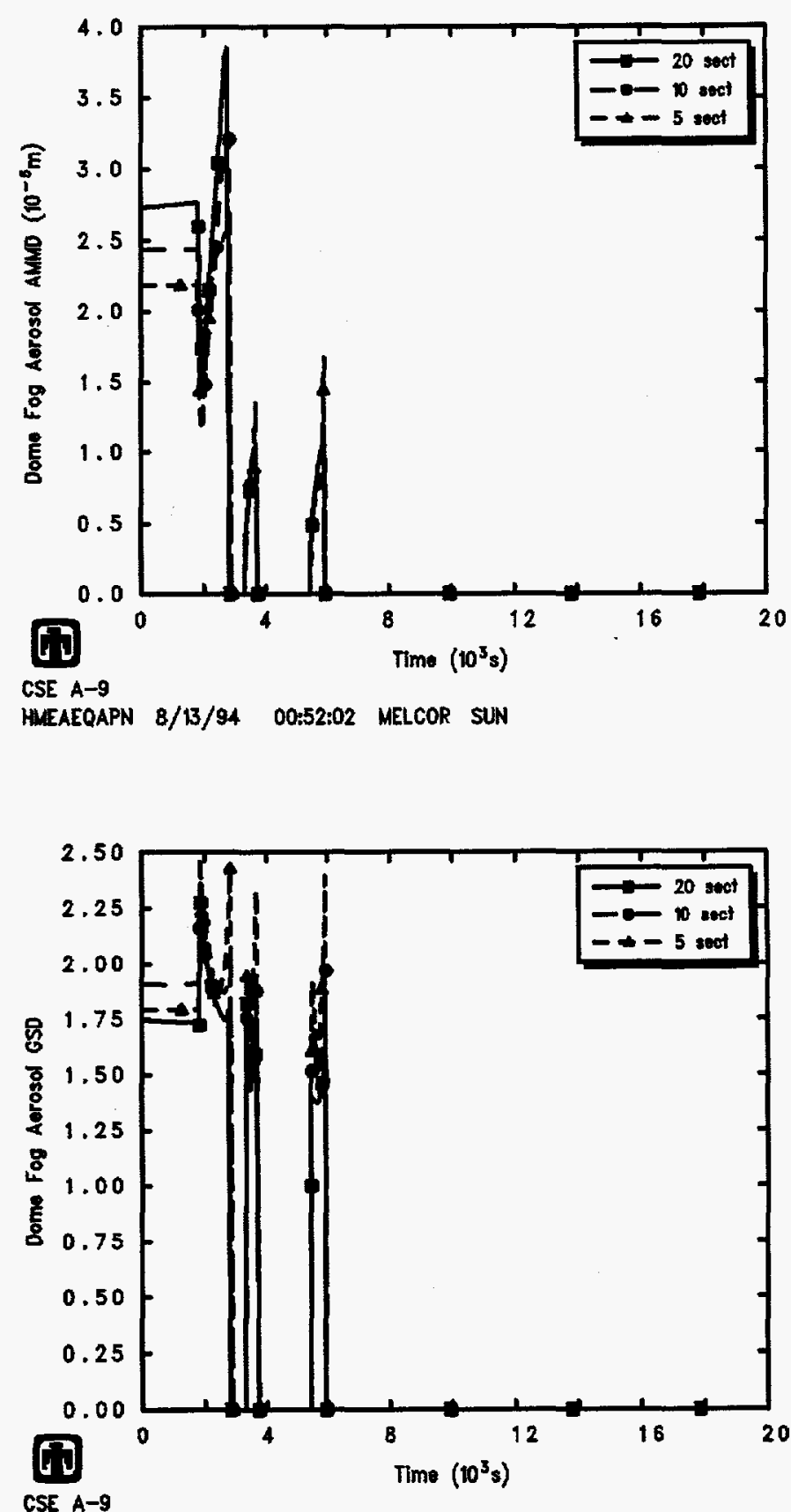

CSE $A-9$

HMEAEQAPN B/13/94 00:52:02 MELCOR SUN

Figure 7.2.5. Fog Water Aerosol Airborne Concentration AMMDs (top) and GSDs (bottom) in Dome for CSE Test A-9 - MAEROS Sections Sensitivity Study 


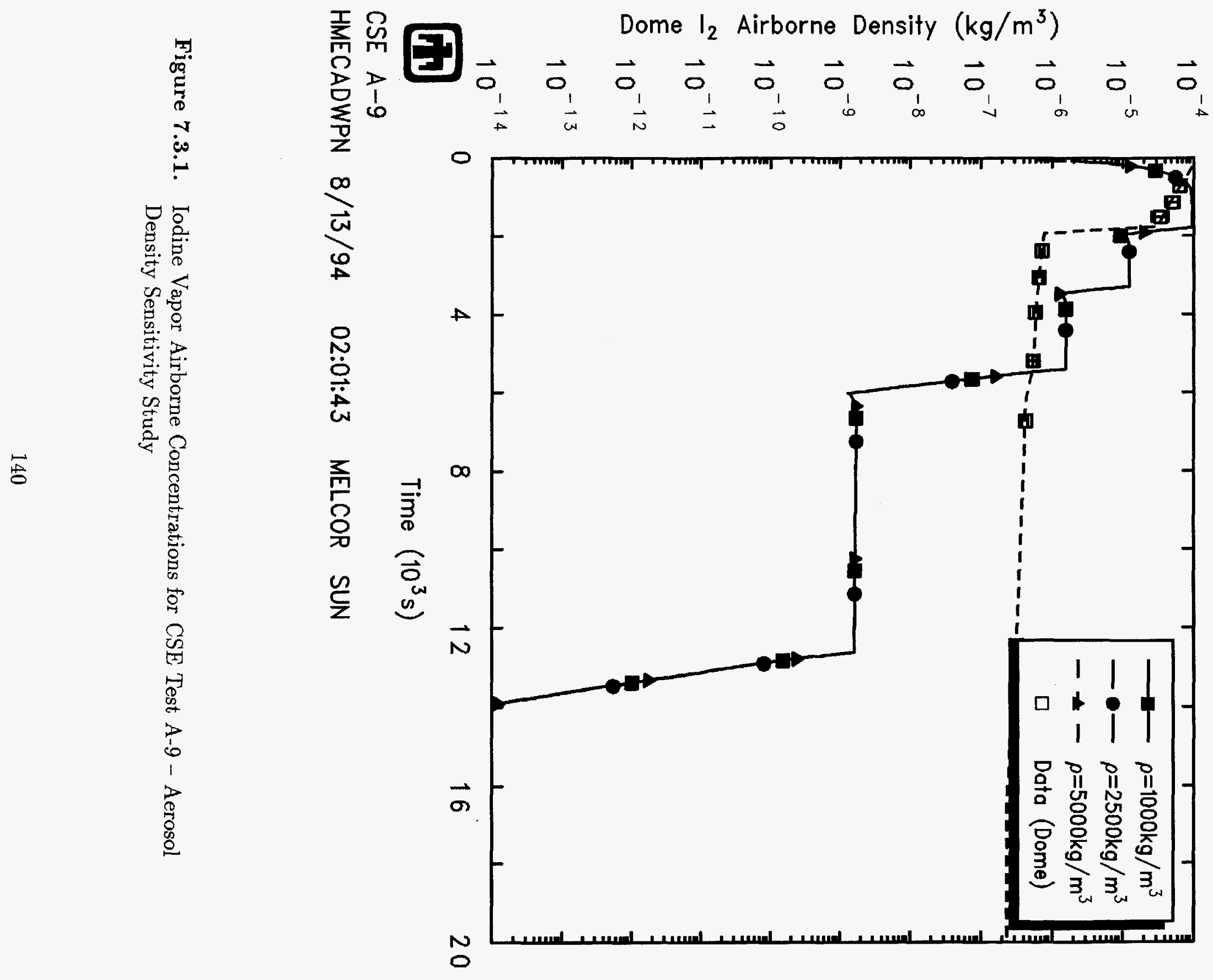


Figures 7.3.2 and 7.3.3 present the concentrations of cesium and uranium aerosols, respectively, in the test vessel dome atmosphere predicted using different aerosol density values, with the test data included for reference. The comparisons are very similar for the upper dome, the lower drywell, the middle room and the lower room or sump. The corresponding washout constants are listed in Table 7.3.1, again including the test data for reference.

On physical grounds, we would expect higher airborne aerosol concentrations for lower aerosol particle densities, and lower airborne aerosol concentrations for larger aerosol particle densities. The calculated results generally are not consistent. Reducing the aerosol density from $2500 \mathrm{~kg} / \mathrm{m}^{3}$ (as specified in the reference calculation) to $1000 \mathrm{~kg} / \mathrm{m}^{3}$ (the default) does result in more aerosol suspension, as expected. However, increasing the aerosol density from $2500 \mathrm{~kg} / \mathrm{m}^{3}$ to $5000 \mathrm{~kg} / \mathrm{m}^{3}$ also results in more aerosol suspension during some periods.

Figure 7.3.4 gives the mass median diameters and geometric standard deviations of the airborne cesium and uranium aerosol particle distributions in the dome atmosphere calculated using different aerosol densities. Figure 7.3.5 presents the same results for the fog (i.e., water aerosol) particles in the dome atmosphere.

On physical grounds, we would expect higher aerosol particle densities to correspond to smaller particles, and lower aerosol particle densities to correspond to larger particles. Again, the calculated results generally are not entirely consistent. In particular, something odd happens in the cesium and uranium particle distributions at the start of the recirculating spray period beginning at $12600 \mathrm{~s}$ in the reference calculation. The aerosol particles' AMMDs fall from between the corresponding results for higher and lower densities to the same value as in the calculation with a greater aerosol density. (This does not correspond to being swept into the smallest section; it corresponds instead to the fourth section out of a total of 10.)

This odd behavior may be related to the inconsistencies seen in some of the results of the sensitivity study on the number of MAEROS sections used and again suggests that some code problems affecting the accuracy and convergence of the results remain and need identification and resolution in the future.

\subsection{Aerosol Particle Initial Size}

There is not much information available in the test reports on the aerosol particle size distribution; based upon data in [19], the cesium aerosol was sourced in as a log-normal particle distribution with an average mass median diameter of $0.5 \mu \mathrm{m}$ and a geometric standard deviation of 1.5 , while the uranium aerosol was sourced in as somewhat larger particles using a log-normal distribution with an AMMD of $1.0 \mu \mathrm{m}$ and GSD of 1.5. Because the data on particle size distribution are considered to have a large uncertainty, a sensitivity study was done varying the initial particle size distributions assumed, from $0.5 \mu \mathrm{m}$ to $1 \mu \mathrm{m}, 2.5 \mu \mathrm{m}$ and $5 \mu \mathrm{m}$, up to $10 \mu \mathrm{m}$, a value more consistent with the particle 


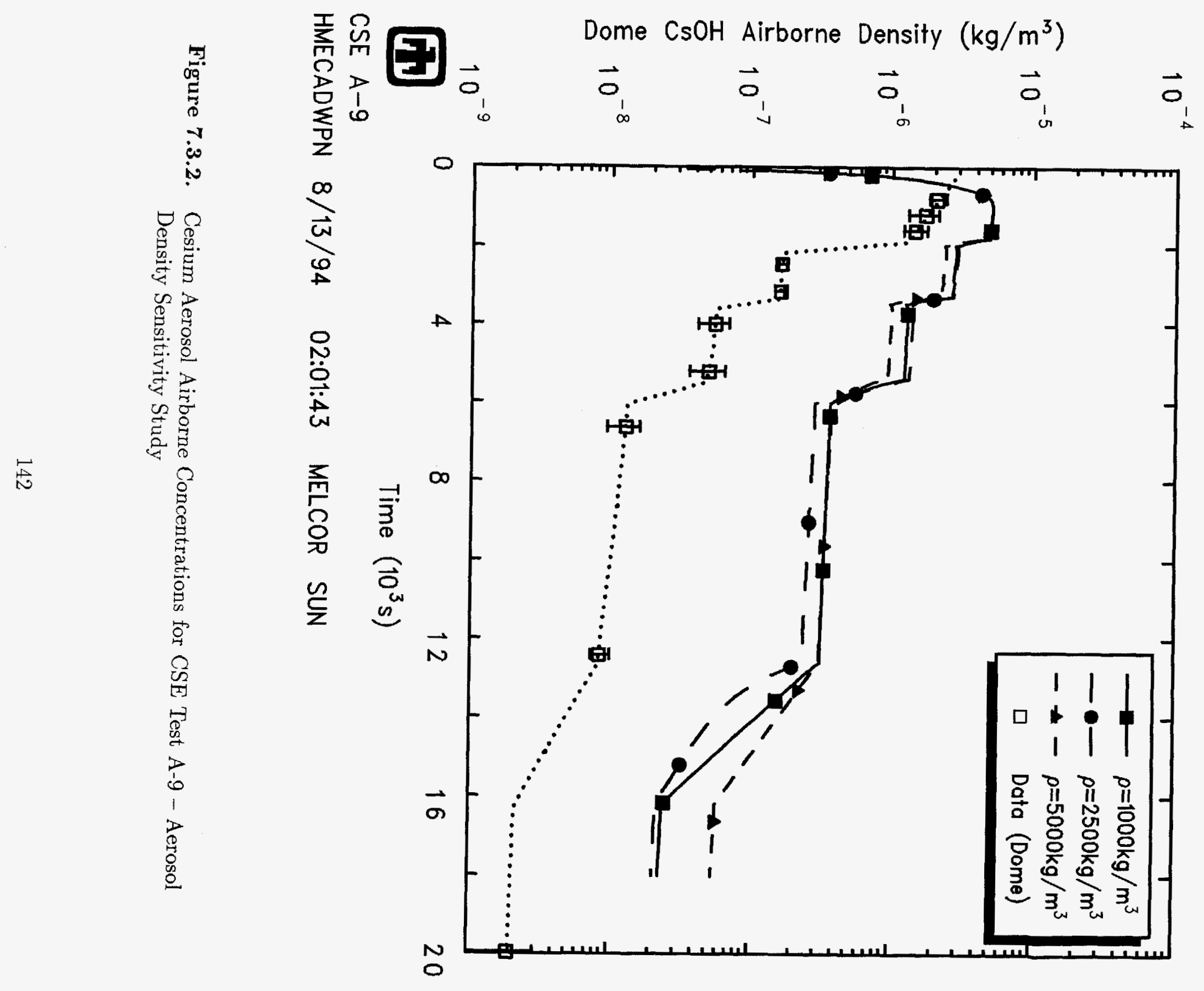




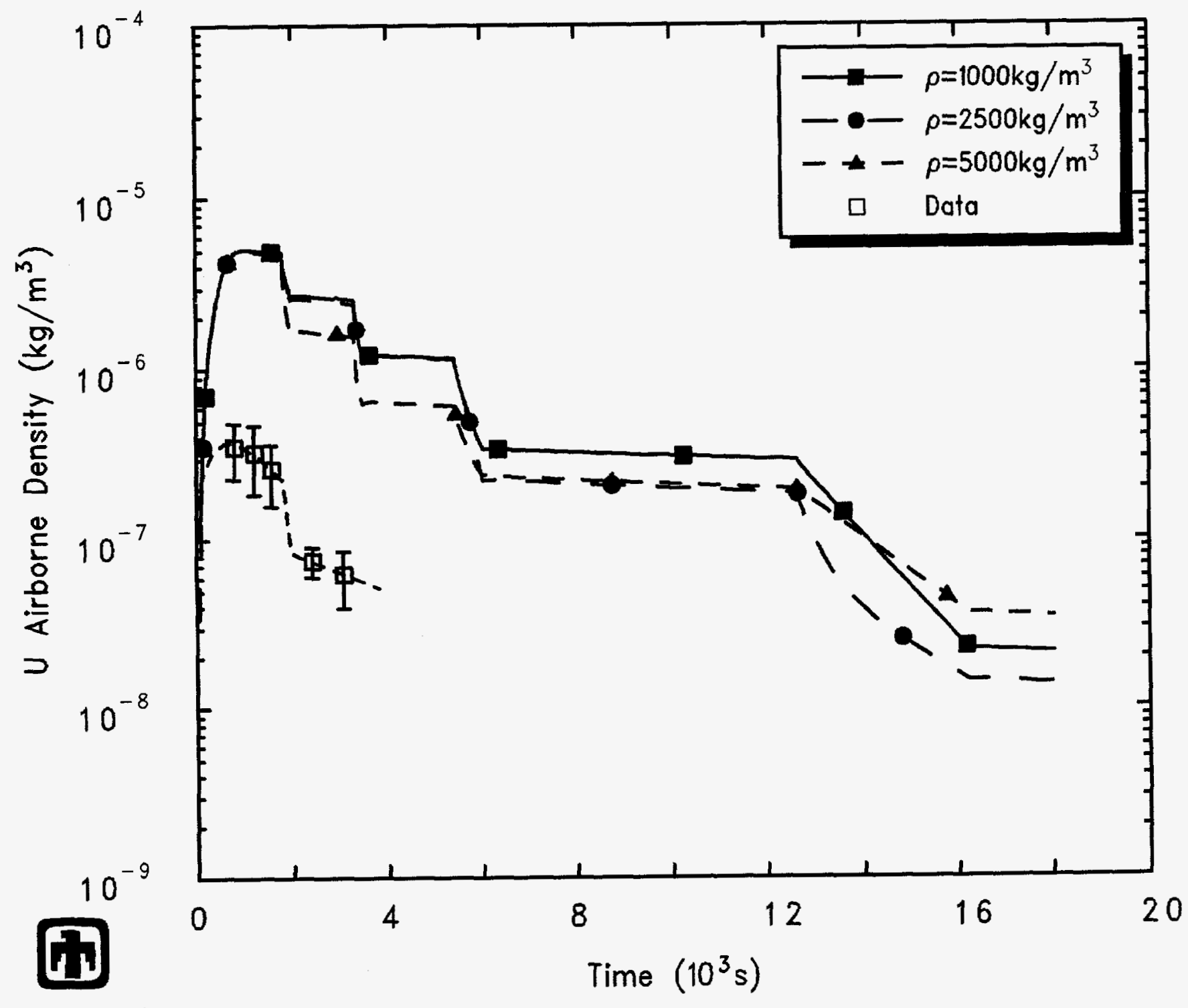

CSE A-9

HMECADWPN $8 / 13 / 94$ 02:01:43 MELCOR SUN

Figure 7.3.3. Uranium Aerosol Airborne Concentrations for CSE Test A-9-- Aerosol Density Sensitivity Study 
Table 7.3.1. Washout Rates for CSE Test A-9 - Aerosol Density Sensitivity Study

\begin{tabular}{lcccc} 
& \multicolumn{5}{c}{$t_{1 / 2}(\mathrm{~min})$} \\
& Measured & \multicolumn{3}{c}{$\rho\left(\mathrm{kg} / \mathrm{m}^{3}\right)$} \\
& & $1000 \quad 2500^{a}$ & 5000 \\
\hline Cesium & & & & \\
First spray & 1.08 & 4.7 & 5.0 & 3.5 \\
Second spray & 2.0 & 3.9 & 4.6 & 3.6 \\
Third spray & 5.4 & 5.2 & 4.3 & 6.6 \\
Fourth spray & 33 & $20^{b}$ & $35^{b}$ & $29^{b}$ \\
& & & & \\
Uranium & & & & \\
First spray & 2.3 & 4.4 & 4.5 & 2.3 \\
Second spray & & 3.8 & 4.6 & 3.2 \\
Third spray & & 5.1 & 4.4 & 6.4 \\
Fourth spray & & $20^{b}$ & $35^{b}$ & $29^{b}$ \\
& & & & \\
Iodine & & & & \\
First spray & 0.58 & 1.1 & 1.1 & 1.1 \\
Second spray & 42 & 1.4 & 1.4 & 1.4 \\
Third spray & 34 & 1.0 & 1.0 & 1.0 \\
Fourth spray & 180 & 1.4 & 1.4 & 1.4 \\
& & & & \\
& ${ }^{a}$ Reference calculation value \\
& ${ }^{b}$ Value at end of recirculating spray
\end{tabular}




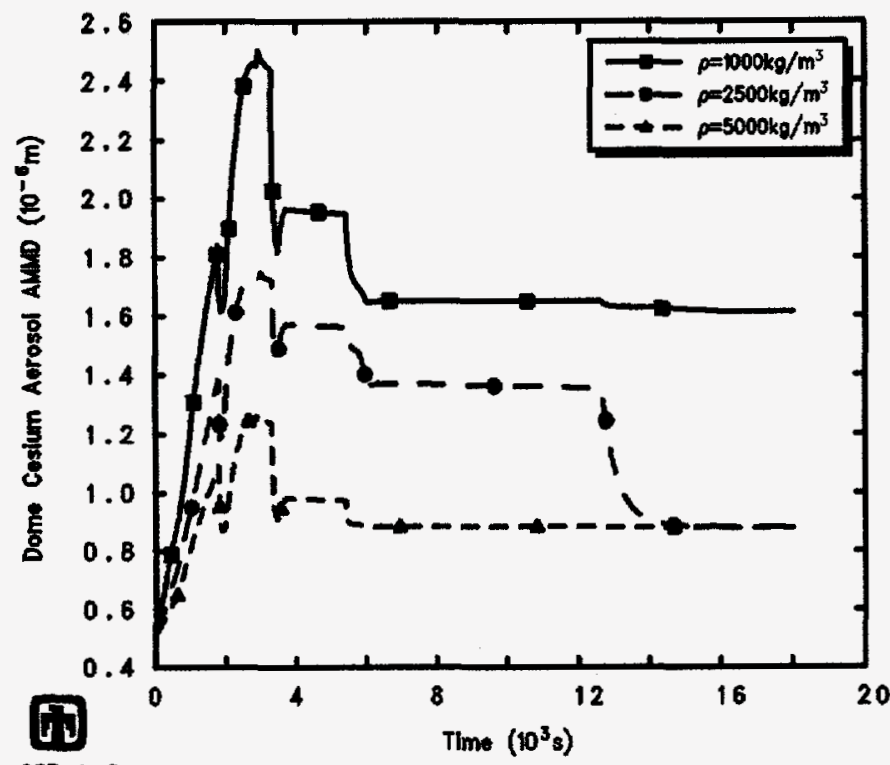

CSE A-9

HUECADWPN 8/13/94 02:01:43 MELCOR SUN

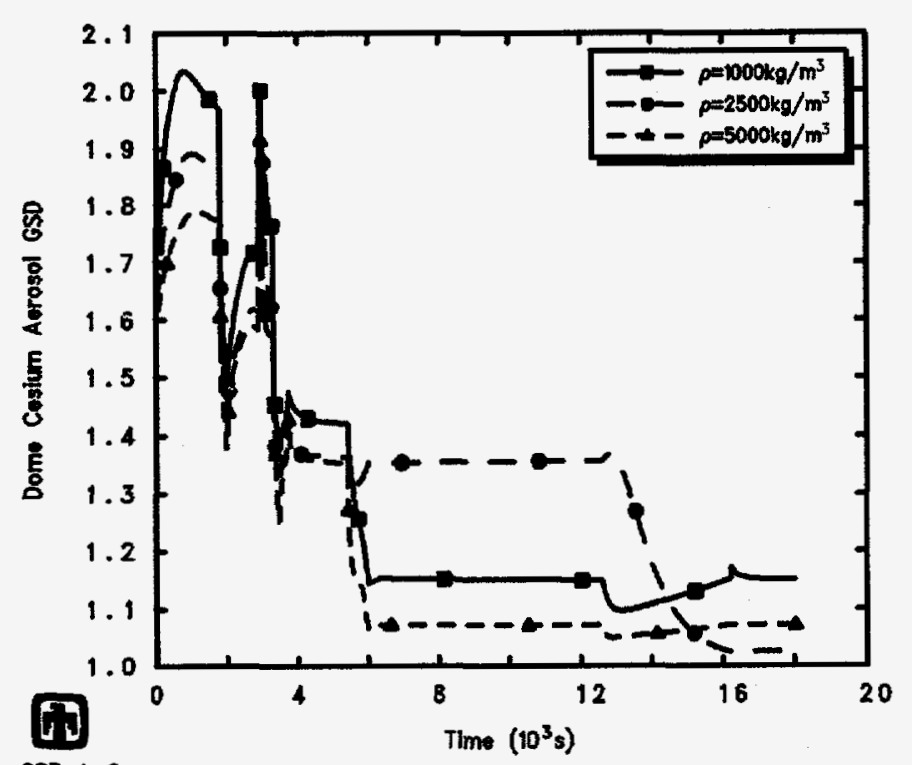

CSE A-9

HMECADWPN 8/13/94 02:0t:43 MELCOR SUN
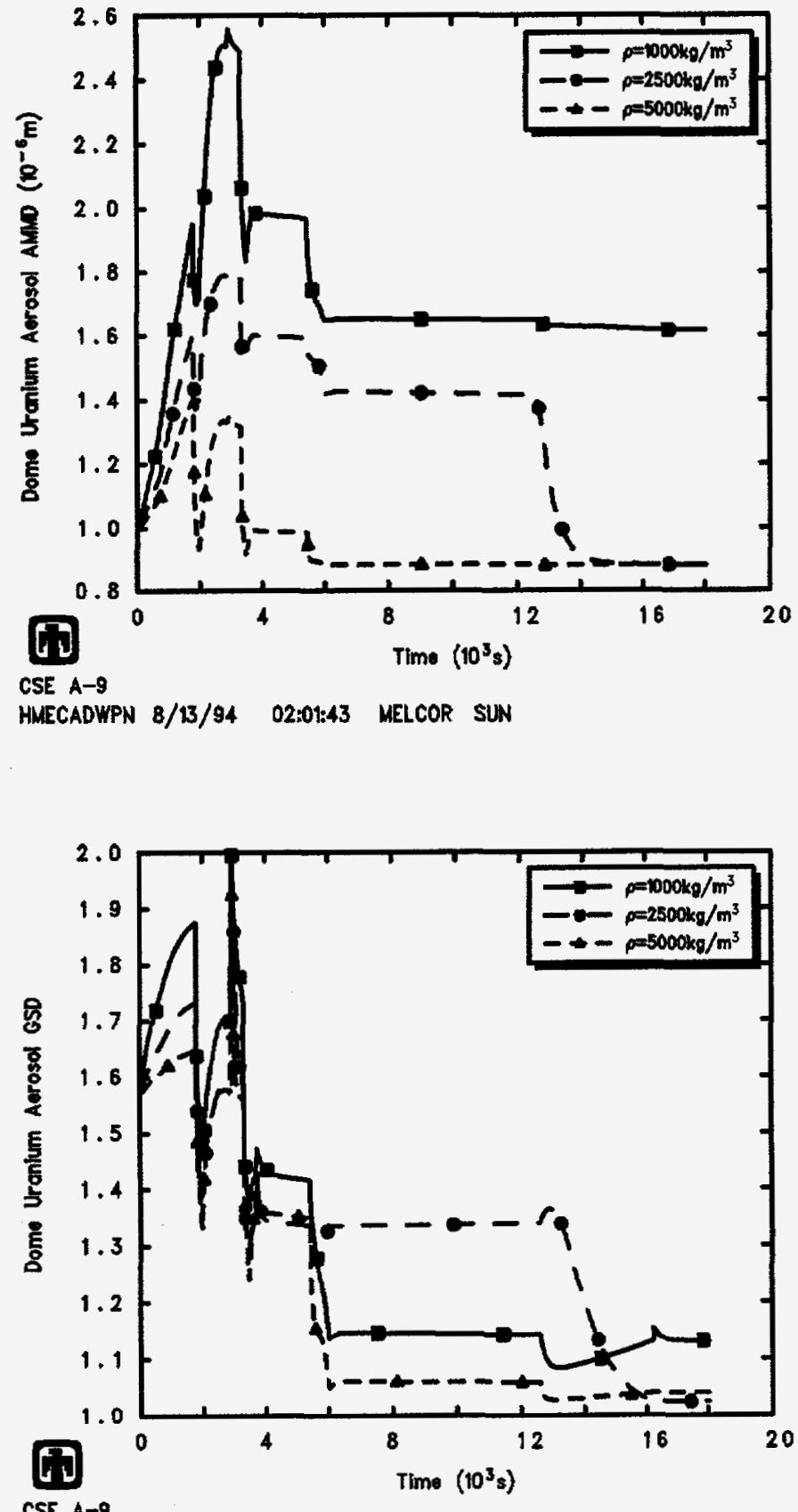

CSE $A-9$

HMECADWPN $8 / 13 / 94$ 02:01:43 MELCOR SUN

Figure 7.3.4. Cesium (left) and Uranium (right) Aerosol Airborne Concentration AMMDs (top) and GSDs (bottom) in Dome for CSE Test A-9 Aerosol Density Sensitivity Study 

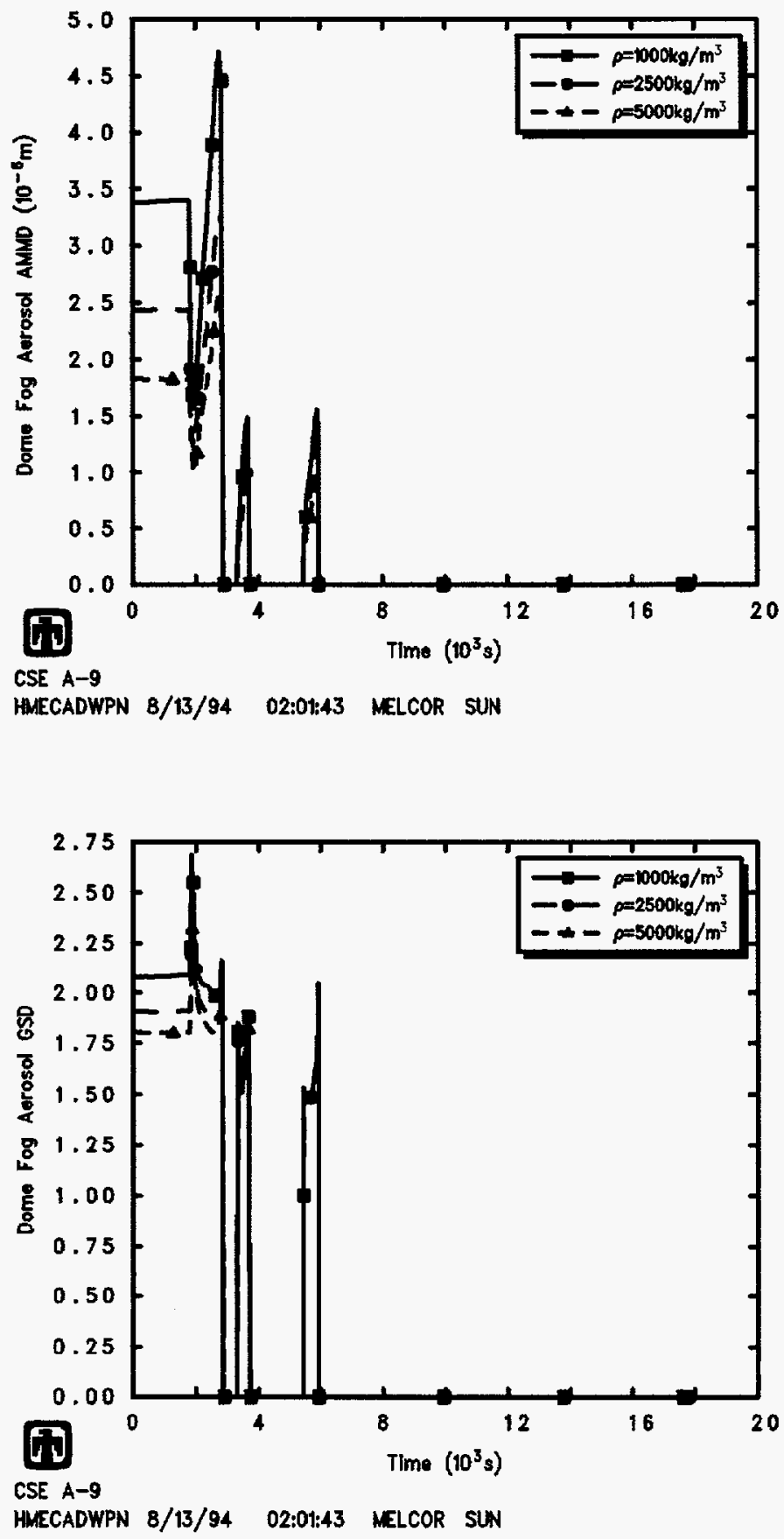

Figure 7.3.5. Fog Water Aerosol Airborne Concentration AMMDs (top) and GSDs (bottom) in Dome for CSE Test A-9 - Aerosol Density Sensitivity Study 
sizes measured during earlier tests in the CSE series on natural removal of suspended cesium and uranium aerosol particles [22]. (The GSD was kept at 1.5 in all these cases.)

The thermal/hydraulic responses calculated when the aerosol particles were injected at larger sizes were virtually identical, as would be expected.

Figure 7.4.1 presents the concentrations of iodine vapor in the test vessel dome atmosphere predicted assuming different aerosol particle initial sizes; the test data are also included for reference. The comparisons are very similar for the upper dome, the lower drywell, the middle room and the lower room or sump. The iodine vapor responses calculated when different initial sizes for the aerosol particles were assumed are virtually identical, also as would be expected.

Figures 7.4.2 and 7.4.3 present the predicted concentrations of cesium and uranium aerosol, respectively, in the test vessel dome atmosphere for different aerosol initial particle sizes, with the test data included for reference. The comparisons are very similar for the upper dome, the lower drywell, the middle room and the lower room or sump. The corresponding washout constants are listed in Table 7.4.1, with the test data included for reference.

Larger aerosol particles should be removed from the containment atmosphere more quickly both by the sprays and by settling out between spray periods. The aerosol removal test data are matched reasonably well by particles with an initial AMMD in the $2.5-5.0 \mu \mathrm{m}$ range. Also, assuming that larger particles are initially present in the simulation better reproduces the experimental observation of progressively slower aerosol washout during the later spray periods.

Figure 7.4.4 gives the mass median diameters and geometric standard deviations of the airborne cesium and uranium aerosol particle distributions in the dome atmosphere calculated using different initial aerosol particle sizes. Figure 7.4.5 presents the same results for the fog (i.e., water aerosol) particles in the dome atmosphere.

As expected, varying the initial size of the cesium and uranium particles has no effect on the water aerosol sizes, since the fog is represented by a separate MAEROS component and since condensation/evaporation is specified to take place only on water aerosols and not on all aerosols present. Cesium and uranium aerosol particles assumed to be injected with larger AMMDs are predicted to keep generally larger AMMDs during the first portion of the transient, but the differences decrease until by the end of the third and last fresh spray injection the cesium and uranium aerosol particle distribution AMMDs are very similar $(\geq 1.5 \mu \mathrm{m})$ regardless of what their initial AMMDs were. The GSDs for the cesium and uranium aerosols during this time remain greater in the cases with smaller aerosol particles injected than in the cases with larger aerosol particles injected.

\subsection{Condensation/Evaporation on Aerosols}

The results of the sensitivity study on aerosol particle initial size presented in the last section showed that the test data on aerosol removal are matched much better by 


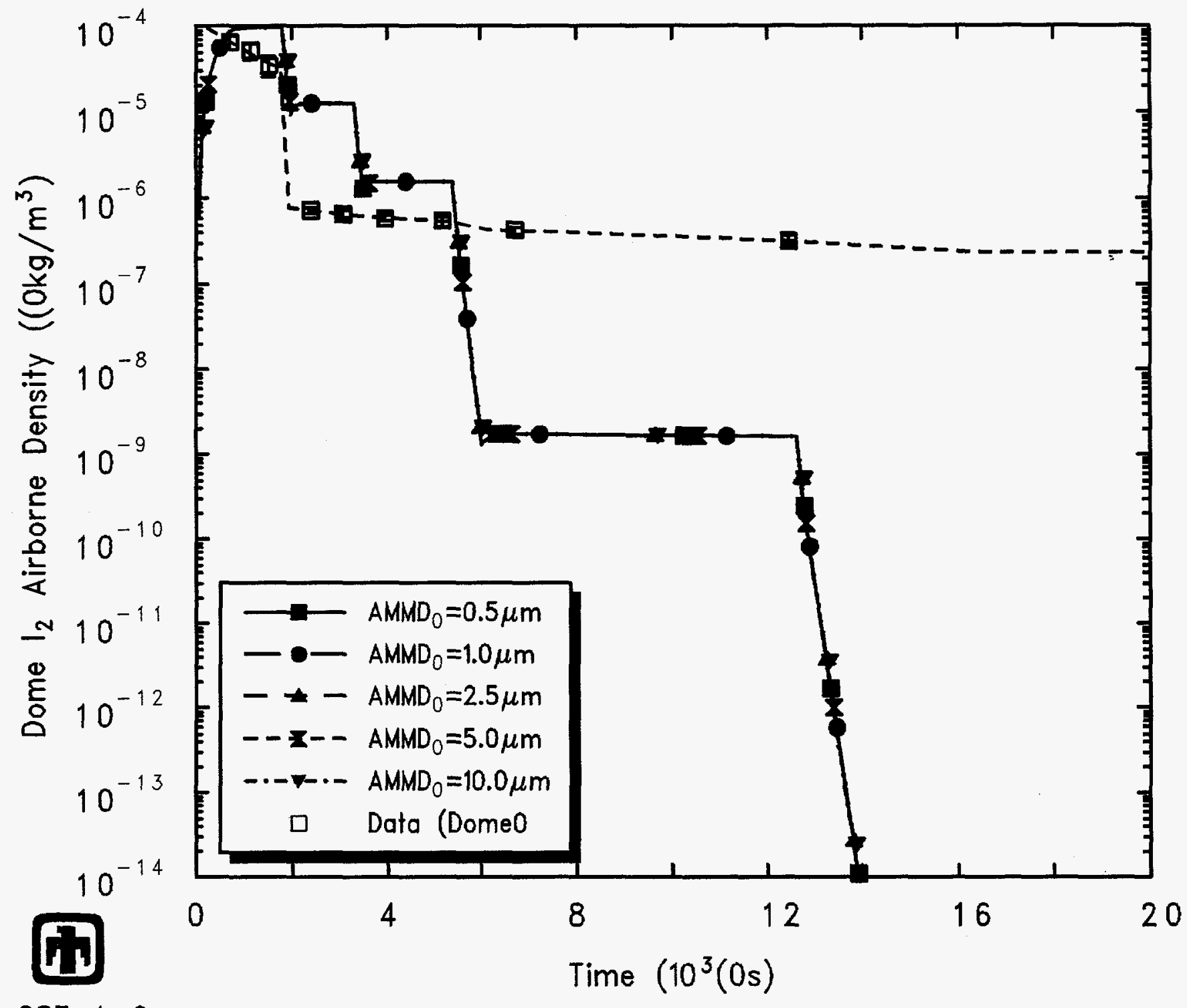

CSE A-9

HLEKCVKPN $8 / 12 / 94 \quad 10: 31: 51 \quad$ MELCOR SUN

Figure 7.4.1. Iodine Vapor Airborne Concentrations for CSE Test A-9 - Aerosol Particle Initial Size Sensitivity Study 


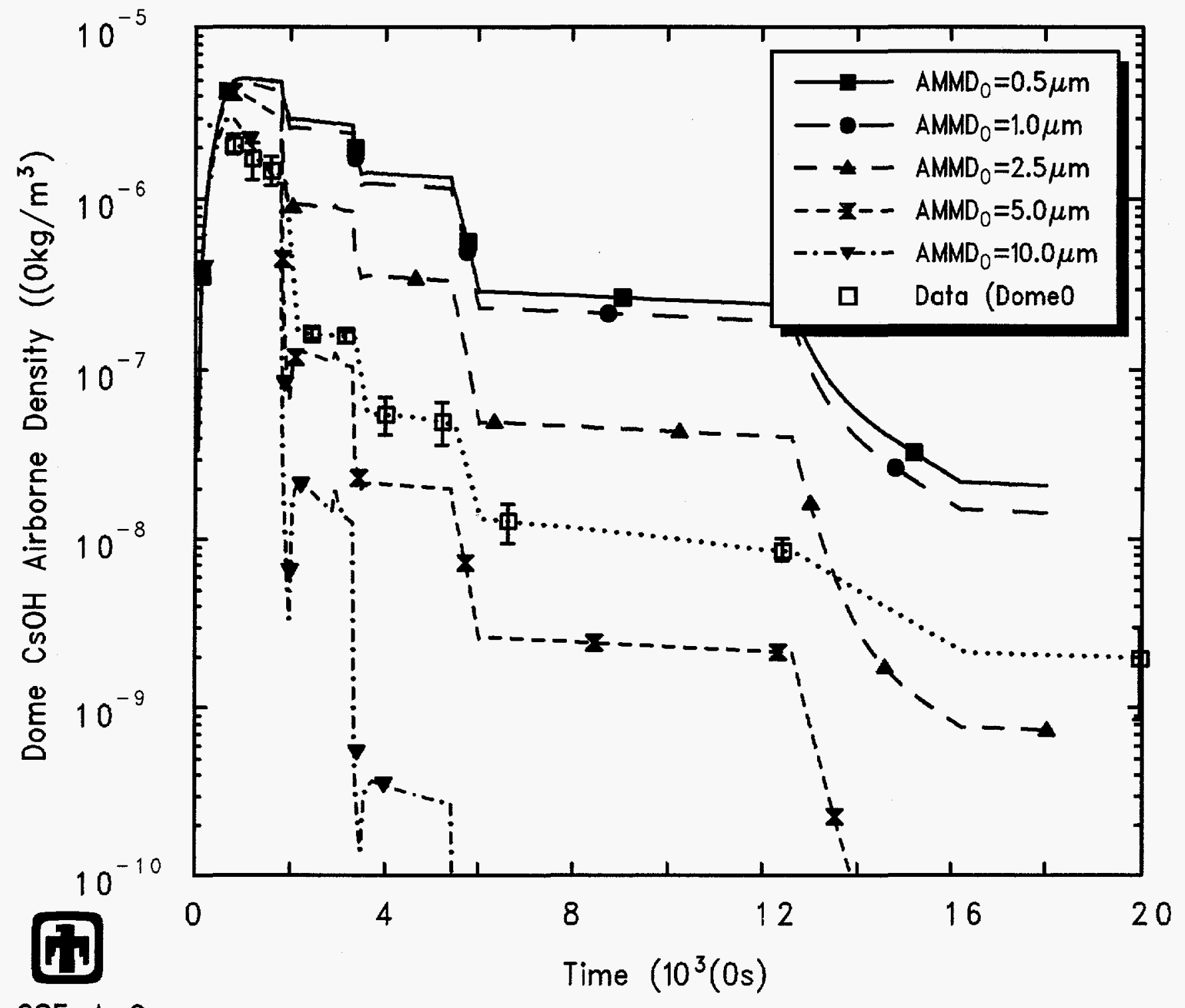

CSE A-9 HLEKCVKPN $8 / 12 / 94 \quad 10: 31: 51 \quad$ MELCOR SUN

Figure 7.4.2. Cesium Aerosol Airborne Concentrations for CSE Test A-9 - Aerosol Particle Initial Size Sensitivity Study 


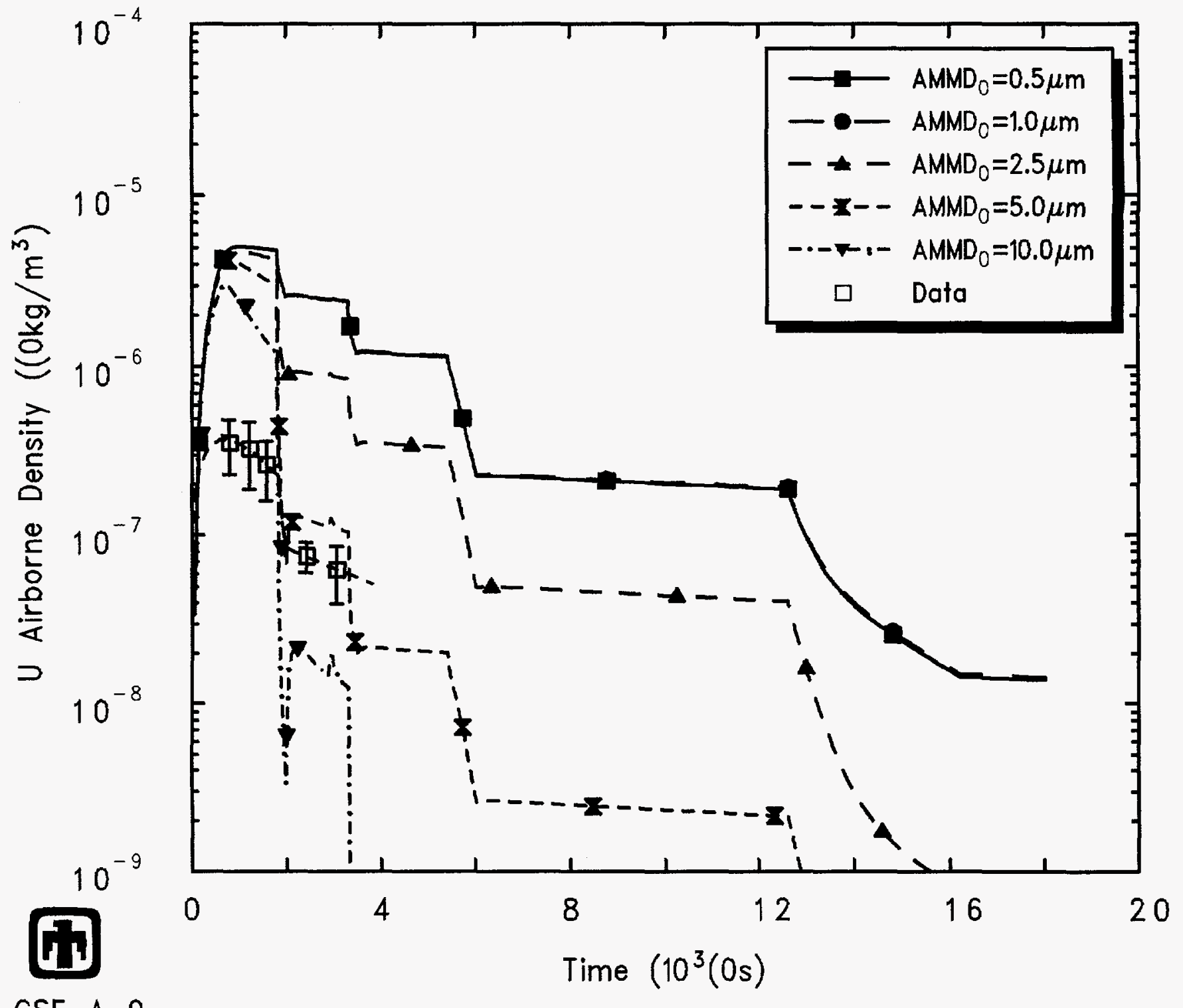

CSE A-9

HLEKCVKPN $8 / 12 / 94 \quad 10: 31: 51 \quad$ MELCOR SUN

Figure 7.4.3. Uranium Aerosol Airborne Concentrations for CSE Test A-9 - Aerosol Particle Initial Size Sensitivity Study 
Table 7.4.1. Washout Rates for CSE Test A-9 - Aerosol Particle Initial Size Sensitivity Study

\begin{tabular}{|c|c|c|c|c|c|c|}
\hline & \multicolumn{6}{|c|}{$t_{1 / 2}(\min )$} \\
\hline & \multirow[t]{2}{*}{ Measured } & \multicolumn{4}{|c|}{$\mathrm{AMMD}_{0}(\mu \mathrm{m})=$} & \multirow[b]{2}{*}{10.0} \\
\hline & & $0.5^{a}$ & 1.0 & 2.5 & 5.0 & \\
\hline \multicolumn{7}{|l|}{ Cesium } \\
\hline First spray & 1.08 & 5.0 & 4.1 & 1.5 & 0.63 & 0.46 \\
\hline Second spray & 2.0 & 4.6 & 4.5 & 3.5 & 2.2 & 0.99 \\
\hline Third spray & 5.4 & 4.3 & 4.3 & 3.6 & 3.5 & 2.3 \\
\hline Fourth spray & 33 & $35^{b}$ & $35^{b}$ & $35^{b}$ & $26^{b}$ & $11^{b}$ \\
\hline \multicolumn{7}{|l|}{ Uranium } \\
\hline First spray & 2.3 & 4.6 & 4.1 & 1.5 & 0.63 & 0.44 \\
\hline Second spray & & 4.3 & 4.5 & 3.5 & 2.1 & 0.98 \\
\hline Third spray & & 4.3 & 4.3 & 3.6 & 3.5 & 1.8 \\
\hline Fourth spray & & $35^{b}$ & $35^{b}$ & $35^{b}$ & $26^{b}$ & $11^{b}$ \\
\hline \multicolumn{7}{|l|}{ Iodine } \\
\hline First spray & 0.58 & 1.1 & 1.1 & 1.1 & 1.1 & 1.1 \\
\hline Second spray & 42 & 1.4 & 1.4 & 1.4 & 1.4 & 1.4 \\
\hline Third spray & 34 & 1.0 & 1.0 & 1.0 & 1.0 & 1.0 \\
\hline Fourth spray & 180 & 1.4 & 1.4 & 1.4 & 1.4 & 1.4 \\
\hline
\end{tabular}




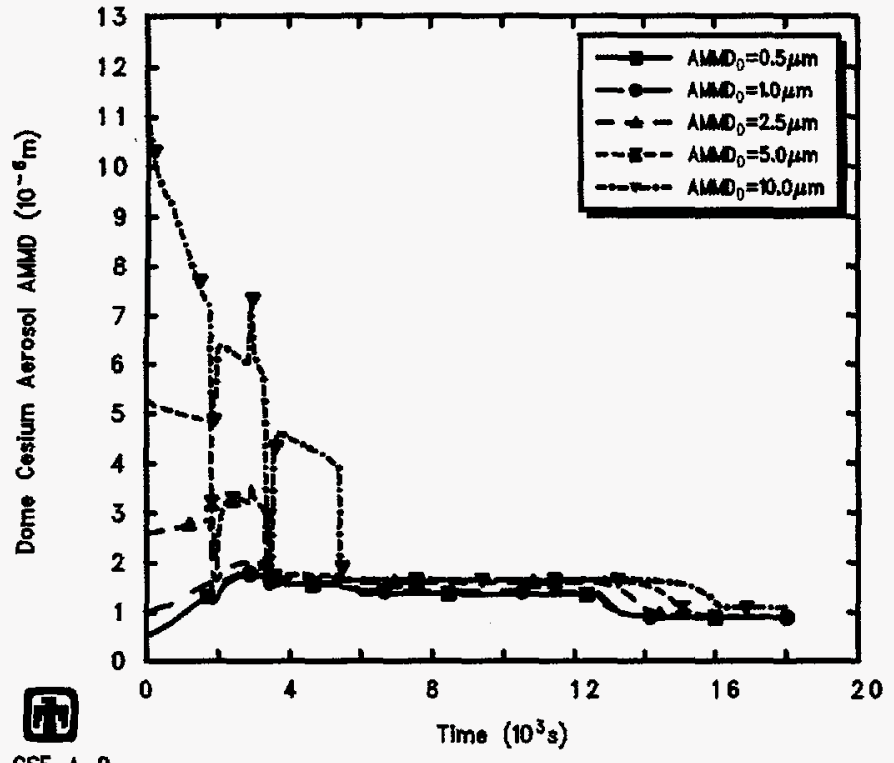

CSE $A-9$

HLEKCVKPN $8 / 12 / 94$ 10:31:51 MELCOR SUN

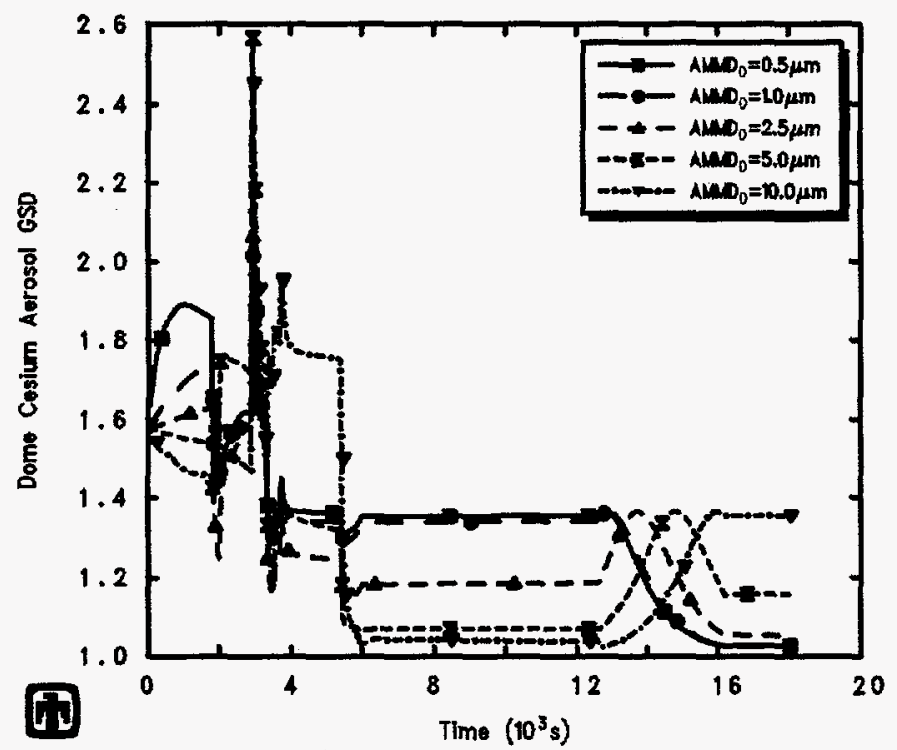

CSE A-9

HLEKCVKPN $8 / 12 / 94 \quad 10: 31: 51$ MELCOR SUN
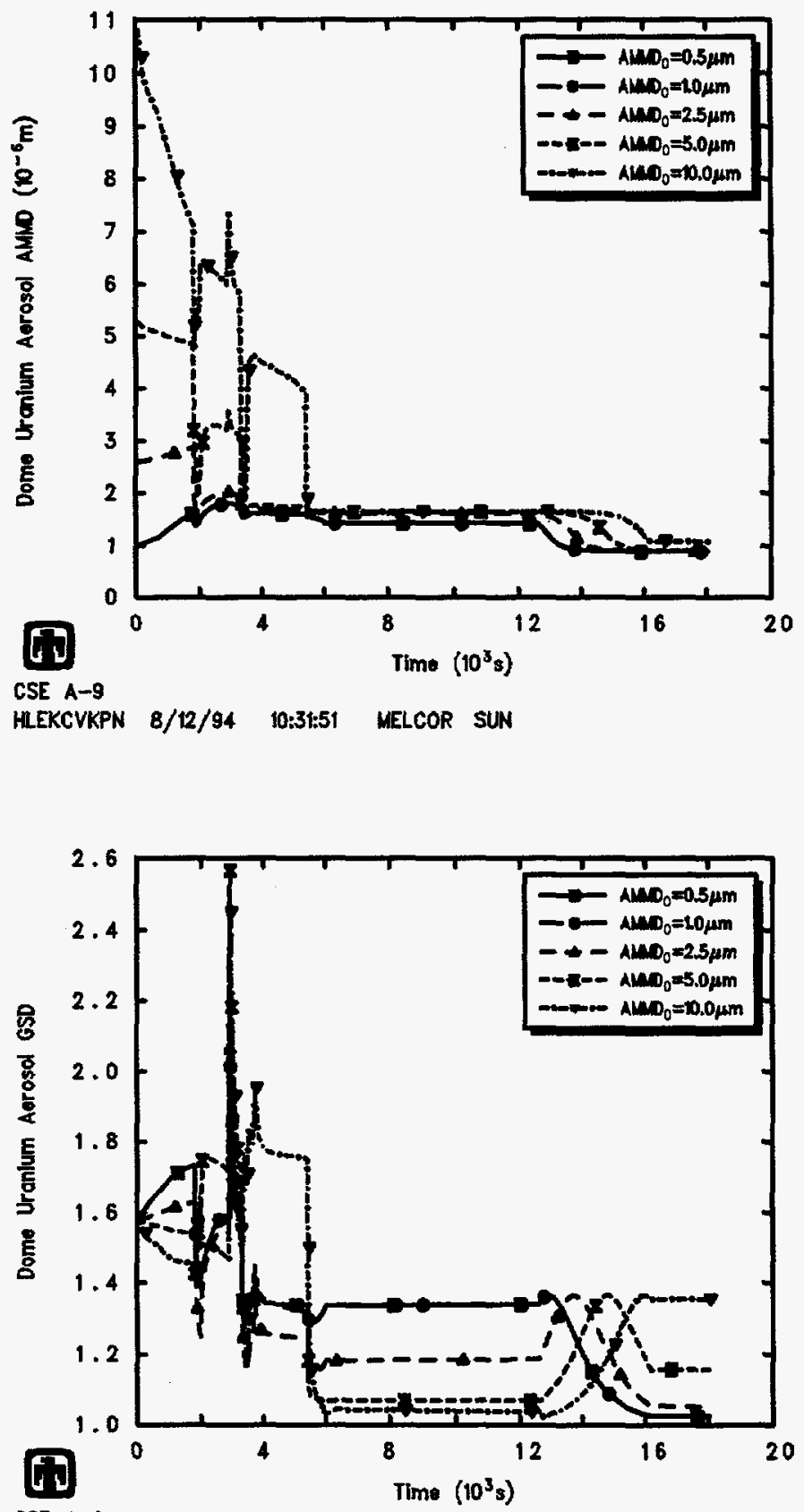

CSE A-9

HLEKCVKPN 8/12/94 0:31:51 MELCOR SUN

Figure 7.4.4. Cesium (left) and Uranium (right) Aerosol Airborne Concentration AMMDs (top) and GSDs (bottom) in Dome for CSE Test A-9 Aerosol Particle Initial Size Sensitivity Study 


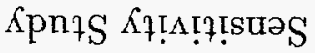

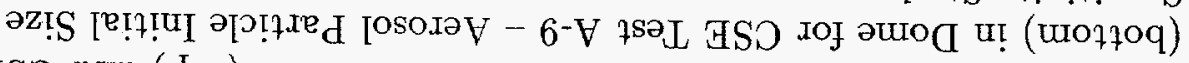

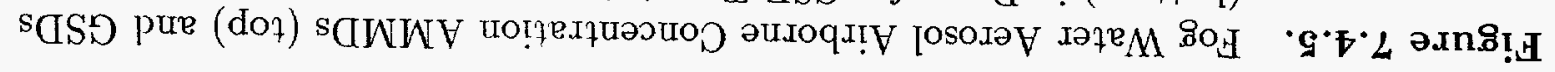
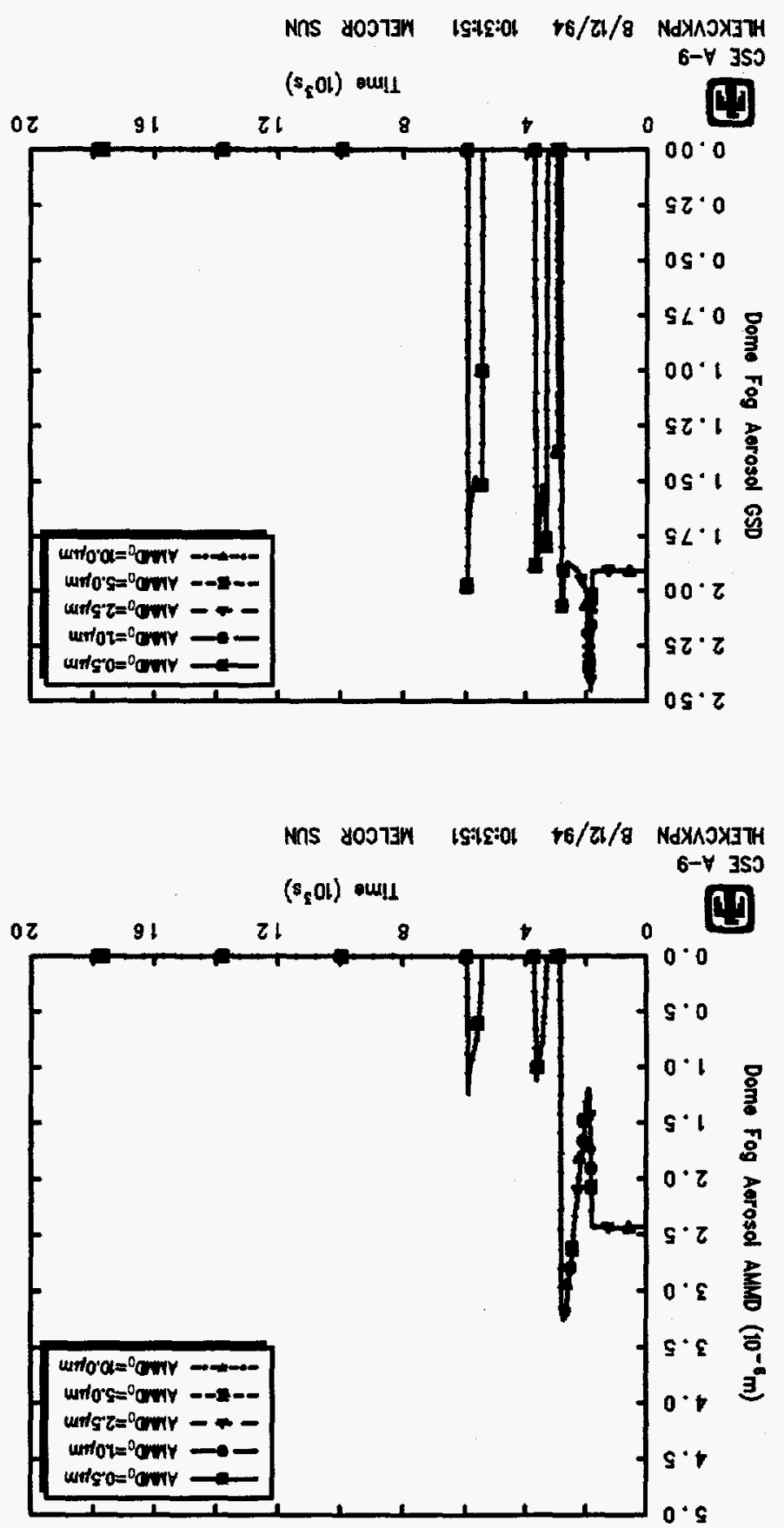
particles with an initial AMMD in the 2.5-5.0 $\mu \mathrm{m}$ range than by injecting the aerosol particles with an initial AMMD in the 0.5-1.0 $\mu \mathrm{m}$ range as specified in the experiment report [21].

In reality, the aerosol particles are probably larger at the start of the spray injection transient, not because large cesium and uranium particles were injected but because the hygroscopic cesium aerosols at least would be expected to very quickly grow to about $5.0 \mu \mathrm{m}$ by condensation of water onto the small particles initially injected; the effect is probably smaller for the uranium particles but still occurs to some extent. [26]

MELCOR models condensation/evaporation effects on aerosol particle size, but does not include any model for such hygroscopic effects. The lack of such a model has been noted in previous MELCOR assessments of aerosol behavior in humid atmospheres [3, 27].

However, in our reference calculation, condensation/evaporation is specified to take place only on water aerosols and not on all aerosols present (as in the code default), to avoid other modelling problems.

Several calculations were done as a sensitivity study on the effects of condensation and evaporation on aerosol response. One calculation simply repeated the reference calculation done for test CSE A-9 using the default condensation/evaporation treatment in MELCOR. Two other calculations were done with condensation/evaporation specified to take place either only on water aerosols or on all aerosols present, but with no calculation of a preconditioning phase to allow growth of pre-existing fog particles.

Figures 7.5.1 and 7.5.2 show the pressures and temperatures predicted in the test vessel dome for test A-9 when the condensation/evaporation modelling and the initial water aerosol particle size were varied. (In the following figures, the reference calculation described in Section 4 with condensation on water aerosols only and with larger fog particles at the start of the transient due to a preconditioning phase in the calculation is labelled "cond on fog, init fog".)

The thermal/hydraulic responses calculated assuming different treatments of condensation/evaporation in the aerosol response modelling were virtually identical, as would be expected. There are some small differences visible in the thermal/hydraulic response calculated depending on whether a preconditioning phase was modelled or not, caused by slightly different vessel atmosphere conditions at the start of the transient, which is reasonable.

Figure 7.5.3 presents the concentrations of iodine vapor in the test vessel dome atmosphere predicted assuming different condensation/evaporation modelling and different initial fog particle sizes; the test data are included for reference. The comparisons are very similar for the upper dome, the lower drywell, the middle room and the lower room or sump. The iodine vapor responses calculated assuming different condensation/evaporation modelling and different initial fog particle sizes are virtually identical, as would be expected.

Figures 7.5.4 and 7.5.5 present the concentrations of cesium and uranium aerosols, respectively, in the test vessel dome atmosphere predicted assuming different condensation/evaporation modelling and different initial fog particle sizes; the test data are 


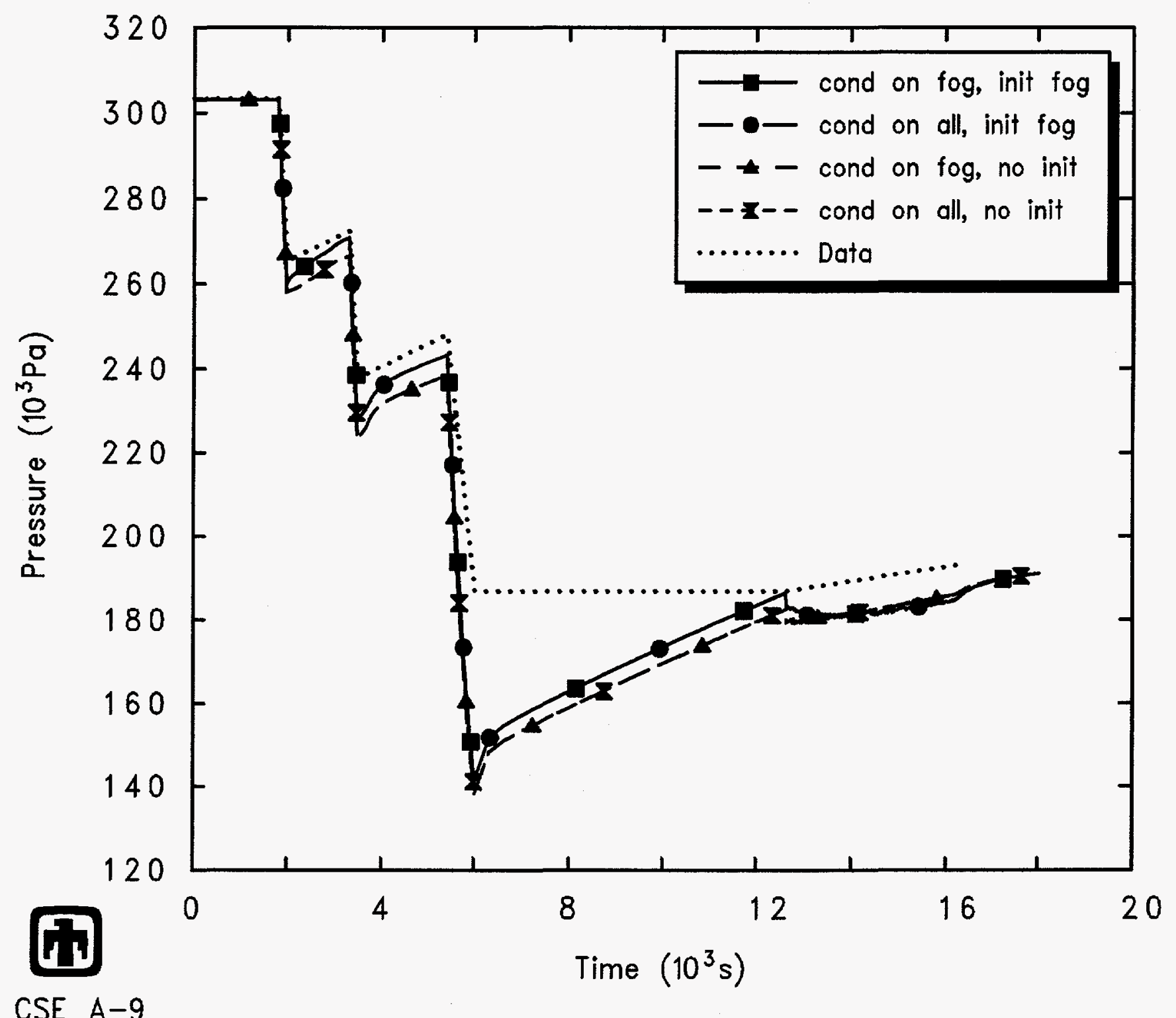

CSE $A-9$

HLEKCVKPN $8 / 12 / 94 \quad 10: 31: 51 \quad$ MELCOR SUN

Figure 7.5.1. Vessel Pressures for CSE Test A-9 - Aerosol Condensation/Evaporation Sensitivity Study 


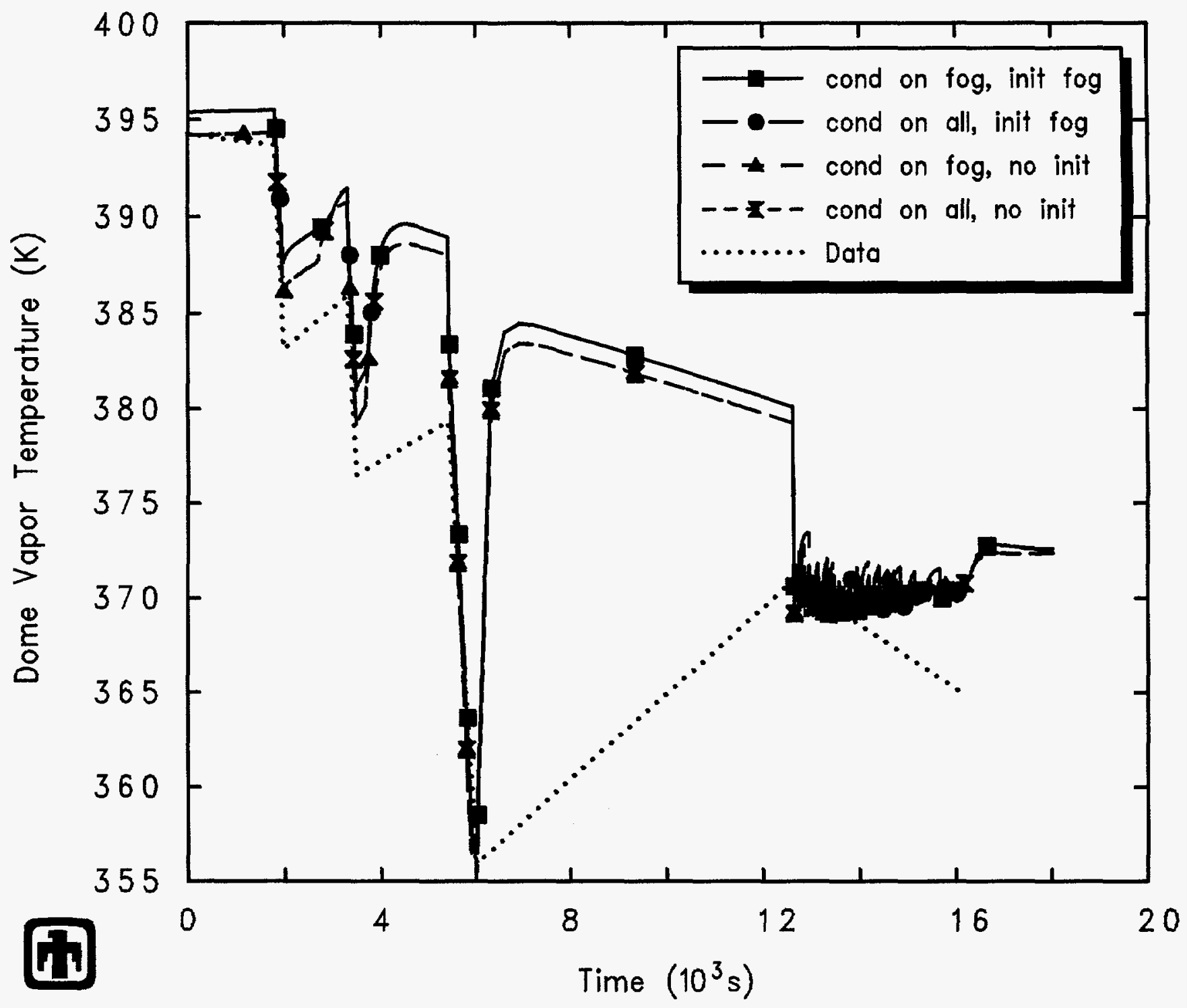

CSE $A-9$

HLEKCVKPN $8 / 12 / 94 \quad 10: 31: 51 \quad$ MELCOR SUN

Figure 7.5.2. Vessel Dome Temperatures for CSE Test A-9 - Aerosol Condensation/Evaporation Sensitivity Study 


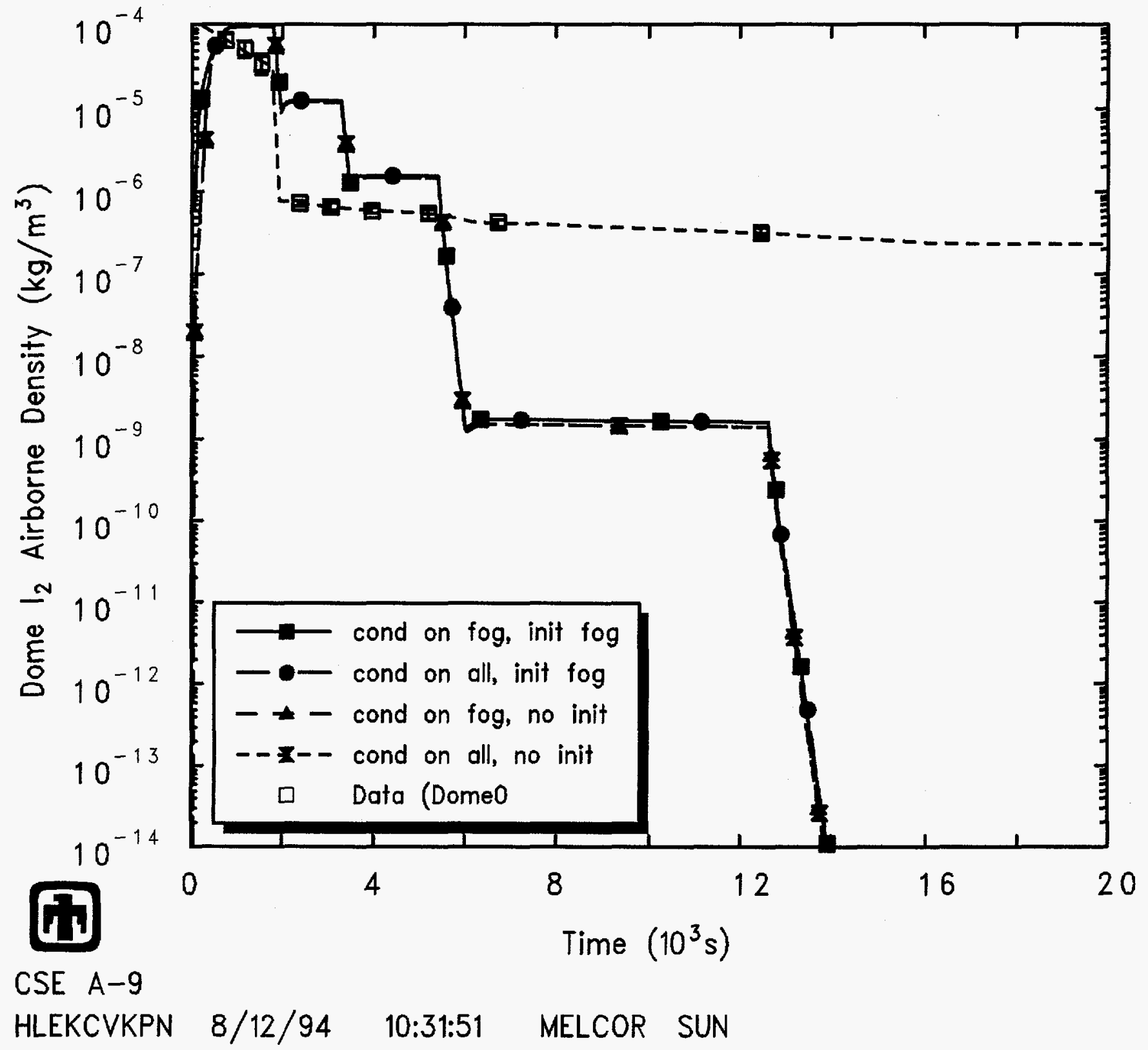

Figure 7.5.3. Iodine Vapor Airborne Concentrations for CSE Test A-9-Aerosol Condensation/Evaporation Sensitivity Study 
included for reference. The comparisons are very similar for the upper dome, the lower drywell, the middle room and the lower room or sump. The corresponding washout constants are listed in Table 7.5.1; again the test data are included for reference.

The reference calculation, with condensation/evaporation specified to take place only on water aerosols and with a preconditioning phase to allow growth of pre-existing fog particles, predicts the most aerosol removal by sprays overall. The calculation allowing condensation/evaporation to take place on any aerosols present does predict more aerosol removal during the first spray, which is in better agreement with test data. This would be expected because the cesium and uranium aerosol particles could grow somewhat before the start of the first spray owing to condensation in this case although, without hygroscopicity taken into account, the growth is not great enough to match the test data. However, allowing MELCOR to treat condensation onto the cesium and uranium aerosols results in greater discrepancies later in the transient, when the water aerosols evaporate.

Figure 7.5.6 gives the mass median diameters and geometric standard deviations of the airborne cesium and uranium aerosol particle distributions in the dome atmosphere calculated using different condensation/evaporation modelling and different initial fog particle sizes. Figure 7.5.7 presents the same results for the fog (i.e., water aerosol) particles in the dome atmosphere.

In the two calculations with condensation onto all aerosols present, the mass median diameters of the cesium and uranium aerosols drop precipitously when the water aerosols evaporate. When condensation occurs on all aerosols, MELCOR assumes that all the aerosol particles are identical. In this case, there is a relatively large amount of water and a relatively large number of water aerosol particles in the humid atmosphere early in the transient, relative to the cesium and uranium. Therefore, all aerosol particles would be assumed to have a thick water film over a small cesium/uranium core, rather than a smaller number of cesium and uranium particles having a thin water film and most of the aerosol particles being pure fog droplets. Thus, after the water film evaporates, a large number of small cesium and uranium aerosol particles have been artificially created from a smaller number of larger cesium and uranium particles. This is a known problem that has been identified in other MELCOR analyses (e.g., [28]). 


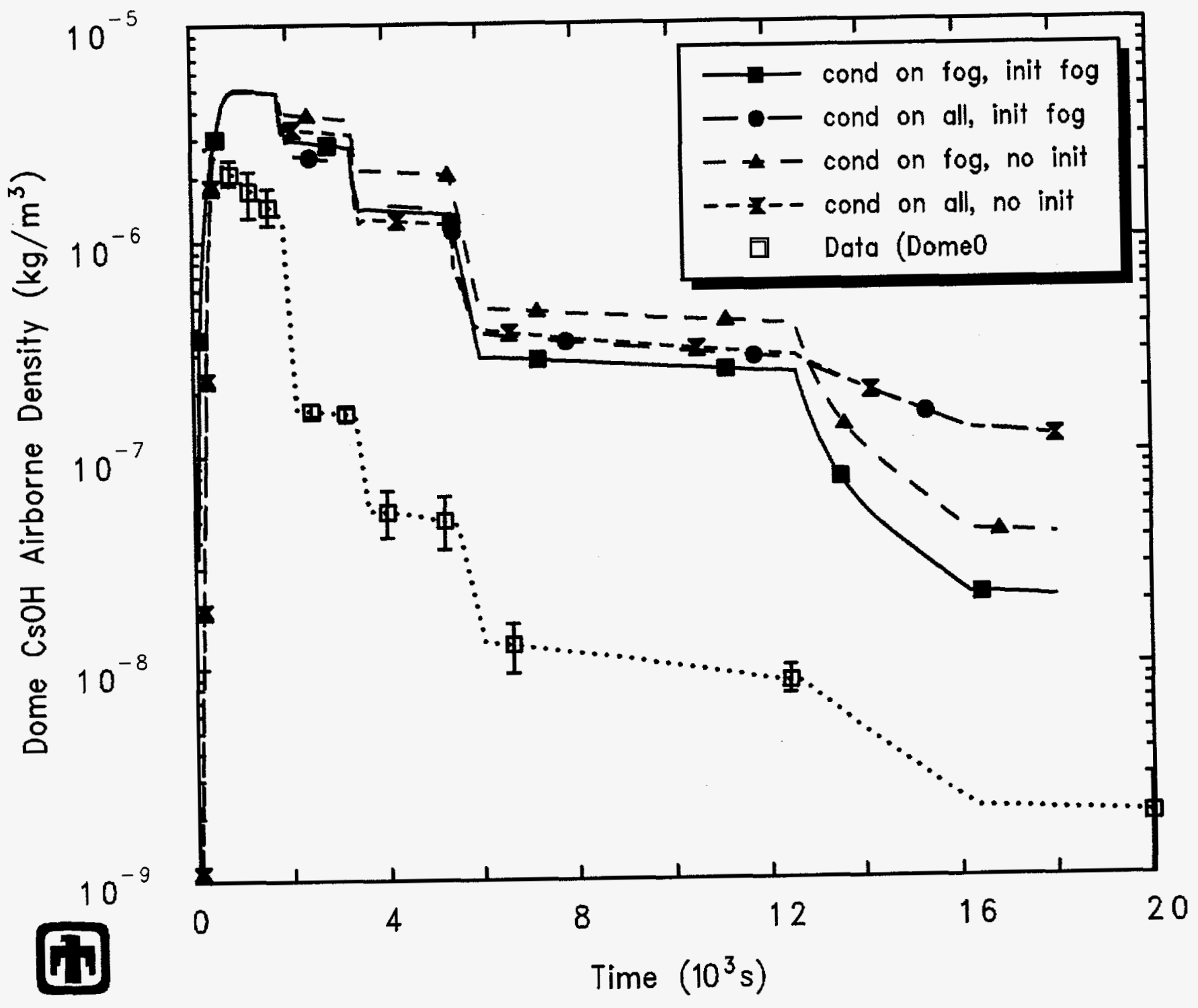

CSE A-9 HLEKCVKPN $\quad 8 / 12 / 94 \quad 10: 31: 51 \quad$ MELCOR SUN

Figure 7.5.4. Cesium Aerosol Airborne Concentrations for CSE Test A-9 - Aerosol Condensation/Evaporation Sensitivity Study 


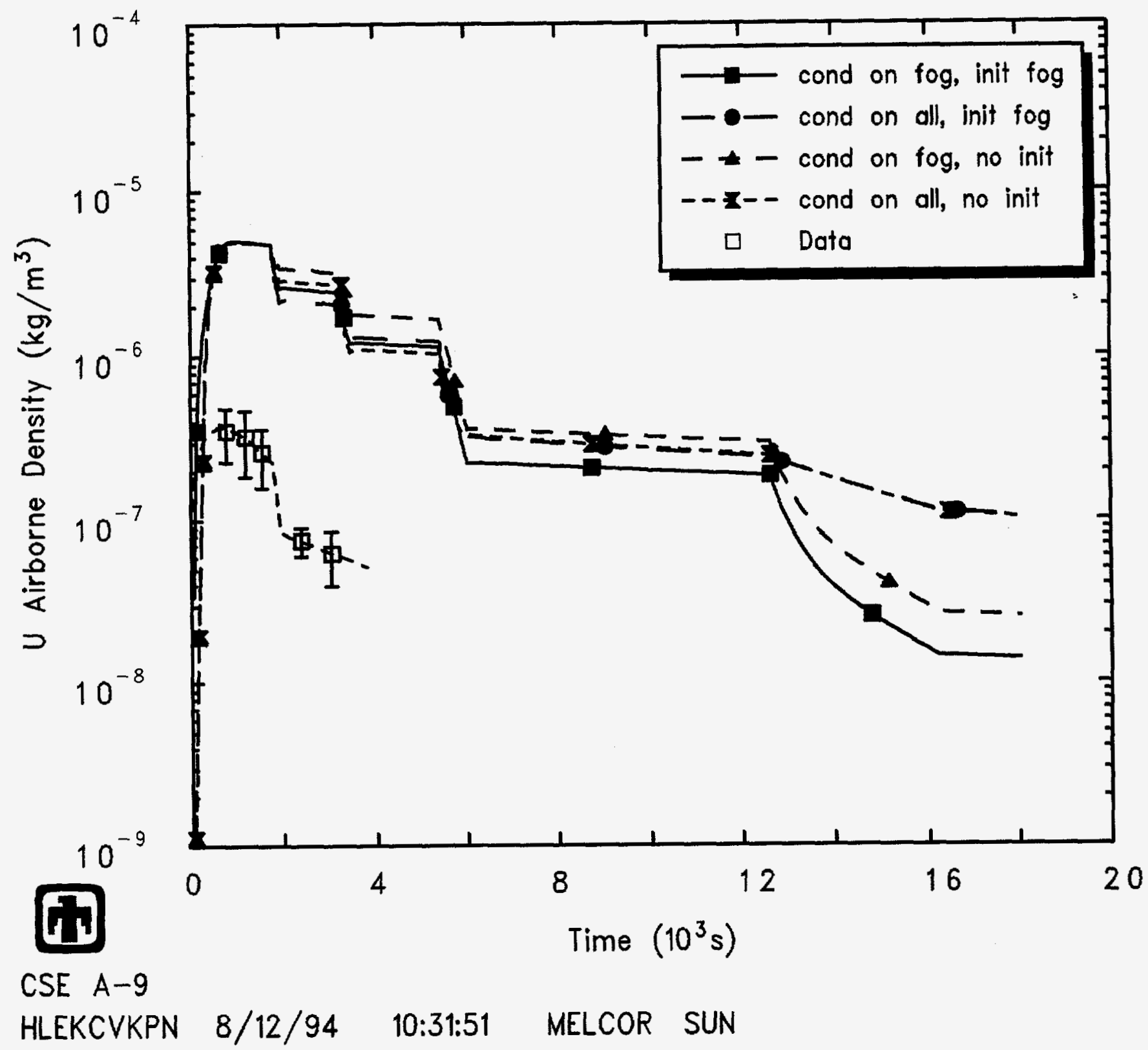

Figure 7.5.5. Uranium Aerosol Airborne Concentrations for CSE Test A-9 - Aerosol Condensation/Evaporation Sensitivity Study 
Table 7.5.1. Washout Rates for CSE Test A-9 - Aerosol Condensation/Evaporation Sensitivity Study

$$
t_{1 / 2}(\min )
$$

Measured cond on fog ${ }^{a}$ cond on all cond on fog cond on all init fog ${ }^{a}$ init fog no init no init

\section{Cesium}

First spray

1.08

Second spray

2.0

5.0

3.6

4.6

4.5

11.7

6.3

Third spray

5.4

Fourth spray

33

4.3

$35^{b}$

3.2

$69^{b}$

\section{5}

1.4

3.6

$35^{b}$

$63^{b}$

\section{Uranium}

First spray

Second spray

2.3

4.6

3.0

7.6

4.6

Third spray

Fourth spray

4.3

4.4

5.0

1.4

4.3

3.2

$35^{b}$

$69^{b}$

4.5

$35^{b}$

3.6

$66^{b}$

\section{Iodine}

First spray

Second spray

Third spray

Fourth spray
0.58

42

34

180
1.1

1.4

1.0

1.4
1.1

1.3

1.0

1.4
1.1

1.4

1.0

1.4

${ }^{a}$ Reference calculation value

${ }^{b}$ Value at end of recirculating spray 


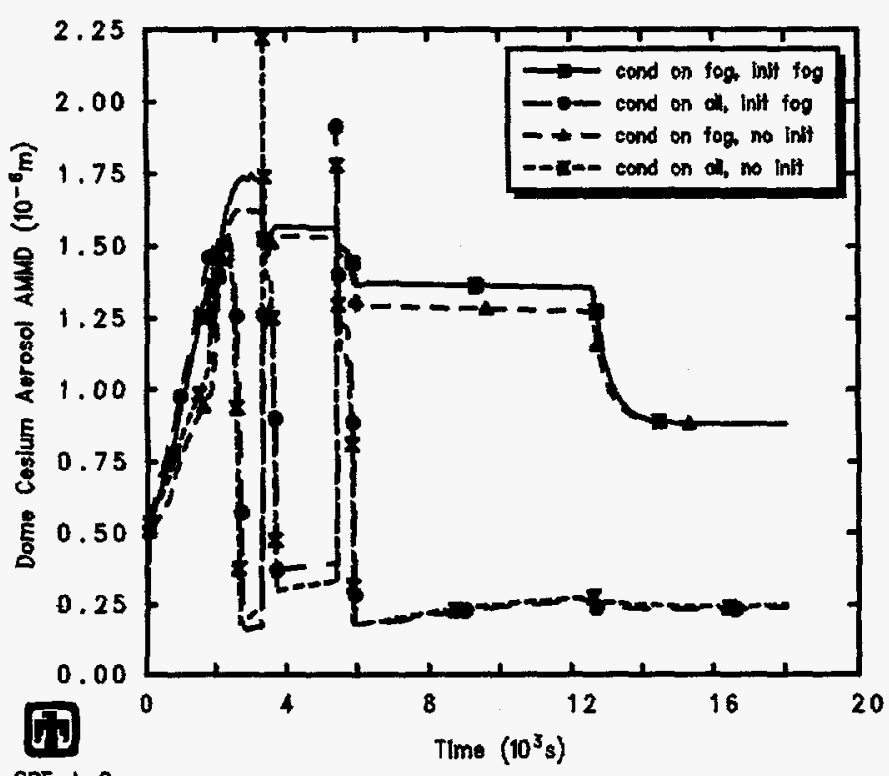

CSE A-9

HLEKCVKPN $8 / 12 / 94$ 10:31:51 MELCOR SUN

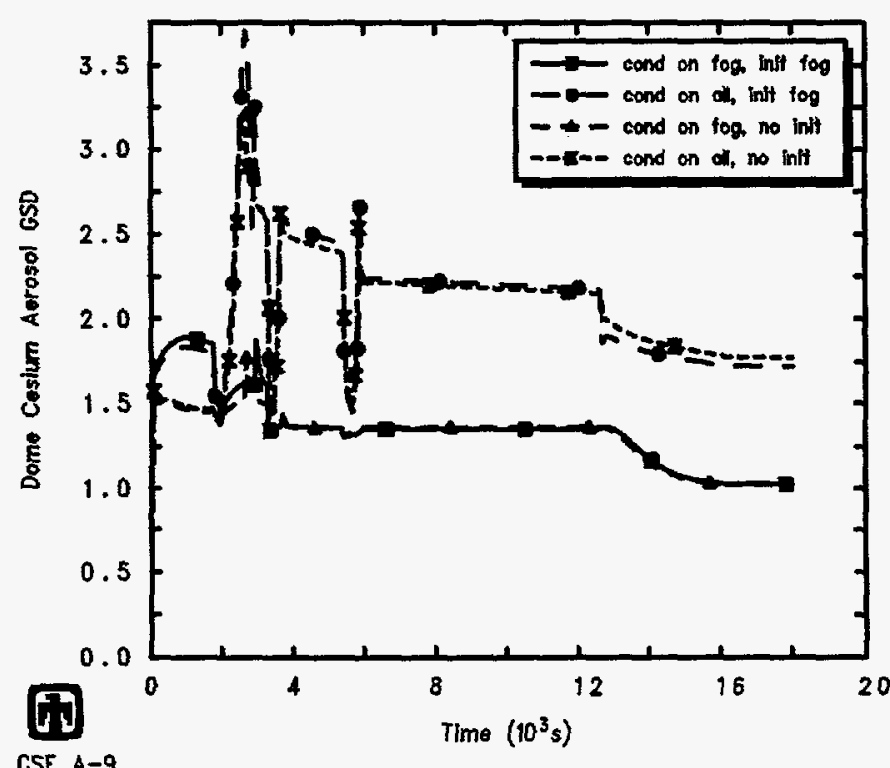

CSE A-9

HLEKCVKPN $8 / 12 / 94 \quad 10: 31: 51$ MELCOR SUN

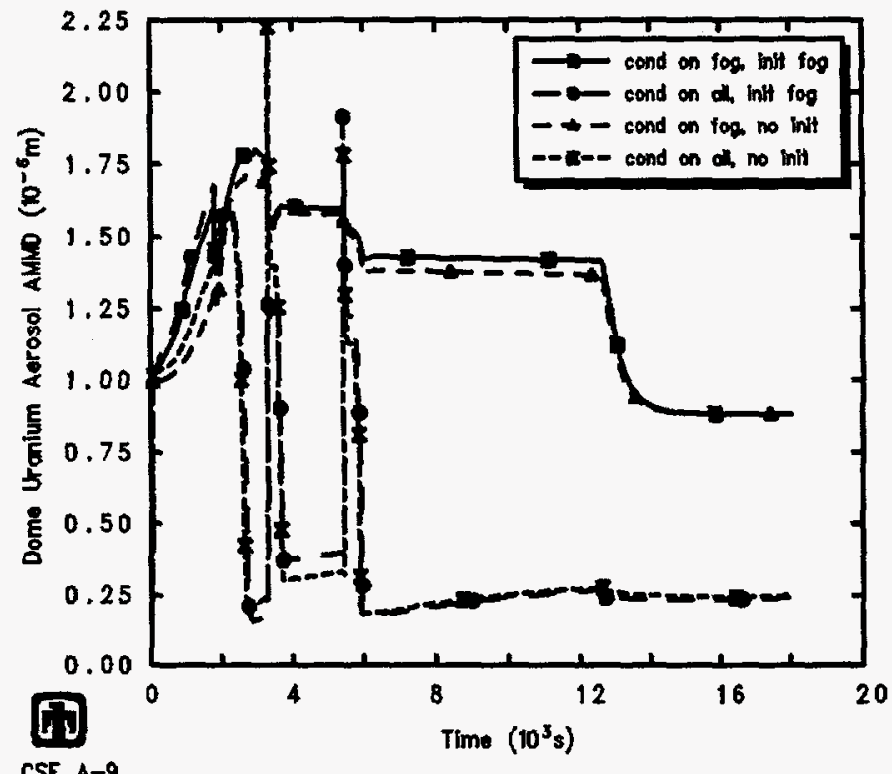

CSE A-9

HLEKCVKPN $8 / 12 / 94$ 10:31:51 MELCOR SUN

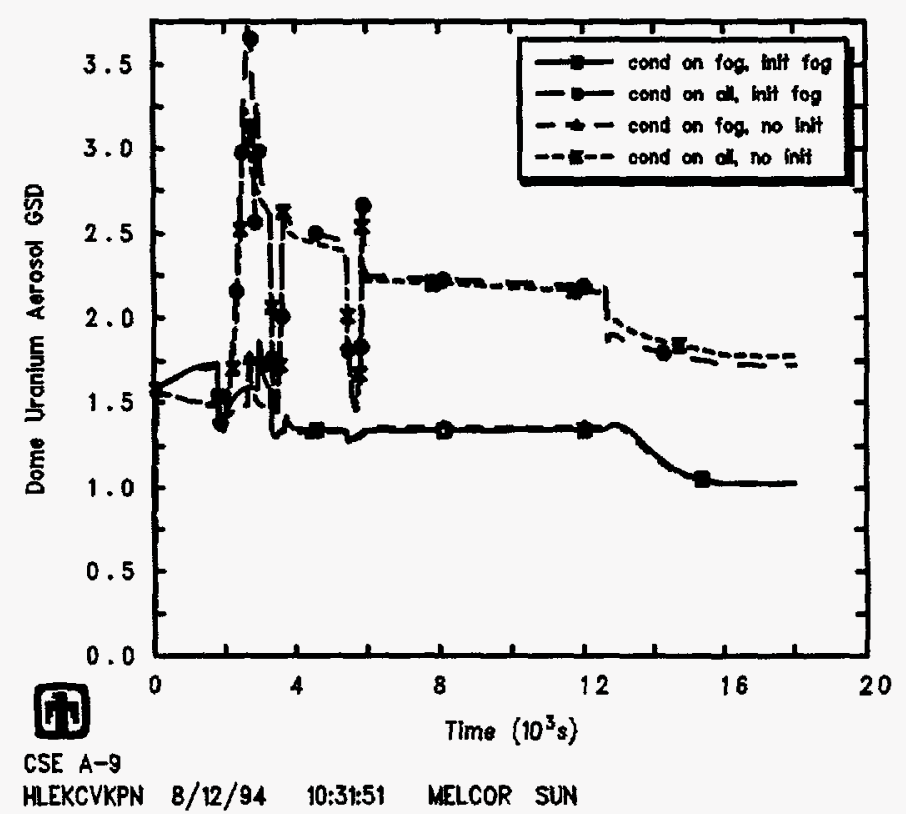

Figure 7.5.6. Cesium (left) and Uranium (right) Aerosol Airborne Concentration AMMDs (top) and GSDs (bottom) in Dome for CSE Test A-9 Aerosol Condensation/Evaporation Sensitivity Study 


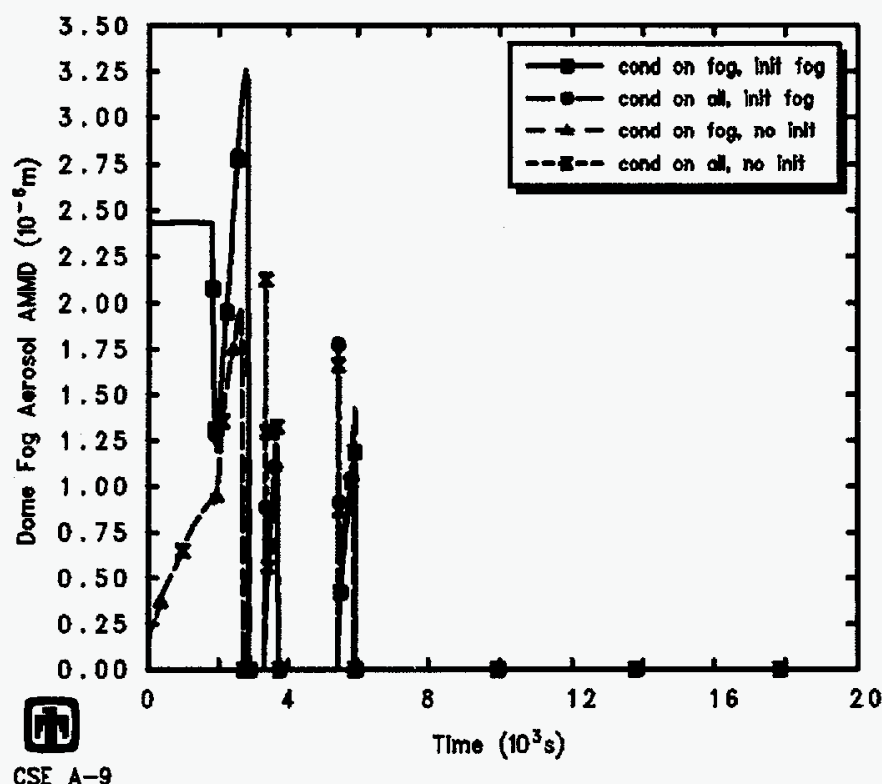

HLEKCVKPN $8 / 12 / 94$ 10:31:51 MELCOR SLN

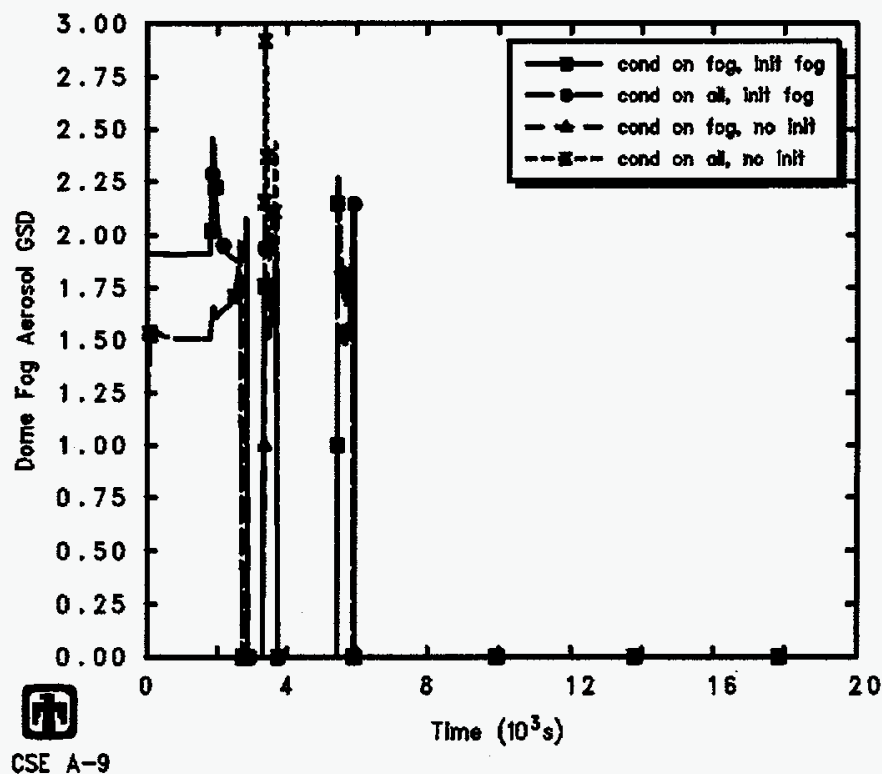

CSE A-9

HLEKCVKPN 8/12/94 10:31:51 MELCOR SUN

Figure 7.5.7. Fog Water Aerosol Airborne Concentration AMMDs (top) and GSDs (bottom) in Dome for CSE Test A-9 - Aerosol Condensation/Evaporation Sensitivity Study 


\section{Vapor Modelling Sensitivity Studies}

There are options and uncertainties both in some MELCOR input values and in the modelling approach taken to represent test conditions. The preceding section investigated how modelling variations would affect the aerosol response predicted by the RN package. This section presents sensitivity studies on parameters affecting the vapor modelling in the RN package used to calculate the iodine response.

\subsection{Partition Coefficient}

MELCOR currently does not model iodine chemistry. However, a user-input parameter is available to define different iodine partition coefficients for different spray types", to help account for chemical interaction effects. The partition coefficient is defined as the ratio of the concentration of iodine in the liquid droplets to the concentration of iodine in the gas under equilibrium conditions. It is normally much greater than 1.0 (the default value in MELCOR), and recommended best-estimate values are 5000 for sodium hydroxide and hydrazine sprays, 100,000 for sodium thiosulfate and 2500 for boric acid sprays.

This parameter was set to 5000 in our reference MELCOR model, for both the fresh and the recirculating sprays. To evaluate the sensitivity of the iodine response to this parameter, a study was done in which the iodine partition coefficient used was varied. Calculations were done with the iodine partition coefficient set to 1 (the default), 2500, 10000 and 100000 .

The thermal/hydraulic responses calculated using various values for the iodine partition parameter were virtually identical to each other, as would be expected. The cesium and uranium aerosol responses calculated using various values for the iodine partition parameter also were virtually identical to each other, as would be expected.

Figure 8.1.1 presents the concentrations of iodine vapor in the test vessel dome atmosphere predicted using different values of the iodine partition parameter, with the test data included for reference. The comparisons are very similar for the upper dome, the lower drywell, the middle room and the lower room or sump.

With the partition coefficient set to 1 (the code default), there is little or no iodine vapor removal predicted, indicating that almost all of the removal is by chemical interaction effects rather than by simple condensation. The calculated iodine vapor removal increases as the user-input iodine partition coefficient is increased, but the removal appears to be approaching a limit as the iodine partition coefficient is made very large.

The corresponding aerosol and vapor washout constants are listed in Table 8.1.1; again the test data are included for reference. 


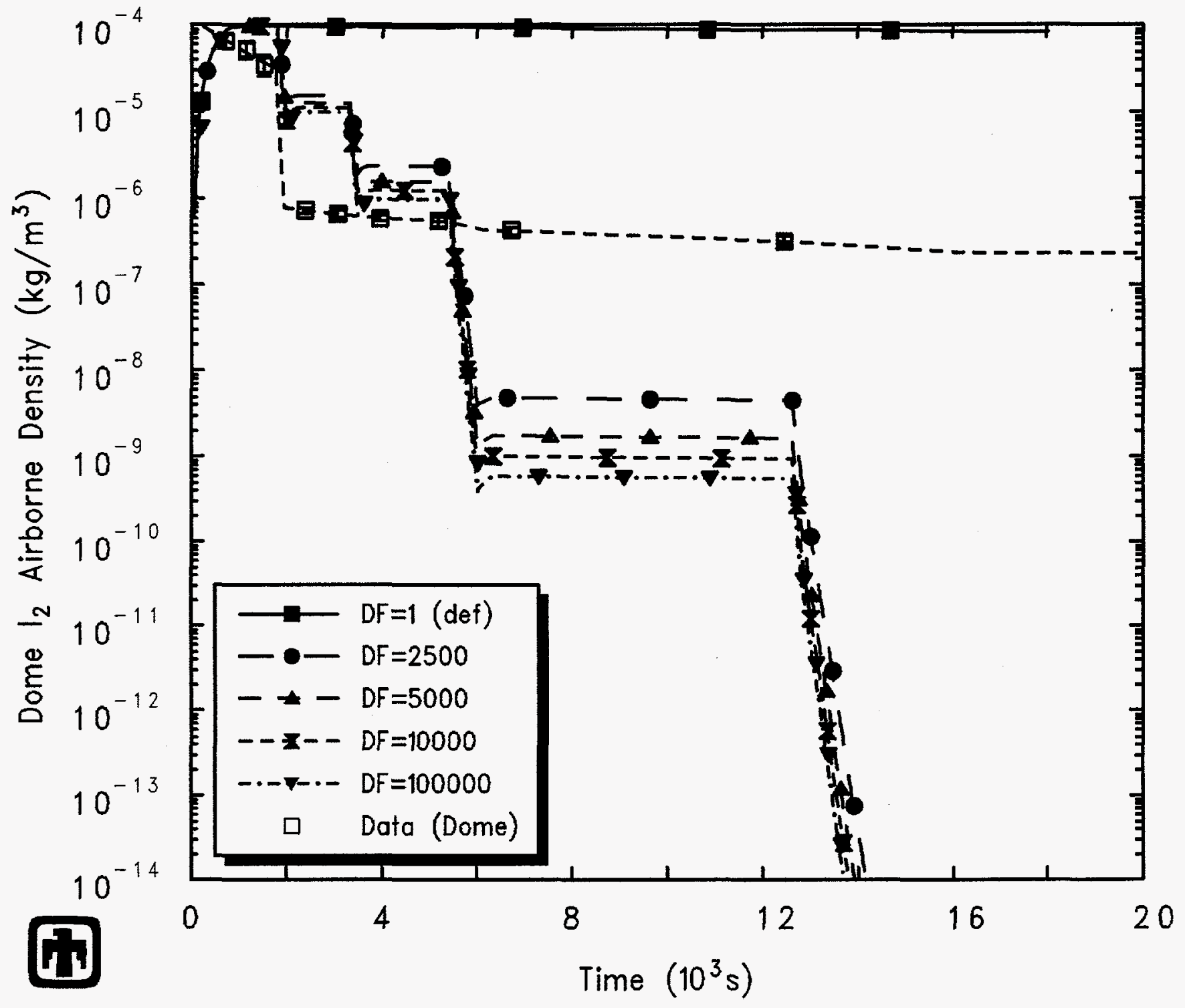

CSE A-9

HLEKDUMPN $8 / 12 / 94 \quad 10: 42: 45$ MELCOR SUN

Figure 8.1.1. Iodine Vapor Airborne Concentrations for CSE Test A-9 - Iodine Partition Coefficient Sensitivity Study 
Table 8.1.1. Washout Rates for CSE Test A-9 - Iodine Partition Coefficient Sensitivity Study

\begin{tabular}{|c|c|c|c|c|c|c|}
\hline & Measured & 1 (def) & 2500 & $\begin{array}{l}\mathrm{DF}_{I_{2}}= \\
5000^{a}\end{array}$ & 10000 & 100000 \\
\hline \multicolumn{7}{|l|}{ Cesium } \\
\hline First spray & 1.08 & 5.0 & 5.0 & 5.0 & 5.0 & 5.0 \\
\hline Second spray & 2.0 & 4.6 & 4.6 & 4.6 & 4.6 & 4.6 \\
\hline Third spray & 5.4 & 4.3 & 4.3 & 4.3 & 4.3 & 4.3 \\
\hline Fourth spray & 33 & $35^{b}$ & $35^{b}$ & $35^{6}$ & $35^{b}$ & $35^{b}$ \\
\hline \multicolumn{7}{|l|}{ Uranium } \\
\hline First spray & 2.3 & 4.6 & 4.6 & 4.6 & 4.6 & 4.6 \\
\hline Second spray & & 4.3 & 4.3 & 4.3 & 4.3 & 4.3 \\
\hline Third spray & & 4.3 & 4.3 & 4.3 & 4.3 & 4.3 \\
\hline Fourth spray & & $35^{b}$ & $35^{b}$ & $35^{b}$ & $35^{b}$ & $35^{b}$ \\
\hline \multicolumn{7}{|l|}{ Iodine } \\
\hline First spray & 0.58 & - & 1.2 & 1.1 & 1.0 & 0.92 \\
\hline Second spray & 42 & - & 1.5 & 1.4 & 1.3 & 1.2 \\
\hline Third spray & 34 & - & 1.1 & 1.0 & 0.92 & 0.87 \\
\hline Fourth spray & 180 & - & 1.5 & 1.4 & 1.3 & 1.3 \\
\hline
\end{tabular}




\subsection{Re-evolution from Pools}

The first calculations for these CSE assessment analyses showed iodine removal by sprays from the test vessel atmosphere as discussed already, but then predicted that the iodine vapor would re-evolve from the liquid pools in the lower drywell and lower room sumps very quickly, returning to near the initial airborne iodine vapor concentration. This occurred because the water in the pool had no capability of continuing to bind the iodine vapor chemically.

This problem was noted by the code developers as DIR 1232, and the implementation of TRAP-MELT modelling for fission product condensation and evaporation was modified as of version $1.8 \mathrm{OU}$ to disallow any evaporation of fission products residing in a control volume pool. This is considered a temporary modification and is expected to be replaced by the iodine chemistry model currently under development for MELCOR. Until this new model is implemented, note that MELCOR versions 1.8.2 and 1.8.3 could have very different fission product vapor responses calculated in control volumes with pools and sprays. 


\section{Time Step Effects and Machine Dependency}

There has been a lot of discussion recently on numeric effects seen in various MELCOR calculations [30], producing either differences in results for the same input on different machines or differences in results when the time step used is varied. Several calculations have been done to identify whether any such effects existed in our CSE assessment analyses. A significant time step dependency due to an error in the spray package coding was identified and eliminated.

\subsection{Machine Dependencies}

The calculations discussed in detail in Section 4, and the majority of our sensitivity study analyses, were run on a SUN Sparc2 workstation. The test A-9 reference calculations were rerun, using the same code version (1.8PN), on an IBM RISC-6000 Model 550 workstation, on an HP 755 workstation, on a CRAY Y-MP8/864, and on a $50 \mathrm{MHz}$ 486 PC.

The predicted test vessel dome pressures and temperatures for the SUN, IBM and HP workstation, and Cray and PC, calculations are presented in Figures 9.1.1 and 9.1.2. There are no machine dependencies visible in the thermal/hydraulic response of the test vessel to the spray injection.

Figures 9.1.3, 9.1.4 and 9.1.5 give the cesium and uranium aerosol and iodine vapor airborne concentrations in the test vessel dome predicted by the SUN, IBM and HP workstation, and Cray and PC, calculations. Again, there are no machine dependencies visible in the aerosol washout or vapor decontamination response of the test vessel to the spray injection.

Figure 9.1.6 presents run times for the CSE A-9 calculations on the various platforms. The SUN and PC are always slowest in run time required; the IBM, HP and Cray are all significantly faster with the HP and IBM workstations the fastest for these analyses. Run times are shown for the $18000 \mathrm{~s}$ transient calculation; the offset at $t=0$ represents the time taken for simulating the $18000 \mathrm{~s}$ preconditioning phase. Figure 9.1 .7 indicates that most of the run time is being used by the HS and RN1 packages, with relatively little used by $\mathrm{CVH}$ or RN2. (RN1 is the portion of the RN package dealing with fission product aerosols and vapors in general; RN2 is the portion dealing specifically with engineered safety features such as containment sprays.)

\subsection{Time Step Effects}

Otherwise identical MELCOR calculations for CSE test A-9 were run on a SUN Sparc2 workstation with the user-input maximum allowed time step progressively set to $99 \mathrm{~s}, 20 \mathrm{~s}, 2 \mathrm{~s}$, and $0.5 \mathrm{~s}$. 


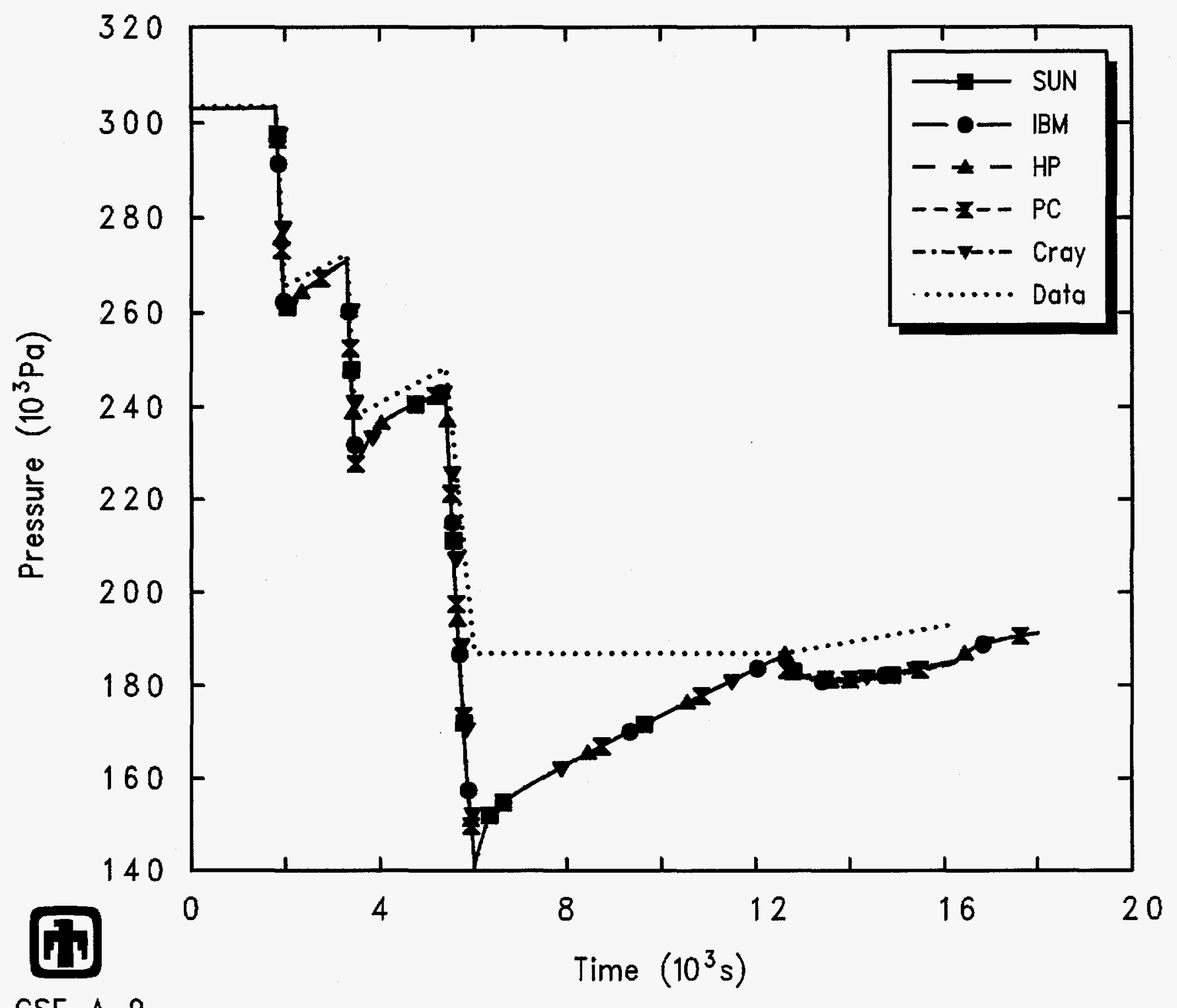

CSE A-9

HLEKCVKPN $8 / 12 / 94 \quad 10: 31: 51 \quad$ MELCOR CRAY

Figure 9.1.1. Vessel Pressure for CSE Test A-9 - Machine Dependency Sensitivity Study 


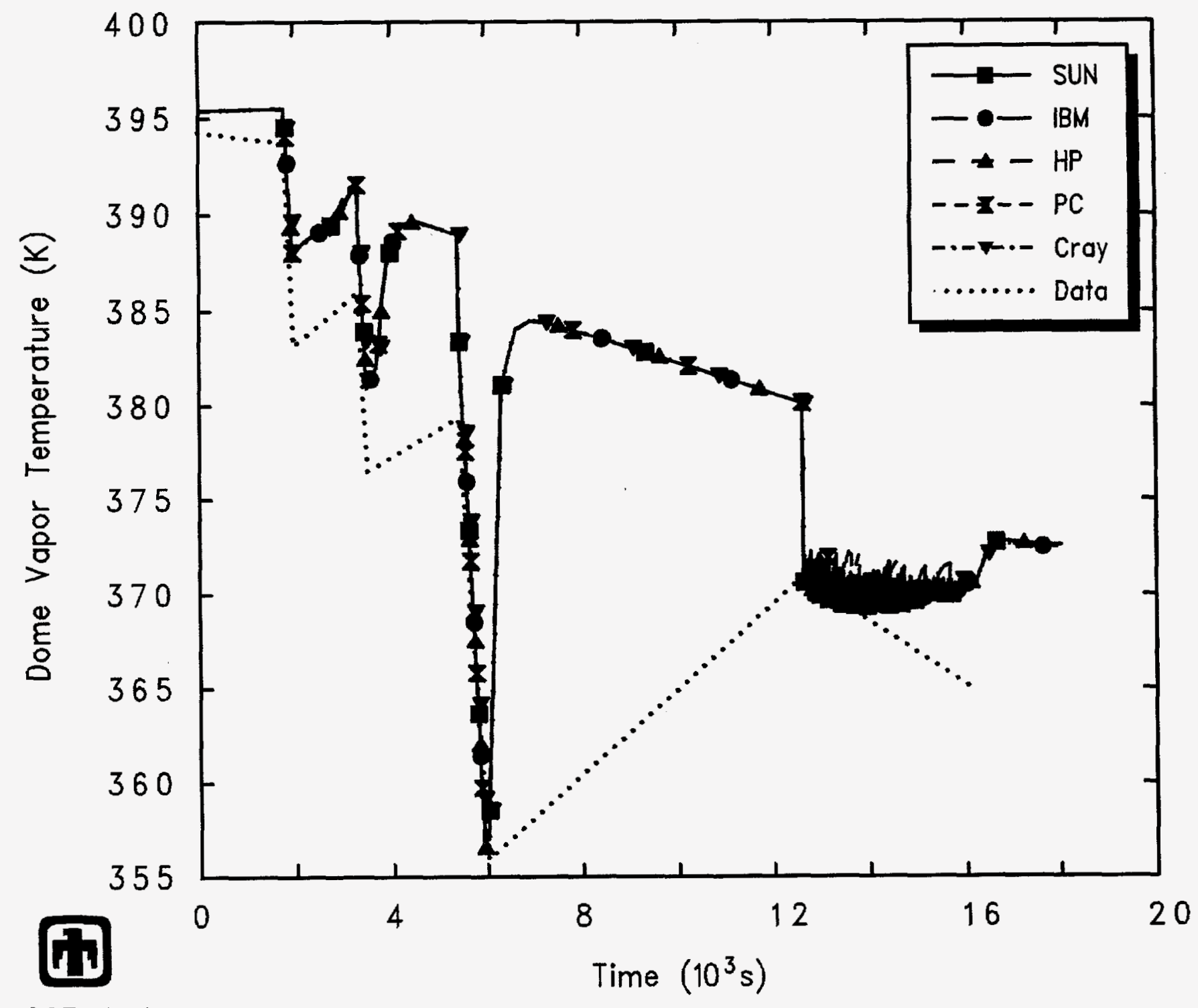

CSE A-9

HLEKCVKPN $8 / 12 / 94 \quad 10: 31: 51 \quad$ MELCOR CRAY

Figure 9.1.2. Vessel Dome Temperature for CSE Test A-9 - Machine Dependency Sensitivity Study 


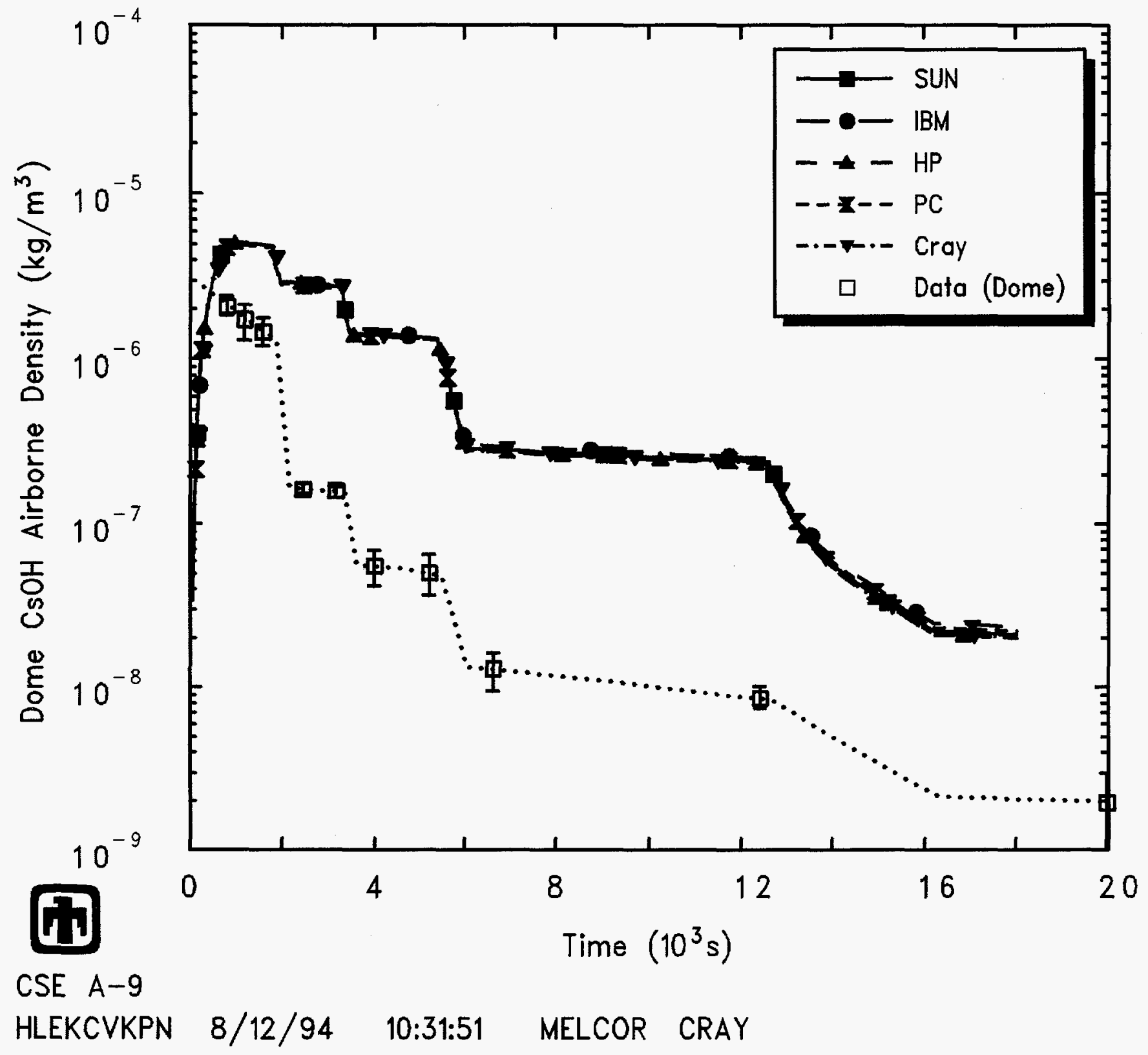

Figure 9.1.3. Cesium Aerosol Airborne Concentrations for CSE Test A-9 - Machine Dependency Sensitivity Study 


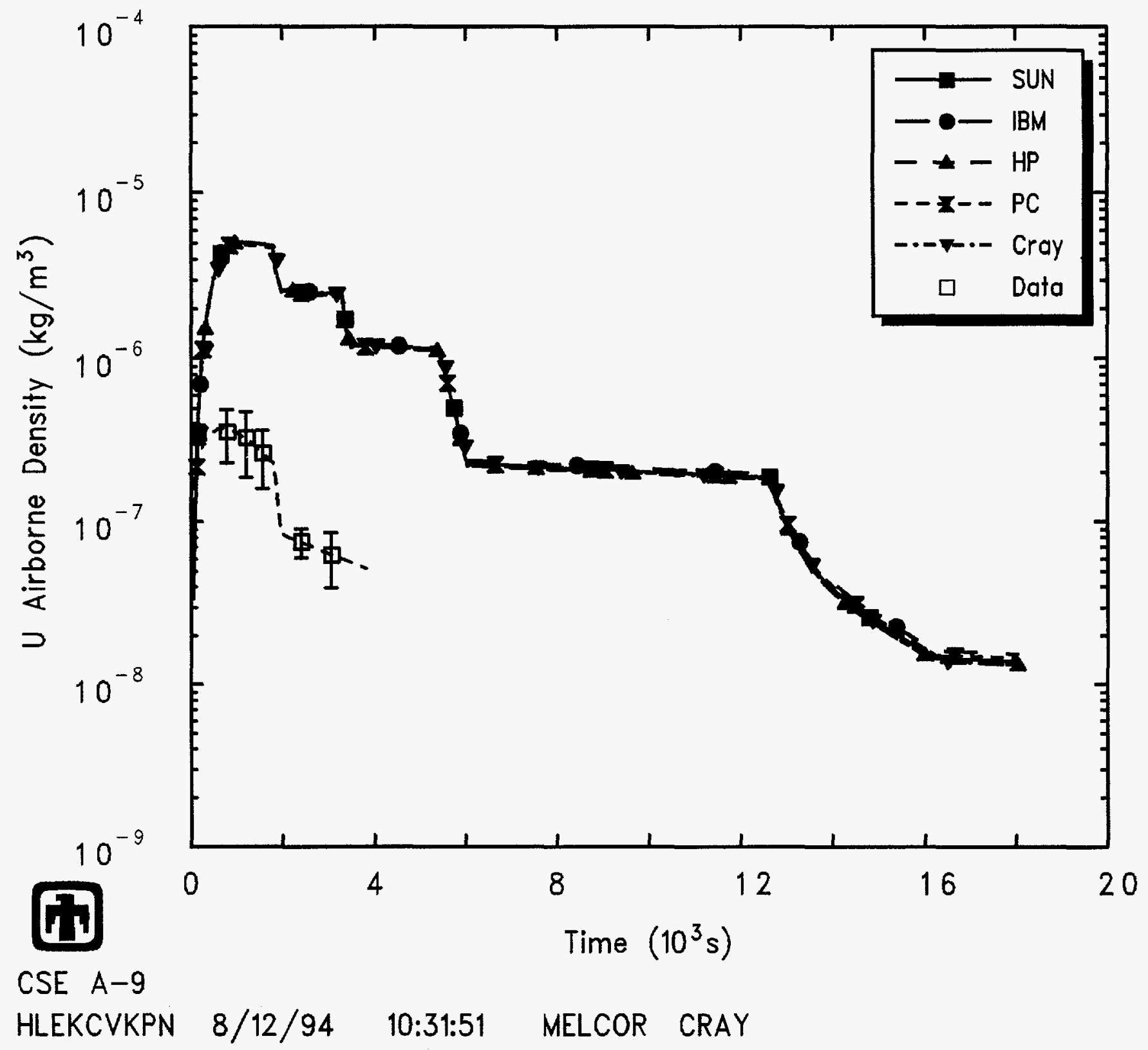

Figure 9.1.4. Uranium Aerosol Airborne Concentrations for CSE Test A-9 Machine Dependency Sensitivity Study 


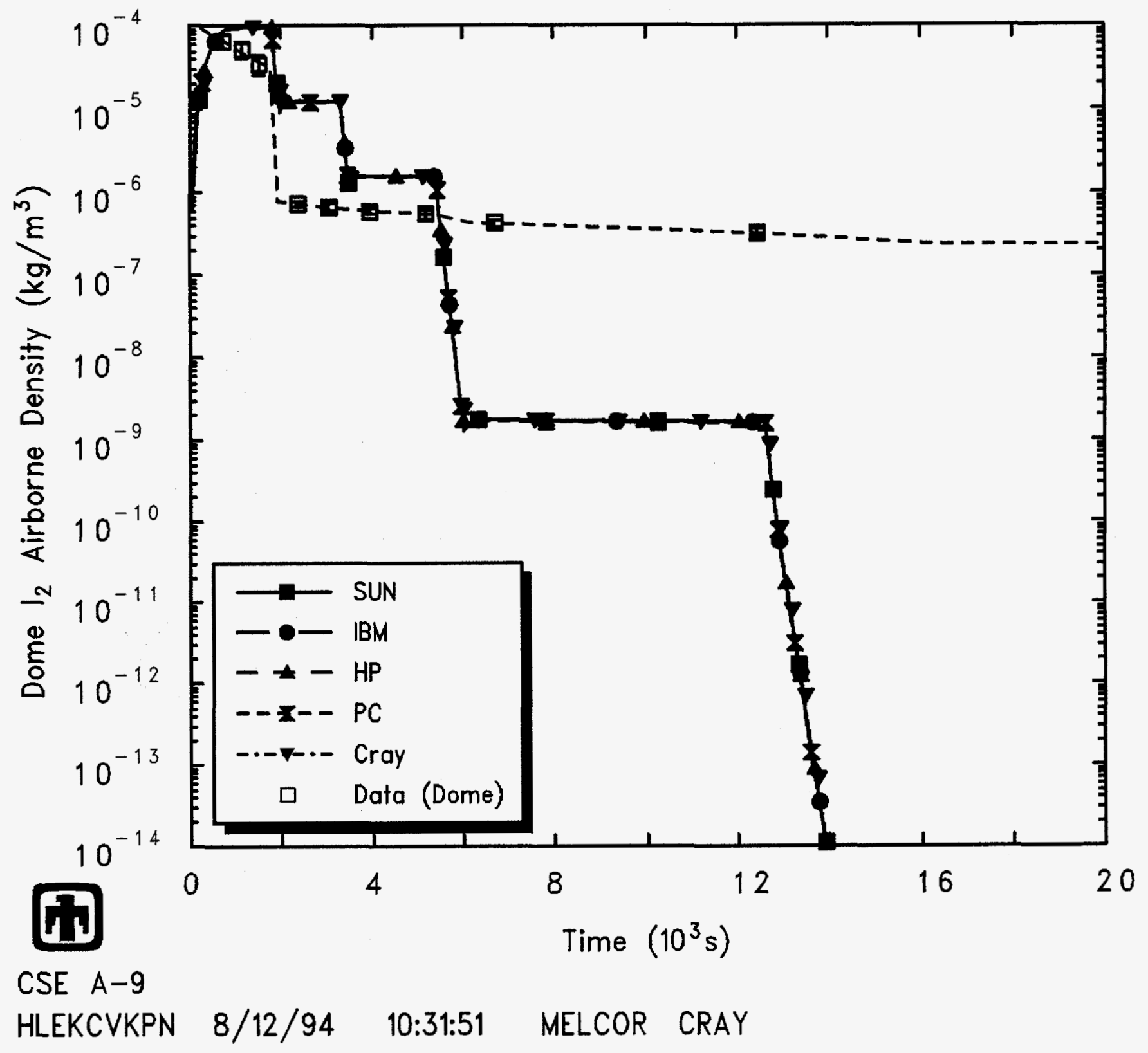

Figure 9.1.5. Iodine Vapor Airborne Concentrations for CSE Test A-9 - Machine Dependency Sensitivity Study 


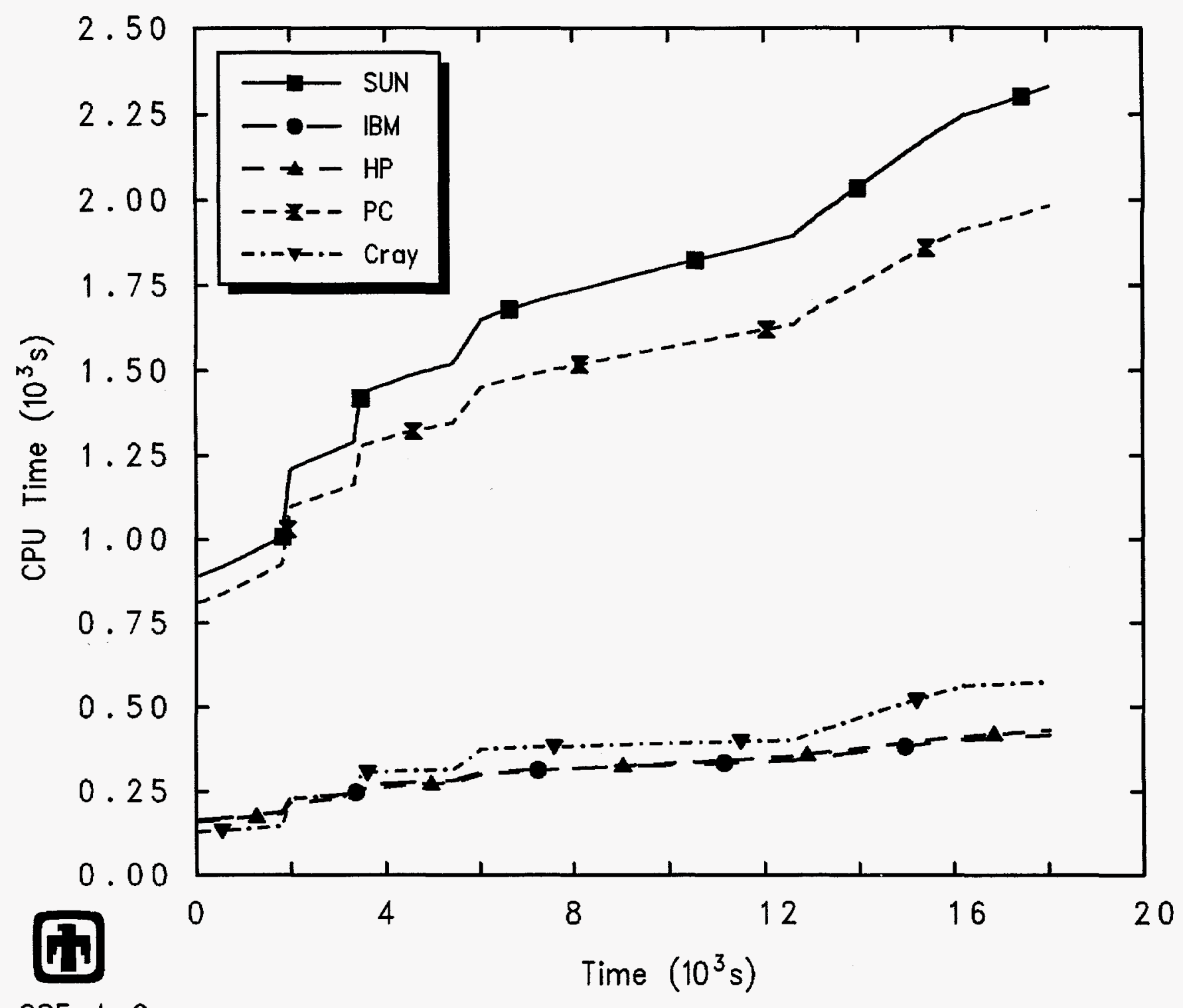

CSE A-9

HLEKCVKPN $8 / 12 / 94 \quad 10: 31: 51$ MELCOR CRAY

Figure 9.1.6. Total Run Times for CSE Test A-9 - Machine Dependency Sensitivity Study 


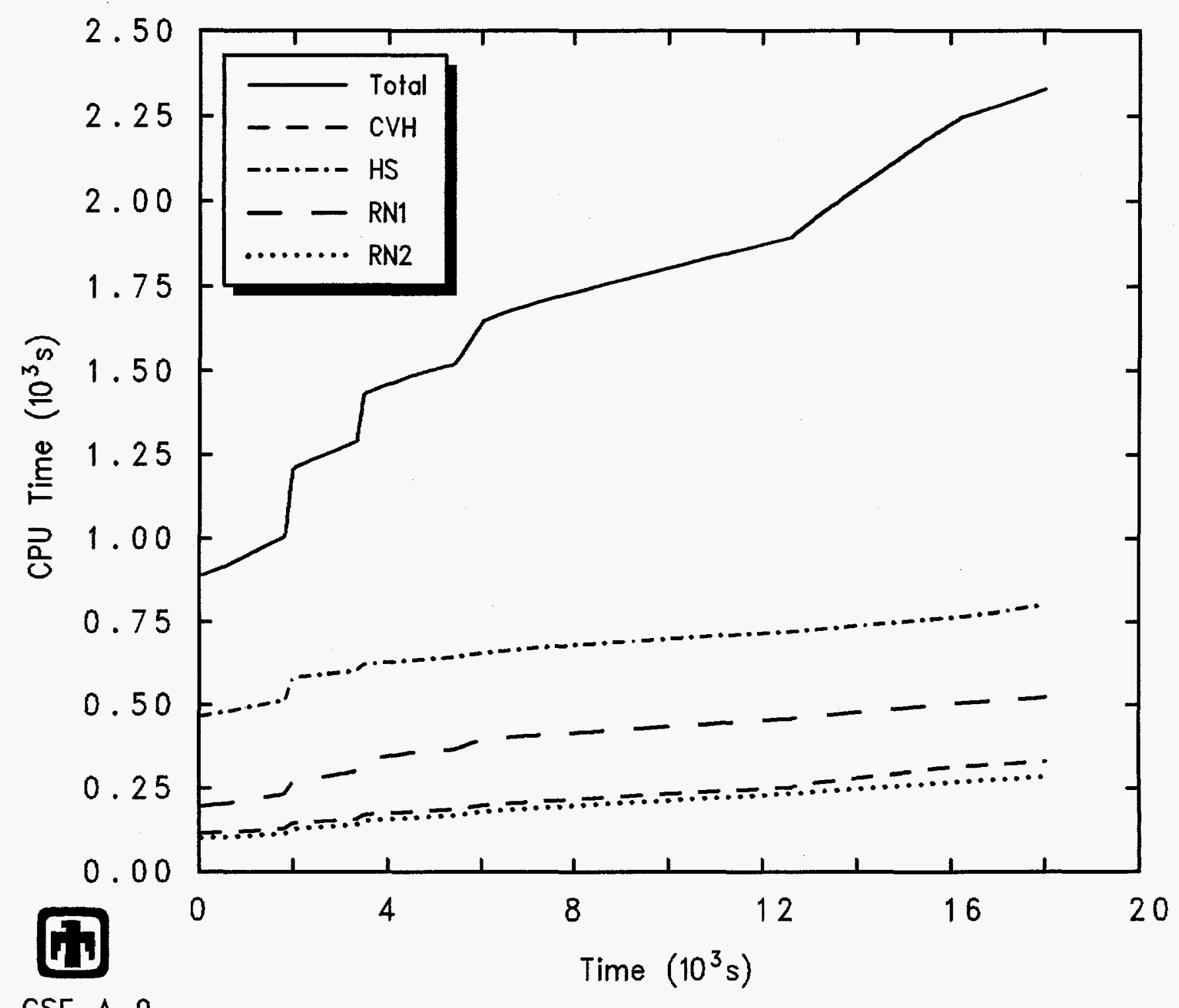

CSE A-9

HLEKCVKPN $8 / 12 / 94 \quad 10: 31: 51$ MELCOR SUN

Figure 9.1.7. Total and Package Run Times for CSE Test A-9 - Reference Calculation 
Figure 9.2.1 shows the time steps actually used in these time step sensitivity study calculations. With a user-specified maximum allowed time step of $99 \mathrm{~s}$, the calculation always ran at the code-determined time step, which was as high as $20-30 \mathrm{~s}$ in the periods between sprays and usually in the 10-20 s when the sprays were on. (The abrupt drops in time step at the start and end of each spray period are due to using "EXACTTIME" records to force the code to begin a cycle at the exact time that a spray was specified to start or stop.) Limiting the user-specified maximum allowed time step to $20 \mathrm{~s}$ generally affected the time step used in the periods between sprays but not when the sprays were on. At the smaller user-specified maximum allowed time steps of $2 \mathrm{~s}$ and $0.5 \mathrm{~s}$, the calculation ran at the user-specified maximum allowed time steps throughout the simulation.

The predicted results from our initial time step sensitivity study calculations are presented in Figures 9.2.2 through 9.2.5. These calculations were run with version 1.8PK.

Figures 9.2.2 and 9.2.3 show the vessel pressures and dome temperatures predicted. While there are no time step effects visible in the test vessel pressure response, there are a few small differences in the temperature behavior predicted, which are particularly noticable during the recirculating spray period where the two smaller, user-limited time steps result in smoother and slightly higher temperatures than the corresponding results with the larger time steps.

Figures 9.2.4 and 9.2.5 give the cesium aerosol and iodine vapor airborne concentrations in the test vessel dome predicted by the first time step study calculations done with version $1.8 \mathrm{PK}$. (The uranium aerosol response predicted is virtually identical to the cesium aerosol response and is not shown separately.) While the iodine response generally echoes the behavior seen in the vessel dome temperature, the cesium aerosol removal increases drastically as the time step is reduced.

This problem was reported to the code developers as DIR 1268. An error was identified and corrected in the implementation of the model for aerosol removal by sprays. In effect, in the calculation of the aerosol removal during a time step from the removal rate, the time step was inappropriately replaced by an approximation to the spray droplet residence time. The effect was to introduce a strong dependency on time step, as well as totally falsifying the aerosol removal.

This problem was corrected in update $1.8 \mathrm{PM}$ (so that it has been eliminated in the release version of MELCOR 1.8.3). The predicted results from our final time step sensitivity study calculations are presented in Figures 9.2.6 through 9.2.9. These calculations were run with version $1.8 \mathrm{PN}$.

Figures 9.2.6 and 9.2.7 show the vessel pressures and dome temperatures predicted. As before, there are no significant time step effects visible in the test vessel pressure response. The temperature behavior predicted is the same as in our initial time step sensitivity study calculations: smoother and slightly higher temperatures during the recirculating spray period for the two smaller, user-limited time steps than the corresponding results with the larger time steps. Correction of an error in calculating the aerosol removal by sprays would not be expected to affect the thermal/hydraulic response calculated in the test vessel. 


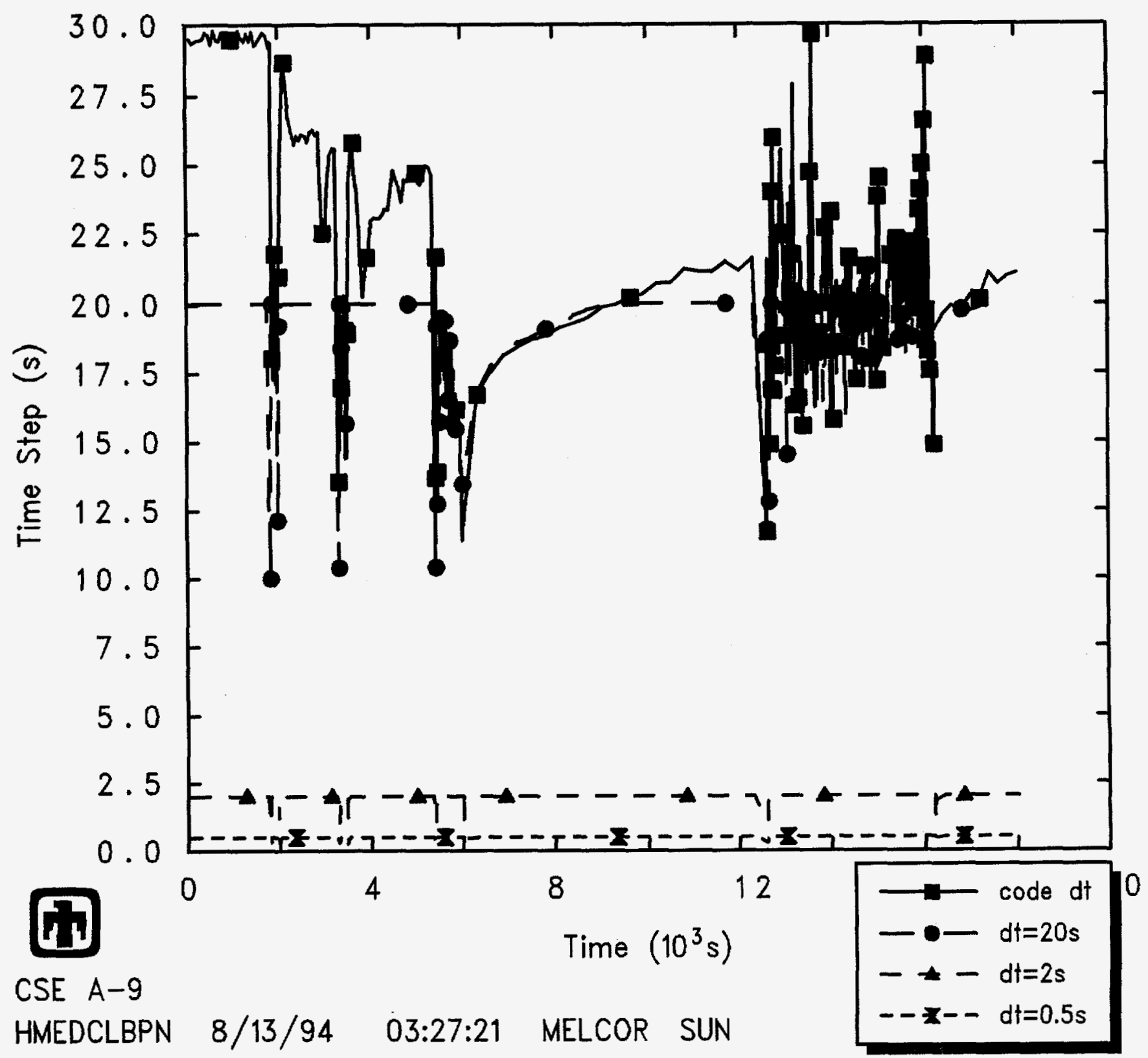

Figure 9.2.1. Time Steps for CSE Test A-9 - Time Step Sensitivity Study 


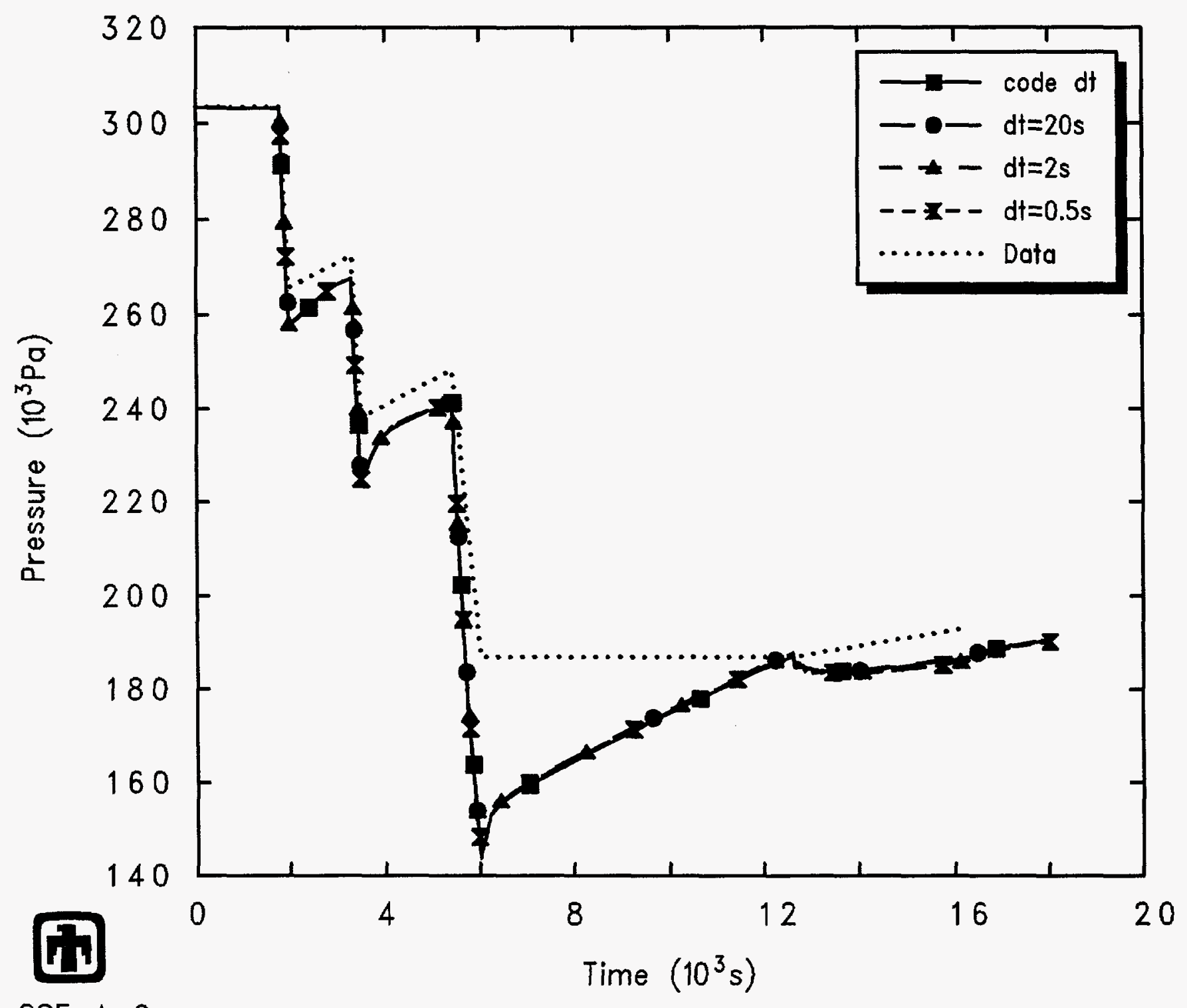

CSE A-9

G2EOBEBPK $7 / 29 / 94 \quad 14: 13: 02 \quad$ MELCOR SUN

Figure 9.2.2. Vessel Pressure for CSE Test A-9 - Time Step Sensitivity Study (Version 1.8PK) 


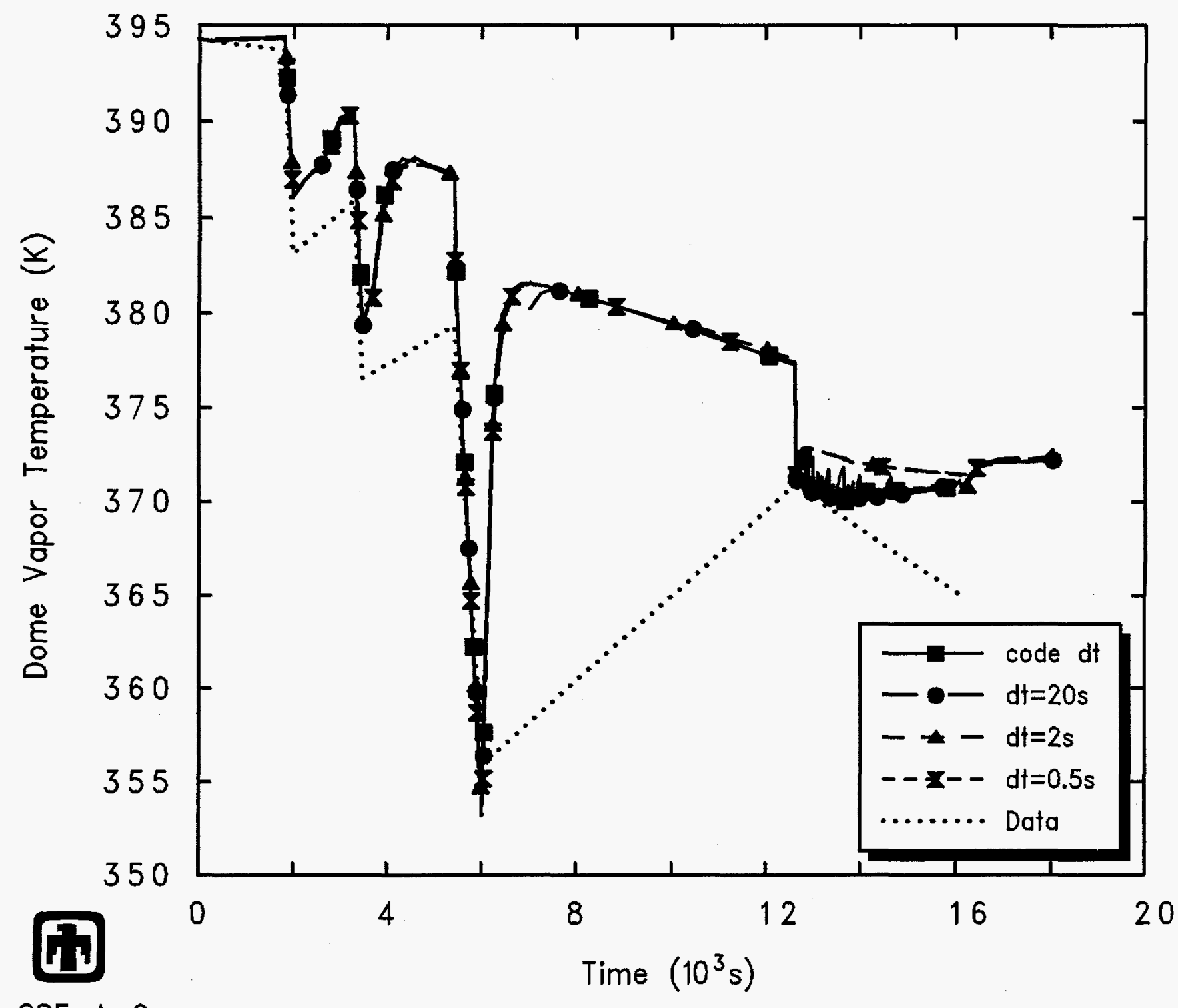

CSE A-9

G2EOBEBPK $7 / 29 / 94 \quad 14: 13: 02$ MELCOR SUN

Figure 9.2.3. Vessel Dome Temperature for CSE Test A-9 - Time Step Sensitivity Study (Version 1.8PK) 


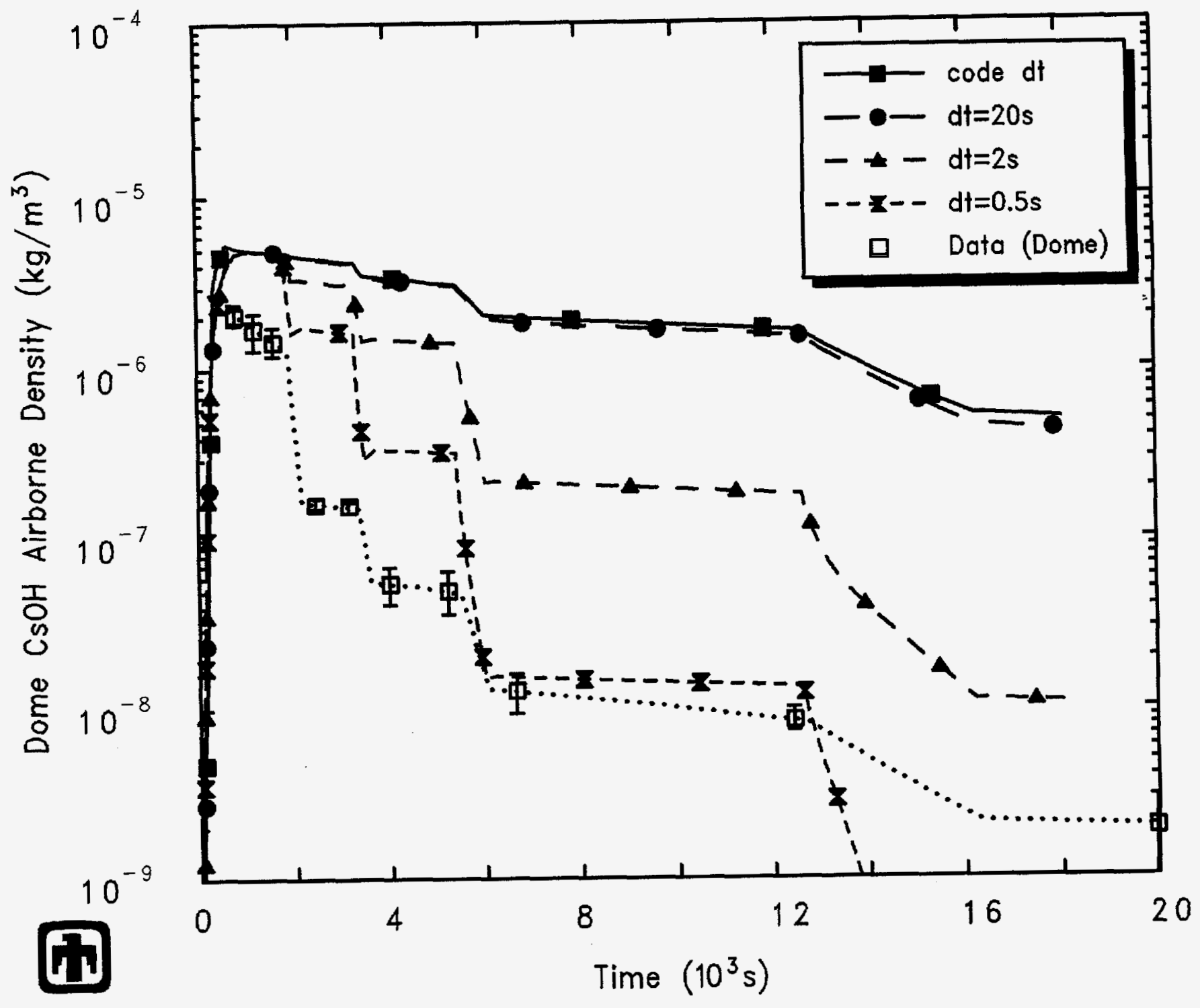

CSE A-9

G2EOBEBPK $7 / 29 / 94 \quad 14: 13: 02$ MELCOR SUN

Figure 9.2.4. Cesium Aerosol Airborne Concentrations for CSE Test A-9 - Time Step Sensitivity Study (Version 1.8PK) 


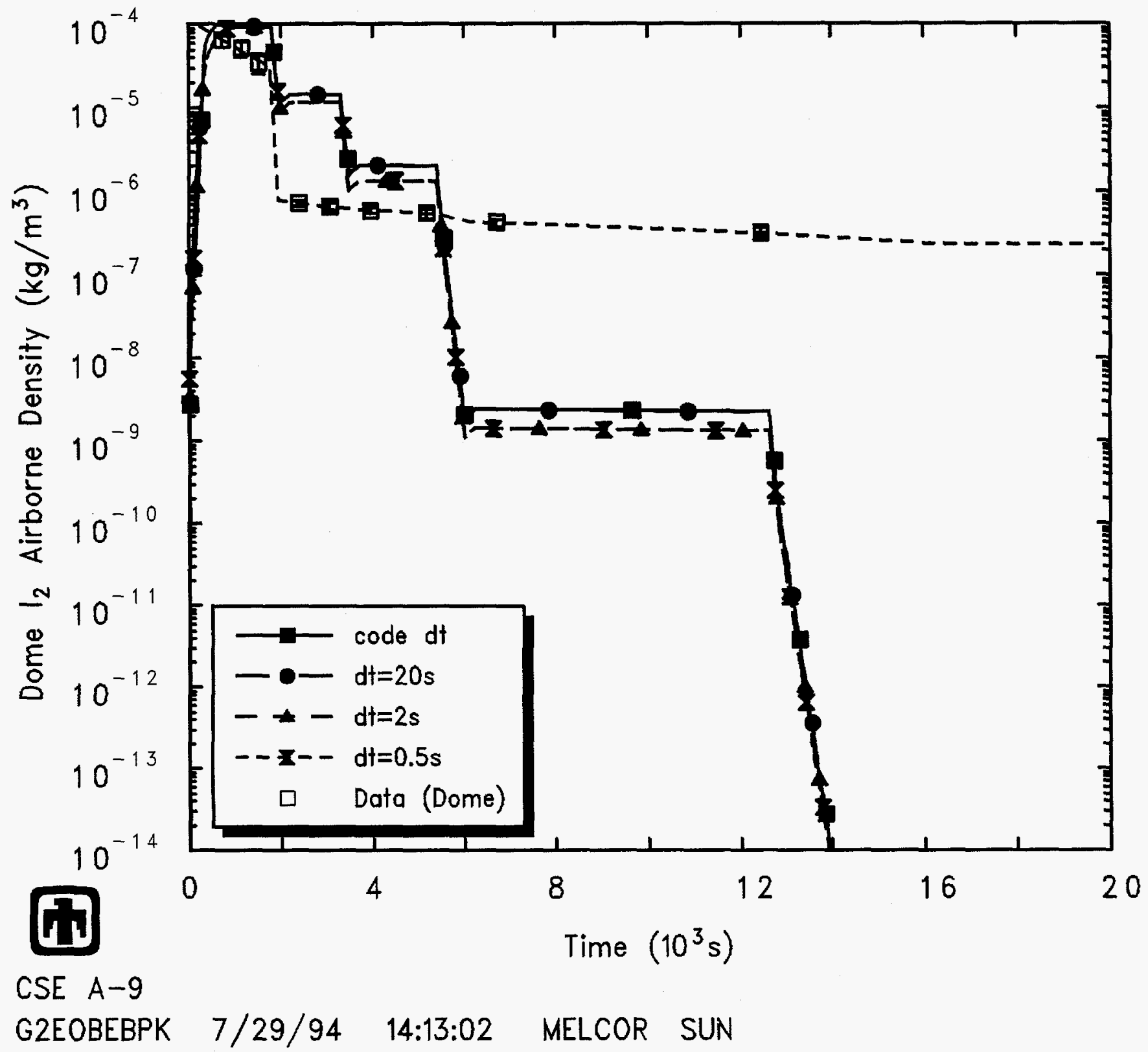

Figure 9.2.5. Iodine Vapor Airborne Concentrations for CSE Test A-9 - Time Step Sensitivity Study (Version 1.8PK) 


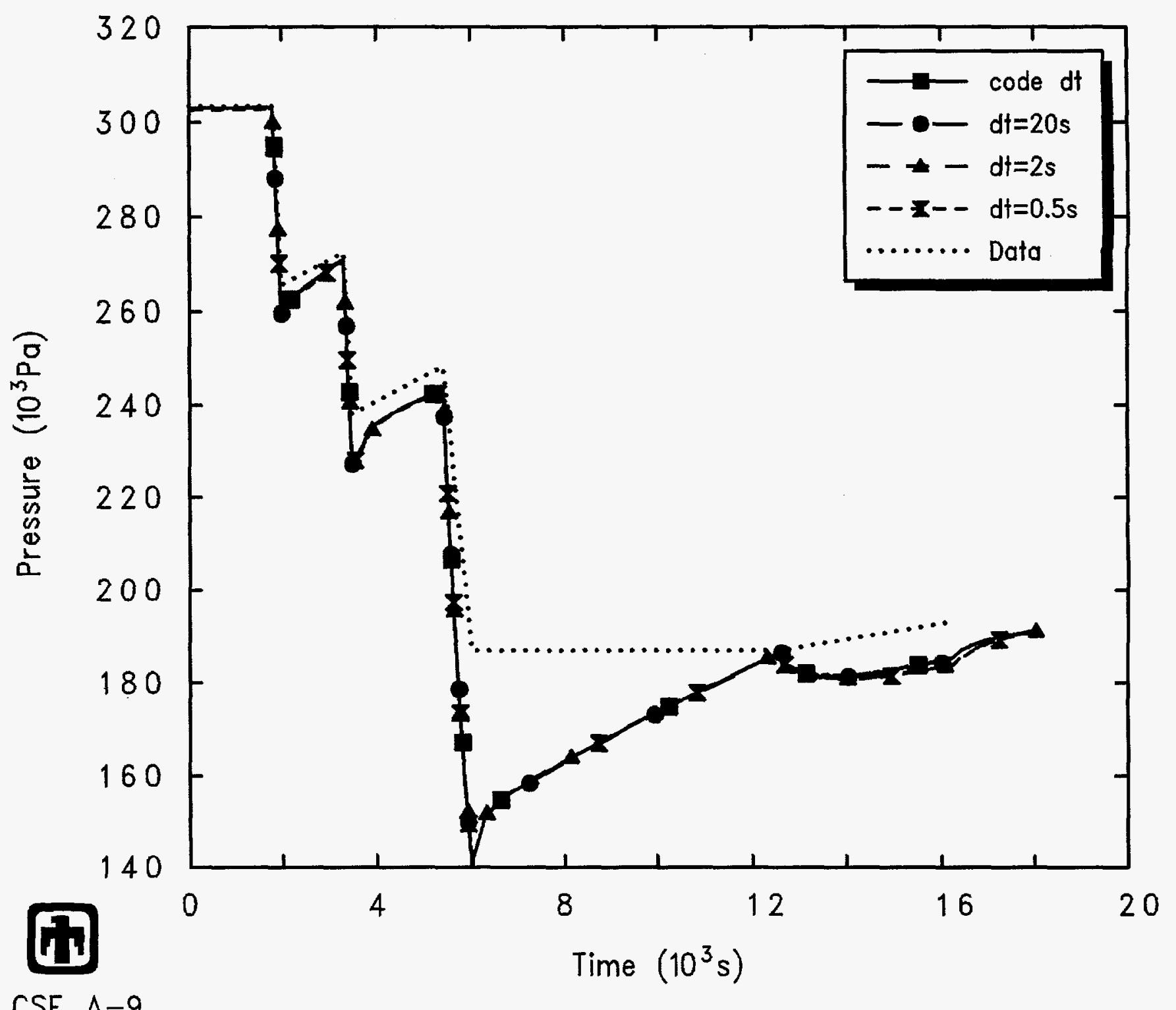

CSE A-9

HMEDCLBPN 8/13/94 03:27:21 MELCOR SUN

Figure 9.2.6. Vessel Pressure for CSE Test A-9 - Time Step Sensitivity Study (Version 1.8PN) 


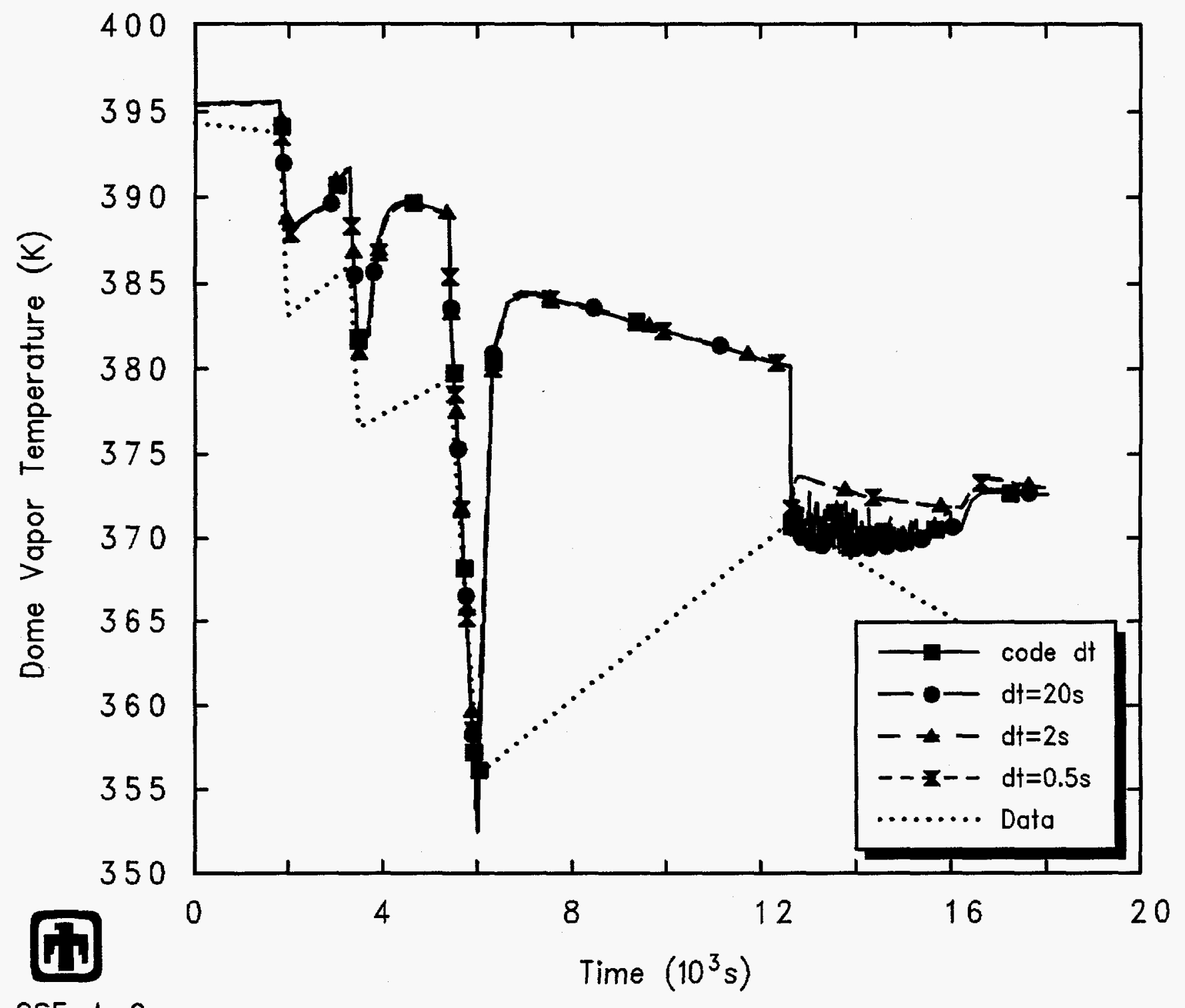

CSE A-9

HMEDCLBPN $8 / 13 / 94 \quad 03: 27: 21$ MELCOR SUN

Figure 9.2.7. Vessel Dome Temperature for CSE Test A-9 - Time Step Sensitivity Study (Version 1.8PN) 
Figures 9.2.8 and 9.2.9 give the cesium aerosol and iodine vapor airborne concentrations in the test vessel dome predicted by the final time step study calculations, done with version $1.8 \mathrm{PN}$. (The uranium aerosol response predicted is virtually identical to the cesium aerosol response and is not shown separately.) Again, the iodine response generally echoes the behavior seen in the vessel dome temperature. However, the cesium aerosol removal predicted has changed significantly with the correction of this error in calculating the aerosol removal by sprays. The aerosol removal predicted now increases slightly as the time step is reduced, owing to residual numeric effects. Such small differences in overall convergence are not surprising in an essentially exponential phenomenon. 


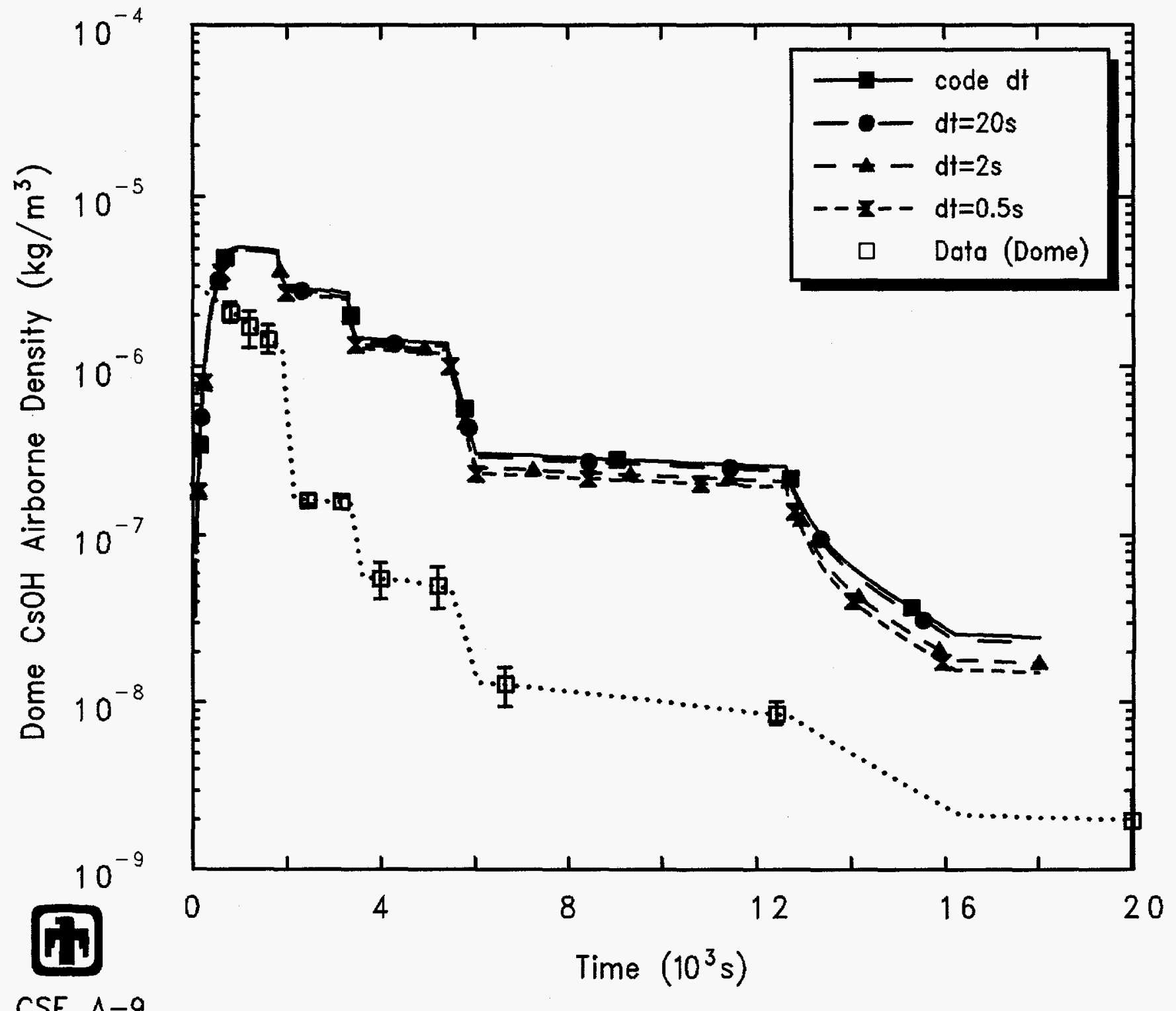

CSE A-9

HMEDCLBPN 8/13/94 03:27:21 MELCOR SUN

Figure 9.2.8. Cesium Aerosol Airborne Concentrations for CSE Test A-9 - Time Step Sensitivity Study (Version 1.8PN) 


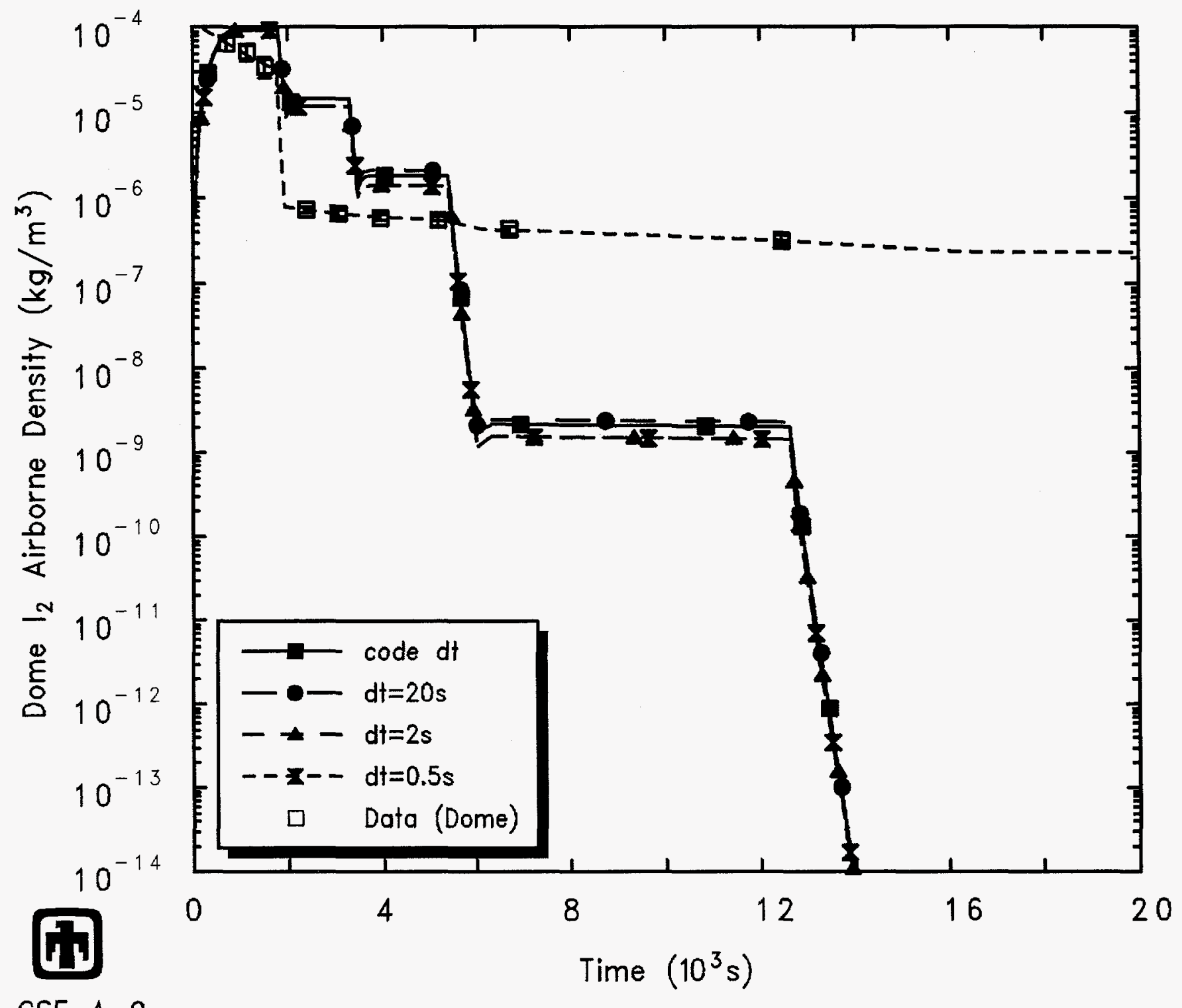

CSE A-9

HMEDCLBPN $8 / 13 / 94 \quad 03: 27: 21 \quad$ MELCOR SUN

Figure 9.2.9. Iodine Vapor Airborne Concentrations for CSE Test A-9 - Time Step Sensitivity Study (Version 1.8PN) 


\section{Code Problems Identified}

\subsection{Recirculating Sprays}

A new feature has been added in MELCOR 1.8.3 in the spray package that allows the user to specify a control volume from which to extract water for recirculating sprays. In previous code versions, this had to be done by the user defining mass and energy sinks via control functions to subtract water from the control volume pool to balance the external spray injection. Since the spray is input as a volumetric flow at a specified temperature (at an undocumented reference pressure) while the mass and energy sinks are defined as a mass flow at a given enthalpy, there was significant potential for mass and energy conservation problems when closed, recirculating spray systems were modelled. This input upgrade was made in update $1.8 \mathrm{OI}$ in response to DIR 1208.

Note, however, that any fission product aerosols and/or vapors already deposited in the pool are left behind in the pool. Thus, MELCOR does not account for the increasing degradation in the capability of the recirculating solution to continually accumulate and remove more iodine as the solution accumulates iodine (owing to the variation of the equilibrium partial pressure of iodine with the square of the iodine concentration in solution).

\subsection{Sensitivity Coefficients Not Connected}

The first sensitivity studies varying the spray droplet terminal velocity and mass transfer correlation yielded identical results even when the sensitivity coefficients were varied over many orders of magnitude. Upon investigation, the code developers identified a coding error in that the spray package sensitivity coefficients listed in the documentation could be input, but then had no effect on the calculation because there was no coding to save and implement the sensitivity coefficients in the SPR package. This was corrected in update $1.8 \mathrm{OM}$ as part of DIR 1216.

\subsection{Iodine Re-evolution}

The first calculations for these CSE assessment analyses showed iodine removal by sprays from the test vessel atmosphere as discussed already, but then predicted that the iodine vapor would re-evolve from the liquid pools in the lower drywell and lower room sumps very quickly, returning to near the initial airborne iodine vapor concentration. This occurred because the water in the pool had no capability of continuing to bind the iodine vapor chemically. (Even without chemistry effects, re-evolution should be reduced because of transport resistance in the pool.)

This problem was noted by the code developers as DIR 1232, and the implementation of TRAP-MELT modelling for fission product condensation and evaporation was modified 
as of update $1.80 \mathrm{U}$ to disallow any evaporation of fission products residing in a control volume pool. This is considered a temporary modification and is expected to by replaced by the iodine chemistry model currently under development for MELCOR. Until this new model is implemented, note that MELCOR versions 1.8.2 and 1.8.3 could have very different fission product vapor responses calculated in control volumes with pools and sprays.

\subsection{Time Step Dependency}

An error was identified and corrected in the implementation of the model for aerosol removal by sprays. The error had resulted in incorrect aerosol removal rates being calculated, with a strong dependency on time step. This problem was reported in DIR 1268: and corrected in update $1.8 \mathrm{PM}$.

\subsection{I/O Improvements Needed}

This CSE assessment was made quite difficult because of a number of limits and omissions in the MELCOR input/output.

The spray package specifies injection in volumetric flow and temperature, for convenience in using plant data usually given in such form. This form, however, is inconsistent with input for other packages in MELCOR (such as mass and energy sources/sinks in CVH or liquid films in HS) where mass and enthalpy are the primary input variables. This leads to possible inconsistencies and problems in defining control functions because specifying volumetric flow and temperature only does not fully define a unique state for water. The documentation does not clarify whether the spray injection is assumed to be at saturation, at atmospheric pressure, or at the local, instantaneous pressure of the volume into which the injection is specified (or some other state).

The output (both printed, plotted and available control functions) for the spray package is also sparse and inadequate. Useful information would be droplet terminal velocity, fall time, equilibration time, temperature and size evolution for each characteristic droplet in the droplet size distribution. Steam condensation, heat transfer to steam and to noncondensibles, aerosol removal amounts and washout rates, and vapor removal amounts and decontamination rates should be given both for each characteristic droplet in the droplet size distribution and for the spray overall. Aerosol and vapor removal by different mechanisms (e.g., impaction, interception, diffusion, etc.) should be provided together with total removal.

The aerosol input requirements are also inconsistent. Aerosol sources vs time are input as distributions with a total mass and an associated AMMD and GSD. However, aerosols initially present can only be input as individual masses in each MAEROS section, with the user forced to make the conversion from a total mass with an associated AMMD and GSD. Further, it is impossible to define an initial aerosol particle size or size distribution for any fog (water aerosol) initially present in the atmosphere. It would have been very 
have been very helpful in our sensitivity studies to be able to define the fog particle size or AMMD/GSD size distribution (while letting CVH define the total fog mass in the atmosphere).

\subsection{Evaporation/Condensation}

The results of the sensitivity study on aerosol particle initial size presented in Section 7.4 showed that the test data on aerosol removal are matched much better by particles with an initial AMMD in the 2.5-5.0 $\mu \mathrm{m}$ range than by injecting the aerosol particles with an initial AMMD in the 0.5-1.0 $\mu \mathrm{m}$ range as specified in the experiment report [21].

In reality, the aerosol particles are probably larger at the start of the spray injection transient, not because larger cesium and uranium particles were injected but because the hygroscopic cesium aerosols at least would be expected to grow very quickly to about $5.0 \mu \mathrm{m}$ by condensation of water onto the small particles initially injected; the effect is probably smaller for the uranium particles but still occurs to some extent [26].

MELCOR does model condensation/evaporation effects on aerosol particle size, but does not include any model for hygroscopic effects. The lack of such a model has been noted in previous MELCOR assessments of aerosol behavior in humid atmospheres [3, 27]. However, in our reference calculation, condensation/evaporation was specified to take place only on water aerosols and not on all aerosols present (as in the code default), to avoid other modelling problems.

The reference calculation, with condensation/evaporation specified to take place only on water aerosols, predicts the most aerosol removal by sprays overall. Allowing condensation/evaporation to take place on any aerosols present does predict more aerosol removal during the first spray, which is in better agreement with test data. This would be expected because the cesium and uranium aerosol particles could grow somewhat before the start of the first spray owing to condensation in this case although, without hygroscopicity taken into account, the growth is not great enough to match the test data. However, allowing MELCOR to treat condensation onto the cesium and uranium aerosols results in greater discrepancies after the water aerosols evaporate.

With condensation onto all aerosols present, the mass median diameters of the cesium and uranium aerosols drop precipitously when the water aerosols evaporate. When condensation occurs on all aerosols, MELCOR assumes that all the aerosol particles are identical. In this case, there is a relatively large amount of water and relatively large number of water aerosol particles in the humid atmosphere early in the transient, relative to the cesium and uranium. Therefore, all aerosol particles would be assumed to have a thick water film over a small cesium/uranium core, rather than a smaller number of cesium and uranium particles having a thin water film and most of the aerosol particles being pure fog droplets. Thus, after the water film evaporates, a large number of small cesium and uranium aerosol particles have been artificially created from a smaller number of larger cesium and uranium particles. This is a known problem that has been identified in other MELCOR analyses (e.g., [28]). 
There are thus several problems in MELCOR with representation of condensation/evaporation effects on aerosol behavior. Hygroscopic effects should be added to the code model to correctly account for aerosol growth in humid atmospheres. Also, some corrections and improvements need to be developed to allow reasonable aerosol particle behavior after evaporation of water in the default MELCOR treatment of condensation/evaporation onto all aerosols present. 


\section{Summary and Conclusions}

The MELCOR computer code has been used to analyze several of the CSE containment spray experiments performed in the Containment Systems Experiment vessel, in order to evaluate the performance of aqueous sprays as a means of decontaminating containment atmospheres. This report documents the results of those analyses.

MELCOR results have been compared with test data, and a number of sensitivity studies on input modelling parameters and options in both the spray package and the associated aerosol washout and atmosphere decontamination by sprays modelled in the $\mathrm{RN}$ package have been done. The results of these assessment analyses demonstrate that MELCOR correctly reproduces the qualitative thermal/hydraulic, aerosol washout and vapor decontamination response to containment spray injection. In particular, MELCOR reproduces the relative responses observed when the spray flow rate and droplet size distribution are varied. Also, the accuracy and reasonableness of the predicted results generally improve as more MAEROS components and sections are used to model the aerosol size distributions, as would be expected. These CSE results confirm the previous MELCOR assessment guideline [3] that water drops in the atmosphere should be represented by a separate component in transients with a humid atmosphere.

There are, however, a number of quantitative differences between the MELCOR results and the test data. Some of the differences are due to uncertainties or inconsistencies in the test data. Others are due to code assumptions or inadequacies, some of which have been or will be addressed.

The quantitative differences in the thermal/hydraulic response are generally attributable to the assumption in MELCOR that the sprays injected into a volume are immediately fully mixed, and that the spray droplets fall through a volume atmosphere at rest, leading to overestimation of steam condensation by the sprays. This is a basic assumption in the HECTR spray model [24] implemented in MELCOR.

The quantitative differences in aerosol washout are attributable primarily to known problems in modelling interaction of aerosol particles with fog (water aerosols) in the atmosphere through evaporation and condensation. The default modelling in the code, with evaporation/condensation on all aerosols provides better agreement with test data during condensation periods but leads to too-small aerosol particles after evaporation (as noted in other MELCOR assessments [28]); an option in MELCOR to consider only condensation/evaporation onto existing water aerosols causes opposite errors. The lack of any model in MELCOR representing hygroscopic effects also affects the ability to correctly predict cesium aerosol response (as noted in other MELCOR assessments [3, 27]).

The quantitative differences in elemental iodine vapor removal are attributable partly to the lack of iodine chemistry modelling in MELCOR. MELCOR currently has no builtin capability to model methyl iodine (a moderately soluble and much less reactive vapor) or other iodine forms, or to model details of iodine chemistry such as interaction with sprays containing different additives. Many of these deficiencies will be addressed in 
the iodine chemistry model being added in the next code version after MELCOR 1.8.3. Among other deficiencies, the first calculations for these CSE assessment analyses predicted that the iodine vapor would re-evolve from the liquid pools very quickly, returning to near the initial airborne iodine vapor concentration because the water in the pool had no capability of continuing to bind the iodine vapor chemically in the current MELCOR coding. There are also effects of pool transport and surface evaporation not captured by TRAP-MELT, such as the effect of dilution in the pool which reduces the vapor pressure. This problem was noted by the code developers and the implementation of TRAP-MELT modelling for fission product condensation and evaporation was modified to totally disallow any evaporation of fission products residing in a control volume pool. This is considered a temporary modification and is expected to be replaced by the iodine chemistry model currently under development for MELCOR. (Because of this change, note that MELCOR versions 1.8.2 and 1.8.3 could have very different fission product" vapor responses calculated in control volumes with pools and sprays. Neither can be expected to be "correct" because the behavior is probably intermediate between the two limiting extremes. The current approximation of retaining vapors in pools is probably a better representation of the real situation, where most of the iodine is expected to be retained in the pool.)

Also, MELCOR does not account for any fission product aerosol or vapor "loading" in recirculating sump water. A new feature was added in MELCOR 1.8.3 in the spray package that allows the user to specify a control volume from which to extract water for recirculating sprays. (In previous code versions, this had to be done by the user defining mass and energy sinks via control functions to subtract water from the control volume pool to balance the external spray injection.) However, any fission product aerosols and/or vapors already deposited in the pool are left behind in the pool. For example, any iodine collected by the sprays remains behind, locked in the liquid pool, as the water from that pool is recirculated by the spray package. Thus, MELCOR does not account for any pre-existing binding of iodine with reactants such as borax or sodium thiosulpfate in recirculating sump water, which might degrade further iodine removal capability.

Time step and machine-dependency calculations were done to identify whether any numeric effects exist in these CSE assessment analyses. A significant time step dependency caused by an error in the spray package coding was identified and eliminated. A number of other code deficiencies and inconveniences also are noted, some of which have been addressed. 


\section{Bibliography}

[1] R. M. Summers et al., "MELCOR 1.8.0: A Computer Code for Severe Nuclear Reactor Accident Source Term and Risk Assessment Analyses", NUREG/CR-5531, SAND90-0364, Sandia National Laboratories, January 1991.

[2] C. D. Leigh, ed.,"MELCOR Validation and Verification - 1986 Papers", NUREG/ CR-4830, SAND86-2689, Sandia National Laboratories, March 1987.

[3] L. N. Kmetyk, "MELCOR 1.8.1 Assessment: LACE Aerosol Experiment LA4", SAND91-1532, Sandia National Laboratories, September 1991.

[4] L. N. Kmetyk, "MELCOR 1.8.1 Assessment: FLECHT SEASET Natural Circulation Experiments", SAND91-2218, Sandia National Laboratories, December 1991.

[5] L. N. Kmetyk, "MELCOR 1.8.1 Assessment: ACRR Source Term Experiments ST1/ST-2", SAND91-2833, Sandia National Laboratories, April 1992.

[6] L. N. Kmetyk, "MELCOR 1.8.1 Assessment: LOFT Integral Experiment LP-FP-2", SAND92-1273, Sandia National Laboratories, December 1992.

[7] R. J. Gross, "MELCOR 1.8.1 Assessment: PNL Ice Condenser Experiments", SAND92-2165, Sandia National Laboratories, June 1993.

[8] L. N. Kmetyk, "MELCOR 1.8.1 Assessment: Marviken-V Aerosol Transport Tests ATT-2b/ATT-4", SAND92-2243, Sandia National Laboratories, January 1993.

[9] T. J. Tautges, "MELCOR 1.8.2 Assessment: the DF-4 BWR Fuel Damage Experiment", SAND93-1377, Sandia National Laboratories, October 1993.

[10] L. N. Kmetyk, "MELCOR 1.8.2 Assessment: IET Direct Containment Heating Tests", SAND93-1475, Sandia National Laboratories, October 1993.

[11] L. N. Kmetyk, "MELCOR 1.8.2 Assessment: Surry PWR TMLB' (with a DCH Study)", SAND93-1899, Sandia National Laboratories, February 1994.

[12] T. J. Tautges, "MELCOR 1.8.2 Assessment: the MP-1 and Mp-2 Late Phase Melt Progression Experiments", SAND94-0133, Sandia National Laboratories, May 1994.

[13] L. N. Kmetyk, "MELCOR 1.8.3 Assessment: GE Large Vessel Blowdown and Level Swell Experiments", SAND94-0361, Sandia National Laboratories, July 1994.

[14] R. K. Hilliard, A. K. Postma, J. D. McCormack, L. F. Coleman, "Removal of Iodine and Particles by Sprays in the Containment Systems Experiment", Nuc. Tech. 10, April 1971, pp. 499-519.

[15] L. F. Coleman, "Preparation, Generation and Analysis of Gases and Aerosols for the Containment Systems Experiment", BNWL-1001, Battelle-Northwest, April 1969. 
[16] J. D. McCormack, "Maypack Behavior in the Containment Systems Experiment: A Penetrating Analysis", BNWL-1145, Battelle-Northwest, September 1969.

[17] C. E. Linderoth, "Containment Containment Systems Experiment, Part I: Description of Experiment Facilities", BNWL-456, Battelle-Northwest, March 1970.

[18] J. G. Knudsen, R. K. Hilliard, "Fission Product Transport by Natural Processes in Containment Vessels", BNWL-943, Battelle-Northwest, January 1969.

[19] R. K. Hilliard, L. F. Coleman, C. E. Linderoth, J. D. McCormack, A. K. Postma, "Removal of Iodine and Particles from Containment Atmospheres by Sprays - Containment Systems Experiment Interim Report", BNWL-1244, Battelle-Northwest, February 1970.

[20] A. K. Postma, L. F. Coleman, "Effect of Continuous Spray Operation on the Removal of Aerosols and Gases in the Containment Systems Experiment", BNWL1244, Battelle-Northwest, December 1970.

[21] R. K. Hilliard, A. K. Postma, "Effect of Spray Flow Rate on Washout of Gases and Particles in the Containment Systems Experiment", BNWL-1591, BattelleNorthwest, July 1971.

[22] R. K. Hilliard, L. F. Coleman, "Natural Transport Effects on Fission Product Behavior in the Containment Systems Experiment", BNWL-1457, Battelle-Northwest, December 1970.

[23] L. F. Parsly, "Spray Program at the Nuclear Safety Pilot Plant", Nuc. Tech. 10, April 1971, pp. 472-485.

[24] S. E. Dingman, "HECTR Version 1.5 User's Manual", NUREG/CR-4507, SAND860101, Sandia National Laboratories, April 1986.

[25] F. Gelbard, "MAEROS User Manual", NUREG/CR-1391, SAND80-0822, Sandia National Laboratories, December 1982.

[26] Private communication from Dana Powers, SNL, August 1994.

[27] F. J. Souto, F. E. Haskin, "MELCOR 1.8.2 Assessment: Aerosol Experiments ABCOVE AB5, AB6, AB7 and LACE LA2" (thesis, University of New Mexico), SAND94-2166, Sandia National Laboratories, October 1994.

[28] N. B. Siccama, "MELCOR Assessment Analyses at ECN", presentation at 2nd MCAP Technical Meeting, Bethesda, April 27-29, 1994.

[29] R. C. Smith et al., "Radionuclide Package Users' Guide", Version 1.8.1, April 30, 1991.

[30] B. E. Boyack, V. K. Dhir, J. A. Gieseke, T. J. Haste, M. A. Kenton, M. KhatibRahbar, M. T. Leonard, R. Viskanta, "MELCOR Peer Review", LA-12240, Los Alamos National Laboratory, March 1992. 


\section{A CSE A-9 Reference Calculation Input Deck}

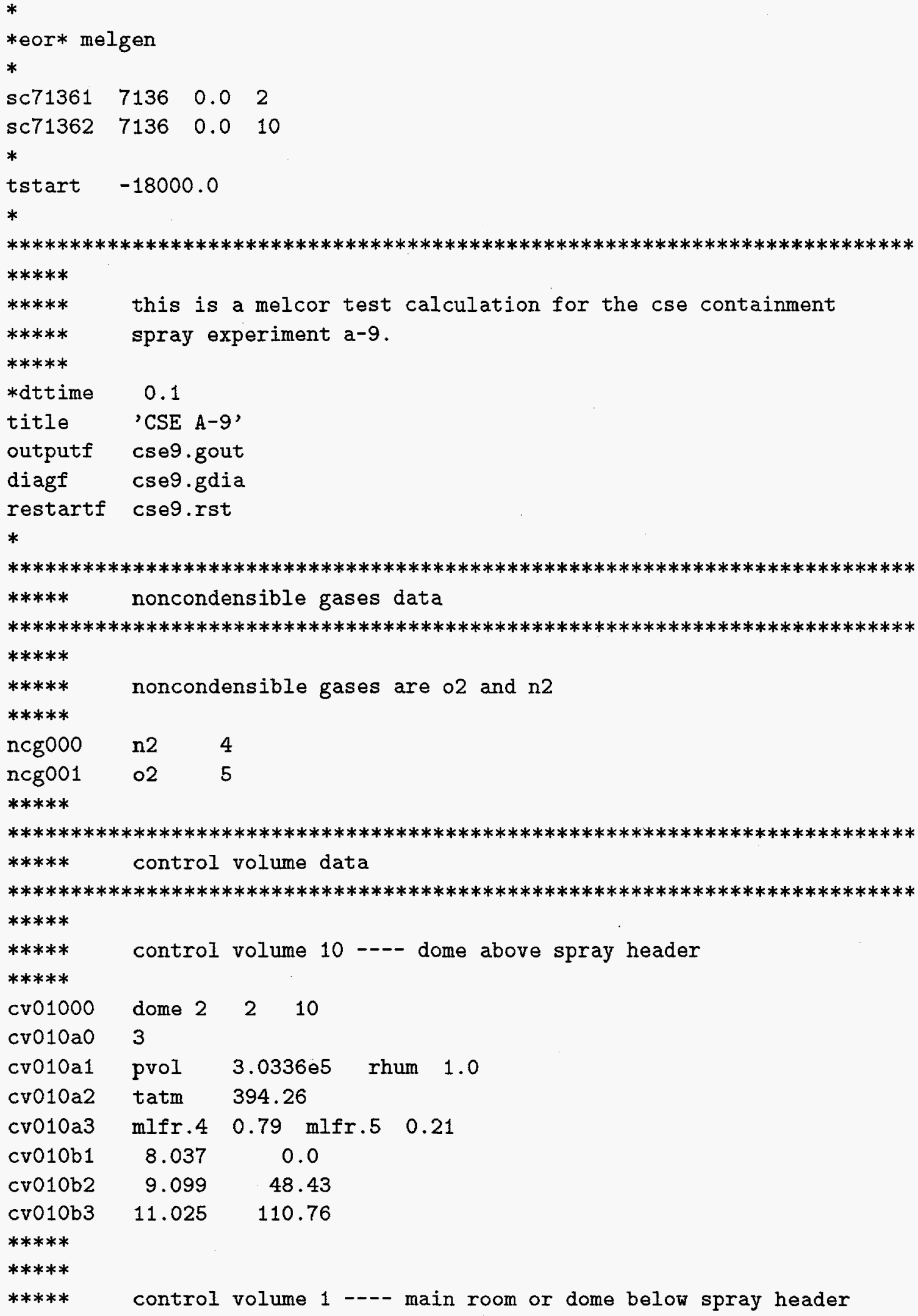




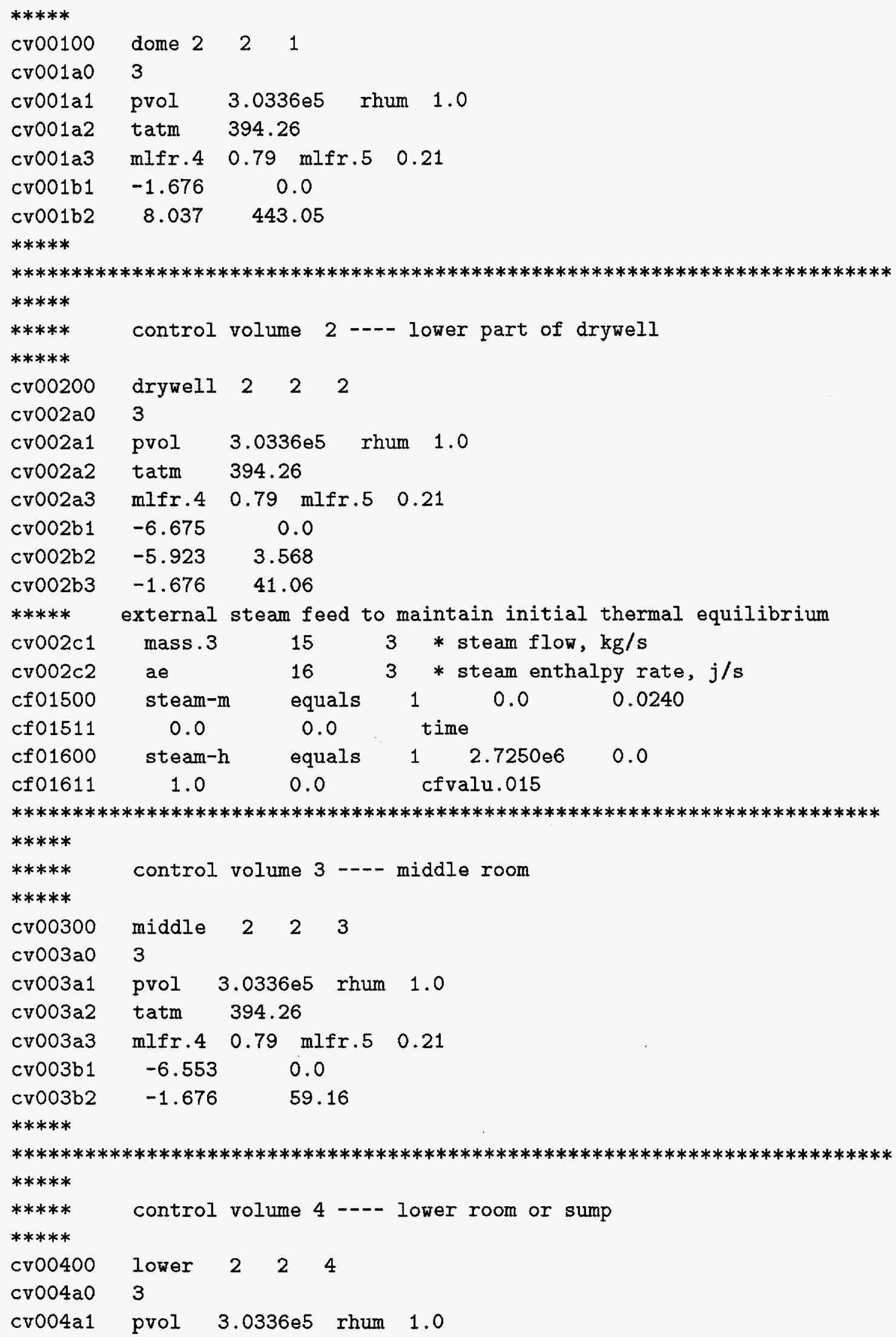




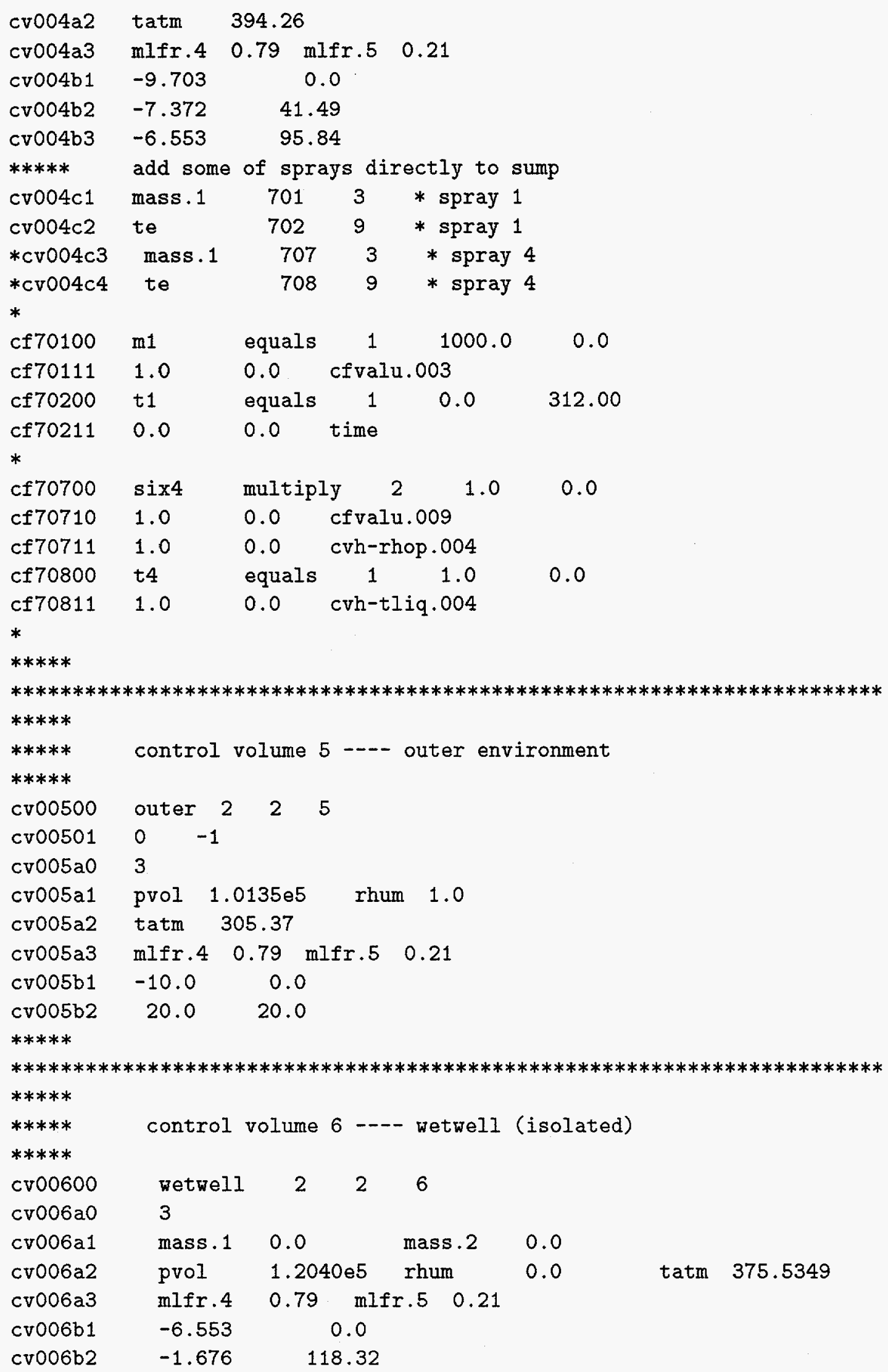




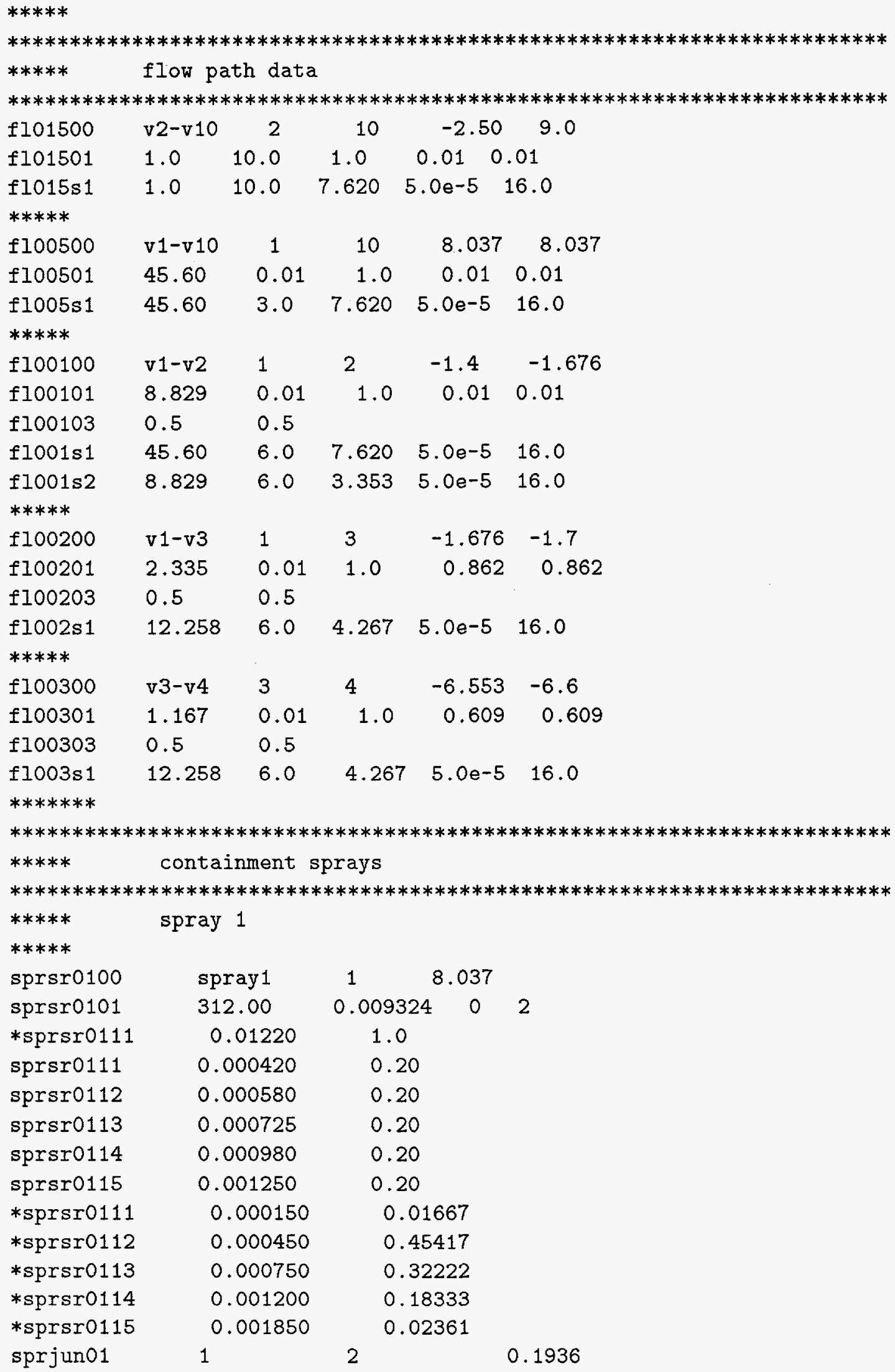




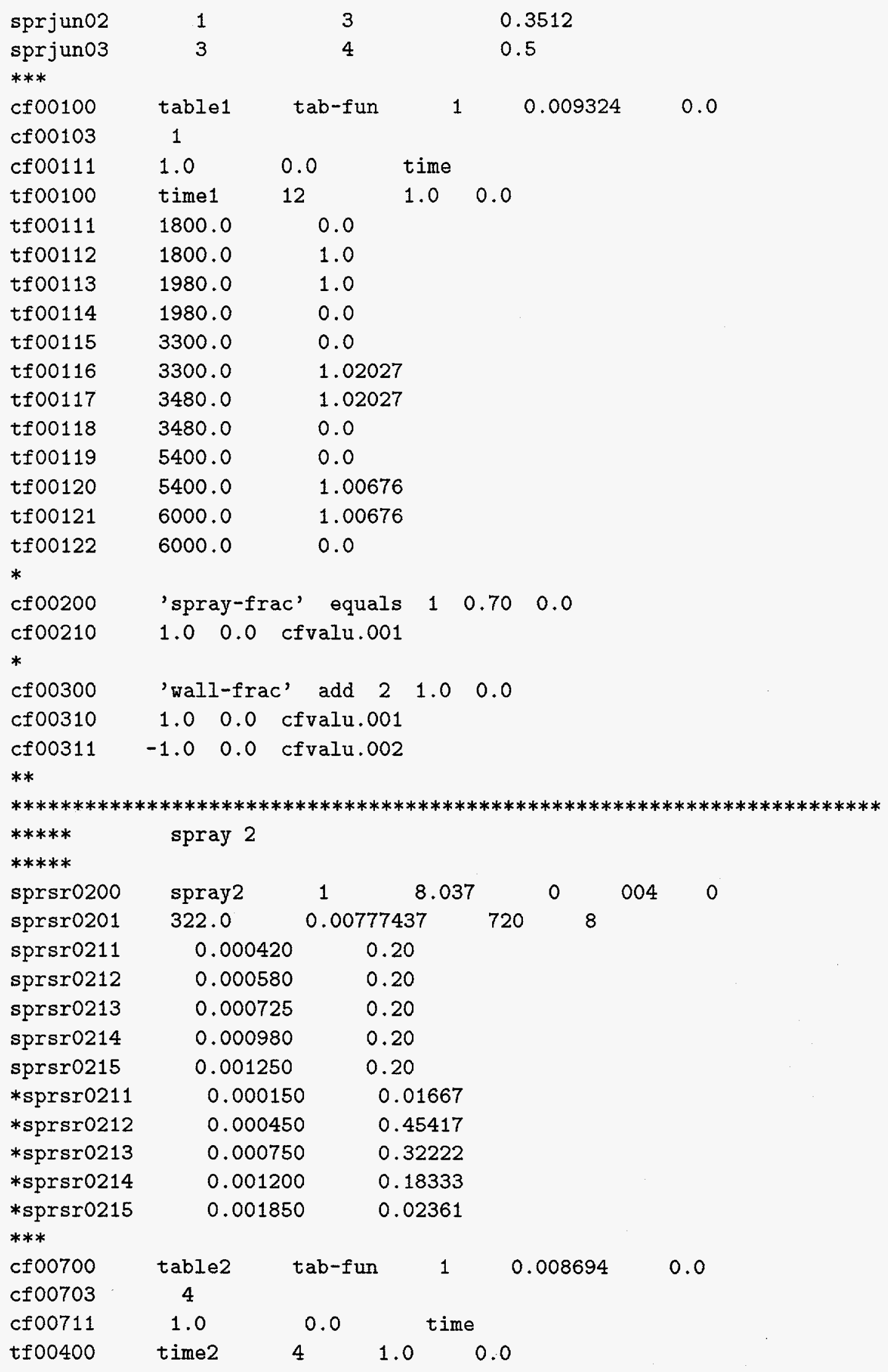




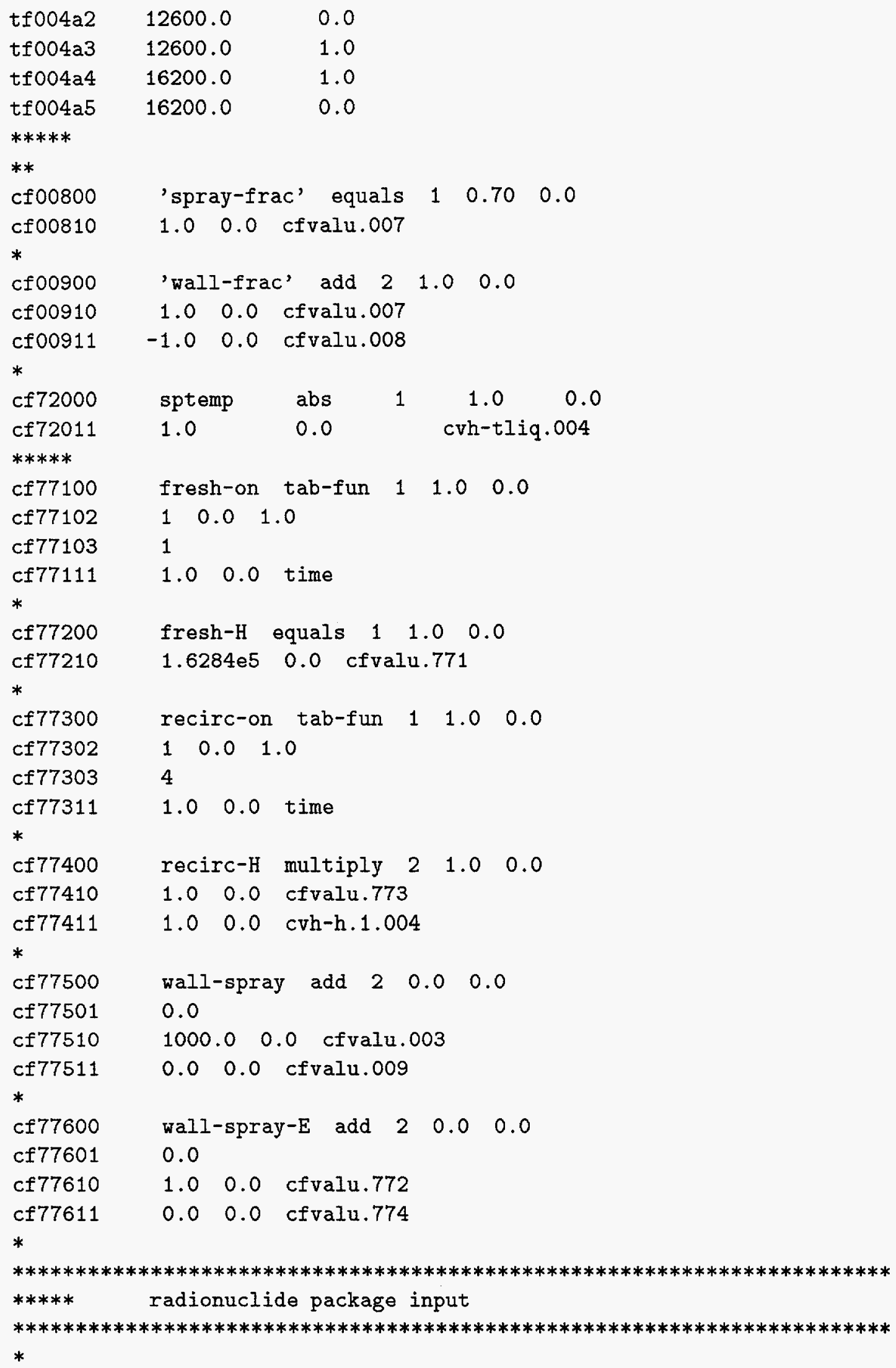




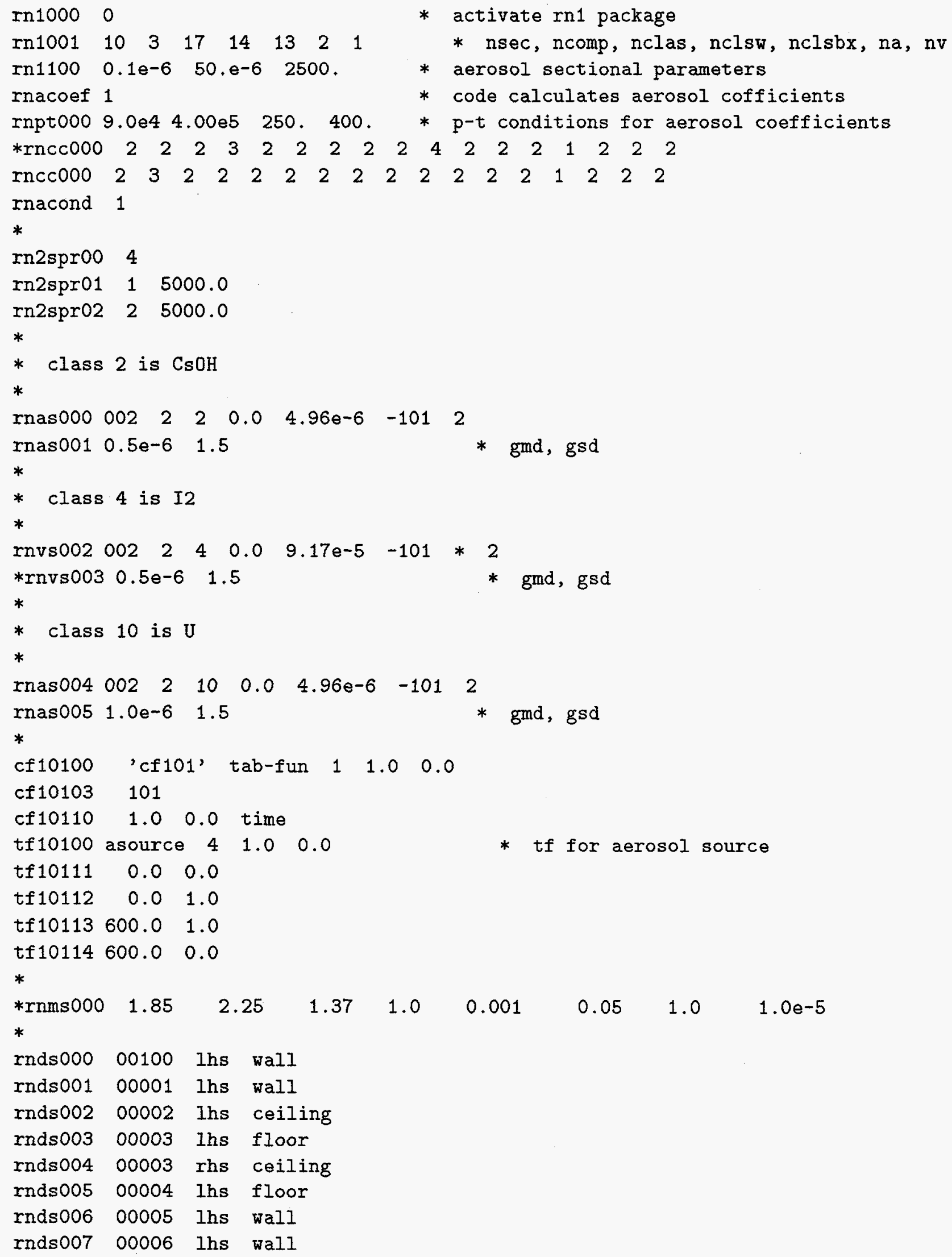




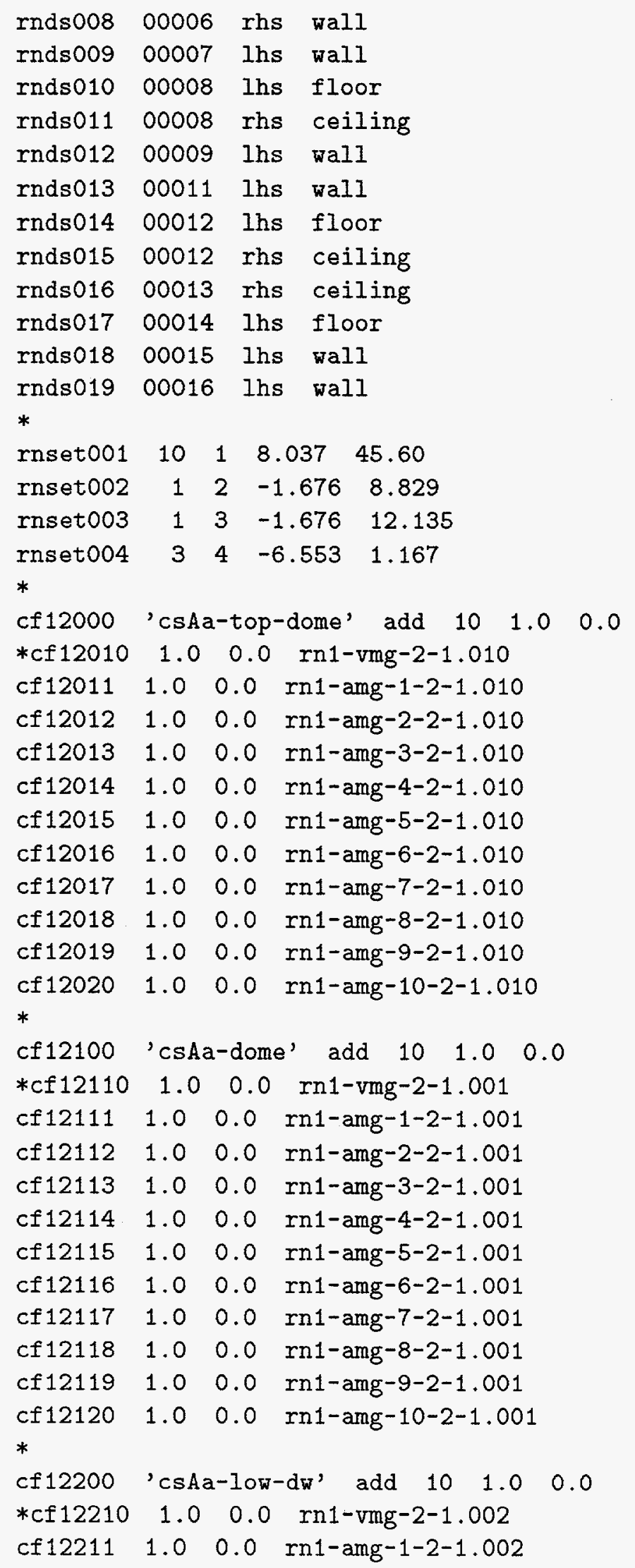




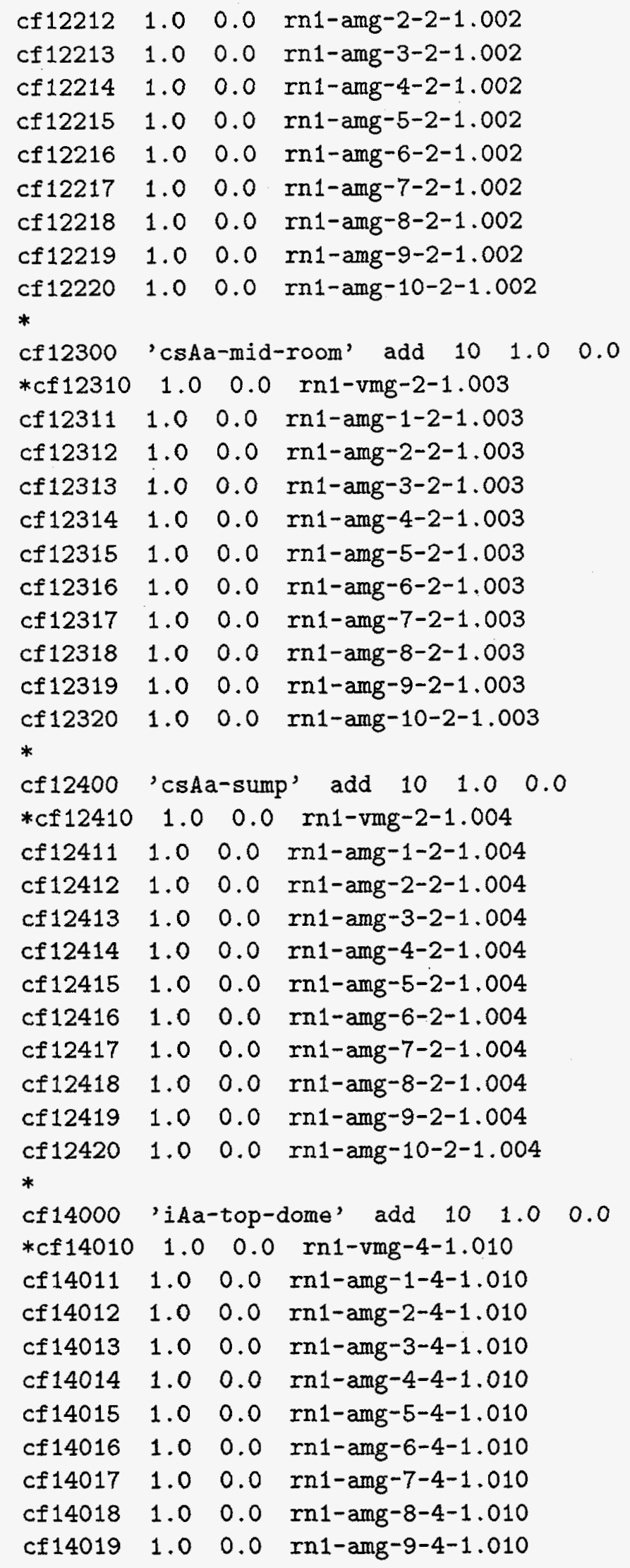




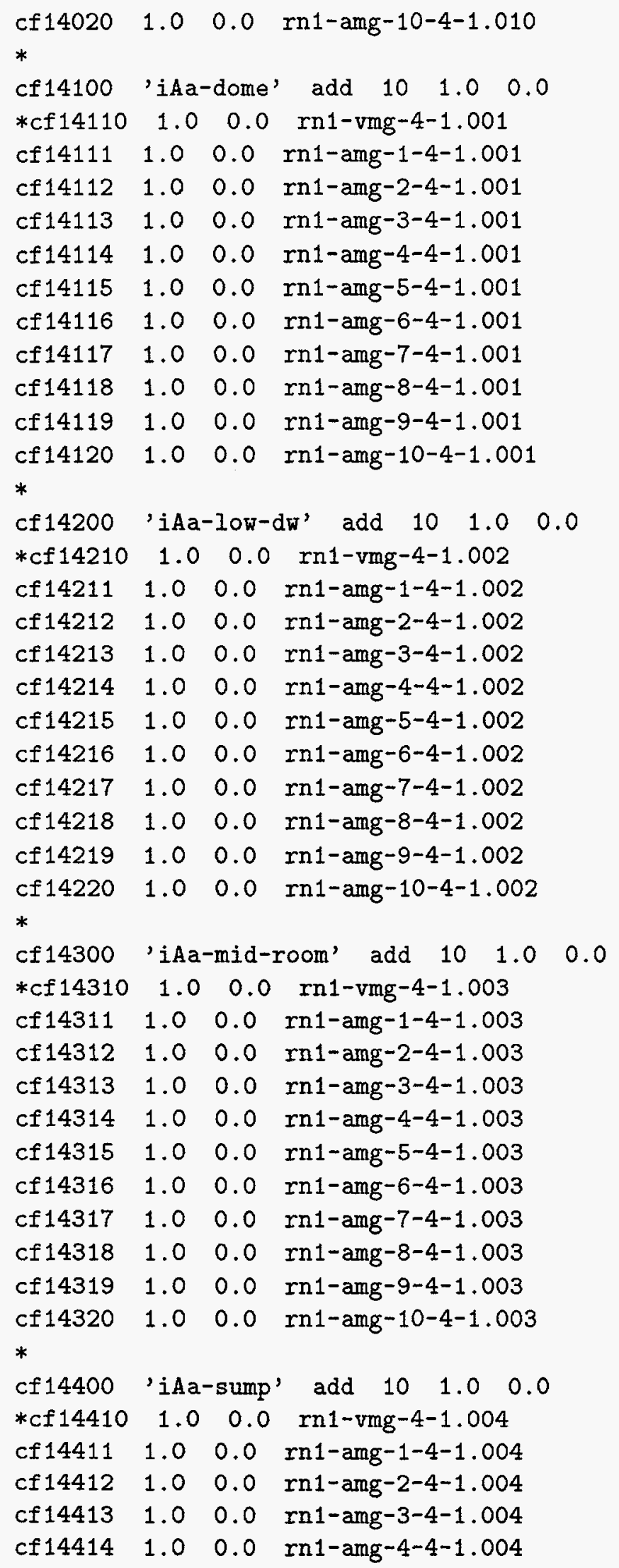




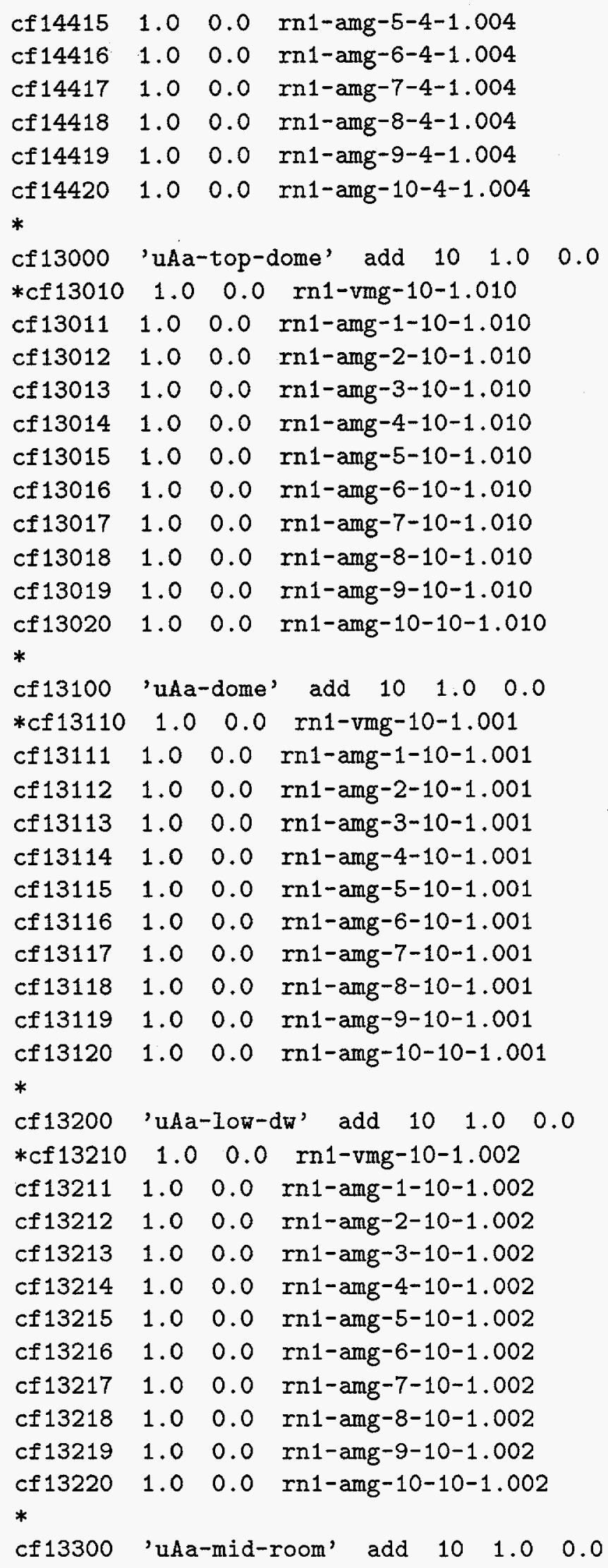




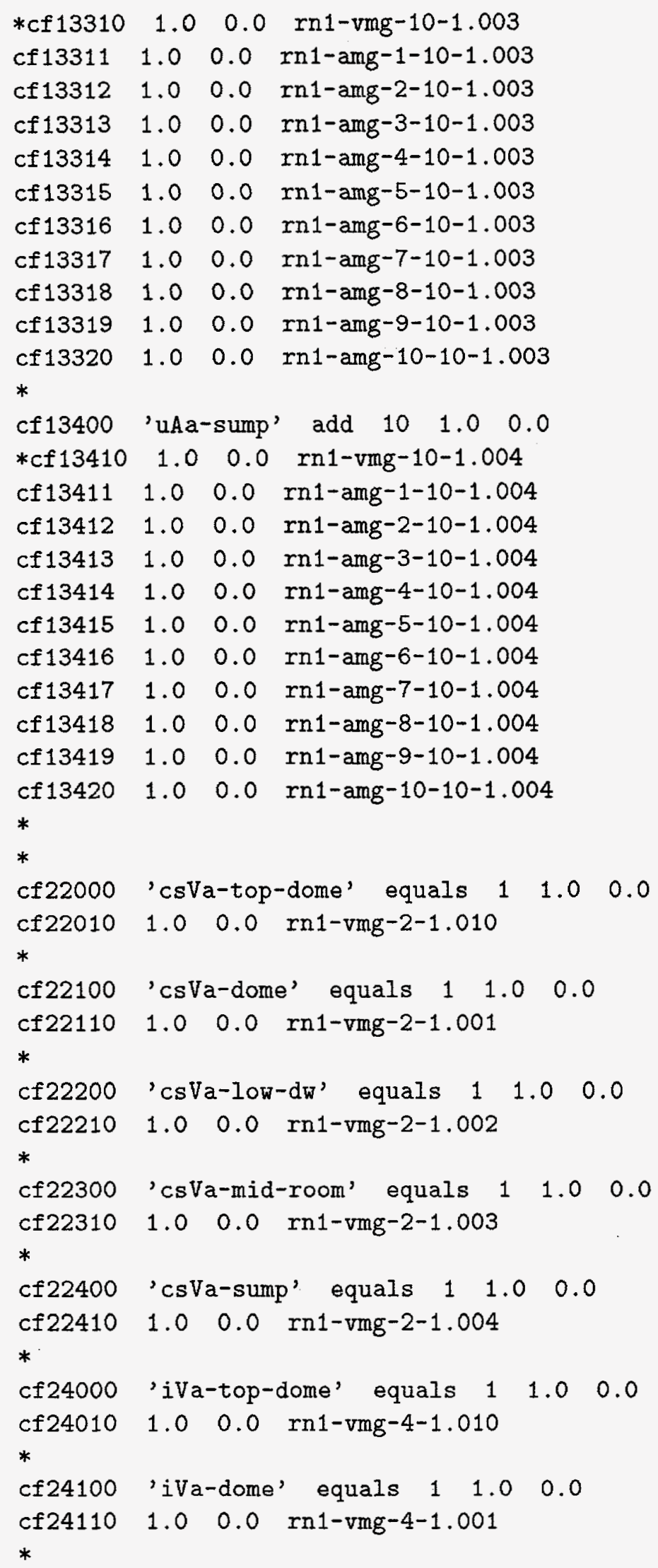




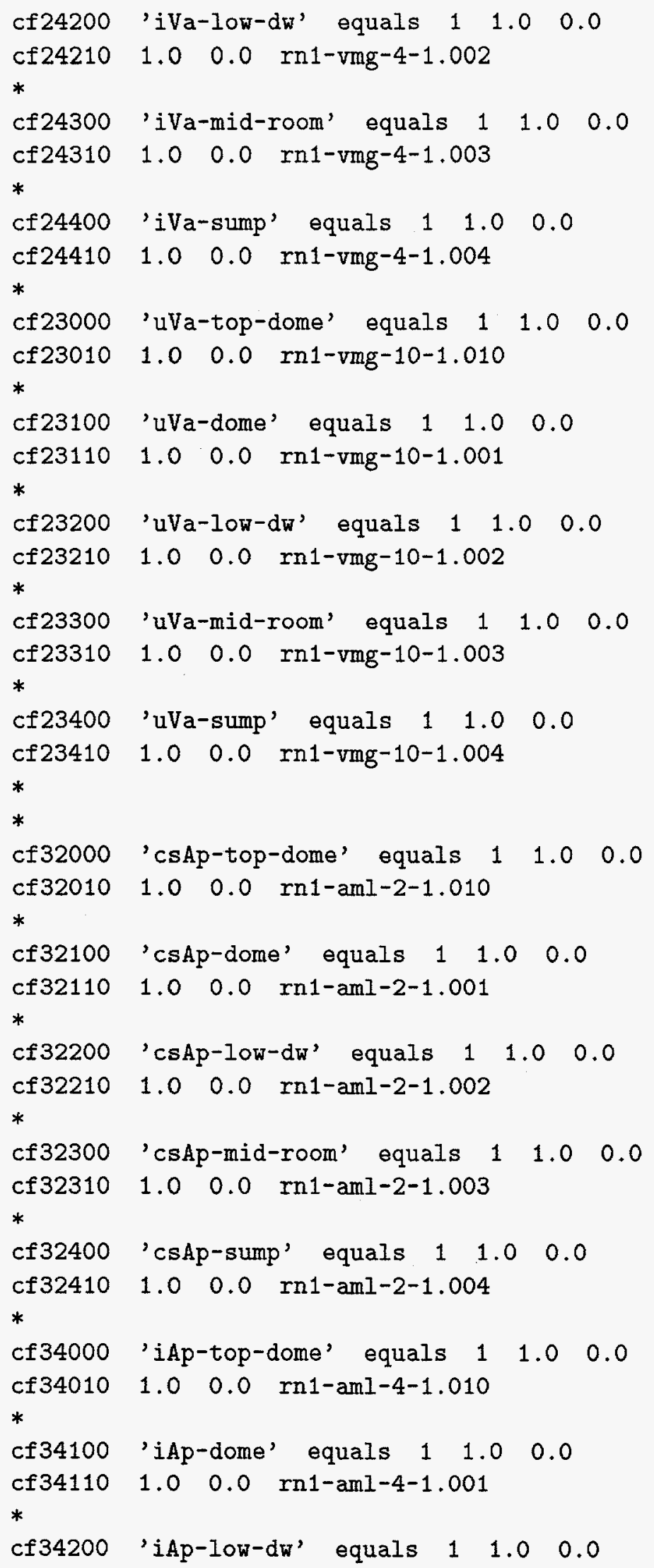




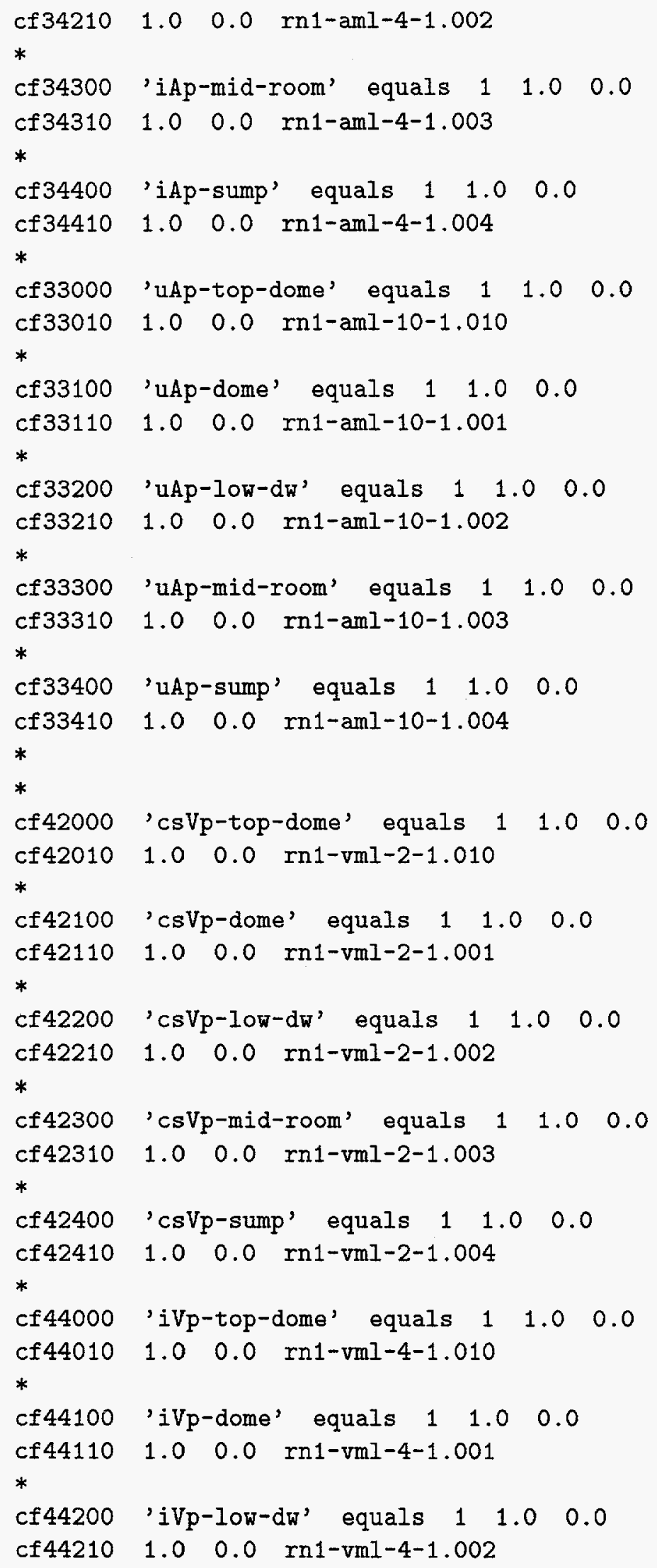




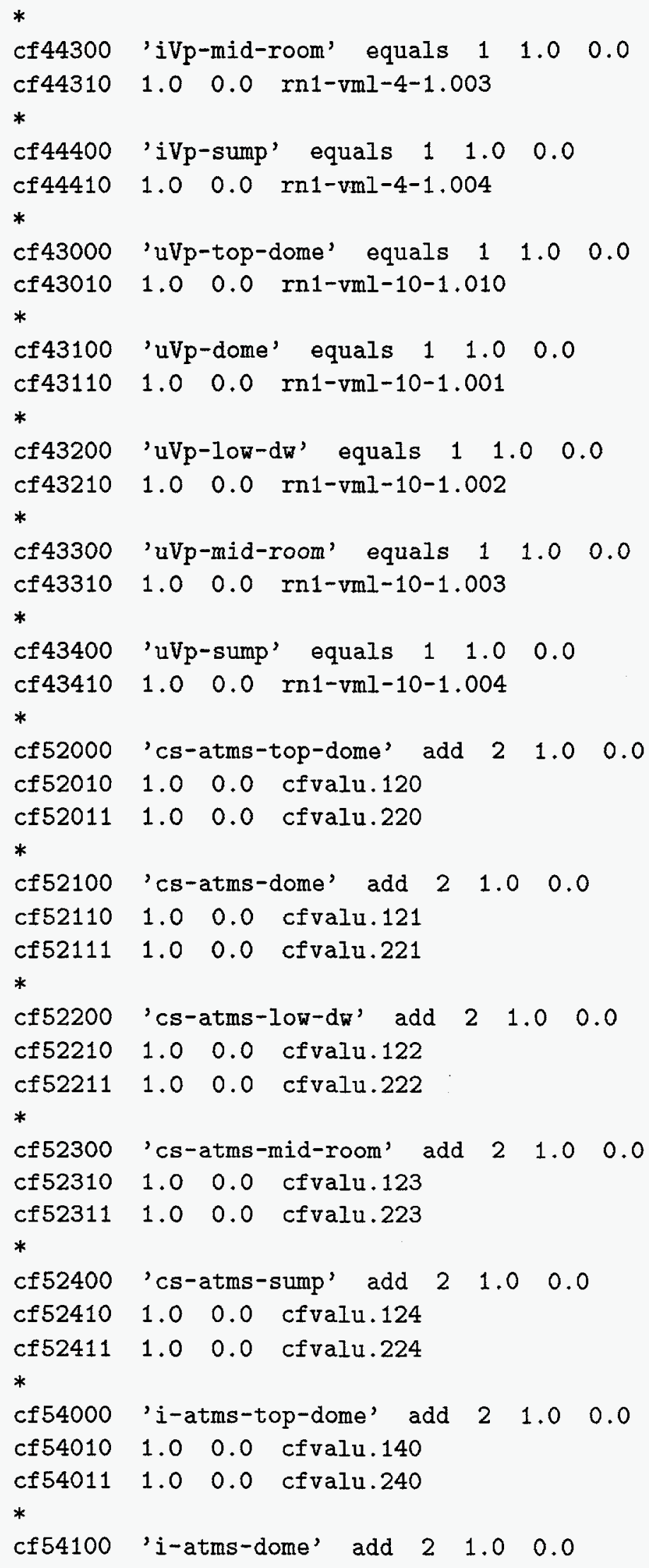




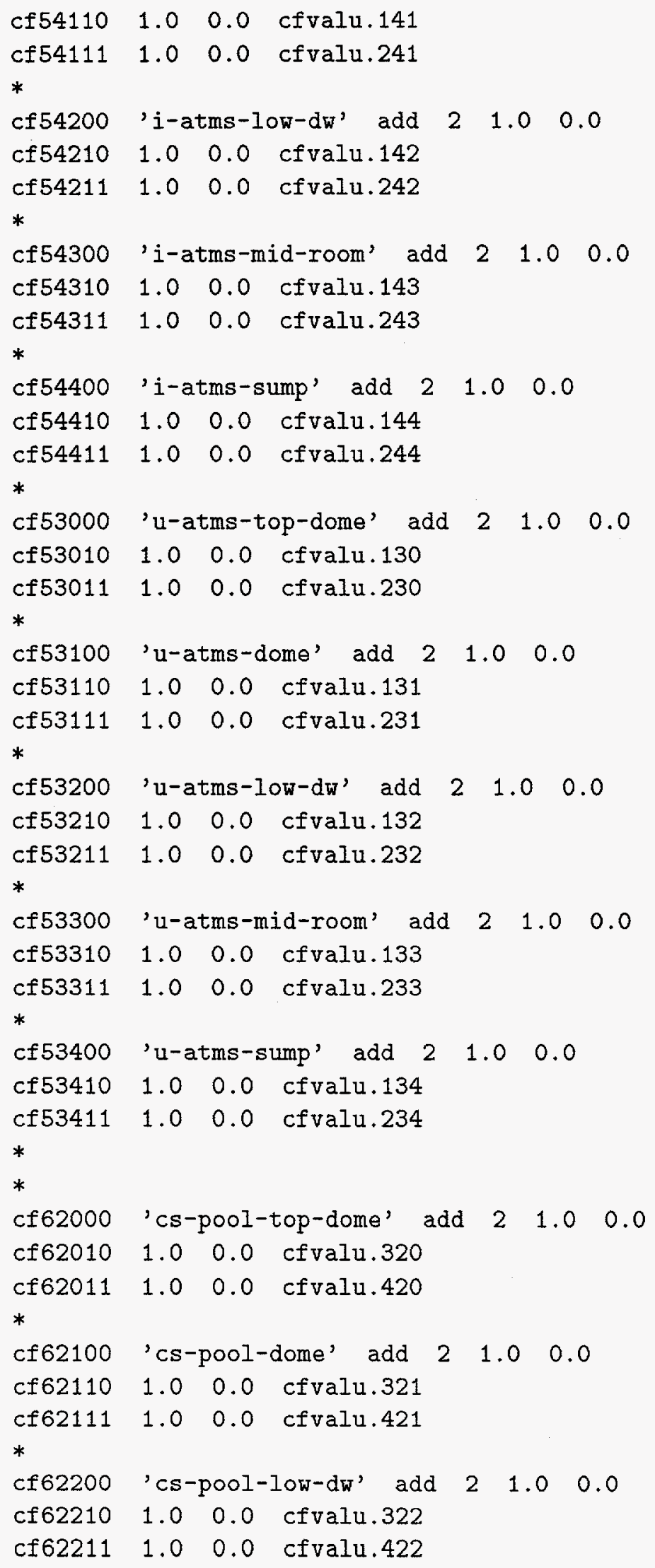




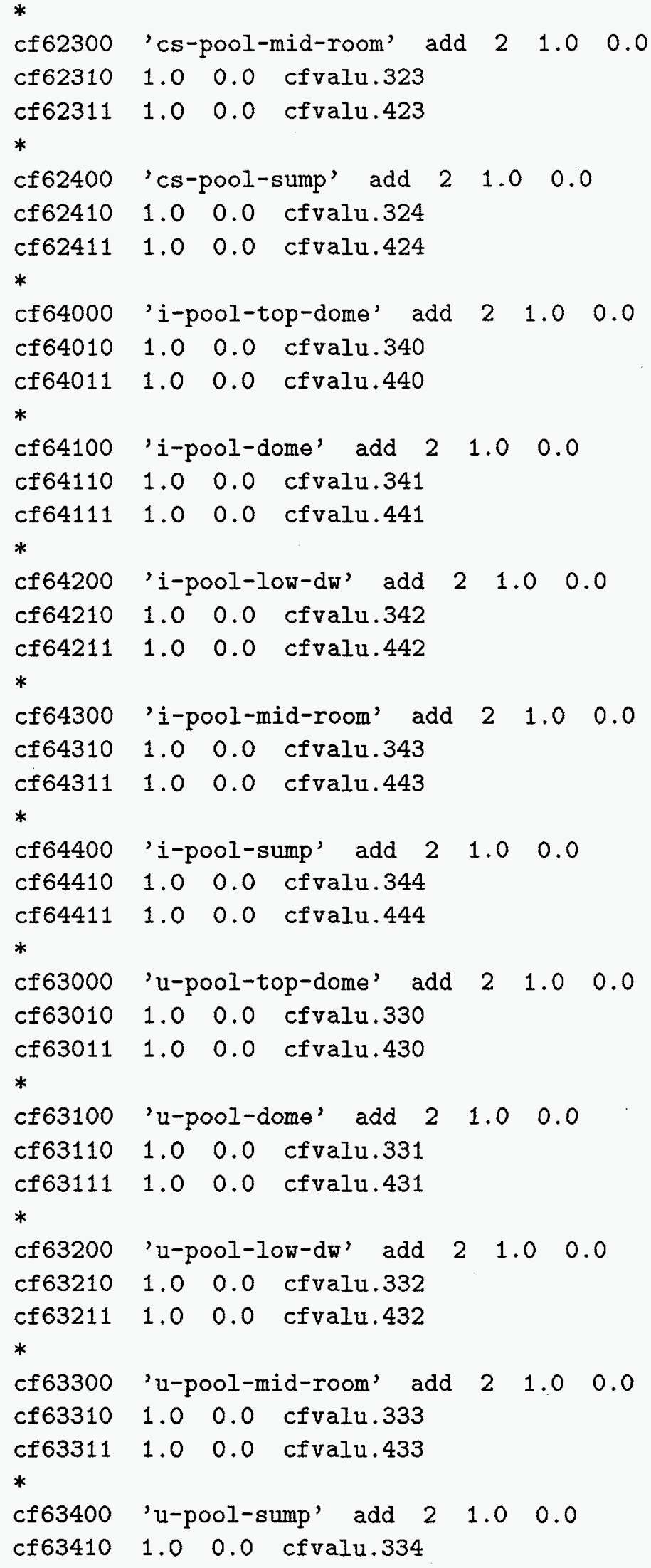




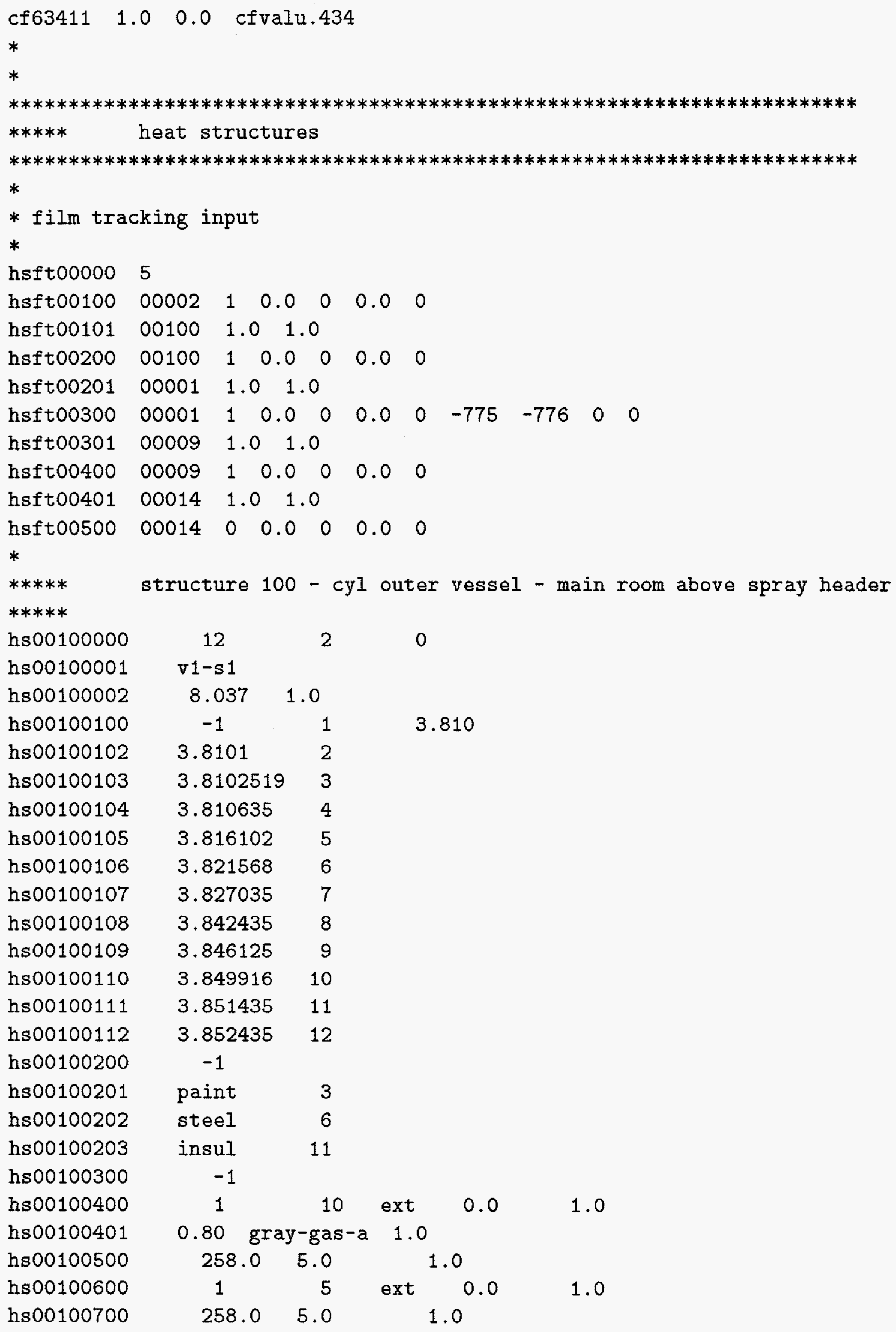




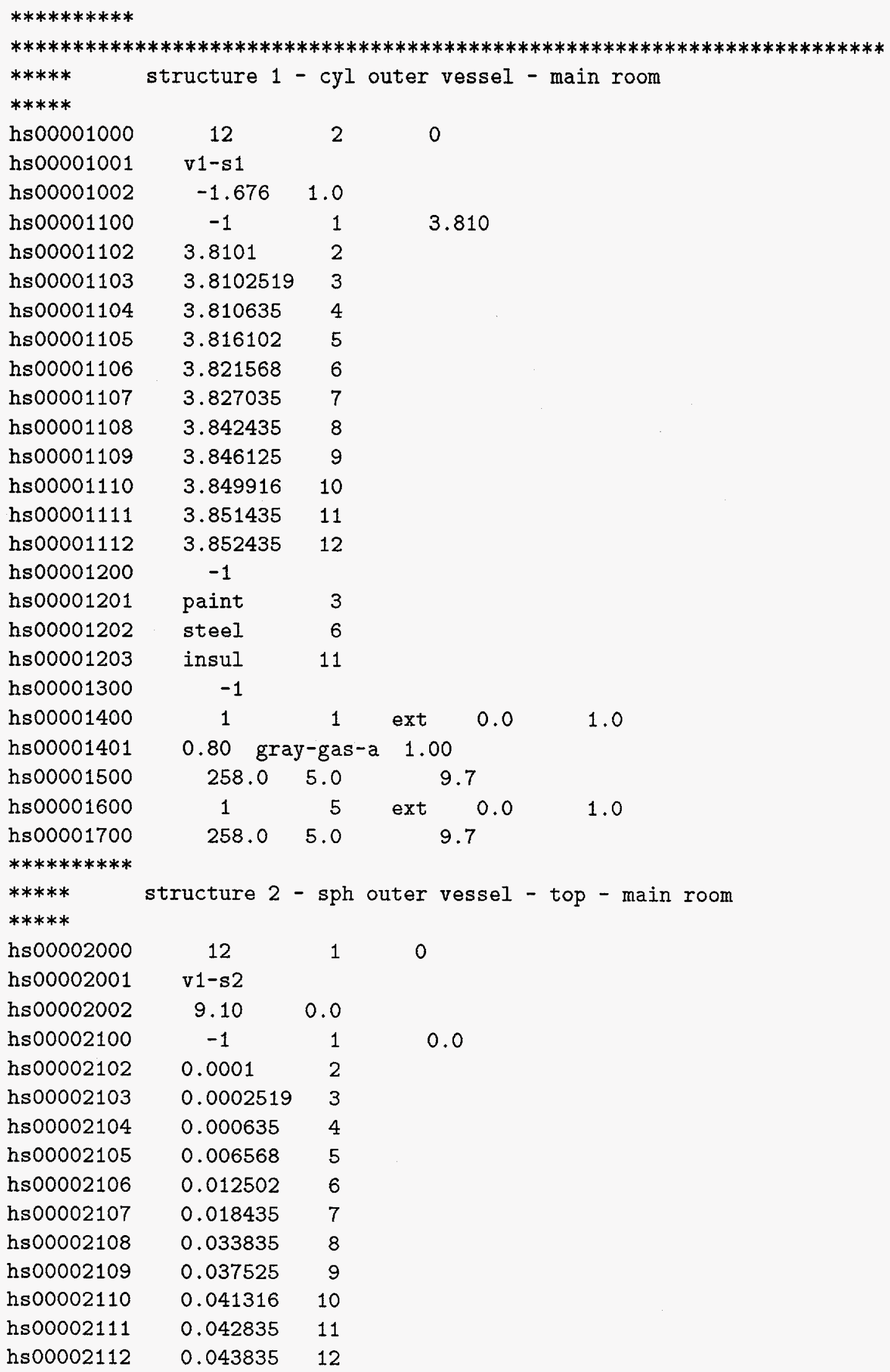




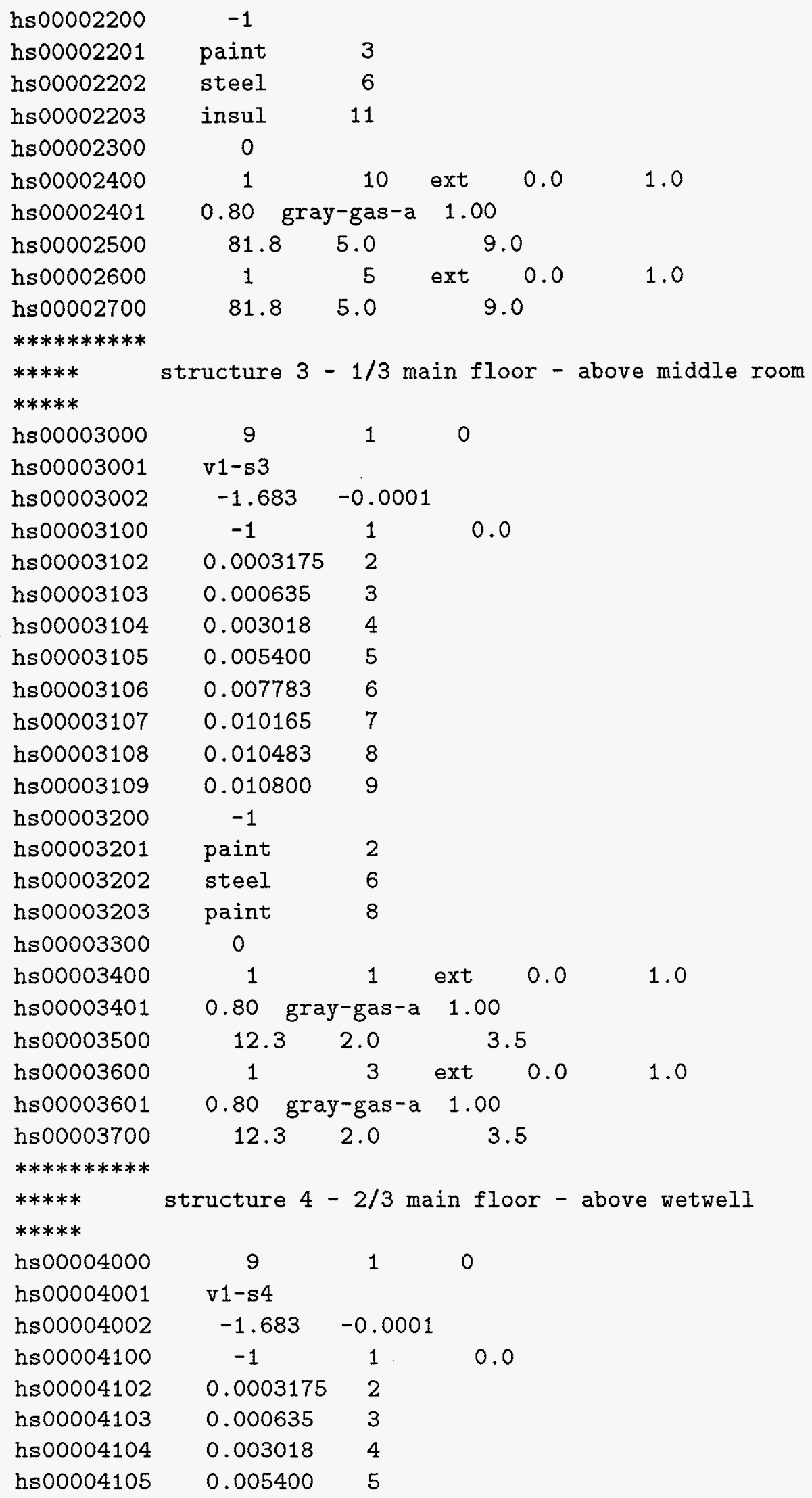




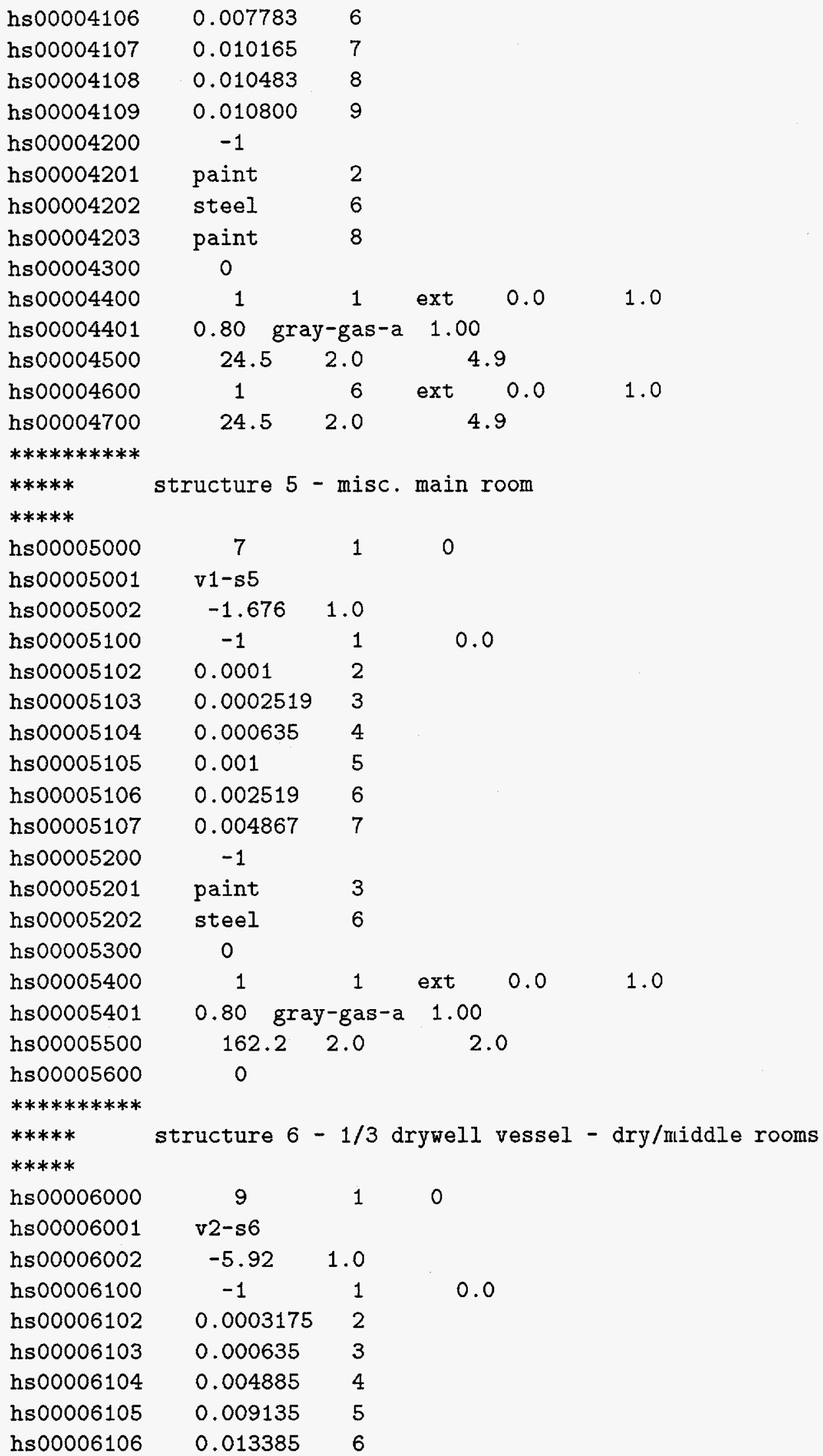




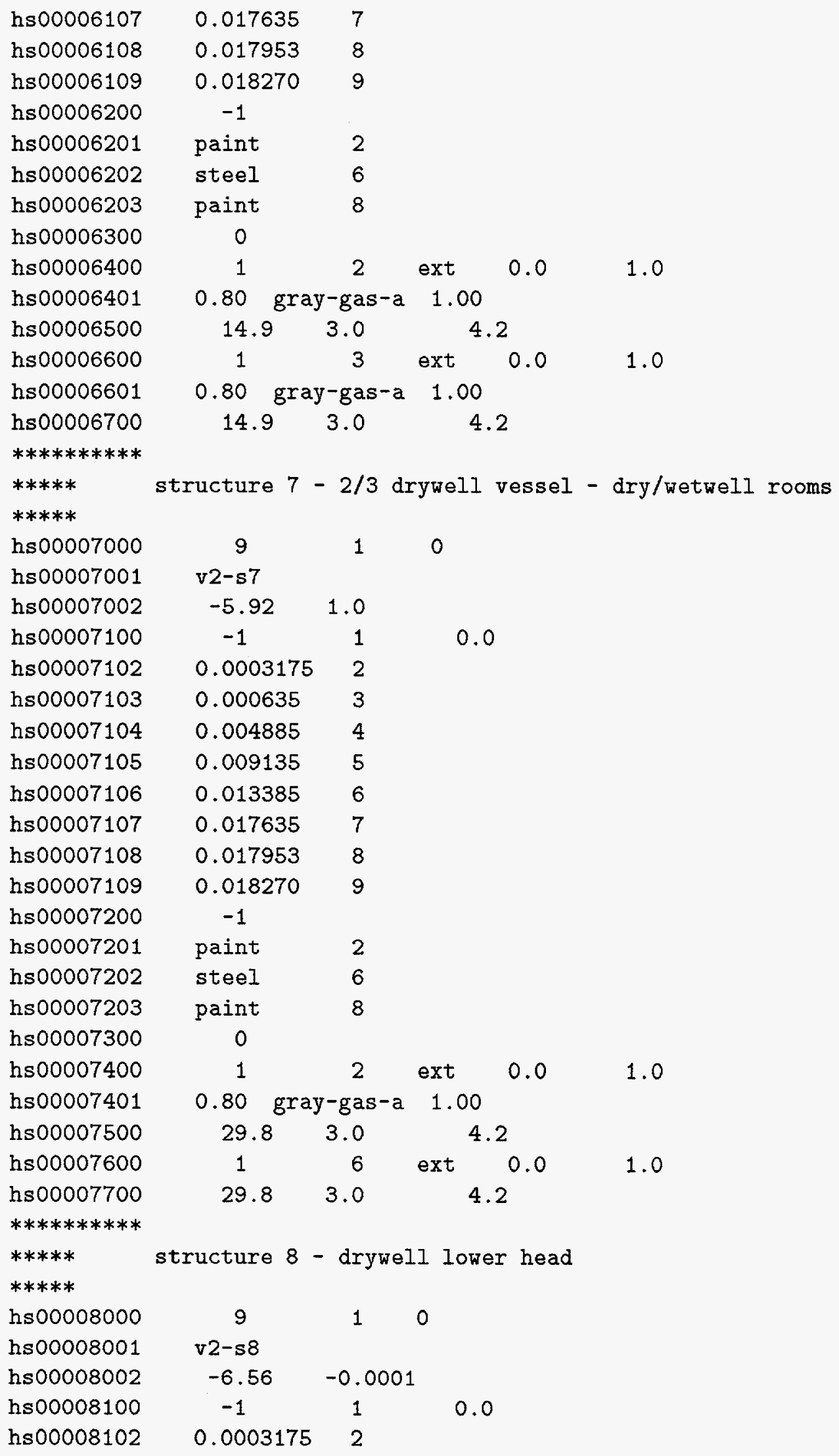




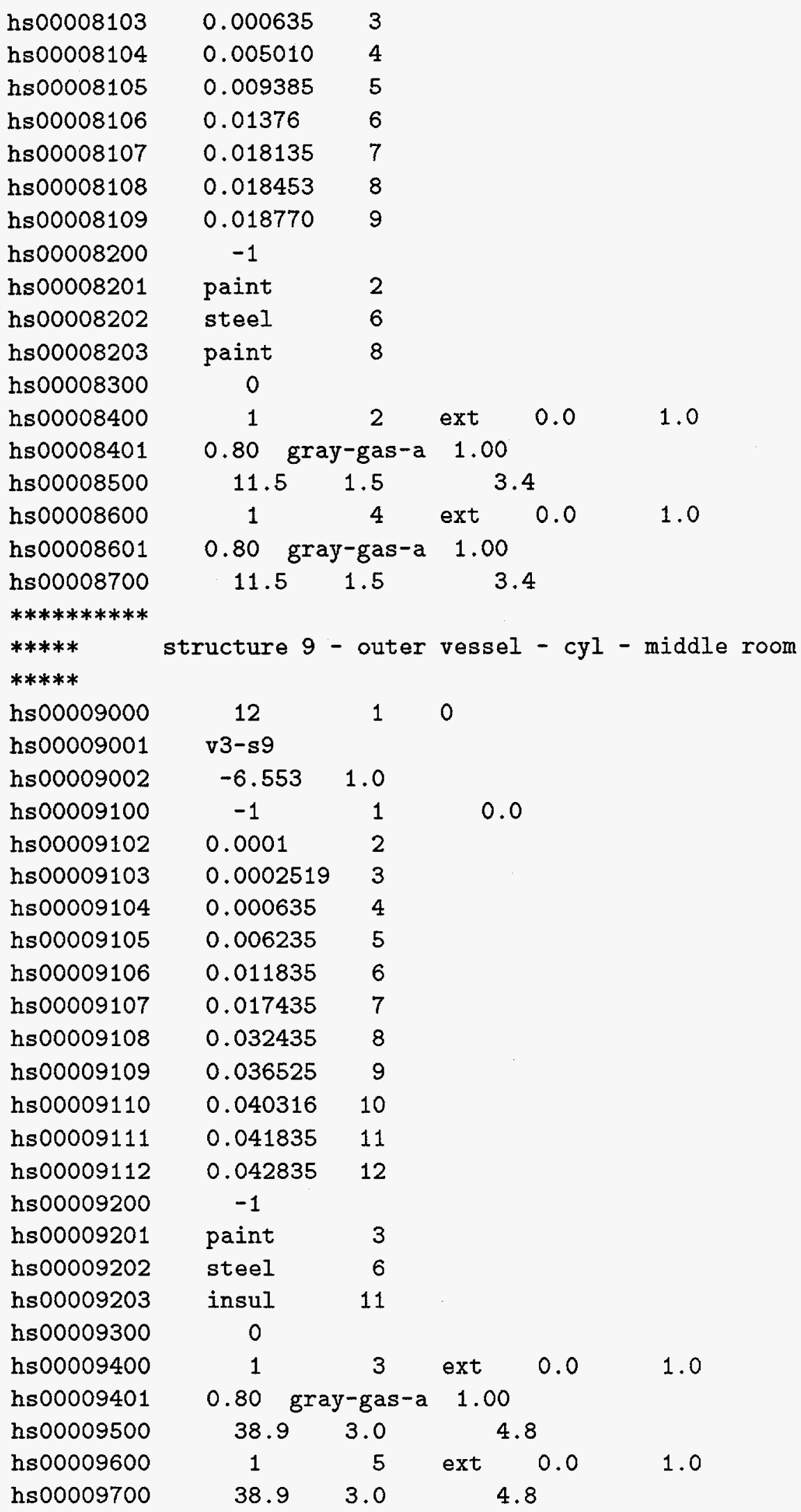




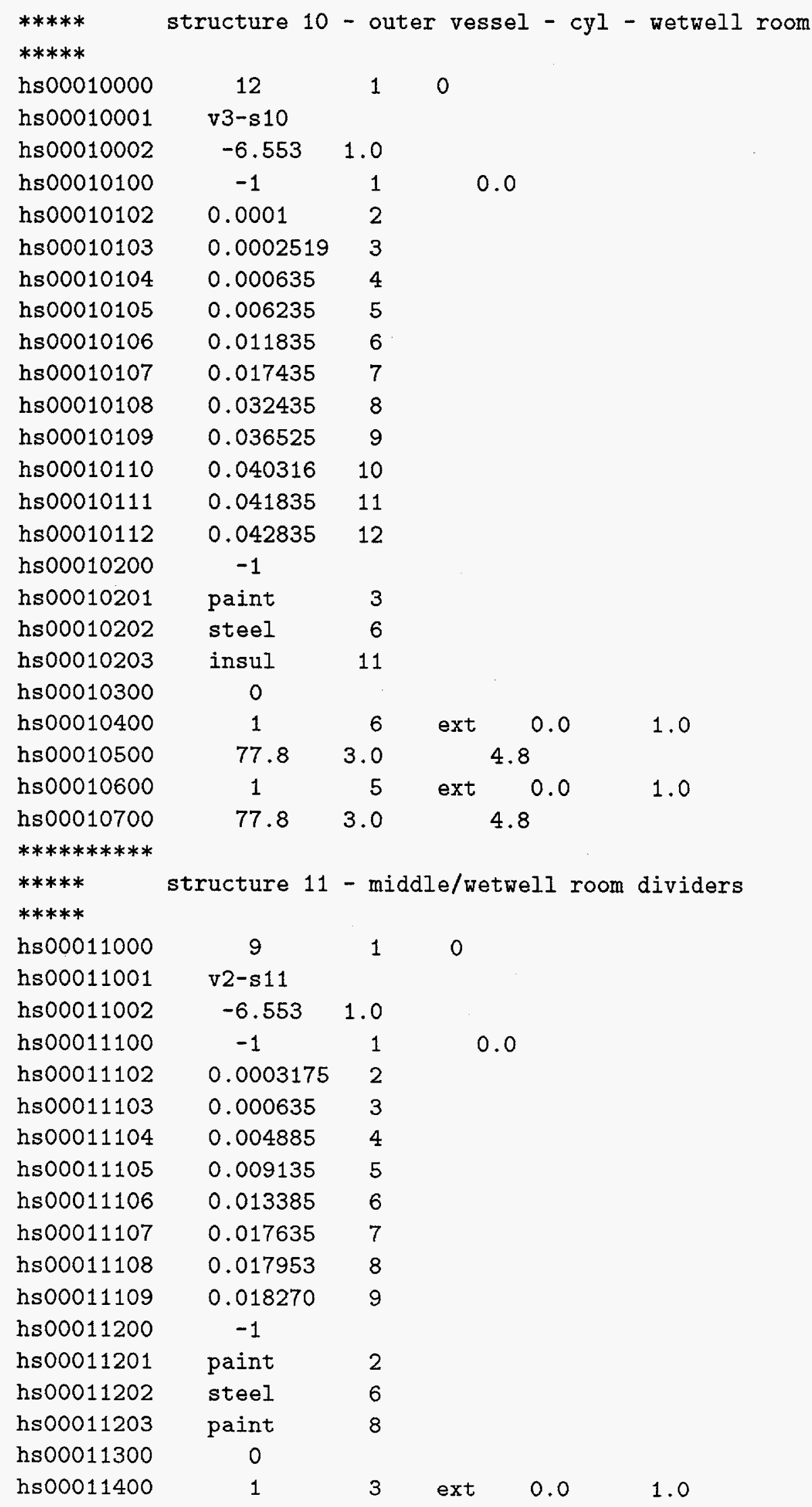




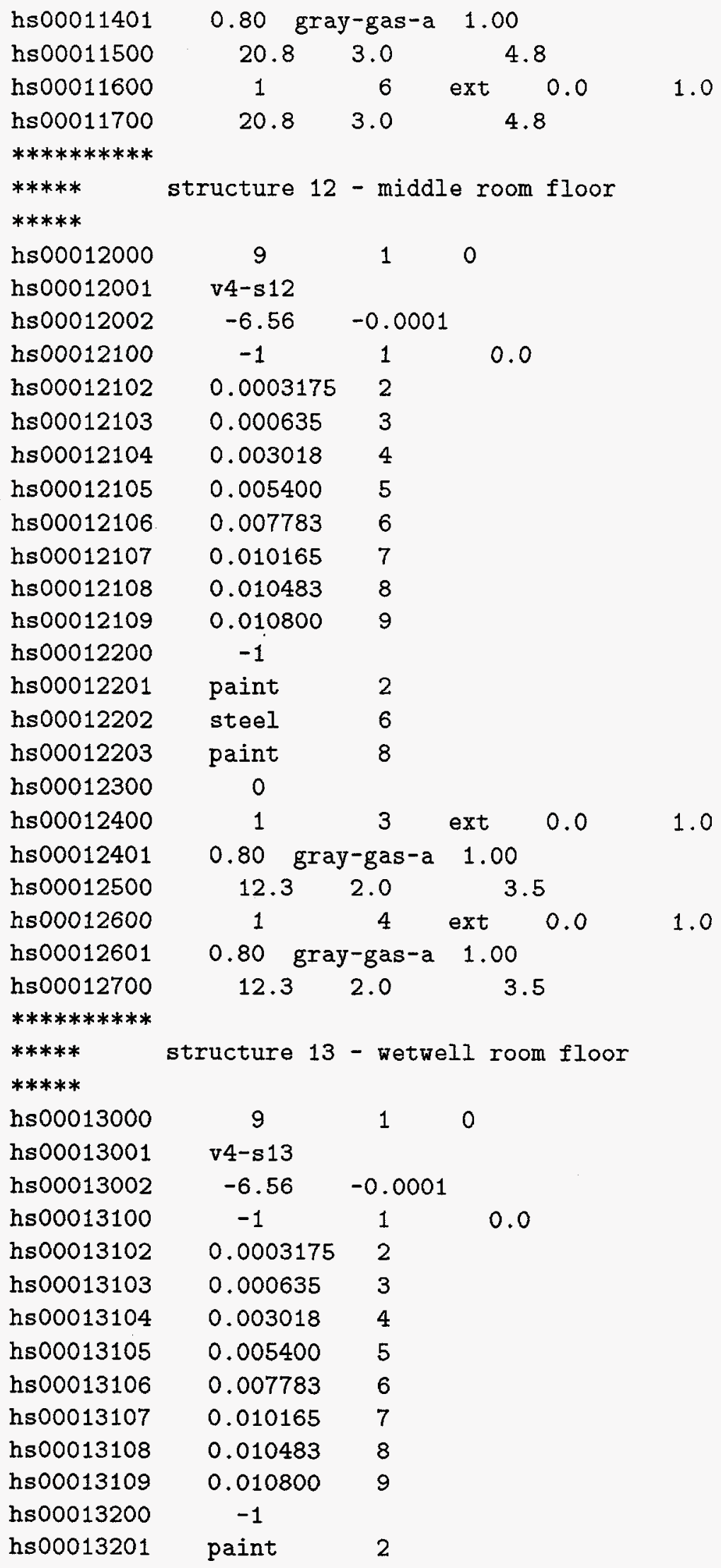




\begin{tabular}{|c|c|c|c|c|}
\hline hs00013202 & steel & 6 & & \\
\hline hs 00013203 & paint & 8 & & \\
\hline hs00013300 & 0 & & & \\
\hline hs00013400 & 1 & 6 & 0.0 & 1.0 \\
\hline hs00013500 & 24.5 & 2.0 & 4.9 & \\
\hline hs00013600 & 1 & 4 & ext $\quad 0.0$ & 1.0 \\
\hline hs00013601 & \multicolumn{2}{|c|}{0.80 gray-gas-a } & 1.00 & \\
\hline hs 00013700 & 24.5 & 2.0 & 4.9 & \\
\hline \multicolumn{5}{|l|}{$* * * * * * * * * *$} \\
\hline$* * * * *$ & \multirow{2}{*}{ structure 14} & - outer & \multirow[t]{2}{*}{ r vessel lower } & head \\
\hline \multicolumn{3}{|c|}{ ( } & & \\
\hline hs00014000 & 12 & 1 & 0 & \\
\hline hs 00014001 & v4-s 14 & & & \\
\hline hs00014002 & -9.69 & -0.01 & \multirow[b]{2}{*}{0.0} & \\
\hline hs00014100 & -1 & 1 & & \\
\hline hs00014102 & 0.0001 & 2 & & \\
\hline hs00014103 & 0.0002519 & 3 & & \\
\hline hs00014104 & 0.000635 & 4 & & \\
\hline hs 00014105 & 0.006568 & 5 & & \\
\hline hs 00014106 & 0.012502 & 6 & & \\
\hline hs 00014107 & 0.018435 & 7 & & \\
\hline hs00014108 & 0.033835 & 8 & & \\
\hline hs00014109 & 0.037525 & 9 & & \\
\hline hs00014110 & 0.041316 & 10 & & \\
\hline hs00014111 & 0.042835 & 11 & & \\
\hline hs00014112 & 0.043835 & 12 & & \\
\hline hs 00014200 & -1 & & & \\
\hline hs00014201 & paint & 3 & & \\
\hline hs00014202 & steel & 6 & & \\
\hline hs00014203 & insul & 11 & & \\
\hline hs 00014300 & 0 & & & \\
\hline hs 00014400 & 1 & 4 & 0.0 & 1.0 \\
\hline hs 00014401 & 0.80 gray & $y$-gas-a & 1.00 & \\
\hline hs00014500 & 101.4 & 3.0 & 9.0 & \\
\hline hs 00014600 & 1 & 5 & ext 0.0 & 1.0 \\
\hline hs00014700 & 101.4 & 3.0 & 9.0 & \\
\hline$* * * * * * * * * *$ & & & & \\
\hline$* * * * *$ & structure 15 & - misc. & middle room & \\
\hline$* * * * *$ & & & & \\
\hline hs00015000 & 7 & 1 & 0 & \\
\hline hs00015001 & $\mathrm{v} 1-\mathrm{s} 15$ & & & \\
\hline hs00015002 & -6.553 & 1.0 & & \\
\hline hs00015100 & -1 & 1 & 0.0 & \\
\hline hs00015102 & 0.0001 & 2 & & \\
\hline hs00015103 & 0.0002519 & 3 & & \\
\hline hs00015104 & 0.000635 & 4 & & \\
\hline hs00015105 & 0.001 & 5 & & \\
\hline
\end{tabular}




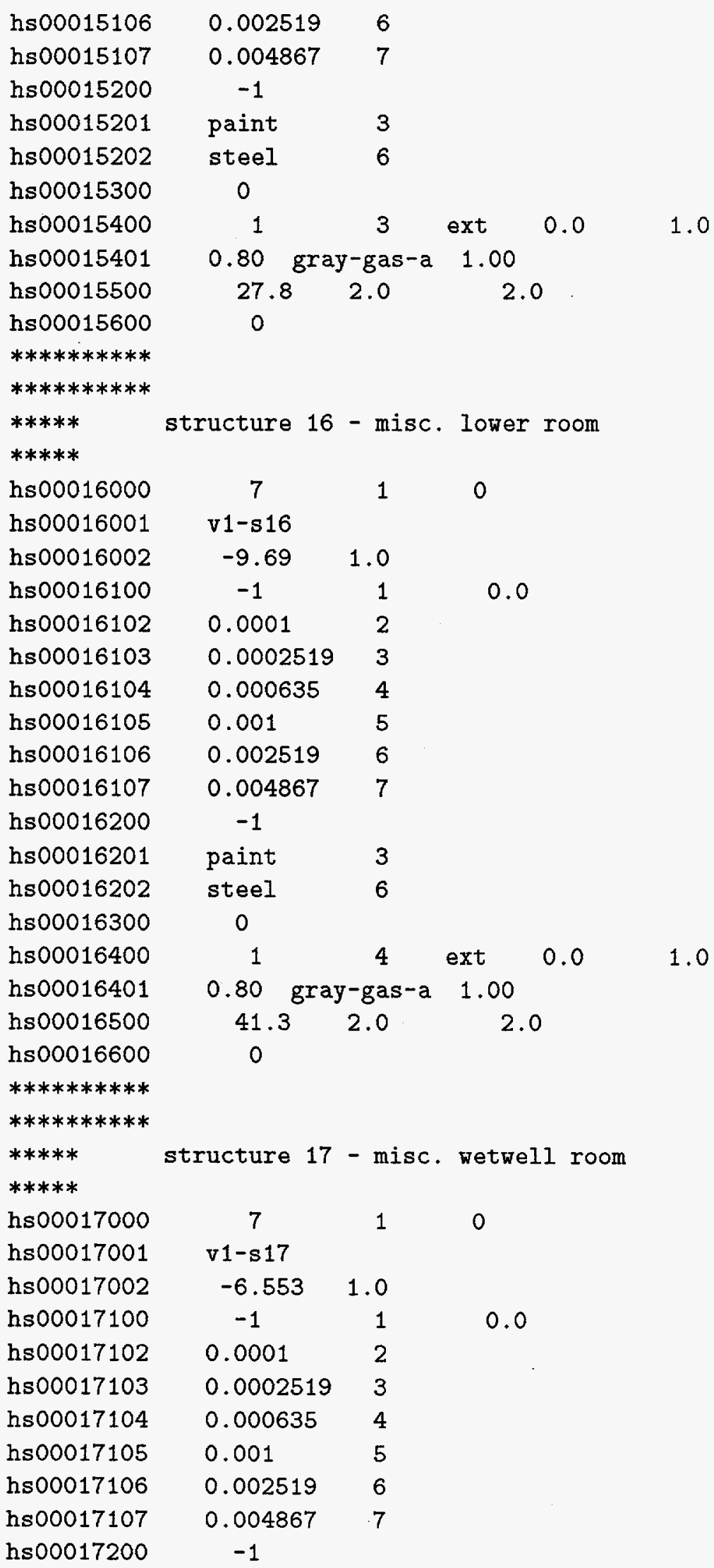




\begin{tabular}{|c|c|c|c|c|}
\hline hs00017201 & paint & 3 & & \\
\hline hs00017202 & steel & 6 & & \\
\hline hs00017300 & 0 & & & \\
\hline hs 00017400 & 1 & ext & 0.0 & 1.0 \\
\hline hs00017500 & 27.8 & \multirow{2}{*}{\multicolumn{2}{|c|}{2.0}} & \\
\hline hs 00017600 & 0 & & & \\
\hline \multicolumn{5}{|l|}{$* * * * * * * * * *$} \\
\hline \multicolumn{5}{|c|}{$* * * * * * * * * * * * * * * * * * * * * * * * * * * * * * * * * * * * * * * * * * * * * * * * * * * * * * * * * * * * * * * * * * * * * * * * * * *$} \\
\hline$* * * * *$ & \multicolumn{4}{|c|}{ material properties } \\
\hline \multicolumn{5}{|c|}{$* * * * * * * * * * * * * * * * * * * * * * * * * * * * * * * * * * * * * * * * * * * * * * * * * * * * * * * * * * * * * * * * * * * * * * * * * * * * * * * *)$} \\
\hline$* * * * *$ & \multicolumn{4}{|l|}{ steel } \\
\hline \multicolumn{5}{|l|}{$* * * * *$} \\
\hline mpmat00100 & steel & & & \\
\hline mpmat00101 & rho & 5 & & \\
\hline mpmat00102 & cps & 6 & & \\
\hline mpmat00103 & the & 7 & & \\
\hline \multicolumn{5}{|l|}{$* * * * *$} \\
\hline tf00500 & rho-steel & 2 & 1.0 & 0.0 \\
\hline$t f 00511$ & 200.0 & 7850.0 & & \\
\hline tf00512 & 5000.0 & 7850.0 & & \\
\hline$t f 00600$ & cps-steel & 2 & 1.0 & 0.0 \\
\hline tf00611 & 200.0 & 500.0 & & \\
\hline$t f 00612$ & 5000.0 & 500.0 & & \\
\hline tf00700 & thc-steel & 2 & 1.0 & 0.0 \\
\hline tf00711 & 200.0 & 47.0 & & \\
\hline tfo0712 & 5000.0 & 47.0 & & \\
\hline \multicolumn{5}{|l|}{$* * * * *$} \\
\hline$* * * * *$ & \multicolumn{4}{|l|}{ paint } \\
\hline \multicolumn{5}{|l|}{$* * * * *$} \\
\hline mpmat 00200 & paint & & & \\
\hline mpmat 00201 & rho & 8 & & \\
\hline mpmat00202 & cps & 9 & & \\
\hline mpmat 00203 & the & 10 & & \\
\hline \multicolumn{5}{|l|}{$* * * * *$} \\
\hline tf00800 & rho-paint & 2 & 1.0 & 0.0 \\
\hline $\operatorname{tf} 00811$ & 200.0 & 1190.0 & & \\
\hline$t f 00812$ & 5000.0 & 1190.0 & & \\
\hline tf00900 & cps-paint & 2 & 1.0 & 0.0 \\
\hline$t f 00911$ & 200.0 & 1880.0 & & \\
\hline$t f 00912$ & 5000.0 & 1880.0 & & \\
\hline $\operatorname{tf} 01000$ & thc-paint & 2 & 1.0 & 0.0 \\
\hline tfo1011 & 200.0 & 0.170 & & \\
\hline $\operatorname{tf0} 01012$ & 5000.0 & 0.170 & & \\
\hline \multicolumn{5}{|l|}{$* * * * *$} \\
\hline$* * * * *$ & insulation & & & \\
\hline$* * * * *$ & insul & & & \\
\hline
\end{tabular}




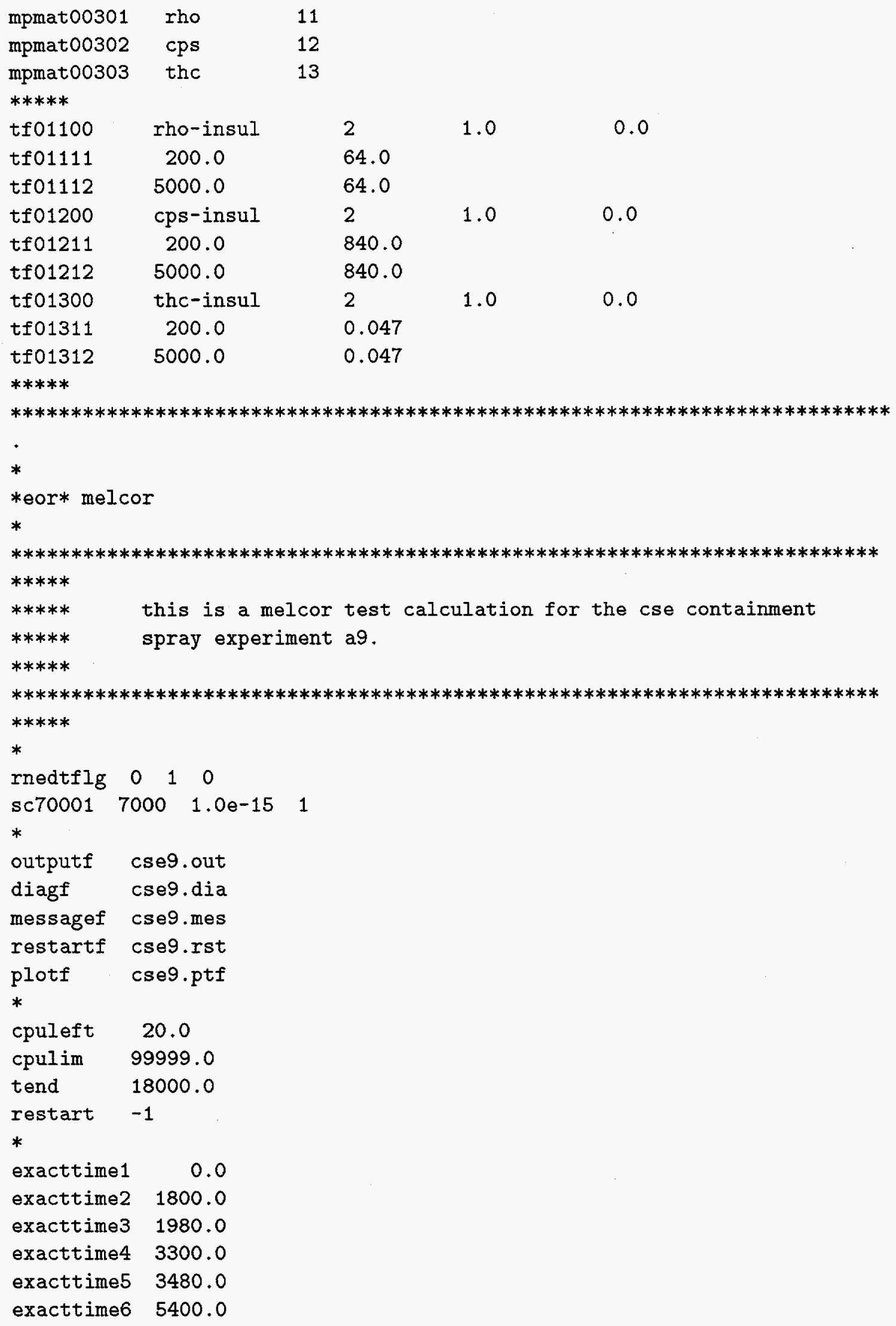




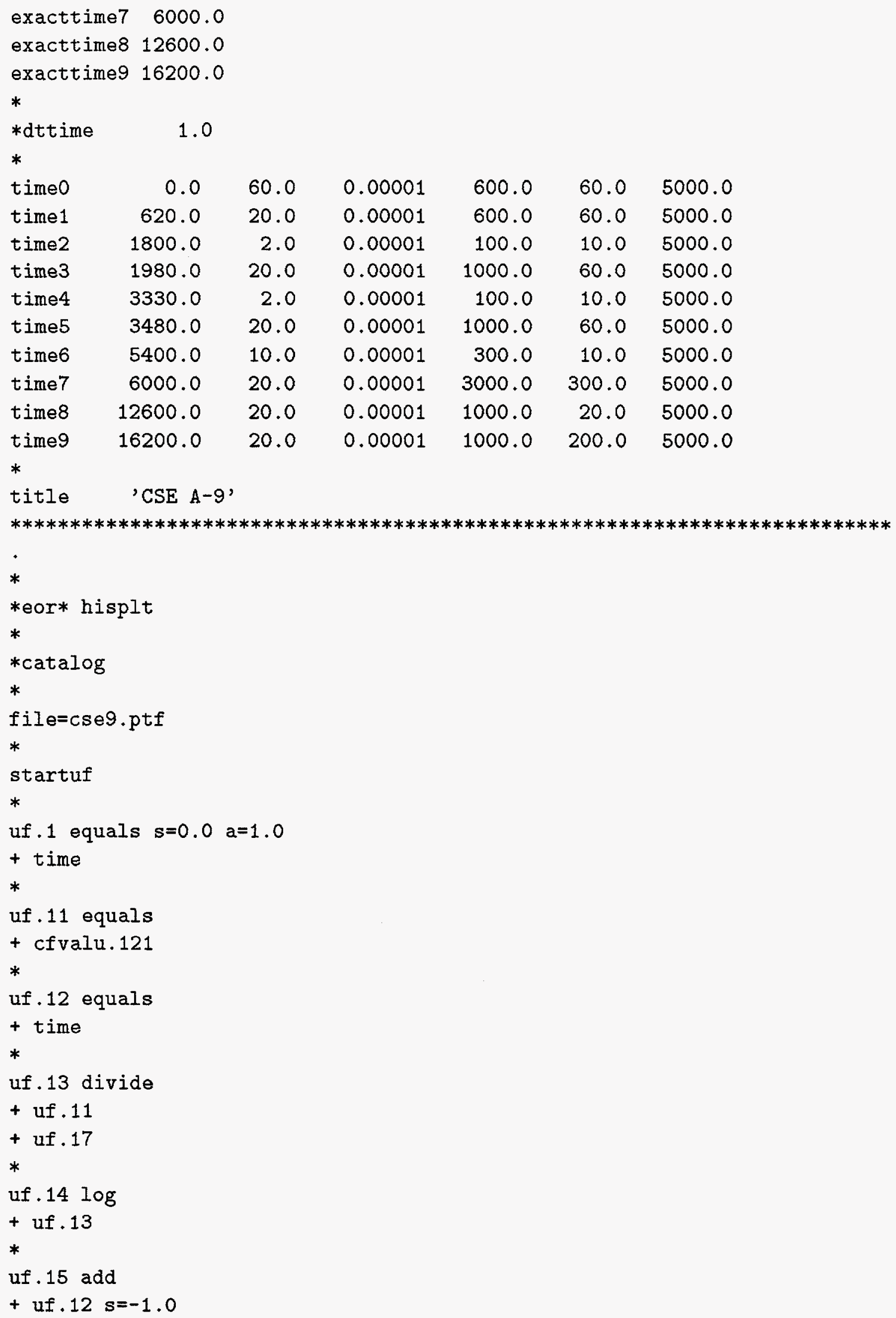




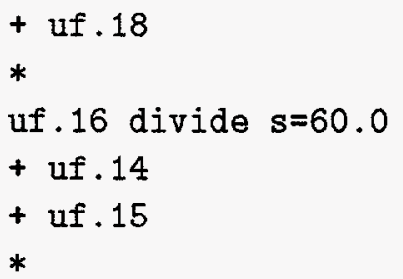

uf. 17 equals init $=1.0$

+ uf. 11

*

uf.18 equals init=1.0

+ uf. 12

*

uf .19 divide $s=0.69315$

+ uf. 1

+ uf 16

*

uf. 21 equals

+ cfvalu.241

*

uf. 22 equals

+ time

*

uf. 23 divide

+ uf. 21

+ uf. 27

*

uf. $24 \log$

+ uf .23

*

uf. 25 add

+ uf. $22 \mathrm{~s}=-1.0$

+ uf. 28

*

uf .26 divide $s=60.0$

+ uf. 24

+ uf. 25

*

uf. 27 equals init $=1.0$

+ uf. 21

*

uf. 28 equals init $=1.0$

+ uf. 22

*

uf. 29 divide $s=0.69315$

+ uf. 1

+ uf. 26

$*$ 


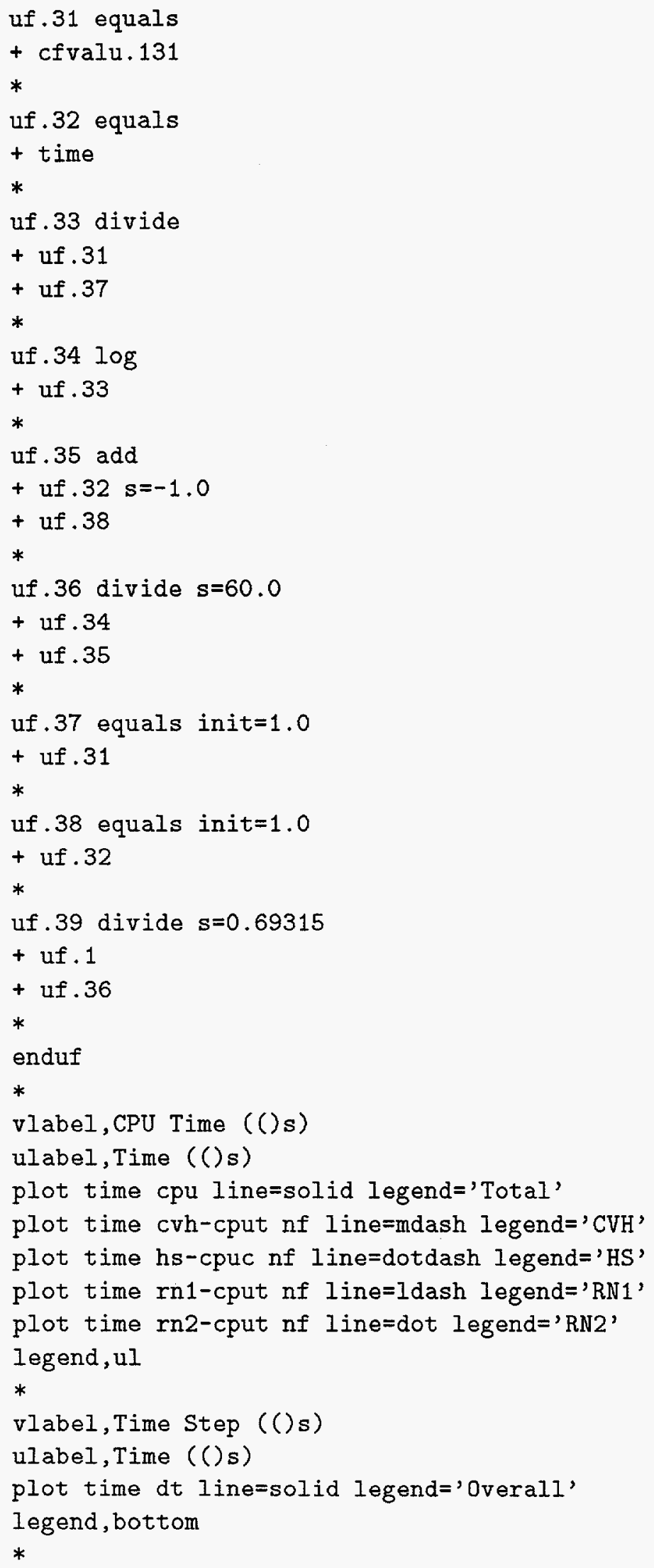




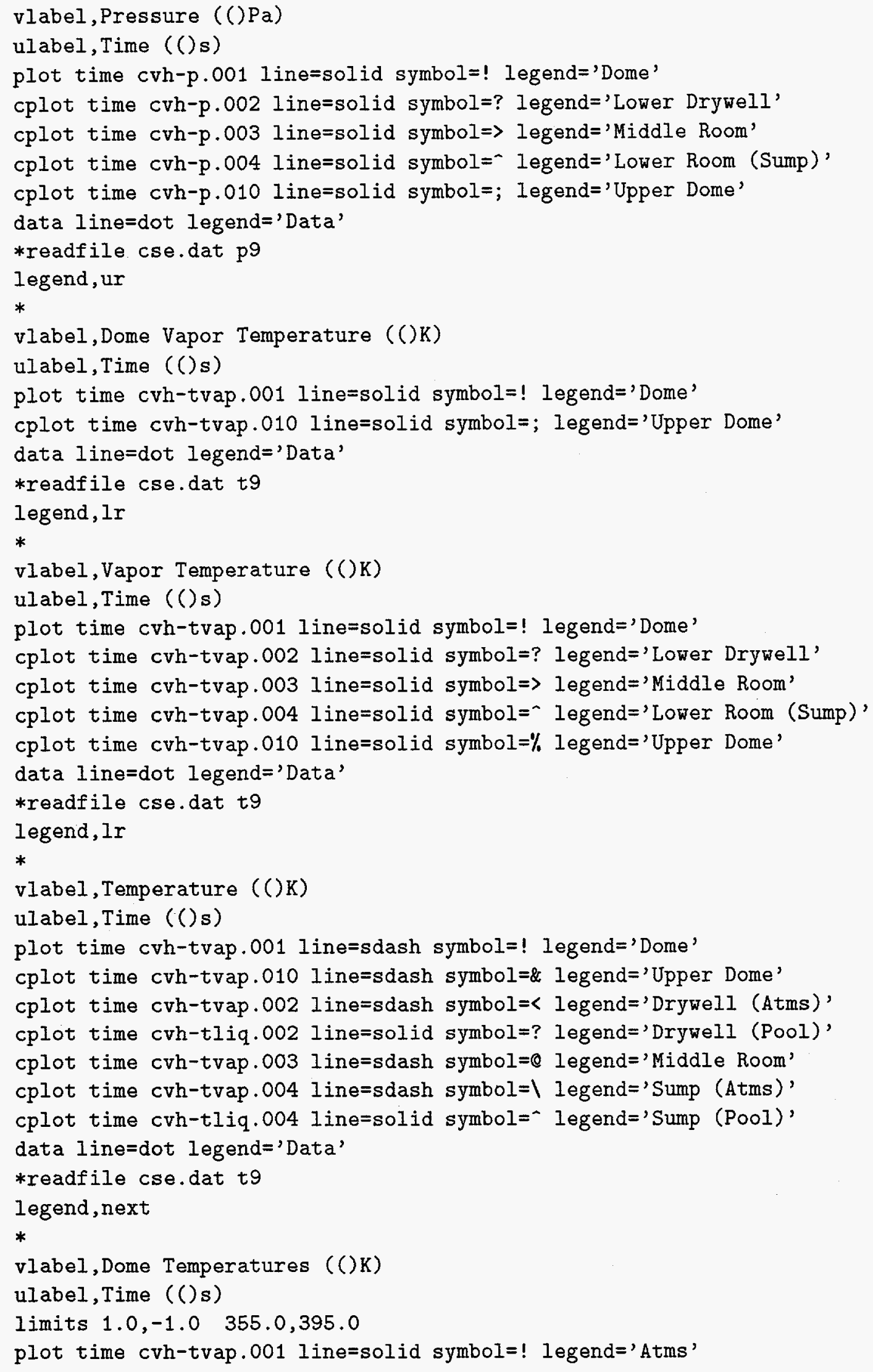


cplot time cvh-tliq.001 line=ldash symbol=< legend='Pool'

cplot time cvh-tsat (a).001 line=sdash symbol=Q legend=' $T$ (sat) $\odot A$ '

cplot time hs-temp.0000101 line=mdash symbol=' legend='Outer Wall'

cplot time hs-temp.0000301 line=mdash symbol=_A legend='Floor-to-MiddleRoom'

cplot time hs-temp.0000401 line=mdash symbol=_B legend='Floor-to-Wetwell'

cplot time hs-temp.0000501 line=mdash symbol=_C legend='Inside Structure'

data line=dot legend='Data'

*readfile cse.dat t9

legend, next

*

vlabel, Lower Drywell Temperatures (()K)

ulabel, Time (()s)

limits $1.0,-1.0 \quad 355.0,395.0$

plot time cvh-tvap.002 line=solid symbol=! legend='Atms'

cplot time cvh-tliq.002 line=ldash symbol=< legend='Pool'

cplot time cvh-tsat(a) .002 line=sdash symbol=@ legend=' $T$ (sat) $\odot A$ '

cplot time hs-temp.0000601 line=mdash symbol=- legend='Wall-to-MiddleRoom'

cplot time hs-temp.Q000701 line=mdash symbol=_A legend='Wall-to-Wetwell'

cplot time hs-temp.0000801 line=mdash symbol=_B legend='Floor'

legend, next

*

vlabel, Middle Room Temperatures (()K)

ulabel, Time ( $(>\mathrm{s})$

limits $1.0,-1.0 \quad 355.0,395.0$

plot time cvh-tvap.003 line=solid symbol=! legend='Atms'

cplot time cvh-tliq.003 line=ldash symbol=< legend='Pool'

cplot time cvh-tsat(a).003 line=sdash symbol=@ legend=' $T$ (sat) $Q A$ '

cplot time hs-temp.0000901 line=mdash symbol=- legend='Outer Wall'

cplot time hs-temp.0000309 line=mdash symbol=_A legend='Roof'

cplot time hs-temp.0000609 line=mdash symbol=_B legend='Inner Wall'

cplot time hs-temp.0001101 line=mdash symbol=_C legend='Wall-to-Wetwell'

cplot time hs-temp.0001201 line=mdash symbol=_D legend='Floor'

cplot time hs-temp.0001501 line=mdash symbol=_E legend='Inside Structure'

legend, next

*

vlabel, Sump Temperatures (()K)

ulabel, Time (()s)

limits $1.0,-1.0 \quad 355.0,395.0$

plot time cvh-tvap.004 line=solid symbol=! legend='Atms'

cplot time cvh-tliq.004 line=ldash symbol=< legend='Pool'

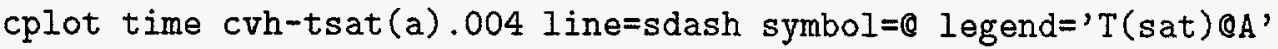

cplot time hs-temp.0001401 line=mdash symbol=- legend='Floor'

cplot time hs-temp.0000809 line=mdash symbol=_A legend='Roof-to-LowerDrywell'

cplot time hs-temp.0001209 line=mdash symbol=_B legend='Roof-to-MiddleRoom'

cplot time hs-temp.0001309 line=mdash symbol=_C legend='Roof-to-Wetwell'

cplot time hs-temp.0001601 line=mdash symbol=_E legend='Inside Structure'

legend, next 


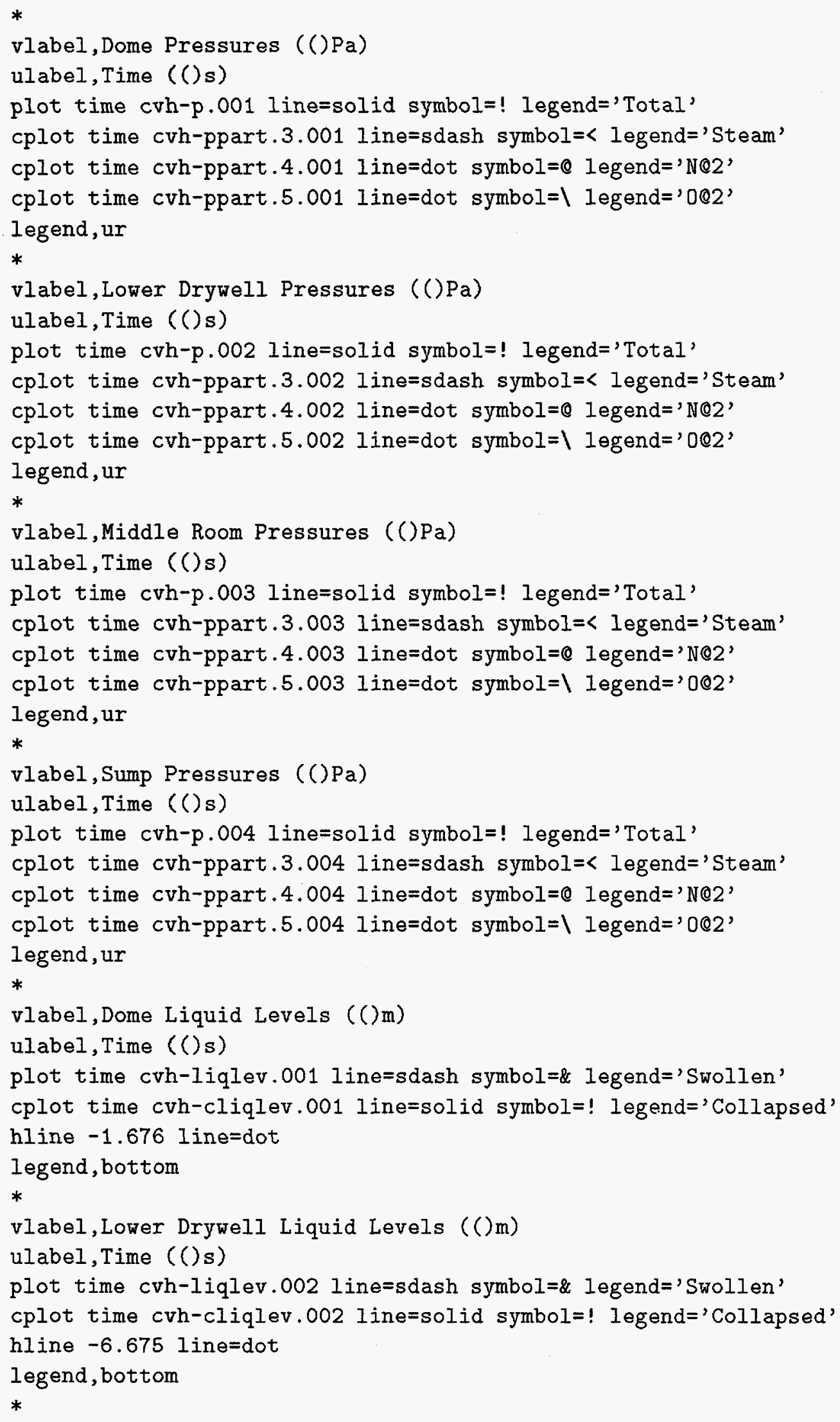


vlabel, Middle Room Liquid Levels (()m)

ulabel, Time (()s)

plot time cvh-liqlev.003 line=sdash symbol=\& legend='Swollen'

cplot time cvh-cliqlev.003 line=solid symbol=! legend='Collapsed'

hline -6.553 line $=$ dot

legend, bottom

*

vlabel, Sump Liquid Levels (()m)

ulabel, Time (()s)

plot time cvh-liqlev.004 line=sdash symbol=\& legend='Swollen'

cplot time cvh-cliqlev.004 line=solid symbol=! legend='Collapsed'

hline -9.703 line $=$ dot

legend, bottom

*

vlabel, Dome Pool Mass (() kg)

ulabel, Time (()s)

plot time cvh-mass.1.001 line=solid symbol=\& legend='Liquid'

legend, bottom

*

vlabel,Lower Drywell Pool Mass (() kg)

ulabel, Time (()s)

plot time crh-mass.1.002 line=solid symbol=\& legend='Liquid'

legend, bottom

*

vlabel, Middle Room Pool Mass (() kg)

ulabel, Time (()s)

plot time cuh-mass.1.003 line=solid symbol=\& legend='Liquid'

legend, bottom

*

vlabel, Sump Pool Mass (() kg)

ulabel, Time (()s)

plot time cvh-mass.1.004 line=solid symbol=\& legend='Liquid'

legend, bottom

*

vlabel,Pool Masses (() kg)

ulabel, Time (()s)

limits $1.0,-1.00 .0,10.0 \mathrm{e} 3$

plot time crh-mass.1.002 line=solid symbol=? legend='Lower Drywell'

cplot time cvh-mass.1.004 line=solid symbol=- legend='Lower Room (Sump)'

vscale, $1.0 \mathrm{e} 3,0.0$

data line=dot symbol=- legend='Data (Lower Drywell)'

*readfile cse-rn.dat pvdw9

vscale, $1.0 \mathrm{e} 3,0.0$

data line=dot symbol=; legend='Data (Sump)'

*readfile cse-rn.dat prcv9

legend, next

* 


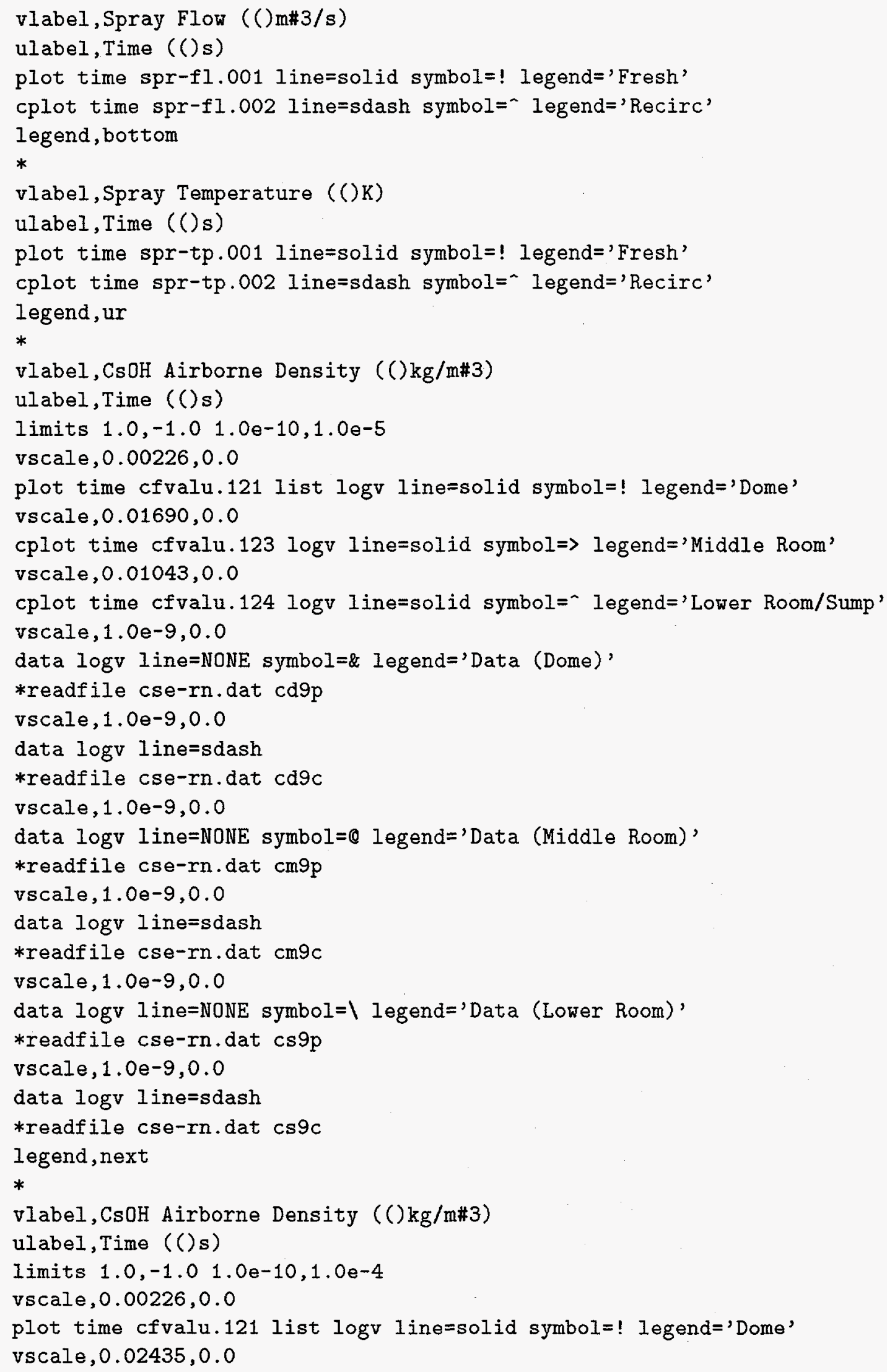




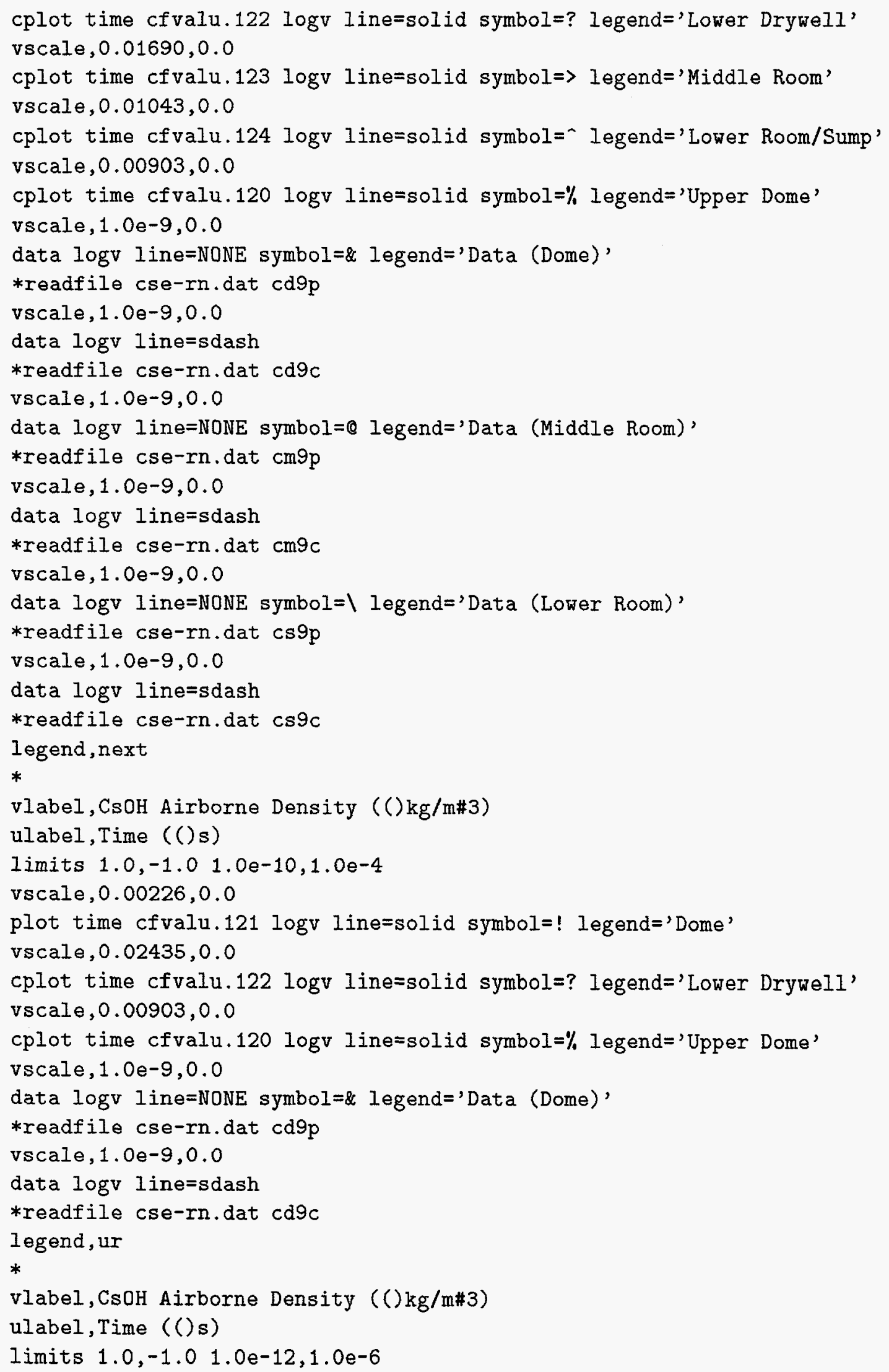




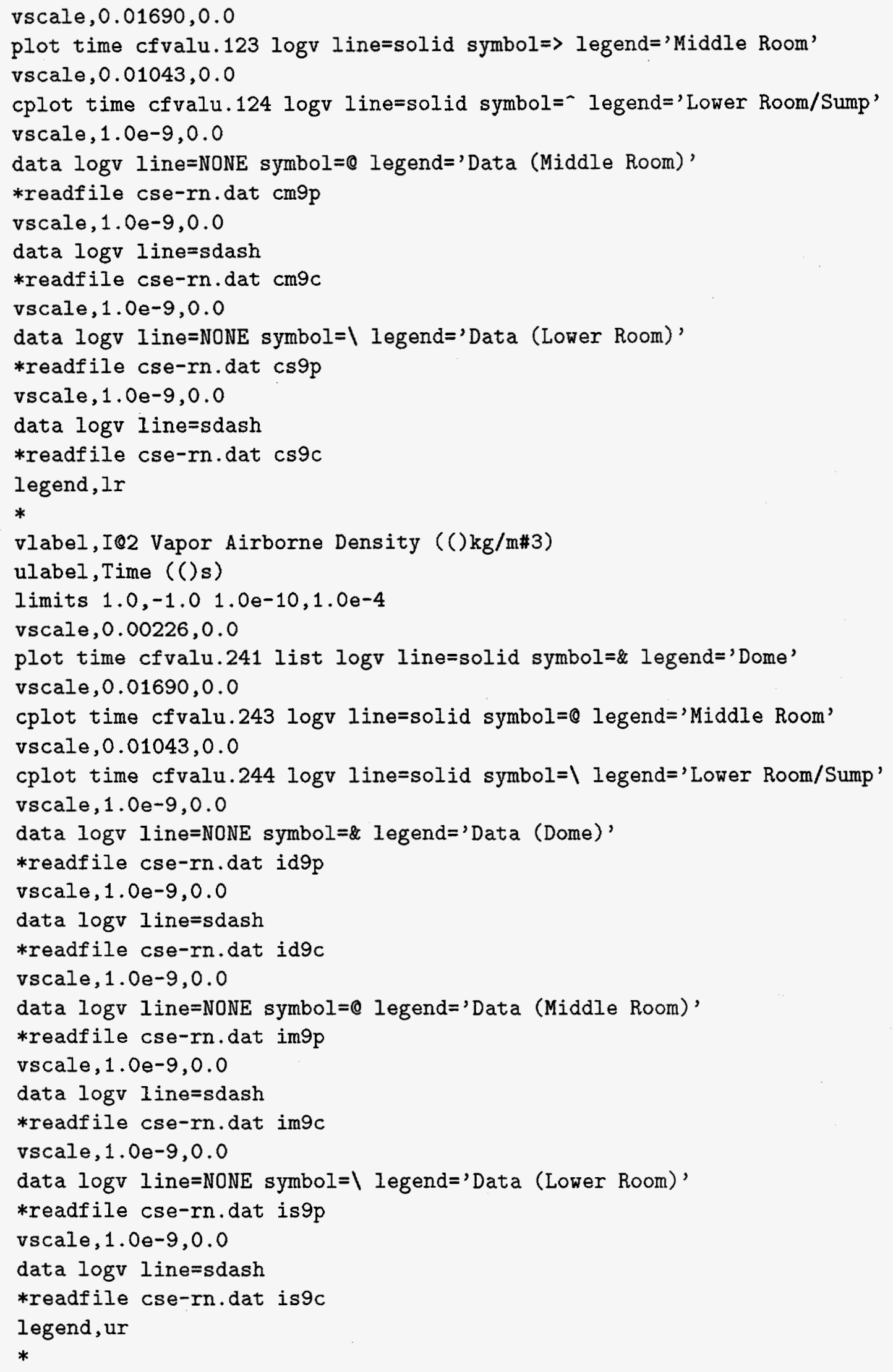




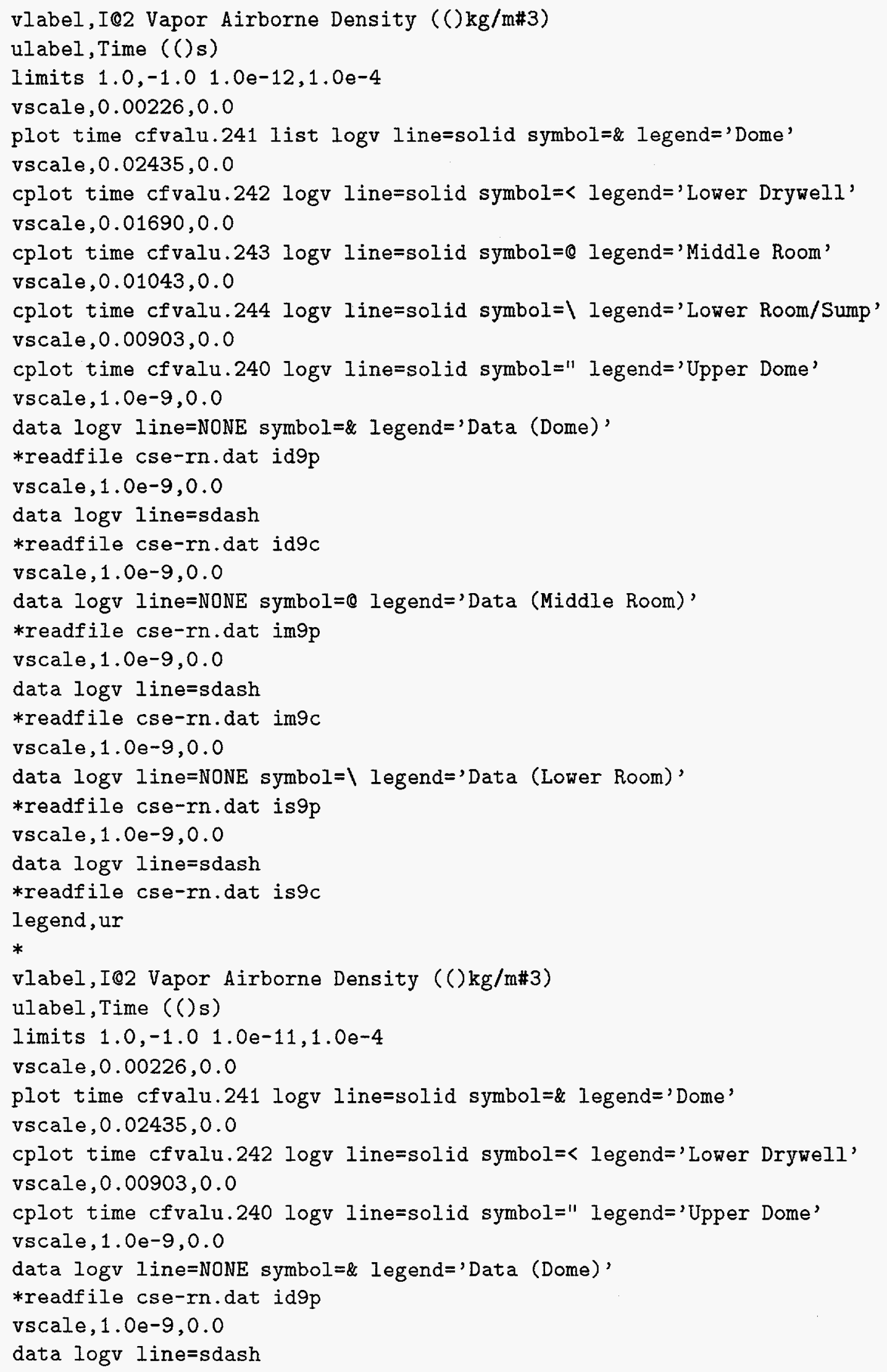




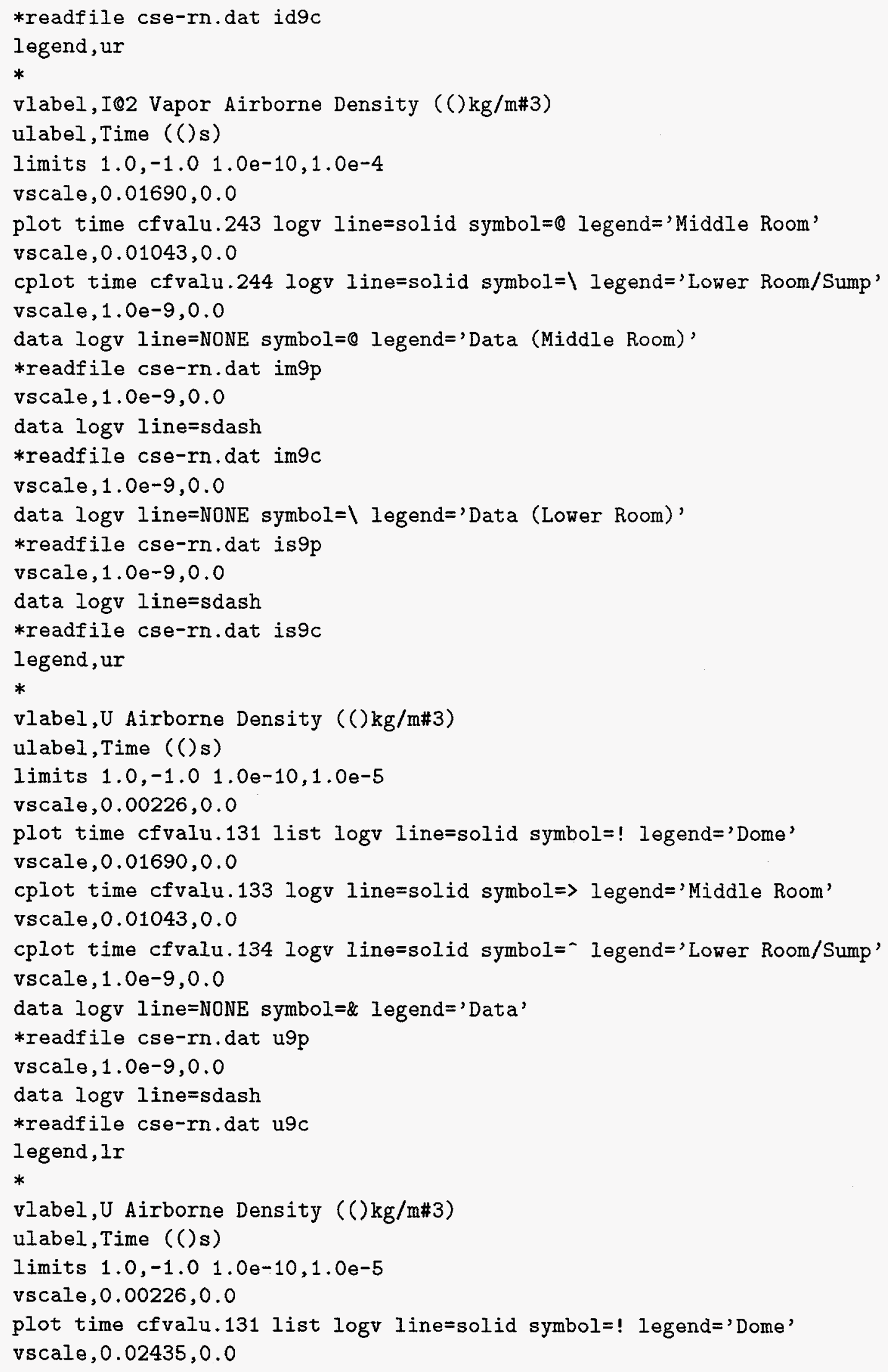


cplot time cfvalu.132 logv line=solid symbol=? legend='Lower Drywell' vscale, $0.01690,0.0$

cplot time cfvalu.133 logv line=solid symbol $\Rightarrow$ legend='Middle Room' vscale, $0.01043,0.0$

cplot time cfvalu.134 logv line=solid symbol=- legend='Lower Room/Sump' vscale, $0.00903,0.0$

cplot time cfvalu.130 logv line=solid symbol=\% legend='Upper Dome'

vscale, $1.0 e-9,0.0$

data logv line=NONE symbol=\& legend='Data'

*readfile cse-rn.dat u9p

vscale, $1.0 e-9,0.0$

data $\log v$ line $=$ sdash

*readfile cse-rn.dat u9c

legend, Ir

*

vlabel,He20 Aerosol Particle AMMD (()m)

ulabel, Time (()s)

limits $1.0,-1.00 .0,4.00-6$

plot time rn1-mmdc-1.001 line=solid symbol=! legend='Dome'

cplot time rn1-mmdc-1.002 line=solid symbol=? legend='Lower Drywell'

cplot time rn1-mmdc-1.003 line=solid symbol $\Rightarrow$ legend='Middle Room'

cplot time rn1-mmdc-1.004 line=solid symbol=- legend='Lower Room'

*cplot time rn1-mmdc-1.010 line=solid symbol=; legend='Upper Dome'

legend, ur

*

vlabel,H@20 Aerosol Particle GSD

ulabel, Time (()s)

plot time rn1-gsdc-1.001 line=solid symbol=! legend='Dome'

cplot time mi-gsdc-1.002 line=solid symbol=? legend='Lower Drywell'

cplot time rn1-gsdc-1.003 line=solid symbol $\Rightarrow$ legend='Middle Room'

cplot time rn1-gsdc-1.004 line=solid symbol=-' legend='Lower Room'

*cplot time rn1-gsdc-1.010 line=solid symbol=; legend='Upper Dome'

legend, ur

*

vlabel,U Aerosol Particle AMMD (()m)

ulabel, Time (Cs)

plot time rn1-mmdc-2.001 line=solid symbol=! legend='Dome'

cplot time rn1-mmdc-2.002 line=solid symbol=? legend='Lower Drywell'

cplot time rn1-mmdc-2.003 line=solid symbol $\Rightarrow$ legend='Middle Room'

cplot time rn1-mmdc-2.004 line=solid symbol=^ legend='Lower Room'

*cplot time rn1-mmdc-2.010 line=solid symbol=; legend='Upper Dome'

legend, Ir

*

vlabel,U Aerosol Particle GSD

ulabel, Time (()s)

plot time rn1-gsdc-2.001 line=solid symbol=! legend='Dome'

cplot time m1-gsdc-2.002 line=solid symbol=? legend='Lower Drywell' 
cplot time rn1-gsdc-2.003 line=solid symbol $\Rightarrow$ legend='Middle Room' cplot time rn1-gsdc-2.004 line=solid symbol=- legend='Lower Room' *cplot time rn1-gsdc-2.010 line=solid symbol=; legend='Upper Dome' legend, ur

*

vlabel, CsOH Aerosol Particle AMMD (()m)

ulabel, Time (C) $\mathrm{s}$ )

plot time rn1-mmdc-3.001 line=solid symbol=! legend='Dome'

cplot time rn1-mmdc-3.002 line=solid symbol=? legend='Lower Drywell'

cplot time rn1-mmdc-3.003 line=solid symbol $\Rightarrow$ legend='Middle Room'

cplot time rn1-mmdc-3.004 line=solid symbol=" legend='Lower Room'

*cplot time rn1-mmdc-3.010 line=solid symbol=; legend='Upper Dome'

legend, Ir

*

vlabel, CsOH Aerosol Particle GSD

ulabel, Time (O)

plot time rn1-gsdc-3.001 line=solid symbol=! legend='Dome'

cplot time rn1-gsdc-3.002 line=solid symbol=? legend='Lower Drywell'

cplot time rn1-gsdc-3.003 line=solid symbol=> legend='Middle Room'

cplot time rn1-gsdc-3.004 line=solid symbol=- legend='Lower Room'

*cplot time rn1-gsdc-3.010 line=solid symbol=; legend='Upper Dome'

legend, ur

*

vlabel, Aerosol Particle AMMD in Dome (()m)

ulabel, Time (()s)

plot time rn1-mmdc-1.001 line=sdash symbol=! legend='H@2O'

cplot time rn1-mmdc-3.001 line=solid symbol=? legend='Cesium'

cplot time $\mathrm{m} 1-\mathrm{mmdc}-2.001$ line=ldash symbol $\Rightarrow$ legend='Uranium'

legend, Ir

*

vlabel,Aerosol Particle GSD in Dome

ulabel, Time (C)s)

plot time $\mathrm{rn} 1-\mathrm{gsdc}-1.001$ line=sdash symbol=! legend='H@20'

cplot time rn1-gsdc-3.001 line=solid symbol=? legend='Cesium'

cplot time rn1-gsdc-2.001 line=ldash symbol $\Rightarrow$ legend='Uranium'

legend, ur

*

vlabel, Aerosol Particle AMMD in Lower Drywell (()m) .

ulabel, Time (C)s)

plot time rn1-mmdc-1.002 line=sdash symbol=! legend='H@20'

cplot time rn $1-m m d c-3.002$ line=solid symbol=? legend='Cesium'

cplot time $r n 1-m m d c-2.002$ line=Idash symbol $\Rightarrow$ legend='Uranium'

legend, Ir

*

vlabel,Aerosol Particle GSD in Lower Drywell

ulabel, Time (()s)

plot time rn1-gsdc-1.002 line=sdash symbol=! legend='H@20' 
cplot time rn1-gsdc-3.002 line=solid symbol=? legend='Cesium' cplot time rn1-gsdc-2.002 line=ldash symbol=> legend='Uranium' legend, ur

*

vlabel,Aerosol Particle AMMD in Middle Room (()m)

ulabel, Time (()s)

plot time $\mathrm{rn} 1-\mathrm{mmdc}-1.003$ line=sdash symbol=! legend=' H@20'

cplot time rn1-mmdc-3.003 line=solid symbol=? legend='Cesium'

cplot time rn1-mmdc-2.003 line=ldash symbol $\Rightarrow$ legend='Uranium'

legend, Ir

*

vlabel, Aerosol Particle GSD in Middle Room

ulabel, Time (C)

plot time rn1-gsdc-1.003 line=sdash symbol=! legend='H@20'

cplot time rn1-gsdc-3.003 line=solid symbol=? legend='Cesium'

cplot time rn1-gsdc-2.003 line=ldash symbol=> legend='Uranium'

legend, ur

*

vlabel, Aerosol Particle AMMD in Lower Room (()m)

ulabel, Time ( ()$s)$

plot time rn1-mmdc-1.004 line=sdash symbol=! legend='H@20'

cplot time rn1-mmdc-3.004 line=solid symbol=? legend='Cesium'

cplot time rn1-mmdc-2.004 line=ldash symbol $\Rightarrow$ legend='Uranium'

legend, ur

*

vlabel,Aerosol Particle GSD in Lower Room

ulabel, Time (()s)

plot time rn1-gsdc-1.004 line=sdash symbol=! legend='H@20'

cplot time rn1-gsdc-3.004 line=solid symbol=? legend='Cesium'

cplot time rn1-gsdc-2.004 line=ldash symbol $\Rightarrow$ legend='Uranium'

legend, ur

*

vlabel,U Aerosol Particle AMMD (()m)

ulabel, Time (C)

plot time rn1-mmdc-2.001 line=solid symbol=! legend='Dome (U)'

cplot time rn1-mmdc-2.002 Iine=solid symbol=? legend='Lower Drywell (U)'

cplot time rn1-mmdc-2.003 line=solid symbol $\Rightarrow$ legend='Middle Room (U)'

cplot time rn1-mmdc-2.004 line=solid symbol=- legend='Lower Room (U)'

*cplot time rn1-mmdc-2.010 line=solid symbol=. legend='Upper Dome (U)'

cplot time rn1-mmdc-3.001 line=mdash symbol=\& legend='Dome (Cs)'

cplot time rn1-mmdc-3.002 line=mdash symbol=< legend='Lower Drywell (Cs)'

cplot time rn1-mmdc-3.003 line=mdash symbol=@ legend='Middle Room (Cs)'

cplot time rn1-mmdc-3.004 line=mdash symbol=1 legend='Lower Room (Cs)'

*cplot time rn1-mmdc-3.010 line=mdash symbol=', ' legend='Upper Dome (Cs)'

legend, Ir

*

vlabel,U Aerosol Particle GSD 
ulabel, Time (()s)

limits $1.0,-1.01 .0,2.0$

plot time rn1-gsdc-2.001 line=solid symbol=! legend='Dome (U)'

cplot time rn1-gsdc-2.002 line=solid symbol=? legend='Lower Drywell (U)'

cplot time rn1-gsdc-2.003 line=solid symbol $\Rightarrow$ legend='Middle Room (U)'

cplot time rn1-gsdc-2.004 line=solid symbol=- legend='Lower Room (U)'

*cplot time $r n 1-g s d c-2.010$ line=solid symbol=. legend='Upper Dome (U)'

cplot time rn1-gsdc-3.001 line=mdash symbol=\& legend='Dome (Cs)'

cplot time rn1-gsdc-3.002 line=mdash symbol=< legend='Lower Drywell (Cs)'

cplot time rn1-gsdc-3.003 line=mdash symbol=@ legend='Middle Room (Cs)'

cplot time rn1-gsdc-3.004 line=mdash symbol=\legend='Lower Room (Cs)'

*cplot time rn1-gsdc-3.010 line=mdash symbol=',' legend='Upper Dome (Cs)'

legend, ur

*

vlabel, Spray Flow (()m\#3/s)

ulabel, Time (()s)

vscale, $1.42857,0.0$

plot time spr-fl.001 line=solid symbol=! legend='Total (Fresh)'

vscale, $1.42857,0.0$

cplot time spr-fl.002 line=solid symbol=^ legend='Total (Recirc)'

cplot time spr-fl.001 line=ldash symbol=! legend='into Atms (Fresh)'

$\mathrm{cplot}$ time spr-fl.002 line=ldash symbol=- legend='into Atms (Recirc)'

vscale, $0.42857,0.0$

cplot time spr-fl.001 line=sdash symbol=! legend='omto Walls (Fresh)'

vscale, $0.42857,0.0$

cplot time spr-fl.002 line=sdash symbol=- legend='omto Walls (Recirc)'

legend, next

*

vlabel, Spray Temperature (()K)

ulabel, Time (()s)

plot time spr-tp.001 line=solid symbol=! legend='Fresh'

cplot time spr-tp.002 line=sdash symbol=- legend='Recirc'

legend, bottom

*

vlabel,Fog Airborne Density ( () $\mathrm{kg} / \mathrm{m \# 3})$

ulabel, Time (O) $s$ )

limits $1.0,-1.01 .0 e-6,1.0 e-1$

vscale, $0.00226,0.0$

plot time cvh-mass.2.001 logv line=solid symbol=! legend='Dome'

vscale, $0.02435,0.0$

cplot time cvh-mass.2.002 logv line=solid symbol=? legend='Lower Drywell'

vscale, $0.01690,0.0$

cplot time cvh-mass.2.003 logv line=solid symbol=> legend='Middle Room'

vscale, $0.01043,0.0$

cplot time cvh-mass.2.004 logv line=solid symbol=- legend='Lower Room (Sump)'

vscale, $0.00903,0.0$

cplot time cvh-mass.2.010 logv line=solid symbol=; legend='Upper Dome' 


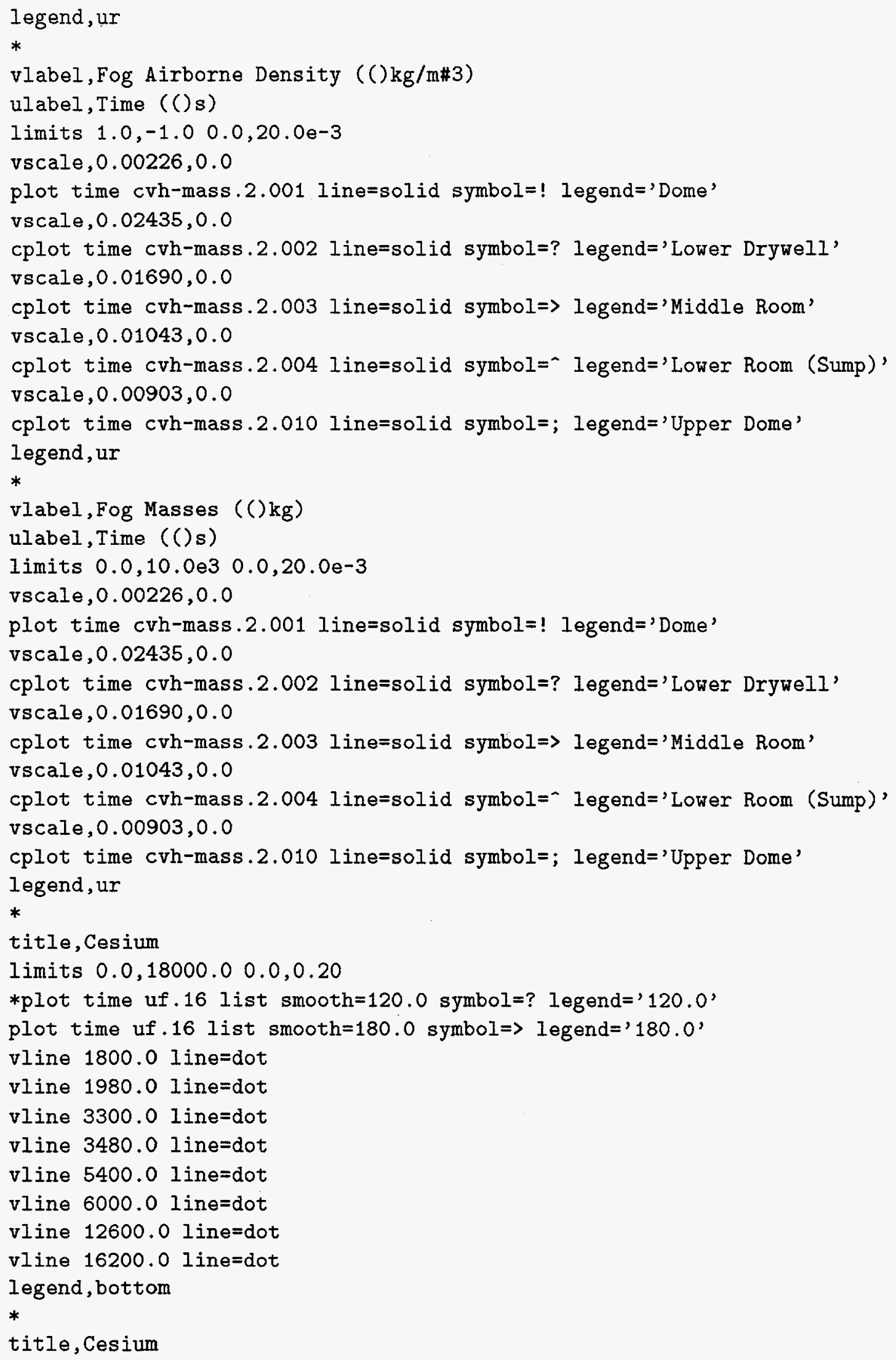


limits $0.0,18000.0 \quad 0.0,1000.0$

*plot time uf.19 list smooth $=300.0$ symbol=? legend=' $300.0^{\prime}$

plot time uf. 19 list smooth $=600.0$ symbol $\Rightarrow$ legend $=' 600.0$ '

vline 1800.0 line $=\operatorname{dot}$

vline 1980.0 line $=$ dot

vline 3300.0 line=dot

vline 3480.0 line=dot

vline 5400.0 line $=\operatorname{dot}$

vline 6000.0 line $=$ dot

vline 12600.0 line=dot

vline 16200.0 line $=$ dot

legend, bottom

*

title,Uranium

limits $0.0,18000.00 .0,0.20$

*plot time uf .36 list smooth $=120.0$ symbol=? legend $=' 120.0$ ' plot time uf. 36 list smooth $=180.0$ symbol=> legend=' 180.0 '

vline 1800.0 line=dot

vline 1980.0 line $=$ dot

vline 3300.0 line $=$ dot

vline 3480.0 line $=$ dot

vline 5400.0 line $=\operatorname{dot}$

vline 6000.0 line $=\operatorname{dot}$

vline 12600.0 line=dot

vline 16200.0 line $=\operatorname{dot}$

legend, bottom

*

title,Uranium

limits $0.0,18000.00 .0,1000.0$

*plot time uf.39 list smooth $=300.0$ symbol=? legend=' 300.0 ' plot time uf. 39 list smooth $=600.0$ symbol $\Rightarrow$ legend $=\prime 600.0^{\prime}$

vline 1800.0 line=dot

vline 1980.0 line=dot

vline 3300.0 line $=$ dot

vline 3480.0 line=dot

vline 5400.0 line=dot

vline 6000.0 line $=\operatorname{dot}$

vline 12600.0 line $=\operatorname{dot}$

vline 16200.0 line=dot

legend, bottom

*

title, Iodine

limits $0.0,18000.00 .0,1.00$

*plot time uf.26 list smooth $=120.0$ symbol=? legend=' $120.0^{\prime}$

plot time uf. 26 list smooth $=180.0$ symbol $=>$ legend=' $180.0^{\prime}$

vline 1800.0 line=dot

vline 1980.0 line=dot 
vline 3300.0 line $=$ dot vline 3480.0 line=dot vline 5400.0 line=dot vline 6000.0 line=dot vline 12600.0 line=dot vline 16200.0 line=dot legend, bottom

*

title, Iodine

limits $0.0,18000.00 .0,2000.0$

*plot time uf. 29 list smooth $=300.0$ symbol=? legend=' $300.0^{\prime}$

plot time uf. 29 list smooth $=600.0$ symbol $\Rightarrow$ legend $=' 600.0^{\prime}$

vline 1800.0 line=dot

vline 1980.0 line=dot

vline 3300.0 line=dot

vline 3480.0 line=dot

vline 5400.0 line $=$ dot

vline 6000.0 line=dot

vline 12600.0 line $=\operatorname{dot}$

vline 16200.0 line $=\operatorname{dot}$

legend, bottom

*

*eor* quit

*

vlabel, Dome Film Thickness (()m)

ulabel, Time (()s)

plot time hs-film-thick-1.00001 line=solid symbol=- legend='Outer Wall'

cplot time hs-film-thick-1.00003 line=solid symbol=_A legend='Floor-to-MiddleRoom'

cplot time hs-film-thick-1.00004 line=solid symbol=_B legend='Floor-to-Wetwell'

cplot time hs-film-thick-1.00005 line=solid symbol=_C legend='Inside Structure'

legend, ur

*

vlabel, Lower Drywell Film Thickness (()m)

ulabel, Time (()s)

plot time hs-film-thick-1.00006 line=solid symbol=- legend='Outer Wall-to-MiddleRoom'

cplot time hs-film-thick-1.00007 line=solid symbol=_A legend='Outer Wall-to-Wetwell'

cplot time hs-film-thick-1.00008 line=solid symbol=_B legend='Floor'

legend, ur

*

vlabel, Middle Room Film Thickness (()m)

ulabel, Time (()s)

plot time hs-film-thick-1.00009 line=solid symbol=- legend='Outer Wall'

cplot time hs-film-thick-r.00003 line=solid symbol=_A legend='Roof'

cplot time hs-film-thick-r.00006 line=solid symbol=_B legend='Inner Wall'

cplot time hs-film-thick-1.00011 line=solid symbol=_C legend='Wall-to-Wetwell'

cplot time hs-film-thick-1.00012 line=solid symbol=_D legend='Floor'

cplot time hs-film-thick-1.00015 line=solid symbol=_E legend='Inside Structure' 
legend, ur

*

vlabel, Sump Film Thickness (()m)

ulabel, Time (()s)

plot time hs-film-thick-1.00014 line=solid symbol=- legend='Floor'

cplot time hs-film-thick-r.00008 line=solid symbol=_A legend='Roof-to-LowerDrywell'

cplot time hs-film-thick-r.00012 line=solid symbol=_B legend='Roof-to-MiddleRoom'

cplot time hs-film-thick-r.00013 line=solid symbol=_C legend='Roof-to-Wetwell'

cplot time hs-film-thick-1.00016 line=solid symbol=_E legend='Inside Structure'

legend, ur

*

title, Vessel Cylinder on Upper Dome

vlabel, HS00100 Temperatures (()K)

ulabel, Time (()s)

plot time cvh-tvap.010 line=sdash symbol=! legend='Inside Atms'

cplot time cvh-tliq.010 line=solid symbol=\& legend='Inside Pool'

cplot time hs-temp.0010001 line=mdash symbol $\Rightarrow$ legend='Inside Surface'

cplot time hs-temp.0010012 line=mdash symbol=\% legend='Outside Surface'

cplot time cvh-tvap.005 line=sdash symbol=? legend='Outside Atms'

cplot time cvh-tliq.005 line=solid symbol=< legend='Outside Pool'

legend, ur

*

title, Vessel Cylinder on Dome (Main Room)

vlabel,HS00001 Temperatures (()K)

ulabel, Time (()s)

plot time cvh-tvap.001 line=sdash symbol=! legend='Inside Atms'

cplot time crh-tliq.001 line=solid symbol=\& legend='Inside Pool'

cplot time hs-temp.0000101 line=mdash symbol $\Rightarrow$ legend='Inside Surface'

cplot time hs-temp.0000112 line=mdash symbol=\% legend='Outside Surface'

cplot time crh-tvap.005 line=sdash symbol=? legend='Outside Atms'

cplot time cvh-tliq.005 line=solid symbol=< legend='Outside Pool'

legend, ur

*

title, Vessel Top on Upper Dome

vlabel,HSO0002 Temperatures (()K)

ulabel, Time $(() s)$

plot time cvh-tvap.010 line=sdash symbol=! legend='Inside Atms'

cplot time crh-tliq. 010 line=solid symbol=\& legend='Inside Pool'

cplot time hs-temp.0000201 line=mdash symbol $\Rightarrow$ legend='Inside Surface'

cplot time hs-temp.0000212 line=mdash symbol=\% legend='Outside Surface'

cplot time crh-tvap.005 line=sdash symbol=? legend='Outside Atms'

cplot time cvh-tliq.005 line=solid symbol=< legend='Outside Pool'

legend, ur

*

title,Deck between Dome and Middle Room

vlabel, HS00003 Temperatures (()K)

ulabel, Time (C)s) 
plot time cvh-tvap.001 line=sdash symbol=! legend='Inside Atms' cplot time cvh-tliq.001 line=solid symbol=\& legend='Inside Pool' cplot time hs-temp.0000301 line=mdash symbol $\Rightarrow$ legend='Inside Surface' cplot time hs-temp.0000309 line=mdash symbol=\% legend='Outside Surface' cplot time cvh-tvap.003 line=sdash symbol=? legend='Outside Atms' cplot time cvh-tliq.003 line=solid symbol=< legend='Outside Pool' legend, ur

*

title,Deck between Dome and Wetwell

vlabel, HSO0004 Temperatures (()K)

ulabel, Time (()s)

plot time cvh-tvap.001 line=sdash symbol=! legend='Inside Atms' cplot time cvh-tliq.001 line=solid symbol=\& legend='Inside Pool' cplot time hs-temp.0000401 line=mdash symbol $\Rightarrow$ legend='Inside Surface' cplot time hs-temp.0000409 line=mdash symbol=\% legend='Outside Surface' cplot time cvh-tvap.006 line=sdash symbol=? legend='Outside Atms' cplot time cvh-tliq.006 line=solid symbol=< legend='Outside Pool' legend, ur

*

title, Structure Inside Dome

vlabel, HSO0005 Temperatures (()K)

ulabel, Time (()s)

plot time cvh-tvap.001 line=sdash symbol=! legend='Inside Atms'

cplot time cvh-tliq.001 line=solid symbol=\& legend='Inside Pool'

cplot time hs-temp.0000501 line=mdash symbol $\Rightarrow$ legend='Inside Surface' cplot time hs-temp.0000507 line=mdash symbol=\% legend='Outside Surface' legend, ur

*

title,Cylinder between Drywell and Middle Room

vlabel, HSO0006 Temperatures (()K)

ulabel, Time (()s)

plot time cvh-tvap.002 line=sdash symbol=! legend='Inside Atms'

cplot time cvh-tliq.002 line=solid symbol=\& legend='Inside Pool'

cplot time hs-temp.0000601 line=mdash symbol $\Rightarrow$ legend='Inside Surface' cplot time hs-temp.0000609 line=mdash symbol=\% legend='Outside Surface' cplot time cvh-tvap.003 line=sdash symbol=? legend='Outside Atms' cplot time cvh-tliq.003 line=solid symbol=< legend='Outside Pool' legend, ur

*

title,Cylinder between Drywell and Wetwell

vlabel, HSO0007 Temperatures (()K)

ulabel, Time (()s)

plot time cvh-tvap.002 line=sdash symbol=! legend='Inside Atms'

cplot time cvh-tliq.002 line=solid symbol=\& legend='Inside Pool'

cplot time hs-temp.0000701 line=mdash symbol $\Rightarrow$ legend='Inside Surface'

cplot time hs-temp.0000709 line=mdash symbol=\% legend='Outside Surface' cplot time cvh-tvap.006 line=sdash symbol=? legend='Outside Atms' 
cplot time cvh-tliq.006 line=solid symbol=< legend='Dutside Pool'

legend, ur

*

title,Floor between Drywell and Lower Room Sump

vlabel, HSO0008 Temperatures (() K)

ulabel, Time (O)s)

plot time cvh-tvap.002 line=sdash symbol=! legend='Inside Atms'

cplot time cvh-tliq.002 line=solid symbol=\& legend='Inside Pool'

cplot time hs-temp.0000801 line=mdash symbol $\Rightarrow$ legend='Inside Surface'

cplot time hs-temp.0000809 line=mdash symbol=\% legend='Outside Surface'

cplot time cvh-tvap.004 line=sdash symbol=? legend='Outside Atms'

cplot time cvh-tliq. 004 line=solid symbol=< legend='Outside Pool'

legend, ur

*

title, Outer Cylinder on Middle Room

vlabel, HS00009 Temperatures (()K)

ulabel, Time (()s)

plot time crh-tvap.003 line=sdash symbol=! legend='Inside Atms'

cplot time cvh-tliq.003 line=solid symbol=\& legend='Inside Pool'

cplot time hs-temp.0000901 line=mdash symbol $\Rightarrow$ legend='Inside Surface'

cplot time hs-temp.0000912 line=mdash symbol=\% legend='Outside Surface'

cplot time cvh-tvap.005 line=sdash symbol=? legend='Outside Atms'

cplot time crh-tliq.005 line=solid symbol=< legend='Outside Pool'

legend, ur

*

title, Outer Cylinder on Wetwell

vlabel, HS00010 Temperatures (()K)

ulabel, Time (C)

plot time cvh-tvap.006 line=sdash symbol=! legend='Inside Atms'

cplot time crh-tliq.006 line=solid symbol=\& legend='Inside Pool'

cplot time hs-temp.0001001 line=mdash symbol $\Rightarrow$ legend='Inside Surface'

cplot time hs-temp.0001012 line=mdash symbol=\% legend='Outside Surface'

cplot time cvh-tvap.005 line=sdash symbol=? legend='Outside Atms'

cplot time cvh-tliq.005 line=solid symbol=< legend='Outside Pool'

legend, ur

*

title,Divider between Middle Room and Wetwell

vlabel,HS00011 Temperatures (()K)

ulabel, Time (Os)

plot time cvh-tvap.003 line=sdash symbol=! legend='Inside Atms'

cplot time cvh-tliq.003 line=solid symbol=\& legend='Inside Pool'

cplot time hs-temp.0001101 line=mdash symbol $\Rightarrow$ legend='Inside Surface'

cplot time hs-temp.0001109 line=mdash symbol=\% legend='Outside Surface'

cplot time crh-tvap.006 line=sdash symbol=? legend='Outside Atms'

cplot time cvh-tliq.006 line=solid symbol=< legend='Outside Pool'

legend,ur

* 
title,Floor between Middle Room and Lower Room Sump

vlabel, HSO0012 Temperatures (()K)

ulabel, Time ( () $\mathrm{s}$ )

plot time cvh-tvap.003 line=sdash symbol=! legend='Inside Atms'

cplot time cvh-tliq.003 line=solid symbol=\& legend='Inside Pool'

cplot time hs-temp.0001201 line=mdash symbol $\Rightarrow$ legend='Inside Surface'

cplot time hs-temp.0001209 line=mdash symbol=\% legend='Outside Surface'

cplot time cvh-tvap.004 line=sdash symbol=? legend='Outside Atms'

cplot time crh-tliq.004 line=solid symbol $=<$ legend='Outside Pool'

legend, ur

*

title,Floor between Wetwell and Lower Room Sump

vlabel, HSO0013 Temperatures (()K)

ulabel, Time (()s)

plot time crh-tvap.006 line=sdash symbol=! legend='Inside Atms'

cplot time cvh-tliq.006 line=solid symbol=\& legend='Inside Pool'

cplot time hs-temp.0001301 line=mdash symbol=> legend='Inside Surface'

cplot time hs-temp.0001309 line=mdash symbol=\% legend='Outside Surface'

cplot time evh-tvap.004 line=sdash symbol=? legend='Outside Atms'

cplot time cvh-tliq.004 line=solid symbol $=<$ legend='Outside Pool'

legend, ur

*

title,Outer Floor on Lower Room

vlabel, HSO0014 Temperatures (()K)

ulabel, Time (()s)

plot time cvh-tvap.004 line=sdash symbol=! legend='Inside Atms'

cplot time cvh-tliq.004 line=solid symbol=\& legend='Inside Pool'

cplot time hs-temp.0001401 line=mdash symbol $\Rightarrow$ legend='Inside Surface'

cplot time hs-temp.0001412 line=mdash symbol=\% legend='Outside Surface'

cplot time cvh-tvap.005 line=sdash symbol=? legend='Outside Atms'

cplot time cvh-tliq.005 line=solid symbol=< legend='Outside Pool'

legend, ur

*

title,Structure Inside Middle Room

vlabel, HS00015 Temperatures (()K)

ulabel, Time (()s)

plot time cvh-tvap.003 line=sdash symbol=! legend='Inside Atms'

cplot time cvh-tliq.003 line=solid symbol=\& legend='Inside Pool'

cplot time hs-temp.0001501 line=mdash symbol => legend='Inside Surface'

cplot time hs-temp.0001507 line=mdash symbol=\% legend='Outside Surface'

legend, ur

*

title,Structure Inside Lower Room

vlabel, HSO0016 Temperatures (O)K)

ulabel, Time (C)s)

plot time cvh-tvap.004 line=sdash symbol=! legend='Inside Atms'

cplot time cvh-tliq.004 line=solid symbol=\& legend='Inside Pool' 
cplot time hs-temp.0001601 line=mdash symbol=> legend='Inside Surface' cplot time hs-temp.0001607 line=mdash symbol=\% legend='Outside Surface' legend, ur

*

title,Structure Inside Wetwell

vlabel, HS00017 Temperatures (()K)

ulabel, Time (Os)

plot time cvh-tvap.006 line=sdash symbol=! legend='Inside Atms'

cplot time cvh-tliq.006 line=solid symbol=\& legend='Inside Pool'

cplot time hs-temp.0001701 line=mdash symbol $\Rightarrow$ legend='Inside Surface' cplot time hs-temp.0001707 line=mdash symbol=\%. legend='Outside Surface' legend, ur

*

*eor* quit

*

vlabel, $\mathrm{CsOH}$ Airborne Mass (() kg)

ulabel, Time (Os)

plot time cfvalu.121 line=solid symbol=! legend='Dome'

cplot time cfvalu.122 line=solid symbol=? legend='Lower Drywell'

cplot time cfvalu.123 line=solid symbol=> legend='Middle Room'

cplot time cfvalu.124 line=solid symbol=- legend='Lower Room/Sump'

cplot time cfvalu.120 line=solid symbol=\% legend='Upper Dome'

cplot time cfvalu.221 line=mdash symbol=\& legend='Dome'

cplot time cfvalu.222 line=mdash symbol=< legend='Lower Drywell'

cplot time cfvalu.223 line=mdash symbol=0 legend='Middle Room'

cplot time cfvalu.224 line=mdash symbol=l legend='Lower Room/Sump'

cplot time cfvalu.220 line=mdash symbol=" legend='Upper Dome'

legend, next

*

vlabel,Dome CsOH Airborne Mass (() kg)

ulabel, Time (()s)

plot time cfvalu.121 line=solid symbol=! legend='(Aerosol)'

cplot time cfvalu.221 line=mdash symbol=\& legend='(Vapor)'

legend, bottom

*

vlabel, Lower Drywell CsOH Airborne Mass (()kg)

ulabel, Time (()s)

plot time cfvalu.122 line=solid symbol=! legend='(Aerosol)'

cplot time cfvalu.222 line=mdash symbol=\& legend='(Vapor)'

legend, bottom

*

vlabel, Middle Room CsOH Airborne Mass (()kg)

ulabel, Time (()s)

plot time cfvalu.123 line=solid symbol=! legend='(Aerosol)'

cplot time cfvalu.223 line=mdash symbol=\& legend='(Vapor)'

legend, bottom

* 


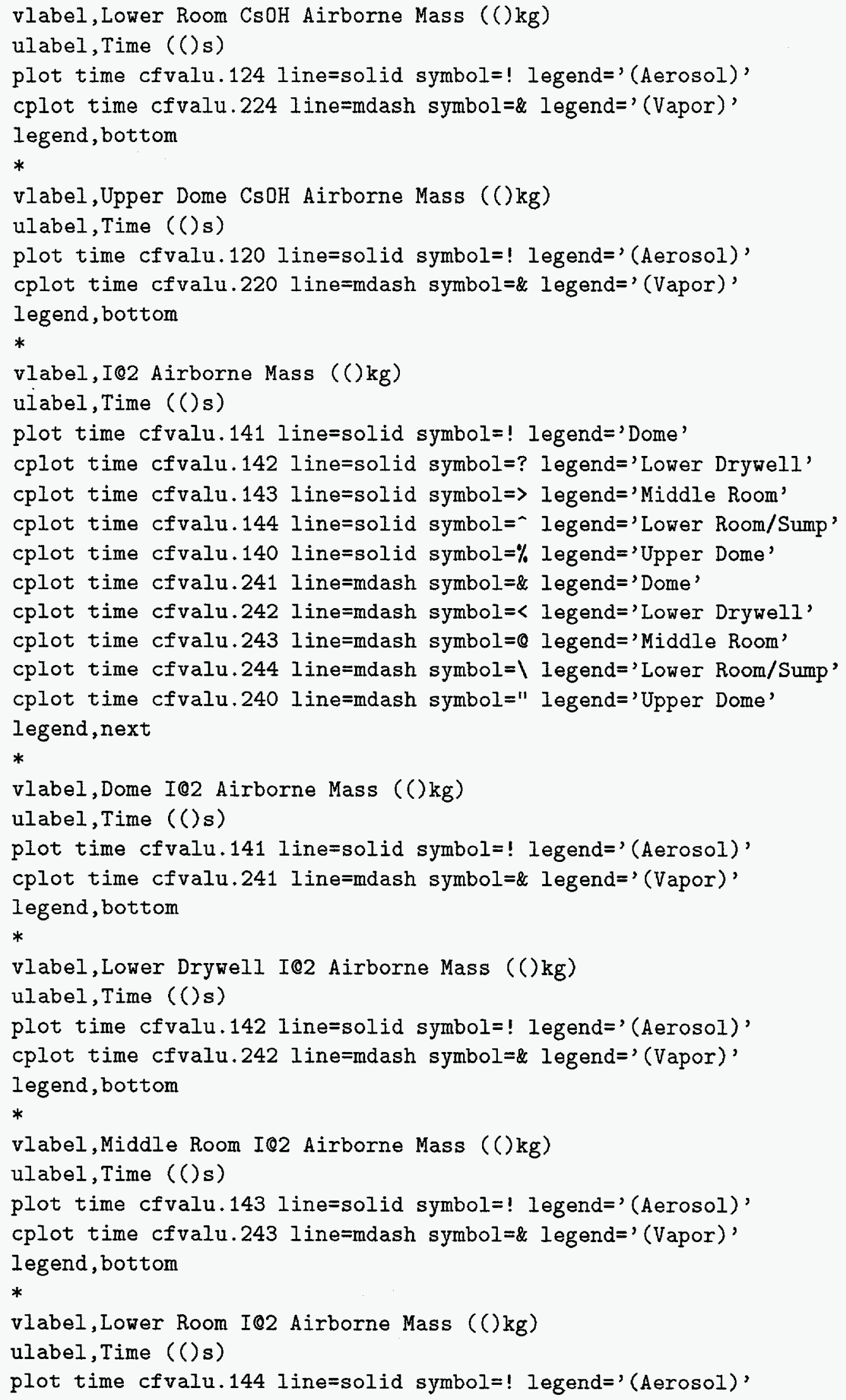


cplot time cfvalu.244 line=mdash symbol=\& legend='(Vapor)'

legend, bottom

*

vlabel, Upper Dome I@2 Airborne Mass (()kg)

ulabel, Time ( ()$s$ )

plot time cfvalu.140 line=solid symbol=! legend=' (Aerosol)'

cplot time cfvalu.240 line=mdash symbol=\& legend='(Vapor)'

legend, bottom

*

vlabel,U Airborne Mass (() kg)

ulabel, Time (()s)

plot time cfvalu.131 line=solid symbol=! legend='Dome'

cplot time cfvalu.132 line=solid symbol=? legend='Lower Drywell'

cplot time cfvalu.133 line=solid symbol $\Rightarrow$ legend='Middle Room'

cplot time cfvalu.134 line=solid symbol=- legend='Lower Room/Sump'

cplot time cfvalu.130 line=solid symbol=\% legend='Upper Dome'

cplot time cfvalu.231 line=mdash symbol=\& legend='Dome'

cplot time cfvalu.232 line=mdash symbol=< legend='Lower Drywell'

cplot time cfvalu.233 line=mdash symbol=0 legend='Middle Room'

cplot time cfvalu.234 line=mdash symbol= $\backslash$ legend='Lower Room/Sump'

cplot time cfvalu.230 line=mdash symbol=" legend='Upper Dome'

legend, next

*

vlabel,Dome U Airborne Mass (() kg)

ulabel, Time (O) $\mathrm{s}$ )

plot time cfvalu.131 line=solid symbol=! legend=' (Aerosol)'

cplot time cfvalu.231 line=mdash symbol=\& legend=' (Vapor)'

legend, bottom

*

vlabel, Lower Drywell U Airborne Mass (() kg)

ulabel, Time ( ( ) s)

plot time cfvalu.132 line=solid symbol=! legend='(Aerosol)'

cplot time cfvalu.232 line=mdash symbol=\& legend='(Vapor)'

legend, bottom

*

vlabel, Middle Room U Airborne Mass (()kg)

ulabel, Time (()s)

plot time cfvalu.133 line=solid symbol=! legend='(Aerosol)'

cplot time cfvalu.233 line=mdash symbol=\& legend=' (Vapor)'

legend, bottom

*

vlabel, Lower Room U Airborne Mass (()kg)

ulabel, Time (()s)

plot time cfvalu.134 line=solid symbol=! legend='(Aerosol)'

cplot time cfvalu.234 line=mdash symbol=\& legend=' (Vapor)'

legend, bottom

* 


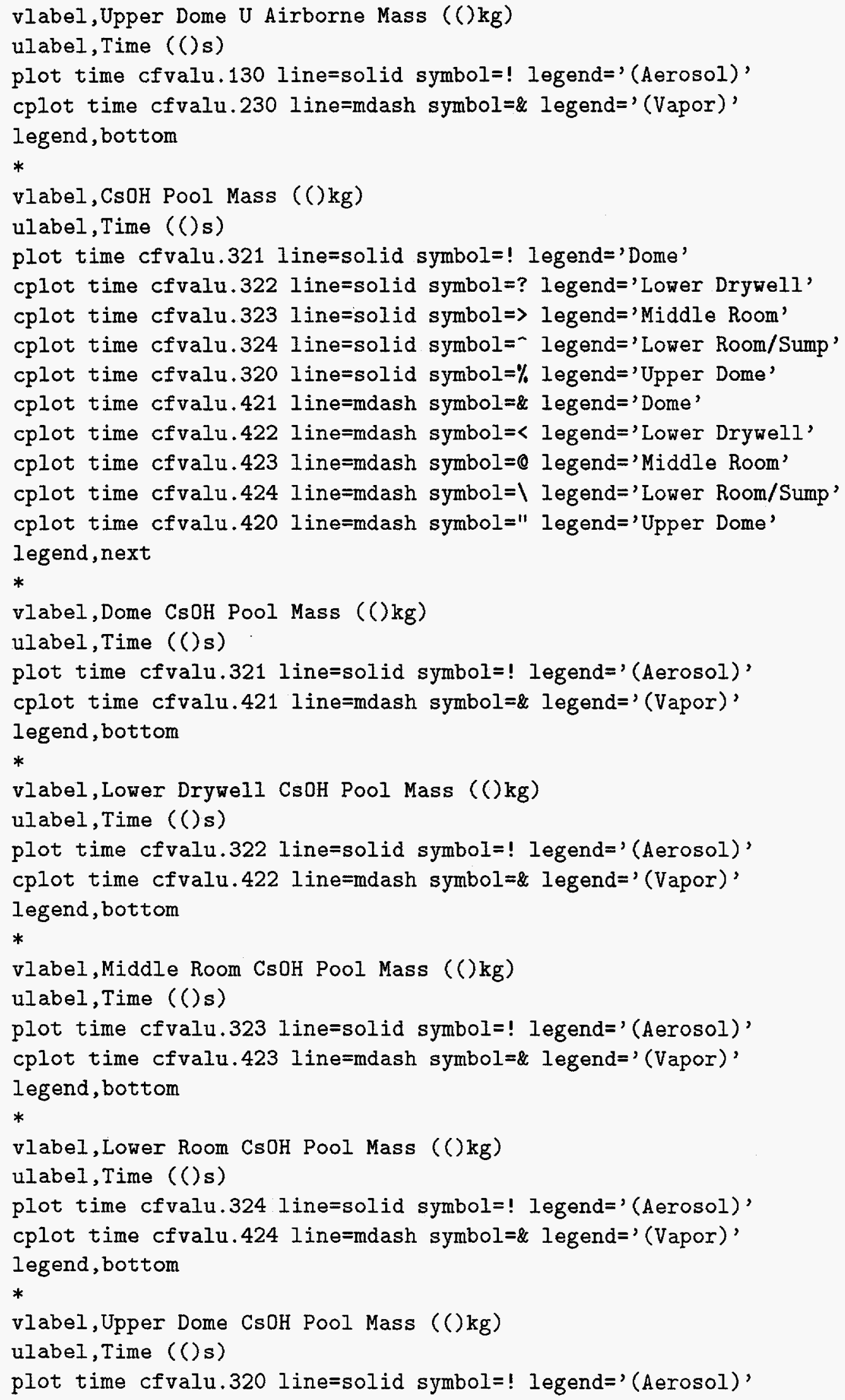


cplot time cfvalu.420 line=mdash symbol=\& legend='(Vapor)' legend, bottom

*

vlabel,I@2 Pool Mass (()kg)

ulabel, Time (()s)

plot time cfvalu.341 line=solid symbol=! legend='Dome'

cplot time cfvalu.342 line=solid symbol=? legend='Lower Drywell'

cplot time cfvalu.343 line=solid symbol=> legend='Middle Room'

cplot time cfvalu.344 line=solid symbol=- legend='Lower Room/Sump'

cplot time cfvalu.340 line=solid symbol=\% legend='Upper Dome'

cplot time cfvalu.441 line=mdash symbol=\& legend='Dome'

cplot time cfvalu.442 line=mdash symbol=< legend='Lower Drywell'

cplot time cfvalu.443 line=mdash symbol=0 legend='Middle Room'

cplot time cfvalu.444 line=mdash symbol=l legend='Lower Room/Sump'

cplot time cfvalu.440 line=mdash symbol=" legend='Upper Dome'

legend, next

*

vlabel, Dome I@2 Pool Mass (()kg)

ulabel, Time (()s)

plot time cfvalu.341 line=solid symbol=! legend='(Aerosol)'

cplot time cfvalu.441 line=mdash symbol=\& legend=' (Vapor)'

legend, bottom

*

vlabel,Lower Drywell I@2 Pool Mass (()kg)

ulabel, Time (()s)

plot time cfvalu.342 line=solid symbol=! legend='(Aerosol)'

cplot time cfvalu.442 line=mdash symbol=\& legend='(Vapor)'

legend, bottom

*

vlabel, Middle Room I@2 Pool Mass (()kg)

ulabel, Time (Os)

plot time cfvalu.343 line=solid symbol=! legend='(Aerosol)'

cplot time cfvalu.443 line=mdash symbol=\& legend='(Vapor)'

legend, bottom

*

vlabel, Lower Room I@2 Pool Mass $(() \mathrm{kg})$

ulabel, Time (()s)

plot time cfvalu.344 line=solid symbol=! legend='(Aerosol)'

cplot time cfvalu. 444 line=mdash symbol=\& legend='(Vapor)'

legend, bottom

*

vlabel,Upper Dome I02 Pool Mass (()kg)

ulabel, Time (()s)

plot time cfvalu.340 line=solid symbol=! legend='(Aerosol)'

cplot time cfvalu.440 line=mdash symbol=\& legend='(Vapor)'

legend, bottom

* 


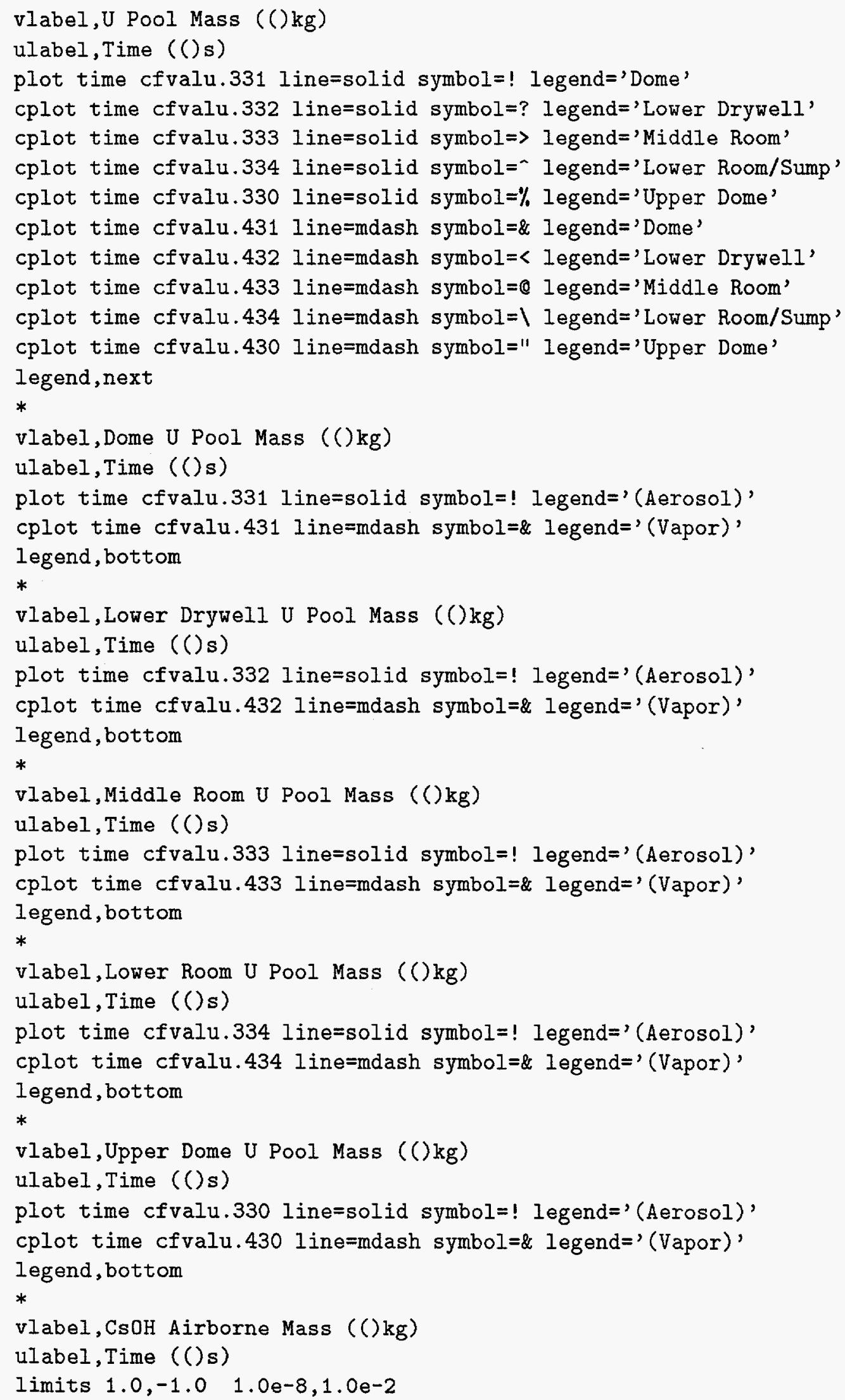


plot time cfvalu.121 logv line=solid symbol=! legend='Dome'

cplot time cfvalu.122 logv line=solid symbol=? legend='Lower Drywell'

cplot time cfvalu.123 logv line=solid symbol=> legend='Middle Room'

cplot time cfvalu.124 logv line=solid symbol=- legend='Lower Room/Sump'

cplot time cfvalu.120 logv line=solid symbol=\% legend='Upper Dome'

legend, Ir

*

vlabel, IQ2 Airborne Mass (() kg)

ulabel, Time (()s)

limits $1.0,-1.0 \quad 1.0 \mathrm{e}-8,1.0 \mathrm{e}-1$

plot time cfvalu.241 logv line=mdash symbol=\& legend='Dome'

cplot time cfvalu.242 logv line=mdash symbol=< legend='Lower Drywell'

cplot time cfvalu.243 logv line=mdash symbol=Q legend='Middle Room'

cplot time cfvalu.244 logv line=mdash symbol=l legend='Lower Room/Sump'

cplot time cfvalu.240 logv line=mdash symbol=" legend='Upper Dome'

legend, Ir

*

vlabel,U Airborne Mass (() kg)

ulabel, Time ( $)$ s)

limits $1.0,-1.0 \quad 1.0 \mathrm{e}-8,1.0 \mathrm{e}-2$

plot time cfvalu.131 logv line=solid symbol=! legend='Dome'

cplot time cfvalu.132 logv line=solid symbol=? legend='Lower Drywell'

cplot time cfvalu.133 logv line=solid symbol=> legend='Middle Room'

cplot time cfvalu.134 logv line=solid symbol=- legend='Lower Room/Sump'

cplot time cfvalu.130 logv line=solid symbol=\% legend='Upper Dome'

legend, lr

*

*eor* quit

$*$

vlabel, Dome to Lower Drywell Flow Rate (() kg/s)

ulabel, Time ( $) s$ )

plot time fl-mflow.1.001 line=solid symbol=! legend='Pool'

cplot time fl-mflow.2.001 line=dotdash symbol=< legend='Fog'

cplot time fl-mflow.3.001 line=sdash symbol=@ legend='Steam'

legend, bottom

*

vlabel, Dome to Lower Drywell Total Flow (() kg)

ulabel, Time (O) $s$ )

plot time fl-i-mflow.1.001 line=solid symbol=! legend='Pool'

cplot time fl-i-mflow.2.001 line=dotdash symbol=< legend='Fog'

cplot time $f l-i-m f l o w, 3.001$ line=sdash symbol=0 legend='Steam'

legend, bottom

*

vlabel, Dome to Middle Room Flow Rate (() kg/s)

ulabel, Time (O)s)

plot time fl-mflow.1.002 line=solid symbol=! legend='Pool'

cplot time fl-mflow.2.002 line=dotdash symbol=< legend='Fog' 
cplot time fl-mflow.3.002 line=sdash symbol=0 legend='Steam' legend, bottom

*

vlabel, Dome to Middle Room Total Flow (() kg)

ulabel, Time (()s)

plot time $f 1-i-m f l o w .1 .002$ line=solid symbol=! legend='Pool'

cplot time fl-i-mflow.2.002 line=dotdash symbol=< legend='Fog'

cplot time fl-i-mflow.3.002 line=sdash symbol=0 legend='Steam'

legend, bottom

*

vlabel, Middle Room to Sump Flow Rate (() $\mathrm{kg} / \mathrm{s})$

ulabel, Time (()s)

plot time fl-mflow.1.003 line=solid symbol=! legend='Pool'

cplot time fl-mflow.2.003 line=dotdash symbol=< legend='Fog'

cplot time fl-mflow.3.003 line=sdash symbol=0 legend='Steam'

legend, bottom

*

vlabel, Middle Room to Sump Total Flow (()kg)

ulabel, Time (()s)

plot time fl-i-mflow.1.003 line=solid symbol=! legend='Pool'

cplot time fl-i-mflow. 2.003 line=dotdash symbol=< legend='Fog'

cplot time $f l-i-m f l o w .3 .003$ line=sdash symbol=0 legend='Steam'

legend, bottom

* 
External Distribution:

U. S. Nuclear Regulatory Commission (18)

Attn: S. Acharya, TWF-9F31

Y. S. Chen, TWF-10K8

M. A. Cunningham, TWF-9F31

F. Eltawila, TWF-10K8

R. B. Foulds, TWF-10K8

S. Basu, TWF-10K8

C. Gingrich, TWF-10K8

C. G. Tinkler, TWF-10K8

R. O. Meyer, TWF-10G6

A. Mitchell, TWF-17G21

C. P. Ryder, TWF-9F31

L. Soffer, TWF-10F13

J. A. Murphy, TWF-10E50

L. M. Shotkin, TWF-10G6

N. Lauben, TWF-10G6

R. Landry, OWFN 11D23

A. Drozd, OWFN 8E1

Washington, DC 20555

S. Y. Chen

Argonne National Laboratory

9700 South Cass Avenue

Argonne, IL 60439

Battelle Columbus Laboratories (3)

Attn: P. Cybulskis

M. Carmel

R. S. Denning

505 King Avenue

Columbus, OH 43201

Brookhaven National Laboratory (2)

Attn: I. K. Madni

T. Pratt

Bldg. 130

32 Lewis

Upton, NY 11973 
Idaho National Engineering Laboratory (5)

Attn: A. Brown

R. J. Dallman

D. W. Golden

S. E. Reed

G. W. Johnsen

EG\&G Idaho

P. O. Box 1625

Idaho Falls, ID 83404

D. Jones

EI International

P. O. Box 50736

Idaho Falls, ID 83405

Electric Power Research Institute (3)

Attn: E. Fuller

R. N. Oehlberg

P. O. Box 10412

Palo Alto, CA 94303

Los Alamos National Laboratory (2)

Attn: B. E. Boyack, K-551

D. R. Liles, K-553

P. O. Box 1663

Los Alamos, NM 87545

Oak Ridge National Laboratory

P. O. Box 2009

Oak Ridge, TN 37831-8057

Attn: S. R. Greene, MS-8057

R. H. Morris, MS-8057

S. E. Fisher, MS-8057

R. Sanders, MS-8057

S. A. Hodge, MS- 8057

C. R. Hyman, MS-8057

R. P. Taleyarkhan, MS-8057

W. P. Barthold

Barthold \& Associates

132 Seven Oaks Drive

Knoxville, TN 37922 
K. C. Wagner

Science Applications Intl. Corp.

2109 Air Park Rd. SE

Albuquerque, NM 87106

Savannah River Laboratory (2)

Attn: B. DeWald

D. Allison

Westinghouse Savannah River Co.

Bldg. 773-41A

Aiken, SC 29808-0001

Westinghouse Hanford Co. (2)

Attn: D. Ogden

O. Wang

P. O. Box 1970

Richland, WA 99352

General Electric Company (3)

Knolls Atomic Pwer Laboratory

Attn: D. F. McMullan

G. H. Epstein

E. Mennard

Bldg. F3, Room 8

P. O. Box 1072

Schenectady, NY 12301-1072

Bettis Atomic Power Laboratory (3)

Attn: Mark Riley

Jow Semanchik

Vincent Baiamonte

P. O. Box 79

West Mifflin, PA 15122

Mohsen Khatib-Rahbar

Energy Research Inc.

P. O. Box 2034

Rockville, MD 20852

V. K. Dhir

2445 22nd Street

Santa Monica, CA 90403 
R. Viskanta

Purdue University

Heat Transfer Laboratory

School of Mechanical Engineering

West Lafayette, IN 47907

Dr. Jim Gieseke

Battelle Memorial Institute

505 King Ave.

Columbus, Ohio 43201

M. A. Kenton

Gabor, Kenton \& Associates

770 Pasquinelli Drive

Suite 426

Westmont, IL 60559

University of California

Attn: T. Theofanous

ERC-CRSS

Santa Barbara, CA 93106

Professor K. B. Cady

Nuclear Science and Engineering

Cornell Universioty

Ward Laboratory

Ithaca, NY 14853-7701

University of New Mexico (2)

Department of Chemical and Nuclear Engineering

Attn: F. E. Haskin

F. J. Souto

Albuquerque, NM 87131

J. C. Lee

University of Michigan

Dept. of Nuclear Engineering

Cooley Building, North Campus

College of Engineering

Ann Arbor, MI 48109-2104 
University of Wisconsin (2)

Dept. of Nuclear Engineering

Attn: M. L. Corradini

G. A. Moses

Engineering Research Building

1500 Johnson Drive

Madison, WI 53706

Ramu K. Sundaram

Manager, LOCA Analysis Group

Nuclear Engineering

Yankee Atomic Electric Company

580 Main Street

Bolton, MA 01740

John Bolin

CEGA

P. O. Box 85608

San Diego, CA 92186-9784

M. Plys

Fauske \& Associates

16W070 West 83rd Street

Burr Ridge, IL 60521

Nick Trikouros

GPU Nuclear Corporation

One Upper Pond Road

Parsippany, NJ 07054

B. Raychaudhuri

Nebraska Public Power District

PRA \& Engineering Review Group

P. O. Box 499

Columbus, NE 68601

Frank Elia

Stone \& Webster Engineering Corp.

245 Summer Street

Boston, MA 02210 
Prof. Dr. Johann Korkisch

Institute of Analytical Chemistry

University of Vienna

A-1090 Vienna, Währingerstrasse 38

AUSTRIA

Samir S. Girgis

Atomic Energy of Canada Limited

CANDU Operations

Sheridan Park Research Community

Mississagua, Ontario

CANADA L5K1B2

Paul J. Fehrenbach

Chalk River Nuclear Laboratories

Fuel Engineering Branch, RSR Division

Chalk River, Ontario

CANADA KOJ1J0

Dr. Bohumír Kujal

Department of Reactor Technology

Nuclear Research Institute $\mathrm{Re}^{2} \mathrm{z}$ plc

25068 Řež

CZECH REPUBLIC

\section{Andrej Mitro}

Institute of Radioecology and Applied Nuclear Techniques

Garbiarska 2

P. O. Box A-41

04061 Košice

CHECHOSLOVAKIA

Shih-Kuei Cheng

Institute of Nuclear Energy Research

P. O. Box 3-3

Lung-Tan, Taiwan

REPUBLIC OF CHINA 
Mr. Yi-Bin Chen

Department of Nuclear Technology

Atomic Energy Council

67, Lane 144

Keelung Road, Section 4

Taipei, Taiwan 106

REPUBLIC OF CHINA

Technical Research Centre of Finland (3)

Nuclear Engineering Laboratory

Attn: Lasse Mattila

Ilona Lindholm

Esko Pekkarinen

P. O. Box 208 (Tekniikantie 4)

SF-002151 Espoo

FINLAND

Jorma V. Sandberg

Finnish Center Radiation \& Nucl. Safety,

Dept. of Nuclear Safety

P. O. Box 268

SF-00101 Helsinki

FINLAND

Akihide Hidaka

Safety Research Department

Reactor Accident Studies and Modelling Branch

DRS/SEMAR

Cadarache Nuclear Center

13108 Saint-Paul-Lez-Durance Cedex

FRANCE

Dr. Lothar Wolf

Battelle Institute EV

AM Romerhof 35

D-6000

Frankfurt/Main90

GERMANY 
Gesellschaft fur Anlagen- und Reaktorsicherheit (3)

Attn: Ulrich Erven

Walter Erdmann

Manfred Firnhaber

Schwertnergasse 1

D-5000 Koln 1

GERMANY

Kernforschungzentrum, Karlsruhe (3)

Attn: P. Hofmann

Werner Scholtyssek

Philipp Schmuck

P. O. Box 3640

D-7500 Karlsruhe 1

GERMANY

Udo Brockmeier

University of Bochum

Energietechnik

IB-4-128

D-4630 Bochum

GERMANY

György Gyenes

Central Research Institute for Physics

Institute for Atomic Energy Research

H-1525 Budapest, P. O. Box 49

HUNGARY

Joint Research Center

Commission of the European Communities

Attn: Alan Jones

Iain Shepherd

Safety Technology Institute

21020 Ispra (Va)

ITALY

Giovanni Saponaro

ENEA

Natl. Comm. for R\&D of Nuclear Energy

Via Vitaliano Brancati, 48

00144 Rome

ITALY 
Japan Atomic Energy Research Institute (3)

Attn: Kunihisa Soda

Jun Sugimoto

Norihiro Yamano

Tokai-mura, Naka-gun, Ibaraki-ken

319-11, JAPAN

Dr. Masayoshi Shiba, Director General

Institute of Nuclear Safety

Nuclear Power Engineering Corporation

Fujita Kankou Toranoman Bldg. $7 \mathrm{~F}$

3-17-1, Toranoman

Minato-Ku, Tokyo, 105

JAPAN

Masao Ogino

Mitsubishi Atomic Power Industries

4-1 Shibakoen 2-Chome

Minatoku Tokyo

JAPAN

Hidetoshi Okada

Nuclear Power Engineering Corporation

3-17-1, Toranomon Bldg. 5F

Minato-ku, Tokyo 105

JAPAN

Hirohide Oikawa

Toshiba Corporation

8, Shin-Sugita, Isogo-ku

Yokohama

JAPAN

Korea Atomic Energy Research Inst. (3)

Attn: Kun-Joong Yoo

Song-Won Cho

Dong-Ha Kim

P. O. Box 7, Daeduk Danji

Taejon

SOUTH KOREA 305-353 
Jae Hong Park

Safety Assessment Department

Korea Atomic Energy Research Institute

P. O. Box 16, Daeduk-Danji

Taejon

SOUTH KOREA 305-353

Netherlands Energy Research Foundation (2)

Attn: Karel J. Brinkmann

E. J. Velema

P. O. Box 1

1755 ZG Petten

THE NETHERLANDS

Dr. Valery F. Strizhov

Russian Academy of Science

Institute of Nuclear Safety

Moscow, G. Tulsky, 52

113191, RUSSIA

Dr. B. Mavko

Institut Josef Stepan

Odsek za Reaktorsko Tehniko

61111 Ljubljana

Jamova 39

P. O. Box 100

SLOVENIA

Universidad Politecnica de Madrid (2)

Attn: Augustin Alonzo Santos

Francisco Martin

E.T.S. Ingenieros Industriales

Jose Gutierrez Abascal, 2

28006 Madrid

SPAIN

Juan Bagues

Consejo de Seguridad Nuclear

Justo Dorado, 11

28040, Madrid

SPAIN 
Oddbjörn Sandervåg

Statens Kärnkraftinspektion

Swedish Nuclear Power Inspectorate

Box 2710610252 Stockholm

SWEDEN

L. Hammar, Director

Division of Research

Swedish Nuclear Power Inspectorate

Statens Karnkraftinspektion

Sehlstedtsgatan 11

Box 27106

S-102-50 Stockholm

SWEDEN

B. Raj Sehgal

Department of Nuclear Power Safety

Royal Institute of Technology

Brinellvagen 60

S-100 44 Stockholm

SWEDEN

Swiss Federal Nuclear Safety Inspectorate (4)

Attn: S. Chakraborty

Sang Lung Chan

U. Schmocker

H. P. Isaak

CH-5232 Villigen-HSK

SWITZERLAND

United Kingdom Atomic Energy Agency (3)

Winfrith Technology Center

Attn: T. Haste

S. R. Kinnersley

D. W. Sweet

Winfrith, Dorchester, Dorset

UNITED KINGDOM, DTS 8DH 
C. Wheatley

United Kingdom Atomic Energy Authority

Safety \& Reliability Directorate

Wigshaw Lane, Culcheth, Warrington

Cheshire, WA3 4NE

UNITED KINGDOM

Geoffrey Brown

AEA Technology

Consultancy Services

Thomson House

Risley, Warrington WA3 6AT

UNITED KINGDOM

Internal Distribution:

MS0736 N. R. Ortiz, 6400

MS0744 D. A. Powers, 6404

MS0747 S. E. Dingman, 6412

MS0748 F. T. Harper, 6413

MS0742 J. E. Kelly, 6414

MS0739 K. D. Bergeron, 6421

MS0739 R. K. Cole, 6421

MS0739 A. A. Elsbernd, 6421

MS0739 R. C. Smith, 6421

MS0739 D. S. Stuart, 6421

MS0899 Technical Library, 13414 (5 copies)

MS0619 Technical Publications, 12613

MS0100 Document Processing for DOE/OSTI, 7613-2 (2 copies)

MS9018 Central Technical Files, 8523-2

MS0966 L. N. Kmetyk, 9217 